
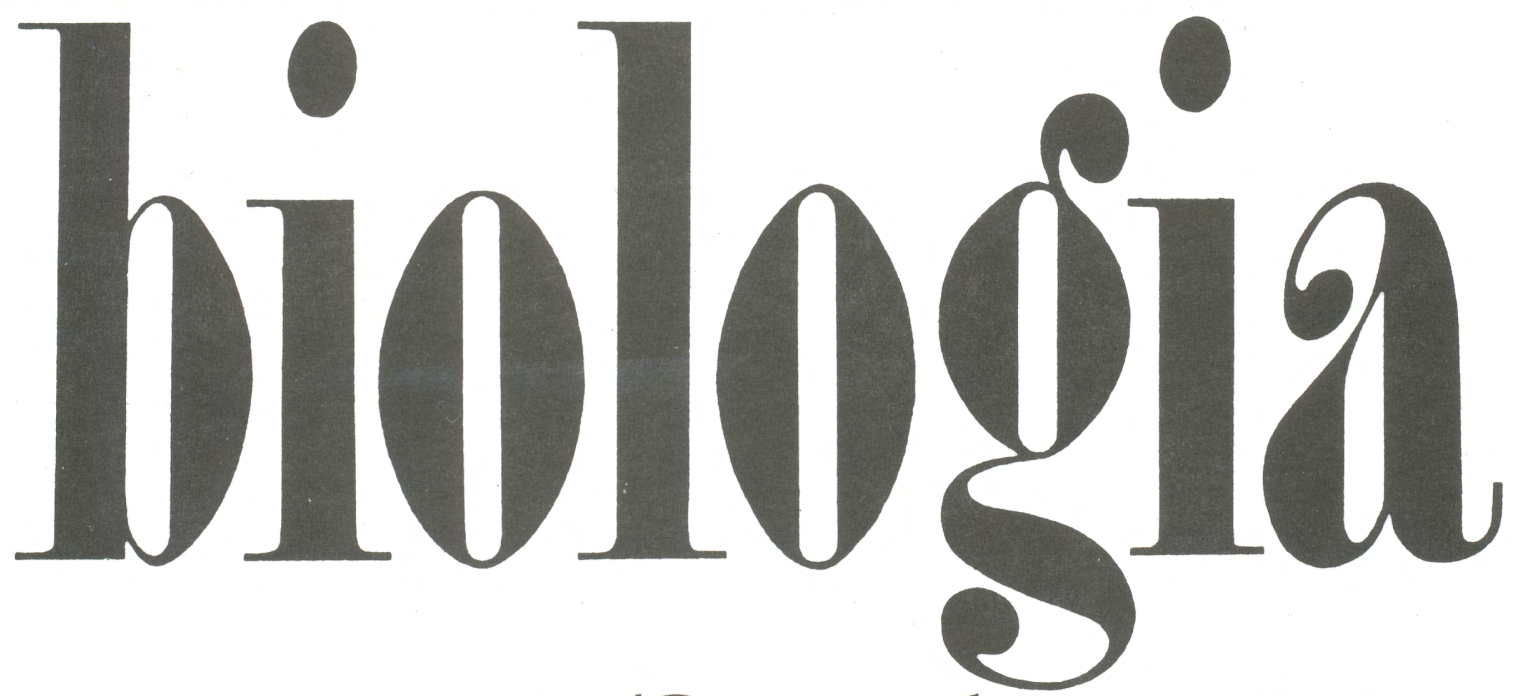

SECTION ZOOLOGY 52/Suppl. 51997

European freshwater fishes

An heuristic checklist of the freshwater fishes of Europe (exclusive of former USSR),

with an introduction for non-systematists and comments on nomenclature and conservation

Maurice KotTelat 


\title{
European freshwater fishes
}

\author{
An heuristic checklist of the freshwater fishes of Europe (exclusive of former USSR), with \\ an introduction for non-systematists and comments on nomenclature and conservation
}

\author{
Maurice Kottelat
}

Case postale 57, 2952 Cornol, Switzerland (address for correspondence) and School of Biological Sciences, National University of Singapore, Kent Ridge, Singapore 119260

The systematics and nomenclature of the European freshwater fishes have been critically reviewed. Using the phylogenetic species concept (PSC), 358 species are recognised in Europe (exclusive of former USSR). Former checklists recognised only about 170 to 213 species for the same area; the increase is in part due to differences in species concepts but also to former checklists overlooking most of the diversity documented for salmonids and coregonids.

The nomenclatural status of the 1931 names applied to European freshwater fishes since 1758 has been reviewed. The original descriptions of 1429 $(99.4 \%)$ of the 1437 nominal species based on material from Europe (exclusive of USSR) have been examined; the original spellings have been checked, type localities are listed as well as information on primary type specimens when available. The correct names of all species have been established in accordance with the International Code of Zoological Nomenclature. It is found that $47-$ $89(28-42 \%)$ of the names of the 170 to 213 species and subspecies recognised by earlier authors were incorrect (incorrect names, incorrect generic placement, incorrect spelling). Many names were found to be very poorly based or with very poorly 'legal' base and in order to stabilise the nomenclature, lectotypes are designated for 18 nominal species and neotypes are designated for 18; the type localities of 8 species are restricted, and the original indications of 5 species are restricted (Table 2). The following species known by previous authors but unnamed or with invalid names are named: Coregonus fatioi, $C$. arenicolus, C. atterensis, and Salmo aphelios.

Despite having being the object of research for more than 450 years (double than the fauna of any other continent), the systematics and nomenclature of European freshwater fishes has been found to be in a chaotic state without equivalent elsewhere. The absence of established nomenclatural rules until the middle of last century is responsible for most of the 'old' nomenclatural problems. Poor practices, poor theoretical concepts, poor writing, absence of international communication and nationalism are responsible for the poor quality of systematic work in recent European ichthyology and ignorance of the rules are responsible for poor taxonomy and unstable nomenclature. The main cause is that contrary to most other fields of zoology and most other parts of the world, fish systematics and fish taxonomy has been a playing ground for fisheries biologists and not a research field for trained taxonomists.

Poor systematics and poor taxonomy are directly responsible for the non-identification of the severe threats to the survival of many species. Twelve of the recognised species are probably extinct: Eudontomyzon sp., Chondrostoma scodrense, Coregonus fera, C. hiemalis, C. confusus, C. restrictus, C. gutturosus, Salmo schiefermuelleri, Salvelinus inframundus, S. profundus, $S$. scharffi, and Gasterosteus crenobiontus. 
"As long as there are active, creative ichthyologists, there will be major disagreements in our classification in the foreseeable future (similarly there is disagreement in almost all important fields of biology). Fish classification is in a dynamic state, and the student pursuing ichthyology will find that all groups can be reworked. There are many challenges, both in developing the theory of classification and in its actual practice. Because particular classifications eventually become obsolete (as will most biological information), they should be regarded as frameworks that will provide a basis for building as advances are made".

J. S. Nelson (1994: x)

"We shall never know how many [... species ... ] inhabited the planet when we began its destruction.
It is past time to get afield and away from minutiae and speculations, back to solid facts of ...
distribution, variation and migration. Successful conservation will require intelligence, realism and
knowledge, based on better collecting of what is to conserve... It can hardly be achieved while
"conservationists" and misled officials impede the collecting, preservation, and study of
our fast vanishing wealth. ... Meanwhile, our scientific "establishment" unanimously ignores
most ... species and concentrates on supporting industry (profitably) by emphasizing computers
and expensive equipment designed to give fantastic Ultimate Answer (based on proteins
and molecules) that, to date, have been largely wrong. Truly, we are in a new Decline and Fall, with
our scientific establishment fiddling while Rome burns, instead of conserving biological diversity." A. R. Phillips (1982: 615)

\section{Prologue}

This essay is not a definitive treatment of the systematics of the European freshwater fishes, it is not a systematic revision, it is not an exhaustive literature review, it is not .... So what is it?

This essay is a review of the (poor) state of the systematics of European freshwater fishes and the related (absence of clear) concepts, (ignorance of) basic biological principles, and (non application of) nomenclature rules. It illustrates a (poor) situation resulting in part from (the neglecting or rejection of) systematics in western (as an outmoded discipline) as well as eastern Europe (until recently, as a static and non-revolutionary discipline), from the (lack of) adequate funding and from the (now virtual absence in most European universities of) competent teaching of systematic methodology (by staff with first hand experience of the subject).

This essay is a brief catalogue of what we know and what we should know, of what has been done and what still has to be done. It aims to provoke thought and to stimulate a new wave of critical and innovative research.

Although a ritual answer and justification for not moving, the 'need to wait for more or better data' is not an acceptable answer to most problems or questions. This essay addresses several of these problems (some known for over a century) and proposes pragmatic ways of handling some unresolved cases.

The efficient conservation and responsible management of the rapidly vanishing European fish diversity requires a sound knowledge of this diversity. This knowledge needs to be based on coherent systematics. To wait for 'more data' is definitively not acceptable when there is no sign that these advocated additional researches will be funded, conducted by experienced staff, completed, published, and will lead to results other than ... 'we need more data'. If the data ultimately arrive, we will certainly be pleased to use them. But we cannot wait indefinitely for them.

Ars longa, vita brevis. 


\section{Introduction}

European ichthyology was born with the Greek philosopher Aristotle (384-322 BC). Even though formal binominal fish nomenclature began with Carl von Linné (Linnaeus, 1758), the history of European fish systematics actually goes back to the sixteenth century with Pierre Bélon (15181564), Hippolyto Salviani (1513-1572), Guillaume Rondelet (1507-1566) and Conrad Gesner (15161565) (see Cuvier, in Cuvier \& Valenciennes, 1828a: 1-270, for a history of ichthyology). The Libri de piscibus and the Universae aquatilium historiae of Rondelet $(1554,1555)$ and the Nomenclator aquatilium and Fischbuch of Gesner $(1560,1575)$ are the corner stones on which European ichthyology was built. Modern ichthyology started with Peter Artedi's (1705-1735) posthumous Ichthyologia (1738) whose system and nomenclature have been adopted by Linné.

Having enjoyed $4 \frac{1}{2}$ centuries of study (about double that for the fauna of any other continent), one would expect our knowledge of the European fish fauna to be very good, and especially that the most basic information would be easily available.

The last reasonably comprehensive bibliography of European freshwater fishes actually is included in Günther's (1859-1870) Catalogue of fishes which is also the last published catalogue of all fishes of the world. The latest checklist of European freshwater fishes apparently dates back to 1971 (Blanc et al., 1971) and is of unequal quality (see Balon, 1974: 802, for criticism; see also Bănărescu \& Hureau, 1976: 414 and Balon, 1976: 416). There are several popular or semi-popular books on European freshwater fishes which have been translated into several languages (e.g., Maitland, 1976; Muus \& Dahlström, 1967); most of them are already quite old (between 20 and 30 years) and out-dated. Newer ones or revised editions tend to be written not by competent authors but by so-called 'science journalists' who uncritically collect data from various sources (or more commonly, paraphrase other's books); sometimes, artists are given photocopies from other books and commissioned to draw the same fishes and give them a new look (in terms of copyright laws or ethics) and this results in a fish with more bars, less spots, longer or shorter barbels etc. For obvious reasons, I cannot list references to such books here.

The only recent technical book which aims at dealing with European fishes is the series The
Freshwater Fishes of Europe which suffers from its more than prohibitive price, unpredictable schedule, heterogeneous editorship, and scanty illustrations (see reviews by Wheeler, 1988: 1099, 1993: 249). The situation with national faunae is very variable; there are good ones, but in many instances, they are poor and very outdated (if still available). For a few countries, there is nothing published this century.

This is more or less the horrifying situation I discovered when I needed to find a checklist from which to extract a few basic data like number of species, distribution, conservation status, etc. for a paper comparing knowledge of the European and Southeast Asian freshwater fish faunae. I thought that the situation was not desperate, and judging from the figure of 215 species recorded by Maitland (1976) for Europe west of the Urals or 393 species and subspecies listed by Blanc et al. (1971) for Europe west of the Urals and the Levant, I decided that it would not take too long to resiew the literature and compile my own list.

This was the second shock. As a systematist, I discovered the astonishingly poor state of European fish systematics and nomenclature. To be short, I discovered that our knowledge of the systematics of European freshwater fishes, though seemingly extensive, is very often of poor quality, that there is an enormous number of bad or very bad publications whose authors have obviously no idea what systematics is or is not and who have probably not even heard of the existence of nomenclatural rules (or at least have not read them). It seems that for decades, most of the 'contributions' to the systematics of European fishes have not been the result of work by systematists, but by fisheries biologists and others without formal training in systematics. Many researchers are just restricted to what happens within national boundaries and do not seem to realise that fish do not recognise political borders. Admittedly, the exchange of information is hampered by language and until recently by political problems.

Is this situation that important or that dramatic? It is certainly irritating to see the money, efforts and time wasted in collecting these data, writing, editing, and publishing all this material for such low quality of output. Basic systematic data are important because they allow communication and exchange of information between scientists: discoveries made by a scientist on the biology 
of a species A are only meaningful to other scientists if they can be sure that their species A is really the same as the species A of other researchers. Basic systematic data are important for conservation; without detailed surveys and accurate taxonomy, it is impossible to identify the various species and evaluate their real conservation status, it is impossible to properly manage their fisheries, it is impossible to evaluate the conservation value of various habitats or areas, it is impossible to establish strategies and it is impossible to set priorities. Without accurate names, it is impossible to list species as endangered or threatened and to take conservation action. The situation, strategy and actions needed for managing European coregonid stocks obviously are not the same if we have a single species with hundreds of local morphs or populations, if we have $7-8$ species ranging across all Europe and present in different combinations in different lakes, or if we have a collection of some 30 species.

It is also dramatic to state that because of this chaotic state of fish taxonomy in Europe, most competent scientists tend to avoid getting involved in the usually sterile controversies which would unavoidably result from innovative papers. As a result, European fish taxonomists work on foreign faunae, while knowing perfectly well that a lot of work still has to be done at home, but predictably under unpleasant (psychological or social) conditions. The influence of the academic social philosophy and rites is not irrelevant to this discussion (Kottelat, 1995).

As a result of these factors, it is extremely difficult to evaluate the real status of the European freshwater fish species. After spending some time compiling lists, evaluating the literature, checking interpretations and re-interpreting data, I put together a more or less reasonable and up-to-date check-list. I certainly do not consider it as a systematic revision but more as a working document on which to base further researches. Many' of my conclusions are still tentative, because a direct ex- amination or re-examination of the material used by earlier authors is essential to solve several of the problems. For the reasons mentioned in the preceding paragraph, I was at first not inclined to publish this list. But, as the situation is so bad, it serves no purpose to postpone this any further.

Even if many points are still imperfect, even if many questions are still unanswered, it seems preferable to have these data instead of wrong data or no data at all. At least readers who are not specialists in the field of fish systematics will know that there are problems. Many nomenclatural problems still have to be solved; I solved all those which could be solved now and have pointed to remaining ones. Some might argue that it would have been better to postpone this publication until all these problems have been addressed. This attitude is not acceptable to me because many of the problems I am pointing to have been known for decades and have not been addressed (subsequent authors often translated tentative conclusions of earlier authors into definitive 'facts', overlooking existing reservations, and recent authors just ignore that problems exist); waiting for external answers would in most cases mean just additional decades of inaction. I do not think that science should be restricted merely to bear witness to problems, but should act on solving them.

Also, the immediate (and tentative) solution of several of the systematic problems we now recognise is important for conservation. Postponing this further could mean that by the time the problem is really addressed, the concerned species will be extinct (and then the problem probably can never be solved). In many cases it seems that we have only two alternatives: 1) to reach a tentative solution leading to adequate conservation and possibly to a definitive solution later; or 2) to wait for a definitive solution, with the risk of extinction of the problem organisms, and ipso facto disappearance of any chance of solving the problem. Conservation-minded scientists will recognise the urgency of the situation.

\section{Results and discussion}

Although unusual, I find convenient to present and discuss in this position the general pattern of the results of my review.
My survey reveals that some 358 fish species can be recognised in European freshwaters West of the former USSR. This compares with the 170 
native species listed by Maitland (1976) or 213 species and subspecies listed by Blanc et al. (1971) for the same area. The difference is partly explained by new taxa described since these books have been published and revalidation of taxa earlier considered as synonyms, but mainly it results from the use of a more objective species concept, the Phylogenetic Species Concept (see discussion and definitions below). Earlier authors have often claimed to have used the so-called 'Biological' Species Concept (other concepts are also 'biological' !) which is now found to be non operational and whose criteria are virtually impossible to apply. Actually most authors have probably been working without really paying much attention to concept formulation and to practical implications.

Most earlier checklists also have avoided as much as possible dealing critically with salmonoid (Salmonidae and Coregonidae) systematics and this resulted in a much underestimated number of species. The species-level systematics of these families is still far from resolved, but even with this limitation a pragmatic handling of their nomenclature is possible. Using a few simple concepts and definition, I tentatively recognise here 44 European monophyletic evolutionary units within the genus Coregonus which can be called species and 27 within the genus Salmo. (Admittedly my treatment of these two families is not geographically even and I apparently deal with the peri-Alpine stocks in greater detail than with the Scandinavian ones. There is an old 'rule' in systematics stating that the number of species in a given area is inversely related to the distance between them and the laboratory of the systematist working on them; this bias seems confirmed here).

At least 1931 names have been applied to these 358 species since the beginning of the modern nomenclatural system in 1758 , or an average of 5.4 names per species, with a maximum of 57 names for Salmo trutta sensu stricto). This profusion of names and the long history of some of them is partly responsible for a relatively frequent occurrence of nomenclatural problems.

Nomenclatural problems are of many types. Grossly, they can be divided into ancient and modern problems. The ancient-type problems are classical cases which taxonomists are accustomed to. $1292(66.9 \%)$ of the 1931 names have been created before 1900 and most of the problems relating to these older names result from the non-existence of precise nomenclatural rules at that time. One of the most frequent problem is species described without explicit designation of type material (a concept which appeared towards the end of last century). For 258 names there is no type material and for 370 the whereabouts of designated types are not known (Table 1); to these should be added types theoretically deposited in an institution where they can no longer be found because they have been misplaced, discarded or lost during wars, fires, etc. The situation is very dramatic for south-eastern Europe where most nominal species have no known extant types, even for species described in recent years.

The modern-type problems are more interesting because they reveal a tragic pattern. They include an incredible number of nomina nuda (see p. 12 for definition), dramatic misunderstanding of nomenclatural rules or obvious signs of ignorance of their existence, poor background knowledge of fish morphology or biology. For obvious reasons, I do not wish to list here precise examples of these cases.

Fundulus heteroclitus, a North American fish introduced in Spain has been described as a new endemic Spanish fish in 1984 and I am aware of another similar case which could be stopped before publication. In 1990, a 'new' subspecies of the North American catfish Ameiurus nebulosus has been described from Hungary on the basis of an introduced stock.

Two species names have first been made available in conference abstracts between 1982 and 1995. Many species (not counted) have been described without explicit mention of type material and the actual existence of this material for most is still unclear. Type material is vanishing from museums and universities. The curators of several European fish collections surveyed in Kottelat et al. (1993) answered that the collections under their care do not include types, while their holdings have earlier been documented in the literature as type repositories.

Table 1 shows that the percentages of new names proposed in the last 50 years which do not present nomenclatural problems (types, availability of the names) is not superior to those of last century. The very high figure $(83 \%)$ for the decade 1980-1989 is to be corelated with the absolute number of 23 new names proposed for that period, the lowest for any decade since 1758 . The proportion of unavailable names (see definition p. 12; in other words, names proposed in ignorance of nomenclature rules) is higher than ever. For the period $1758-1830$, at the beginning of the history of zoological nomenclature, the main causes of unavailability were nomina nuda and the inconsistent use of the then not fully established binominal system. From 1850 to 1970 , the main cause is the 
Table 1. Number of names (total, available, not available) proposed between 1758 and 1996, in 10-years intervals, including indication of problems with type specimens of available names (NT, no types; LU, location of types unknown [not including types subsequently lost]) and cause of unavailability (nomina nuda, infrasubspecific names, or others). Percentages refer to the total number of names for a given 10-years interval.

\begin{tabular}{|c|c|c|c|c|c|c|c|c|c|c|c|c|c|c|c|}
\hline & \multicolumn{15}{|c|}{ names } \\
\hline & \multirow[t]{3}{*}{ total } & \multicolumn{6}{|c|}{ available } & \multicolumn{8}{|c|}{ not available } \\
\hline & & \multicolumn{2}{|c|}{ no type problems } & \multicolumn{4}{|c|}{ type problems } & \multicolumn{2}{|c|}{ total } & \multicolumn{2}{|c|}{ nomina nuda } & \multicolumn{2}{|c|}{ infrasubspecific } & \multicolumn{2}{|c|}{ others } \\
\hline & & & $\%$ & total & $\%$ & NT & $\mathrm{LU}$ & & $\%$ & & $\%$ & & $\%$ & & $\%$ \\
\hline $1758-1769$ & 93 & 30 & 32 & 59 & 63 & 58 & 1 & 4 & 4 & & & & & 4 & 4 \\
\hline $1770-1779$ & 25 & 7 & 28 & 9 & 36 & 5 & 4 & 9 & 36 & 1 & 4 & & & 8 & 32 \\
\hline $1780-1789$ & 41 & 20 & 49 & 19 & 46 & 17 & 2 & 2 & 5 & & & & & 2 & 5 \\
\hline 1790-1799 & 33 & 11 & 33 & 15 & 45 & 11 & 4 & 7 & 21 & 4 & 12 & & & 3 & 9 \\
\hline 1800-1809 & 52 & 18 & 35 & 33 & 63 & 20 & 13 & 1 & 19 & 1 & 19 & & & & \\
\hline 1810-1819 & 65 & 4 & 6 & 51 & 78 & 12 & 39 & 10 & 15 & 9 & 14 & & & 1 & 15 \\
\hline $1820-1829$ & 82 & 32 & 39 & 42 & 51 & 34 & 8 & 8 & 10 & 8 & 10 & & & & \\
\hline 1830-1839 & 184 & 85 & 46 & 69 & 38 & 18 & 51 & 30 & 16 & 25 & 14 & 3 & 2 & 2 & 1 \\
\hline $1840-1849$ & 194 & 108 & 56 & 48 & 25 & 14 & 34 & 38 & 20 & 20 & 10 & & & 18 & 9 \\
\hline $1850-1859$ & 119 & 85 & 71 & 22 & 18 & 6 & 16 & 12 & 10 & 5 & 4 & 4 & 3 & 3 & 3 \\
\hline $1860-1869$ & 158 & 108 & 68 & 21 & 13 & 5 & 16 & 29 & 18 & 7 & 4 & 12 & 8 & 10 & 6 \\
\hline $1870-1879$ & 91 & 47 & 52 & 31 & 34 & 8 & 23 & 13 & 14 & 6 & 7 & 2 & 2 & 5 & 5 \\
\hline 1880-1889 & 93 & 43 & 46 & 30 & 32 & 15 & 15 & 16 & 17 & 1 & 1 & 13 & 14 & 2 & 2 \\
\hline 1890-1899 & 62 & 32 & 52 & 4 & 6 & 2 & 2 & 26 & 42 & 6 & 10 & 17 & 27 & 3 & 5 \\
\hline 1900-1909 & 84 & 49 & 58 & 10 & 12 & 4 & 6 & 25 & 30 & 2 & 2 & 12 & 14 & 11 & 13 \\
\hline 1910-1919 & 36 & 20 & 55 & 6 & 16 & & 6 & 10 & 28 & & & 10 & 27 & & \\
\hline 1920-1929 & 110 & 42 & 38 & 50 & 45 & 1 & 49 & 18 & 16 & 1 & 1 & 17 & 15 & & \\
\hline 1930-1939 & 141 & 38 & 27 & 40 & 28 & 8 & 32 & 63 & 45 & 4 & 3 & 54 & 38 & 5 & 4 \\
\hline 1940-1949 & 71 & 27 & 38 & 20 & 28 & 1 & 19 & 24 & 34 & & & 22 & 31 & 2 & 3 \\
\hline 1950-1959 & 62 & 21 & 34 & 17 & 28 & 6 & 11 & 24 & 39 & & & 24 & 39 & & \\
\hline 1960-1969 & 44 & 18 & 42 & 9 & 21 & & 9 & 17 & 39 & 1 & 2 & 15 & 34 & 1 & 2 \\
\hline 1970-1979 & 48 & 24 & 50 & 17 & 35 & 11 & 6 & 7 & 15 & & & 1 & 2 & 6 & 13 \\
\hline 1980-1989 & 23 & 19 & 83 & 4 & 17 & & 4 & & & & & & & & \\
\hline \multirow[t]{2}{*}{ 1990-1996 } & 20 & 10 & 50 & 2 & 10 & 2 & & 8 & 40 & 8 & 40 & & & & \\
\hline & 1931 & 898 & 47 & 628 & 33 & 258 & 370 & 401 & 21 & 109 & 6 & 206 & 11 & 86 & 4 \\
\hline
\end{tabular}


proposal of infrasubspecific names (see definition p. 18). New infrasubspecific names peaked in the period 1930-1970 and then abruptly disappeared; most were proposed by Russian and eastern European authors and the abrupt drop in the 1970s probably reflects a change in emphasy of biological research and, more recently, the sad shrinking support to research. Since 1970, the main causes of unavailability of new names is again nomina nuda, use of non-binominal system, etc. Tragically, these are the same main causes as for the period 1758-1830 and this simply reflects ignorance of the nomenclature rules and possibly (in the case of the nomina nuda) the stress to publish.

It is now well known that systematics as a whole is not doing well in Europe, but it does not seem that any other animal group is in such a chaotic situation, with so few species, but of concern for so many specialists. I think that part of the explanation lies exactly there. In other geographic areas, in other groups, systematic researches are conducted by systematists, usually with the appropriate background and based in research or teaching institutions. Any research on fish, due to their economic and food significance, has too often been appropriated by fisheries or agriculture agencies (I really mean appropriated; for many agencies, to pause as 'the' specialists does not involve a moral or ethical responsibility and commitment to produce sound research but merely taping at the money source to keep the administration running and, not seldom, to prevent other institutions or agencies from entering the field). Fisheries biologists might certainly be competent and efficient for some kind of fisheries management, but few only have been successful as taxonomists.

I think it is greatly time that fish taxonomists gain control again on taxonomy of European fishes. It is vital for their conservation that we can rely on sound data obtained by critical and experienced taxonomists. A failure to understand this need now will soon have irreversible impact on the survival chances of many species. The recognition and correct identification of species is not just an academic problem. Sound management and conservation depend of correct identification. We have great potential of seeing maybe up to 50 species going extinct in the next 10-20 years. Failure of competent demonstration of their distinctness, of recognition of their distribution and of identification of their ecological requirements is the best guarantee for seeing them disappearing. Recognising them may not save them, but definitely is a first, big step towards their survival.

At least $12(3.4 \%)$ of the European freshwater fish species are probably extinct: Eudontomyzon sp., Chondrostoma scodrense, Coregonus fera, C. hiemalis, C. confusus, C. restrictus, C. gutturosus, Salmo schiefermuelleri, Salvelinus inframundus, S. profundus, S. scharffi, and Gasterosteus crenobiontus. The population of Alosa vistonica has decreased very sharply in recent years and the species has not been seen for 2 years ( $P$. Economidis, pers. comm., 1996). The taxonomic status of several populations is not clear and it is likely that some of them, now extinct, might actually have represented valid species (e.g., Gobio antipai, see p. 62). But the lack of record should not be taken as an absolute indication that a fish species is extinct; Mayden \& Kuhajda (1996: 267) list several examples of fishes inhabiting readily accessible and easily collected habitats which have only been rediscovered after a hiatus of 23 to 110 years in North America. On the other hand, it is likely that species went extinct unnoticed in historical times (see Hoffmann, 1996, for discussion of environmental changes in aquatic ecosystems in medieval Europe).

At least 14 species reported in the literature have no valid scientific names, either because they have not been recognised as valid species before, because the proposed names are invalid or unavailable, or because wrong names have been applied to them. I here provide new names for four of these species (Coregonus fatioi, C. arenicolus, C. atterensis, Salmo aphelios). This does not mean that I consider their systematic status to be definitively solved, but that with the existing data or evidences they are treated as species; for handling them and for consistency they require a name. This is also a way to ensure that the names are properly established and that types are properly designated and lodged in suitable institutions. The descriptions of four other species are in press. There are evidences or strong indication of the existence of 6 additional unnamed species (Eudontomyzon sp., Coregonus spp., Salmo spp., Salvelinus sp.), but despite many attempts I could not personally examine material of these species and I do not wish to name them without personally examining them.

Similarly, when I met a nomenclatural problem which could reasonably be solved in the present context by mean of simple nomenclatural acts, I did them. This includes 8 restrictions of type localities, 5 restrictions of orig- 
Table 2. Nomenclatural acts included in the present work.

act taxon

page

Restriction of type locality

Clupea sardinella Vallot, $1837 \quad 38$

Cobitis taenia Linnaeus, $1758 \quad 89$

Cobitis trichonica Stephanidis, $1974 \quad 90$

Fario argenteus Valenciennes, $1848 \quad 142$

Salmo lacustris Linnaeus, $1758 \quad 143$

Salmo punctatus Cuvier, $1829 \quad 131,153$

Salmo salvelinus profundus Schillinger, $1901 \quad 154$

Salmo saxatilis Paula Schrank, $1798 \quad 137,141$

Restriction of original citation

Cyprinus albula Nardo, 1827

Cyprinus cephalus Linnaeus, $1758 \quad 66$

Gardonus wyrozub Walecki, $1863 \quad 77$

Salmo cenerinus Chiereghini, $1847 \quad 134$

Salmo maraena gutturosus Gmelin, $1818 \quad 112$

\section{Lectotype designation}

$\begin{array}{lr}\text { Barbus caninus Valenciennes, } 1846 & 37 \\ \text { Blent } & 48\end{array}$

Blennius cagnota Valenciennes, $1836 \quad 175$

Cobitis caspia romanica Bacesco, $1943 \quad 92$

Coregonus balleus Fatio, $1885 \quad 108$

Coregonus exiguus albellus Fatio, $1890 \quad 108$

Coregonus restrictus bondella Fatio, 1885 105

$\begin{array}{ll}\text { Coregonus schinzii alpinus Fatio, 1885 } & 108\end{array}$

$\begin{array}{ll}\text { Coregonus wartmanni alpinus Fatio, } 1890 & 109\end{array}$

Cyprinus barbus Linnaeus, $1758 \quad 48$

$\begin{array}{ll}\text { Cyprinus umbra Walbaum, } 1792 & 68\end{array}$

Leuciscus leuciscus roulei Bertin \& Estève, $1948 \quad 69$

Petromyzon japonicus Martens, $1868 \quad 28$

Petromyzon lampetra Pallas, 1814 29

Petromyzon marinus Linnaeus, $1758 \quad 29$

Salmo ferra Walbaum, $1792 \quad 113$

Salmo maraena ferra Gmelin, $1818 \quad 113$

$\begin{array}{ll}\text { Salmo nigripinnis Günther, 1866 } & 138\end{array}$

Neotype designation

Acipenser kostera Fitzinger, $1832 \quad 32$

Acipenser schypa Gmelin, $1788 \quad 34$

Acipenser schypa Bonnaterre, $1788 \quad 34$

$\begin{array}{ll}\text { Acipenser shyp Forster, } 1767 & 34\end{array}$

Chondrostoma rysela Agassiz, $1835 \quad 71$

Coregonus candidus Goll, $1883 \quad 105$

$\begin{array}{ll}\text { Coregonus nobilis Haack, } 1882 & 109\end{array}$

$\begin{array}{lr}\text { Cyprinus agone Scopoli, } 1786 & 37\end{array}$

Cyprinus morella Leske, $1774 \quad 34$

$\begin{array}{ll}\text { Petromyzon branchialis Linnaeus, } 1758 & 27\end{array}$

Petromyzon fluviatilis Linnaeus, $1758 \quad 27$

Petromyzon lumbricalis Pallas, 1814 28

Petromyzon marinus camtschaticus Tilesius, $1811 \quad 28$

$\begin{array}{ll}\text { Phoxinus laevis punctatus Benecke, } 1881 & 59\end{array}$

$\begin{array}{lr}\text { Phoxinus percnurus gdaniensis Berg, } 1932 & 59\end{array}$

Salmo carpio Linnaeus, $1758 \quad 134$

$\begin{array}{ll}\text { Salmo lavaretus Linnaeus, } 1758 & 134 \\ & 105\end{array}$

$\begin{array}{lr}\text { Telestes rysela Heckel, } 1852 & 71\end{array}$ 
inal indications, 18 lectotype designations and 18 neotype designation (Table 2) to definitively link some (sometime potentially) problem-making names with their present acceptation or to definitively eliminate them from the nomenclature.

The nomenclature used in this list is as upto-date as known to me by 31 December 1996 and strictly in agreement with the International Code of Zoological Nomenclature. Compared to earlier work of similar scope, the names of $89(42 \%)$ of the 213 species and subspecies recognised by Blanc et al. (1971) for the same area had to be changed and $143(67 \%)$ additional species are recognised; the names of $47(28 \%)$ of the 170 species recognised by Maitland (1976) are changed and there are $186(109 \%)$ additional species. Noteworthy changes are:

present name

earlier

Lethenteron camtschaticum Lethenteron japonicum

Alosa agone

Alosa lacustris

Alosa immaculata

Alosa pontica

Eupalasella perenurus

Phoxinus percnurus

Salmo cettii

Sander lucioperca

Sander volgensis

Padogobius bonelli

Salmo macrostigma

Stizostedion lucioperca

Stizostedion volgense

Padogobius martensii

Most of these name changes are justified by the new name being the oldest available; thus according to nomenclature rules, they are the valid name of the species. Some of these names had been overlooked by previous authors while others were regularly cited in synonymies by authors who for some reasons had decided to ignore the rules. That quite so many names have been overlooked would be surprising in other fields or in other areas, and again I am tempted to link this with a lack of knowledge of the practice of nomenclature, a lack of knowledge of the literature and a blind acceptance of previous data. The role of large, then exhaustive works cannot be ignored; they often lead people to think that everything has been done. For example there is probably some kind of "Günther effect". Günther's eight volume Catalogue of fishes (1861-1870) is the last attempt at providing a more or less complete bibliography of world fishes; while there have been very detailed and quite exhaustive bibliographies for some areas since (e.g., Africa, north-eastern Atlantic) there still is nothing more recent for European freshwater fishes. Works which were not available to Günther were not cited, and they have largely been ignored since and mistakes introduced by Günther (e.g., misspellings) have been repeated by compilers who did not check the original sources. This phenomenon is not unique to Günther, and is paralleled in most national faunae.

The suggestion arose that the International Commission on Zoological Nomenclature should be petitioned to suppress these older, valid names in favour of those more commonly used. Such proposals are usually made on the ground of preserving stability, but too often the sense of such applications is somewhat dubious except for their authors (see Kottelat, 1995: 568).

In the present case, I think there is ample evidence that the apparent stability is an illusion; there is no strong base on which to build a robust, stable nomenclature at this stage. The fact that between 28 and $42 \%$ of the names used in relatively recent monographs and checklists are incorrect demonstrates the lack of previous detailed and critical literature review, the first basic step for sound nomenclature and systematics. (The real number actually would be much higher if I had not been able to eliminate some 15 names by judicious neotype and lectotype designations). The changes resulting from a strict application of nomenclature rules concern only a few species in regard of the number of name changes or new names potentially resulting from normal, critical systematic research. Finally, the conservation of each name would require a separate application and a separate decision of the Commission. The whole process would span over years and the uncertainty about each decision for sure is creating instability, not to mention that the Commission has an impressive and extensive catalogue of cases on which it never decided anything or which have been authoritatively or arbitrarily blocked by its secretariat.

Is there a risk that I overlooked some earlier names for other 'established' species ? I cannot estimate how many, but some possibly exist. 


\section{Concepts}

This and the following sections on species concepts, etc. may seem superfluous to systematists as they summarise more extensive discussions published elsewhere. As stated in the Introduction, most of the publications on European fish systematics during the last decades have been authored by non-systematists. As a result, many problems appear to be due to out-dated, poor or wrong concepts, poor translation of terminology, poor definition and misunderstanding. Such basic rules as the International Code of Zoological Nomenclature are obviously unknown to many authors and key concepts (e.g., species) are often misunderstood. As this check-list is aimed at a general readership, it seems appropriate to start by providing brief summaries of some of the fundamental principles of systematics and nomenclature and to clarify what is a species. A brief summary of the evolution of the species concept is presented in order to explain how this concept evolved and how presently accepted concept(s) differ from earlier ones and the implications for the present review.

Although aberrant, systematics and nomenclature are no longer part of the biology curriculum in most European universities (or are too often taught by lecturers without personal experience in that field) and this has a clear and significant impact on the ability of non-systematists to understand and interpret systematic data as well as the ability of systematists to understand theoretical discussions on systematics. While it appears normal that a student conducting a $\mathrm{PhD}$ research involving genetics or any specialised field will have a geneticist or the appropriate specialists in his advisory board, it now just seems normal, if the thesis involves systematics, to consider it superfluous to involve a competent systematist. This trendy but unprofessional approach to systematics also has obvious negative side-effects for conservation, as the systematists (the specialists on biodiversity) are becoming extinct even faster than most of the threatened animal groups themselves.

\section{Systematics, taxonomy, classification and phylogeny}

Systematics is the study of the diversity of life and the relationships between living organisms, using all available information. Taxonomy is the theory and practice of describing, naming and clas- sifying living organisms. Nomenclature is the system of names and the provisions for their formation and use (see below).

Classification is the grouping of objects into a hierarchical system. Classifications are usually based on shared similarity and objects with similar appearance are likely to be placed in the same group. Grouping criteria can be very diverse, depending of the needs or tastes of the observer. This is how the first classifications were made. Gesner (1560), in his Nomenclator aquatilium, put together fish, whales and mermaids, while eels were grouped with snakes, etc. This kind of classification can be satisfactory for inanimate objects but, in today's standards, is not meaningful for living beings. Individuals are members of species which have evolved over time; they are related to other species not only by general appearance but by genealogical links. Biological classifications aim to depict these phylogenetic (genealogical) relationships between organisms. Phylogeny is the history of the evolution of a given lineage.

There are different ways of reconstructing phylogenies and the different schools supporting them have been at times engaged in colourful conflicts. Ironically, one regrets that many of the most vociferous participants at these debates were not known as practising systematists (an important distinguo in my eyes). The main schools have been called 'evolutionary systematics', 'numerical taxonomy' (or pheneticism; apparently a dead school in its original form) and 'phylogenetic systematics' (or cladism) (this terminology is inappropriate as cladistics is as evolutionary as 'evolutionary systematics', but it is quite firmly established). Of course, these schools can be subdivided in narrower groups. Most practising systematists possibly belong to a fourth school, 'opportunistic or applied systematics'. For the following discussions, I shall follow a minimal cladistic approach in that I agree that lineages can only be defined by derived character states and not by primitive character states. [A derived (or apomorphic) character state is a novelty which appeared in the common ancestor of all members of the lineage and which is passed down to its descent; the primitive (or plesiomorphic) character state is the situation which then existed in the other, related taxa and which may still be retained in other lineages. Note the distinction between character and charac- 
ter state; e.g., the number of branched dorsal-fin rays is a character which may have a plesiomorphic state [e.g., 7 in a whole family] and an apomorphic state [e.g., 9 in a given lineage]; the plesiomorphic state is useless to define any evolutionary lineage within the family, while the apomorphic one [ideally in conjunction with concordant apomorphic states of other characters] could be used to define a lineage within the family or within a genus in this family; refer to systematic text book for further discussion, e.g., Wiley, 1981]. I have doubts about some of the other axioms of the cladistic theory (e.g., the dichotomy principle) or do not believe in some corollary tools like parsimony. [If phylogeny has to depict evolution it should depict it, not find a mathematically parsimonious way of representing it; a parsimonious lie is not the truth. Admitting the reality of evolution and hypothesizing that the most parsimonious explanation is the most likely to correctly describe evolutionnary patterns, is the creationist theory not the most parsimonious evolutionnary theory as it requires a single assumption? If this is correct (I do not know), then, the most parsimonious explanation is the negation of the reality, thus, ad absurdum, the most parsimonious explanation is not acceptable]. Although sophisticated numerical approaches may look more scientific they are not the only testable ones; it remains to be demonstrated that they are better descriptors of reality or if they are merely preferred because of the psychological need to buy new toys, or for their impact when courting funding agencies. Anyway, most of this theoretical background is not needed here because the present discussion deals with species and only marginally with phylogeny (although, of course, the species are the result of evolution and phylogeny is relevant in that context).

Finally, it must be stated that unfortunately one of the negative point of cladistics is that it sometime appropriate terms widely used and uses them with a different sense, creating confusion. Stability of scientific terms is needed to allow communication: this is demonstrated at will by the endless polemics between cladists and other schools, many of which result only from the insidious use of the same terminology with different meaning (e.g., phylogeny, monophyly). Some of these polemics could have been easily avoided. The latest example is the use of the word diagnosis. If some are unhappy with its classical meaning (a statement of characters that distinguish a taxon from other similar or related taxa), they should create another name for their new concept, but not use the same name for a different concept. The reason is very simple: one may like it or not, but the classical concept behind the word diagnosis is very important for applied systematics, for other fields of biology, and for identification, where the other concept is of limited use or sense. If the diagnosis concept is not satisfactory for some, they are right to replace it, but also they should be logical and coin a new name for their new concept. It is not for the rest of the world to adjust to the idiosyncrasy of some and create new names for old concepts. No school has the monopole of the vocabulary.

"While seeming eclectic in its choice of biological data, systematics organizes those data through taxonomy and nomenclature in its own particular way, to arrive at a yet more meaningful interpretation. .... No branch of biology can advance to more general principles without comparative studies, which would be meaningless unless based on sound systematics. ... Surprisingly large areas of biological investigation still pay mere lip service to up-to-date identifications and nomenclature in the belief that these are unnecessary refinements ... Far from losing its role as biology becomes more complex, systematics has become even more necessary" (Whitehead, 1990: 181).

\section{Nomenclature}

To give names to objects and living beings appears to be natural and important for humans because it allows them to communicate with others. This explains why most things which play a role in their life, positive or negative, have been given names. Animals are no exception. Each language, each country has its own set of names. In order to reach a high level of precision, zoologists developed a nomenclatural (naming) system which gives one (and only one !) name to every different animal species. This system is governed by a set of rules known as the International Code of $\mathrm{Zo}$ ological Nomenclature (here the Code or ICZN), which is revised from time to time, the last revision having being published in 1985 (Anon., 1985). This code prescribes a neat and regulated system of naming which is of vital importance to all biologists because unambiguous communication depends on it.

Zoological nomenclature formally starts with the tenth edition of Linnaeus's Systema naturae (1758). This starting date was agreed on much later, with the introduction of the 1886 Code of Zoological Nomenclature, so earlier authors were at times quite inconsistent in their nomenclat- 
ural practices. Linnaeus's nomenclature was derived from Aristotelian philosophy and Logical Division; in Linnaeus's view, the genus was the key element of classification (see Cain, 1957). Although these Aristotelian principles are no longer used or disagree with today's principles and although the species is now broadly accepted as the real unit of systematic discussion, we still use the Linnaean binominal nomenclatural system; Aristotelian logic is still surfacing in (and biasing) some taxonomic treatment, especially in poorly known groups (e.g., the definition of clear-cut discrete groups which have no theoretical justification in evolutionary taxonomy but was a practical necessity under the Logical Division [and still is for keys], Cain, 1957: 150; the erroneous idea that some characters are indicative of species, genus or family-rank; symmetrical or parallel classifications in different lineages such as the quinary system). From both philosophical and practical points of view, there would be arguments to abandon this binominal nomenclature, but its replacement by an other nomenclature for several million names already proposed is probably not realistic and could only result in a long lasting chaos. Similarly, there would be arguments to abandon naming any supraspecific category and especially to abandon naming long successions of hierarchies (subtribe, tribe, supertribe, infrafamily, subfamily, etc.).

These weaknesses being stated, the zoological nomenclature system is quite dynamic in order to allow the incorporation of results of new research. Examples of data which have to be incorporated into the system are:

- the discovery of new species or new genera which need to be given names; the Code includes provisions on how the names have to be created;

- the discovery that some species in a genus actually belong to another genus or even represent an entirely new genus; again, the Code includes provisions on how these names have to be treated;

- the discovery that two different names have been given to a single species; such names are called synonyms; the Code has provisions that the oldest name (senior synonym) has to be retained (this is the principle of priority);

- the discovery that the same name had been given to two different species; such names are called homonyms; the Code has provisions that the first named species (senior homonym) has to retain the name while the junior homonym has to be replaced by a new name (this, again, is the principle of priority).

Several words will be used here in a very for- mal way which may differ from other meanings they might have in other contexts or in everyday usage. New names which satisfy the conditions of the Code are called available names and they can be used in zoological nomenclature. A name which does not follow these rules is an unavailable name and cannot be used. A taxon (plural: taxa) is any taxonomic unit (e.g., a family, a genus, a species). The valid name of a taxon is the oldest available name applied to it, unless it is invalidated by a provision of the Code; all the other names applied to the taxon are invalid. A name can thus be available but invalid. Junior synonyms and junior homonyms are invalid names. A name proposed without any description is called a nomen nudum (plural: nomina nuda) and is unavailable.

A nominal species is a nomenclatural concept denoted by an available name; it may be a valid species (a species distinguished by taxonomic characters), a synonym of a valid species or a name of uncertain status. The phrase 'nominal species' is commonly used to mean 'a species or a subunit of a species which at some time had been recognised as distinct but whose actual status is not relevant for the present discussion'.

Each family has a type genus which gives its name to the family (Cyprinus is the type genus of Cyprinidae), each genus has a type species and each species has one (or more) type specimen(s) (termed holotype, lectotype, neotype, paratype, paralectotype, or syntypes, depending of technical criteria - other 'type' categories have no nomenclatural status, e.g., allotypes, iconotypes, topotypes, etc.). Contrary to what non-systematists often perceive or state, this is not a typologist misconception; a type is not an individual selected because it is thought to represent an idealised conception of the species. A type is merely a namebearer or a standard for a given name, and nothing else. That means that if one discovers that two species have been confused under a single name, the type specimen for that specific name determines to which of the two species the name has to apply; the other species must be designated by another name. Name-bearing types have an international validity and are the 'property' of the whole scientific community. They are kept and curated by institutions or museums which are responsible for maintaining them in a good state and for making them accessible to researchers; they are sensitive objects, are easily damaged, and should be curated with great care and handled only if justified. Authors should only deposit name-bearing types in internationally recognised collections with 
large holdings and permanent ichthyological staff. A new potential threat to the survival of old types is that they can now be used for 'biochemical systematics'; it is the responsibility of curators to ensure that types be used for such analyses only if justified by outputs relevant for nomenclature and if the resulting data can be used in a systematic context (see p. 19 for discussion).

A potential and significant source of nomenclatural instability in European ichthyology is the absence of types for many species. Systematics started in Europe, and logically, the first naturalists were interested in the European fauna and described a great number of species in the very early days of European ichthyology. The rules of nomenclature were very unstable at the beginning and, in a way, every researcher had his own rules (using the word loosely). Different 'rules' and concepts appeared (and disappeared) with time and it is only in this century that nomenclature became formally codified. The concept of a type, as explained above, only appeared around 1870. As a result, there are no formal type designations for most species described in the 18th and 19th centuries; in many cases it is possible to find out on which specimens the various new species descriptions were based and these can be considered as types (the Code also has rules about type definitions). But in a large number of cases, there is no extant type material. This particularly applies to the earlier authors who usually were also those giving the shortest or least detailed descriptions, and in several cases, it appears that these descriptions either were based on a mixture of several species, or could apply to a variety of species. This is potentially problematic and could lead to a great amount of nomenclatural instability if not handled properly. This is a field that workers without a serious background in the practice of systematics should refrain from entering.

\section{Species concepts}

I shall not discuss here if species have an actual existence in nature, independently of the judgement of an observer, or if they are mere concepts designed for the purposes of our subjective understanding of nature. The reader is referred to Griffiths (1974), Hull (1976) and Ghiselin (1975) for discussions on this subject.

I consider here that species are real entities. This means that "they may be discovered and recognised with greater or lesser success, depending on whether suitable techniques have been applied. Such species exist, whether we can recognise them or not, and the naming of such a species represents a hypothesis that the named unit corresponds to an actual entity in nature" (Brothers, 1985: 35). (I am not convinced that categories above the level of species exist or are objectively defined - but perhaps they are necessary; anyway the present work is essentially concerned with species and current nomenclatural rules require that species be given a generic and a specific names).

If species or higher categories were considered to be imaginary constructs, their naming would not be a hypothesis, and they would be "classes [concepts constructed from similarities], the membership of which is defined by the possession of essential characteristics specified by man" (Brothers, 1985: 35). The typological and nominalistic concepts (for history of systematics, see Mayr, 1982b) considering species as classes, they cannot be applied to real species.

Three main species concepts have been successively proposed which refer to real units in nature: the biological species concept, the evolutionary species concept, and the phylogenetic species concept. Clearly, the group of organisms studied or the aims of the research influences a researcher's view of what a 'species' is (Mishler \& Donoghue, 1982: 493; Endler, 1989: 625), as do the genesis of this researcher's education, his social background, etc. (e.g., city born and educated biologists are unlikely to perceive nature's [un]organisation the same way as those born in country-side). It should be remembered that the earlier (and present ?) concepts were based on just a handful of groups like Drosophila flies and birds which cannot be taken as representative of the whole animal kingdom.

The biological species concept (BSC) appeared in the 1930s; in its classical form (Mayr, 1969: 26), it is restated as a "group of actually or potentially interbreeding populations, which are reproductively isolated from other such groups". The BSC has been criticised for theoretical (e.g., Sokal \& Crovello, 1970) and practical reasons. The criticisms are:

- The BSC confuses pattern and process in speciation (Cracraft, 1982: 411, 1983: 170; Donoghue, 1985: 173), and it biases toward a particular type of process (Cracraft, 1987, 1989: 32). In other words, before identifying species, one has to identify a given process of speciation. But we wish to reach conclusions, even if the speciation process is not known (anyway, a speciation process can never be objectively known with certainty). From a pragmatic point of view, in complex groups 
with uncertain speciation histories (e.g., Coregonus, Salvelinus) there is an obvious advantage in having a process-free species concept. The BSC emphasises the process of reproductive disjunction and not the discovery of taxonomic differentiation, excluding the recognition of a large number of evolutionary taxa that are diagnostically distinct but not completely reproductively isolated from other such populations (Cracraft, 1983: 163-165, 1989).

- The BSC is difficult to apply in case of the existence of a contact zone between differentiated taxa (e. g. Warren, 1992) or can lead to the recognition of illogical taxa. Rosen (1979: 276-277) considered that it has logical flaws associated with its criterion of sympatry used to test for reproductive incompatibility. species.

- The BSC is not applicable to uniparental

The practical problems with the use of the $\mathrm{BSC}$ are even more serious than the theoretical ones. The key element in identifying 'biological species' is reproductive isolation; it can rarely be applied and researchers have to deduce reproductive isolation from other criteria (morphological, ecological, biochemical, ethological, etc.) which are assumed to reflect reproductive isolation or incompatibility; obviously, this is very subjective. Characters assumed to be adequate are likely to change with better knowledge and experience with similar cases. Additionally, there is not obligatorily a relationship between the potentiality of interbreeding and phenotypic or genotypic differentiation. Interbreeding is not therefore evidence of conspecificity (see discussion on the nonsignificance of hybrids, p. 17).

The BSC is only applicable in the case of sympatry; its inapplicability to allopatric units is "an underemphasised problem because there are thousands of allopatric ... populations" (McKitrick \& Zink, 1988: 3). In order to incorporate allopatric populations, Mayr developed the polytypic species concept, in which disjunct populations would be considered conspecific if assumed to be reproductively compatible if brought into contact. Again this is a subjective decision. (A polytypic species should not be confused with a polymorphic species as is often done in literature; a polymorphic species is a species whose members can exhibit permanent or temporary different morphologies depending on sex, ontogenetic stage, social status, substrate, etc.).

As reproductive isolation is a criterion almost impossible to apply, more and more workers have had difficulties with standard practices or defini- tions of the BSC. Some have abandoned it (e.g., in ichthyology: Etnier \& Starnes, 1986: 836; Warren, 1992), paid less or no attention to the criterion of reproductive isolation (e.g., Etnier \& Bailey, 1989: 22 ) or concluded that using magnitude of differentiation as an argument for 'lumping' taxa "serves no purpose and would not be indicative of the biological factors involved in differentiation and speciation" (Buth \& Mayden, 1981: 589). Common sense seems to require that a concept which is inapplicable, or applicable only if the key criterion is overlooked, has no great value and should be abandoned. In practice, systematic ichthyologists have generally considered morphologically distinct but allopatric populations as species; in North America, this has been applied for the Cyprinidae and Percidae, but not for the Salmonidae (Cavender, 1980: 296); this apparently corresponds more or less to areas and groups worked out by taxonomists, as against those worked out by nontaxonomists.

The BSC has been used mainly in ornithology and was felt not workable in most other fields, where it has been abandoned (Donoghue, 1985: 173). Although people frequently mentioned that they used the BSC, actually it seems that its basic criterion (reproductive isolation) could only be used in a handful of cases. Although irrelevant to the discussion of the BSC itself, it is worth mentioning that in many instances the authors who claim to have used the BSC probably did not understand it; for example, Steinmann's work on coregonids is supposed to be based on the BSC (Steinmann, 1952).

The argument (e.g., May \& Ashlock, 1991: 27) that the BSC is important because it is widely accepted in a large number of biological disciplines is at best irrelevant in a discussion of species concepts. The supposed popularity of the context is not that obvious (see previous paragraph; also Mayden \& Wood (1995: 101) and, anyway, popularity (or fashion, wide-acceptance, etc.) is not sufficient to justify the use of a scientific concept. Many once popular concepts, theories or philosophies lead to scientific fiasco, social disasters or mass murders; many unpopular ones later gained wide acceptance.

The above discussion of the BSC is based on Donoghue (1985), Cracraft (1983, 1987, 1989), Frost \& Hillis (1990), Mayden \& Wood (1995); McKitrick \& Zink (1988), and Warren (1992: 33).

A variant of the BSC is the 'Recognition' species concept (RSC) for which a species is the "most inclusive population of individual bi- 
parental organisms which share a common fertilisation system" (Paterson, 1985: 25).

The RSC changes the perspective from reproductive isolation to specific mate recognition and should correspond as closely as possible to the species as perceived by the organisms themselves, that is the actual unit in nature. My perception of nature makes me consider the RSC as making more sense than the BSC. However, it suffers from the same practical problem; that is mate recognition is usually inferred from other criteria. Also, the RSC is not process-independent (see Chandler \& Gromko, 1989: 122).

The evolutionary species concept (ESC) was first proposed by Simpson (1961). As now understood, an 'evolutionary species' is "a lineage of ancestral descendant populations which maintains its identity from other such lineages and which has its own evolutionary tendencies and historical fate" (Wiley, 1978: 17). The ESC has been considered as non-operational and Rosen (1978: 176) considered that it "conforms, in practice, with Regan's (1926) definition that a species is what a competent taxonomist says it is". (Several authors quote Regan as having written "a species is what a competent taxonomist says it is"; they obviously have not checked the original. Regan [1926: 75] actually was more subtle and wrote "a species is a community ... whose distinctive morphological characters are, in the opinion of a competent systematist, sufficiently definite to entitle it ... to a specific name". Probably, this is still the most popular species concept).

In a review of species concepts and biodiversity, Mayden \& Wood (1995: 108) and Mayden (1997) consider the ESC as the most flexible of the species concepts and recommend its use as a conceptual basis for units of biodiversity. They retain the species as being "an entity composed of organisms which maintains its identity from other such entities through time and over space, and which has its own independent evolutionary fate and historical tendencies". These works have been received too late for including a discussion of details here, but it seems that for practical purposes in the present context, use of the ESC or the PSC (see below) are equivalent and that many of the advantages of the PSC over the BSC listed below apply to the ESC vs. BSC. The differences mainly concern the recognition of ancestral species and fossil biodiversity.

Considering the difficulty or impossibility of measuring reproductive isolation as required by the $\mathrm{BSC}$ and the unclear role of reproductive isolation in speciation, a more objective concept, the phylogenetic species concept (PSC), has been proposed, based on the generally accepted statement that evolution occurs and produces differentiated taxa (Cracraft, 1983, 1987, 1989; Donoghue, 1985). A species is thus "... an irreductible (basal) cluster of organisms diagnosably distinct from other such clusters, and within which there is a parental pattern of ancestry and descent" (Cracraft, 1989: 34-35).

Species are recognised in terms of their hypothesised status as diagnosable evolutionary units (Warren, 1992: 34). It is definitively more objective to decide whether characters are diagnostic or not than to speculate on whether allopatric population could potentially hybridise or not. "The grouping criteria of the PSC are diagnosability and monophyly ... The smallest diagnosable cluster is given the rank of species" (McKitrick \& Zink, 1988: 2). An advantage of the PSC is that it removes the necessity "to interpret [intraspecific] variation from a taxonomic point of view" (Cracraft, 1983: 171).

The most common objection to the application of PSC or ESC is that they result in an increase of the number of recognised species. I doubt that this argument makes sense: if these units are real, they should be recognised and I do not see the advantage of systematics which actually would conceal biological diversity. The potential 'increase' in number of species is compensated for by the removal from our classification of the numerous arbitrarily-defined subspecies (see Subspecies section, p. 17). I fully agree with Frost \& Hillis (1990: 94) when they state "We believe that prior to phylogenetic analyses, or studies of geographic variation, it is better to overestimate biological diversity (...); we see no theoretical or operational advantage in underestimating biological diversity". Lets give the benefit of the doubt to unresolved stocks for which names are available.

The argument that recognition and naming of (let us say) 50 species of Salmo and 200 species of Coregonus would lead to a chaotic systematics of course is irrelevant. Under a given species concept, stocks or population satisfy or not the species criteria and are or not distinct species. The chaos does not result from the number of named species, but from the poor quality of the concepts, from their inconsistent application and from poor base-line work. I do not perceive a system which recognises 4 species of Salmo with 50 Evolutionarily Significant Units (ESU) and 1 species of Coregonus with $200 \mathrm{ESU}$ as less chaotic, especially if 
all or most ESUs satisfy the species criteria (for further discussions of ESUs, see Mayden \& Wood, 1995; Mayden, 1997). Speciose genera are known in most groups of organisms and unless unnatural (polyphyletic) they do not create problems; why should it be different for a few genera of fishes ? It is noteworthy that these discussions always centre around salmonids, coregonids and a few species of concern for fisheries. If the problem is merely administrative, biology simply cannot help; at best administrators may fund sound research by competent biologists to produce sound systematics as soon as possible.

Considerations on 'lumping' or 'splitting' (qualified as "traditional inanities" by Frost \& Hillis, 1990: 94; I concur) do not contribute anything relevant to the discussion (incidentally, these considerations seldom originate from workers with practical experience of systematics); under the PSC or ESC, species are defined by objective criteria and are testable hypotheses. This is not the case with the BSC.

Considering the recent concern for biodiversity documentation and conservation, application of the ESC or PSC allows a better and more objective measure of biodiversity (see also Mayden \& Wood, 1995). The use of a species concept which does not allow to recognise significant part of the biodiversity obviously reduces our ability to inventorise and manage this biodiversity.

Another argument against PSC is that its application may result in recognising and naming potentially unstable evolutionary units or short-lived species. Actually, all but very few species are probably short lived (one just need to consider the fossil record) and with this logic one should question the sense of naming fossil species or of naming just about anything. Anyway, short-lived species certainly exist in nature and I do not see any justification not to recognise and name them. If it could be demonstrated that a given species has been separated from its sister-species for only 300 years and evolved in this time span, it would certainly become the subject of much research, and even just for communication it would certainly need a formal name. Anyway, 300 years would already be a time-span longer than our present systematic practices, and something like six times older than the BSC or any 'modern' species concept. Such species are likely to survive a few more revolutions in biological thinking before ending their 'unstable' existence (if mankind gives them this opportunity). But I admit that there is probably no real practical point in naming species which can only be identified by biochemical subtleties.
Any species, of course, has the potential to split into several independent lineages, but this does not mean that significant divergence must occur (Wiley, 1978: 23); when two populations are separated and diverge, it is subjective to use criteria such as magnitude of difference to deduce if they are conspecific or not, because this relies on experience with other taxa; in stressing diagnosability, the PSC is more objective because it relies on intrinsic characters. In freshwater fish, each basin or sub-basin has a potential for weakly differentiated populations; this possibly relates to the linear nature of their distributions (a drainage is a set of lines organised in an arborescent way and dispersal usually implies moving along the lines; movements across the lines often leads to speciation) while most terrestrial and aerial organisms have a two-dimensional distribution and dispersal possibilities.

Speciation is not necessarily linked with morphological (Larson, 1989: 583) or genetic divergence (Templeton, 1981: 24; see also example under Gasterosteus, p. 164) or adaptive radiation. Morphological divergence generally seems to be induced by the availability of new or non-exploited habitats or resources (but this does not mean 'environmentally induced', as too often translated); adaptive radiation generally seems to be a response to competition, but not directly a result of speciation. (In this context, one should not underestimate the influence of subjective sociological components in science: the psychic need to explain diversity and the perceived superiority of a paper creating a theory over those reporting diversity are responsible for many teleological theories [as well as unnecessary theorisation, rules, axioms, and wasted paper]; to be complete, one should blend these psychological components with nationalistic, political or religious idiosyncrasy, the semantic requirements of different languages and their influence on translation or perception of other's languages).

Finally, our ability or inability to perceive different lineages which exist in nature is not the key criterion for deciding whether they are real. The key criterion is the organism's ability to meet and recognise its conspecifics and several criteria are likely to be used which escape our sensory capabilities. Fish do not read our books and do not always know what they are supposed to do. If they could read they would certainly die of laughing (just as non-western populations can be shocked when they read ethnographic books on themselves or as I am surprised when I read a tourist guide on my country). 'Cryptic species' are only cryptic 
for our eyes or our tools, they certainly are not cryptic for individuals of these particular species (humans and apes are probably cryptic species for most deep-sea fishes).

The pragmatic species concept which I have mentioned elsewhere (Kottelat, 1995: 567; Kottelat \& Ng, 1994) serves a quite different purpose, is much less sophisticated and has a much less elaborate theoretical base; but in application it would reach about the same result. In the precise case of the two systematic and nomenclatural nightmares of European ichthyology, coregonids and salmonids, the PSC could not be used consistently because the available data are too confusing, incomplete and incompatible, and I have had to resort to a blend of 'pragmatic species' taking into account the blurred result of the conjunction of a century of confused nomenclature, chaotic publications, teleological or metaphysical species concepts, incompatible methodologies and, on top of it, irremediable damage to the original populations resulting from translocation, introductions, hybridisation and extinction. This is discussed under the families concerned.

A few words seem justified here to point to the non-significance of hybrids for systematic analysis. Hybrids have often been used as indicator of genetic compatibility or as a measure of relationships (e.g., Dubois, 1982; Daget, 1983). On the contrary, Rosen (1979: 277) considered reproductive compatibility as a plesiomorphy marked by mosaic distribution within a group ("a primitive attribute for the members of a lineage and has ... no power to specify relationship within a genealogical framework"). As concluded by Howes (1981: 48), "this hypothesis would certainly account for those conflicting cases ... where supposedly phylogenetically closely related taxa fail and distantly related ones succeed in being reproductively compatible". While the inability to hybridise is a useful information, especially for species-level analysis, the ability to hybridise indicates that the two stocks have had a common ancestor sometime and somewhere, but does not allow any other conclusion and especially not a dating of the age of this ancestor neither in absolute nor in relative terms. The greatest use of hybridisation is not as a systematic but as an environmental indicator as it usually reflects environmental stress (natural or man-made) or introductions (Hubbs, 1955; Wheeler \& Easton, 1978; Howes, 1981: 49; 1991: 8).

In the following checklist, I have tried to apply the PSC whenever possible. In most cases there is no significant difference between application of the different species concepts. The main difference concerns the way polytypic species and subspecies are treated (this also points to inconsistency of earlier concepts) as they are recognised as real entities by neither the PSC nor the ESC.

The subspecies seems to be a convenience construct. Many authors see the use of subspecies as a simplification of the classification. For example, Mayr (1982a: 594) sees "the downgrading to subspecies rank of every isolate, even when not clearly connected by intermediates, ... [as] an extraordinary simplification of taxonomy at the species level". I doubt the relevance of this kind of argument; I think that the aim of systematics is to describe nature as it is, not to find a simplified way; its task is not to introduce order, but to understand the existing order (if any, or the existing disorder). In any case, I do not perceive as real simplification the use of trinominals instead of binominals (the ad absurdum extrapolation of this simplification is Berg's [1932a] nomenclature for European coregonids in which a single species is recognised together with several subspecies, and more than a hundred infrasubspecies, natios, subnatios, morphs, varieties, etc.). I do not understand the purpose of simplifying a classification for convenience by denying the existence of real diversity. Anyway, within the framework of phylogenetic systematics, the subspecies is unobservable and undefinable (Rosen, 1979: 277). If a species is the smallest unit, it is logically not possible to construct something smaller than the smallest unit; subspecies are not evolutionary units and have no status under the PSC.

Mayr (1982a: 594) concluded that "the primary use of subspecies is as a sorting device in [museum] collections". If it is a clerical tool used for filing and shelving, then it is not more relevant for biological discussion than the size of the jars or the management software. After all, local populations can be talked about by geographic names.

Similarly, the hierarchy species, infraspecies, subspecies, infrasubspecies, natio, infranatio, subnatio, infrasubnatio, subsubnatio, etc. (e.g., Berg, 1935) cannot be adopted as the categories are not based on intrinsic characters of the units, but on a similarity with other units and subjective decision of the degree of distinctness.

Like the subspecies, the polytypic species is a taxonomic construct or a category of convenience, not an evolutionary one. Under the polytypic biological species concept, there is no consistent, objectively defined unit of evolution and the concept does not provide a sound ontological 
basis for the study of the pattern or process of speciation (Cracraft, 1989: 32).

Hereunder, the status of subspecies of earlier authors has been evaluated individually on the basis of available data. Where conclusions could be reached, the logical nomenclatural changes have been made. In other cases, heuristic comments are given on the data missing for reaching a conclusion or about existing problems.

Application of the PSC or ESC results in the disappearance of those subspecies which were nothing but arbitrarily delimited sections of clines, etc. Phenetic distances, which have often been used to justify recognition of subspecies, are irrelevant because they do not by themselves make a unit diagnosable; at best, they can be regarded as an additional tool to confirm the results of a character analysis. ("Some enthusiasts of similarity analysis seem to assume that this study alone is the key to understanding the world and all else is non-sense. They are consequently led to futile efforts to construct concepts of other kinds as similarity extension. [The] book by Sokal \& Sneath (1963) [...] consists largely of an exegesis of methods unsuited to the apparent purpose"; Griffiths (1974: 89). This apparently also applies to any similar indices and genetic distances. McKitrick \& Zink (1988: 9) note that an individual discriminant score might be a diagnostic 'character' but that it is not clear if it is heritable and indicates a monophyletic group.

As a result of the strict application of the Code, of the application of the PSC and in some cases the critical review of the available data, the resulting check-list differs from those of earlier authors. There are some modifications in nomenclature, but the most striking difference is in the number of recognisable species resulting from the reevaluation of the subspecies of earlier authors (the subspecies concept completely disappears) and of a pragmatic and heuristic handling of salmonoid nomenclature to replace biological nonsense, systematic heresy and nomenclatural chaos.

Some may argue that such abrupt changes as in the case of salmonoids is threatening the stability of nomenclature. Frankly, this cannot be the case when one considers 150 years of nomenclatural anarchy and that names presently in use do not even follow the most basic principles of zoological nomenclature. The proposed nomenclature follows the conclusions of such sound systematic data as are available and is not very different from what is presently done by sensible fisheries managers with the use of scientific names followed by a river or a lake name to distinguish particular stocks. A correct formal nomenclature therefore should facilitate communication. It points to evolutionary uniqueness of several species/populations (as well as the uniqueness of several communities) and attracts attention to their threatened status, biodiversity value and conservation needs. Coregonid and salmonid populations have been so much impacted by translocations, introductions and extinctions that it is difficult to clarify further their systematics on an European scale. This might be possible but would require a fair funding and that the funding goes to trained and competent systematists.

Proofs of the need for a sound and coherent formal nomenclature abound and the consequences of poor taxonomic practices can be dramatic. Gerdeaux (1993: 23) reports that in 1982, following a decrease of coregonid catches in lake Genève, eggs have been imported as Coregonus lavaretus and later turned out to be $C$. peled. The pre-existing situation is confusing (the two names are likely to be incorrectly used; the endemic species of lake Genève are extinct; the local stock is the result of introductions) and accidental introductions might have devastating effects in many watersheds and lakes. A formal nomenclature recognising unambiguously the different stocks contributes to reduce such risks.

In the following taxonomic discussions, I have tried to avoid using distribution patterns as evidence in favour of one or the other decision. Sound zoogeographic analysis has to be based on systematics and phylogeny, and to use the zoogeographic conclusions of others as the base for systematic decisions which will in turn be used by others to support zoogeographic discussions quickly leads to circular reasoning. This circular reasoning is only avoided by the sole use of intrinsic characters of the taxa. The same kind of circular reasoning also seems to be present in some genetic or morphometric analyses where distances are considered as indicating a given taxonomic level or rank.

Infrasubspecific taxa are taxa of a rank lower than that of a subspecies, i.e. subdivisions of the subspecies. Infrasubspecific names are a significant source of potential future nomenclatural problems. The Code is very precise about them: they are not available for nomenclatural purposes (ICZN art. 1(b)(5)). This explicitly applies to the race, natio, subnatio, infrasubnatio, morph, aberratio, etc. categories frequently used in eastern European literature (ICZN art. 45(f) and exam- 
ple). The exceptions are 'forms' and 'varieties' described explicitly as such; if published before 1961 they can be considered as subspecies under certain conditions (ICZN art. 45(g)(i)). Those proposed after 1960 are infrasubspecific and therefore not available (ICZN arts. 16, 45(g)(ii)). A name which is not available cannot be used in nomenclature.

The problems is that an infrasubspecific name becomes available if it is used subsequently as a name for a species or a subspecies; its author is the one who first uses it as a species or subspecies (ICZN arts. 10(c), 50(c)(i)); its publication date, types and type locality are determined by that usage (if done before 1985 and if precise conditions are satisfied, the name might be available with its original author and date [ICZN art. 45(g)(ii)(1)]). In practice, this means that an author who inadvertently uses an earlier infrasubspecific name as a subspecific one is deemed to be the author of a new subspecies (or makes available an unavailable name). Example: Leuciscus leuciscus natio roulei Vladykov (1931) is an infrasubspecific name and thus is not available; but the inadvertent use of the name with the form of a subspecies (Leuciscus leuciscus roulei) by Bertin \& Estève (1948) in a type catalogue where it is treated as available and adopted as the name of a subspecies makes the name available. As the use of infrasubspecific categories has been quite chaotic and inconsistent, and often the work of non-taxonomists, such inadvertent new names or validations occur not infrequently. The problem in most instances is to trace the oldest use of a given combination and I see there a great potential for nomenclatural instability. In order to avoid the inadvertent creation of nomenclatural problems, authors who use names for units of a rank lower than the subspecies or for morphs or varieties should never use a latinised or an italicised name. It is wrong to use latinised and italicised trinomens for a morph or a variety because a trinomen means a subspecies (see also Species Concepts, p. 13).

The use of infrasubspecific names should be discontinued, even if the involved stocks are later considered as valid species. In such cases, new names should be created to avoid confusion.

\section{Biochemical and genetic techniques}

The development of biochemical and molecular systematics has often led to what is yet the fantasy that herein lies the ultimate answer. These new techniques certainly contribute to our understanding of evolution and phylogeny of fishes, but until now the number of papers really contributing to this knowledge and leading to actual conclusions is limited. The problems are not in the techniques themselves, but more in the philosophy with which they are used and the way results are interpreted. The weakest point is that these techniques are often applied not by systematists working on a precise group of which they already have a minimum knowledge, but by researchers more interested in the technique itself. It is diagnostic that in several of these studies one can read that the authors' experience of the species is limited to tissue samples sent by correspondents. One is always tempted to equate discrepancies between these and morphological data with misidentification, and only too often this cannot be contested as there is no voucher of the examined material. Most of the difficulties arising in analysis of morphological systematic data are also encountered with molecular data (Avise, 1983: 104). "Not all species should be expected to differ from one another for either allozymes or DNA sequences that we are currently able to visualize" (Mayden \& Wood, 1995: 85).

A recurrent problem is that results are often presented as 'distances' which may be of interest for phenetic reconstruction (admitting that all taxa within a lineage have been investigated, which is only exceptionally the case; a situation hardly acceptable for phylogenetic studies), but uninformative for systematic analysis as there is no theory to support the link of a given distance with a given systematic relationship or taxonomic rank. Moreover, distances like Nei's (1978) genetic distances, are based upon allele frequencies and are not strongly affected by unique alleles at low frequencies (see, e.g., Beaumont et al., 1995: 887), In a phylogenetic study, this means that the phylogeny would be based mainly on plesiomorphic character states and that apomorphies would be neglected; this disagrees with the accepted principles (p. 10) that a reconstructed phylogeny has to be supported by apomorphies and that plesiomorphies are uninformative.

The non-significance of distances is evidenced by the available data on African Cichlidae. Mitochondrial DNA data (Meyer et al., 1990; Avise, 1990) on the speciose cichlid species-flocks of lake Victoria, Africa showed that the flock contains less genetic variation than the human species which itself exhibits less intraspecific variation than many other vertebrates. See Moran et al. (1994: 282-5) for restriction enzyme analysis of mtDNA of lake Malawi cichlids with similar results and discussion of limits of mtDNA analysis and problems associated with time estimations based on sequence divergence. 
Doyle (1992: 161) commented that "molecular systematics .... ironically may represent an insidious new form of one-character taxonomy" and this should be seriously considered, as well as the distinction between phylogeny of organisms and phylogeny of genes. A gene-tree is not a speciestree. The reader is also referred to Miyamoto \& Cracraft (1991), Minelli (1993: 59-61) and Avise (1994) for a more detailed summary of biochemical and molecular systematics.

On 'classical' vs. 'modern' taxonomy. Some authors have been tempted to distinguish between what they perceived as 'classical' taxonomy and 'modern' taxonomy and statements like "classical taxonomy alone cannot solve this question" are not rare (see also discussion of Salmo, p. 130). Beside the fact that there is no definition of what 'classical' and 'modern' taxonomies are, a few comments are in order.

There is not a dichotomy between a 'classical' and a 'modern' taxonomy. There is a taxonomy which can use morphological, ecological, biochemical, ethological or any other type of data, providing that they are presented in a usable format. They are not alternative taxonomies, they are complementary data sets. The main problem is that most of the 'modern' taxonomic data are presented in a way which makes them impossible to incorporate in taxonomic analysis; in other words, the 'modern' data are too often presented in an antiquated fashion which makes them useless for modern systematic analysis.

In most cases (and all those I looked at) where it is advertised that 'classical taxonomy' could not solve a given systematic problem, it appears that: 1) no competent taxonomist ('classical' or 'modern') investigated the case; and 2) the case has not been solved by 'modern' taxonomy either. (The trivial case that the 'problem' is already solved for a long time also occurs).

Certainly the molecular techniques (and other still to be discovered techniques ...) will progress and hopefully most of the conceptual shortcomings can be addressed. But still, this does not mean that a single technique alone will answer all questions and it does not mean that morphological methods are becoming obsolete. Biochemical and molecular data form, at best, an additional set of characters, nothing more. "We will have to be content, for centuries if not millennia, with imprecise verbal descriptions of many biological characters" (Griffiths, 1974: 113).

Morphology and comparative anatomy, among others, share the positive aspect that students need to 'meet' the organism they study and to describe with words (a psychical process of the utmost importance) what they see and the patterns of relationships they perceive; these aspects have to be incorporated into biochemical systematics too, or they will not be systematics but mere button-pushing science, expensive machine testing or computer games.

It is not rare to read comments, usually emanating from uninformed biologists or from lay persons, that taxonomic decisions should not be based on morphology alone but should wait for information on genetics, biochemistry, ethology, etc. Indeed, taxonomic hypotheses should ideally include all possible data. But 'all possible data' means an endless catalogue of possible additional research and methods. While this may be intellectually desirable, for any practical purposes it does not make sense. This criticism sometimes also hide other, less scientific arguments (an inability to conclude or to switch to another project, rivalry, etc.). One must be able to take decisions and to once close a case (it can always be re-opened if needed).

With the limited funding available for systematic research, priorities have to be set (Kottelat, 1995) and I doubt that an exhaustive survey with all possible tèchnologies is reasonable. Methods have to be selected according to the needs and the questions to be answered. While it can be justified that a plethora of methods be used to investigate a particularly interesting case, it is not desirable (and financially feasible) to routinely apply all possible methods to every case. In most instances, a trained eye and morphological analysis will always be the fastest, most efficient and cost-effective means of answering identification problems, especially outside of a laboratory. More sophisticated and expensive methods should be used in priority to investigate cases where these methods really have something to contribute. One should also distinguish between using a method to solve a problem or using a problem to test a method. 


\section{Checklist}

Content and limits of the present checklist. First of all, the following checklist is not an authoritative or ultimate treatment of the systematics of European freshwater fishes. A greatly underestimated amount of work remains to be done before we reach an acceptable level of knowledge. In several instances where data were ambiguous or contradictory, I have tended to chose unconventional alternatives, not for the pleasure of being provocative (criticism will surely focus on these few unconventional alternatives and overlook the overwhelming number of cases where I followed conventional conclusions) but partly because unconventional positions will attract more attention and hopefully generate the much needed detailed studies.

Taxonomy and systematics have two main goals. One is primarily of academic interest, to elucidate phylogenetic relationships among living organisms. The other is of immediate practical interest: inventories, surveys, documentation of biodiversity, and compilation of identification tools. For proper management of natural resources, we need information on number of species and their identification now, not in 50 years. If definitive conclusions are not possible with the available data, then tentative decisions are needed. As for other components of environment management strategies, the precautionary approach should be the rule. In the present context, in case of doubt on the distinctness of two species, the precautionary approach is to treat them as distinct awaiting (possible) further research.

The checklist includes references to original descriptions of all freshwater fish species naturally occurring in Europe (exclusive of the former Soviet Union) as well as their synonyms. Introduced species which became established are listed, but neither references nor synonymies are provided, except for those synonyms based on material from European localities. Marine species regularly entering freshwaters are included. These references have been checked in the original publications. Publication years in bold face indicate references which were not accessible and which I could not check personally; in such cases the source of the record is usually indicated. It might be argued that it would be better to postpone such a publication until all of these references could be checked or until all the problems can be solved. From a strictly academic point of view, this is possibly correct.
But, again, from a practical point of view and when considering the needs and use of systematic data by other fields, it seems desirable to publish now. The present state is a compromise between the justified desire for exhaustiveness, urgency of needs, and time needed to reach the missing literature because clearly the point of diminishing returns has been reached and the efforts to examine the few remaining references have been vain or would represent travel and time expenses far greater than those needed for all the already examined literature.

Families and genera. Families are listed following the sequence in Nelson (1994). When there is disagreement between authors about limits of genera or higher categories, I generally followed common practice, but have noted alternatives.

Transliteration of non-Latin alphabets. Author names, place names, and journal names in non-Latin alphabets have been transliterated; titles of books and papers have been translated. When a transliteration is used in the original paper, the same spelling is used here. As it happens that different transliterations have been used in the literature for the same name, some inconsistencies may result. Frequently, transliteration systems have changed with time and no standardisation has been attempted here. Older bibliographies or indexes used earlier transliterations systems and I consider that a standardised use could actually complicate data retrieval, especially for those not familiar with these languages.

Unfortunately, some accents and diacritic marks disappeared as standard western European softwares do not support them.

Geographic coverage. All species occurring in freshwaters of Europe west of the former Soviet Union are included. Species known only from the countries of the former Soviet Union have not been included, not because of lack of interest, but because of the near impossibility of locating a significant amount of both recent and ancient literature in libraries accessible to me and also my inability to read it, thus to objectively extract data from it. Synonyms of European species based on material from European Russia, Ukraine, Moldova, Belarus, Latvia, Lithuania, Estonia, Armenia, Georgia, Azerbaidjan, etc. are also given; these have 
not been checked methodically, but only whenever I came across relevant literature. Synonyms of European species based on Asian, North African material are listed when I encountered them and when the synonymy seemed reasonable. Synonyms based on North American material have usually been omitted. In most cases, I suggest that these synonymies should be examined very carefully and should be confirmed by critical revision. The purpose of the inclusion of these extra-European synonyms is to illustrate the geographical dimension of the systematic problems to be solved.

Listed names. I list all names which I found, including infrasubspecific names which are given in their original form. The only names which are not included are those subspecific or infrasubspecific epithets typicus when they are just intended to denote the nominotypical subspecies; such names are usually not nomenclaturally correct and should not be used; in one case (Salmo letnica typicus), this epithet has been 'widely' used and I traced its origin.

Spellings. In the synonymies, all names are given with their original combination (except that interpolated subgeneric names are omitted) and spelling, including misspellings, capitalised letters, and diacritic marks (which have to be emended, ICZN arts. 27, 28, 32(c), 32(d)).

Publication dates. Publication years have usually been accepted as they appear in the publications, unless a different, actual date is well documented. For nomenclatural purposes the actual publication date might differ from the published one, but I did not search exhaustively for effective publication dates; this would have been very time and effort consuming and the expected return justifies it only in cases where respective priority of two names is involved or if one has to determine if a given work appeared before or after some of the dates important to the ICZN. Additionally, dates as they appear in the publication are much more important than actual dates for efficient retrieval on library shelves or interlibrary loans.

Type localities. The type locality is the locality where the holotype, lectotype or neotype was collected. In cases where there is only a series of syntypes, the various localities are listed (separated by a slash [ / ] where clarity required it). In a few cases when the list of localities is too extensive, I have merely given a general description of the localities. Localities are usually given with the original spelling; this sometimes results in the same name being spelt differently under different headings; I have tried to introduce some consistency, but only in cases where I was certain that different authors were really referring to the same place under different spellings. Alternative spellings or modern equivalents are occasionally given in square brackets. Locality descriptions have been translated into English when possible; in some cases, words meaning river, lake, etc. are part of the name in the original language and they have not been deleted in order to avoid ambiguities when using local maps [but the name river, lake, etc. has been added]. Local names have been used, except for a few well known rivers (e.g., Rhine, Danube) and lakes. For larger topographic features which have several different names, a single one has been consistently used; this especially applies to several of the central European lakes; I usually retained the name easiest to find for readers not familiar with local toponymy (e.g., lake Genève instead of Léman, lake Konstanz instead of Bodensee, lake Garda instead of Benaco, lake Maggiore instead of Verbano or lake Locarno).

Type localities are given only for those original descriptions which I have seen personally. For the others I list the 'locality' as indicated in my sources and wait for direct examination before confirmation of the type locality. Infrasubspecific names and nomina nuda having no nomenclatural status, they do not have a type locality and I thus list only a 'locality'. Localities are usually not indicated for infrasubspecific names based on aberrant specimens; they are given only if the name has been created for a particular geographical form.

FYROM stands for Former Yugoslavian Republic of Macedonia.

Primary types. Primary types only are listed. Primary types are holotype, lectotypes, neotypes and syntypes; holotypes are recognised only if explicitly designated as such (alternatively as 'type', singular) by the original author, or if there is clear evidence that the author based the species on a single specimen. In all other cases, type specimens are syntypes. References to the designation of lectotypes or neotypes are given. Paratypes and paralectotypes are not listed as they have no significance as name-bearing types. Other 'type' categories (e.g., allotypes, topotypes, paratopotypes, paraneotypes) are not recognised by the ICZN, should not be used and are ignored. When known, institutions where the primary types are deposited have been listed, together with accession register number. The source for the catalogue number is 
given whenever it is not the original description; data on material in NMW, ZMB and NRM have been provided by B. Herzig, H.-J. Paepke and S. O. Kullander, respectively. When there is a series of syntypes, I listed those which I could trace in the literature, but made no effort to trace the whole series; this would have been tedious, a good number of them having been used for later exchanges between institutions, etc. NT indicates that there is apparently no preserved type material, $\mathrm{LU}$ that there was apparently type material but that the location is not known. A question mark (?) following the acronym indicates that the material is probably deposited in this institution but that it should be confirmed. Institution acronyms follow those in Kottelat et al. (1993) which are those provided by the concerned institutions; for institutions for which no acronyms have been given, acronyms follow current use by workers at that institution (when possible) or Leviton et al. (1985; Leviton \& Gibbs, 1988). (I did not follow Leviton et al. as a standard because for non-US collections, the acronyms they list often are not those actually used by the institutions themselves). The present status of collections earlier stated to be in Ferrara, Pavia (Italy), Istambul (Turkey), Metz, Poitiers (France), and Valencia (Spain) is not known.

AMNH, American Museum of Natural History, New York, USA;

ANSP, Academy of Natural Sciences, Philadelphia, USA;

BELUM, Ulster Museum, Belfast, U.K.;

BFSU, Department of Hydrobiology and Ichthyology, Biology Faculty, Sofia University, Sofia, Bulgaria;

BMNH, Natural History Museum (formerly British Museum, Natural History), London, U.K.; BZF, Bioloski Zavod, Titograd, Montenegro;

CUP, Charles University, Praha, Czech Republic; DZAUT, Department of Zoology, Aristotle University, Thessaloniki, Greece;

EAWAG, EAWAG Forschungszentrum für Limnologie, Kastanienbaum, Switzerland;

GNM, Naturhistoriska Museum, Göteborg, Sweden;

HZM, Hrvatski Zooloski Muzek, Zagreb, Croatia; IMNH, Iceland Museum of Natural History, Reykjavik, Iceland;

ISBB, Institutul Stiinte Biologice, Bucuresti, Romania;

ISNB, Institut Royal des Sciences Naturelles, Bruxelles, Belgium;

IZA, Dipartimento di Scienze Ambientali, Universita, L'Aquila, Italy;
IZPAN, Zoology Institute, Polish Academy of Sciences, Warszawa, Poland;

LJG, Landesmuseum Joanneum, Graz, Austria;

LSL, Linnean Society, London, U.K.;

MB, Museu Bocage, Lisboa, Portugal;

MCZ, Museum of Comparative Zoology, Cambridge, USA;

MCVR, Museo Civico di Storia Naturale, Verona, Italy;

MGAB, Museul de Istorie Naturala 'Gr. Antipa', Bucuresti, Romania;

MHNG, Muséum d'Histoire Naturelle, Genève, Switzerland;

MHNN, Musée d'Histoire Naturelle, Neuchâtel, Switzerland;

MMB, Moravské Muzeum, Brno, Czech Republic; MMNHS, Macedonian Museum of Natural History, Skopje, FYROM;

MNCN, Museo Nacional de Ciencias Naturales, Madrid, Spain;

MNH, Magyar Nemzeti Múzeum, Budapest, Hungary;

MNHN, Muséum National d'Histoire Naturelle, Paris, France;

MNHV, Museum of Natural History, Varna, Bulgaria;

MNHW, Museum of Natural History, University, Wroclaw, Poland;

MSNG, Museo Civico di Storia Naturale, Genova, Italy;

MSNM, Museo Civico di Storia Naturale, Milano, Italy;

MZH, Zoological Museum, Helsinki, Finland;

MZUT, Museo Zoologico, Universita, Torino, Italy;

NMC, National Museum of Canada, Ottawa, Canada;

NMI, National Museum, Dublin, Ireland;

NMNHS, National Museum of Natural History, Sofia;

NMW, Naturhistorisches Museum, Wien, Austria;

NRM, Naturhistoriska Riksmuseet, Stockholm, Sweden;

OLL, Oberösterreichisches Landesmuseum, Linz, Austria;

RMNH, Nationaal Natuurhistorisch Museum (earlier Rijksmuseum van Natuurlijke Historie), Leiden, Netherlands;

SMF, Forschungsinstitut Senckenberg, Frankfurt, Germany;

SMNS, Staatliches Museum für Naturkunde, Stuttgart, Germany;

SNM, Slovenské Národné Múseum, Bratislava, Slovakia; 
USNM, National Museum of Natural History, Washington, USA;

UUZM, Uppsala University, Zoological Museum, Uppsala, Sweden;

ZISP, Zoological Institute of the Academy of Sciences, St. Petersburg, Russia;

ZMB, Zoologisches Museum, Humboldt Universität, Berlin, Germany;

ZMH, Zoologisches Museum, Hamburg, Germany; ZMMSU, Zoological Museum, Moscow State University, Moscow, Russia;

ZMT, Georgian State Museum (earlier Caucasus Museum), Tbilisi, Georgia;

ZMUC, Zoologisk Museum, København, Denmark;

ZSM, Zoologische Staatssammlung, München, Germany.

Infrasubspecific names. For infrasubspecific names, I have indicated the locality stated by the original author, but have not listed material. Even if these have been called type localities and types, as the names are not valid (in the sense of the ICZN), these type localities and types have no nomenclatural validity.

Whenever I found an infrasubspecific name validated by a subsequent use as a subspecies I noted it; if such subsequent uses are not listed, it does not mean there is none, just that I did not find one. Most of these 'validations' have been accidental (the authors were not aware of the nomenclatural implications of their decisions) and appeared in non-systematic publications and usually escaped indexing. A search through the whole ichthyological literature was simply not feasible during the time available. An other obvious bias of this search is that I have had only a limited access to eastern European (especially Russian) literature.

Stocks. In discussions, I generally use the neutral word 'stock' to designate groups of individuals whose taxonomic status or entity is not ascertained. This has the advantage of avoiding confusion with other categories regularly used in systematic discussions, like population, species, form, etc.

Vernacular or common names. Common names are not listed, because there is no list of standard and accepted vernacular names for European fishes. Lists of common names exist for some countries (e.g., USA and Canada, see Robins et al., 1991) but it is not certain that this is easily possible (or really needed) for Europe. While it makes sense in a large area with one or two official lan- guages to have a single common name for each species, in the European context (with some 25 official languages) it is less obvious. If the need arises, I feel that this should be achieved by a representative society or committee, and European ichthyologists would be advised to establish a list by themselves before some list be imposed on them from outside or, worst, by bureaucrats. At present, the most universal common names are still the scientific names. Blanc et al. (1977) provides a list of local names in most European languages for the species then recognised.

I only occasionally use vernacular names in this text. To follow English usage in scientific literature, common names are not capitalised; when non-English local names are used, they are consistently without capital initial, even if derived from languages (e.g., German) which require that nouns be capitalised. Vernacular names are only used where this makes comprehension easier or removes risks of ambiguities. The nomenclature of species of Coregonus has long been so confused that most workers still distinguish them mainly by their vernacular names and in order to clarify the link with earlier works, I usually give the main local names in the various lakes; these local names should be used carefully because a single name may be used in different lakes for different species, or different names may be used in different places for a single species.

For species with restricted distribution for which a single, unambiguous name is locally used, this name should be retained as vernacular name, unless a well accepted English name already exists.

Linnaean species. The synonymy of species described by Linnaeus (Linnaean species) and other early authors present particular problems. In his Systema naturae, Linnaeus (1758) based most species on earlier accounts by himself or others. These have been traced and the exact bibliographic references are given. Those titles I could access have been examined; very often this actually creates additional problems because these authors themselves refer to older publications, etc. I have usually not included such 'secondary' citations. Additionally, several of these earlier works exist in various editions and Linnaeus's bibliographic references are not detailed enough to decide which one he used; for example, I have had the rare opportunity of examining side by side nine different editions of Gesner's Nomenclator aquatilium and Fischbuch but could not find all texts to which Linnaeus referred (it might be best to use Linnaeus's own copies in the library of the Lin- 
nean Society of London; A. Wheeler, pers. comm., 1996). As these books usually are considered to be antiquities or collector items, interlibrary loans or photocopies are not possible; to examine and compare various editions would mean travelling to many different libraries and quite some time and money and it was beyond the limits of the present work. The utility of the exercise also is not obvious in most cases. In such instances, I merely list the reference as given by the original author. References to unpublished data are omitted as they are not relevant for nomenclatural purposes. Type localities listed are those given by Linnaeus; but the actual type localities are those listed by the authors on which Linnaeus based his accounts. Where holotypes are extant, or if lectotypes or neotypes have been designated the localities of these specimens of course becomes the type localities. To identify Linnaeus's type specimens, one has to follow about the same procedure, that is to find the specimens on which the accounts cited by Linnaeus are based (for examples, see Wheeler, 1958, 1985, 1991; Fernholm \& Wheeler, 1983). This is a very tedious and time consuming task; unless already documented in the literature,
I made no attempt to this, but obviously this work has to be done for some of the Linnaean species in order to stabilise their nomenclature and clarify their identity.

Systematic and nomenclatural notes. I have distinguished between comments on systematics and comments on nomenclature. I have found that a frequent source of confusion in earlier works resulted from the mixing in the same discussion of two completely different topics, species and names. Comments on systematics relate to species (their number and distinctness, their relationships, their generic position, their distribution, etc.). Comments on nomenclature relate to names (their validity, their authors, their publication date, their name-bearing types, their type localities, etc.). Keeping the two issues distinct eases the discussion and also draws the line between biology (on which research should focus) and nomenclature (a marginal, but necessary concern). Systematic problems should be resolved before addressing nomenclatural aspects. The resolution of systematic problems is independent of nomenclatural issues, while nomenclature depends of systematics.

\section{Petromyzontidae}

1.1.1 Eudontomyzon danfordi Regan, 1911

Lampetra minor Grossinger, 1794: 207 (not binominal, name not available)

Eudontomyzon danfordi Regan, 1911a: 200 (type locality: Transylvania and river Sebés, Romania; syntypes [total 13]: BMNH 1951.5.22:1-5 [6], Renaud \& Holčík, in Holčík, 1986: 147)

Lampetra Bergi Vladykov, 1925a: 251 (type locality: not explicitly stated, but can be assumed to be the upper Tisza basin, Ukraine; syntypes: LU [about 100])

Lampetra (Eudontomyzon) gracilis Kux, 1967: 294

(type locality: stream Topla, Lukov [river Tisza

basin, Slovakia]; holotype: MMB 1247/7)

Systematic notes. Eudontomyzon gracilis is treated as a synonym of $E$. danfordi by Renaud \& Holčík (1988: 127).

1.1.2 Eudontomyzon hellenicus Vladykov, Renaud, Kott \& Economidis, 1982

Eudontomyzon hellenicus Vladykov, Renaud, Kott
\& Economidis, 1982: 2898, fig. 1 (type locality: brook Kefalárion, a tributary of river Struma, near Kefalárion, Greece; holotype: NMC 771773)

Systematic notes. This species has earlier been recorded with a disjunct range; it appears that two species might be involved (Economidis, 1995: 201).

\subsubsection{Eudontomyzon mariae (Berg, 1931)}

? Petromyzon ponticus Yashchenko, 1895: 95 (nomen nudum; Noworossijsk, Russia) from Berg, 1911: 40

Lampetra mariae Berg, 1931: 94 (type locality: river Kharkov near Kharkov, river Donets basin, Ukraine; syntypes: ZISP 23124 [36])

Systematic notes. See Eudontomyzon danfordi for status of Lampetra minor.

Hubbs \& Potter (1971: 46) mention that the non-parasitic Eudontomyzon (E. vladykovi, E. mariae) may comprise an irregularly distributed complex of variable forms. Eudontomyzon vla- 
dykovi is considered as a valid species by Hubbs \& Potter (1971: 45) and Holčík (1963). Holčík \& Renaud (in Holčík, 1986: 166, 174) treat it as a synonym of $E$. mariae but without giving arguments; they further comment that three subspecies might be recognised after further studies: E. m. mariae (Neman and Vistula basins, and Black Sea drainage), E. m. vladykovi (Danube basin) and E. m. stankokaramani (Drin and Vardar basins). Considering that they are diagnosable and have discrete and disjunct distributions, I treat them as valid species. Contrary to Holčík \& Renaud (in Holčík, 1986: 172), I cannot treat them as a cline because they are discrete populations without gene flow. I am willing to admit a relation between temperature (as inferred from latitude) and number of myomeres if it is demonstrated for a given population, but not as a rule or implied from data for another species.

The trunk myomere counts of E. stankokaramani only slightly overlap those of E. vladykovi (58-62, mean 60.2, vs. 61-67, mean 63.1) and only marginally overlap those of E. mariae (6273, mean 66.1) (data extracted from Holčík \& Renaud, in Holčík, 1986: 169). The only 'E. mariae' population whose myomere counts overlap to some extent with E. stankokaramani is the Kuban one (59-65, mean 61.8). River Kuban is on the northern slope of the Caucasus, at the eastern extremity of the range of E. mariae. The population of the next basin to the west, the Donets, has very distinctive counts (64-73). To me, the distribution of these counts is not suggestive of a single, variable species, but more of the existence of four species with discrete and disjunct distributions. There is apparently no name available for the Kuban species.

The main weakness of the present argument is the same as can be used against Holčík \& Renaud (in Holčík, 1986): these counts are compiled from many different sources and there is no certainty that all have been obtained with the same method. Awaiting better data, we have no choice but to use them. Information on additional characters would be needed, too.

\subsubsection{Eudontomyzon stankokaramani Kara- man, 1974}

Eudontomyzon vladykovi stankokaramani Karaman, 1974b: 2, fig. 2 (type locality: brook Istočka Reka near Istok (vicinity of Peć) and brook Rastavički Pateek near Peć, tributaries of the river Beli Drim, Serbia; holotype: LU)
Systematic notes. See Eudontomyzon mariae for discussion of systematic status.

\subsubsection{Eudontomyzon vladykovi Oliva \& Zanandrea, 1959}

Eudontomyzon danfordi vladykovi Oliva \& Zanandrea, 1959: 2 (type locality: river Danube near Čilistovo, Bratislava, Slovakia / Linz, Prater Krieau, Grünau [Wien], Graz, Styrien, Austria / river Drava [Croatia] / lake Maksimir, Zagreb, Croatia / Sava and Drava basins, Serbia / stream Ljubjana in Ljubljana, Slovenia / creek Topla, Tibisco [Tisza] basin near Lukov, Slovakia; syntypes [total 348, see list in Zanandrea, 1959: 239]: CUP 4525 [1, Cilistovo], other specimens in CUP, MCVR, OLL, NMW 50013 ? [1], 50020 [1], 76823:1 [1], LJG, ? HZM)

Systematic notes. See Eudontomyzon mariae for discussion of systematic status.

Nomenclatural notes. According to Holčík (1963), the paper by Oliva \& Zanandrea (1959) appeared before Zanandrea's (1959) original 'description' of Eudontomyzon danfordi vladykovi and the name is thus available from this earlier paper. Oliva \& Zanandrea referred explicitly to a specimen from the Danube river in Cilistovo, Slovakia and illustrated a specimen from Graz, Austria; they also explicitly mentioned having examined lampreys from the Danube basin in Austria and Yugoslavia for which they state having used the name $E$. $d$. vladykovi; there is a reference to Zanandrea (1959) which was then in press. Finally (p. 4) there is mention of one more locality, Lukov. No holotype is clearly stated and thus all specimens included by Oliva \& Zanandrea constitute the type series (ICZN art. 72(b)(i)), that is the Cilistovo and Graz specimens as well as those which Zanandrea (1959a) had examined. Holčík (1963: 57) designated a neotype but this designation is not valid because it does not fulfil the conditions of ICZN art. 75(d), especially condition (iii), the demonstration that all the syntypes are lost. Holčík examined the Čilistovo specimen which is a syntype; the argument that the oral disc is damaged (although critical for an accurate identification) is nomenclaturally irrelevant to discard this specimen as a syntype.

\subsubsection{Eudontomyzon (?) sp.}

Systematic notes. According to Holčík \& Renaud (in Holčík, 1986: 174), the large (up to 280 $\mathrm{mm}$ TL), anadromous, parasitic lamprey from the Prut (the river making the border between Romania and Ukraine), the Dniester and the Dnieper reported by Wajgel (1884: 317) and Zhukov (1965a: 
$73,1965 \mathrm{~b}, 1968, \mathbf{1 9 7 5})$ is a still unnamed species apparently related to $E$. mariae. It is now extinct (Holčík, 1995: 27) and the causes of the extinction are not known. Wajgel's specimens are apparently not preserved (they have not been found in NMW; B. Herzig, pers. comm. 1995).

\subsubsection{Lampetra fluviatilis (Linnaeus, 1758)}

Petromyzon fluviatilis Linnaeus, 1758: 230 (based on Linnaeus [1746: 102, n. 273], Artedi [1738: gen. 64 [99], syn. 89, spec. 99 [64], Petromyzon unico ...], Gesner [p. 597, Lampetra alterum genus; edition not stated, seen in Gesner, 1558: 705, 1563: 181, 1604: 597], Salviano [1554-58: fol. 63, lampetra], Jonston [1649: pl. 24 fig. 6, Pricka], Willughby [1686: 106, Lampetra medium genus] and Ray [1710: 35 , idem] type locality: "in fluviis Europae", restricted here to river Rhine in Basel, Switzerland, by neotype designation; neotype: MHNG 816.18, present designation)

Petromyzon branchialis Linnaeus, 1758: 230 (based on Linnaeus [1746: 102, n. 274], Rondelet [1555: 202, Lampetra parva \& fluviatilis; locality: "tales in Arvernie"], Gesner [p. 598, idem; edition not stated, seen in Gesner, 1558: 706, 1604: 598], Willughby [1686: 104, idem], Ray [1710: 35, idem], and Salviano [1554-58: fol. 63, Lampetra minor]; type locality: "aquis dulcibus Europae", restricted here to river Rhine in Basel, Switzerland; neotype: MHNG 816.18, present designation)

Petromyzon argenteus Bloch, 1795: 74, pl. 415 fig. 2 (type locality: Tranquebar, India [obviously an error]; syntypes: ZMB 4683 [2], Paepke \& Schmidt, 1988: 159)

Pétromyzon pricka La Cepède, 1798: 18 (not latinised, context shows that the name was not used as a scientific name [ICZN art. 11(b) and second example under 11(b)(ii)], name not available)

Petromyzon sanguisuga La Cepède, 1800: 101, pl. 1 fig. 3 (type locality: river Seine, France; syntypes: LU)

Petromyzon Jurae MacCulloch, 1819: 187, pl. 29 (type locality: island Jura, Scotland, U.K.; holotype: LU)

? Petromyzon macrops Blainville, 1825: 7, pl. 2 fig. 2 (type locality: "all large rivers" [of France]; type material: NT ?)

? Petromyzon argenteus Chiereghini, in Nardo, 1847: 111 (type locality: Venezia, Italy; syntypes: LU; junior primary homonym of $P$. argenteus Bloch, 1795: 74)

Ammocoetes communis Gistel, in Gistel \& Brom- me, 1850: 340 (unnecessary replacement name for Petromyzon branchialis Linnaeus, 1758)

Petromyzon Omalii Van Beneden, 1857: 551, pl. figs. 1-3 (type locality: shores of Belgium; syntypes: LU [3])

Petromyzon fluviatilis forma major Smitt, 1895: 1189 (infrasubspecific, name not available)

Lampetra opisthodon Gratzianow, 1906: 18 (type locality: river Neva near St. Petersburg, Russia; syntypes: ZMMSU ?) from Berg, 1907b: 368, 1911: 26

Lampetra fluviatilis f. praecox Berg, 1931: 111 (infrasubspecific, name not available; localities: several localities in rivers Neva and Kavash, lakes Ladoga and Onega, and other rivers falling into the Finnish Gulf, Russia)

Lampetra fluviatilis morpha ladogensis IvanovaBerg, 1966: 565 (infrasubspecific, name not available; locality: lake Ladoga, Russia)

Nomenclatural notes. There is no extant type material for Linnaeus's Petromyzon fluviatilis and P. branchialis (Fernholm \& Wheeler, 1983; Wheeler, 1985, 1989: 157). Linnaeus accounts are also based on earlier authors who are not known to have preserved specimens. Specimen MHNG 816.18 from river Rhine in Basel, Switzerland, is here designated as neotype of both $P$. fluviatilis and $P$. branchialis. Both are simultaneous objective synonyms and as first reviser I retain $P$. fluviatilis as having priority.

According to Hardisty (in Holčík, 1986: 249, 278), Petromyzon branchialis Linnaeus, 1758 includes ammocoetes of both $L$. fluviatilis and $L$. planeri; no supporting data are provided but this seems a possibility. Linnaeus's account is very brief and quite uninformative; this is also true of his bibliographic sources. Earlier authors (e.g., Günther, 1870: 504) had considered $P$. branchialis as conspecific with $L$. planeri. If this were followed, $P$. branchialis would have priority and the name of the present species, the subject of numerous researches, would have to be changed. The above neotype designation definitively rules out such a nomenclatural change.

Berg (1931: 111; 1948: 49) reports as Lampetra fluviatilis $\mathrm{f}$. praecox a stock sympatric with $P$. fluviatilis in streams draining to the Gulf of Finland and southern Norway. They differ apparently by size at reproduction. Berg (1948: 50) seems to suggest that they might be a single breeding population. If it turns out that the praecox characteristics are hereditary traits, it may then be likely that two species are involved; the name praecox would not be available for the small stock because it is infrasubspecific (ICZN art. 45(g)) and it should be 
redescribed. See Vladykov (in Hureau \& Monod, 1973: 4) for further comments.

Petromyzon sanguisuga La Cepède (1800) and P. macrops Blainville (1825) are referred to the present species, although it seems likely that the original material might have been a mixture of $L$. fluviatilis and P. marinus (N. da Costa Pereira, pers. comm.).

\subsubsection{Lampetra planeri (Bloch, 1784)}

Petromyzon Planeri Bloch, 1784: 47, pl. 78 fig. 3 (type locality: creeks of Thüringen, Germany; syntypes: ZMB 4686 [3], Paepke \& Schmidt, 1988: 160)

Petromyzon septoeuil La Cepède, 1802: 667, pl. 15 fig. 1 (type locality: rivers Seine, Epte and Audelle, Pont de l'Arche, France; syntypes: LU ?)

Petromyzon niger La Cepède, 1802: 667, pl. 15 fig 2 (type locality: rivers Seine, Epte and Audelle, Pont de l'Arche, France; syntypes: LU ?)

Petromyzon Plumbeus Shaw, 1804: 263 (unnecessary replacement name for Petromyzon septoeil [sic, septoeuil] La Cepède, 1802: 667)

Petromyzon Bicolor Shaw, 1804: 263 (unnecessary replacement name for Petromyzon niger La Cepède, 1802: 667)

? Petromyzon caecus Couch, 1832: 23, figs. 9-10 (type locality: Cornwall, U.K.; type material: NT?)

? Petromyzon anomalum De la Pylaie, 1835: 526 (nomen nudum; locality: stream Nanson at Fougères, Ille-et-Vilaine, France)

Petromyzon fluviatilis forma minor Smitt, 1895: 1189 (infrasubspecific, name not available)

Petromyzon fluviatilis forma larvalis Smitt, 1895: 1189 (infrasubspecific, name not available)

\subsubsection{Lethenteron camtschaticum (Tilesius, 1811)}

Petromyzon marinus Camtschaticus Tilesius, 1811: 240, pl. 9 (type locality: Jeddo and Yokohama, Japan, by present neotype designation [originally "portus Divi Petri et Pauli Camtschatici" (Petropavlovsk Kamtchatskii, Siberia)]; neotype: ZMB 6475 , present designation)

Lampetra variegata Tilesius, 1811: 247 (not proposed as a new name, not binominal, name not available; locality: "in sinu Bolschaja fluvii prope ostium")

Petromyzon lumbricalis Pallas, 1814: 69 (type locality: Jeddo and Yokohama, Japan, by present neotype designation [originally river Ienissei and Kamchatka, Siberia]; neotype: ZMB 6475, present designation)
Petromyzon borealis Girard, 1858: 377 (available by indication to Petromyzon fluviatilis of Richardson, 1836: 294; type locality: Great Slave Lake, Canada; syntypes: BMNH ?)

Petromyzon Japonicus Martens, 1868: 3, pl. 1 fig. 2 (type locality: Jeddo and Yokohama, Japan; lectotype: ZMB 6475, present designation)

Petromyzon Kameraticus Dybowski, 1869: 948 (nomen nudum)

Petromyzon Ernstii Dybowski, 1872: 220 (type locality: mouth of river Amur, Siberia; syntypes: IZPAN?)

Lampetra mitsukurii major Hatta, 1911: 268, pl. 9 figs. 1-2 (type locality: Sapporo, Japan; syntypes: LU)

Lampetra japonica septentrionalis Berg, 1931: 100, pl. 5 fig. 4 (type locality: river Onega at Podporozhye, Siberia, Russia; syntypes: ZISP $12159[10])$

Lampetra japonica f. praecox Ioganzen, 1935: 3 (infrasubspecific, name not available) from Holčík, 1986: 199

Systematic notes. In the area covered by the present review, L. camtschaticum is recorded only from the Pasvik river which forms the border between Norway and Russia (Holčík, 1986: 207).

Nomenclatural notes. Holčík (1986: 199) treated Petromyzon marinus camtschaticus Tilesius, 1811 as unavailable because the work is not using binominal nomenclature (ICZN art. 11(c)). The sections of the work that I have examined clearly use the binominal systems and new names proposed in it are available. This appears to be the first available name for $L$. japonicum and it has priority. The types are not extant in ZISP (N. Bogutskaya, pers. comm.). Specimen ZMB 6475 (the lectotype of Petromyzon japonicus Martens, 1868) is designated as neotype of P. m. camtschaticus.

According to Holčík (1986: 199, 220), P. lumbricalis Pallas, 1814 is based on ammocoetes of both L. japonicum (material from Kamchatka) and $L$. kessleri (Anikin, 1905) (material from river Ienissei). I could not find any nomenclatural act restricting the name to one or the other species. Such an act is needed and one of these two species has to become a junior synonym of $L$. lumbricalis. Pallas's material of this species is no longer extant (Svetovidov, 1978, 19981; Paepke \& Schmidt, 1988: 159) and there are not even traces of it in ZISP files (N. Bogutskaya, pers. comm., 1995). As first reviser, I designate specimen ZMB 6475 (the lectotype of Petromyzon japonicus Martens, 1868) as neotype of $P$. lumbricalis Pallas, 1814. I prefer to restrict the name $P$. lumbricalis to $P$. japonicus 
because this name is invalid anyway (a junior synonym of $P$. camtschaticum, see above). If $P$. lumbricalis is treated as synonym of $L$. kessleri, both $L$. kessleri and $L$. japonicum would have to change their names. By changing a single name instead of two, the solution adopted here thus creates the smallest amount of nomenclatural changes.

Holčík (1986: 199) listed Petromyzon borealis Girard, 1858 as a senior synonym of $L$. japonicum (Martens, 1868) but did not use it on the ground that it is a nomen oblitum. He did not indicate by whom and when this name has been treated as a nomen oblitum and I could not find such a nomenclatural act. Names can only be rejected as nomina oblita if they have been declared as such between 6 November 1961 and 1 January 1973 (see ICZN art. 79(c)(iii), Glossary 'nomen oblitum') and if they had not previously been used for 50 years. The name has been used by Jordan, Evermann \& Clark (1930: 10; a work reprinted since) and by Hubbs \& Potter (1971: 51) and does not qualify as a nomen oblitum.

\subsubsection{Lethenteron zanandreai (Vladykov, 1955)}

Lampetra zanandreai Vladykov, 1955: 216, pls. 14 (type locality: river Guà de Lonigo, Vicenza, Italy; holotype: NMC 86-0001)

Systematic notes. See also comments by Bailey (1980, 1982) and Vladykov \& Kott (1982).

\subsubsection{Petromyzon marinus Linnaeus, 1758}

Petromyzon marinus Linnaeus, 1758: 230 (based on Artedi [1738: gen. $64[-]$, syn. 90, [descr. 64], Petromyzon maculosus ...], "Bel" [Belon, 1553: 76, Mustela f. lampetra, 1555: 6667, la lamproye], Gesner [p. 590, idem; edition not stated, seen in Gesner, 1558: 696, 1604: 590; figured specimen from Basel, Switzerland], Rondelet [1554: 398, Lampetra; locality: France], Willughby [1686: 105, Lampretra] and Ray [1710: 35, Lampetra]; type locality: Basel, Switzerland, by lectotype designation [original locality information: "in mari Europaeo"]; lectotype: specimen illustrated by Gesner, 1604: 590 , by present designation)

? Petromyzon ruber La Cepède, 1800: 99, pl. 1 fig. 2 (type locality: river Seine, France; holotype: $\mathrm{LU})$
Petromyzon lampetra Pallas, 1814: 66 (type locality: Basel, Switzerland, by lectotype designation; lectotype: specimen illustrated by Gesner, 1604: 590, by present designation)

? Petromyzon Adriaticus Chiereghini, in Nardo, 1847: 109 (type locality: Quarnero [? = Carnero $=$ Kvarner, Croatia (strait between Cres island and Istra)]; syntypes: LU)

Petromyzon maculosus Gronow, 1854: 2 (available by indication to Linné [1766: 394, Petromyzon ore intus papilloso ...], Artedi [1738: gen. $42 \mathrm{n}$. 2 [sic; desc. 64, syn. 90], Petromyzon maculosus ...] and Willughby [1686: pl. G.2, Petromyzon major]; type locality: "in fluminibus majoribus Angliae"; type material: NT)

Petromyzon marinus dorsatus Wilder, in Jordan \& Gilbert, 1883: 869 (type locality: lake Cayuga, New York, USA; syntypes: USNM ?)

Nomenclatural notes. Holčík (1986: 119, 198) considers that Petromyzon lampetra Pallas (1814: 66) is based on both L. japonicum and Caspiomyzon wagneri (Kessler, 1870). If this were correct, it would have to be the valid name for one of these species. Actually P. lampetra is also based on earlier accounts (Marsili, 1726: pl. 1 [Lampetra], Linnaeus, 1766: 394 [Petromyzon marinus], Bloch, 1784: 38 pl. 87 [Die Lamprete (Petromyzon marinus)], Pennant, 1776: vol. 3: 67 n. 27, pl. 8 [Sea-lamprey]). This apparently includes two species as Marsili's Danube species is unlikely to be conspecific with the material of the other authors. As first reviser I restrict the use of the name to Linnaeus's (1766) reference; this is the same text as Linnaeus's (1758: 230) original description of Petromyzon marinus. As there is no type material for both species, the specimen illustrated by Gesner (1604: 590 [page referred to by Linnaeus, 1758]) is designated as lectotype of $P$. marinus Linnaeus, 1758 and $P$. lampetra Pallas, 1814; this makes $P$. lampetra an objective junior synonym of $P$. marinus Linnaeus, 1758 and restricts the type locality. Gesner explicitly states that the illustrated specimen is from Basel, Switzerland. The lectotype is lost (it probably has not been preserved).

Petromyzon maximus in Cuvier (1816: 118) is a misprint for $P$. marinus, but the name has been repeated by several 19th century French authors (e.g., Ogérien, 1863: 374, fig. 87). 


\section{Acipenseridae}

Due to their large size, museum samples of most species of Acipenseridae are rare. As a result, many species descriptions were based on few specimens only and their variability is not very well known. On the other hand, the commercial interest in these species and their peculiar morphology have attracted considerable attention. For various reasons (usually unsuitable concepts and methods), this resulted in only few data usable in a systematic analysis. One of the significant problems (recurrent in eastern European fish systematics) is that authors very rarely compare specimens, but only compare data compiled from a variety of sources, whose consistency can always be doubted.

Many sturgeon stocks are already extinct or seriously endangered. At this stage, it cannot be excluded that the actual number of species could be seriously underestimated. For most 'species', we are badly missing critical, careful and first hand comparisons of material from the various basins, the different seasonal 'races' and the migratory and non-migratory 'races'.

\section{Acipenser baeri Brandt, 1869 introduced}

\subsubsection{Acipenser gueldenstaedtii Brandt \& Ratzeburg, 1833}

Acipenser pygmaeus Reisinger, 1830: 93 (type locality: river Danube, Hungary; syntypes: LU; junior homonym of Acipenser pygmaeus Pallas, 1814: 102)

Acipenser Güldenstädtii Brandt \& Ratzeburg 1833: 13, pl. 3 fig. 2 (description apparently based on several earlier accounts [including Lepechin, 1771b: 161 [Schip-Kostera] and Marsili, 1726: 37 [Antaceus stellatus]], including material from rivers Volga, Ural, Terek, Kura and their main tributaries, Azov Sea, rivers Don, Dnieper and Danube; type material: NT)

Acipenser aculeatus Lovetzki, 1834: 262, pl. 19 fig. 2 (type locality: "fluvius Borysthenes ... locis restrictis Gubern. Catherinoslawl" [river Dnieper in Yekaterinoslav province; Berg, 1911: 247]; holotype: LU; author stated as Fischer, but Lovetzki is actual author of conditions making name available)

Acipenser tücka Brusina, 1902: 49 (listed in synonymy, name not available)

Acipenser medius Brusina, 1902: 50 (listed in synonymy, name not available; author stated to be Heckel, reference not found)
Acipenser brevirostris Brusina, 1902: 50 (listed in synonymy, name not available; author stated to be Heckel, reference not found)

Acipenser macrophthalmus Brusina, 1902: 50 (listed in synonymy, name not available; author stated to be Heckel, reference not found)

Acipense [sic] Güldenstaedtii var. scobar Antipa, 1909: 260, figs. 101-102 (infrasubspecific, name not available; locality: river Danube)

Acipenser Güldenstaedtii var. golis Antipa, 1909: 261, figs. 103-104 (infrasubspecific, name not available; locality: river Danube and Black Sea at mouth of Portitza)

Acipenser Güldenstaedtii var. longirostris Antipa, 1909: 262, fig. 106 (infrasubspecific, name not available; locality: river Danube and Black Sea)

Acipenser Güldenstaedtii var. acutirostris Antipa, 1909: 262, fig. 107 (infrasubspecific, name not available; locality: river Danube)

Acipenser güldenstädti var. tanaica Marti, 1940: 869 (Azov Sea) from Berg, 1948: 84

Acipenser güldenstädti var. colchica Marti, 1940: 869 (river Rioni and pre-estuarine area of river Inguri, Georgia) from Berg, 1948: 84

Acipenser güldenstädti colchicus natio danubica Movchan, 1967: 31 (infrasubspecific, name not available; locality: river Danube)

Systematic notes. See family introduction for general comments. Vlasenko et al. (in Holčík, 1989: 298-305) report biochemical and morphological differences (but do not state explicitly which differences) between various stocks. Migratory and non-migratory 'races' are known from the Danube basin.

\subsubsection{Acipenser naccarii Bonaparte, 1836}

Accipenser lutescens Rafinesque, 1820: 79 (nomen nudum; locality: Old continent)

Acipenser Heckelii Brandt \& Ratzeburg, 1833: 29 (nomen nudum)

Acipenser Naccarii Bonaparte, 1836: [fasc. 1617, p. 377], pl. 129 fig. 2 (type locality: rivers Brenta, Adige, Piave, Livenza, Bacchiglione, Tagliamento / Firenze, Italy; syntypes: ANSP 624-625 [2], Böhlke, 1984: 17)

Acipenser Heckelii Fitzinger, in Fitzinger \& Heckel, 1836: 303, pl. 26 fig. 4, pl. 29 figs. 11-12 (type locality: Adriatic Sea, rivers Po, Etsch [Adige] and Brenta, Italy; syntypes: NMW 76784 [1], 92877 ? [1])

Acipenser platycephalus Bonaparte, 1846: 21 (list- 
ed in synonymy, name not available, ICZN art. $11(\mathrm{c}))$

Acipenser Nardoi Heckel, 1851c: 557, pl. 24 fig. 2 (type locality: Venezia, Italy; holotype: NMW 63076)

Acipenser nasus Heckel, 1851c: 551, pl. 23 (type locality: Venezia, Italy; holotype: NMW 63093)

Acipenser Ladanus Nardo, 1847: 111 (cited in synonymy, name not available), Molin, 1853: 367 (cited in synonymy, name not available)

Acipenser sturionellus Nardo, 1860: 71 (cited in synonymy, name not available)

Acipenser ladanus Ninni, 1872: 33 (unnecessary replacement name for Acipenser naccarii Bonaparte, 1836; could possibly also be treated as available by indication to 9 earlier works)

Acipenser sturionaster Brusina, 1902: 52 (listed in synonymy, name not available; author stated to be Nardo according to Bonaparte, but reference not found)

Nomenclatural notes. Although Bonaparte (1846: 21) indicated Heckel as author of Acipenser platycephalus, this was only a manuscript name (Fitzinger \& Heckel, 1836: 304).

Both Acipenser naccarii Bonaparte and $A$. heckelii Fitzinger appeared in 1836. In his account of A. naccarii, Bonaparte explicitly provides a full bibliographic reference to the description of $A$. heckelii in Fitzinger \& Heckel (1836), and obviously the latter name has priority. As the species is endangered and listed in several national and international legal instruments, the conservation of the commonly accepted name seems justified and Kottelat \& Wheeler (in press b) have petitioned the International Commission on Zoological Nomenclature to conserve the name A. naccarii. Awaiting the decision, the existing usage has to be maintained (ICZN art. 80(a)), that is A. naccarii.

\subsubsection{Acipenser nudiventris Lovetzky, 1828}

? Schypa: Acipenser Gueldenstaedt, 1772: 533 (name sometime listed in synonymy, but not available because not binominal)

Acipenser nudiventris Lovetzky, 1828: 78, pl. 6 fig. 2 (type locality: Aral Sea [Berg, 1948: 66]; type material: LU)

Acipenser glaber Fitzinger, 1832: 340 (available by indication to Marsili [1726: 34, pl. 10 fig, 2, Antaceus glaber]; type locality: river Danube in Austria; syntypes: NMW 91292 [1]; author given as Heckel, but Fitzinger is actual author); see Fitzinger \& Heckel (1836: 270, pl. 25 fig. 3, pl. 28 figs. 5-6) for illustration of possible syntypes Acipenser turritus Brusina, 1902: 61 (published in synonymy, name not available; based on a museum label)

Acipenser nudiventris derjavini Borzenko, 1950 (Caspian Sea) from Sokolov \& Vasilev, in Holčík, 1989: 209

Systematic notes. The type locality of $A$. nudiventris is the Aral Sea. Borzenko (1950, cited by Sokolov \& Vasilev, in Holčík, 1989: 209) considered that the Caspian Sea specimens belong to a distinct subspecies, a conclusion rejected by Sokolov \& Vasilev. However, as their conclusion is based solely on a coefficient of difference, it is irrelevant to a systematic discussion (see p. 18) and inconclusive. The status of the Black Sea specimens is simply not discussed and no data are provided in their table 19. I could not find a critical, first hand comparison of the Black Sea, Caspian Sea and Aral Sea specimens. The hypothesis that more than one species is involved should be envisaged. A detailed comparison of the diadromous and non-migratory 'races' would also be justified.

\subsubsection{Acipenser ruthenus Linnaeus, 1758}

Acipenser ruthenus Linnaeus, 1758: 237 (based on Linnaeus [1746: 102, n. 272; 1754: 54, pl. 27 fig. 2, pl. 28 fig. 1]; type locality: "Habitat in Rutheno; introductus a Rege Friderico I in Maelerum, Sveciae"; holotype: NRM 96)

Sterleta: Acipenser Gueldenstaedt, 1772: 533 (sometime listed in synonymy but not available because not binominal)

Acipenser pygmaeus Pallas, 1814: 102 (type locality: Black, Caspian and White seas, Arctic Ocean, rivers Danube, Mesen, Petschora, Volga, Kama, Yenissei, Ob, Irti and Tom, lakes Ladoga and Neva; syntypes: LU)

Acipenser Kostera Fitzinger, 1832: 340 (available by indication to Lepechin [1771b: 161, Schip-Kostera] and Marsili [1726: 37, pl. 12 fig. 1, Antaceus stellatus, river Danube]; type locality: river Danube at Wien [Vienna], Austria [by neotype designation; original locality: river Danube [Marsili, Fitzinger; restricted by Fitzinger \& Heckel, 1836: 298)]; neotype: BMNH 1898.4.11:26, present designation)

Acipenser marsiglii Brandt \& Ratzeburg, 1833: 352 (type locality not stated [river Ob ?, Siberia, according to Berg, 1911: 201]; holotype: ? ZISP 11825 [1], Berg, 1911: 201)

Acipenser Kamensis Lovetzky, 1834: 262, pl. 16 fig. 2 (type locality: river Kama, Russia; holotype: LU; spelt $A$. camensis on plate, spelling A. kamensis retained by Berg, 1911: 201 as first reviser)

Acipenser Gmelini Fitzinger, in Fitzinger \& Heckel, 
1836: 276, pl. 25 fig. 2, pl. 30 figs. 17-18 (type locality: "Black and Caspian Sea" [general distribution based on earlier records; actual type locality depends of holotype data once it can be located]; holotype: NMW [not yet located, B. Herzig, pers. comm., 1995])

? Acipenser Aleutensis Fitzinger, in Fitzinger \& Heckel, 1836: 285 (based on Pallas [1814: 107, "Acipenser rutheno affinis"; actually "affinem Acip. rutheno"; type locality: island Canaga, Aleutian islands, Alaska; syntypes: LU)

Acipenser ruthenus var. leucotica Brandt, 1851: 13 , pl. fig. 1 (infrasubspecific, name not available; locality: Nizhnyi-Novgorod, Russia)

Acipenser ruthenus var. grisescens Brandt, 1851: 16, pl. fig. 2 (infrasubspecific, name not available; locality: Nizhnyi-Novgorod, Russia)

Acipenser Lovetzkyi Duméril, 1870: 254 (type locality: Russia; syntypes: MNHN [2]))

Acipenser Kankreni Duméril, 1870: 256 (type locality: probably Russia; holotype: MNHN)

Sterledus ruthenus var. sibiricus Dybowski, 1874: 394 (type locality: middle and lower course of river Angara, Siberia; syntypes: IZPAN ?)

Acipenser Jenisensis Herzenstein, in Anonym, 1895: 79 (nomen nudum; locality: Turukhansk Province) from N. Bogutskaya, pers. comm., 1995

Acipenser ruthenicus Brusina, 1902: 63 (published in synonymy, name not available; author stated as Linnaeus, but reference not found)

Acipenser sterlet Brusina, 1902: 63 (published in synonymy, name not available; author stated as Daubenton, but reference not found)

Acipenser dubius Brusina, 1902: 63 (published in synonymy, name not available; author stated as Heckel, but reference not found)

Acipenser ruthenus var. albinea Brusina, 1902: 64 (infrasubspecific, name not available; localities: river Sava at Rugvica, river Kupa at Sisak, Croatia)

Acipenser ruthenus var. birostrata Brusina, 1902: 65 (infrasubspecific, name not available; locality: Croatia)

Acipenser ruthenus var. obtusirostris Brusina, 1902: 66 (infrasubspecific, name not available; locality: river Una, Croatia)

Acipenser ruthenus var. septemcarinata Brusina, 1902: 66 (infrasubspecific, name not available; locality: Budapest, Hungary)

Acipenser ruthenus var. alba Antipa, 1909: 249, pl. 31 fig. 93a (infrasubspecific, name not available)

Acipenser ruthenus var. erytraea Antipa, 1909: 250, pl. 31 fig. 93 (infrasubspecific, name not available)
Acipenser ruthenus var. brevirostris Antipa, 1909: 250, fig. 94 (infrasubspecific, name not available)

Acipenser ruthenus ruzskyi Ioganzen, 1946: 171

(Novosibirsk-Baturino, river Ob basin, Siberia) from Berg, 1949: 1318

Acipenser ruthenus ruzskyi natio baschmakovae Ioganzen, 1946: 173 (Narym, river Ob basin, Siberia) from Berg, 1949: 1318

Nomenclatural notes. Some authors (e.g., Fowler, 1972: 169) considered Acipenser sturio var. kostera as available from Pallas (1814: 96). Pallas's uses of the name kostera was as a vernacular name, not as a scientific name. The name appears in the phrase " $\beta$. Varietas nondum adulta, Kosterâ Rossorum" ( $\beta$. Variety non adult, the kosterâ of the Russians).

"Acipenser rutheno affinis Pallas, 1814: 107" is sometime listed in synonymies. This name is not binominal and thus is not available. It is actually part of a phrase meaning " $A$. aff. ruthenus"; actual phrase is "Affinem Acip. rutheno in sinu maris etc..."

Specimen BMNH 1898.4.11:26, $250 \mathrm{~mm}$ SL, from the Danube in Wien (Vienna), Austria, is designated as neotype for A. kostera Fitzinger, 1832. Fitzinger's original material cannot be located in NMW. The name is available by indication to Lepechin (1771b) and Marsili (1726) and material of these authors is not known to exist. The original description is ambiguous and the nominal species could possibly be interpreted as a senior synonym of $A$. gueldenstaedtii, threatening a well established name for an endangered species listed in several national and international legal instruments. The present neotype designation definitively rules out this possibility. Marsili's material was from the Danube. The neotype conforms to the description of $A$. ruthenus by Sokolov \& Vasiljev (in Holčík, 1989: 227).

Berg (1911: 202) lists specimen ZISP 13549 as holotype of Acipenser ruthenus var. leucotica Brandt, 1851. As the name is infrasubspecific and not available, this specimen has no type status.

\subsubsection{Acipenser stellatus Pallas, 1771}

Acipenser stellatus Pallas, 1771: 460 (type locality: Caspian Sea and tributary streams; syntypes: LU)

Seuruga: Acipenser Gueldenstaedt, 1772: 533 (not binominal, name not available)

Acipenser Seuruga Bonnaterre, 1788: 17 (after Giildenstädt, 1772: 533; type locality: Caspian Sea; type material: NT ?)

Acipenser Helops Pallas, 1814: 97 (type locality: 
Black, Caspian and Aral Seas, rivers Danube, Dnieper, Don, Volga, Ural and Arax; syntypes: LU)

Acipenser ratzeburgii Brandt \& Ratzeburg, 1833: 351 , pl. 1a fig. 3 (description based on Lepechin [1795: 35, pl. A, Acipenser stellatus var.]; type locality: Caspian Sea at the mouth of river Emba; holotype: ZISP 11760 [1], Berg, 1911: 291)

? Acipenser rostratus Brandt \& Ratzeburg, 1833: 29 (published in synonymy, name not available; author stated as Fischer, but reference not found)

Acipenser stellatus donensis Lovetzki, 1834: 263, pl. 19 fig. 1 (type locality: river Don, Ukraine; holotype: LU)

Acipenser stellatus danubialis Brusina, 1902: 59, fig. 3 (type locality: river Danube; syntypes: $\mathrm{LU})$

Acipenser stellatus illyricus Brusina, 1902: 60, figs. 1-2 (type locality: Dalmatia, Montenegro, Albania; syntypes: LU)

Acipenser stellatus stellatus natio cyrensis Berg, 1932c: 65 (infrasubspecific, name not available; locality: river Kura, Azerbaidjan [Berg, 1948: 100])

\subsubsection{Acipenser sturio Linnaeus, 1758}

Acipenser Sturio Linnaeus, 1758: 237 (based on Linnaeus [1746: 101, n. 271, Acipenser corpore tuberculis ...; 1754: 54, pl. 18 fig. 2 1751: 187, Acipenser], Artedi [1738: gen. 65 [-] syn. 91 [descr. 65], Acipenser corpore tuberculis ...], Gronovius [1756: 42, idem]; type locality: "in mari Europaeo"; syntypes: BMNH 1853.11.12:210 [1], NRM LP uncat., Wheeler, 1958: 206, Fernholm \& Wheeler, 1983: 209)

Sturio accipenser Strøm, 1784: 129 (type locality: Eger [in Boskerud District, south west of Oslo], Norway; type material: NT)

Acipenser lichtensteinii Bloch, in Schneider, 1801: 348, pl. 69 (type locality: "habitat in mari germanico"; holotype ?: ZMB 4411)

Accipenser attilus Rafinesque, 1820: 79 (nomen nudum; locality: Old Continent); Bonaparte, 1846: 21 (listed in synonymy, name not available)

Acipenser latirostris Parnell 1838b: 405, pl. 39 (type locality: near Alloa, Firth of Forth and river Tay, Scotland; syntypes: LU [probably only one head preserved]); also 1839a: 137, pl. 4

Acipenser attilus Gray, 1851: 13 (unnecessary replacement name for Acipenser sturio Linnaeus, 1758)

Acipenser Thompsonii Ball, in Thompson, 1856:
245 (published as a junior synonym, name not available [ICZN art. 11(c)]; locality: Corelaine, Donaghadee, Belfast, Cushendall, Dundrum, Dundalk, Carrick-on-Suir, Wexford, rivers Blackwater and Bandon, and Dublin, Ireland)

Acipenser hospitus Kröyer, 1852: 780 (type locality: northern Öresund [sound between Sweden and Seeland, Denmark]; holotype: LU)

Acipenser sturioides Malm, 1861: 618 (type locality: Göta Elf near Göteborg, Sweden; holotype: GNM)

Acipenser Yarrellii Duméril, 1867: 164 (type locality: no data; holotype: MNHN)

Acipenser Fitzingeri Duméril, 1870: 97, pl. 20 fig. 4 (type locality: Russia; holotype: MNHN)

Acipenser ducissae Duméril, 1870: 98, pl. 20 fig. 5 (type locality: Russia; holotype: MNHN)

Acipenser Nehelae Duméril, 1870: 100, pl. 20 fig. 6 (type locality: Russia; holotype: MNHN)

Acipenser podapos Duméril, 1870: 102 (no locality data; syntypes: MNHN [2])

Acipenser Valenciennii Duméril, 1870: 180 (type locality: Atlantic Ocean at Sables-d'Olonne and mouth of river Seine, France; syntypes: MNHN [2])

Acipenser europaeus Brusina, 1902: 54 (published in synonymy, name not available; author stated as Linnaeus, reference not found)

Sturio vulgaris Brusina, 1902: 54 (published in synonymy, name not available; author stated as Rafinesque, reference not found)

Systematic notes. See family introduction for general comments. Seasonal, migratory and nonmigratory 'races' are known. Holčík et al. (in Holčík, 1989: 298-305) report differences between various populations and a detailed comparison is needed. The Baltic Sea population is noteworthy as it differs from the Atlantic and Mediterranean populations in having significantly fewer dorsal and lateral scutes; future research may show that they represent distinct species.

Nomenclatural notes. Rafinesque (1820: 79) listed Acipenser attilus without description and Bonaparte (1846: 21) listed it in synonymy, indicating Rafinesque as author, but without giving references. I could not find other uses of this name in Rafinesque's papers available to me and therefore list the name as nomen nudum; it may have been used in some overlooked Rafinesque's paper. The earliest use of the name making it available is by Gray (1851).

\subsubsection{Huso huso (Linnaeus, 1758)}

Acipenser Huso Linnaeus, 1758: 238 (based on Artedi [1738: gen. $65[-]$, syn. 92 [descr. 65], 
Acipenser tuberculis carens]; type locality: "Habitat in Danubio, fluviisque Imperii Ruthenici"; type material: NT)

Acipenser Beluga Forster, 1767: 354 (type locality: rivers Volga and Yaiki, Russia; type material: NT)

Acipenser Albula Forster, 1767: 354 (type locality: rivers Volga and Yaiki, Russia; type material: NT; listed as an alternative name to $A$. beluga Forster, 1767)

Acipenser Shyp Forster, 1767: 353 (type locality: Bosphore or Sea of Marmara, Turkey, by neotype designation; neotype: NRM 8946, present designation)

? Acipenser Schypa Gmelin, 1788: 1484 (type locality: Bosphore or Sea of Marmara, Turkey, by neotype designation; neotype: NRM 8946 , present designation)

? Acipenser Schypa Bonnaterre, 1788: 16 (type locality: Bosphore or Sea of Marmara, Turkey, by neotype designation; neotype: NRM 8946 , present designation)

Huso ichthyocolla Bonaparte, 1846: 22 (unnecessary replacement name for Acipenser huso Linnaeus, 1758)

Acipenser Vallisnerii Molin, 1853: 366 (type locality: Adriatic Sea, Italy; holotype: LU)

Huso huso maeoticus Sal'nikov \& Malyatskii, 1934: 44 (Azov Sea) from Berg, 1948: 61

Huso huso ponticus Salnikov \& Malyatskii, 1934: 44 (Black Sea) from Berg, 1948: 61

Huso huso ponticus natio occidentalis Salnikov \& Malyatskii, 1934: 45 (infrasubspecific, name not available; locality: western part of Black Sea) from Berg, 1948: 61

Huso huso ponticus natio orientalis Salnikov \& Malyatskii, 1934: 45 (infrasubspecific, name not available; locality: Caucasian coast of Black Sea) from Berg, 1948: 61

Huso huso caspicus Babushkin, 1942: 131 (Caspian Sea) from Berg, 1948: 61

Huso huso caspicus natio curensis Babushkin, 1942: 131 (infrasubspecific, name not avail- able; river Kura, Caspian Sea basin) from Berg, 1948: 65

Huso huso orientalis Lelek, 1987: 55 (available by indication to Salnikov \& Malyatskii [1934]; type locality: Caucasian coast of Black Sea; syntypes: LU)

Systematic notes. See family introduction for general comments. Pirogovskii et al. (in Holčík, 1989: 298-305) report differences between various stocks. Two seasonal races were known from the Danube basin.

Nomenclatural notes. Specimen NRM 8946, $320 \mathrm{~mm} \mathrm{SL}$, from the Bosphore or the Sea of Marmara, Turkey, is designated as neotype for A. shyp Forster, 1767, A. schypa Gmelin, 1788 and A. schypa Bonnaterre, 1788. Forster's original material cannot be located. Gmelin and Bonnaterre's names are based on different literature accounts (Gueldenstaedt, 1772: 532; Gmelin, 1774: 238; Lepechin. 1771b: 54) and the material of these authors is not known to have been preserved (N. Bogutskaya, pers. comm.). The original descriptions are ambiguous and these nominal species could possibly be interpreted as senior synonyms of $A$. nudiventris, threatening a well established name for an endangered species listed in several national and international legal instruments. The present neotype designation definitively rules out this possibility. Material on which Forster's, Gmelin's and Bonnaterre's accounts are based was from the Caspian Sea; no Caspian Sea material could be located in museum collections accessible to me and the present neotype comes from as close as possible. It conforms to the description of Huso huso by Pirogovskii et al. (in Holčík, 1989: 157).

Acipenser schypa is available from both Gmelin (1788) and Bonnaterre (1788). No precise publication date is available and both have to be considered as published on 31 December 1788 (ICZN art. 21(c)(ii)) and they are simultaneous homonyms. As first reviser I retain A. schypa Gmelin, 1788 as having priority. Bonnaterre's account is based on the same source as Gmelin and his species is an objective synonym of Gmelin's species.

\section{Anguillidae}

\subsubsection{Anguilla anguilla (Linnaeus, 1758)}

Muraena Anguilla Linnaeus, 1758: 245 (based on Artedi [1738: spec. 66 [24], gen. 24 [66], syn.
39, Muraena unicolor], Linnaeus [1746: 109, n. 290, idem], Fahlberg [1750: 194]; type locality: "Habitat in Europa; maxima in lacu Cornachio 
Ferrariensi"; type material: NT)

Anguilla Vulgaris Shaw, 1803: vol. 4 (1): 15, pl. 1 (unnecessary replacement name for Muraena anguilla Linnaeus, 1758)

Anguilla vulgaris var. marina Rafinesque Schmaltz, 1810b: 38 (nomen nudum; locality: Sicilia)

Anguilla vulgaris var. fluviatilis Rafinesque Schmaltz, 1810b: 38 (nomen nudum; locality: Sicilia)

Anguilla vulgaris var. lacustus Rafinesque Schmaltz, 1810b: 38 (nomen nudum; locality: Sicilia)

Anguilla acutirostris Risso, 1826: 198 (type locality: not stated, probably around Nice, France; type material: NT)

Anguilla latirostris Risso, 1826: 199 (type locality: not stated, probably around Nice, France; type material: NT)

Anguilla mediorostris Risso, 1826: 199 (type locality: not stated, probably around Nice, France; type material: NT)

Anguilla fluviatilis Anslijn, 1828: 226, pl. 93 (unnecessary replacement name for Muraena anguilla Linnaeus, 1758)

Muraena oxyrhina Ekström, 1832: 287 (type locality: Mörkö Island [near Stockholm], Sweden; syntypes: LU)

Muraena platyrhina Ekström, 1832: 287 (type locality: Mörkö Island [near Stockholm], Sweden; syntypes: LU)

Muraena [anguilla] var. macrocephala De la Pylaie, 1835: 529 (type locality: shores of Atlantic Ocean, France; type material: NT ?)

Muraena [anguilla] var. ornithorhyncha De la Pylaie, 1835: 529 (type locality: shores of Atlantic Ocean, France; type material: NT ?)

Muraena [anguilla] var. oxycephala De la Pylaie, 1835: 529 (type locality: shores of Atlantic Ocean, France; type material: NT ?)

Muraena [anguilla] var. platyura De la Pylaie, 1835: 529 (type locality: shores of Atlantic Ocean, France; type material: NT ?)

Anguilla canariensis Valenciennes, 1843: 88, pl. 20 fig. 1 (type locality: barrancos or deep ravines of Teneriffa, Canaria Is., Atlantic Ocean; holotype: MNHN 3783, Bauchot et al., 1993: 94)

Anguilla septembrina Bonaparte, 1846: 38 (nomen nudum)

Anguilla cloacina Bonaparte, 1846: 38 (available by indication to Cuvier [1829: 349, pimperneaux]; type locality: France; type material: NT ?)

Muraena Anguilla maculata Chiereghini, in Nardo, 1847: 115 (nomen nudum)

Anguilla marina Nardo, 1860: 73 (nomen nudum)
Anguilla migratoria Kröyer, 1849: 616 (type locality: all over Denmark; syntypes: LU)

Anguilla platyrhynchus Costa, 1850: 50, pl. 58, pl. 60 fig. 3 (type locality: lake Ligola, Italy; syntypes: LU)

Anguilla callensis Guichenot, 1850: 111, pl. 7 fig. 1 (type locality: La Calle, Algeria; syntypes: MNHN 3160 [1], 3220 [1], 3774 [4], Bauchot et al., 1993: 93)

Anguilla Kieneri Kaup, 1856a: 54 (type locality: Toulon, France; holotype: MNHN 3757, Bauchot et al., 1993: 95)

Anguilla Cuvieri Kaup, 1856a: 54 (type locality: Abbeville, France; syntypes: MNHN 3758 [2], 3759 [7], Bauchot et al., 1993: 94)

Anguilla Bibroni Kaup, 1856a: 54 (type locality: Sicilia, Italy; syntypes: MNHN 3760 [1], 3779 [3], Bauchot et al., 1993: 93)

Anguilla Savignyi Kaup, 1856a: 55 (type locality: Napoli, Italy; holotype: MNHN 3131, Bauchot et al., 1993: 97)

Anguilla platycephala Kaup, 1856a: 55 (available by indication to Cuvier [1829: 349, Anguille plat-bec], type locality: Abbeville, France; holotype: MNHN 3210, Bauchot et al., 1993: 97)

Anguilla capitone Kaup, 1857: 34, pl. 3 fig. 17 (type locality: Napoli, Italy; holotype: MNHN 3762, Bauchot et al., 1993: 94)

Anguilla morena Kaup, 1857: 35, pl. 3 fig. 18 (type locality: Napoli, Italy; holotype: MNHN 3763, Bauchot et al., 1993: 96)

Anguilla melanochir Kaup, 1857: 35, pl. 4 fig. 19 (type locality: river Tibre [Tevere], Italy; holotype: MNHN 3764, Bauchot et al., 1993: 96)

Anguilla marginata Kaup, 1857: 36, pl. 4 fig. 20 (type locality: Valencia, Spain; holotype: MNHN 3765, Bauchot et al., 1993: 95)

Anguilla microptera Kaup, 1857: 36, pl. 4 fig. 21 (type locality: Bay of Algesiras, Spain; holotype: MNHN 3766, Bauchot et al., 1993: 96)

Anguilla ancidda Kaup, 1857: 37, pl. 4 fig. 22 (type locality: Sicilia, Italy; syntypes: MNHN 3767 [1], 3780 [1], Bauchot et al., 1993: 93)

Anguilla altirostris Kaup, 1857: 37, pl. 4 fig. 24 (type locality: estuary of river Seine, France; holotype: MNHN 3768, Bauchot et al., 1993: 93)

Anguilla nilotica Kaup, 1857: 40, pl. 5 fig. 28 (type locality: river Nile, Egypt; syntypes: MNHN 3769 [1], 3770 [1], 3771 [1], Bauchot et al., 1993: 96 [Kaup explicitly mentions only two specimens])

Anguilla aegyptiaca Kaup, 1857: 40 (type locality: river Nile, Egypt; holotype: MNHN 3772 [1] or 3773 [1], Bauchot et al., 1993: 93 [Kaup explicitly mentions only one specimen]) 
Leptocephalus brevirostris Kaup, 1857: 150, pl. 18 fig. 15 (type locality: Messina, Sicilia; holotype: $\mathrm{MNHN})$

Anguilla fluviatilis Heckel \& Kner, 1858: 319, fig. 167 (unnecessary replacement name for $\mathrm{Mu}$ raena anguilla Linnaeus, 1758; junior homonym of Anguilla fluviatilis Anslijn, 1828)

Anguilla eurystoma Heckel \& Kner, 1858: 325, fig. 168 (type locality: Dalmatia; holotype: NMW 9284-5?)

Anguilla Hibernica Couch, 1865: 328, pl. 235 (type locality: river Liffey, Ireland; syntypes: LU)

Anguilla oblongirostris Blanchard, 1866: 496 (type locality: stream Huveaune near Marseille, river Lot, and lake Bourget, France; syntypes: MNHN 1880-343 [5], 9985 [1], Bauchot et al., 1993: 96)
Muraena anguilla var. maculata Chiereghini, in Ninni, 1872: 50 (type locality not stated, probably Adriatic Sea; syntypes: LU)

Anguilla Linnéi Malm, 1877: 590 (unnecessary replacement name for Muraena anguilla Linnaeus, 1758)

Anguilla brevirostris Cisternas, 1877: 108 (type locality: Valencia, Spain; syntypes: LU)

Systematic notes. See Smith (in Böhlke, 1989: 41-46) for discussion of status of American ( $A$. rostrata Lesueur, 1817) and European eels.

Nomenclatural notes. Linnaeus (1758: 245) used both spellings angvilla (line 1) and anguilla (line 3 of Muraena caeca account). As first reviser, I retain anguilla as the correct spelling.

\section{Clupeidae}

\subsubsection{Alosa agone (Scopoli, 1786)}

Ciprinus [sic] agone Scopoli, 1786: 71 (type locality: lake Lugano, Switzerland, by neotype designation [originally: "Lacuum insubricorum" (lakes of northern Italy), especially lakes Verbano (Maggiore) and Lario (Como)]; neotype: MHNG 656.48, present designation; description repeated by Vallot, 1837: 279)

Clupea Alosa major Pollini, 1816: 21 (nomen nudum)

Clupea Alosa minor Pollini, 1816: 21 (nomen nudum)

Clupea Alosa parvula Pollini, 1816: 21 (nomen nudum)

Alosa Finta var. lacustris Fatio, 1890: 51 (type locality: lake Lugano, Switzerland, by lectotype designation [originally: lakes of Ticino, Switzerland and Lombardia, Italy]; lectotype: MHNG 656.48 , present designation)

Alosa lacustris forma ceresio-verbana Barbieri, 1907: 140, pl. 1 fig. 4 (type locality: lakes Maggiore and Lugano, Italy and Switzerland; syntypes: MSNM ?)

Alosa lacustris forma lariana Barbieri, 1907: 140, pl. 1 fig. 3 (type locality: lake Como, Italy; syntypes: MSNM ?)

Alosa lacustris forma benacensis Barbieri, 1907: 140, pl. 1 fig. 5 (type locality: lake Garda, Italy; syntypes: MSNM ?)
Alosa finta gracilis Regan, 1916: 10 (type locality: lake Garda, Italy; syntypes: BMNH [3])

Systematic notes. This species has usually been treated as a subspecies or variety of $A$. fallax by earlier authors (e.g., Ferrero, 1951). The differences in morphology, habitat, life history, and ontogeny (compare figures by Chiappi, 1933, reproduced in Hoestlandt, 1991: 263, 287) are strongly indicative that it is a distinct species.

Localities of this species in Sardegna in Quignard \& Douchement's map (in Hoestlandt, 1991: 260) actually are their rhodanensis, part of it tentatively treated here as $A$. algeriensis.

Nomenclatural notes. The earliest available name for this species is Cyprinus agone Scopoli, 1786. Although the format used by Scopoli is rather unusual, it is clearly binominal. The original citation is in the following format and fonts: "CIPRINUS (Agone) lanceolatus, quinqueuncialis, compressus; pinna dorsalis" etc. A comparison of the other fish and bird species described in the same volume clearly shows that Scopoli used the generic names in capital letters followed by the specific name in brackets, and the diagnosis in italics. This is clear by his use of Gadus lota p. 71, Cyprinus phoxinus, C. rutilus p. 72, C. leuciscus, C. barbus p. 73, Perca fluviatilis, Esox lucius, Salmo trutta, and Muraena anguilla p. 74, all species named by Linnaeus (1758). In earlier 
pages, he clearly used the same format, under a more classical heading; e.g., p. 68, under the heading Turbo obtusus, he also has "TURBO (obtusus) testa turrita etc." The description and discussion may not be very accurate (e.g., his placement of the present fish in the genus Cyprinus, or the contradictions in his dorsal-fin ray counts), but his colour pattern description seems unambiguous. As no material of Scopoli is known to be extant, specimen MHNG 656.48 is designated as neotype; this specimen is also the lectotype of Alosa finta lacustris Fatio, 1890 and the two names are objective synonyms.

Svetovidov (1952) lists Pirola as author of 'Alosa finta lariana' without giving a reference; I located a paper by Pirola (1930) dealing with this fish, but published after Barbieri's (1907) original description of A. 1. lariana.

\subsection{2? Alosa algeriensis Regan, 1916}

Alosa finta algeriensis Regan, 1916: 10 (type locality: Algeria and Mogadore [now Essaouira], Morocco; syntypes: BMNH [2])

Alosa africana Regan, 1916: 8 (type locality: Algeria and Mogadore [now Essaouira], Morocco; syntypes: BMNH [2])

Alosa fallax bolivari Lozano Rey, 1929: 660, pl. 30

fig. 3 (type locality: Melilla, Spanish enclave in Morocco; syntypes: LU)

Systematic notes. This taxon was treated as a subspecies of A. fallax by Quignard, Douchement and Kartas (in Hoestlandt, 1991: 210-224). It is apparently distinguished from $A$. fallax (as recognised here) by more gill-rakers on the first gill arch (p. 212): 42-54 in A. algeriensis (but 40-60 on p. 213 ), vs. $31-46$ in $A$. fallax (but $28-60$ in other parts of the text, see account of $A$. fallax below). I tentatively recognise it as distinct from A. fallax.

Kartas (in Hoestlandt, 1991: 217) considered it to be restricted to the Mediterranean coast of Algeria and Tunisia, but the species seems to be present in Sardegna. Quignard \& Douchement (in Hoestlandt, 1991: 277) considered that "the populations [of their rhodanensis] on Sardegna have affinities with those of North Africa, and they probably constitute an independent insular group. Nevertheless, these populations are included in this [account of rhodanensis] because they live within the range of [rhodanensis], which is the northern part of western Mediterranean". They quote data from Cottiglia (1963a-b, 1968, 1970) reporting gill-raker counts on the first arch of $38-$ 54 , which seems to be in agreement with those of A. algeriensis.

Admitting that $A$. algeriensis is a distinct species, I cannot follow them in considering Sardegna as "within the range of [rhodanensis]", their rhodanensis being restricted to the coast of Italy, France, north-eastern Spain, and Corsica. Sardegna is definitively at the edge of this range, and adjacent to the range of $A$. algeriensis. The mentioned Sardegna populations are geographically almost closer to Tunisia than to the nearest rhodanensis population (if a direct distance has a biological meaning). In this context, information on Corsican populations would be of interest.

Nomenclatural notes. Alosa algeriensis and $A$. africana are possibly simultaneous synonyms and Kartas's treatment (in Hoestlandt, 1991: 213) can be considered to be a first reviser's action to determine priority of $A$. algeriensis. Furnestin \& Vincent (1958: 3378) considered Alosa africana to be an hybrid between $A$. alosa and $A$. fallax.

\subsubsection{Alosa alosa (Linnaeus, 1758)}

Clupea Alosa Linnaeus, 1758: 318 (based on Artedi [1738: gen. 7 [34], syn. 15, spec. 34 [7], Clupea apice maxillae ...], Gronovius [1754: 6, n. 23, idem], and Hasselqvist [1757: 388, idem]; type locality: "Habitat in Oceano Europaeo"; syntypes: LSL 48 [1], BMNH 1853.11.12:179 [1], Wheeler, 1958: 207; 1985: 24)

Alosa communis Yarrell, 1836: vol. 2: 136 (type locality: rivers Thames and Severn, U.K.; syntypes: LU)

Alausa vulgaris Valenciennes, in Cuvier \& Valenciennes, 1847: 391 (type locality: "Halles de Paris" [central market of Paris], France; lectotype: MNHN 3676, designated by Whitehead, 1967: 78)

Alosa rusa Mauduyt, 1848: 49 (type locality: river Vienne at Châtellerault, France; syntypes: Musée de Poitiers ?)

Alosa Cuvierii Malm, 1877: 654 (unnecessary replacement name for Clupea alosa Linnaeus, 1758; localities: Hjärbek at Limfjorden, Denmark / Bordeaux, France)

Systematic notes. Boisneau et al. (1992) found no electrophoretic differentiation between $A$. alosa and A. fallax and discussed the possibility that the two are conspecific. Their "morphological analysis" actually considered only gill-raker counts. Their alternative explanation (p. 737) that they are two "species derived from a nearly [electrophoretically] monomorphic" ancestor seems more likely, judging from the differences in distribution, habitat, spawning areas, seasons and mode which they report and the morphological differences reported by earlier authors. There is no theory to request that species have to differ 
electrophoretically or that stocks not distinguishable electrophoretically are conspecific (see also Mayden \& Wood, 1995: 85). The number of characters (and character states) which can be observed, measured or counted by electrophoretic techniques is much smaller than those which are accessible through morphological or anatomical examination. Thus the probability of having related species indistinguishable electrophoretically is greater than of having them indistinguishable morphologically.

Nomenclatural notes. The specimen listed as lectotype of Clupea alosa by Quignard \& Douchement (in Hoestlandt, 1991: 89) is actually the lectotype of Alausa vulgaris.

\subsubsection{Alosa fallax (La Cepède, 1803)}

Alosa ficta Duhamel du Monceau, 1772: 320 (not binominal, suppressed, ICZN, Opinion 859, 1968a: 92)

Clupea fallax La Cepède, 1803: 424, 452 (type locality: river Seine at Rouen, France; neotype: MNHN 3188, designated by Whitehead, 1967: 79)

Clupea rufa La Cepède, 1803: 452 (type locality: river Seine between Pointe du Hode to Aisiers, France; type material: NT ?)

Clupea nilotica Geoffroy Saint-Hilaire, 1808: pl. 10 (type locality: river Nile, Egypt; holotype: LU)

Clupea finta Cuvier, 1829: 320 (type locality: not stated; also available by indication to Clupea ficta [sic, probable lapsus for C. fallax] La Cepède, 1803; syntypes: ? MNHN)

? Clupea Alosa var. elongata De la Pylaie, 1835: 533 (infrasubspecific, name not available; locality: shores of Atlantic Ocean, France)

Clupea sardinella Vallot, 1837: 277 (type locality: lakes of Savoie, France [by present restriction; original type locality included also: Dauphiné, France / lakes Garda, Lugano, Como and Maggiore, Italy]; available by very short diagnosis and indication to numerous earlier authors; type material: NT)

? Alosa minor Bonaparte, 1846: 34 (cited in synonymy, name not available; author given as Risso, but Risso apparently never published this name)

Alosa finta rhodanensis Roule, 1924: 266 (type locality: river Rhône, France; syntypes: MNHN ?)

Systematic notes. The latest synopsis of $A$. fallax is by Quignard, Douchement and Kartas (in Hoestlandt, 1991: 211-296) who recognised six subspecies. Three of them (A. f. fallax, A. f. nilotica, A. f. rhodanensis) are anadromous and widely distributed, one (A.f. algeriensis) is anadromous with a restricted distribution and the remaining two (A.f. lacustris [here A. agone], A.f. killarnensis) have a more restricted distribution in freshwaters. The last three taxa are morphologically distinct, have different ecology and inhabit widely disjunct areas and are here treated as species.

The remaining subspecies are reported to differ in gill-raker counts as follow (p. 212): fallax 33-46 gill-rakers on the first arch (but 35-60 on p. 230), rhodanensis 31-43(46) (but 30-49 on p. 275), and nilotica $31-40$ (but $28-40$ on p. 265). The only other difference explicitly stated is: "members of [rhodanensis] seem to have less numerous, thicker, and coarser gill-rakers than fallax" (p. 274). Awaiting unambiguous and explicit data, it seems reasonable to consider that a single species is involved.

The northern Italian landlocked populations of A. f. nilotica on Quignard \& Douchement's map (p. 268) are not mentioned in the text but seem to be their A.f. lacustris, here treated as a distinct species ( $A$. agone). The northern Italian landlocked populations of $A$. $f$. rhodanensis on their map (p. 279) are not mentioned in the text, but appear to be the same $A$. agone populations. Those from Sardegna, also appearing on their map of $A$. f. lacustris (p. 260), are probably those mentioned in the text as A. f. rhodanensis (p. 277) and discussed here under $A$. algeriensis.

\subsubsection{Alosa immaculata Bennett, 1835}

Alosa immaculata Bennett, 1835: 92 (type locality: Black Sea at Trebizond, Turkey; holotype: LU)

Clupea pontica Eichwald, 1838: 135 (type locality: Black Sea near Odessa, Ukraine; type material: NT)

Clupea Eichwaldii Grimm, 1901: 67 (type locality: Kerch Strait, river Don at Rostow, Kalmius, Russia; syntypes: LU)

Alosa pontica var. nigrescens Antipa, 1904: 300 (type locality: Romanian coast of Black Sea and Danube delta; syntypes: NMW 92939 ? [3]); also Antipa, 1905: 21, pl. 1 figs. 1-3

Alosa pontica var. Danubii Antipa, 1904: 300 (type locality: several localities in western Black Sea and Danube [as far upriver as Braila, Antipa, 1906: 17] and Constantza, Romania; syntypes: NMW 4239 ? [4]); also Antipa, 1905: 17, pl. 1 figs. $4-8$

Alosa pontica var. Russac Antipa, 1904: 301 (type locality: Romanian coast of Black Sea and limans at mouth of Danube [lake Razim, Antipa, 1905: 22], Romania; syntypes: NMW 4247 ? [1]); also Antipa, 1905: 22, pl. 2 figs. 1-5 
Caspialosa pontica var. chtamalocephala Isachenko, 1925: 110 (delta of river Dnieper, Ochakov) from Berg, 1948: 124 (probably infrasubspecific; if so, name is available from Fowler, 1973: 235; from Costa Pereira, in litt.)

Caspialosa pontica var. hypselocephala Isachenko, 1925: 112 (delta of river Dnieper, Ochakov) from Berg, 1948: 124 (probably infrasubspecific; if so, name is available from Fowler, 1973: 236; from Costa Pereira, in litt.)

Alosa kessleri pontica natio borysthenis Pavlov, 1959: 18 (infrasubspecific, name not available; locality: river Dnieper, Ukraine) from Kolarov, in Hoestlandt, 1991: 337

Alosa kessleri pontica natio issatschenkovi Pavlov, 1959: 38 (infrasubspecific, name not available; locality: river Dnieper, Ukraine) from Kolarov, in Hoestlandt, 1991: 337

Alosa kessleri pontica natio moriac Pavlov, 1959 (infrasubspecific, name not available; locality: rivers Danube and Dnieper, Ukraine and Romania) from Kolarov, in Hoestlandt, 1991: 343

Systematic notes. Hoestlandt, Heckman and Kolarov (in Hoestlandt, 1991: 321-387) recognised three subspecies in A. pontica. Nowhere do they provide an argument to justify this status. Different habitats, disjunct distribution and morphological differences suggest that three species are involved: A. pontica in the Black Sea basin and A. kessleri (Grimm, 1887) and A. volgensis (Berg, 1913) in the Caspian Sea basin.

Nomenclatural notes. Kolarov (in Hoestlandt, 1991: 337) listed A. immaculata in the synonymy of A. pontica and Whitehead (1985: 204) included it with a question mark. If the synonymy is true, then the correct name of the species is A. immaculata which has priority. Bennett's (1835) material is apparently no longer extant and a neotype designation is needed.

The publication date of Antipa's subspecies is usually given as 1905 or 1906 , but an abstract of this monograph appeared in 1904, making the names available from this date.

Kolarov (in Hoestlandt, 1991: 342) lists an "A. p. var. moreac (Antipa, 1936)". Antipa (1936: 165) never created such a name. After listing the 3 varieties which he described earlier, he states that he has a fourth one which the local fishermen call moreac. This does not make moreac an available scientific name.

\subsubsection{Alosa killarnensis Regan, 1916}

Alosa finta killarnensis Regan, 1916: 9 (type locality: Killarney, Ireland; syntypes: BMNH [3])

Systematic notes. Considered as a subspecies of
A. fallax by earlier authors. Its discrete distribution, particular ecology and morphological distinctiveness seem to justify that it be considered as a distinct species. It is reported to be distinguished from the adjacent $A$. fallax populations by size, coloration, and gill-raker counts: 43-53 gill-rakers on the first gill arch in A. killarnensis (Quignard \& Douchement, in Hoestlandt, 1991: 212), vs. 33-46 in A. fallax from the Atlantic basin (but see above account of $A$. fallax for different values given by the same authors).

O'Maoileidigh et al. (1988) found no overlap in gill-raker counts between A. killarnensis and an adjacent $A$. fallax populations, but found a high degree of genetic similarity (but still slight differences). They commented that the differences between the two (size and number of gill-rakers) are "thought to be due to environmental selection" and that "retention of the subspecific name deserves serious consideration" but did nor argue further. Under the PSC, A. killarnensis is a distinct species. That selection may have played a role seems a truism under any species concepts and is irrelevant in the present context.

\subsubsection{Alosa macedonica (Vinciguerra, 1921)} Clupea macedonica Vinciguerra, 1921: 323, pl. 4 fig. 2 (type locality: lake Besikia [Volvi], Macedonia, Greece; syntypes: MSNG 11571 [5], Tortonese, 1961: 181)

\subsubsection{Alosa maeotica (Grimm, 1901)}

Clupea maeotica Grimm, 1901: 67 (type locality: Black Sea, Kerch Strait [along Caucasus shores]; neotype: ZISP 32230, designated by Svetovidov, 1952: 254 ?)

Caspialosa brauneri Nikolski, 1923: 5 (Akkerman and Ovidiopol, liman of river Dniester) from Berg, 1948: 119

Caspialosa brauneri m. elongata Issachenko, 1925: 128 (infrasubspecific, name not available; locality: Ochakov) from Berg, 1948: 119

Nomenclatural notes. Kolarov (in Hoestlandt, 1991: 309) reported that Svetovidov (1952: 254) considered a specimen collected on 8-10 December 1948 as the holotype of A. maeotica. This is obviously not possible, the species having been originally described in 1901. I could not check Svetovidov (1952), but in the 1963 translation, he clearly states that Grimm's material is lost and that specimen ZISP 32230 should be regarded as type. Formally, this specimen cannot be the holotype, but is here considered as a neotype designation, even though the word neotype is not used (at least in the translation), as it fulfils the conditions of a 
neotype designation and as ICZN art. 75(a) allows for the use of both the words type or neotype.

\subsubsection{Alosa tanaica (Grimm, 1901)}

Clupea tanaica Grimm, 1901: 67 (type locality: lower reaches of river Don near Rostow, Ukraine; neotype: ZISP 16125, designated by Svetovidov, 1952: 235)

Alosa Nordmanni Antipa, 1904: 301 (type locality: Black Sea and lower Danube [as far upriver as the Iron Gate; Antipa, 1906: 28], Romania; syntypes: NMW 88576 ? [6] [invalid neotype: ZISP 15497, designated by Svetovidov, 1952: 233; locality: river Danube near Vilkov]); also Antipa, 1905: 28 , pl. 2 figs. $6-10$

Caspialosa tanaica palaeostomi Sadovskii, 1934: 135 (type locality: lake Paleostom, Georgia; syntypes: 4, apparently not preserved; neotype: ZISP 31470, designated by Svetovidov, 1952: 231)

Caspialosa palaeostomica Maiorova, 1934: 22 (lake Paleostom, river Moltakva and Black Sea off Poti, Georgia) from Berg, 1948: 136

Alosa bulgarica Drensky, 1934: 79, fig. 1 (type locality: river Resovska south of Burgas, Bulgaria; lectotype: NMNHS 300/2, designated by Karapetkova, in Hoestlandt, 1991: 129 [see comment below])

? Caspialosa tanaica etemi Battalgil, 1941: 171 (type locality: lake Manyas, Turkey; syntypes: LU)

Alosa caspia tanaica natio asovi Pavlov, 1959: 106 (infrasubspecific, name not available; locality: Azov Sea) from Mogilchenko, in Hoestlandt, 1991: 180

Systematic notes. Various authors in Hoestlandt (1991) recognise eight subspecies of $A$. caspia (Eichwald, 1838) [although, Kolarov (p. 156) seems to imply that he recognises only three]. Nowhere do they present arguments to justify recognition of these subspecies or to explain why they are given that rank. There is also no evidence that the different authors are referring to the same fish by the same name. There is no general treatment of the species presenting the relationships between the different 'subspecies'. As the Caspian and Black Sea taxa seem morphologically distinct and occur in disjunct areas, I consider here that C. caspia is restricted to the Caspian Sea; I have not investigated the status of the three Caspian 'subspecies' because the data available to me do not allow it.

The A. c. nordmanni and A. c. tanaica 'subspecies' seem very close and indeed have been considered identical by some authors (e.g., Borcea,
1934: 383, 1936: 26). Kolarov (in Hoestlandt, 1991: 158) stated by which characters they differ, but did not give the character states of both taxa. His table gives values for $A$. c. nordmanni but nowhere in that volume are there similar data for A. c. tanaica. Interestingly, the characters used in the key are different, and the character states supposed to distinguish the two taxa almost completely overlap. In addition, the supposedly diagnostic eye diameter is given as $22-27 \%$ of head length in the key (p. 128) while in the species account it is $18-30 \%$ (p. 157) or $18.0-26.5 \%$ (p. 158) for A. c. nordmanni. Gill-rakers on first gill arch are given as $62-85$ in the key (p. 128) and as $62-$ 85 (p. 182) and 58-93 (p. 185) in the text for $A$. c. tanaica, as 66-88 in the key (p. 128), 66-96 (p. 156) in the text and 60-97 (p. 160) in the table for A. c. nordmanni. Morphometric data cannot be compared because they are given in places as percentages of body length ( = standard length or length to last scales on caudal fin ?), fork length and total length. Alosa c. tanaica occurs in the eastern Black Sea, east of the Crimean Peninsula and A. c. nordmanni west of the Crimean Peninsula; their ranges are adjacent and contiguous. Under these circumstances, I doubt that the two taxa can be distinguished and I consider them as a single species. Alosa tanaica, being the oldest name, has priority.

The 'subspecies' A. c. palaeostomi is supposed to be distinguished from $A$. tanaica by a body depth of $25-31 \%$ of "standard body length" [= SL ?] (vs. 20.5-27 in A. tanaica) (key in Hoestlandt, 1991: 128). But data by Mogilchenko (in Hoestlandt, 1991) are $18.6-21.3 \%$ of "body length" [= SL ?] on p. $172,24.5-30.7$ and $25.2-30.8$ on p. 173 ; figure 42 shows a body depth of about $30 \%$ of SL or about $24 \%$ of total length. The number of gillrakers on the first arch is $73-87$ according to the key (p. 128) and 61-90 according to the text (p. 172). Alosa c. palaeostomi is sympatric with $A$. c. tanaica in most of its range (Mogilchenko, in Hoestlandt, 1991: 186, fig. 51). Much good faith is required to admit the distinctness of the two nominal taxa and awaiting the presentation of coherent and conclusive data, I do not see how they could be distinguished.

Similarly, A. c. bulgarica has a distribution restricted to the Black Sea coast of Bulgaria (Karapetkova, in Hoestlandt, 1991: 132). According to the key (p. 128), it is distinguished from the pair A. c. tanaica - A. c. nordmanni by a deeper body (25-31, vs. $24-28.5 \%$ of "standard body length" [= SL ?]). According to the text, body depth is $25.7-31.7 \%$ of "fork length" in A. c. bulgarica (p. 
130 ) and $20.4-28.3 \%$ of "fork length" in A. c. nordmanni (p. 157) and maximum $24.2 \%$ of "body length" in A. c. tanaica (p. 183). The gill-raker count is reported to be critical in the key (p. 128): 87-107 in A. c. bulgarica vs. $62-88$ in A. c. tanaica - A. c. nordmanni. Again the text data are somewhat different: 81-107, 72-102 and 87-102 in $A$. c. bulgarica (p. 130), 62-85 (p. 182) and 58-93 (p. 185 ) in A. c. tanaica, 66-96 (p. 156) and 60-97 (p. 160 ) in $A$. c. nordmanni. It seems that the number of gill-rakers is somewhat higher in bulgarica, but again the supporting data (and their presentation) is so ambiguous that for the time being it seems justified to consider it as a synonym of $A$. tanaica.

The accounts in Hoestlandt (1991) are very confusing and often not compatible. Most seem to rely heavily (in some cases apparently exclusively) on data compiled from other sources, themselves possibly not even first-hand and compatible. Clearly, the status of all these forms deserves a careful and critical review, based on the actual examination of specimens of all of them by the same researcher, and not by an exploration of the literature.

Data in Economidis \& Sinis (1986) seem to indicate that $A$. vistonica has a distribution disjunct from other $A$. caspia 'subspecies', a different biology and is morphologically distinct. It is therefore treated as a valid species.

Nomenclatural notes. The publication date of Antipa's Alosa nordmanni is usually given as 1905 or 1906, but an abstract of this monograph appeared in 1904, making the names available from this date.

Svetovidov (1952: 233) designated what appears to be a neotype for $A$. nordmanni (see $A$. maeotica for similar discussion). He does not discuss the whereabouts of Antipa's specimens; 6 specimens (NMW 88576) still exist and are likely to be syntypes; therefore Svetovidov's designation seems invalid.

Karapetkova (in Hoestlandt, 1991: 129) designated a lectotype for $A$. bulgarica. The specimen was collected by Drensky on 15 May 1934 while Drensky (1934) explicitly states that he collected material in 1930 and his figure 1 shows a specimen collected on 15 May 1931. I assume that 1934 is a lapsus for 1931 and tentatively consider the lectotype designation as valid, but this requires confirmation.

\subsubsection{Alosa vistonica Economidis \& Sinis, 1986}

Alosa caspia vistonica Economidis \& Sinis, 1986:

729, fig. 3 (type locality: lake Vistonis, Greece; holotype: DZAUT D-351)
Systematic notes. See Alosa tanaica for a general discussion of taxa earlier considered to be subspecies of A. caspia.

\subsubsection{Clupeonella cultriventris (Nordmann, 1840)}

Clupea cultriventris Nordmann, 1840: 522 (type locality: Pont-Euxin [Black Sea]; syntypes: MNHN 3681 [3, Odessa], Whitehead \& Bauchot, 1985: 10)

Clupea delicatula Nordmann, 1840: 524 (type locality: market in Odessa [probably from Black Sea]; lectotype: ZISP 2254, designated by Svetovidov, 1952: 194; junior homonym of Clupea delicatula Bennett, 1831)

Clupeonella delicatula azovi Vladimirov, 1950: 126 (type locality: Azov Sea at Berdyansk Spit; syntypes: LU)

Systematic notes. Kozlovsky and Heckman (in Hoestlandt, 1991: 55, 67, 71) considered C. cultriventris to consist of two subspecies and one "morph". Nowhere do they present a clear argument to justify these status and ranks and their accounts are quite confusing. In addition to the two subspecies and the morph, several "forms" (precoastal, marine, common, lake forms) are mentioned (e.g., p. 58) and it is difficult to make taxonomic sense of much of their data. They seem to present data suggesting that the Black Sea and Caspian populations are morphologically and genetically distinct and that they have discrete and disjunct distribution. Although confusingly presented, the electrophoretic data seem to suggest that the two subspecies are not even each other's closest relatives.

Given this confusing situation, the only objective statement seems to be that the two population are morphologically distinct and occur in different areas. There is no evidence that they are more closely related to each other than to any other species of the genus. In such circumstances, the proposal of a subspecific nomenclature is misleading and it seems justified to consider them as different species. Clupeonella cultriventris is the Black Sea species and C. caspia (Svetovidov, 1941) is the Caspian Sea species; their respective status should be further investigated.

The "morph" tscharchalensis from the Volga basin is distinct from C. caspia by morphological and life history characters, habitat and distribution, and considering the available data it seems to actually be a distinct species. As the name tscharchalensis was originally proposed for a variety by Borodin (1896: 82), it is available with that author and date, despite the fact of having subsequently been treated as an infrasubspecific name. 


\section{Cyprinidae}

Many of the genera commonly recognised among European Cyprinidae (Abramis, Leuciscus, Rutilus, Scardinius, to cite only the main ones) are very poorly established. To simplify, these genera are not properly defined and are justified more because of tradition than as real phylogenetic entities (see, e.g., Bogutskaya, 1994: 618, 1995: 153). Most of these genera remained more or less untouched since the beginning of the 19th century. In the absence of critical phylogenetic studies it is not possible to propose a better classification at this stage and this system is followed here.

It seems obvious that several large genera are composite assemblages, while the recognition of others is artificial. For example, the 'Leuciscus souffia group' could possibly be recognised as a genus distinct from Leuciscus. But the situation is not that simple, and several species occasionally placed in Rutilus, Phoxinellus and Iberocypris have been suggested to be closely related or to belong to this lineage (Bianco, 1988b: 9), but a formal definition has not been proposed. Similarly, the distinction between Scardinius and Rutilus relies on few characters of arguable value; it might well be that Scardinius and some Rutilus are congeneric, but as there are also indications that $R u$ tilus itself might be polyphyletic, there does not seem to be advantages at placing the few species of Scardinius in the presently poorly defined Rutilus.

\subsubsection{Abramis ballerus (Linnaeus, 1758)}

Cyprinus Ballerus Linnaeus, 1758: 326 (based on

Linnaeus [1746: 122, n. 323, Cyprinus ... radiis

40] and Artedi [1738: gen. 3 [23], syn. 12, spec.

23 [3], Cyprinus admodum latus ...; "in Melaro

et aliis Uplandiae lacubus"]; type locality: "in

Europae lacubus"; type material: NT)

Cyprinus Farenus Linnaeus, 1758: 316 (based on

Artedi [1738: spec. [gen.] 23, Cyprinus ... radiis

37 (27 in title, 37 in text)] and Linnaeus [1746:

123, n. 327, idem]; type locality: "in Sveciae

Lacu Maelero"; type material: NT)

Systematic notes. Hensel (1978) considers the present species and A. sapa to constitute a distinct genus Ballerus.

Nomenclatural notes. Cyprinus ballerus and C. farenus are subjective simultaneous synonyms. The first reviser action to determine respective priority apparently is Smitt (1895: 819) who retained C. ballerus. Earlier authors considered C. farenus as a synonym of Abramis brama.

\subsubsection{Abramis bjoerkna (Linnaeus, 1758)}

Cyprinus Bjoerkna Linnaeus, 1758: 326 (based on Linnaeus [1746: 124, n. 328, Cyprinus .. radiis 35] and Artedi [1738: gen. 3 [20], syn. 13, spec. 20 [3], Cyprinus quincuncialis ...]; type locality: "in Sveciae lacubus"; type material: NT)

Cyprinus gieben Wulff, 1765: 50 (type locality: Borussia [Prussia]; syntypes: NT)

Cyprinus Plestya Leske, 1774: 69 (type locality: Leipzig, Germany; type material: possibly LSL 72 ?; Wheeler, 1985: 5, 29)

Cyprinus Blicca Bloch, 1782: 65, pl. 10 (type locality: "in allen unsern Landseen" [in all our interior lakes, thus Germany]; syntypes: ZMB, lost H.-J. Paepke, in litt., 1995)

Cyprinus latus Gmelin, 1788: 1438 (available by description [apparently compiled] and indication to earlier authors, especially Bloch [1782: 65, pl. 10, Cyprinus blicca]; type locality: Germany, Borussia [Prussia], Silesia, Belgium and France; syntypes: ZMB, lost [Bloch's material]) Cyprinus meckel Hermann, 1804: 328 (type locality: Strasbourg, France; type material: NT ?)

Cyprinus gibbosus Pallas, 1814: 324 (type locality: rivers Sura and Volga, Russia; syntypes: LU)

Cyprinus Laskyr Pallas,1814: 326 (type locality: "Tanaï inferiore et fl. Lipewiza regionis Voronicensis [river Don and Seim, a tributary of the Desna; Berg, 1949: 763]; syntypes: LU)

Abramis micropteryx Agassiz, 1835b: 39 (nomen nudum)

Abramis erythropterus Agassiz, 1835b: 39 (nomen nudum)

Abramis micropteryx Valenciennes, in Cuvier \& Valenciennes, 1844: 44 (type locality: not stated; type material: based on a drawing sent by Agassiz; material on which the drawing is based is possibly MHNN 1080 [1, river Rhine ?], 1081 [1, river Rhine ?])

Abramis erythropterus Valenciennes, in Cuvier \& Valenciennes, 1844: 58 (type locality: not stated; type material: based on a drawing sent by Agassiz; material on which the drawing is based is possibly MHNN 1079-B [1, river Rhine ?])

Blicca argyroleuca Heckel, 1843: 1007, pl. 1 (available by indication [ICZN arts. 12(b)(7), 14(b)]; no locality data; syntypes: ? NMW [used as a replacement name for Cyprinus blicca Bloch, 1782 by Heckel \& Kner, 1858: 120])

? Blicca intermedia Fatio, 1882: 355 (type lo- 
cality: river Rhône in Lyon, France; holotype: MHNG 656.16)

Blicca bjoerkna transcaucasica Berg, 1916a: 306 (type locality: lower reaches of the Kura, Araks, Lenkoran district [from Berg, 1949: 767]; syntypes: ZISP ?)

Blicca bjoerkna derjavini Dadikyan, 1970: 550 [of translation] (type locality: river Sevdzhur, basin of river Araks, Armenia; syntypes: LU [30])

Systematic notes. This species was previously considered to belong to a distinct genus, Blicca. Generic position follows Shutov (1969), Hensel (1978) and Howes (1981: 46).

Nomenclatural notes. Abramis micropteryx and $A$. erythropterus as used by Agassiz (1835b) are nomina nuda. Valenciennes (in Cuvier \& Valenciennes, 1844) described them on the basis of drawings sent by Agassiz. The specimens used as models for the drawings are the types of these species. Specimens identified by Agassiz under these names are in MHNN and are potential types; this could be established by comparing them with Agassiz's drawings which possibly are in Cuvier \& Valenciennes's files in MNHN (Pietsch, 1985; Bauchot et al., 1990).

\subsubsection{Abramis brama (Linnaeus, 1758)}

Cyprinus Brama Linnaeus, 1758: 326 (based on Linnaeus [1746: 121, n. 318, Cyprinus ... radiis 27], Artedi [1738: gen. 6 [20], syn. 4, spec. 22 [6], Cyprinus pinnis omnibus nigrescentibus ...; "lacus et stagna pleraque in Suecia"], Gronovius [1754: 3, n. 14, idem]; type locality: "in Europae lacubus"; syntypes: NRM 48 [1], BMNH 1853.11.12:147 [1], Wheeler, 1958: 211)

Abramis microlepidotus Agassiz 1835b: 39 (nomen nudum)

Abramis argyreus Agassiz 1835b: 39 (nomen nudum)

Abramis melaenus Agassiz, 1835b: 39 (nomen nudum)

Abramis vetula Heckel, 1836: 230, pl. 20 fig. 6 (type locality: lake Neusiedler, Austria; syntypes: NMW 55259 [1], 55260 [2], 91131 ? [1])

? Abramis media Koch, in Koch, Herrich-Schäffer \& Forster, 1840: 40 (type locality: stream Naab, Regensburg, Germany; type material: NT ?)

Abramis microlepidotus Valenciennes, in Cuvier \& Valenciennes, 1844: 43 (type locality: river Danube; type material: based on a drawing sent by Agassiz, material on which the drawing is based is possibly MHNN 1060 [1, river Rhine ?])

Abramis argyreus Valenciennes, in Cuvier \& Valenciennes, 1844: 45 (type locality not stated; type material: based on a drawing sent by Agassiz, material on which the drawing is based is possibly MHNN 1061 [2, river Rhine ?])

Abramis vulgaris Mauduyt, 1848: 30 (unnecessary replacement name for Cyprinus brama Linnaeus, 1758)

Abramis gehini Blanchard, 1866: 355, fig. 74 (type locality: river Moselle at Metz, France; lectotype: MNHN 3927, Bertin \& Estève, 1948: 61)

Abramis brama var. sinegorensis Lukash, 1925 (locality: river Kobra, tributary of river Vyatka, Volga basin, Russia) from Hensel, 1978: 137

Abramis brama bergi Grib \& Vernidub, 1935: 112 (type locality: Aral Sea at Muinak / lake Yaskhan in Uzboi / river Sary-su; syntypes: LU; junior primary homonym of Abramis sapa bergi Belyaev, 1929) from Berg, 1949: 774

Abramis brama orientalis Berg, 1949: 774 (replacement name for Abramis brama bergi Grib \& Vernidub, 1935)

Abramis brama danubii Pavlov, 1956: 894 (type locality: limans Yalpug and Khitay, Ukraine; syntypes: LU [at least 63])

Nomenclatural notes. Abramis microlepidotus and $A$. argyreus as used by Agassiz (1835b) are nomina nuda. Valenciennes (in Cuvier \& Valenciennes, 1844) described them on the basis of drawings sent by Agassiz. The specimens used as models for the drawings are the types of these species. Specimens identified by Agassiz under these names are in MHNN and are potential types; this could be established by comparing them with Agassiz's drawings which possibly are in Cuvier \& Valenciennes's files in MNHN (Pietsch, 1985; Bauchot et al., 1990). Valenciennes indicated that A. microlepidotus is from the Danube, but the MHNN specimen is labelled as possibly from the Rhine.

Bertin \& Estève (1948: 61) list a specimen which they claim to be the holotype of Abramis gehini Blanchard, 1866. As Blanchard has not explicitly stated that he had a single specimen, and as it seems possible that he had more than one specimen (although he does not state so) the specimen has to be treated as the lectotype (ICZN art. 74(b)) [I refer to the French version of Art. 74 , which is not equivalent to the English version; "même si un autre" does not mean "should another" but "even if another"].

\subsubsection{Abramis sapa (Pallas, 1814)}

Cyprinus Kleweza Güldenstädt, 1791: 86 (nomen nudum)

Cyprinus Sapà Pallas, 1814: 328 (type locality: streams Sura, Samara, Kinel [tributaries of river 
Volga], Russia; syntypes: LU)

Cyprinus Cleveza Pallas, 1814: 329 (type locality:

"in alveo Tanais versus Maeoticam" [mouth of river Don; Berg, 1949: 780], Russia; syntypes: LU)

Abramis balleropsis Agassiz, 1835b: 39 (nomen nudum)

Abramis schreibersii Heckel, 1836: 227, pl. 20 fig. 4 (type locality: rivers Danube downriver of Wien and March, Austria; syntypes: NMW 16584 [1], 79462 [1], 79463 [1])

Abramis sapa bergi Belyaev, 1929: 80 (river Kura, Caspian Sea basin) from Berg, 1949: 783

Abramis sapa aralensis Tyapkin, 1939: 144 (Aral Sea) from Berg, 1949: 784

Abramis sapa bergi natio ferganensis Maksunov, 1961 (infrasubspecific, name not available; Farkhad reservoir [near Fergana], Uzbekistan) from Opalatenko, 1967: 33

5.2.1 Alburnoides bipunctatus (Bloch, 1782) Cyprinus bipunctatus Bloch, 1782: 50, pl. 8 fig. 1 (type locality: Minden, Germany; holotype: ZMB 3357 [1])

Cyprinus Blochii Nau, 1791: 33 (unnecessary replacement name for Cyprinus bipunctatus Bloch, 1792)

Cyprinus Annoni Walbaum, 1792: 32, 705 (description based Gronovius [1763: 108 n. 341]; type locality: river Rhine in Basel, Switzerland; type material: NT ? [Gronovius's material])

? Cyprinus tinca notha Walbaum, 1792: 33 (type locality: river Rhine; syntypes: material of Sander [1784:' 252])

? Cyprinus sanderi Bloch, in Schneider, 1801: 445 (type locality: river Rhine; syntypes: material of Sander [1784: 252])

? Cyprinus gronovii Bloch, in Schneider, 1801: 446 (type locality: river Rhine in Basel, Switzerland; syntypes: material of Gronovius [1763: 108, n. 341])

Cyprinus spirlin La Cepède, 1803: 499, 588, 595 (unnecessary replacement name for Cyprinus bipunctatus Bloch, 1782: 50, pl. 8 fig. 1)

Cyprinus Punctatus Shaw, 1804: vol. 5 (1): 220 (unnecessary replacement name for Cyprinus bipunctatus Bloch, 1782)

Cyprinus Riemling Hermann, 1804: 320 (type locality not stated [Strasbourg, France ?]; type material: NT ?)

Aspius fasciatus Nordmann, 1840: 497, pl. 23 fig. 2 (type locality: rivers of the east coast of the Black Sea ["torrents et rivières des pays situés le long de la côte orientale du Pont-Euxin et habités par les Tcherkesses, les Chapsoughes, les
Abases et les Mingréliens"]; syntypes: MNHN 3897 [4], Bertin \& Estève, 1948: 79)

Leuciscus Baldneri Valenciennes, in Cuvier \& Valenciennes, 1844: 262, pl. 497 (type locality: based on specimens from lake Genève at Genève, Switzerland, and tentatively on several published and unpublished accounts and figures; syntypes: MNHN 1969 [2], Bertin \& Estève, 1948: 79)

? Alburnus maculatus Kessler, 1859a: 535 (type locality: small streams of Crimea, especially near Salghir / market in Simpheropol, Crimea, Ukraine; syntypes: LU [10])

Alburnus Eichwaldii Filippi, 1863: 392 (type locality: river Kur near Tiflis [Tbilisi], Georgia; syntypes: MZUT 677 [4], Tortonese, 1940: 140) Alburnoides bipunctatus rossicus Berg, 1924: 56 (river Kama basin, a tributary of the Volga, Russia) from Berg, 1949: 757

Alburnus bipunctatus var. prespensis Karaman, 1924b: 54 (type locality: lake Prespa and its tributaries, FYROM and Greece; syntypes: LU)

Alburnus bipunctatus ohridanus Karaman, 1928: 153 (type locality: lake Ohrid, FYROM; syntypes: LU); repeated in 1929: 172

Alburnoides bipunctatus var. smyrnaea Pellegrin, 1927: 37 (type locality: stream Mélès near Smyrna, Turkey; syntypes: MNHN 27-64 [16], Bertin \& Estève, 1948: 80, MSNM 4412 [1], Conci \& Michelangeli, 1974: 223))

Alburnoides bipunctatus rossicus natio kubanicus Berg, 1932a: 150 (infrasubspecific, name not available; river Kuban); has priority over Berg, 1932c: 493

Alburnoides bipunctatus tzanevi Chichkoff, 1934: 375, fig. 1 (type locality: river Riesova, entering Black Sea at $42^{\circ} \mathrm{N}$, Bulgaria; syntypes: LU [at least 96])

Alburnoides bipunctatus strymonicus Chichkoff, 1940: 113, 141, pl. 1 fig. 3, pl. 2 fig. 6 (type locality: river Toplitza, a tributary of river Mesta / river Struma, Bulgaria; syntypes: LU [45])

Alburnoides bipunctatus v[ar]. thessalicus Stephanidis, 1950: 204 (type locality: rivers Pinios and Sperchios, Greece; syntypes: lost?)

Alburnoides bipunctatus armeniensis Dadikyan, 1972: 520 [of translation] (type locality: rivers Arpa, Vorotan, Vedi, Marmarik, Kasakh and their tributaries, basin of river Araks, Armenia; syntypes [total 434]: ZISP 37502)

Systematic notes. Economidis \& Bănărescu (1991: 261, 264) consider A. b. tzanevi, A. b. strymonicus and $A$. b. thessalicus as valid subspecies. The canonical analysis by Daget et al. (1977) suggests that two groups can be distin- 
guished among Greek populations. Their data (p. 22 ) indicate an almost complete overlap (but with different means) for those characters they consider as most useful to distinguish their subspecies, but it is not clear whether they are diagnosable. So, with the available data, it does not seem that they can be recognised as species under the PSC.

\subsubsection{Alburnus albidus (Costa, 1838)}

Leuciscus albidus Costa, 1838: fasc. 19: 16, pl. 14 (type locality: river Alento near Fasana (SA), Campania, Italy; neotype: IZA 7899, designated by Bianco, 1980: 32)

Leuciscus Vulturius Costa, 1838: fasc. 19: 17, pl. 15 (type locality: lakes Vulture and Monticchio, Italy; syntype: MNHN 195 [1], Bertin \& Estève, 1948: 79)

? Leuciscus cordilla Valenciennes, in Cuvier \& Valenciennes, 1844: 291 (type locality: Italy; holotype: MNHN)

Systematic notes. See Bianco (1980: 33) for discussion of synonymy.

\subsubsection{Alburnus alburnus (Linnaeus, 1758)}

Cyprinus Alburnus Linnaeus, 1758: 325 (based on Linnaeus [1746: 134, n. 330, Cyprinus ... radiis 30], Artedi [1738: gen. 6 [17], syn. 10, spec. 17 [6], Cyprinus quincuncialis pinna ...; Sweden], Gronovius [1754: 3, n. 10, idem; 1746: 75, n. 58, Cyprinus Koning van Asterling; river Rhine]; type locality: "in Europae aquis dulcibus"; type material: NT)

Cyprinus Albor Scopoli, 1786: 73 (type locality: lakes of Insubria, northern Italy [particularly lake Como]; type material: NT)

Cyprinus lanceolatus Bloch, in Schneider, 1801: 446 (type locality: lakes of Insubria, northern Italy [particularly lake Como]; type material: NT [material of Scopoli, 1786: 73, Cyprinus albor])

Aspius ochrodon Fitzinger, 1832: 335 (nomen nudum; locality: river Danube, Austria)

Aspius arborella Bonaparte, 1841: [fasc. 30, p. 253], pl. 116 fig. 5 (type locality: lakes of northern Italy; syntypes: ANSP 6075-6076 [11], Bianco, pers. comm., 1985)

Aspius alburnoides Selys-Longchamps, 1842: 214 (type locality: rivers Vesdre, Ourthe, Moselle and tributaries, Bruxelles, river Escaut, Belgium; syntypes: ISNB 109 [31], MHNN 1087 [2], Kottelat, 1984: 147)

Alburnus alborella Filippi, 1844: 402 (type locality: Verbano [lake Maggiore] and Lario [lake Como], Italy; syntypes: LU)
Alburnus lucidus Bonaparte, 1844: 437 (nomen nudum)

Alburnus Ausonii Bonaparte, 1844: 437 (nomen nudum)

Alburnus avola Bonaparte, 1844: 437 (nomen nudum)

Alburnus strigio Bonaparte, 1844: 437 (type locality: Italy; syntypes: LU)

Alburnus obtusus Bonaparte, 1845: 12 (type locality: "Pannonia"; syntypes: LU)

Alburnus acutus Bonaparte, 1845: 12 (type locality: "Pannonia"; syntypes: LU)

Alburnus scoranza Bonaparte, 1845: 12 (type locality: Montenegro; syntypes: LU)

Alburnus avola Bonaparte, 1845: 12 (type locality: northern Italy; syntypes: ANSP 6066-6073 [8], Böhlke, 1984: 69)

Alburnus gracilis Bonaparte, 1845: 12 (listed in synonymy, name not available); 1846: 33 (nomen nudum)

Alburnus fracchia Bonaparte, 1845: 12 (listed in synonymy, name not available); 1846: 33 (nomen nudum)

Alburnus lucidus Heckel, 1853: 30 (nomen nudum; locality: river Danube, Hungary)

Leuciscus alburnellus Martens, 1857: 179, pl. 9 fig. 6 (unjustified emendation of Alburnus alborella Filippi, 1844)

Alburnus lucidus Heckel \& Kner, 1858: 131, figs. 67-68 (type locality: river Danube near Wien, Aspern and Achau, river Salzach, river Mur near Czakaturn, rivers Sala, Teufelsbach and Czerna, river Stry in Galicia, Siebenbürgen, Mehadia, Krakau, river Töpel near Eger, lake Balaton, Augsburg, river Ocker, Breslau, Pommern, Bergen and Serbia; syntypes: NMW 20701-20708 [8], 55536 [1], 55538 [2], 55540 [2], 55543 ? [2], 55549 [1], 55551 [11], 55564 [1], 55565 [6], 55566 [1], 55567 [3], 55568 [1], 55569 [6], 55601 [1], 55615 [3], 55616 [1], 55736 ? [1], $78073[2])$

Alburnus lucidus var. lacustris Heckel \& Kner, 1858: 134 (type locality: lakes Neusiedler and Balaton, Austria and Hungary; syntypes: NMW 55530 [3], 55531 [3], 55532 [3], 55533 [3], 55534 [2], $55535[3])$

Alburnus breviceps Heckel \& Kner, 1858: 134, fig. 69 (type locality: river Danube [in Wien, Austria]; holotype: NMW 55539)

Alburnus fracchia Heckel \& Kner, 1858: 138, fig. 72 (type locality: Treviso, Italy; holotype: NMW $55522)$

Alburnus scoranza Heckel \& Kner, 1858: 138 (type locality: lake Scutari, Montenegro and Albania; syntypes: NMW 55700 [2]; junior homonym of 
Alburnus scoranza Bonaparte, 1845: 12)

Alburnus scoranzoides Heckel \& Kner, 1858: 139

(type locality: Montenegro; syntypes: NMW $55702[2])$

Alburnus lucidus var. angustior Walecki, 1863: 358

(infrasubspecific, name not available; spelt augustior in Walecki, 1864: 53)

Alburnus lucidus var. latior Walecki, 1863: 358 (infrasubspecific, name not available)

Alburnus mirandella Blanchard, 1866: 369, fig. 80 (type locality: lake Genève, France and Switzerland / lake Bourget, France; syntypes: ? MNHN)

Alburnus fabraei Blanchard, 1866: 370, fig. 81 (type locality: river Rhône near Avignon, France; syntypes: MNHN ?)

Alburnus alborella var. lateristriga Canestrini, 1864: 103 (type locality: Castelfranco, Bolognese, Italy; syntypes: LU [minimum 6])

Alburnus Linnéi Malm, 1877: 568 (unnecessary replacement name for Cyprinus alburnus Linnaeus, 1758)

Alburnus arquatus Fatio, 1882: 433 (proposed in synonymy, name not available; locality: river Rhine, Basel, Switzerland)

Alburnus lucidus var. elata Fatio, 1882: 429 (infrasubspecific, name not available)

Alburnus lucidus var. elongata Fatio, 1882: 429 (infrasubspecific, name not available)

Alburnus lucidus var. oxycephala Fatio, 1882: 429 (infrasubspecific, name not available)

Alburnus lucidus var. colobocephala Fatio, 1882: 429 (infrasubspecific, name not available)

Alborella maxima Fatio, 1882: 449 (type locality: lake Lugano, Switzerland; holotype: MHNG 815.80)

Alburnus lucidus var. ilmenensis Warpachowski, 1886b: 63, fig. (lake Ilmen, Russia) from Berg, 1949: 746

Alburnus alburnus macedonicus Karaman, 1928: 151 (type locality: lake Dojran and river Vardar until downriver of Skopje, FYROM; syntypes: LU); repeated in 1929: 171

Alburnus alburnus [charusini] natio dagestanicus Petrov, 1930: 146, 149 (infrasubspecific, name not available; locality: Caucasus shore of the Caspian Sea)

Alburnus alburnus thessalicus Stephanidis, 1950: 205 (type locality: basin of river Pinios and lakes Viviis and Xiniada, Greece; syntypes: lost ?)

Alburnus alburnus strumicae Karaman, 1955: 184 (type locality: river Strumica, FYROM; syntypes: LU)

Systematic notes. Sce Bianco (1980: 36) for dis- cussion of synonymy of Italian and Balkan populations.

Nomenclatural notes. Contrary to the opinion of Gandolfi \& Zerunian (1990: 298) and Gandolfi et al. (1991: 173) and as correctly pointed out by Bianco (1988a: 151), the name Aspius arborella Bonaparte, 1841 is available and has priority over Alburnus alborella Filippi, 1844. The Code is clear on this matter (see ICZN art. 52) and the case is straightforward. Bonaparte described Italian specimens identified as $A$. alburnus. At the beginning of the account, he stated that he was not sure whether this Italian material was conspecific with the northern European species or should be treated as a distinct species under the name [A.] arborella. The fact that the name was proposed conditionally does not make it unavailable (only names proposed conditionally after 1960 are unavailable; ICZN arts. 11(d)(i), 15). Clearly, it refers to the Italian material just described by Bonaparte and thus the name is available.

If the Italian stock is considered as a species distinct from the northern European one, the earliest available name is Cyprinus albor Scopoli, 1786 .

Böhlke (1984: 69) listed syntypes of Alburnus avola Bonaparte, 1846 (a name actually available from Bonaparte, 1845). Bonaparte indicated the distribution as "Ital. s.", that is Italia septentrionalis [northern Italy], while the assumed syntypes are from "southern Italy", possibly a translation lapsus.

\subsubsection{Anaecypris hispanica (Steindachner, 1866)}

Phoxinus hispanicus Steindachner, 1866d: 268, pl. 1 fig. 1 (type locality: creek entering river Guadiana near Merida, Spain; syntypes: NMW 51168 [4; 3 according to original description])

\subsubsection{Aspius aspius (Linnaeus, 1758)}

Cyprinus Aspius Linnaeus, 1758: 325 (based on Linnaeus [1746: 121, n. 319, Cyprinus ... radiis 16] and Artedi [1738: gen. 6 [14], syn. 14, spec. 14 [6], Cyprinus maxilla inferiore longiore ...; "lacus Melerus in Uplandia"]; type locality: "in Sveciae lacubus"; type material: NT)

Cyprinus Rapax Leske, 1774: 56 (type locality: Leipzig, Germany; type material: NT ?)

Cyprinus taeniatus Eichwald, 1831: 102 (type locality: "in fluvio Cyro prope Mingischaur" [river Kura at Mingetschaur, Azerbaidjan; Berg, 1949: 606]; syntypes: LU)

Aspius vulgaris Leiblein, 1853: 122 (unnecessary replacement name for Cyprinus aspius Linnaeus, 1758) 
Alburnus iblioides Kessler, 1872: 63, pl. 6 fig. 27 (type locality: springs at Jany-kurgan, Syrdarja basin, Kazakhstan; syntypes: LU)

Aspius rapax var. jaxartensis Kessler, 1874a: 27 (type locality: Syr-darja, Kazakhstan; syntypes: LU) from Berg, 1912: 319, 1949: 607

Aspius Linnéi Malm, 1877: 567 (unnecessary replacement name for Cyprinus aspius Linnaeus, 1758)

Aspius erytrostomus Kessler, 1877a: 143 (type locality: river Kura, Caspian Sea, restricted by Berg, 1949: 606; syntypes: LU)

Aspius transcaucasicus Warpachovski, 1895: 29 (river Lenkoranka and lake Bussadagny in Lenkoran district, Azerbaidjan; syntypes: ZISP 10497 [5], 10498 [2], 10488 [2], Berg, 1912: 316) from Berg, 1949: 606

Aspius aspius taeniatus natio iblioides morpha phragmiteti Berg, 1932c: 382 (infrasubspecific, name not available)

Nomenclatural notes. Bertin \& Estève (1948: 78) lists 7 syntypes of "Leuciscus aspius Cuv. Val.". Valenciennes (1844: 265), under the heading "Leuciscus aspius, nob.", did not describe a new species but merely made a new combination of Cyprinus aspius Linnaeus, 1758, which is explicitly mentioned p. 266. Thus the material listed by Bertin \& Estève has no type status.

\subsubsection{Aulopyge huegelii Heckel, 1842}

Aulopyge Huegelii Heckel, 1842: 74 (type locality: Dalmatia, Croatia; syntypes: NMW 55723 [11], MHNN 946 [1], Kottelat, 1984: 148)

Systematic notes. See Howes (1987) for a discussion of anatomy and phylogeny.

Nomenclatural notes. Although the generic name Aulopyge is available from Heckel (1841a: 384 [published 30 April 1841]; also 1841b: 523 [published 1842 according to Eschmeyer, 1990]), the species name $A$. huegelii is only available from his 1842 description. In the 1841 paper, Heckel only mentioned that the species would be named for Hügel, but did not use the name huegelii. The description is repeated in Heckel (1843: 1021).

\subsubsection{Barbus albanicus Steindachner, 1870} Barbus albanicus Steindachner, 1870: 630, pl. 3 fig. 1 (type locality: lake Scutari, Albania; syntypes: NMW 53651 [2], MSNG 9043 [2])

Nomenclatural notes. The type locality is indicated as lake Scutari in the original description, but later assumed to be a mistake and corrected to lake Janina by Steindachner (1896: 184).

\subsubsection{Barbus barbus (Linnaeus, 1758)}

Cyprinus Barbus Linnaeus, 1758: 320 (based on Artedi [1738: gen. [spec.] 4, syn. 8, Cyprinus maxilla superiore longiore ...] and Gronovius [1754: 5, n. 20. idem; 1756: 3, n. 20, idem]; type locality: river Ijssel at Deventer, Netherlands [by lectotype designation; original locality: "in Europa australis"]; lectotype: BMNH 1853.11.12:144, by present designation)

Barbus vulgaris Fleming, 1828: 185 (type locality: "English rivers"; available by description and by indication to earlier sources; type material: NT ?)

Barbus communis Perty, 1832: 718 (unnecessary replacement name for Cyprinus barbus Linnaeus, 1758)

Barbus fluviatilis Fitzinger, 1832: 333 (unnecessary replacement name for Cyprinus barbus Linnaeus, 1758; author indicated as Agassiz, but Fitzinger is actual author)

Barbus communis Nordmann, 1840: 472 (unnecessary replacement name for Cyprinus barbus Linnaeus, 1758; junior homonym of Barbus communis Perty, 1832; authorship indicated as Pallas, 1814: 291, but Pallas used Cyprinus barbus)

Barbus Mayori Valenciennes, in Cuvier \& Valenciennes, 1842: 138, pl. 461 (type locality: lake Zug, Switzerland; holotype: MNHN 3822, Almaça, 1969: 1130)

Barbus microphthalmus Bonaparte, 1846: 27 (listed in synonymy, name not available; Heckel is indicated as author, but it is a manuscript name and Bonaparte is actual author)

Barbus fluviatilis var. tyrasensis Dybowski, 1862: 77 (type locality: river Dniester, Ukraine; available by description and by indication to Kessler, 1856a: 30; syntypes: IZPAN ?)

Barbus fluviatilis var. borysthenicus Dybowski, 1862: 77 (type locality: river Dnieper, Ukraine; available by description and by indication to Kessler, 1856a: 30; syntypes: IZPAN ?)

Barbus fluviatilis var. aurata Fatio, 1882: 249 (infrasubspecific, name not available; locality: confluence of rivers Sarine and Aar, Switzerland)

Barbus fluviatilis var. alba Krauss, 1882: 346 (type locality: river Enz at Lomersheim, BadenWürttemberg, Germany; holotype: SMNS 14332 , Fricke, 1995: 11; could possibly be treated as infrasubspecific)

Barbus fluviatilis var. aurata Veesenmayer, 1884: 325 (type locality: river Danube at Rottenacker, $5 \mathrm{~km}$ SSW of Ehingen, Germany; holotype: SMNS 14331, Fricke, 1995: 11; could possibly be treated as infrasubspecific) 
Barbus barbus gallicus Karaman, 1971: 194 (type locality: Toulouse, France; holotype: NMW 54112)

Nomenclatural notes. See Wheeler (1958: 212) and Fernholm \& Wheeler (1983: 217) for a discussion of the syntypes of $B$. barbus and associated problems. The specimen examined by Linnaeus (1758) is from Spain and those examined by Gronovius (1754: 5), on which Linnaeus's description is also based and one of which is still extant, are from the river Ijssel at Deventer, Netherlands and they are unlikely to be conspecific. Gronovius's specimen BMNH 1853.11.12:144 is here designated as lectotype.

\subsubsection{Barbus bocagei Steindachner, 1865}

Barbus Bocagei Steindachner, 1865: 3 (type locality: river Mondego in Coimbra, river Collares in Cintra, river Tejo in Lisboa, Portugal; syntypes: MB ?, NMW 3362-3368 [7], 5311 [1], 5876 [1], 18580 [2], 53763 [2], 53779-788 [18], 53791-797 [19], 53799-823 [more than 47], 53825-838 [more than 21], 53840-844 [11], 53846-849 [11], 53876876 [3], 53903-904 [6], 53906-907 [5], 53913-915 [6], 53924-932 [11], 53936-938 [13], 79069 [2], 80119 [2], 80152 ? [1], MSNM 4657 [1], Conci \& Michelangeli, 1974: 223)

\subsubsection{Barbus caninus Bonaparte, 1839}

Barbus caninus Cuvier, 1829: 273 (nomen nudum); repeated by Valenciennes, in Cuvier, 1842: 217 (nomen nudum)

Barbus Caninus Bonaparte, 1839: [fasc. 25, p. 217], pl. 110bis fig. 2 (type locality: Piemont, Po basin, Italy; lectotype: ANSP 6142, designated by Bianco, 1995b: 318)

Barbus caninus Valenciennes, in Cuvier \& Valenciennes, 1842: 142 , pl. 464 (type locality: lake Maggiore, Switzerland \& Italy; lectotype: MNHN 1408, present designation; junior homonym of Barbus caninus Bonaparte, 1839)

Systematic notes. Barbus caninus was earlier considered as a synonym of $B$. meridionalis. Genetic data (Berrebi, 1995: 244) show that French and northern Italian material are genetically well separated; Bianco (1995b) treats them as distinct species.

Nomenclatural notes. Almaça (1969: 1132, 1133) designated two different specimens as "lectotype" for B. caninus Valenciennes (in Cuvier \& Valenciennes, 1842: 142); as there can be only one lectotype for any nominal species, as first reviser I retain MNHN 1408 as lectotype.

\subsubsection{Barbus comizo Steindachner, 1865}

Barbus comizo Steindachner, 1865: 4 (type locality: river Tejo [Portugal and Spain]; syntypes: MB ?, NMW 5306 [1], 54062-066 [6], 54070 [2], MSNM 4658 [1], Conci \& Michelangeli, 1974: 223); see Steindachner, 1866c: pl. 2 for illustration of a possible syntype

Nomenclatural notes. Note that correct original spelling is $B$. comizo and not $B$. comiza; $B$. comiza is an incorrect subsequent spelling (by Steindachner, 1866c: 8) and cannot be used (ICZN art. 33(c)).

\subsubsection{Barbus cyclolepis Heckel, 1837}

Barbus communis var. cyclolepis Heckel, 1837: 155 (type locality: river Marizza near Philippoli in Rumelia [southern Bulgaria]; syntypes: NMW 54734 [1], 54736 [5]; on the same page, Heckel also uses the combination Barbus cyclolepis)

? Barbus barbus bergi Chichkoff, 1935: 305, pl. 1 (type locality: river Riesova Rieca, Bulgaria; syntypes: LU)

Barbus tauricus polylepis Battalgil, 1941: 177 (type locality: creek entering the Bosphorus (Keçe suyu) near Eyub, European Turkey; syntypes: LU)

Barbus euboicus sperchiensis Stephanidis, 1950: 202 (type locality: river Sperchios, Greece; syntypes: lost?)

Barbus cyclolepis strumicae Karaman, 1955: 187 (type locality: Monospitovo swamp and river Strumica, FYROM; syntypes: LU)

Barbus plebejus pergamonensis Karaman, 1971: 203 (type locality: Bergama, Turkey; syntypes: ZMH 3602 [8], 3898 [4], see below)

Barbus cyclolepis cholorematicus Stephanidis, 1971b: 223, fig. 4 (type locality: river Cholorema north of Almyros, Thessaly, Greece; syntypes: lost ? [24])

Systematic and nomenclatural notes. The position of Barbus plebejus pergamonensis follows Bogutskaya (pers. comm.), who also points to discrepancies between catalogue and published data of the type series. Karaman (1971: 203) listed 12 syntypes as ZMH 3602 (8) and 3889 (4). Fourteen specimens are labelled as types in ZMH: 3602 (9), 4207 (1) and 3898 (not 3889) (4). As specimen ZMH 4207 is not mentioned in the original description, it does not seem to be part of the type series. The discrepancy in number of specimens in ZMH 3602 is not explained, and one of the specimens may not be part of the type series. Specimen ZMH 4207 is labelled as holotype, but as no holotype is mentioned in the original publication it cannot have 
this status and the type series includes only syntypes.

The B. plebejus escherichi Steindachner, 1897 (p. 688, pl. 2 fig. 1) reported from European Turkey (e.g. Geldiay \& Balik, 1988: 328) is apparently the present species.

\subsubsection{Barbus euboicus Stephanidis, 1950}

Barbus euboicus Stephanidis, 1950: 202 (type locality: small streams on island Evia, Greece; syntypes: lost?)

\subsubsection{Barbus graecus Steindachner, 1896}

Barbus graecus Steindachner, 1896: 187, pl. 2 fig. 4 (type locality: river Aspropotamos (Acheloos) and lake of Wrachori [Trichonis], Greece; syntypes: NMW 10702 [1])

\subsubsection{Barbus graellsii Steindachner, 1866}

Barbus graellsii Steindachner, 1866b: 198 (type locality: river Ebro near Tortosa, Zaragoza and Logrono, creeks near Arenas north of Bilbao, Nervion near Bilbao, Spain; syntypes: NMW 5295-5298 [4], 5340 [1], 5359-5366 [8], 52394 [1], 54112 [1], 54139-141 [3], 54143-143 [3], 54146 [10], 54148-157 [33], 54159 [2], 79353 [5]); see Steindachner (1866c: pl. 3 fig 1) for illustration of one of the syntypes

5.7.10 Barbus guiraonis Steindachner, 1866 Barbus guiraonis Steindachner, 1866c: 11, pl. 5 (type locality: river Jucar near Cuenca which enter the sea near Alcira south of lake Albufera, Spain; syntypes: NMW 5291 [1], 5307-5310 [4], 54124-54133 [18])

Systematic notes. Valid species according to Doadrio (1990: 270) and Machordom et al. (1995: 231).

\subsubsection{Barbus haasi Mertens, 1924}

Barbus haasi Mertens, 1924: 1 (type locality: stream Noguera Pallaresa near Pobla de Segur, Prov. Lérida, Spain; holotype: SMF 3254)

Systematic notes. Valid species according to Doadrio \& Garzon (1987) and Machordom et al. (1995: 231).

5.7.12 Barbus macedonicus Karaman, 1928 Barbus barbus macedonicus Karaman, 1928: 151 (type locality: river Vardar, FYROM; syntypes: LU); repeated in 1929: 171

Barbus barbus thessalus Stephanidis, 1971b: 213, fig. 1 (type locality: river Pinios, Thessaly, Greece; syntypes: lost ? [14 ?])

Systematic notes. Mitochondrial DNA data
(Tsigenopoulos, 1996) and morphological comparison show that this species is distinct from B. barbus.

\subsubsection{Barbus meridionalis Risso, 1826}

Barbus meridionalis Risso, 1826: 437 (type locality: river Var and Département des Alpes Maritimes, France; lectotype: MNHN 70, designated by Bianco, 1995b: 319)

Systematic notes. As recognised here this species is restricted to southern France and north-eastern Spain. Material from Italy formerly referred to it represents a distinct species, B. caninus (Bianco, 1995b).

\subsubsection{Barbus microcephalus Almaça, 1967}

Barbus microcephalus Almaça, 1967: 321, figs. 1-2 (type locality: Rio Guadiana, about $4 \mathrm{~km}$ from Moura, Portugal; holotype: MB B.m.1)

\subsubsection{Barbus peloponnesius Valenciennes,} 1842

Barbus Peloponnesius Valenciennes, in Cuvier \& Valenciennes, 1842:143 (type locality: Morée [Peloponnese], Greece; holotype: MNHN 3824, Almaça, 1969: 1134)

Barbus Petenyii Heckel, 1848: 194 (nomen nudum; localities: rivers Maros [Mures], Szamos [Samos], Czerna [Cerna], Hungary [now Romania])

Barbus Petenyi Heckel, 1852c: 29 (type locality: river Maros [Mures] in Siebenbürgen [now Romania], as restricted by Bănărescu (1957: 72); tentative syntypes: NMW 5318-5324 [7], 10875876 [2], 54436 [2], 54440 [1], 54442 [2], 54446-448 [4], 54450 [9])

Pseudobarbus Leonhardi Bielz, 1853: 179 [? pl. 3 fig. 1, missing in examined copy] (type locality: Marosch [Mures], Alt [Oltul], Samosch [Samos], streams Tscherna [Cerna] and Zibin [Sibiu], Siebenbürgen [now Romania]; syntypes: $\mathrm{LU})$

Barbus Rebeli Koller, 1926a: 178 (type locality: creek Fandi Vogelj in Miriditen area, Albania; syntypes [total 3]: NMW 5333-5334 [2])

Barbus cyclolepis waleckii Rolik, 1970: 401 (type locality: stream Oslawa, a tributary of river San near Zagorz, district Sanok, Poland; holotype: IZPAN 25(66)3)

Systematic notes. Synonymy tentatively follows Karakousis et al. (1993). Mitochondrial DNA data (Tsigenopoulos, 1996) suggest that more work is needed on the nominal species included in the synonymy of $P$. peloponnesius before final conclusions can be reached.

Nomenclatural notes. Koller published two pa- 
pers in 1926 with the description of $B$. rebeli, once as a species (1926a), once as a variety (1926b). The description of the species appeared first, as seems to be indicated by the $1926 \mathrm{~b}$ paper which refers to the earlier one (although with incorrect bibliographic data), but does not mention 'new species' and lists an additional specimen received in between. In a third paper, Koller (1926c: 175) refers only to the $1926 \mathrm{~b}$ paper.

The first use of the name Barbus petenyi by Heckel in 1848 is a nomen nudum. His 1852 diagnosis is very brief but makes the name available. Although the title page is dated 1853 , it is explicitly stated to be the volume for 1852 . Heckel's paper was presented at a meeting of 5 May 1852 and appeared in the first fascicle of the volume. No exact publication date is available, but it is reasonable to assume that the fascicle appeared in 1852 and that the last fascicle(s) and cover page appeared in 1853. In any case, they seem to predate Bielz's paper which appeared in September 1853.

\subsubsection{Barbus plebejus Bonaparte, 1839}

Barbus plebeius Cuvier, 1829: 273 (nomen nudum), repeated by Valenciennes, in Cuvier, 1842: 217 (nomen nudum)

Barbus Plebejus Bonaparte, 1839: [fasc. 25, p. 216], pl. 110 fig. 1 (type locality: lake Como, Italy; lectotype: ANSP 6183, designated by Bianco, 1995b: 307)

Barbus Eques Bonaparte, 1839: [fasc. 25, p. 215], pl. 110 fig. 2 (type locality: river Arno, Italy; lectotype: ANSP 6145, designated by Bianco, 1995b: 307)

Barbus plebeius Valenciennes, in Cuvier \& Valenciennes, 1842: 139, pl. 462 (type locality: river Po, Italy; lectotype: MNHN A.3825, designated by Almaça, 1969: 1130; junior homonym of Barbus plebejus Bonaparte, 1839, ICZN art. 58 (3))

? Barbus plebejus ercisianus Karaman, 1971: 204 (type locality: Ercis, lake Van, and road from Ercis to Patnos, Turkey; syntypes: ZMH 3566 [13], 3567 [13])

? Barbus plebejus kosswigi Karaman, 1971: 206 (type locality: creek Hamann, Beytüssebap, Hakkari Prov., Tigris basin, Turkey; syntypes: ZMH 1159 [6])

Systematic notes. Bianco (1995b: 312) considers that B. plebejus is restricted to the Adriatic drainage of Italy and Croatia and that the material reported from the Vistula basin (Krupka \& Holčík, 1976) is possibly B. cyclolepis. Stratil et al. (1983) showed that these Slovakian "B. plebejus" are possibly hybrids between $B$. barbus and
"B. meridionalis petenyi". The status of these individuals or stocks is still open to question, but zoogeography would suggest that none is likely to be conspecific with $B$. plebejus.

The two nominal subspecies from Turkey tentatively listed above are unlikely to be conspecific with $B$. plebejus but their status is unresolved; a third one (B. p. pergamonensis) is a synonym of B. cyclolepis (Bogutskaya, pers. comm.). Similarly, I doubt that B. scincus Heckel, 1843 (p. 1049; type locality: Aleppo, Syria) is conspecific with $B$. plebejus as proposed by Almaça (1991: 70); Coad (1991: 14) tentatively treats it as a synonym of B. lacerta Heckel, 1843 (p. 1045, pl. 2 fig. 1; type locality: stream Kueik near Aleppo, Syria).

Nomenclatural notes. Barbus plebejus kosswigi Karaman, 1971 is a secondary homonym of $C y$ clocheilichthys kosswigi Ladiges, 1960 (p. 135, fig. 7), placed in Barbus by Coad (1982: 263, 1991: 14).

\subsubsection{Barbus prespensis Karaman, 1924}

Barbus graecus var. prespensis Karaman, 1924b: 32 (type locality: lake Prespa and its tributaries, FYROM and Greece; syntypes: LU)

\subsubsection{Barbus sclateri Guinther, 1868}

Barbus sclateri Günther, 1868: 93 (type locality: Guadalquivir [Spain] and Portugal; syntypes: BMNH [6]).

Systematic notes. According to Doadrio (1990: 271 ), this is a polytypic species consisting of three very distinct populations; further study may show that they are different species.

\subsubsection{Barbus steindachneri Almaça, 1967}

Barbus steindachneri Almaça, 1967: 263, figs. 1-2

(type locality: rio Guadiana, 4 km from Moura, Portugal; holotype: MB B.s.1)

Systematic notes. Doadrio (1988b: 26) considers $B$. steindachneri to be a synonym of $B$. comizo, while Almaça (1990: 590) and Costa Pereira (1995: 487) consider it a distinct species.

\subsubsection{Barbus tyberinus Bonaparte, 1839}

? Barbus Eques Cuvier, 1829: 273 (nomen nudum), repeated by Valenciennes, in Cuvier, 1842: 217 (nomen nudum)

Barbus Fluviatilis Tyberinus Bonaparte, 1839 [fasc. 25], pl. 110 fig. 3 (type locality: river Tevere near Roma, Italy; lectotype: ANSP 6152, designated by Bianco, 1995b: 313)

Barbus eques Valenciennes, in Cuvier \& Valenciennes, 1842: 141, pl. 463 (type locality: Firenze, Italy; lectotype: MNHN A.6913, designated by 
Almaça, 1969: 1131; junior homonym of Barbus eques Bonaparte, 1839)

Barbus Canalii Valenciennes, in Cuvier \& Valenciennes, 1842: 143 (type locality: rivers Topico [Topino] and Tevere near Perugia, Italy; lectotype: MNHN 1412, designated by Almaça, 1969: 1132)

Barbus fucini Costa, 1853: fasc. 79: index to plates p. 4, pl. 11 (type locality: lake Fucino, Italy; syntypes: ? MNHN 192, Bianco, 1995b: 313, ? NMW 54799 [1])

Systematic notes. Valid according to Bianco (1994a, 1995b).

Nomenclatural notes. Bianco (1994a) used B. fucini Costa, 1838 as the name of the species, but the name did not appear in 1838. Costa only used it in the captions of the plates (Spiegazioni delle tavole che accompagnano la parte prima) which appeared in 1853 (Sherborn, 1937: 36-37; Bianco, 1995b: 313). Thus the earliest available name is $B$. tyberinus.

\subsubsection{Carassius auratus (Linnaeus, 1758) in- troduced}

Carassius encobia Bonaparte, 1845: 3 (listed in synonymy, name not available)

Leuciscus auratus Mauduyt, 1848: 32 (type locality: Vienne, France, introduced from southern China; syntypes: Musée de Poitiers ?; a secondary homonym of Cyprinus auratus Linnaeus, 1758)

\subsubsection{Carassius carassius (Linnaeus, 1758)}

Cyprinus Carassius Linnaeus, 1758: 321 (based on Artedi [1738: gen. 4 [29], syn. 5, spec. 29 [4], Cyprinus pinnae dorsi ...], Linnaeus [1746: 122, n. 322, idem], Gronovius [1754: 3, n. 11, idem; 1746: 75, n. 55, Cyprinus hamburger; Wawerveen, Belgium]; type locality: "in Europae stagnis"; type material: NT)

Cyprinus moles Agassiz, 1835b: 37 (nomen nudum) Carassius humilis Heckel, 1837: 156, pl. 9 fig. 4 (type locality: Lago nella Piana della Stippa near Palermo, Sicilia, Italy; syntypes [total 15]: NMW 56108 [8])

C[arassius] charax Lesniewski, 1837: 53 (type locality: Poland; syntypes: LU)

Carassius vulgaris Nordmann, 1840: 479 (unnecessary replacement name for Cyprinus carassius Linnaeus, 1758)

Carassius moles Nordmann, 1840: 480 (nomen nudum; locality: Danube basin)

Cyprinus moles Valenciennes, in Cuvier \& Valenciennes, 1842 [August]: 89 (type locality: river Danube; syntypes: MNHN 3406 [1], MNHN
B.2591 [1], Almaça, 1969: 1142)

Cyprinus moles Selys-Longchamps, 1842 [December]: 200 (type locality: Longchamps, Belgium; syntypes: ? ISNB [not found])

Carassius linnaei Bonaparte, 1845: 3, 1846: 27 (unnecessary replacement name for Cyprinus carassius Linnaeus, 1758)

Carassius oblongus Heckel \& Kner, 1858: 73, fig. 33 (type locality: Lemberg, Galicia [now Lvov, Ukraine] and river Stry near Podhoroce, Carpathes, Ukraine; syntypes: NMW 56103 [2], 56133 ? [1])

Carassius gibelio var. minutus Kessler, 1856a: 42 (type locality: ponds near Kiew, Ukraine; syntypes: ZISP 7192, Berg, 1932b: 18) from Berg, 1932b: 18

Carassius vulgaris var. gibbosus Walecki, 1863: 358 (infrasubspecific, name not available)

Carassius vulgaris var. șubventrosus Walecki, 1863: 358 (infrasubspecific, name not available)

Carassius Linnéi Malm, 1877: 556 (unnecessary replacement name for Cyprinus carassius Linnaeus, 1758)

Carassius Linnéi forma lacustris Malm, 1877: 556 (infrasubspecific, name not available; locality: river Goeta Elf near Göteborg, Tingstad, Göteborg, Sweden)

Carassius Linnéi forma piscinarum Malm, 1877: 556 (infrasubspecific, name not available; locality: Göteborg, Sweden and København, Denmark)

? Carassius carassius jacuticus Kirillov, 1956 (locality: Yakutia, Siberia) from Kirillov, 1962: 52 Nomenclatural notes. Carassius Nilsson and Cyprinopsis Fitzinger were both published in 1832. The exact publication dates are not known and in the absence of contrary evidences they have to be considered as simultaneously published on 31 December 1832 (ICZN art. 21(c)(ii)) and as first reviser I retain Carassius as having priority. The authorship of Carassius is sometime attributed to Jarocki (1822: 54, 71), but it is considered as not being available from that publication by Eschmeyer (1990: 76).

The first use of C. moles by Agassiz (1835b) is a nomen nudum, as is also Nordmann's (1840) use. The species is made available independently twice, by Valenciennes (in Cuvier \& Valenciennes, August 1842) and Selys-Longchamps (1842). The exact publication date of Selys-Longchamps's work is not known, so the date to be adopted is 31 December 1842 (ICZN art. 21(c)(ii)). Valenciennes's description appeared first and has priority. Cyprinus moles Selys-Longchamps is a primary junior homonym and is not available. 
Almaça (1969: 1142) considered Agassiz as author of C. moles in Valenciennes (1842) and commented that only one of the two specimens mentioned by Valenciennes is a syntype because it was received from Agassiz. According to ICZN art. 50 (a), the author of the name is Valenciennes and the two specimens are syntypes because he used both for his discussion.

\subsubsection{Carassius gibelio (Bloch, 1782)}

Cyprinus Gibelio Bloch, 1782: 71, pl. 12 (type locality: Churmark, Pommern, Schlesien, Preussen (Prussia, now Germany and Poland); syntypes: ZMB 3203 [2])

? Carassius bucephalus Heckel, 1837: 157 (type locality: warm springs near Salonik, Macedonia, Greece; syntypes: NMW [2])

? Cyprinus amarus Koch, in Koch, Herrich-Schäffer \& Forster, 1840: 39 (type locality: Regensburg, Germany; type material: NT ?; junior homonym of Cyprinus amarus Bloch, 1782)

Carassius ellipticus Heckel, 1848: 194 (nomen nudum; locality: Hungary; possibly a lapsus for Carassius bucephalus)

Carassius vulgaris var. ventrosus Walecki, 1863: 358 (infrasubspecific, name not available)

Carassius vulgaris var. kolenty Dybowski, 1877: 11 (river Amur basin) from Berg, 1949: 826

Carassius auratus gibelio morpha vovkii Ioganzen, 1945: 12 (infrasubspecific, name not available; locality: lakes of Baraba steppe and Narym region, Siberia)

Systematic notes. The status of this species has long been discussed, several authors just considering it as a wild form, native to Europe or introduced, of the well known goldfish $C$. auratus, or as feral stock of introduced goldfishes, or as a result of hybridisation. Available data lead me to tentatively conclude that it is native to eastern Central Europe from where it has been reported before the introduction of the goldfish into European waters; it can possibly be equated with a stock from northern Asia extending eastwards to northeastern China, but with none of the East Asian domesticated cyprinids; awaiting resolution of the systematics of the Chinese and Japanese Carassius species, we are left with no choice but to treat it as a distinct species.

Valenciennes (1842: 108) summarised the history of the introduction of Carassius auratus to Europe. He states that some authors (but does not say which ones) consider that it was introduced to Europe as early as 1611 or 1691 and that Yarrell reports that it had been introduced by the Portuguese from Java [in which case, might Yarrell's account in part be based on the Javanese longfinned Cyprinus sp. bred in Java ?] to South Africa and from there to Lisboa. The first introduction to England dates to 1691 (Pennant, 1812: 490) and to France to 1755 (Hervey \& Hems, 1968, cited by Scott \& Crossman, 1973). It was bred in northern Europe for the first time in Holland in 1728 according to Sterba (1987: 272). Prior to this date, all those goldfishes existing in Europe had to be imported by boat from Asia (or some port between Asia and Europe where they could have been already acclimatised) and they were thus imported in limited numbers and must have been very expensive. It seems thus unlikely that before 1728 their owners would have willingly released them into European waters and if this happened accidentally [some of them were kept in outside pools], probably only very few individuals were concerned.

The earliest published record of $C$. gibelio apparently dates from Gesner (1560: 298) as 'Klein Karas (Chiblichen)' from river Elbe, Germany (but Gesner's figure of 'Klein Karas' p. 298 likely shows C. carassius). Gesner's account is based on Kentmann's codex (1549; see Hertel, 1978: 92). "Cyprinus tertius" (a pre-Linnaean, thus unavailable name) in Marsili (1726: 61; reproduced in Hensel, 1971: 188, pl. 1) from the Danube is apparently the present species. There are several reports of it in the 17-18th centuries literature from central Europe (see references in Bloch, 1782: 71) suggesting that it was relatively well known and distributed. Thus, its supposed origin as feral goldfish seems to have no sound base. Admittedly, the earliest records are quite vague and could apply to C. carassius as well.

As its distribution includes areas where no population of Cyprinus carpio, Carassius carassius or introduced $C$. auratus exist, the theory that $C$. gibelio individuals are mere hybrids, has no support (Heuschmann, 1957: 75). Carassius gibelio seems to be a gynogenetic (all-female) triploid species (Pelz, 1987: 125). The species may have an hybrid origin, but as it consitutes a distinct lineage and is diagnosable, it is a distinct species under the PSC (for brief review of other fish species of hybrid origin, see Stauffer et al., in press)

The systematics of the genus Carassius in East Asia is confusing. The ancestor of today's domesticated goldfishes was introduced to Japan from China at a date between 1502 and 1748 (Okada, 1959-60: 531). Available data show that at least five genetically and morphologically distinct stocks are known in Japan which are considered as distinct species or subspecies (Teitler \& 
Fujita, 1993; Hosoya, in Nakabo, 1994: 212-213) and are treated as species here under the PSC. At present the European species can possibly be considered as conspecific only with a stock extending eastwards to northeastern China (see, e.g., Kimura et al., 1992: 95, pl. 3) which is definitively not feral.

Bănărescu (1993: 95) "believes that [C. gibelio, as a subspecies of $C$. auratus] has been introduced as culture fish from China, possibly during the Middle Age, first in the Middle Asian moslim countries, later in eastern Europe". No supporting data is given.

\subsubsection{Chalcalburnus belvica (Karaman, 1924)}

Alburnus alburnus belvica Karaman, 1924b: 50

(type locality: lake Prespa, FYROM and Greece; syntypes: LU)

Systematic notes. Valid species (see Economidis, 1986).

\subsubsection{Chalcalburnus chalcoides (Guelden- staedt, 1772)}

Cyprinus chalcoides Gueldenstaedt, 1772: 540, pl. 16 (type locality: river Terek, Caspian Sea basin, Russia [restricted by Berg, 1949: 733]; syntypes: LU)

Cyprinus clupeoides Pallas, 1776: 704 (available by indication to Gueldenstaedt, 1772: 540; type locality: river Terek, Caspian Sea basin, Russia; syntypes: LU)

Aspius Mento Perty, 1832: 720 (nomen nudum)

Aspius Heckelii Fitzinger, 1832: 335 (nomen nudum; locality: stream Traun, upper Austria)

Aspius mento Heckel, 1836: 225, pl. 19 fig. 3 (type locality: stream Traun near Gmunden, upper Austria / München, Germany / river Danube near Wien, Austria; syntypes: NMW 16441 [1], 16261 [1], 50440 ? [1], 55629 [4], 55630 [1], 55650 [2], 55652 [1], MNHN [1]; author given as Agassiz, but actually is Heckel)

Alburnus mentoides Kessler, 1859a: 531 (type locality: Salghir and Alma rivers, market in Simpheropol, Crimea, Ukraine; syntypes: ZISP ? [17])

Alburnus longissimus Warpachovski, 1892: 154 (Lenkoran District, Caspian Sea basin) from Berg, 1949: 734

Alburnus latissimus Kamensky, 1901: 120, 191 (type locality: mouth of river Kura, Caspian Sea basin; syntypes: ZMT ?)

Alburnus chalcoides var. danubicus Antipa, 1909, fig. 64: 162 (type locality: lakes Razim, Zaton and Sinoe-Liman in river Danube delta and river Danube at Chernavody, Romania; syn- types: LU)

Alburnus chalcoides derjugini Berg, 1923: 272 (river Coruh basin, Transcaucasia) from Berg, 1949: 740

Chalcalburnus chalcoides aralensis Berg, 1923: 272, 506 (Vozrozhdenie island, Aral Sea) from Berg, 1949: 742

Chalcalburnus chalcoides mentoides natio longicephala Tseeb, 1930: 163 (infrasubspecific, name not available; river Chernaya, Crimea) from Berg, 1949: 741

Alburnus chalcoides sapancae Battalgil, 1941: 179 (type locality: lake Sapanca, Turkey; syntypes: LU)

Alburnus chalcoides nicaeensis Battalgil, 1941: 179 (type locality: lake Iznik, Turkey; syntypes: LU)

Alburnus chalcoides carinatus Battalgil, 1941: 179 (type locality: lake Manyas, Turkey; syntypes: LU)

Alburnus chalcoides istanbulensis Battalgil, 1941: 180 (type locality: stream Kâathane, draining to the Bosphorus near Istanbul, Turkey [European part]; syntypes: LU)

Chalcalburnus chalcoides mandrensis Drensky, 1943: 349 (type locality: lake Burgas near Burgas, Bulgaria; syntypes: LU)

Chalcalburnus chalcoides schischkovi Drensky, 1943: 353, fig. 1 (type locality: mouth of the rivers Resowska and Weleka, Bulgaria; syntypes: LU)

Chalcalburnus chalcoides iranicus Svetovidov, 1945: 142 (type locality: vicinity of town Shakhi, basin of river Talar, entering the Caspian Sea west of Gorgan Bay, Iran; holotype: ZISP)

Chalcalburnus chalcoides macedonicus Stephanidis, 1971b: 224, fig. 5 (type locality: lake Volvi, Macedonia, Greece; syntypes: lost ? [18])

Systematic notes. Chalcalburnus is greatly in need of a critical revision and it seems likely that several species might be recognised. I tentatively consider that the material from the Danube basin is conspecific with that from the Volga basin, where the type locality of $C$. chalcoides is located. Berg (1949: 737) and Bănărescu (1964: 367) treat them as distinct subspecies. If future studies show that they are distinct species, the name C. mento (Heckel, 1836) should be used for the Danube species. Among the names listed above, heckelii and danubicus would be synonyms of C. mento. Bănărescu (1961a) discussed the status of Alburnus chalcoides var. danubicus.

Berg (1949: 738), Bănărescu (1964: 368) and Economidis \& Bănărescu (1991: 261) consider C. c. schischkovi and C. c. mandrensis as valid subspecies. Meristic data in Drensky (1943: 357) show 
them obviously diagnosable from material of the Danube basin and this would suggest that they are specifically distinct, but data of Danube material in Bănărescu (1961a: 1490) differ from those of Drensky and show some overlap with those of schichkovi and mandrensis and I retain them as conspecific awaiting a critical revision.

Economidis \& Bănărescu (1991: 261) consider C. c. macedonicus as a valid subspecies (as part of a list of subspecies, "some of which [unspecified] ... however questionable"). Stephanidis (1971b: 240) distinguished it from C. chalcoides (unspecified locality; apparently based on literature) by having more gill-rakers on the first gill arch (26-30, vs. 19-25). Comparative data for other populations/species are not available and in their absence it is not possible to reach a decision.

The status of the four nominal subspecies described by Battalgil (1941) will remain in limbo until material from their respective type localities is carefully described.

Nomenclatural notes. The author of Aspius mento is often given as Agassiz, 1832 or Agassiz in Perty, 1832. Perty (1832: 720) listed 'Aspius Mento Agassiz' but there is no indication that Agassiz should be considered as author. A footnote (p. 716) indicates that he had only read the manuscript. Anyway, the 'description' of the species reads as follows (my translation): "In [lake] Chiemsee. Is deceptively sold as Renke [Coregonus] and most commonly comes to our market (if I am not mistaken) packed in barrels, that is, dead, in November". I do not think that there is anything in this 'description' which can make a name available. The name is first made available by Heckel (1836).

Bertin \& Estève (1948: 78) list a holotype of Aspius mento Agassiz (1839: "pl. 28 fig. 2") (MNHN 3894, river Danube in Austria, $190 \mathrm{~mm}$ ); I could not find this plate in Agassiz (1839) which includes only 27 plates (see also Surdez, 1971). Bertin \& Estève may have seen proofs of an unpublished plate [Agassiz frequently distributed such proofs]. I do not consider this specimen as having type status.

\subsubsection{Chondrostoma arrigonis (Steindach- ner, 1866)}

Leuciscus Arrigonis Steindachner, 1866c: 16, pl. 4 fig. 1 (type locality: Rio Jucar near Cuenca and Laguna of Una, Spain; syntypes: NMW 52162 207 [more than 128], 52209-232 [179], 53409 [5])

? Chondrostoma toxostoma turiensis Elvira, 1987: 133 (type locality: river Turia, Chulilla, Valencia, Spain; holotype: UZA 1983.12.06.01)
Systematic notes. Collares-Pereira (1980a) considers C. arrigonis as a valid species, while Elvira (1987: 131) considers it a subspecies of C. toxostoma. Collares-Pereira's data (1980a: 276) show them quite distinct in number of lateral-line scales and gill-raker counts and her data are in agreement with those of Elvira for material from the same areas. Elvira considers them to be the two extremities of a clinal variation, but his data are condensed in such a way that I cannot reach a conclusion. From his data, C. arrigonis inhabits a separate basin and is morphologically diagnosable and I treat it as a valid species; I tentatively consider C. toxostoma turiensis as conspecific: the two seem to be very similar, differing only slightly in gillraker and pharyngeal teeth counts. The characters distinguishing C.t. turiensis from C. t. miegii (sensu Elvira) are roughly those distinguishing $C$. t. arrigonis from C. t. miegii; C. t. miegii seems to be in general agreement with C.t. toxostoma and is considered conspecific with it.

See Costa Pereira (1995: 492) for a potential, alternative interpretation.

\subsubsection{Chondrostoma genei (Bonaparte, 1839)}

Leuciscus Genei Bonaparte, 1839: [fasc. 24, p. 243], pl. 114 fig. 2 (type locality: lakes of Piemont, Italy; syntypes: ANSP 6110-6115 [6], Böhlke, 1984: 77)

Chondrostoma jaculum Filippi, 1844: 397 (type locality: rivers Ticino and Po, Italy; syntypes: NMW 52244 [4])

Chondrostoma Genei var. albicans Gatti, 1896: 215 (type locality: river Vomano near Fontanelle, Italy; syntypes: LU)

\subsubsection{Chondrostoma knerii Heckel, 1843}

Chondrostomus Knerii Heckel, 1843: 1030 (type locality: river Narenta, Dalmatia; syntypes: NMW 52265 [2], 52269-52271 [6], 52280-281 [4])

5.10.4 Chondrostoma nasus (Linnaeus, 1758) Cyprinus Nasus Linnaeus, 1758: 325 (based on Artedi [1738: gen. [spec.] 5, syn. 6, Cyprinus ... pinnae ani radiis 14] and Gronovius [1756: 2, n. 147, idem]; type locality: "in Rheno fluvio" [river Rhine]; type material: NT)

Chondrostomus Nasus auratus Schäfer, 1844: 305 (type locality: river Moselle in Trier, Germany; syntypes: LU; proposed as a trinominal name, but treated as a variety in the text; could be treated as an infrasubspecific name, thus not available)

Chondrostoma nasus var. hernadiensis Jeitteles, 
1862: 306 (type locality: river Hernad [Hornád], Kaschau [Košice], Slovakia; syntypes: NMW 52475 [4])

Chondrostoma caerulescens Blanchard, 1866: 416, fig. 104 (type locality: rivers Doubs and Ognon, France; syntypes: MNHN ?)

Chondrostoma nasus var. taeniata Fatio, 1882: 691 (infrasubspecific, name not available; locality: river Rhine in Basel, Switzerland)

Chondrostoma Nasus var. macrolepidotus Veesenmayer, 1884: 325 (nomen nudum; locality: river Danube near Ulm, Germany)

? Chondrostoma nasus var. borysthenica Dybowski, in Sinitzyn, 1900: 46 (nomen nudum; locality: river Dnieper, Ukraine) from Berg, 1914: 369

? Chondrostoma nasus borysthenicum Berg, 1914: 373, fig. 33 (type locality: river Dnieper near Neyasit, Ukraine; syntypes: ZISP 11985 [2])

Chondrostoma nasus ohridanum Karaman, 1924b: 71 (type locality: lake Ohrid and its tributaries, FYROM; syntypes: LU)

? Chondrostoma nasus lumi Poljakov, Filipi \& Basho, 1958: 88 (type locality: Pogradec, Albania; syntypes: LU)

? Chondrostoma nasus angorensis Elvira, 1987: 117, fig. 3a (type locality: Eskishir [Eskisehir], Turkey; holotype: NMW 52234:1)

Systematic notes. Under the PSC, the subspecies C. n. angorensis Elvira (1987) from Turkey probably has to be recognised as a valid species.

Nomenclatural notes. Although Chondrostoma is a neutral name, the specific name nasus does not have to agree in grammatical gender: it is a noun in apposition, thus invariable [ICZN art. 31 (b) (ii)].

Fricke (1995: 11) listed Chondrostoma nasus var. macrolepidotus Krauss, in Veesenmayer (1884: 325$)$ as an available name. As used in in Veesenmayer (1884: 325), the name is not accompanied by a description or an indication and it is a nomen nudum, thus not available. Fricke stated that the name is based on the description by Krauss (1879: 348); indeed, the specimen described by Krauss most likely is the specimen referred to by Veesenmayer, but as there is no bibliographic reference (ICZN art. 13(a)(ii)) to Krauss's paper, the name is not available. An infrasubspecific rank is explicitely meant and the name would not be available anyway (ICZN art. $45(\mathrm{~g})(\mathrm{ii})(1))$. As the name is not available, authorship of the name is not relevant. (If the name were available, Veesenmayer would have to be considered as only author by virtue of ICZN art. $50(\mathrm{a}))$.
5.10.5 Chondrostoma phoxinus Heckel, 1843

Chondrostomus Phoxinus Heckel, 1843: 1031 (type locality: creeks around Livno, Bosnia; syntypes: NMW 52481 [10], MHNN 1045 [1], Kottelat, 1984: 148)

\subsubsection{Chondrostoma polylepis Steindach-} ner, 1865

Chondrostoma polylepis Steindachner, 1865: 5

(type locality: Crato, Portugal; syntypes: MB, NMW 52589 [2])

Chondrostoma polylepis duriensis Coelho, 1985:

33, fig. 1c (type locality: rivers Tambre and Douro basins, Portugal; syntypes: MB [33])

Systematic notes. Elvira (1987: 124) does not consider C. p. duriensis as distinct from C. p. polylepis and mentions that both forms occur in the Douro basin. As pointed out by Costa Pereira (1995: 490) the published information does not allow to rule out the possibility of two distinct species occurring in the Douro basin. See also data on genetic differentiation in Coelho (1992).

\subsubsection{Chondrostoma prespense Karaman, 1924}

Chondrostoma nasus prespensis Karaman, 1924b:

73 (type locality: lake Prespa, FYROM and Greece; syntypes: LU)

5.10.8 Chondrostoma scodrense Elvira, 1987 Chondrostoma scodrensis Elvira, 1987: 129 (type locality: Scutari, Montenegro; holotype: NMW 52099:1)

Remarks. Extinct (Crivelli \& Rosecci, 1994: 14).

\subsubsection{Chondrostoma soetta Bonaparte,} 1840

Chondrostoma Soetta Bonaparte, 1840: [fasc. 28, p. 251], pl. 115 fig. 3 (type locality: rivers of Lombardy, Italy; syntypes: ANSP 6424-6426 [3], Bianco, pers. comm., 1985)

Chondrochylus nasicus Heckel, 1843: 1031 (type locality: lake Garda, Italy; syntypes: NMW 52638 [4], 52639 [3])

Chondrostoma seva Valenciennes, in Cuvier \& Valenciennes, 1844: 396 (type locality: river Po in Torino, Italy; holotype: MNHN 3147, Elvira, 1987: 130, Almaça, 1969: 1128)

Nomenclatural notes. The heading of Bonaparte's (1840) account is Chondrostoma rysela and the subheading is "Condrostoma soetta". Throughout the work, subheadings consistently are the Italian names. In the text, Bonaparte says that he doubts that the Italian stock is conspecific with the C. rysela of northern Europe and that 
it should have the specific name soetta if it turns out specifically distinct. Although proposed conditionally, the name $C$. soetta is available because it was proposed before 1961 (ICZN arts. 11(d)(i), 15).

\subsubsection{Chondrostoma toxostoma (Vallot, 1837)}

Cyprinus toxostoma Vallot, 1837: 188 (type locality: villages on river Saône near Pontailler, France; syntypes: LU)

Chondrostoma dremaei Blanchard, 1866: 418 , fig. 105 (type locality: river Lot near Cahors, France; lectotype: MNHN 3142, designated by Almaça, 1969: 1128)

Chondrostoma rhodanensis Blanchard, 1866: 420, fig. 108 (type locality: river Rhône near Avignon, France; lectotype: MNHN 3145, designated by Almaça, 1969: 1129)

Chondrostoma Miegii Steindachner, 1866b: 202 (type locality: river Ebro near Logrono and Zaragoza, river Nervion near Bilbao, stream near Arenas north of Bilbao, Laguna de Pulguer [Purguel, near Tudela; Elvira, 1987: 133], Spain; syntypes: NMW 52258, 52393-52414 [105])

Chondrostoma Peresi La Blanchère, 1872: 1632 (type locality: confluence of rivers Lot and Trueyre, near Entraygues / river Aveyron at Mouline, near Rodez, France; syntypes: LU [about 50])

Systematic notes. See Chondrostoma arrigonis for discussion.

\subsubsection{Chondrostoma vardarense Kara- man, 1928}

Chondrostoma nasus vardarensis Karaman, 1928: 160, fig. 3a (type locality: river Vardar, FYROM; syntypes: LU); repeated in 1929: 172

\subsubsection{Chondrostoma willkommii Stein- dachner, 1866}

Chondrostoma Willkommii Steindachner, 1866d: 266, pl. 2 (type locality: river Guadiana and its tributaries near Merida and Mertola, river Guadalquivir near Sevilla and Cordova [Cordoba], rivers Guadaira, Genil and Guadalete, Spain; syntypes: NMW 52664-666 [6], 52668-707 [100], 79744 [2], Elvira, 1987: 125)

Systematic notes. Elvira (1987: 124-125) considers Chondrostoma polylepis and C. willkommii as conspecific while Coelho (1985) considers them to be distinct species. The data in Elvira are too summarised and their presentation in Coelho is too confusing (especially in the absence of raw data) to reach a conclusion. Coelho (1992) showed that they are genetically distinct and therefore I treat $C$. willkommii as a valid species.

\section{Ctenopharyngodon idella (Valenciennes, 1844) introduced}

\subsubsection{Cyprinus carpio Linnaeus, 1758}

Cyprinus Carpio Linnaeus, 1758: 320 (based on Artedi [1738: gen. 4 [25], syn. 3, spec. 25 [4], Cyprinus cirris quatuor ...], Linnaeus [1746: 120, n. 317, idem] and Gronovius [1754: 5, n. 19, idem.]; type locality: "in Europa"; syntype: BMNH 1853.11.12:139 [1], Wheeler, 1958: 212)

Cyprinus cirrosus Schaeffer, 1760: 18, figs. 1-3 (name not available because work is on Index of rejected works, ICZN, 1955, Opinion 345)

Cyprinus rex cyprinorum Bloch, 1782: 107, 193, pl. 17 (type locality: near Torgau, Dresden, Germany / Franken, Germany / Böhmen, Czech Republic; syntypes: ZMB 3195 [2])

Cyprinus nudus Bloch, 1784: 178 (type locality: Schlesien, Poland; type material: NT, H.-J. Paepke, in litt., 1995)

Cyprinus alepidotus Bloch, 1784: 178 (proposed in synonymy, name not available)

Cyprinus regius Nau, 1791: 29 (unnecessary replacement name for Rex cyprinorum Bloch, 1782)

Cyprinus carpio var. caspicus Walbaum, 1792: 17 (available by indication to Lepechin, 1771b: 321, pl. 23 and Güldenstaedt, 1778: 253; type locality: mouths of rivers Volga and Tanais [Don], Russia and Ukraine; type material: NT ?)

Cyprinus rex Walbaum, 1792: 24 (type locality: near Torgau, Dresden, Germany / Franken, Germany / Böhmen, Czech Republic [localities listed by Bloch, 1782: 107], probably Regensburg, Germany [material of Schaeffer, 1760]; syntypes: ZMB 3195 [2] [Bloch's material])

Cyprinus macrolepidotus Meidinger, 1794: iii, pl. 40 (type locality: river Danube in Austria; syntypes: LU)

Cyprinus specularis La Cepède, 1803: 528 (unnecessary replacement name for Rex cyprinorum Bloch, 1782)

Cyprinus coriaceus La Cepède, 1803: 528 (unnecessary replacement name for Cyprinus nudus Bloch, 1784)

Cyprinus viridescens La Cepède, 1803: 541, 543, pl. 17 fig. 1 (type locality: Rouen, France; holotype: LU)

Cyprinus Rondeletii Shaw, 1804: vol. 5 (1): 194, pl 123 (type locality: Lyon, France; type material: NT) 
Cyprinus macrolepidotus Hartmann, 1827: 183 (type locality: lake Konstanz, Switzerland; type material: NT ?; junior homonym of Cyprinus macrolepidotus Meidinger, 1794: pl. 41)

Cyprinus carpio var. lacustris Fitzinger, 1832: 333 (available by indication to Kramer [1756: 390, Cyprinus 1 var.]; type locality: lake Neusiedler [lacu Nischiteriensi, Kramer], Austria; syntypes: NMW ? [not yet located; B. Herzig, pers. comm., 1995]; junior primary homonym of Cyprinus lacustris Pallas, 1814)

Cyprinus Regina Bonaparte, 1836: [fasc. 17-18, p. 195], pl. 108 fig. 1 (type locality: rivers Arno, Tevere and Aniene, lakes Trasimeno and Bracciano, Italy; syntypes: MNHN 751 [1], Almaça, 1969: 1142, ANSP 6616-6623 [8], Böhlke, 1984: 88)

Cyprinus hungaricus Heckel, 1836: 222, pl. 19 fig. 1 (type locality: lake Neusiedler, Hungary; syntypes: NMW 10958-959 [2])

Cyprinus Elatus Bonaparte, 1836: [fasc. 17-18, p. 203], pl. 108 fig. 3 (type locality: lake Bracciano and lake Vico near Ronciglione and ditches leading to lake Trajano, Italy; holotype: ANSP 6614, Böhlke, 1984: 75)

Cyprinus Nordmannii Valenciennes, in Cuvier \& Valenciennes, 1842: 66, pl. 456 (type locality: market in Odessa, from rivers Dniester and Bug, Ukraine; holotype: MNHN 3375, Almaça, 1969: 1141)

Cyprinus angulatus Heckel, 1843: 1013 (nomen nudum; locality: Hungary)

Cyprinus thermalis Heckel, 1843: 1013 (nomen nudum; locality: Hungary)

Cyprinus festetitzii Bonaparte, 1845: 3 (proposed in synonymy, name not available); 1846: 26 (proposed in synonymy, name not available)

Carpio vulgaris Rapp, 1854: 141 (type locality: lake Konstanz, Germany-Switzerland-Austria; syntypes: LU)

Cyprinus carpio var. gibbosus Kessler, 1856b: 357 (type locality: Ekaterinoslaw, Kiev, Ukraine; syntypes: ZISP ?; junior primary homonym of Cyprinus gibbosus Pallas, 1814)

Cyprinus Bithynicus Richardson, 1856: 372 (type locality: lake Apollonitis, Asia Minor; holotype: BMNH, Günther, 1868: 28)

Cyprinus acuminatus Heckel \& Kner, 1858: 58, fig. 22 (type locality: river Danube and lakes Neusiedler and Balaton, Austria and Hungary; syntypes: NMW 52846 [2], 52854-855 [2], 52927 929 [3], 52950 [9], 53403 [2]; junior primary homonym of $C$. acuminatus Richardson, 1846: 289)

Cyprinus carpio var. elongatus Walecki, 1863: 359 (unnecessary replacement name for Cyprinus hungaricus Heckel, 1836)

Cyprinus carpio var. monstrosus Walecki, 1863: 359 (nomen nudum and infrasubspecific, name not available)

Cyprinus carpio var. oblongus Antipa, 1909: 117, pl. 8 fig. 36 (type locality: delta of river Danube, Romania; syntypes: LU)

Cyprinus carpio anatolicus Hanko, 1924: 150, pl. 3 fig. 10 (type locality: river Pursak near EskiChehir and Kötschke-Kissik, Turkey; syntypes: MNH ? [6])

Cyprinus carpio morpha aralensis Spiczakow, 1935: 428, fig. 7a (infrasubspecific, name not available; Aral Sea at St. Nikolaus and Lasarew islands)

Cyprinus carpio fluviatilis Pravdin, 1945: 11 (type locality: flood-plain lakes of river Volga near Saratov, Russia) from Berg, 1949: 1327

Cyprinus carpio morpha brevicirri Misik, 1958: 70

(infrasubspecific, name not available)

Cyprinus carpio morpha longicirri Misik, 1958: 70

(infrasubspecific, name not available)

Systematic notes. The above synonymy includes only nominal species based on European and western and central Asian material.

Balon $(1974,1995)$ discussed the origin of domesticated carps and concluded that the European ones originated from a rheophilic stock from the Danube. I agree with him and Hoffmann (1995) that it is unlikely to have been imported from China, as is often assumed. Balon (1995: 15) does not exclude the possibility that the Chinese carps are derived from introduced European carps and that the many species of Cyprinus recognised by Chinese authors are merely domesticated or feral forms. There is no evidence for this. The hypothesis of a radiation in southern China and adjacent areas (especially in lakes of Yunnan and Myanmar) accompanied by specialisation to occupy different feeding niches is at least as reasonable (ongoing DNA analysis could confirm or refute this phylogenetic hypothesis); a very similar zoogeographic pattern is observed in Yunnanilus and Anabarilius (Kottelat \& Chu, 1988: 89; Chen, 1986). I doubt that the non-feral Chinese species reported as C. carpio really is conspecific with the European one and except for their treatment as two distinct subspecies by Berg (1949), I could not find evidence that the two have ever been the subject of a detailed comparison. Misik (1958) compiled data from different sources but did not compare actual material from the different geographic areas. Ivasik (1968) reports that the two stocks have different parasite faunae. See Zhou (1989), 
Zhou \& Chu (1986) and Wang (1979) for recent revisions of Chinese species, but it does not seem that they compared them with actual specimens of the European species.

As noted by Balon, the assumed original wild population in the Danube is now seriously threatened, and the loss of the wild stock of such an economically important species would be a great loss of genetic material. The alternative possibility that additional original wild stocks exist(ed) in other European basins (e.g., Rhine) has apparently not been investigated but, a priori, cannot be excluded. Lelek (1987) reports on a 'feral' carp from the Rhine similar in shape to the Danube one, but explicitly did not exclude the possibility that it might be a native Rhine carp (Balon [1995: 31] considers that data in Köhler \& Lelek [1992: 131] identifies it as feral; I see no data to support or refute this conclusion). If, as concluded by Balon, wild carps are only known from the Danube, considering this species as potentially threatened in areas or countries where only cultivated or introduced stocks exist (e.g., Switzerland; Pedroli et al., 1991: 65) is not justified and may even be prejudicial to the conservation of native species. Additional wild stocks exist in rivers draining to the Black, Caspian and Aral seas.

Nomenclatural notes. Spiczakov (1935) created the name Cyprinus carpio morpha aralensis. In the same paper the same fish is also called $C$. aralensis. The text and the context makes it clear that he considered this fish as a morph of $C$. carpio and that ' $C$. aralensis' is just a convenient, short way of referring to C. c. morpha aralensis.

It is not clear yet which name should be used for the East Asian carp. The name C. carpio haematopterus Temminck \& Schlegel, 1845: pl. 96 (type locality: Kiusin [Kyushu] island, Japan; type material: NT; text, p. 189, published in 1846; see Hureau \& Monod, 1973: vol. 2: 149) has commonly been used, but as Nagasaki (on Kyushu island) was the earliest European settlement in Japan, that very population could have been introduced (Balon, 1995: 15). DNA analysis may be able to show the relationships between this material, East Asian and European carps. If $C$. haematopterus turns out as an invalid name for the East Asian carp, there are at least 10 other names which should be considered as the potential valid name (listed by Wu et al., 1977: 412-414). Several of these names actually are anterior to $C$. haematopterus and it seems there has never been a justification for the use of $C$. haematopterus rather than these older names.
5.12.1 Eupallasella perenurus (Pallas, 1814)

Cyprinus Perenurus Pallas, 1814: 299 (type locality: lakes and swamps around river Lena, Siberia; syntypes: LU)

Phoxinus jelskii Dybowski, 1869: 952 (type locality: lakes of Darasun, But-Durutaj, Ila and Makhojtowa valleys [river Onon basin], Siberia; syntypes: IZPAN, Oliva, 1963 327)

Leucaspius Fischeri Sabanejew, 1871: 277 (nomen nudum [conclusion also reached by Berg, 1912: 198]; locality: east slope of Ural range, Siberia)

Phoxinus perenurus var. dauricus Dybowski, 1877: 17 (lake in basin of river Onon, Siberia) from Berg, 1949: 574

Phoxinus laevis var. punctatus Benecke, 1881: 140 (original locality: Danzig [Gdansk], Poland; type locality: swampy lake Jasien near Gdansk, Poland [by neotype designation]; neotype: CUP 32106, present designation)

Phoxinus stagnalis Warpachowski, 1886a: 76, fig. (type locality: lake Schumjer [in basin of river Malaya Kokschaga, Berg, 1949: 578], Kazan prov., Russia; syntypes: ZISP 7782-7783 [8], Berg, 1912: 211)

Phoxinus Sabanejewi Warpachowski, 1887: 535 (type locality: lakes on eastern slope of Urals range [district Schadrinsk, Tscheljabinsk; Berg, 1912: 199]; syntypes: ZISP 7779 [2], Berg, 1912: 199)

Phoxinus altus Warpachowski, 1887: 535 (type locality: tributaries of river Yenissei, Siberia [lower Tunguska, Berg; 1912: 199]; syntypes: ZISP 3235 [6], Berg, 1912: 199)

Phoxinus variabilis Warpachowski, 1887: 535 (type locality: tributaries of river Ob, Siberia [river Tscharysch; Berg, 1912: 199]; syntypes: ZISP 7742-3, 3339 [20], Berg, 1912: 199)

Phoxinus percnurus mantschuricus Berg, 1907a: 204 (type locality: Da-tschu-an, a tributary of river Sungari, Amur basin, China; syntypes: ZISP 13890 [2])

Phoxinus Dybowskii Lorec \& Wolski, 1910: 115 (type locality: Buchnik and Piaseczno, small lakes in Warsaw District, Poland [Berg, 1949: 579]; syntypes: IZPAN ? [20 ?])

Phoxinus percnurus sarykul Ruzskii, 1926: 112, fig. (lake Sarykul south of Chelyabinsk, Siberia) from Berg, 1949: 575

Phoxinus percnurus gdaniensis Berg, 1932a: 140 (nomen nudum; locality: near Danzig [Gdansk], Poland)

Phoxinus percnurus posnaniensis Berg, 1932a: 140 (nomen nudum; locality: near Posen, river Oder basin, Poland)

Phoxinus percnurus gdaniensis Berg, 1932c: 359 
(type locality: swampy lake Jasien near Gdansk, Poland; neotype: CUP 32106, present designation [designation by Oliva, 1963: 320 not valid; ICZN, art. 75(d)(3)])

Phoxinus czekanowskii posnaniensis Berg, 1932c: 362 (type locality: Poznan, basin of river Oder, Poland; holotype: ZISP ?)

Phoxinus percnurus occidentalis Kaj, 1953: 66, fig. 2 (type locality: Zlotkowo, Poznan district and Serock, near Bydgoszcz, Poland; syntypes: LU) Systematic notes. Formerly placed in Phoxinus. Gasowska (1979) discussed the generic differences between $P$. phoxinus and $P$. perenurus and placed the second species in Moroco Jordan \& Hubbs, 1925. Howes (1985: 61) agreed with her conclusion but showed that Moroco actually is a synonym of Lagowskiella Dybowski, 1916 (p. 101) and that Eupallasella Dybowski, 1916 (p. 101) is the correct generic name for the present species.

Oliva (1963) recognised three subspecies in Poland; E. p. gdaniensis, E. p. occidentalis and E. p. dybowskii, differing in details of morphometry and meristics showing a kind of mosaic distribution. All seem to have very restricted and broadly disjunct distributions. Data available to me do not allow to decide whether a single variable or several species are involved. Comparative data on detailed distribution and variability of Russian populations is especially badly needed.

The weak point in Oliva's data is that except for his original data on E. p. gdanensis all the data he tabulated and used apparently are compiled from six different sources. Among the characters which diagnose the subspecies are the number of branched anal and dorsal rays and the length of caudal peduncle, characters well known to have been counted and measured in very different and incompatible ways by different authors.

For the time being, it seems reasonable to consider that a single species is involved but obviously each of the European populations, if they are clearly isolated, deserves a detailed reexamination. Considering their stenotypic habitat requirements, their conservation deserves urgent attention.

Phoxinus percnurus sachalinensis Berg, 1907a: 204 (type locality: river Arakul near Tschepisan, southern Sachalin, Siberia; syntypes: ZISP 13879 [14]) is a synonym of Rhynchocypris oxycephalus Sauvage \& Dabry de Thiersant, 1874 (Howes, 1985: 72).

Nomenclatural notes. Note that the correct spelling of the specific name is perenurus and not percnurus as used by most contemporary authors. Although Eupallasella is a feminine noun, perenu- rus does not have to agree in grammatical gender because it is a noun in apposition.

If different species were to be recognised among the Polish populations, it should be noted that E. punctata (Benecke, 1881) has priority over E. gdaniensis (Berg, 1932). Benecke (1881: 140) recognised a variety of Phoxinus phoxinus from Gdansk which he called "punctatus Zadd."). "Zadd." is probably Ernst Gustav Zaddach (18171881 ) and I could not find a publication by him making the name punctatus available. It was probably a personal communication to Benecke, and Benecke being responsible of the conditions making the name available, he alone is author of the name. Although the description is very brief, it does make the name available. The whereabouts of Benecke's material are not known. In order to remove any potential ambiguity, specimen CUP 32106 is designated as neotype of Phoxinus phoxinus punctatus Benecke, 1881; this specimen is also the neotype of $P$. perenurus gdaniensis.

Oliva (1963: 320) designated specimen CUP 32106 as neotype of P. p. gdaniensis. However, this designation is not valid because it does not fulfil all conditions of the Code, especially art. $75(c)(3)$ as Oliva did not state his reasons for believing that Berg's type material was lost and steps taken to trace it. This material is not present in ZISP and there is not even trace of it in ZISP registers and catalogues (N. Bogutskaya, pers. comm., 1995). In order to avoid future confusion, specimen CUP 32106 is here formally designated as neotype of Phoxinus percnurus gdaniensis Berg, 1932. See Oliva (1963) for diagnostic characters of the species, description of the neotype, and discussion of identity and locality.

\subsubsection{Gobio albipinnatus Lukash, 1933}

Gobio albipinnatus Lukash, 1933: 57, fig. 12 (type locality: river Vyatka, Volga basin, Russia; lectotype: ZISP 25069, designated by Bănărescu \& Nalbant, 1973: 150)

Gobio Belingi Slastenenko, 1934: 350, pl. 1 figs. 2-3 (type locality: upper reaches and rapids of river Dnieper, Ukraine; syntypes: LU)

Gobio vladykovi Fang, 1943: 403 (type locality: liman Cahoul, Romania; holotype: MNHN 19254)

\subsubsection{Gobio banarescui Dimovski \& Grup-} če, 1974

Gobio kessleri banarescui Dimovski \& Grupče, 1974: 83, fig. 1 (type locality: river Vardar south 
of Véles [from Nogaevtsi to Guevguélia] and stream Bregalnitsa from Solifari to confluence with Vardar, FYROM; syntypes: MMNHS [75]). Systematic notes. Originally described as a subspecies of G. kessleri, G. banarescui constitutes an independant lineage, is diagnosable (Bănărescu, 1992a: 321) and is treated as a valid species under the PSC.

\subsubsection{Gobio benacensis (Pollini, 1816)}

Cyprinus benacensis Pollini, 1816: 21, pl. 1 fig. 2 (type locality: lake Garda, Italy; type material: NT)

Gobio Venatus Bonaparte, 1839: [fasc. 25, p. 218], pl. 110bis, fig. 5 (type locality: Piemonte / stream Savena near Bologna, Italy; syntypes: ANSP 6560-8564 [5], Böhlke, 1984: 94)

Gobio lutescens Filippi, 1844: 393 (type locality: Lombardia, Italy; syntypes: LU)

Gobio pollinii De Betta, 1862: 77 [of reprint] (type locality: lake Garda, Isola della Scala and Montorio, Italy; syntypes: LU)

Systematic notes. Data in Bianco \& Taraborelli (1984) and Pizzul et al. (1993) provide evidence that the northern Italian Gobio constitutes an independent and diagnosable lineage and thus is a distinct species, as already treated by Bianco (1994b: 458, 1995: 166). The argument of Gandolfi \& Zerunian (1987: 29) is not relevant under the PSC; in addition, as discussed under Salvelinus (p. 147; see also Mayden \& Wood, 1995: 85), there is no theoretical basis for their requirement that a given magnitude of difference is necessary to be of historical or taxonomic value.

\subsubsection{Gobio elimeius Kattoulas, Stephani-} dis \& Economidis, 1973

Gobio albipinnatus elimeius Kattoulas, Stephanidis \& Economidis, 1973: 179, fig. 2 (type locality: Aliakmon river by the bridge near Ammudara village (Kastoria), Greece; holotype: DZAUT ?)

Gobio persus stankoi Karaman, 1974b: 4 (type locality: river Vardar near Skopje, FYROM; syntypes: ? MMNHS [13])

Gobio uranoscopus stankoi Dimovski \& Grupče, 1976: 82, 89, fig. 1 (type locality: upper course of streams Tsrna Reka and Bregalnitsa, streams Treska and Lepenets, and river Vardar from Gostivar to Skopje, FYROM; syntypes: MMNHS [79]; junior homonym of Gobio persus stankoi Karaman, 1974b: 4)

Systematic notes. This species has been considered as a subspecies of G. uranoscopus by Dimovski \& Grupče (1976: 89) and Bănărescu (1992a: 320). As it constitutes an independent and diagnosable lineage, it is a distinct species under PSC.

\subsubsection{Gobio gobio (Linnaeus, 1758)}

Cyprinus Gobio Linnaeus, 1758: 320 (based on Artedi [1738: gen. 4 [13], syn. 11, spec. 13 [4], Cyprinus quinculialis maculosus ...] and Gronovius [1756: 2, n. 149. idem]; type locality: "in Anglia \& adjacentibus"; type material: NT)

? Cobitis fundulus Wulff, 1765: 32 (type locality: Borussia [Prussia]; type material: NT ?)

Gobio fluviatilis Fleming, 1828: 186 (type locality: "gentle streams in England"; available by description and by indication to earlier sources; type material: NT ?)

Gobio Phoxinoïdes De la Pylaie, 1835: 533 (nomen nudum; locality: Yon and other streams of Vendée, France)

Gobio vulgaris Heckel 1836: pl. 21 (no stated locality; syntypes: NMW ?)

Gobio saxatilis Koch, in Koch, Herrich-Schäffer \& Forster, 1840: 40 (unnecessary replacement name for Cyprinus gobio Linnaeus, 1758)

Gobio obtusirostris Valenciennes, in Cuvier \& Valenciennes, 1842: 311 (type locality: München, Germany; syntypes: MNHN 8 [6], Bertin \& Estève, 1948: 87)

? Bungia nigrescens Keyserling, 1861: 22, pl. 8 (type locality: river Heri-rud near Herat, Afghanistan; holotype not preserved)

Gobio fluviatilis var. cynocephalus Dybowski, 1869: 951 (type locality: rivers Onon and Ingoda, Amur basin, Siberia; syntypes: LU); synonymy follows Bogutskaya, pers. comm.

Gobio fluviatilis var. lepidolaemus Kessler, 1872: 59 (type locality: Ak-darja and Chodshaduk in Serafschan basin, Uzbekistan / Syr-darja at Chodshent [Leninabad], Tadzhikistan; syntypes: ZISP 2706 [25], Berg, 1914: 453)

Gobio lepidolaemus var. caucasica Kamensky, 1901: 6, 126, 142 (type locality: northern Caucasus in Podkumok and Sulak and in Transcaucasus near Choni [Sunzha near Groznyí / Podkumok near Georgijewsk / Choni, Tzchenistzchali basin, a tributary of river Rioni, $\mathrm{Ku}-$ tais district; Berg, 1914: 458, 459], Russia, restricted to Rion basin by Berg, 1949: 649; syntypes: ZMT 130c, Berg, 1914: 458, 469; primary simultaneous homonym of Gobio uranoscopus var. caucasica Kamensky, 1901: 9, 145)

Gobio latus Anikin, 1905: 18 (lake Issyk-Kul at mouth of river Ulachol, Kirghizistan) from Berg, 1914: 453 
Gobio gobio morpha brevicirris Berg, 1914: 442, fig. 56 (infrasubspecific, name not available; various localities in Ukraine, Russia and Siberia)

Gobio gobio morpha longicirris Berg, 1914: 442 (infrasubspecific, name not available)

Gobio gobio morpha prosopyga Berg, 1914: 442 (infrasubspecific, name not available; various localities in Russia)

Gobio gobio morpha katopyga Berg, 1914: 446 (infrasubspecific, name not available; various localities in Russia and Siberia)

Gobio gobio lepidolaemus natio holurus Berg, 1914: 457, fig. 61 (infrasubspecific, name not available; locality: streams Martan and Tschernorjetschje [Chernorech'e], tributaries of river Sunzha near Groznyí, Chechnia, Russia)

Gobio gobio var. ohridana Karaman, 1924b: 39 (type locality: lake Ohrid, FYROM; syntypes: LU)

Gobio gobio carpathicus Vladykov, 1925a: 248 (type locality: upper river Theiss [Tisza] basin, Danube basin, Ukraine; syntypes [total: several hundreds]: MNHN 30-195, 30-196 [2], Bertin \& Estève, 1948: 87)

Gobio gobio bulgarica Drensky, 1926: 131, fig. 2 (type locality: river Maritsa, Bulgaria; syntypes: NMNHS)

Gobio gobio carpathicus natio sarmaticus Slastenenko, 1934: 352, pl. 1 figs. 4-5 (infrasubspecific, name not available; locality: tributaries of rivers Dniester, southern Bug and Dnieper, Ukraine)

Gobio gobio lepidolaemus forma skadarensis Karaman, 1936: 60 (infrasubspecific, name not available; usage in Bănărescu \& Nalbant, 1973: 117 makes name available as G. g. skadarensis Karaman, 1936 [ICZN art. 45(g)(ii)(1)]; type locality: lake Skutari, Drim basin, Montenegro and Albania; syntypes: LU)

Gobio gobio sibiricus Nikolsky, 1936: 470 (type locality: river Nura, Siberia; syntypes: LU)

Gobio gobio kovatschevi Chichkoff, 1937: 257 (type locality: river Provadiiska, entering the Black Sea near Varna, Bulgaria; syntypes: LU)

Gobio gobio carpathicus natio krymensis Delyamure, 1937 (infrasubspecific, name not available; locality: river Biyuk-Karassu, South Crimea, Ukraine) from Bănărescu \& Nalbant, 1973 119

Gobio gobio acutipinnatus Menschikov, 1938: 121 (lake Marka-Kul, river Irtysh basin, Altai, Siberia; syntypes: ZISP 26865 [6], Bănărescu \& Nalbant, 1973: 126) from Berg, 1949: 645

Gobio gobio microlepidotus Battalgil, 1942: 294 , fig. 5 (type locality: lake Beysehir, Turkey; syn- types: ZMH 1127 [2], Wilkens, 1977: 158)

Gobio gobio intermedius Battalgil, 1944: 130, fig. 3 (type locality: lake Eber, Vilâyet Afyon Karahisar; Turkey; syntypes: ZMH 1128, 1135 [2], Wilkens, 1977: 158)

Gobio gobio sarmaticus Berg, 1949: 643 (available by description aud reference to $G$. g. carpathicus natio sarmaticus Slastenenko, 1934; type locality: rivers Dniester and Yuzhnyi Bug, Ukraine; syntypes: LU)

Gobio gobio muresia Jaszfalusi, 1951: 119, pl. 1 fig. 4 (type locality: river Maros [Mures] near Gödemesterháza and at confluence with creeks Zebrak and Göde, Hungary [now Romania]; syntypes: LU)

Gobio gobio lepidolaemus natio lindbergi Turdakov \& Piskarev, 1955: 89 (infrasubspecific, name not available)

Gobio gohio nikolskyi Turdakov \& Piskarev, 1955: 89 ( \ınu Darya basin)

Gobio gobio insuyanus Ladiges, 1960: 136, fig. 8 (type locality: creek Insuyu, Cihanbeyli, Turkey; holotype: ZMH 1133, missing, Wilkens, 1977: 157)

Gobio gobio gymnostethus Ladiges, 1960: 137, fig. 9 (type locality: creek Kizilcak, Nigde, Turkey; holotype: ZMH 1131, Wilkens, 1977: 157)

? Gobio gobio magnicapitata Gundrizer, 1967: 69 (lake Kara-khol, Kharnusovoe, Khamsari, Tadzhikistan) from Gundrizer, 1979: 9

Gobio gobio albanicus Oliva, 1961: 42, pl. 2 fig. 3 (type locality: river Kiri, Albania; holotype: CUP 30408)

Gobio gobio balcanicus Dimovski \& Grupče, 1977: 95, fig. 1 (type locality: river Vardar and its tributaries, FYROM; syntypes: ? MMNHS [100])

Gobio gobio krymensis Bănărescu \& Nalbant, 1973: 119 (type locality: rivers Alma and Kacha, Crimea [see below]; syntypes: LU [100])

Gobio gobio feraeensis Stephanidis, 1973: 192, fig. 1 (type locality: Kefalovrysson [fountain] of Velestinon, Thessaly, Greece; holotype: lost ?)

Gobio gobio brevicirris Fowler, 1976: 103 (available by indication to Gobio gobio morpha brevicirris Berg, 1932c: 407, fig. 317 [=1914: 442]; type locality: various localities in Ukraine, Russia and Siberia; syntypes: ZISP)

Gobio gobio holurus Fowler, 1976: 104 (available by inclication to Gobio gobio lepidolaemus natio holurus Berg, 1914: 457, fig. 61; type locality: streams Martan and Tschernorjetschje, tributaries of river Sunsha near Grosnyj, Chechnia, Russia; syntypes: ZISP)

Systematic notes. Bănărescu \& Nalbant (1973: 110) recognise 19 subspecies and Bănărescu (1992a) 
later retained only 17 , with bulgaricus, gobio, obtusirostris, benacensis, feraeensis and ohridanus in Europe, and 11 in Asia. It seems likely that future analysis could lead to recognition of several forms as distinct species. Wu et al. (1977), followed by Bănărescu (1992a: 319), recognised G. soldatovi Berg, 1914 as valid. Some of the nominal taxa from Turkey seem to represent distinct species. Bănărescu (1992a: 317) comments that there is a gradual and continuous transition between G. g. obtusirostris and G. g. gobio in the Baltic Sea and the Dnieper basins and between $G$. g. gobio and G. g. bulgaricus in the southern part of the Danube basin; although data are not provided, I tentatively accept this as evidence that a single species is involved. Gobio gobio benacensis is treated here as a valid species.

Nomenclatural notes. Gobio gobio carpathicus natio krymensis Delyamure, 1937 is not available because it is infrasubspecific. The use of this name as a subspecies by Bănărescu \& Nalbant (1973: 119) makes this name available from this last publication and with Bănărescu \& Nalbant as authors. Their description is exclusively based on Berg (1949: 643), itself apparently exclusively based on Delyamure (1937); the only locality mentioned by Berg is thus the type locality and the specimens mentioned are syntypes.

\subsubsection{Gobio kesslerii Dybowski, 1862}

Gobio Kesslerii Dybowski, 1862: 71 (type locality: river Dniester near Mohilew [Mogilev], Ukraine; available by brief diagnosis and indication to Kessler [1856a: 30, Gobio uranoscopus ?; repeated in Kessler, 1856b: 353]; syntypes: ZISP 3436 [5], Berg, 1914: 464)

Gobio uranoscopus carpathorossicus Vladykov, 1925a: 250 (type locality: river Theiss [Tisza] between Buštino and Velky Byčkiv villages, Ukraine; syntypes [total 10]: MNHN 30-197 [1], Bertin \& Estève, 1948: 88)

Gobio similis Chichkoff, 1929: 159, pl. 1 fig. 1 (type locality: river Isker, Danube basin, Bulgaria, restricted by Bănărescu \& Nalbant, 1973: 158; syntypes: LU)

Gobio kessleri kessleri natio banaticus Bănărescu, 1953: 318, fig. 3 (infrasubspecific, name not available; locality: river Timis near Timisoara [between Albina and Urseni, Bănărescu, 1961b: 343], Banat, Romania; "holotype" MGAB 49913 listed by Mihai-Bardan, 1984: 456 has no nomenclatural status and thus is not holotype) Gobio kessleri antipai Bănărescu, 1953: 318, fig. 4 (type locality: mouth of river Danube at Sulina, Romania; syntypes [total 13]: MGAB 49908 [1],

\section{Mihai-Bardan, 1984: 456)}

Gobio kessleri banaticus Bănărescu, 1960: 121 (available by indication to Bănărescu, 1953; type locality: river Bega at Timisoara and lower Arges basin, Romania; syntypes [total 13] MGAB 49913 [1])

Systematic notes. Bănărescu \& Nalbant (1973: 157) recognise 3 subspecies "connected by intergrades”. Bănărescu (1992a: 321, 1994a: 53) considers that $G$. antipai might be a distinct species "reproductively isolated from the true riverine $G$. kessleri." Awaiting supporting evidence, I treat them as conspecific; it might be too late to clear this problem as $G$. antipai might be extinct (Bănărescu, 1994b: 12).

Nomenclatural notes. Mihai-Bardan (1984: 456) lists specimen MGAB 49908 as holotype of G. k. antipai. Bănărescu (1953) based his description on 12 specimens from Sulina and one from the lower Arges; he did not designate a holotype and all 13 specimens are syntypes. The type locality is explicitly restricted to Sulina by Bănărescu (1953: 319); it should be confirmed by a lectotype designation, maybe MGAB 49908, in order to avoid future possible confusion.

\subsubsection{Gobio uranoscopus (Agassiz, 1828)}

Cyprinus uranoscopus Agassiz, 1828: 1048, pl. 12 figs. 1a-d (type locality: river Isar in München, Germany; lectotype: MNHN 5825, designated by Bănărescu, 1970: 165)

Gobio frici Vladykov, 1925a: 249 (type locality: stream Teresovka, a tributary of river Teiss [Tisza], near Podplesa village, Ukraine; syntypes: LU [3])

Systematic notes. Bănărescu \& Nalbant (1973: 138) recognise $G$. frici as a valid subspecies and Bănărescu (1992a: 320) recognises G. u. elimeius as a third subspecies. Gobio elimeius is considered here as a valid species.

\section{Hypophthalmichthys molitrix (Valenciennes, 1844) introduced}

\section{Hypophthalmichthys nobilis (Richardson, 1845) introduced}

\subsubsection{Iberocypris palaciosi Doadrio, 1980}

Iberocypris palaciosi Doadrio, 1980: 7 (type locality: Río Jándula, Lugar Nuevo, Andujár (Jaén), Spain; holotype: MNCN A 790413162).

Systematic notes. The generic position and relationships of this species deserve further studies. Bianco (1988b: 9) suggested that it might be a Leuciscus related to the L. souffia complex (genus 
or subgenus Telestes of earlier authors); I agree with him that too much weight has been given to the number of rows of pharyngeal teeth in the definition of genera of European cyprinids.

\subsubsection{Ladigesocypris ghigii (Gianferrari, 1927)}

Leucaspius Ghigii Gianferrari, 1927: 123 (type locality: stream Mulini, Coschino [Coschinou], Rhodos Island, Greece; holotype: MSNM 4385, Conci \& Michelangeli, 1974: 225); also in Gianferrari, 1929: 39, fig. 1

Leucaspius Prosperi Gianferrari, 1927: 124 (type locality: stream of Mulini, in Coschino [Coschinou], Rhodos Island, Greece; holotype: MSNM 4386); also in Gianferrari, 1929: 39, fig. 3

Systematic notes. Karaman (1972: 143) considers L. prosperi, L. irideus (Ladiges, 1960) and L. mermere (Ladiges, 1960) as synonyms or subspecies of $L$. ghigii. Ladigesocypris irideus and $L$. mermere from Turkey are apparently distinctive, geographically isolated forms and are not considered conspecific.

Leucaspius prosperi was placed in Pseudophoxinus by Bianco \& Taraborelli (1988: 251).

Nomenclatural notes. Gianferrari clearly based her description of $L$. prosperi on a holotype; she mentions additional specimens which may be considered as paratypes. Specimens listed as syntypes by Bertin \& Estève (1948: 78) and Kottelat \& Sutter (1988: 53) are paratypes [additional paratypes have certainly been distributed to other museums]. Conci \& Michelangeli (1974: 225) list two syntypes as MSNM 4386 while this is the number given by Gianferrari, apparently for the holotype. Hopefully her published data on the holotype should allow its identification; if not, then all specimens have to be treated as syntypes. Gianferrari (1927) lists the type locality of L. prosperi as stream Mulini, while in 1929 she reported it as Arghiro; Conci \& Michelangeli merely report the locality of the 'syntypes' as Rhodos Island.

The original spelling of the name is $L$. prosperi. It has been emended by Gianferrari (1929) into L. prosperoi. As there is no explanation for this emendation, it has to be considered as an unjustified subsequent spelling and has no validity.

Leucaspius ghigii Gianferrari, 1927 and L. prosperi Gianferrari, 1927 are simultaneous synonyms. Karaman (1972: 143) acted as first reviser and retained $L$. ghigii as having priority over $L$. prosperi.

5.16.1 Leucaspius delineatus (Heckel, 1843) Squalius delineatus Heckel, 1843: 1041 (type locality: Marchfelds near Wien and Mähren, Austria; syntypes: NMW 49783 [7], 50794 [6], 50796 [11])

Cyprinus Fischeri Valenciennes, in Cuvier \& Valenciennes, 1844: 378 (nomen nudum; locality: creek Beresofka [Beresovka], tributary of river Ingoul, Cherson [Kherson] government, Russia; author stated to be Arendt, but Valenciennes is actual author)

Aspius Ovsianka Czernay, 1851: 281, pl. 7 (type locality: Charkowschen [Kharkov county], Russia; syntypes: LU)

Leucaspius abruptus Heckel \& Kner, 1858: 145, fig. 76 (type locality: Lemberg [now Lviv], Ukraine; syntypes: NMW 49741 [2])

Cyprinus Pallasii Arendt, in Kessler, 1860: 121 (nomen nudum)

Leuciscus pigmeus Plater, 1861: 41, 66 (Dwina [Dvina], Latvia) from Berg, 1912: 326

Owsianka Czernayi Dybowski, 1862: 147, 148 (type locality: in rivers Dnieper, Dniester, Don, Weichsel [Vistula] and Düna [Dvina], Poland, Ukraine, Russia and Latvia; syntypes: LU)

Leucaspius relictus Warpachowsky, 1889b: 60, fig. 36 (lake Svetloyar at Svetlaya Lusha (Ozero) near Vladimirskoe in Makarevskii District, Niznyi Novgorod Prov., Russia; syntypes: ZISP 7956 [6], Berg, 1912: 328) from Berg, 1949: 610

Leucaspius delineatus dimorphus Ruzsky, in Gratzianow, 1907b: 132 (nomen nudum; locality: river Sura, Simbirsk Prov., Russia; Berg, 1912: 327)

Leucaspius delineatus dimorphus Ruzskii, 1914: 1 (lakes in river Sura basin, Kurmysh district, Russia) from Berg, 1949: 610

? Phoxinellus thracicus Battalgil, 1942: 301, fig. 9 (type locality: Kagithane Deresi, a creek entering the Bosphorus, Turkey [European side]; syntypes: LU)

Leucaspius delineatus natio caucasicus Berg, 1949: 612 (infrasubspecific, name not available; locality: North Caucasus and Transcaucasia)

Systematic notes. Ladiges (1960: 123) considered Phoxinellus thracicus as a synonym of Leucaspius delineatus. Bănărescu (1992b: 755) considered $P$. thracicus as distinct, placing it in "Pseudophoxinus" and commenting that it might "actually be a Rutilus (Leucos) and does perhaps not deserve specific rank". Its status can only be solved by examination of the types (apparently not extant) or fresh material from the type locality. The species is not mentionned by Geldiay \& Balik (1988). It is not a Phoxinellus as defined by Bogutskaya (1992: 264) because it has too many branched anal rays (12-14, vs. 6$9)$. 


\subsubsection{Leuciscus borysthenicus (Kessler,} 1859)

Squalius borysthenicus Kessler, 1859a: 545 (type locality: river Dnieper near Aleschki [Aleshki], Ukraine; syntypes: ZISP ?)

Telestes leucoides Filippi, 1863: 392 (type locality: Batum, Georgia; syntypes: MZUT 671 [2], Tortonese, 1940: 140)

Leuciscus heterandrius Battalgil, 1940: 74, figs. 1-2 (type locality: a swift flowing tributary of lake Iznik, Turkey; holotype: Zool. Mus. Istanbul 2500)

Systematic notes. Leuciscus smyrnaeus Boulenger, 1896 (p. 154; type locality: rivers and streams between the north coast of Smyrna and Troy, Turkey; syntypes: BMNH 1895.12.28: 19-28 [10]), sometimes listed as a synonym of $L$. borysthenicus, is a valid species according to Bogutskaya (1994: $600,602)$.

Bogutskaya (1994: 618; 1995: 153) comments that $L$. borysthenicus and a few other species from southwest Asia stand apart from other species of Leuciscus and are possibly most closely related to Ladigesocypris and Pseudophoxinus.

\subsubsection{Leuciscus burdigalensis Valenciennes, 1844}

Leuciscus burdigalensis Valenciennes, in Cuvier \& Valenciennes, 1844: 218 (type locality: river Gironde, France; lectotype: MNHN 3104, designated by Almaça, 1969: 1121)

Squalius bearnensis Blanchard, 1866: 400, fig. 95 (type locality: lake Mariscot, near Biarritz, France; lectotype: MNHN A.2265, designated by Almaça, 1969: 1117)

Squalius oxyrrhis La Blanchère, 1873: 662, fig. (type locality: rivers Aveyron, upper Lot, Trueyre, Argence and Viaur, France; syntypes: LU)

Aturius Dufourii Dubalen, 1878: 157, pl. (type locality: river Adour [St. Sever, Landes, according to manuscript note by Dubalen in reprint in MNHN, Laboratoire d'Ichtyologie], France; type material: NT?)

Systematic notes. Spillmann (1961a: 146) mentions various populations of $L$. leuciscus from south-western France which differ morphologically (head shape, body shape, structure of gill-rakers) from other populations. His text is somewhat ambiguous about distribution; he seems to imply that in part of their range they co-exist with 'normal' L. leuciscus (p. 13), but at the same time he treats them as subspecies (p. 146); he also seems to imply that there is a correlation between habitat and morphology (he calls them ecological types, p. 140). If they were subspecies of a single species, then, by definition, two of them cannot occur in sympatry (the maps in Allardi \& Keith, 1991: 115116 also show two of them apparently in sympatry). The kind of morphological differences reported by Spillmann (especially the shape of the gill-rakers) seems to indicate that they are not conspecific with $L$. leuciscus and I tentatively recognise them here as valid species; judging from the data in Spillmann, it is even possible that more species should be recognised. If my hypothesis is correct, the supposed relation between habitat and "morphological types" would then be differences in preferred habitats of different species, a possibility not considered by Spillmann.

This is apparently the species recognised valid as L. rostratus by Roule (1925: 174).

\subsubsection{Leuciscus carolitertii Doadrio, 1988}

Leuciscus carolitertii Doadrio, 1988a: 302, fig. 1 (type locality: river Cega, Rebollo, Segovia, Spain; holotype: MNCN 82050157)

5.17.4 Leuciscus cephalus (Linnaeus, 1758) Cyprinus Cephalus Linnaeus, 1758: 322 (based on Linnaeus [1754: 77, pl. 30, Cyprinus ... radiis 11], Artedi [1738: gen. [spec.] 5, syn. 7, Cyprinus oblongus macrolepidotus ...] and Gronovius [1754: 3, n. 12, idem; 1756: 3, n. 12, idem]; type locality: "in Europa meridionali"; type material: syntypes: NRM LP 81 [1, Fernholm \& Wheeler, 1983: 212], UUZM 213 [Wheeler, 1991: 161])

? Cyprinus Capito Scopoli, 1786: 73 (type locality: lakes of Insubria, northern Italy; type material: NT; junior homonym of Cyprinus capito Gueldenstaedt, 1778: 248)

Cyprinus Chub Bonnaterre, 1788: 195, pl. 77 fig. 323; type locality: rivers of Europe [based on the chub of Pennant (edition not stated; see e.g., 1776: vol. 3: 222 n. 175 , pl. 73 ; no stated locality)]; type material: NT)

Cyprinus Lugdunensis Walbaum, 1792: 29: 705 (type locality: river Rhine near Leiden, Netherlands; holotype: BMNH 1853.11.12:138, Wheeler, 1958: 212 [material of Gronovius, 1763: 106, n. 335])

Cyprinus orthonotus Hermann, 1804: 322 (type locality not stated [Strasbourg, France ?]; type material: NT ?)

Leuciscus cabeda Risso, 1826: 438 (type locality: Nice, France; lectotype: MNHN B.846, designated by Almaça, 1969: 1124)

Cyprinus Albula Nardo, 1827a: 482, 488 (type locality: France [original locality: Adriatic Sea basin / France]; available by description and by indication to Jonston [1649: pl. 26 fig. 2, Al- 
bula] and "Bell" [Belon, 1555: 315, chevesne, 319 , rosse]; restricted here to 'chevesne' of Belon, 1555: 315 ; syntypes: some then in Nardo's collection in Chioggia, LU) or 1827b: 34, 40 (same data)

Cyprinus kietaibeli Reisinger, 1830: 77 (type locality: lake Balaton, Hungary; syntypes: LU)

Leuciscus Chub var. Pictava De la Pylaie, 1835: 533 (nomen nudum; locality: Napoléon, Vendée, France)

Cyprinus rufus Vallot, 1837: 185 (type locality: Dijon, Pontailler and Auxonne, France; syntypes: LU)

Leuciscus Squalus Bonaparte, 1837: [fasc. 19, p. 225], pl. 111 fig. 1, pl. 112 fig. 2 (type locality: rivers Tevere and Arno, almost all streams of Toscana, Stato Romano and Regno di Napoli, Italy; syntypes: ANSP 6273-6309 [37], Böhlke, 1984: 91, MNHN 5421 [4], Bertin \& Estève, 1948: 61, NMW 48920 [1]; a secondary junior homonym of Cyprinus squalus Walbaum, 1792)

Lenciscus Albus Bonaparte, 1838: [fasc 22, p. 237], pl. 113 fig. 2 (type locality: lake Trasimeno, Italy; syntypes: ANSP 6335-6336 [2], Böhlke, 1984: 68, MNHN 3903 [1], Almaça, 1969: 1117)

Leuciscus Cavedanus Bonaparte, 1838: [fasc .22, p. 239], pl. 113 fig. 3 (type locality: stream l'iccolo Reno, Bolognese, Italy; syntypes: ANSP 63136318 [6], Böhlke, 1984: 72)

Leuciscus brutius Costa, 1838: fasc. 19: 22, pl. 18 (type locality: "river Crati which flows through Cosenza", Italy; lectotype: MNHN 198, designated by Almaça, 1969: 1126)

Leuciscus Rissoi Schinz, 1840: 326 (type locality: brooks around Nice, France; based on Leuciscus bulatmai [non Gmelin, 1784: 135] of Risso, 1810: 361 ; syntypes: MNHN ?)

Leuciscus orientalis Nordmann, 1840: $48 \overrightarrow{4}$ (type locality: Abasie [Abkhasia]; syntypes: LU)

Squaliuls tyberinus Bonaparte, 1841: [fasc. 30, introduction p. 5] (available by indication to Leuciscus squalus of [fasc. 19. p. 221] pl. 111 fig. 1, pl. 112 fig. 2; type locality: rivers Tevere and Arno, almost all streams of Toscana, Stato Romano and Regno di Napoli, Italy; syntypes: ANSP 6273-6309 [37], Böhlke, 1984: 91, MNHN 5421 [4], Bertin \& Estève, 1948: 61, NMW 48920 [1])

Leuciscus Nothulus Bonaparte, 1841: [fasc. 30, introduction p. 5], pl. 112 fig. 2 (type locality: not stated [in text, part of Leuciscus squalus]; syntypes: LU)

Squalius Pareti Bonaparte, 1841: [fasc. 30, introduction p. 16] (type locality: Liguria, Italy; syntypes: LU); predates actual description in Bona- parte, 1842: 148 (locality: fish market of Scoglietto del Durazzo, Genova, Italy)

Squalius cephalopsis Heckel, 1843: 1080 (type locality: Aleppo, Syria; syntypes: NMW 49438 [3], 49440 [2])

Leuciscus squalius Valenciennes, in Cuvier \& Valenciennes, 1844: 191 (type locality: Italy; lectotype: MNHN 3914, designated by Almaça, 1969: 1124)

Leuciscus Albiensis Valenciennes, in Cuvier \& Valenciennes, 1844: 194 (type locality: river Elbe, Germany; holotype: MNHN)

Leuciscus peloponensis Valenciennes, in Cuvier \& Valenciennes, 1844: 197 (type locality: Morée [Peloponnese], Greece; holotype: MNHN 3905, Almaça, 1969: 1127)

Leuciscus frigidus Valenciennes, in Cuvier \& Valenciennes, 1844: 234 (type locality: "probably from Germany because our specimens has been received from the Vienna Museum"; holotype: MNHN)

Cyprinus salmoneus Gronow, 1854: 186 (type locality: river Rhine near Leiden, Netherlands; holotype: BMNH 1853.11.12: 138, Wheeler, 1958: 212)

Leuciscus latifrons Nilsson, 1855: 309 (type locality: river Helgeă at Broby and Glimăkra / Götheborg / Lake Hjelmaren, river Storå / Norrköping, Sweden / Finland [based on material in NRM; S. O. Kullander, in litt. 1995; syntypes: NRM)

Leuciscus Cii Richardson 1856: 375 (type locality: river Gemlek, anciently named Cius, entering the Propontis near the promontory of Posidium, Asia Minor; holotype: BMNH ?)

Squalius meunier Heckel, 1852b: 68 (available by indication to Cyprinus jeses of Jurine [1825: 207] and Valenciennes [in Cuvier \& Valenciennes, 1836: 74], Leuciscus dobula of SelysLongchamps [1842: 206], Valenciennes [in Cuvier \& Valenciennes, 1844: 172] and Bonaparte [1846: 30]; syntypes: material used or listed by these authors)

Squalius turcicus Filippi, 1865: 359 (river Arax near Erzerum, Turkey) from Berg, 1912: 135

Squalius meridionalis Blanchard, 1866: 396, fig. 93 (type locality: river Sâve [Lot-et-Garonne] and river Sorgue near Avignon, France; lectotype: MNHN A.2257, designated by Bertin \& Estève, 1948: 63)

Squalius clathratus Blanchard, 1866: 398, fig. 94 (type locality: river Lot in Cahors, France; lectotype: MNHN A.2260, designated by Almaça, 1969: 1125)

Squalius turcicus var. platycephala Kamenskii, 
1897: 85, pl. 4 figs. 6-8 (lakes Toporovan [Paravani] and Tuman-Göl in upper Kura and Araks basins, Georgia) from Berg, 1949: 559

Squalius agdamicus Kamensky, 1901: 49, 160 (type locality: river Agdam, Shusha district, Azerbaidjan; hototype: ZMT ?)

Squalius cephalus var. Athurensis Roule \& Cardaillac de Saint-Paul, 1903: 732 (type locality: river Adour, France; syntypes: LU)

Leuciscus cephalus orientalis natio kaznakovi Berg, 1912: 143, pl. 2 fig. 3 (infrasubspecific, name not available; locatity: lake Nour near Vandam, Tur'yan-chai basin [river Kura basin], Nuchinskii District, Elisavetopol'skaya Prov., Russia)

Squalius cephalus cavedanus var. prespensis Karaman, 1924b: 67 (infrasubspecific [ICZN art. 45 (f) (iii)], name not available; locality: lake Prespa and tributaries, FYROM and Greece)

Leuciscus orientalis var. pursakensis Hanko, 1924: 140, pl. 3 fig. 1 (type locality: Kara-Chehir, Kötschke-Kissik and Eski-Chehir, Turkey; syntypes: MNH ? [6])

Leuciscus cephalus var. wjatkensis Lukash, 1925: 9, 48 (type locality: river Vyatka near Mulino, Volga basin, Russia) from Costa Pereira, in litt.

Squalius cephalus vardarensis Karaman, 1928: 156 (type locality: basin of river Vardar, FYROM; syntypes: LU); repeated in 1929: 172

Leuciscus svallize zrmanjae natio risae Vladykov \& Petit, 1930: 395 (infrasubspecific, name not available; locality: lake Risa, draining to southern shore of lake Butrinto, Albania)

Leuciscus cephalus orientalis natio aralychensis Barach, 1934: 109 (infrasubspecific, name not available; river Karasu at the foot of $\mathrm{Mt}$. Ararat) from Berg, 1949: 557

Leuciscus cephalus orientalis natio zangicus Barach, 1934: 111 (infrasubspecific, name not available; river Zanga at Erivan [Yerevan], Armenia) from Berg, 1949: 557

Leuciscus cephalus orientalis natio ardebilicus Barach, 1934: 114 (infrasubspecific, name not available; river Balyk-chai near Ardebi [Ardabil], Iran) from Berg, 1949: 557

Leuciscus cabeda var. pamvoticus Stephanidis, 1939a: 30, pl. 6 (type locality: lake Pamvotis, central Greece; syntypes: lost? [2])

Leuciscus cephalus orientalis natio thracicus Drensky, 1930: 672 (infrasubspecific, name not available; locality: river Maritza, Bulgaria)

Leuciscus cephalus macedonicus Karaman, 1955: 185 (type locality: river Strumica, FYROM; syntypes: LU; a primary junior homonym of Leuciscus macedonicus Steindachner, 1892)

Leuciscus cephalus moreoticus Stephanidis, 1971a:
197, fig. 8 (type locality: lake Stymphalia, Greece; syntypes: lost ? [11])

Leuciscus cephalus prespensis Fowler, 1977: 28 (available by indication to Squalius cephalus cavedanus var. prespensis Karaman, 1924b: 67; type locality: lake Prespa and tributaries, FYROM; syntypes: LU)

Leuciscus cephalus ruffoi Bianco \& Recchia, 1983: 16 (type locality: river Savuto, Calabria, Italy; holotype: IZA 830a)

Systematic notes. Bianco \& Recchia (1983) considers four valid subspecies in Italy: L. c. cabeda, L. c. albus, L. c. brutius and L. c. ruffoi; later, Bianco (1988a: 151) recognises a single taxon in Italy, L. cephalus. Additional work is needed to determine their status under the PSC, especially L. c. albus from lake Trasimeno which, according to Bianco \& Recchia's data, seems to have a distinctive head shape.

Nomenclatural notes. Wheeler \& Fernholm (1983: 212) and Wheeler (1991: 161) noted that Linnaeus's Cyprinus cephalus is bared on literature accounts (Artedi, 1738; Gronivius, 1754, 1756) of the species now called L. cephalus and on specimens of the South American erythrinid Hoplerythrinus unitaeniatus (Agassiz, in Spix \& Agassiz, 1829). This has apparently been noted by other authors, but as there is apparently no previous restriction of the use of the name, as first reviser I restrict C. cephalus to the species described by Artedi (1738).

The description of Cyprinus kietaibeli Reisinger, 1830 from lake Balaton is not very informative and the total size of the individuals on which it is based is not even indicated. His material is not present in NMW and Reisinger's specimens have not been reported from any other museum. Valenciennes (in Cuvier \& Valenciennes, 1844: 380) considers that this taxon is probably a variety of Leuciscus leuciscus, but the species does not occur in lake Balaton (Bíró \& Paulovits, 1994: 2165) while Reisinger described C. kietaibeli as abundant. Schinz (1840: 327) reports the size as 68 inches, but there is no evidence that he had seen specimens. The species has been sometime identified with Leucaspius delineatus (a name over which it would have priority). The size of Schinz's fish is apparently too large for being $L$. delineatus. Also, Leucaspius delineatus has a short, incomplete lateral line; Reisinger and Schinz mention only a curved lateral line. To remove ambiguity and threat on the name $L$. delineatus, a specimen of $L$. cephalus could be designated as neotype of Cyprinus kietaibeli Reisinger, 1830.

Böhlke (1984: 86) lists syntypes of "Leuciscus 
pareti Bonaparte, 1846". Bonaparte (1846: 31) provided neither description, diagnosis nor indication. This species is actually described by Bonaparte (1842), but the name was already made available in 1841 .

Almaça (1969: 1129) lists a syntype MNHN 2623 from Roma for Leuciscus cavedanus Bonaparte, 1838. The type locality being explicitly stated as Piccolo Reno by Bonaparte, this specimen has no type status.

Bertin \& Estève (1948: 63) list a holotype for Squalus meridionalis Blanchard. This specimen is a syntype; Blanchard (1866: 398) explicitly indicated that he had several specimens.

The name L. cephalus albus Bonaparte, 1838 is sometime used for a population from Greece (e.g., Economidis, 1991: 14). As this taxa has been originally described from lake Trasimeno, where a distinctive population occurs, it does not seem that the name can be used for a Greek fish. If one really wishes to use a subspecific name for this population, L. c. pamvoticus Stephanidis, 1939, is the earliest available name. The name $L$. c. macedonicus Karaman, 1955 cannot be used for a subspecies of $L$ cephalus as the name is a primary homonym of L. macedonicus Steindachner, 1892, now Pachychilon macedonicum

\subsubsection{Leuciscus idus (Linnaeus, 1758)}

Cyprinus Idus Linnaens, 1758: 324 (based on Linnaeus [1746: 121, n. 320, Cyprinus ... radiis 13], Artedi [1738: gen. 5 [6], syn, 14, spec. 6 [5], Cyprinus iride sublutea ...] and Gronovius [1754: 3, n. 13, idem]; type locality: "in Europae aquis dulcibus"; type material: NT)

Cyprinus Jeses Linnaeus, 1758: 325 (based on Artedi [1738: syn. 7, Cyprinus cubitalis ...]; type locality: "in Germania"; type material: NT)

Cyprinus Orfus Linnaeus, 1758: 324 (based on Artedi [1738: syn. 6, Cyprinus orfus dictus], Ray [1710: 118, Rutilus latior ...] and Meyer [1748-56: pl. 94, Orf]; type locality: "in Rheno, Angliae fluviis, lacubus"; type material: NT)

Cyprinus idbarus Linnaeus, 1758: 324 (type locality: "in Sveciae lacubus"; type material: NT)

Cyprinus microlepidotus Ekström, 1835: 18, pl. 2 (type locality: "Scheeren von Mörkö"[Strait of Mörkö Island, near Stockholm], Sweden; syntypes: LU)

Leuciscus neglectus Selys-Longchamps, 1842: 208 (type locality: Bruxelles, Belgium; syntypes [total 4]: ? ISNB [not found], MHNN 1008 [1], Kottelat, 1984: 148)
Idus melanotus Heckel, 1843: 1008, pl. 1 (available by indication [ICZN art. 12(b)(7), 14(b)]; no locality data; syntypes: ? NMW [used as a replacement name for Cyprinus idus Linnaeus. 1758 by Heckel, 1852 b: 49, 66])

Idus miniatus Bonaparte, 1845: 9 (type locality: eastern Europe; syntypes: NMW 53432 ?, probably based on information provided by Heckel, thus material subsequently listed by Heckel \& Kner [1858: 151] could be syntypes)

Idus miniatus Heckel \& Kner, 1858: 151 (type locality: Tirol, Austria; syntypes: NMW 53432 [1]; junior homonym of Idus miniatus Bonaparte, 1845: 9)

Lenciscus idus var. lapponicus Günther, 1868: 230 (type locality: river Munio, Sweden and Finland; syntypes: BMNH [6])

Squalius oxianus Kessler, 1877a: 124 (type locality: mouth of river Amu and Kunja-Urgentsch in delta of river Amu, Uzbekistan [Berg, 1949: 567]; syntypes: Univ. St Petersburg 463, Berg. 1912: 179)

Idus melanotus var. orientalis Sinitzyn, 1900: 45 (nomen nudum; lake Baikal, Siberia) from Berg, 1912: 166

Lenciscus idus var. auratus Bade, 1901: 128 (infrasubspecific, name not available)

Leuciscus idus idus natio sibiricus Kirillov, 1958 (infrasubspecific, name not available: locality: river Leny, Siberia) from Kirillov, 1962: 47

\subsubsection{Leuciscus illyricus (Heckel \& Kner, 1858)}

Squalius illyricus Heckel \& Kner, 1858: 195, fig. 108 (type locality: rivers Isonzo, Knin and Sign, Dalmatia; syntypes: NMW 49313 [7], 49315 [3], 49322 [1], 49324 [1], 49331 [1], 49339-341 [3, illustrated by Bianco \& Knežević, 1987: 50, fig. 1])

Systematic notes. Bianco \& Knežević (1987: 49) considered the validity of this species as doubtful.

5.17.7 Leuciscus keadicus Stephanidis, 1971 Leuciscus soufia keadicus Stephanidis, 1971a: 198, fig. 9 (type locality: river Eurotas near Sparta, and its small tributaries Kelefinas [Inous], Magoulitsas and Retzinas, Greece; syntypes: lost? [21])

Systematic notes. Originally described as a subspecies of L. souffia, this taxon is considered as a valid species by Economidis (1991: 15), Economidis \& Bănărescu (1991: 266) and Tsigenopoulos \& Karakousis (1996), who, however, do not discuss its status. A direct comparison of both taxa shows that they are easily distinguished by body 
shape and colour pattern (details will be presented elsewhere).

\subsubsection{Leuciscus leuciscus (Linnaeus, 1758)}

Cyprinus Leuciscus Linnaeus, 1758: 323 (based on Artedi [1738: syn. 9, Cyprinus novem digitorum ...]; type locality: "in Europae media"; type material: NT)

Cyprinus Grislagine Linnaeus, 1758: 323 (based on Linnaeus [1746: 123, n. 325, Cyprinus ... radiis 11], Artedi [1738: gen. 5 [12], syn. 5, spec. 12 [5], Cyprinus oblongus ...] and Gronovius [1756: 2, n. 148, idem]; type locality: "in Europae lacubus"]; type material: NT)

Cyprinus Dobula Linnaeus, 1758: 323 (based on Artedi [1738: syn. 10, Cyprinus pedalis gracilis ...]; type locality: "in Europa media"; type material: NT)

Cyprinus squalus Walbaum, 1792: 28, 705 (type locality: river Rhine [Gronovius, 1763: 106, n. 334; see also Gronow, 1854: 185]; type matcrial: NT)

Cyprinus graining Walbaum, 1792: 36 (type locality: river Mersey [near Warrington], U.K.; based on Pennant [1769: vol. 3: 367 [1776: 331], Graining]; type material: NT); also Bloch, in Schneider, 1801: 450 (same data)

Cyprinus Umbra Walbaum, 1792: 30, 705 (based on Ausonius [ca. 370: 90, river Moselle], $=$ Thymallus thymallus according to Valenciennes, in Cuvier \& Valenciennes, 1848: 429] and Gronovius, 1763: 106, n. 337 [= Cyprinus salax Gronow, 1854: 186]; here restricted to Gronow's account; type locality: lakes of Europe; lectotype (present designation): $\mathrm{BMNH}$ 1853.11.12:157 [holotype of C. salax, Wheeler, 1958: 210])

Cyprinus Lancastriensis Shaw, 1804: vol. 5 (1): 234 (based on Pennant [edition not stated, e.g., 1776: 321, Grayning]; type locality: river Mersey near Warrington, U.K.; type material: NT)

Cyprinus simus Römer-Büchner, 1827: 68, pl. 2 (type locality: river Main near Frankfurt, Germany; syntypes: LU; junior primary homonym of Cyprinus simus Hermann, 1804)

Leuciscus vulgaris Fleming, 1828: 187 (type locality: "rivers in England"; available by description and by indication to earlier sources; type material: NT ?)

Leuciscus argenteus Fitzinger, 1832: 336 (unnecessary replacement name for Cyprinus leuciscus Linnaeus, 1758; author indicated as Agassiz, but Fitzinger is actual author)

Leuciscus rostratus Agassiz, 1835b: 38 (nomen nudum)
Leuciscus rodens Agassiz, 1835b: 39, pl. 1 figs. 12 (type locality: lake Neuchâtel, Switzerland; syntypes: MHNN 964 [2], Kottelat, 1984: 148, MNHN)

Leuciscus majalis Agassiz, 1835b: 38, pl. 1, figs. 3-7 (type locality: lake Neuchâtel, Switzerland; syntypes: MHNN 969 [4], 970 [1], 971 [1], Kottelat, 1984: 148, MNHN)

Cyprinus mugilis Vallot, 1837: 196 (type locality: river Saône, France; syntypes: LU)

Leuciscus rostratus Valenciennes, in Cuvier \& Valenciennes, 1844: 201 (type locality not stated; syntypes: based on a drawing sent by Agassiz [based on specimen possibly in MHNN] and MNHN 256 [1] from river Meuse at Liège, Belgium [Bertin \& Estève, 1948: 62])

Leuciscus saltator Bonaparte, 1845: 8 (type locality: western Europe; holotype: ANSP 6364, Böhlke, 1984: 90)

Squalius lepusculus Heckel, 1852b: 109, pl. 11 figs. 1-4 (type locality: small tributaries of river Danube near Wien, river Moldau [Vltava] near Budweis [České Budějovice], river Olsa [Olše] in Teschen [Těšín]/upper rivers Elbe [Czech Republic] and Oder [Odra] [Poland]; syntypes: NMW 49345 [2], 49347-348 [3], 49352 [2], 49393 [2])

Squalius chalybeius Heckel, 1852b: 111, pl. 12 figs. 1-4 (type locality: stream Kamp near Zwetl monastery, Austria; syntypes: NMW 49353-354 [2], 49382 [1], 49391 [1])

Cyprinus salax Gronow, 1854: 186 (type locality: lakes of Europe; holotype: BMNH 1853.11.12: 157, Wheeler, 1958: 210)

Squalius vulgaris var. robustior Walecki, 1863: 363 [line 5] (unnecessary replacement name for Squalius chalybeius Heckel, 1852b)

Squalius vulgaris var. robustior v. nasutus Walecki, 1863: 363 (infrasubspecific, name not available; unnecessary replacement name for Squalius chalybeius Heckel, 1852b)

Squalius vulgaris var. minor Walecki, 1863: 363 (infrasubspecific, name not available; unnecessary replacement name for Squalius lepusculus Heckel, 1852b)

Squalius vulgaris var. argenteus Walecki, 1863: 363 (infrasubspecific, name not available)

Squalius vulgaris var. leptorhinus Walecki, 1863: 342 (nomen nudum), 1864b: 50 (infrasubspecific, name not available)

Squalidus baicalensis Dybowsky, 1874: 388 (type locality: lake Baical [rare] and all its tributary streams, Siberia; syntypes: LU)

Squalius leuciscus var. elongata Fatio, 1882: 603 (infrasubspecific, name not available) 
Squalius leuciscus var. elata Fatio, 1882: 603 (infrasubspecific, name not available)

Squalius leuciscus var. lateristriga Fatio, 1882: 603 (infrasubspecific, name not available)

Squalius suworzewi Warpachovski, 1889a: 17 (river Irtysh at Semipalatinsk, Siberia) from Berg, 1949: 546

Squalius mehdem Warpachovski, 1897: 255, pl. 12 fig. 1 (type locality: river Ob at Atlym, Siberia; holotype: ZISP 11295)

Leuciscus leuciscus baicalensis natio kirgisorum Berg, 1912: 112, pl. 1 fig. 3 (infrasubspecific, name not available; localities: rivers Chu and Nura, Kirghizistan)

Idus stagnalis Dubalen, 1913: 77 (type locality: Vendays, Cazaux, Sanguinet, Parentis, Léon, Pissos, France; syntypes: LU)

Leuciscus leuciscus natio roulei Vladykov, 1931: 311, fig. 18 (infrasubspecific, name not available; localities: lower course of rivers Tisza, Rieka, Borjava [Borzhava], Latorica, Ouge [Uh], and Laboretz [Laborec], Slovakia and Ukraine; specimen MNHN 30-206, designated as lectotype by Almaça, 1969: 1122 has no type status)

Leuciscus leuciscus baicalensis natio teletzkensis Ioganzen, 1945: 10 (infrasubspecific, name not available; locality: lake Teletzk, Siberia) from Berg, 1949: 546

Leuciscus leuciscus roulei Bertin \& Estève, 1948: 63 (available by indication to Leuciscus leuciscus natio roulei Vladykov, 1931: 311; type locality: [river] Uh, Slovakia and Ukraine; lectotype: MNHN 30-206, present designation)

Nomenclatural notes. Cyprinus leuciscus, C: grislagine and $C$. dobula are simultaneous subjective synonyms. I could not find which first reviser formally established priority between these names; C. leuciscus seems to have been universally accepted as having priority. Linnaeus's description of C. grislagine is based on several earlier accounts which probably refer to different species (see Heckel, 1853: 32); grislagine is the vernacular name which Willughby (1686: 263, pl. Q.1) reports for L. souffia in Augsburg, Germany.

Bertin \& Estève (1948: 62) list a paratype of "Leuciscus argenteus Selys-Longchamps, 1842". This name is actually an unnecessary replacement name for Cyprinus leuciscus introduced by Agassiz (1835b) and the specimen listed by Bertin \& Estève has no type status.

\subsubsection{Leuciscus lucumonis Bianco, 1983}

Leuciscus lucumonis Bianco, 1983: 82, fig. 1 (type locality: Fosso delle Lene, stream between Campagnatico and Istia d'Ombrone, river Ombrone basin, Grosseto, Toscana, Italy; holotype: IZA 8238)

5.17.10 Leuciscus microlepis (Heckel, 1843) Squalius microlepis Heckel, 1843: 1042 (type locality: Imosky, Croatia; syntypes: NMW 49414416 [5], 49421 [1], MHNN 973 [1], Kottelat, 1984: 147)

Squalius tenellus Heckel, 1843: 1042 (type locality: waters around Livno, Bosnia; syntypes: NMW 16001-002 [3], 49613 [2])

\subsubsection{Leuciscus montenigrinus Vukovic, 1963}

Leuciscus souffia montenigrinus Vukovic, 1963: 206 (type locality: river Moraca near Titograd, Montenegro; syntypes: LU); repeated in Vukovic, 1965: 217

Systematic notes. Considered as a subspecies of Leuciscus souffia by Bănărescu (1992: 680). Data in Vukovic (1963) suggest that under the PSC L. s. montenigrinus is specifically distinct from the adjacent $L$. souffia populations. Lateral line scale counts (44-50 in L. montenigrinus, vs. $50-57$ in $L$. souffia) and vertebrae counts (37-38, vs. 42-43) seem conspicuously diagnostic. Vukovic's morphometric data are uninformative.

Nomenclatural notes. Vukovic (1963) described the species as L. s. montenigrinus and in 1965 he published the diagnosis again as L. s. montenegrinus; the second spelling is an incorrect subsequent spelling and is not valid (ICZN art. 33(c)).

\subsubsection{Leuciscus muticellus Bonaparte, 1837}

Leuciscus Muticellus Bonaparte, 1837: [fasc. 20, p. 231], pl. 112 fig. 3 (type locality: streams of Viterbo, Terni and Roma provinces, Italy; syntypes: MNHN 3891 [5], Almaça, 1969: 1117; ANSP 6374-6411 [38], Böhlke, 1984: 84)

Leuciscus comes Costa, 1838: fasc. 19: 26, pl. 18bis (type locality: Kingdom of Napoli [now in Italy]; syntypes: LU)

Telestes Savigny Bonaparte, 1840: [fasc. 28, p. 247], pl. 115 fig. 1 (type locality: lake Lugano and various small lakes of Piemonte, Italy; syntypes: ANSP 6417-6423 [7], Böhlke, 1984: 90)

Systematic notes. See Leuciscus souffia for discussion.

\subsubsection{Leuciscus pleurobipunctatus (Ste-} phanidis, 1939)

Rutilus pleurobipunctatus Stephanidis, 1939a: 19, pl. 2 (type locality: streams Thiamos, Lourou, Acheron and Evinos, lake Trichonis / creek 
Messoghi, area of Kalamictisa (1 hour from Aghios Matheus village), Corfou [Kerkira island], Greece; syntypes: lost? [96])

Rutilus pleurobipunctatus alfiensis Stephanidis, 1971a: 183 (type locality: river Alfios basin [Alfios, Enipeus, Erymanthos, Ladon, Tragos], river Pinios and its tributary Langadi near Aghios Dimitrios, rivers Vergas and Peristeras [Kyparissia], Greece; syntypes: lost ? [22])

Systematic notes. Placed in Phoxinellus by Karaman (1972: 128) and Economidis (1991: 12) and in Pseudophoxinus by Bianco (1986: 293). Bogutskaya (1992: 283) excluded it from Pseudophoxinus. Placement in Leuciscus follows Bianco (1988b: 9) but needs to be confirmed by a proper phylogenetic revision and diagnosis of this genus.

\subsubsection{Leuciscus polylepis (Steindachner,} 1866)

Telestes polylepis Steindachner, 1866e: 300, pl. 1 (type locality: streams Mresniza [Mresnica] and Dobra and creek in Josefthal [Josipdol, about $10 \mathrm{~km}$ SSE of Ogudin], Croatia; syntypes [total 6 , but presently not possible to find out which ones among following series]: NMW 18931-941 [11], 49709-715 [41], MSNM 4660 [1], Conci \& Michelangeli, 1974: 225)

\subsubsection{Leuciscus pyrenaicus Günther, 1868}

Leuciscus pyrenaicus Günther, 1868: 223 (type locality: river Mondego and Cintra [Sintra], Portugal, restricted to Sintra by Costa Pereira, 1995: 495; syntypes: BMNH [8])

Nomenclatural notes. Günther (1868: 223) based his description of $L$. pyrenaicus on material from two localities, Sintra and river Mondego. Costa Pereira (1995: 495) noted that these represent two species and restricted the type locality to Sintra; the material from river Mondego belongs to $L$. carolitertii. The syntype series cannot be restricted and all of Günther's specimens are still syntypes. A lectotype designation would be in order to definitively link the name $L$. pyrenaicus to the present concept of the species.

An electrophoretic study of Portuguese L. pyrenaicus and $L$. caroliterii shows that populations from southern Portugal are only distantly related to these two species (Coelho et al., 1995). Other data (e.g., morphology) relevant to elucidate their status are not available.

\subsubsection{Leuciscus souffia Risso, 1826}

Leuciscus Souffia Risso, 1826: 438 (type locality: river Var, France: type material: NT)

Chondrostoma rysela Agassiz, 1835b: 38 (avail- able by indication to Gesner [1560: 290, Risele, Ryserle, Ryssling]; type locality: "in silo torrento ad urbem nostram" = stream Sihl in our city [Zürich], Switzerland; neotype: MNHN 712, present designation)

Leuciscus agassii Valenciennes, in Cuvier \& Valenciennes, 1844: 254, pl. 495 (type locality: Danube [basin] in München, Germany; lectotype: MNHN 712, designated by Almaça, 1969: 1123)

Telestes Rysela Heckel, 1852a: 387 (type locality: stream Sihl in Zürich, Switzerland; neotype: MNHN 712, present designation; a secondary junior homonym of Chondrostoma rysela Agassiz, 1835)

Systematic notes. Spillmann (1959, 1961b, 1962 , 1966, 1967; d'Aubenton et al., 1971) recognised three subspecies in L. souffia: L. s. souffia from the Var basin, France, L. s. agassii from the Rhône, Rhine and Danube basins, and L. s. muticellus from Italy. Bănărescu (1992: 680) recognised five subspecies (without providing the supporting data): L. s. souffia from the Var and Rhône basins, L. s. agassii from the Rhine and Danube basins, L. s. muticellus from Italy, an unnamed subspecies from the upper Tisza basin in Romania and Ukraine, and L. s. montenigrinus from Montenegro. Leuciscus montenigrinus is considered as a valid species here. Spillmann's data show that his material of L. s. agassii is easily diagnosed from his L. s. muticellus by lateral line scale count. Geographically, the two stocks come very close in southern France. Spillmann's L. s. souffia occurs in a single basin (river Var) between the two other subspecies, but is in quite close agreement with his L. s. agassii. Instead of 3 subspecies, I see in this case 2 diagnosable species with extensive, discrete distributions and history (Italian and RhôneRhine-Danube stocks) and a narrowly delimited population probably representing past introgression between them (see Hewitt, 1989 for review of hybrid zones and Warren, 1992: 25-28 for a summarised discussion) or of independent origin (Var stock).

Nomenclatural notes. There is a slight nomenclatural pol, lem resulting from the fact that the oldest name available for the L. souffia complex is based on material from the possible introgression zone. As the work of Spillmann has shown that it has greater similarity with the RhôneRhine-Danube species than with the Italian one, I consider the Var material as conspecific with the Rhône-Rhine-Danube species and the name $L$. souffia is to be used for this species. The earliest available name for the Italian species is $L$. muticellus. Names applied to hybrids cannot be used 
(ICZN art. 23(h)) but this does not apply here as the name $L$. souffia is not based on a hybrid individual but on a population possibly of hybrid origin.

If future research were to demonstrate that the population from river $\mathrm{Var}$ is specifically (or subspecifically under the BSC) distinct from the Rhône-Rhine-Danube species, note that the earliest available name for the latter is L. rysela (Agassiz, 1835), not L. agassii Valenciennes, 1844.

The correct spelling of the name is Leuciscus souffia and not L. soufia; Risso (1826: 438) unambiguously used $L$. souffia as the scientific name and soufia as the local name. The correct spelling of the subspecies sometimes recognised from the Danube basin is L. s. agassii and not L. s. agassizii (e.g., Spillmann, 1961b, 1962, 1966, 1967), because the specific name is based on a latinised form of Agassiz's name (probably Agassius) and probably also because of euphonic usage at the time [the final ' $z$ ' of Agassiz is not pronounced in French - his native language].

The status and history of the use of Chondrostoma rysela is somewhat complicated. As originally proposed by Agassiz (1835b) the name is not accompanied by a description but is merely available by indication to the rysele of Gesner [edition not stated; see, e.g., 1560: 290]. Gesner's species has been identified as L. souffia by Heckel (1849: 288; 1852a: 377). Difficulties arise because Agassiz sent (unpublished) drawings of a fish labelled C. rysela to various correspondents (including at least Valenciennes, Heckel, Bonaparte and SelysLongchamps) and the fish in the drawings likely was not the same as Gesner's species. This does not enter into consideration for the identification of Agassiz's (1835b) species as the name is available by indication, but these drawings have been used by several of his correspondents as a reference for the identification of various Chondrostoma. As the name rysela is technically available only for Gesner's rysele, these identifications have to be treated as misidentifications and the name is not available for a Chondrostoma species. This clearly applies to the use of C. rysela by Bonaparte (1840: [p. 251] pl. 115 fig. 3).

Valenciennes (1844) used both Chondrostoma rysela (p. 395) and Leuciscus ryzela (p. 199). His C. rysela is based on Agassiz's drawing and paper and is merely a subsequent use of that name. Valenciennes's L. ryzela is a description of a new species based on original specimens which Valenciennes considered to be the same as Bonaparte's (1840) C. rysela. The name L. ryzela is available and is treated here as a synonym of Rutilus pi- gus. Bonaparte's C. rysela has been identified as C. soetta by Heckel (1852a: 388) and as an hybrid C. nasus x L. souffia by Günther (1868: 235).

Heckel $(1852 \mathrm{a}: 378,388)$ noted the problem of the identification of Gesner's rysele and considered two valid species with the specific name rysela, a Telestes and a Chondrostoma. He considered his Chondrostoma to be the same as Agassiz's (based on the drawing) and his Telestes was a name for the Rysele of Gesner. This was logical in Heckel's context and probably correct under the then prevailing nomenclatural practices, but cannot be followed under our present nomenclatural rules. Formally, under the present ICZN, Heckel's C. rysela has to be considered a misidentification of C. rysela of Agassiz and has no nomenclatural status. [Although technically not the case, if one would alternatively argue that C. rysela of Heckel be considered as the description of a new species, it would have no validity because it would be a primary homonym of Agassiz's species].

Heckel's Telestes rysela is a name available because it is accompanied by a brief diagnosis and reference to Gesner's rysele (1560: 290) and Heckel (1849: 288 [given as p. 186 by Heckel, 1852a; probably based on an offprint]). Heckel (1852a: 388) seems to imply that he had used the name T. rysela in his 1849 paper. The combination is not used in the text, only in the index; but as the index refers merely to page 288 without further detail, the name cannot be available from that work. As Agassiz's C. rysela is conspecific with T. rysela, the latter name is a junior secondary homonym. There is no type material for Agassiz's species and Heckel's material is lost (B. Herzig, pers. comm., 1995) and a neotype designation is the only method of definitively solving the identity of these nominal species. I have not been able to examine fresh material from river Sihl and specimen MNHN 712 (lectotype of Leuciscus agassii Valenciennes, 1844) is here designated as neotype of Chondrostoma rysela Agassiz, 1835 and Telestes rysela Heckel, 1852. Telestes is now placed in the genus Leuciscus, but L. rysela (Agassiz) and L. ryzela Valenciennes are not homonyms as they differ by one letter (ICZN art. 57(f)).

\subsubsection{Leuciscus svallize (Heckel \& Kner, 1858)}

Squalius svallize Heckel \& Kner, 1858: 197, fig. 110 (type locality: lakes near Vergoraz and river Narenta [Neretva], Dalmatia [Croatia]; syntypes: NMW 49593 [1], 49595-597 [3], 49612 [1, illustrated by Bianco \& Knežević, 1987: 51, fig. 2]) 
Systematic notes. Bianco \& Knežević (1987: 51) recognise this species as valid and restricted to the Neretva and Trebinje basins, Bosnia. They comment that all the Greek specimens of the $L$. cephalus complex examined by them belong to $L$. cephalus. Economidis (e.g., 1991: 14; Economou et al., 1991) reported a second species from Greece as L. cf. svallize which agrees with the diagnostic characters listed by Bianco \& Knežević but has a more slender appearance (pers. obs.). The broadly disjunct distribution ranges suggest that it might be distinct and on-going work should soon clarify its taxonomic status.

\subsubsection{Leuciscus turskyi (Heckel, 1843)}

Squalius Turskyi Heckel, 1843: 1041 (type locality: creek ["Felsenbach"] Cicola [Cikola] near Dernis [Drniš], Dalmatia [Croatia]; syntypes: NMW $49629[18])$

\subsubsection{Leuciscus ukliva (Heckel, 1843)}

Squalius Ukliva Heckel, 1843: 1042 (type locality: river Cettina [(Cetina), near Sign (Sinj); Heckel \& Kner, 1858: 201], Dalmatia [Croatia]; syntypes: NMW 49635 [4], 496339 [3])

5.17.20 Leuciscus zrmanjae (Karaman, 1928) Squalius svallizze zrmanjae Karaman, 1928: 159

(type locality: lower course of river Zrmanja, Dalmatia [Croatia]; syntypes: LU); repeated in 1929: 172

Systematic notes. The Leuciscus sp. of Bianco \& Knežević (1987: 53, fig. 3d) is tentatively identified as L. zrmanjae, as implicitly suggested by these authors. Once material from the Zrmanja becomes available, a neotype designation is desirable to stabilise this use of the name.

\section{Mylopharyngodon piceus (Richardson, 1846) introduced}

\subsubsection{Pachychilon macedonicum (Stein-} dachner, 1892)

Leuciscus macedonicus Steindachner, 1892: 377, pl. 2 fig. 2 (type locality: lake Dojran, FYROM and Greece; syntypes: NMW 7025-7028 [4], 49811-813 [8])

Systematic notes. Howes (1981: 46) considers Pachychilon as a synonym of Rutilus and Karaman (1972) considers it as a subgenus of $R u$ tilus. The osteological study by Šorić (1992) shows that Pachychilon is a distinct genus, including two species. Generic placement of $P$. macedonicum follows Karaman (1972: 145), Bianco (1986: 297) and Šorić (1992).

\subsubsection{Pachychilon pictum (Heckel \& Kner,} 1858)

Squalius pictus Heckel \& Kner, 1858: 196 (type locality: Rieka creek, Montenegro; syntypes: NMW 49310 [2]])

\subsubsection{Pelecus cultratus (Linnaeus, 1758)}

Cyprinus cultratus Linnaeus, 1758: 326 (based on Linnaeus [1754: 82, pl. 2]; type locality: "in M. Baltico"; type material: holotype: UUZM 224, Wheeler, 1991: 162)

Clupea ziga Wulff, 1765: 40 (type locality: "in lacu Curonico", Borussia [Prussia]; type material: NT?)

Nomenclatural notes. Bertin \& Estève (1948: 85) list two syntypes (MNHN 3913) of "Leuciscus cultratus Cuv. Val.". Valenciennes (1844: 330) under the heading "Leuciscus cultratus nob" did not describe a new species, but merely established a new combination of Cyprinus cultratus Linnaeus, 1758 , a name which is explicitly mentioned (p. 337). Several other citations of this name and its combinations are quoted in this account, and the material listed by Bertin \& Estève obviously has no type status.

Cyprinus clupeoides Bloch (1795:49, pl. 408 fig 2 ), sometimes listed as a synonym of Pelecus cultratus (e. g. Heckel \& Kner, 1858: 127), is an Indian fish of the genus Salmostoma (see Bănărescu, 1968: 5). In any case, this name is a junior primary homonym of Cyprinus clupeoides Pallas, 1776 (see above Chalcalburnus chalcoides) and should be replaced by Salmostoma balookee (Sykes, 1839) (Kottelat, 1996).

Parabramis pekinensis (Basilewsky, 1855) introduced

Pimephales promelas Rafinesque, 1820 introduced

5.20.1 Phoxinellus adspersus (Heckel, 1843) Leucos adspersus Heckel, 1843: 1038 (type locality: Imosky [Imotski], Croatia; syntypes: NMW not yet located; B. Herzig, pers. comm., 1995])

Systematic notes. All species placed here in Paraphoxinus were considered as subspecies of $P$. adspersus by Karaman (1972). They are all distinct species under the PSC.

Nomenclatural notes. The type locality was originally stated as Imostki. Heckel \& Kner (1858: 168) later state that they first collected it in Jessero Rosso, a lake near Imotski. 
5.20.2 Phoxinellus alepidotus Heckel, 1843

Phoxinellus alepidotus Heckel, 1843: 1040 (type locality: waters around Livno, Bosnia; syntypes: NMW 51061 [3], 51106 [2], MHNN 1018 [1]. Kottelat, 1984: 149)

Systematic notes. Howes (1985: 69) commented that the Croatian Phoxinellus actually belongs to one or more distinct genera, retaining $P$. adspersus in Phoxinellus. But he is quite ambiguous, stating that Phoxinellus includes "those species distributed throughout the Levant (adspersus, zeregi)...". No mention is made of the type species $P$. alepidotus. Until the genus is critically revised. it seems reasonable to retain the Croatian species in Phoxinellus.

Nomenclatural notes. Trewavas (1971: 359) showed that $P$. alepidotus is the type species of the genus Phoxinellus Heckel, 1843 and that Paraphoxinus Bleeker, 1863b: 263 (repeated in 1863c: 209 and 1863d: 31) is an objective junior synonym. Therefore Phoxinellus has to be used for the present and the following species instead of Paraphoxinus. Eschmeyer (1990: 313) apparently overlooked Trewavas's argument.

\subsubsection{Phoxinellus croaticus Steindachner, 1866}

Phoxinellus croaticus Steindachner, 1866a: 594, pl.

1 (type locality: streams Licca and Novchicza [Novcica] near Gospich [Gospič], stream Ottucha [Otešica] near Grachaez [Gračac], stream Richicza [Ričina] near Stikada, Croatia; syntypes: NMW 51063-064 [14], 51115-116 [3]. 51167 [45], MSNG 9076 [3])

\subsubsection{Phoxinellus epiroticus (Steindachner, 1896)}

Paraphoxinus epiroticus Steindachner, 1896: 185, pl. 1 fig. 3 (type locality: lake of Janina and river Luros, Albania [now Greece]; syntypes: NMW 12982-990 [9], 51119-132 [more than 100], 51133-143 [more than 69], 51146-155 [more than 80], MSNG 9054 [2], Tortonese, 1961: 187)

Nomenclatural notes. Steindachner (1896: 185) indicated that part of his material was from river Luros. Economidis (1991: 12) considered that the presence of the species in Luros is doubtful.

\subsubsection{Phoxinellus fontinalis Karaman, 1972} Phoxinellus adspersus fontinalis Karaman, 1972: 134, fig. 5 (type locality: source in Hrnjakova Pecina cave, Krbavsko polje in Lika, Croatia; syntypes: LU [6])
5.20.6 Phoxinellus ghetaldii (Steindachner, 1882)

Paraphoxinus ghetaldii Steindachner, 1882b: 16 . pl. 5 fig. 2 (type locality: cave in the plain of Popovo, Herzegovina [Bosnia]; syntypes: NMW 51158-159 [4], 51161-162 [4], 51164-165 [3])

\subsubsection{Phoxinellus metohiensis (Steindach-} ner, 1901)

Paraphoxinus metohiensis Steindachner, 1901: 197 (type locality: karstic waters and springs near Gacko, rivers Gračanica and Musica, stream Zalomska in the Nevesinje plain in Herzegovina, and river Ljuta near Grinda east of Ragusa Vecchia [Cavtat], Bosnia; syntypes: NMW 12972975 [4], 51171-176 [14], BMNH 1911.9.22:12-13 [2], Howes, 1985: 57)

\subsubsection{Phoxinellus prespensis (Karaman, 1924)}

Paraphoxinus epiroticus forma] prespensis Karaman, 1924a: 32, fig. 6b [not 6a, see Karaman, 1924b: 63] (type locality: lake Prespa and backwaters, FYROM and Greece; syntypes: LU)

\subsubsection{Phoxinellus pstrossii (Steindachner, 1882)}

Paraphoxinus Pstrossii Steindachner, 1882a: 73, pl. 5 fig. 3 (type locality: stream Trebinschitza [Trebišnjica] near Trebinje, Bosnia; syntypes: NMW 51177 [2])

Nomenclatural notes. Specimens BMNH 1911.9.28:4-5 [2] listed as syntypes by Howes (1985: 57) cannot be syntypes. Steindachner explicitly indicated that his description is based on two specimens (p. 74: "... beiden Exemplaren ...") and these are in NMW.

\subsubsection{Phoxinus phoxinus (Linnaeus, 1758)} Cyprinus Phoxinus Linnaeus, 1758: 322 (based on Artedi [1738: syn. 12, Cyprinus tridactylus ...] type locality: "in Europa"; type material: NT) Cyprinus aphya Linnaeus, 1758: 323 (based on Linnaeus [1746: 125, n. 331, Cyprinus ... radiis 9; 1747: 232, Cyprinus minimus], Artedi [1738: gen. 4 [30], syn. 13, spec. 30 [4], Cyprinus biuncinalis ...]; type locality: "in Europae rivulis"; type material: NT)

Cyprinus rivularis Pallas, 1773: 717 (type locality: small streams in the Altail range [p. 616 Zmeinogorsk, basin of river Alei], Siberia; syntypes: ZISP ?)

Cyprinus Morella Leske, 1774: 47 (type locality: creek Bode near Rübeland, $103 \mathrm{~km} \mathrm{NW}$ of 
Leipzig, Germany [by neotype designation; original locality: Leipzig, Germany]; neotype ZMB 32661, present designation)

Cyprinus isetensis Georgi, 1775: 621 (available by indication to Lepechin [1771a: 491, pl. 26 figs. $2-3$ ]; type locality: Catharinopolis, Siberia; syntypes: LU)

Cyprinus Galian Gmelin, 1788: 1421 (type locality: Catharinopolis, Siberia; based on Lepechin [1771a: 491, pl. 26 figs. 2-3; 1772: pl. 9 figs. 4-5]; syntypes: LU)

Cyprinus chrysoprasius Pallas, 1814: 318 (type locality: hill streams on Mount Chersones. Crimea, Ukraine; syntypes: LU)

Phoxinus varius Perty, 1832: 719 (unnecessary replacement name for Cyprinus phoxinus Linnaeus, 1758)

Phoxinus laevis Fitzinger, 1832: 337 (unnecessary replacement name for Cyprinus phoxinus Linnaeus, 1758; author indicated as Agassiz, but Fitzinger is actual author)

? Leuciscus obtusus De la Pylaie, 1835: 533 (nomen nudum; locality: Vendée, France)

Phoxinus marsilii Heckel, 1836: 232 (type locality: all clear streams around Wien, Austria; syntypes: NMW [not yet located; B. Herzig, pers. comm., 1995])

Cyprinus Lumaireul Nordmann, 1840: 482 (proposed in synonymy, name not available; locality: river Po, Italy; author indicated as Bonelli, but this is apparently based on unpublished sources)

Phoxinus Lumaireul Schinz, 1840: 331 (type locality: river Po, Italy; type material: NT ?)

Phoxynus montanus Ogérien, 1863: 362 (type locality: river Ain near Champagnole / waterfall Queue de Cheval, near Saint-Claude, France; type material: NT ?)

Phoxinus laevis var. balchaschana Kessler, 1879: 283 (type locality: river Ajagus [Ayaguz] near Sergiopol [Ayaguz], lake Balkhash basin, Kazakhstan; syntypes [total "almost 100"]: ZISP 4149, 4150 [6], Berg, 1912: 252)

Phoxinus laevis ujmonensis Kashchenko, 1899: 144 (river Katun' near Uimon village, Altai, river Ob basin, Siberia) from Berg, 1949: 591

Phoxinus laevis mikrosquamatus Kashchenko, 1899: 145 (lake Karalachinskoie in basin of river Argut [basin of river Katun], Altai, Siberia) from Berg, 1912: 251

Phoxinus saposchnikowi Kashchenko, 1899: 146 (lake on plateau of Ukök, source of river Kalguty, a tributary of river Argut, Altai, Siberia) from Berg, 1912: 251

Phoxinus czekanowskii sedelnikowi Berg, 1908a: 226 (type locality: lake Saissan [Zaisan], up- per Irtysch basin, Karasuat-Busen, Kazakhstan; syntypes: ZISP 14361 [9])

Phoxinus phoxinus colchicus Berg, 1910: 0169 (available by indication to Kamensky, 1901: 63, 165; type locality: river Bachwis-tzchali in district Osurgety, Transcaucasia; syntypes: ZMT 141a, Berg, 1912: 263)

Phoxinus Csikii Hankó, 1922: 1, pl. 1 fig. 1 (type locality: near Korita [Donje Korito], $50 \mathrm{~km}$ north-west of Ipek, $40 \mathrm{~km}$ westsouthwest of Novipazar, Montenegro; syntypes: MNH [2])

Phoxinus phoxinus strandjae Drensky, 1926: 137 (type locality: Strandscha [Istrandzhha] range, Bulgaria; syntypes: NMNHS)

Phoxinus phoxinus carpathicus Popescu-Gorj \& Dumitriu, 1950: 94, fig. 6 (type locality: lake Rosu, Romania; syntypes: LU)

Systematic notes. From a morphometric analysis Repa \& Pivnička (1980) concluded that there is a single species in the genus Phoxinus. As this conclusion was based on the BSC, on coefficients of differences and in part on literature data, it seems desirable to reevaluate the status of the different nominal species on the basis of fresh material, direct comparison, additional characters and a more appropriate approach.

Nomenclatural notes. Cyprinus phoxinus and C. aphya are simultaneous subjective synonyms. I did not trace the first reviser action determining respective priority. Cyprinus phoxinus seems to have been universally accepted as having priority.

Cyprinus morella Leske, 1774 could be the earliest available name for the fish usually called $\mathrm{Al}$ burnoides bipunctatus (Bloch, 1782). Bloch (1782: 52) himself noted that C. morella is possibly the same as his C. bipunctatus, but that the references in Leske's synonymy refer's to Phoxinus phoxinus [and also Rhodeus amarus]. Leske's material has been reported by Karsten (1789) and had been purchased by the Dublin Society and brought to Ireland (O'Reilly, 1813). The Dublin Society's collection became part of the National Museum of Ireland but no fish specimen is extant; some Leske's fish specimens may be part of Linnaeus collection in the Linnean Society of London (Wheeler, 1985: 5), but it will probably never be possible to demonstrate it. No specimen identifiable as Cyprinus morella is present in the LSL. Specimen ZMB 32661, 65 mm SL, from creek Bode near Ruibeland, $103 \mathrm{~km}$ NW of Leipzig, Germany, is designated as the neotype of C. morella Leske, 1774 ; it is unambiguously identifiable as $P$. phoxinus and the two names are thus objective synonyms. 
Berg (1912: 263) lists Phoxinus phoxinus colchicus Berg, 1910 as a nomen nudum. But the name is available in Berg (1910) by indication to Kamensky (1901).

\subsubsection{Pseudophoxinus beoticus (Stephani-} dis, 1939)

Rutilus beoticus Stephanidis, 1939b: 57, pl. 2

(type locality: lake Yliki, Greece; syntypes: lost [16])

Systematic notes. Placed in Pseudophoxinus by Economidis (1991: 11) and Economidis \& Bănărescu (1991: 266, 269), in Pachychilon by Karaman (1972: 145), and as a subspecies of Phoxinellus pleurobipunctatus [here a Leuciscus] by Stephanidis (1974b: 241).

\subsubsection{Pseudophoxinus minutus (Karaman, 1924)}

Paraphoxinus minutus Karaman, 1924a: 33, fig. 6a [not 6b, see Karaman, 1924b: 61] (type locality: lake Ohrid and backwaters, FYROM; syntypes: LU)

? Phoxinellus stimphalicus montenigrinus Karaman, 1972: 131 (type locality: creek Boka Kotorska near Radanovci, at cross road KotorTivat, Montenegro; syntypes: LU [50])

Systematic notes. Maitland (1976) considers Paraphoxinellus minutus as a valid species of his Phoxinellus (which is not identical to Phoxinellus here). Bănărescu (1992b: 753) considers it a valid species of Pseudophoxinus.

\subsubsection{Pseudophoxinus stymphalicus (Va-} lenciennes, 1844)

Lenciscus stymphalicus Valenciennes, in Cuvier \& Valenciennes, 1844: 295 pl. 498 (type locality: lake Zaraco, earlier known as lake Stymphale, Greece; syntypes: MNHN 5821, Fang, 1942b: 168)

Leucaspius marathonicus Vinciguerra, 1921: 328, pl. 4 fig. 4 (type locality: small creek near spring Sterna, near Kato-Souli village, Maratona plain, Greece; lectotype: MSNG 17154-A, designated by Tortonese, 1961: 187)

Leucaspius stymphalicus var. thesproticus Stephanidis, 1939a: 24, pl. 8 fig. 1 (type locality: marsh of Paramythias, villages Scoupitsa and Grica, district Thesprotia, Greece; syntypes: lost ? [32])

Systematic notes. Placed in Pararhodeus by Fang (1942b: 168). Placed in Phoxinellus by Karaman (1972: 128-131) who considered marathonicus, minutus, thesproticus and montenigrinus as subspecies; he gave no argument to justify this sta- tus and his descriptions are too vague to provide information useful in deciding on their status. Leucaspius marathonicus and L. s. thesproticus are tentatively considered as conspecific with $P$. stymphalicus; this deserves further research. The geographically isolated and diagnosable $P$. minutus is treated as a valid species. Placed in Pseudophoxinus by Bianco \& Miller (1989: 385) and Bănărescu (1992b: 757).

\section{Pseudorasbora parva (Temminck \& Schlegel, 1842 ) introduced}

\subsubsection{Rhodeus amarus (Bloch, 1782)}

Cyprinus Amarus Bloch, 1782: 52, pl. 8 fig. 3 (type locality: lake Müggelsee near Köpenik, Berlin, Germany; syntypes: ZMB 3393 [3])

Rhodeus Lucinae Walecki, 1863: 364 (apparently an unnecessary replacement name for Cyprinus amarus Bloch, 1782)

Rhodeus genitalis Walecki, 1863: 364 (apparently an unnecessary replacement name for Cyprinus amarus Bloch, 1782)

Rhodeus amarus var. meridionalis Karaman, 1924b: 41 (type locality: river Vardar near Skopje and lake Dojran, FYROM and Greece; syntypes: MNHN 35 - 358-360 [3], Bertin \& Estève, 1948: 75)

Rhodeus sericeus sericeus forma strumicae Karaman, 1955: 183, fig. 2 (infrasubspecific, name not available)

Rhodeus sericeus amarus natio danubicus Holčík, 1959: 50 (infrasubspecific, name not available; locality: river Danube basin, Slovakia)

Rhodeus sericeus amarus natio svetovidovi Holčík, 1959: 50 (infrasubspecific, name not available; locality: rivers Dnieper and Bug, Ukraine)

Systematic notes. Rhodeus amarus has long been considered a subspecies of the East Asian $R$. sericeus (Pallas, 1776 [p. 704; type locality: Dauria (river Amur basin)]) (e.g., Holčík \& Duyvené de Wit, 1964; Arai \& Akai, 1988: 211). The two species are broadly disjunct, $R$. amarus occurring (roughly) in central and eastern Europe and northern Asia Minor, and R. sericeus in the Amur basin and Sakhalin Island. Holčík \& Jedlička (1994: 147) discussed the variability of three characters and concluded that the two stocks are conspecific and that there is a West-East clinal variation. Indeed, the stocks reportedly are diagnosable only by modal values of lateral-line pored scales (4-6 in $R$. amarus, vs. 6-7 in $R$. sericeus, ranges $0-9$ and $4-10$ respectively) and gill-rakers (10-12, vs. 12-14; ranges 9-13, vs. 9-16) and apparently have similar ecology and ontogeny, ex- 
cept that eggs are incubated in different hosts (the East Asian hosts do not occur in Europe and vice versa).

The reported West-East clinal variation may appear only if $R$. amarus and $R$. sericeus are analysed together and if average values are used; if taken separately, the number of $R$. sericeus populations is too limited to show a cline. For the character with greatest interest (pored lateral line scales), the clinal variation is not obvious (p. 158, fig. 4) and the data in table 3 (p. 152) shows that: 1) while the average number of pored scales should increase from West to East, the lowest counts have been observed in the Elbe and Danube basins; 2) the Western Europe values are completely included within those of the Elbe, Danube, Black Sea and Middle East; and 3) the Eastern Europe and Asia Minor values are completely included within those of Western Europe. The number of examined populations is probably too low to demonstrate the existence of clinal variation.

[In a more general contex, while clinal variation can possibly be observed in terrestrial or marine organisms, the dispersal constraints in the freshwater habitats (linear, vs. bi-dimensional dispersal; see p. 16) certainly make clinal variation a concept not applicable to freshwater fishes without adjustment. Analyses extending across several basins should place emphasis on the hydrographic network and its historical evolution. Geographic variation should be analysed within a basin framework instead of within a coordinate grid. Of course, clines may exist within a given basin.]

The European and East Asian stocks are unarguably distinct lineages separated for an estimated 2 to 4 million years (Holčík \& Jedlička, 1994: 160) by about $4000 \mathrm{~km}$ and they are diagnosable; they are treated here as species. Admittedly the differences between the two stocks are slight, but they exist [with large overlap]. Holčík \& Jedlička's (p. 165) conclusion that $R$. amarus and $R$. sericeus "cannot be separated as the [coefficients of difference] values $\ldots$ are $0.75,0.09$ and 0.58 respectively, which is far below the accepted value of 1.28 " was valid under the BSC, but is definitively not acceptable under other species concepts; as discussed in the introduction (pp. 12, 147), it is subjective to use magnitude of difference to deduce conspecificity or distinctness. To be objective, in this particular case my judgement might be somewhat biased by the huge geographic distance between the two species.
The above discussion assumes that $R$. amarus and $R$. sericeus constitute monophyletic lineages. The possibility that they are not monophyletic lineages has not yet been considered (e.g., that some of the European populations are more closely related to the East Asian populations than to the other European ones).

\subsubsection{Rutilus alburnoides (Steindachner, 1866)}

Leuciscus alburnoides Steindachner, 1866d: 263, pl. 1 fig. 3 (type locality: river Guadiana and its tributaries near Merida and Mertola, river Guadalquivir near Sevilla and Cordova [Cordoba], river Genil near Ecija, river Guadaira near Alcala de Guadaira, Spain; syntypes: NMW 49749 [15], 49778 [12])

Systematic notes. Generic placement doubtful. Placed in Tropidophoxinellus by Stephanidis (1974b: 248) because he assumed that "it is obvious that ... a keel exist". Actually, the abdomen is not keeled and the generic placement is not correct. Present placement follows Collares-Pereira (1984) who also notes the presence of two forms in Portugal, a diploid bisexual one and an all-female triploid one (1985: 69).

\subsubsection{Rutilus arcasii (Steindachner, 1866)}

Leucos Arcasii Steindachner, 1866b: 199 (type locality: river Cailes near Tudela, river Ebro near Zaragoza and streams of Galicia, Spain; syntypes: NMW 49755-775 [54], 50646 [5], 60485 [1], 60743 [1]); see Steindachner (1866c: pl. 3 figs. 2-3) for illustration of syntypes

Systematic notes. Bianco (1988b: 9) suggested that this species might be a Leuciscus related to the L. souffia complex.

\subsubsection{Rutilus aula (Bonaparte, 1841)}

Squalius Aula Bonaparte, 1841: [fasc. 30], pl. 116 fig. 4 (type locality: Venetian Province, Italy; holotype: ? part of ANSP 6434-6445 [12], Böhlke, 1984: 69, MNHN 3934, Bertin \& Estève, 1948: 58 [see Nomenclatural notes, below])

Squalius Elatus Bonaparte, 1841: [fasc. 30], pl. 116 fig. 3 (type locality: Lombardia, Italy; holotype: ? part of ANSP 6647-6649 [2], Böhlke, 1984: 75 [see Nomenclatural notes, below])

Leuciscus henle Bonaparte, 1841: [fasc. 30, introduction p. 15] (nomen nudum; locality: lake Lugano, Italy and Switzerland)

Leucos cisalpinus Heckel, 1843: 1038 (nomen nudum)

Leuciscus altus Valenciennes, in Cuvier \& Valenciennes, 1844: 237 (type locality: lake Maggiore, 
Italy; holotype: MNHN)

Leuciscus pagellus Filippi, 1844: 400 (type locality: Verbano [lake Maggiore], Italy; syntypes: LU)

Leuciscus scardinus Filippi, 1844: 400 (type locality: canals near Milano, Italy; syntypes: LU)

Leuciscus pauperum Filippi, 1844: 401 (type locality: Lombardia, Italy; syntypes: LU)

Leucos rubellicus Bonaparte, 1845: 6 (type locality: "Dalmatia"; syntypes: LU)

Leucos cisalpinus Bonaparte, 1845: 6 (listed in synonymy, name not available)

Leucos cisalpinus Heckel, 1851b: 315 (available by indication to Leuciscus scardinus Filippi, 1844)

Rutilus erythrophthalmus Zerunian, 1982: $\mathrm{n}^{\circ} 346$ (type locality: lake Bracciano, Italy; syntypes: LU)

Nomenclatural notes. Böhlke (1984: 69, 75) lists 12 syntypes ANSP 6434-6445 for Squalius aula and 2 syntypes ANSP 6647-6649 for S. elatus. As these names are available from Bonaparte's (1841) plate 116, the illustrated specimen is the holotype and there are no syntypes. Bertin \& Estève (1948: 58) list a holotype MNHN 3934 from Torino for $S$. aula; its status is doubtful as Bonaparte in the text, under a different name, indicated that his material came from the "Venetian Province".

Rutilus erythrophthalmus Zerunian, 1982, was originally described in a conference abstract. This suffices to make the name available from that publication, even if the 'description' is rudimentary. Rutilus erythrophthalmus Zerunian is a secondary junior homonym of Cyprinus erythrophthalmus Linnaeus, 1758, if this species is placed in Rutilus as done by some authors (e.g., Howes, 1981). In any case, there are several earlier names applied to this species (see Bianco \& Taraborelli, 1985: 143 as well as Zerunian, 1984: 233) and, as a junior synonym, $R$. erythrophthalmus is not valid; the comments by Zerunian (1991) and Gandolfi et al. (1991: 133) are irrelevant and can only have been induced by a lack of familiarity with the ICZN. Zerunian's arguments against the use of $R$. aula are that the name has been used only as a caption of a poor-quality plate, without description, and that $R$. aula was a nomen oblitum when he described $R$. erythrophthalmus. A name published only as a caption of a plate is available; ICZN art. 12 (b)(7) is very explicit and leaves no opportunity for discussion on this; the absence of a description for names published before 1931 or the quality of the plate are irrelevant to the argument. Rutilus aula cannot be rejected as a nomen oblitum on the ground advocated by Zerunian; the only names which can be rejected are those which have been explicitly designated as nomina oblita between 6 November 1961 and 1 January 1973 (see ICZN art. 79(c)(iii), Glossary 'nomen oblitum'). I could not find such a rejection and Zerunian gives no reference to one; thus $R$. aula is not a nomen oblitum and cannot be rejected. The fact that the name has not been used for 50 years by itself does not make a name a nomen oblitum.

Anyway, even if $R$. aula were a nomen oblitum (which is not the case), there are seven other names published between 1841 and 1851 which apparently refer to the same species and thus potentially have priority over $R$. erythrophthalmus. To make $R$. erythrophthalmus the valid name of the species, one would need to demonstrate that all of these names are not available or are invalid for the species, which has not been done.

Leuciscus aula and L. elatus are simultaneous synonyms and the first reviser's action is decisive to establish which name has priority (ICZN art. 24). The first reviser is Canestrini (1866: 95, 99) and he retained $L$. aula has having priority (as clearly stated by Bianco \& Taraborelli, 1985: 143)

\subsubsection{Rutilus basak (Heckel, 1843)}

Leucos Basak Heckel, 1843: 1006, pl. 1 (type locality: Dalmatia [Vergoraz and lake Drusino near Imosky [Imotski], Croatia; Heckel \& Kner, 1858: 166, figs. 88-89]; syntypes: NMW 50723 [2], $50725[2])$

Systematic notes. Valid according to Bianco \& Taraborelli (1985: 149).

Nomenclatural notes. The authors of the name are usually indicated as Heckel \& Kner (1858: 166). The name is actually available from Heckel (1843) where it is used for the caption of a plate illustrating pharyngeal teeth.

\subsubsection{Rutilus frisii (Nordmann, 1840)}

Cyprinus Wiresuba Güldenstädt, 1791: 169 (nomen nudum; locality: Yuzhnyi Bug, Ukraine [Berg, 1949: 532])

Leuciscus Frisii Nordmann, 1840: 487 (type locality: market in Odessa and rivers Danube and tributaries, Dniester, Bug, Dnieper and Don; syntypes: ? NMW 50456 [2])

Gardonus wyrozub Walecki, 1863: 360 (available by indication to Leuciscus Frisii Nordmann, 1840 and four other, ambiguous references; here restricted to reference to Leuciscus Frisii Nordmann, 1840; type locality: market in Odessa and rivers Danube and tributaries, Dniester, Bug, Dnieper and Don; syntypes: ? NMW 50456 [2])

? Leuciscus frisii var. kutum Kamenskii, in Radde. 
1899: 317 (nomen nudum; localities: BoshyPromysl [Bozhii Promysel on lower river Kura] and Lenkoran, Azerbaidjan)

? Leuciscus frisii caspius Lönnberg, 1900: 15 (type locality: mouth of river Volga, Russia; holotype: NRM)

? Leuciscus frisii var. kutum Kamensky, 1901: 23,154 , pl. 12 (type locality: southern part of Caspian Sea, rivers Kura, Arax, Terek, lower Volga and streams of the Lenkoran area; syntypes: ZMT ?)

Rutilus frisii velecensis Chichkoff, 1932: 355, pl. 1 fig. 1 (type locality: streams Veleca and Rezova Rieca [Rezovska Reka, Rezvaia], Bulgaria; syntypes: LU)

Systematic notes. It seems that the population from the Caspian Sea basin, usually called $R$. $f$. kutum, actually has to be treated as a distinct species. Berg (1912: 47) considered R. f. kutum and $R$. f. caspium as belonging to the same taxon but retained the name $R$. f. kutum although $R$. f. caspium has priority. Economidis \& Bănărescu (1991: 261) list $R$. f. velecensis as a valid subspecies. The status of these taxa needs to be reevaluated once usable data become available.

\subsection{6 ? Rutilus heckelii (Nordmann, 1840)} Leuciscus heckelii Nordmann, 1840: 491, pl. 23 fig. 1 (type locality not stated [Black Sea basin]; syntypes: ZISP 3008-3009 [3], Berg, 1912: 65)

Rutilus rutilus heckeli natio dono-kubanensis Pavlov, 1961: 250 (infrasubspecific, name not available; locality: Sea of Azov)

Rutilus rutilus morpha migratorius Holčík \& Skořepa, 1971: 49 (infrasubspecific, name not available)

Systematic notes. Data in Holčík \& Skořepa (1971) suggest that the various subspecies, natios and morphs earlier recognised within $R$. rutilus are conspecific, but these authors recognised the migratory forms of the Black, Azov and Caspian Seas as a distinct morph migratorius. The Black Sea stock occurs sympatrically with the freshwater one in the lower Dniester, Bug, Dnieper and Don (presence in Danube is open to question) where it migrates into freshwaters (Holčík \& Skořepa, 1971: 41). It has been recognised as $R$. r. heckeli by earlier authors who had reported morphological differences between the two stocks (e.g., Berg, 1949: 493; Bănărescu, 1964: 305) but Holčík \& Skořepa could not find any striking difference; however, they list differences in the early ontogeny (based on a compilation of published data). Their table 1 does not provide much comparison data except for gill-raker counts for $R$. heckeli; their table 2 could indicate that $R$. heckeli and the adjacent $R$. $r$. carpathorossicus differ in lateral-line scale and gill-raker counts, but as it is based on their own as well as compiled data, the table's reliability depends of the consistency of the methods used by the various authors. Awaiting a detailed comparison of sympatric and adjacent populations of the different stocks and considering the differences reported by earlier authors and what seems to be different life histories, it seems reasonable to retain them as two valid species.

Bănărescu (1991: 296, 1992: 732) suggested that $R$. heckeli might be a subspecies of $R$. pigus, but did not provide data.

If future studies confirm that the Black Sea basin $R$. heckeli is specifically distinct from $R$. rutilus, the status of other migratory stocks (e.g., those of the Caspian drainage) should be investigated too, as they are then unlikely to be conspecific with $R$. heckeli.

\subsection{7 ? Rutilus karamani Fowler, 1977}

Rutilus aula natio karamani Vladykov \& Petit, 1930: 391 (infrasubspecific, name not available; locality: lake Ohrid near Pogradec, Albania)

Rutilus rubella karamani Fowler, 1977: 85 (available by indication to Rutilus aula natio karamani Vladykov \& Petit, 1930: 391; type locality: lake Ohrid at Pogradec, Albania; syntypes [total 4 ?]: MNHN 1977.281 [3], B. Elvira, pers. comm., 1995)

Rutilus prespensis vukovici Maric, 1989: 65, figs 2d, 4 (type locality: lake Skadar, Montenegro; holotype: BZT)

Systematic notes. See discussion under Rutilus ohridanus. Tentatively considered valid.

Nomenclatural notes. Rutilus aula natio karamani Vladykov \& Petit, 1930 is infrasubspecific and unavailable, but its use as a subspecific name in Fowler (1977: 85) makes it available.

\subsubsection{Rutilus lemmingii (Steindachner, 1866)}

Leuciscus Lemmingii Steindachner, 1866d: 265, pl. 1 fig. 2 (type locality: river Guadiana and its tributaries near Merida, rivers Guadalquivir and Guadaira near Sevilla, Spain; syntypes: NMW 52356-357 [6], 52363 [2], 52366 [2], 52373 [4])

Systematic notes. Generic position follows Elvira (1987: 138).

\subsubsection{Rutilus lusitanicus (Collares-Pereira, 1980)}

Chondrostoma lusitanicum Collares-Pereira, 
1980a: 275 (type locality: Rio Xarrama, Sado basin, Portugal; holotype: MB c.1.1)

Systematic notes. Generic position follows Elvira (1987: 138).

\subsubsection{Rutilus macrolepidotus (Steindach- ner, 1866)}

Leuciscus macrolepidotus Steindachner, 1866c: 15 (type locality: creeks near Alcobazar, and Cintra, Portugal; syntypes: NMW 49815 [4; 3 according to original description]); see Steindachner (1866d: pl. 1 fig. 4) for illustration of a syntype

Systematic notes. Bianco (1988b: 9) suggested that this species might be a Leuciscus related to the L. souffia complex.

\subsubsection{Rutilus meidingeri (Heckel, 1851)}

Leuciscus Meidingeri Heckel, 1851a: 147 (available by indication to Cyprinus grislagine in Meidinger, 1790: pl. 40 ["lacu Kamerano"]; type locality: lake Attersee, Austria; syntypes: NMW 49826 [1], 50451 [1], 50452 [1])

Nomenclatural notes. Although Heckel described this species in $1852 \mathrm{~b}$ (p. 88, pl. 9), his $1851 \mathrm{a}$ account suffices to make the name available. He also published a second account (1851b: 290) which would fulfil conditions to make the name available.

The earliest of these citations (1851a) makes the name available because it is accompanied by a reference to Meidinger (1790: pl. 40). As it is obvious from the content of the paper that Heckel had examined specimens, these are syntypes.

5.24.12 Rutilus ohridanus (Karaman, 1924) Leucos aula var. ohridana Karaman, 1924b: 56 (type locality: lake Ohrid, FYROM; syntypes: LU)

Systematic notes. I tentatively follow the opinion of Bianco (1986) and Economidis (1991: 9) that $R$. ohridanus is a valid species. According to Maric (1989) and Bănărescu (1992b: 753) it is a subspecies of $R$. basak.

Maric (1989) considers $R$. ohridanus as a subspecies of $R$. basak, and $R$. prespensis as valid with 2 subspecies, R. p. prespensis and R. p. vukovici. Language and semantic problems make it very difficult to understand his argument; nowhere is it explained how the two 'species' differ and why the two pairs of 'subspecies' are assumed to be each other's closest relative. Awaiting more explicit data and clearer information, I tentatively accept that the four taxa are diagnosable and thus treat them as four distinct species. To treat some of them as conspecific (e.g., ohridanus and basak, prespensis and vukovici) could be acceptable only if it is demonstrated that they constitute a distinct lineage; there is no such demonstration. The reported sympatric occurrence of two of them seems to refute the hypothesis that a single species is involved.

From Maric's data, it seems clear that " $R$. $b$. ohridanus" and "R. p. vukovici" are sympatric in lakes Skadar and Ohrid and that they are distinguished by lateral line scale counts (41-45, vs. $37-$ 41 [based only on material of lake Skadar]). If the same pair of species really occurs in both lakes, then it seems that R. p. vukovici is the same as Rutilus karamani, and this is the name retained here.

It seems that Maric's $R$. ohridanus is the same as Karaman's (but Maric had no material from lake Ohrid and there is a striking difference in number of branched dorsal rays, apparently the result of an error in Karaman [the simple rays are probably included again in his count of branched rays]). It also seems that it differs from $R$. basak in dorsal and anal ray counts and body depth. $R u$ tilus prespensis seems to have a smaller eye than all others and different modal ray counts.

\subsubsection{Rutilus pigus (La Cepède, 1803)}

Cyprinus pigus La Cepède, 1803: 607, 610 (type locality: lakes Como and Maggiore, Italy [based on Rondelet (1555: 153, pigus, Lario [lake Como] and Verbano [lake Maggiore]), Artedi (1738: syn 13), Salviani (1554-58: fol. 83, pl. 17, pigo, Lario [lake Como], Verbano [lake Maggiore]), Valmont de Bomare [edition not stated]); syntypes: LU)

Leuciscus Roseus Bonaparte, 1839: [fasc. 24, p. 241], pl. 114 fig. 1 (type locality: "an unknown lake in Piemonte", Italy; holotype: ANSP 6525, Böhlke, 1984: 89)

Leuciscus ryzela Valenciennes, in Cuvier \& Valenciennes, 1844: 199 (type locality: Torino, Italy; syntypes: MNHN 3935 [2], Bertin \& Estève, 1948: 58)

Leuciscus virgo Heckel, 1852b: 69, pls. 11-12 (type locality: river Danube near Wien, Austria; syntypes: NMW 22373 [1], 50626 [1])

Systematic notes. Bănărescu (1992: 682, 732) considers $R$. pigus as introduced from the Danube into Italy and that $R$. heckeli is conspecific with $R$. pigus. Bianco (1995c: 254) considers that the species might have been introduced in Italy during the Middle Age. The populations from northern Italy and from the Danube are now considered as conspecific, but more data are needed on this matter.

The Italian and Danube populations have been 
earlier considered as different species, subspecies or natio. There does not seem to have been much actual comparisons of the two populations. Mišík (1957b) treated them as conspecific on the ground that $R$. virgo "does not differ much in the meristic characters" from $R$. pigus, but without providing data on the type of differences. He has not examined material of the Italian population and, beside Heckel \& Kner (1858), does not cite authors who have done so. A direct comparison of the two species seems highly desirable.

Nomenclatural notes. See Leuciscus souffia for discussion of the status of Leuciscus ryzela Valenciennes, 1844 .

Bertin \& Estève (1948: 57) list a specimen MNHN 3916 from Roma as paratype of Leuciscus roseus Bonaparte, 1839, but there is no information in Bonaparte's text to indicate that he might have had more than one specimen.

Should future research show that the Italian and Danube populations are distinct taxa, the name $R$. pigus has to be used for the Italian one and $R$. virgo for the Danube one.

\subsubsection{Rutilus prespensis (Karaman, 1924)}

Leucos aula var. prespensis Karaman, 1924b: 57 (type locality: lake Prespa, FYROM and Greece; syntypes: LU)

Systematic notes. See discussion under Rutilus ohridanus. Tentatively considered valid. Economidis \& Bănărescu (1995: 266) and Maric (1989) consider $R$. prespensis as a valid species, Economidis (1995: 211) as a subspecies of $R$. ohridanus.

\subsubsection{Rutilus rubilio (Bonaparte, 1837)}

Leuciscus Rubilio Bonaparte, 1837: [fasc. 19, p. 225], pl. 111 fig. 2 (type locality: lakes Bracciano and Nemi, Italy; syntypes: ANSP 65096519 [11], Böhlke, 1984: 89, NMW 49860 [1])

Leuciscus Trasimenicus Bonaparte, 1837: [fasc. 20, p. 233], pl. 112 fig. 4 (type locality: lake Trasimeno, Italy; syntypes: NMW 50789 [1], ANSP 5039-5059 [20], Böhlke, 1984: 93)

Leuciscus Rubella Bonaparte, 1837: [fasc. 20, p. 229], pl. 112 fig. 1 (type locality: river Tevere and all swamps and wetlands, Italy; syntypes: MNHN 3924, Almaça, 1969: 1117, ANSP 64876508 [22], Böhlke, 1984: 89, NMW 50772 [1])

Leuciscus Fucini Bonaparte, 1838: [fasc. 22, p. 235], pl. 113 fig 1 (type locality: lake Fucino, Italy; syntypes: ANSP 6448-6454 [7], Böhlke, 1984: 77)

Leuciscus lascha, Costa, 1838: fasc. 19: 19, pl. 16 (type locality: not stated, but title implies Kingdom of Napoli [now in Italy]; syntype: MNHN 194 [1], Almaça, 1969: 1119)

Leuciscus sardella Valenciennes, in Cuvier \& Valenciennes, 1844: 243 (type locality: river Sarno at confluence with Garigliano, Italy; based on Leuciscus dobula of Costa, 1838: fasc. 19: 23, pl. $19[=R$. rubilio according to Bianco \& Taraborelli, 1985: 136])

Rutilus italicus Calderoni, 1980: 459 (type locality: lake Trasimeno, Italy; syntypes: LU [no type designated, so that all specimens of "laschetta" used for the paper are syntypes])

Nomenclatural notes. Leuciscus lascha is considered a synonym of Scardinius erythrophthalmus by Zerunian (1984: 221) without discussion. The above synonymy follows the revision of Bianco \& Taraborelli (1985: 136).

\subsubsection{Rutilus rutilus (Linnaeus, 1758)}

Cyprinus Rutilus Linnaeus, 1758: 324 (based on Linnaeus [1746: 124, n. 329, Cyprinus ... radiis 12], Artedi [1738: gen. 3 [10], syn. 10, spec. 10 [3], Cyprinus iride pinnis ...], Gronovius [1754: 2, n. 8, idem; 1746: 74, n. 51, idem, n. 52, Cyprinus Rex van Ruy: Waverveen, Belgium]; type locality: "in Europae lacubus"; syntype: LSL 44 [1], Wheeler, 1985: 32)

Cyprinus Rubellio Leske, 1774: 53 (type locality: Leipzig, Germany; type material: possibly LSL 63, 64, Wheeler, 1985: 5, 31)

? Cyprinus simus Hermann, 1804: 325 (type locality: not stated; holotype: LU)

Cyprinus lacustris Pallas, 1814: 314 (type locality: Siberia as far as river Lena, lake Baikal [Berg, 1949: 499]; syntypes: LU)

Cyprinus jaculus Jurine, 1825: 221, pl. 14 (type locality: Léman [lake Genève], Switzerland and France; type material: NT ?)

Leuciscus decipiens Agassiz, 1835b: 38 (nomen nudum)

Leuciscus prasinus Agassiz, 1835b: 46, pl. 2 (type locality: lake Neuchâtel, Switzerland; syntypes: MHNN 994 [2], 994-B [2], 995 [1], Kottelat, 1984: 148, ? MNHN)

? Cyprinus xanthopterus Vallot, 1837: 147 (type locality: stream Ouche, below the park [in Dijon], France; syntypes: LU)

? Cyprinus fulvus Vallot, 1837: 183 (type locality: market [in Dijon?, France]; holotype: LU)

Gardonus pigulus Bonaparte, 1841:[fasc. 30, introduction p. 16] (nomen nudum)

Leucos cenisophius Bonaparte, 1841: [fasc. 30, introduction p. 16] (nomen nudum)

Leuciscus selysii Selys-Longchamps, 1842: 210, pl. 
6 fig. 1 (type locality: Longchamps-sur-Geer, Belgium; syntypes: ? ISNB [not found], MHNN 996 [1], Kottelat, 1984: 148)

Leuciscus rutiloides Selys-Longchamps, 1842: 212, pl. 7 fig. 1 (type locality: river Meuse in Liège, Belgium; holotype: ISNB 108)

Leuciscus Pausingeri Heckel, 1843: 1039 (type locality: lake Egelsee, Austria; syntypes: NMW 49884-886 [3])

Leuciscus lividus Heckel, 1843: 1039 (type locality: Plattensee [lake Balaton], Marizza, Hungary; syntypes: NMW 49978-979 [5])

Leucos pigulus Bonaparte, 1844: 436 (type locality: lakes of Switzerland; holotype: ANSP 6450, Böhlke, 1984: 87)

Leucos cenisophius Bonaparte, 1845: 7. (type locality: "Sequana" [part of former Gaul drained by rivers Saône and Doubs, France]; holotype: ANSP 6430)

Cyprinus pigus Gronow, 1854: 183 (type locality: rivers and lakes of Europe; holotype: BMNH 1853.11.12:154, Wheeler, 1958: 211; junior homonym of Cyprinus pigus La Cepède, 1803: 607, 610)

Leuciscus rutilus var. daugawensis Dybowski, 1862: 92, 101, pl. 6 (type locality: river Düna in Riga, Latvia; syntypes: LU)

Leuciscus Jurinii Dybowski, 1862: 94 (type locality: lake Genève, Switzerland \& France; available by indication to L. rutilus Jurine, 1825 , L. prasinus of Valenciennes, in Cuvier \& Valenciennes, 1844: 153 and Leuciscus nov. spec. Heckel, 1852b: 79; syntypes: MNHN [material listed by these authors; Jurine's specimen are not extant in MHNG (C. Weber, pers. comm., 1995), Heckel's ones cannot be found in NMW (B. Herzig, pers. comm., 1995)])

Gardonus ruboculus Walecki, 1863: 364 (apparently an unnecessary replacement name for Cyprinus rutilus Linnaeus, 1758)

Leuciscus pallens Blanchard, 1866: 386, fig. 88 (type locality: Annecy [river Rhône basin], France; syntypes: MNHN ?)

Leuciscus rutilus var. caspicus Yakovlev, 1870: 103 (delta of river Volga, Russia) from Berg, 1949: 513

Leuciscus rutilus var. communis Yakovlev, 1873: 333 (delta of river Volga, Russia) from Berg, 1912: 50

Leuciscus rutilus var. fluviatilis Yakovlev, 1873: 334 (delta of river Volga, Russia) from Berg, 1949: 497

Leuciscus rutilus var. auratus Yakovlev, 1873: 334 (delta of river Volga, Russia; junior primary homonym of Leuciscus auratus Mauduyt, 1848) from Berg, 1912: 67

Leuciscus rutilus forma bolmensis Malm, 1877: 560 (type locality: lake Bolmen in Småland, Sweden; syntypes: LU)

Leuciscus rutilus var. elata Fatio, 1882: 498 (infrasubspecific, name not available)

Leuciscus rutilus var. elongata Fatio, 1882: 499 (infrasubspecific, name not available)

Leuciscus rutilus var. crassa Fatio, 1882: 499 (infrasubspecific, name not available)

Leuciscus rutilus var. aurata Fatio, 1882: 506 (infrasubspecific, name not available)

Leuciscus rutilus var. terekensis Rossikov, 1895: $\mathbf{n}^{\circ} \mathbf{5 4}$ (river Terek, Mosdok) from Berg, 1912: 52

Leuciscus rutilus var. communis Rossikov, 1895: $\mathbf{n}^{\circ} \mathbf{5 4}$ (river Terek, Mosdok) from Berg, 1912: 52

Leuciscus rutilus var. wobla Grimm, 1896: 67, 114 (nomen nudum; river Volga at Tzaritzyn, Russia) from Berg, 1912: 68

Leuciscus rutilus var. vobla Dikson, 1909: 31 (Saratow, Russia) from Berg, 1912: 68

Leuciscus rutilus var. erytraea Antipa, 1909: 181, fig. 71 (infrasubspecific, name not available)

Rutilus rutilus aralensis Berg, 1916a: 134 (type locality: Aral Sea [Berg, 1949: 505]; syntypes: ZISP?)

Rutilus rutilus mariza Drensky, 1926: 133, fig. 3 (type locality: river Mariza, Bulgaria; syntypes: NMNHS)

Rutilus rutilus schelkovnikovi Derjavin, 1926: 162, fig. (river Karasu, a tributary of river Araks, a tributary of river Kura, Caspian Sea basin) from Berg, 1949: 529

Rutilus rutilus caspius natio knipowitschi Pravdin. 1927: 84 (infrasubspecific, name not available; south-western Caspian Sea, Astrabad Bay) from Berg, 1949: 528

Leuciscus pigus dojranensis Karaman, 1928: 153, fig. 2a (type locality: lake Dojran, FYROM and Greece; syntypes: LU); repeated in 1929: 172

Rutilus rutilus carpathorossicus Vladykov, 1930: 103 (type locality: river Laborec, a tributary of Bodrog, Slovakia; lectotype: MNHN 30-211, designated by Almaça, 1969: 1119)

Rutilus rutilus aralensis morpha phragmiteti Berg, 1932c: 314, fig. 235 (infrasubspecific, name not available; Aral Sea at Muinak [Berg, 1949: 510])

Rutilus rutilus uzboicus Berg, 1932c: 315, fig. 237 (type locality: lake Yashkan, river Uzboi basin [Berg, 1949: 512]; syntypes: ZISP ?)

Rutilus rutilus caspicus natio kurensis Berg, 1932c: 323 (infrasubspecific, name not available; locality: river Kura, Azerbaidjan [Berg, 1949: 
527])

Rutilus rutilus caspicus natio tscharchalensis Berg, 1932c: 325 (infrasubspecific, name not available; locality: lake Charkhal, river Ural basin [Berg, 1949: 528])

Rutilus rutilus bucharensis Nikolsky, 1933: 264 (type locality: area of Termes, upper AmuDarja basin, Turkmenistan; holotype: ZMMSU P-1390)

Rutilus rutilus sucharensis Stangenberg, 1938: 47, 104 (type locality: lake Suchar Wielki, Poland; syntypes: LU)

Rutilus rutilus goplensis Stangenberg, 1938: 51, 104 (type locality: lake Goplo, Poland; syntypes: LU)

Rutilus rutilus caspicus natio geoktshaicus $\mathrm{Ba}-$ rach, 1941 (infrasubspecific, name not available)

Rutilus rutilus v[ar]. vegariticus Stephanidis, 1950: 203 (type locality: lake Vegaritida, Greece; syntypes: lost?)

Rutilus rutilus frici Misik, 1957a: 17, fig. 1 (type locality: Poltruba, an oxbow lake of river Elbe [Elbaltwasser] near Celákovice, Czech Republic; syntypes: ? CUP [80])

Rutilus rutilus lacustris natio menschikowi Kirillov, 1962: 40 (infrasubspecific, name not available; locality: river Vilyui basin, Yakutia, Siberia)

Systematic notes. The clarification of the status of several nominal taxa placed in the synonymy of R. rutilus by Holčík \& Skořepa (1971) requires more study. This particularly applies to populations of isolated basins of southeastern Europe. These should not be compared to the gobal range of variation of a few characters of a ' $R$. rutilus' encompassing all northern and central European Rutilus populations. On the contrary, the effort should focus in thorough comparisons of populations inhabiting adjacent basins. In northeastern Greece, Economidis \& Sinis (1982: 312) show that two group of populations (corresponding to the nominal taxa mariza and dojranensis) differ in gillraker counts.

Rutilus vegariticus is considered a valid species by Economidis \& Bănărescu (1991: 274) and as a subspecies of $R$. rutilus by Economidis (1995: 211). Not knowing on which characters this decision is based, I am left with no choice but to include it in the synonymy of $R$. rutilus. Hopefully this can be re-evaluated soon.

The migratory stocks of the Black Sea basin are tentatively considered as a distinct species; see above under Rutilus heckeli. Nominal species based on migratory stocks from the Caspian Sea basin are listed under the present species, but their status needs to be examined too.

Nomenclatural notes. Selys-Longchamps (1842: 210) considers Heckel as author of Leuciscus selysii. Heckel nowhere published this name and is responsible for none of the conditions which make it available in Selys-Longchamps (1842) and cannot be the author. Selys-Longchamps is alone responsible for the conditions making the name available and is thus author of the name (ICZN art. 50) (Kottelat, 1984: 148). This was probably a manuscript name of Heckel.

\subsubsection{Rutilus ylikiensis Economidis, 1991}

Rutilus aula rubella var. ylikiensis Stephanidis, 1939b: 55, pl. 3 (infrasubspecific, name not available; locality: lake Yliki, Greece)

Rutilus ylikiensis Economidis, 1991: 10 (available by reference to Stephanidis, 1939b; type locality: lakes Yliki and Paralimni / Aetolia and Akarnania (lakes Trichonis, Lyssimachia, Ozeros and Amvrakia, river Acheloos) / lake Pamvotis (Jannina), Greece; syntypes: lost ?)

Systematic notes. Listed as a valid species by Economidis \& Bănărescu (1991: 269).

Nomenclatural notes. As published by Stephanidis (1939b: 55), the name $R$. aula rubella var. ylikiensis is not available. The next use of the name I could find is by Economidis (1991); it is used as a species name and refers to Stephanidis (1939b) and this makes the name available; as Economidis is author of the conditions which make the name available, he is author of the name.

\subsubsection{Scardinius acarnanicus Economidis, 1991}

Scardinius scardafa plotizza [forma] acarnanicus Stephanidis, 1939a: 26, pl. 4 (infrasubspecific, name not available; locality: lakes Trichonis and Lyssimachia, river Acheleoos, Greece)

Scardinius acarnanicus Economidis, 1991: 16 (available by reference to Stephanidis, 1939b; type locality: river Acheleoos basin [river Acheleoos and lakes Trichonis, Lyssimachia, Ozeros and Amvrakia], Greece; syntypes: lost ?)

Systematic notes. See fliadou et al. (1996) for a recent discussion of the status of the species.

Howes (1981: 46) considers that Scardinius is not distinguishable from Rutilus. As presently understood, Rutilus is probably not a monophyletic lineage; awaiting an analysis of the interrelationships of its nembers, I conservatively retain the species of Scardinius as a distinct genus.

Nomenclatural notes. As published by Stephanidis (1939b: 26), the name S. scardafa plotizza 
f. acarnanicus is not available. The next use of the name I could find is by Economidis (1991); it is used as a species name and refers to Stephanidis (1939b) and this makes the name available; as Economidis is author of the conditions which make the name available, he is author of the name.

\subsubsection{Scardinius erythrophthalmus (Lin- naeus, 1758)}

Cyprinus Erythrophthalmus Limnaeus, 1758: 324

(based on Linnaeus [1746: 123, n. 324, Cyprinus ... radiis 14 pinnis rubris] and Artedi [1738: gen. 3 [9], syn. 4, spec. 9 [3], Cyprinus iride pimis omnibus ...]; type locality: "in Europae septentrionali"; syntypes: LU)

Cyprinus erythrops Pallas, 1814: 317 (type locality: all streams of Russia and Siberia; syntypes: LU)

Cyprinus Compressus Hollberg, 1822: 66 (type locality: canals in Götheborg, Sweden; syntypes: GNM ?)

Cyprinus Scardula Nardo, 1827a: 482, 488 (type locality stated as Adriatic Sea basin but actually lake Zürich, Switzerland / lakes of Auvergne, France; available by indication to Cyprinus latus of Aldrovandri, 1613: 641, 642 [localities explicitly stated are: lake Zürich (after Gesner) and lakes of Auvergne (after Rondelet)]; type material: NT) or 1827b: 34, 40 (same data)

Cyprinus caeruleus Yarrell, [1833]: 8, pl. 2 fig. 2 (type locality: Knowsley Park, Lancashire, U.K.; syntypes: LU)

? Cyprinus fuscus Vallot, 1837: 146 (type locality: market [in Dijon ?, France]; syntypes: LU [2])

Scardinius hesperidicus Heckel, 1843: 1037 (nomen nudum); Bonaparte, 1844: 431 (nomen nudum)

Scardinius hesperidicus Bonaparte, 1845: 10 (available by indication to Scardinius erythrophthalmus of Bonaparte, 1840: [fasc. 28, pl. 115 fig. 2], 1840: [fasc. 30, pl. 116 fig. 2]; type locality: lakes of Piemonte, Italy; syntypes: LU)

Scardinius platizza Bonaparte, 1846: 32 (nomen nudum)

Scardinius macrophthalmus Heckel, 1853: 30 (nomen nudum; locality: Kizbüehl [sic], Tirol, Austria)

Leuciscus Apollonitis Richardson, 1856: 374 (type locality: lake Apollonitis [near Brussa], Asia Minor; holotype: BMNH ?)

Scardinius macrophthalmus Heckel \& Kner, 1858: 160, fig. 85 (type locality: lake Hechtsee near Kupfstein, Tirol, Austria; syntypes: NMW 51678-684 [9])

Scardinius dergle Heckel \& Kner, 1858: 156, fig.
81 (type locality: streams Kerka and Zermagna. Dalmatia [Croatia] / Livno, Bosnia; syntypes: NMW 51676 [6], 51753 [2]. 51754 [3])

Scardinius plotizza Heckel \& Kner, 1858: 159, fig. 84 (type locality: Jessero Grande near Vergoraz and near Imosky, Dalmatia [Croatia] / Livno, Bosnia; syntypes: NMW 51816 [8], 51817 [2])

Scardinius crocophthalmus Walecki, 1863: 364 (apparently an unnecessary replacement name for Cyprinus erythrophthalmus Linnaeus, 1758) Scardinius erythrophthalmus var. dojranensis Karan, 1924b: 60 (type locality: lake Dojran, FYROM and Greece; syntypes: LU)

Scardinius scardafa natio ohridana Vladykov \& Petit, 1930: 399 (infrasubspecific, name not available: locality: lake Ohrid near Pogradec, Albania)

Scardinius erythrophthalmus morpha rutiloides Vladykov, 1931: 306 (infrasubspecific, name not available: locality not explicitly stated: "with the typical forms"; includes a long list of localities throughout Europe)

Scardinius erythrophthalmus v[ar]. achrus Stephanidis, 1950: 206 (type locality: river Cholorema near Almiros, Thessalia, Greece; syntypes: lost ?)

Systematic notes. Howes (1981: 46) considered Scardinius as part of Rutilus.

The morphological analysis by Grupče \& Dimovski (1984) indicates the presence of two species in FYROM (called S. e. scardafa and S. e. dojranensis by them). Comparison of the first one with Italian material is needed to confirm its identification; the second species should be compared with Danube or northern European material to confirm that it is distinct from them.

Economidis \& Bănărescu (1991: 269) treat S. plotizza as a valid species, without further information.

Nomenclatural notes. The publication year of Yarrell's description of Cyprinus caeruleus is usually given as 1837 . However, the name is cited (with Yarrell as author) by Jenyns (1835a: 26) and the paper is cited with correct page numbers by Jenyns (1835b: 413) and Yarrell (1836, vol. 1: 365). The paper was read June 19th, 1832 to the Linnean Society (Yarrell, [1833]: 5) and it seems obvious that volume 17 of their Transactions spans several years and that the title page, issued once the volume was complete has been printed in 1837 . The last paper of vol. 16 was read on April 3rd, 1832 and the title page of vol. 16 is dated 1833. Yarrell's paper being at the beginning of volume 17 was probably printed much earlier than 1837 , may be as early as 1832 . A publication date in 
1833, shortly after the title page of vol. 16 , seems reasonable.

\subsubsection{Scardinius graecus Stephanidis, 1937}

Scardinius graecus Stephanidis, 1937: 266, pl. 2

(type locality: lake Yliki (Likerni), near Thebes,

Beotia, Greece; syntypes: lost?)

Systematic notes. See Iliadou et al. (1996) for a recent discussion of the status of the species.

\subsection{4 ? Scardinius racovitzai Müller, 1958}

Scardinius racovitzai Müller, 1958: 165, fig. 1 (type locality: pond Petzea, a tributary of Cris Repede, Romania; holotype: MGAB 49917, MihaiBardan, 1984: 453)

Systematic notes. This species is known only from a thermal spring in Romania. Bănărescu (1964: 355, 1966: 381) treated this taxon as a subspecies of S. erythrophthalmus, but as far as I can understand, without giving reasons. Later (1981: 477), he commented that "it is possible that in the lower course there might be intergrades between this subspecies and the nominal one" [my translation from Romanian]. He further commented on the differences between the two taxa that "one cannot rely on the phenotype, because it has no genotypic base", but does not provide supporting evidence. Considering the differences between the two taxa reported by Müller (1958) and Bănărescu (1964) (maximum size, number of vertebrae, general appearance) and the absence of concrete data showing the existence of intergrades or phenotypic plasticity, at present there is no choice but to consider this species as valid. Clearly, more accurate information is needed to clear the case; it is true that the differences may be due to differences in temperature, but it is not correct to imply it from the case of some other species; this explanation is only acceptable if it is demonstrated case by case.

\subsubsection{Scardinius scardafa (Bonaparte,} 1837)

Rutilus heegeri Agassiz, 1835b: 38 (nomen nudum) Leuciscus Scardafa Bonaparte, 1837: [fasc. 19, p. 227], pl. 111 fig. 3 (type locality: lakes Nemi, Ronciglione, Bracciano, Fogliano and others, and many ditches, Italy; syntypes: ANSP 62116270 [59], 17002 [1], Böhlke, 1984: 90)

Leuciscus scarpata Bonaparte, 1837: [fasc. 19, p. 227] (nomen nudum; locality: lake Trasimeno, Italy)

Leuciscus marrochius Costa, 1838: fasc. 19: 12, pl. 13 (type locality: not stated [lake Fuccino, Italy, according to Tortonese, 1970: 261, supporting evidence not given]; syntypes: LU)
? Leuciscus Heegeri Bonaparte, 1839: [fasc. 24, p. 245], pl. 114 fig. 3 (type locality: some streams in Istria, Italy; holotype: LU)

? Heegerius typus Bonaparte, 1844: 435 (unnecessary replacement name for Leuciscus heegeri Bonaparte, 1839)

Leuciscus scarpetta Valenciennes, in Cuvier \& Valenciennes, 1844: 126 (type locality: lake Trasimeno, Italy; syntypes: MNHN 3890 [2], Bertin \& Estève, 1948: 80)

Systematic notes. Considered as valid by Bianco (1990b, 1994b: 455, 1995a: 165) and Economidis \& Bănărescu (1991: 269).

\subsubsection{Tinca tinca (Linnaeus, 1758)}

Cyprinus Tinca Linnaeus, 1758: 321 (based on Artedi [1738: gen. 4 [27], syn. 5, spec. 27 [4], Cyprinus mucosus ...] and Linnaeus [1746: 122, n. 321, Cyprinus ... ossiculorum [1]; type locality: "in Europae stagnis, lacubus"; type material: NT)

Cyprinus Tinca Auratus Bloch, 1782: 90, pl. 15 (type locality: pond in Schönhausen castle, Germany, introduced from Schlesien [now Poland]; holotype: ZMB 3312)

Tinca aurea Gmelin, 1788: 1414 (not binominal, name not available; refers to Bloch, 1782: 90, pl. 15)

Cyprinus zeelt La Cepède, 1803: 550, 552 (type locality: not stated; holotype: MNHN ?)

Cyprinus tincauratus La Cepède, 1803: 541, 542 (unnecessary replacement name for Cyprinus tinca auratus Bloch, 1782)

Cyprinus Tincaurea Shaw, 1804: vol. 5 (1): 217, pl. 130 (unnecessary replacement name for Cyprinus tinca auratus Bloch, 1782)

Tinca vulgaris Fleming, 1828 (type locality: "lakes and rivers, England"; available by description and by indication to earlier sources; type material: NT?)

Tinca chrysitis Fitzinger, 1832: 337 (unnecessary replacement name for Cyprinus tinca Linnaeus, 1758; author indicated as Agassiz, but Fitzinger is actual author)

Tinca Italica Bonaparte, 1836: [fasc. 18, p. 205], pl. 109 fig. 1 (type locality: Italy; syntypes: ANSP 6119-6122 [4], Böhlke, 1984: 79)

Tinca vulgaris var. maculata Costa, 1838: fasc. 19: 10, pl. 12 (infrasubspecific, name not available; localities: lakes Vulture and Matese, Italy)

Tinca communis Swainson, 1839: 285 (available by indication to Bloch, 1782, pl. 14 [Cyprinus tinca])

Tinca limosa Koch, in Koch, Herrich-Schäffer \& Forster, 1840: 40 (unnecessary replacement 
name for Cyprinus tinca Linnaeus, 1758)

Tinca Linnéi Malm, 1877: 564 (unnecessary replacement name for Cyprinus tinca Linnaeus, 1758)

Tinca vulgaris var. Cestellae Segre, 1904: 2 (type locality: lake Cestella, Padola, Cadore, Alpi Venete, Italy; syntypes [total 38]: MZUT 1448 [3], Tortonese, 1940: 140)

\subsection{1 'Tropidophoxinellus' hellenicus (Ste-} phanidis, 1939)

Rutilus alburnoides hellenicus Stephanidis, 1939a: 23, pl. 3 (type locality: lakes Trichonis and Lyssimachia, Greece; syntypes: lost ? [40])

Systematic notes. The generic position of this species is not clear. Bianco (1988b: 9) suggests that it might belong to the genus Alburnus. A direct comparison of the two species placed in Tropidophoxinellus shows that they are not closely related. They differ in body and head shape, mouth structure, etc. The only character they share is the presence of a ventral keel between pelvic fins and anus, a character present in many other genera. The species shows some resemblance with Alburnus, but it differs in having a very weak symphysal knob on the dentary (vs. well developed), the upper jaw protactible (vs. only slightly) with premaxilla with a very long ascending process (vs. short), and apparently a different structure of the keel. It apparently also differs from Alburnus species in having fewer branched anal rays (8-10, vs. 10-20).

It is provisionally retained in Tropidophoxinellus, avaiting for a more detailed study of its relationships. Transferring it provisionally to Alburnus (where is is likely not to remain) presents no advantage at this stage.

Nomenclatural notes. This name is available from Stephanidis (1939a) and the types and type locality are those specimens and locality mentioned in this paper. Stephanidis referred to another '1939' paper as containing the original description, but this paper did not appear until 1971 (see comment under T. spartiaticus). Stephanidis (1971a) actually is this '1939' paper in its original form, with footnotes updating it. The 'original' description (1971a: 180) has obviously no validity having appeared much later than the 'accidental' description of 1939a.

\subsubsection{Tropidophoxinellus} (Schmidt-Ries, 1943)

Rutilus spartiaticus Schmidt-Ries, 1943: 332 (type locality: Peloponese, Greece; type material: NT ?)
Rutilus spartiaticus Stephanidis, 1971a: 176, fig. 3 (type locality: river Eurotas near Sparta, Kelefinas, Mangoulitsa, Rezinas and Vassilopotamos, Greece; syntypes: MNHN 1975-849, B. Elvira, pers. comm., 1995 [total: 46, rest apparently lost]; a junior primary homonym of $R$. spartiacus Schmidt-Ries, 1943)

Rutilus spartiacus var. megalophthalmus Stephanidis, 1971a: 180, fig. 4 (infrasubspecific, name not available)

Systematic notes. Bianco (1988b: 9) suggested that this species might belong to the genus Scardinius, but without providing data.

Nomenclatural notes. Schmidt-Ries's (1943) brief diagnosis makes the name $R$. spartiaticus available. His account refers to a publication by Stephanidis dated 1939 in Acta Instituti et Musei Zoologici Universitatis Atheniensis, vol. 2, fasc. 5/6. Stephanidis (1971a: 163) explains that the paper was ready for printing when the journal was discontinued and that this is the reason why he mentions the paper in his own 1939 publications. Schmidt-Ries, who had seen Stephanidis's manuscript, probably believed that the paper was published inbetween. The paper was finally published in 1971 (Stephanidis, 1971a).

5.28.1 Vimba elongata (Valenciennes, 1844) Abramis elongatus Agassiz, 1835b: 39 (nomen nudum)

Abramis elongatus Valenciennes, in Cuvier \& Valenciennes, 1844: 75 (type locality: river Danube; type material: not stated; based on a drawing sent by Agassiz, the material on which the drawing is based is possibly in MHNN)

Systematic notes. Vimba is considered as a synonym of Abramis by Howes (1981: 46).

Uiblein \& Winkler (1994) analysed the morphometry of Austrian Vimba. Their conceptual base is quite confusing and blurred by semantic problems: e.g., the dichotomy between a so-called taxonomic hypothesis and a functional hypothesis, the labelling of species as populations, the poor morphological definitions of body landmarks used. the neglect of many other potentially useful characters, and a unusual understanding of fish morphology (p. 57-58: “... common variation in morphometric and meristic traits such as ... the number of gill-rakers are functionally interconnected with specific habitat parameters, such as current velocity and the availability of certain food types"; as a rule, meristic characters have a genetic basis). Uiblein \& Winkler reached no taxonomic conclusion, but observed a "high variability in morphometric characters among ecologically separa- 
ble groups of Vimba which are best explained by ongoing processes of adaptation to spatial food preference" (p. 57). To the systematist, this kind of argument is unlikely to yield an answer to the question 'is $V$. elongata specifically distinct from V. vimba ?' According to the authors, the two "forms" clearly differ in distribution, habitat, feeding, reproduction, spawning migrations, etc., and, as they pointed out (p. 63), lake forms in most fish groups have a deeper body, etc., while it is the reverse in the present case. From the available information, I see no reason to doubt that each form fits into what has been defined as a species in the Introduction.

Nomenclatural notes. This species is often listed as "Abramis elongata Agassiz, 1835". Actually, Agassiz merely listed the name and did not provide any description, diagnosis or indication (in the sense of ICZN art. 12(b)(1)) and it is thus a nomen nudum.

\subsubsection{Vimba melanops (Heckel, 1837)}

Abramis melanops Heckel, 1837: 154, pl. 9 fig. 3 (type locality: river Marizza [near Philippoli] in Rumelia [southern Bulgaria]; holotype: NMW $55270)$

Abramis elongatus var. asianus Steindachner, 1897: 689, pl. 4 fig. 1 (type locality: KiutahiaSu near Eskischehir, Turkey; syntypes: NMW $55251[5])$

Systematic notes. Bănărescu (1964: 403; Bănărescu et al., 1970) considers Vimba elongata and $V$. melanops as two valid species.

\subsubsection{Vimba vimba (Linnaeus, 1758)}

Cyprinus Vimba Linnaeus, 1758: 325 (based on Linnaeus [1746: 123, n. 326, Cyprinus ... radiis 24] and Artedi [1738: gen. 6 [18], syn. 14, spec. 18 [6], Cyprinus rostro nasiformi ...]; type locality: "in Sveciae lacubus"; type material: NT)

Cyprinus Zerta Leske, 1774: 44 (type locality: Leipzig, Germany; type material: possibly LSL 71, Wheeler, 1985: 5, 33)

Cyprinus persa Gmelin, 1774: 233, 241 (nomen nudum; locality: Caspian Sea)

Cyprinus vimpa Strøm, 1784: 120 (type locality: Eger [in Boskerud District, south west of Oslo], Norway; type material: NT)

Cyprinus Serta Shaw, 1804: vol. 5 (1): 232 (unnecessary replacement name for Cyprinus vimba Linnaeus, 1758)

Cyprinus Persa Pallas, 1814: 310 (type locality: "Persa; in lacubus ad Cyrum"[Iran, in lakes along river Kura]; syntypes: LU)

Cyprinus carinatus Pallas, 1814: 323 (type local- ity: river Don and Azov Sea; syntypes: LU)

Abramis tenellus Nordmann, 1840: 510 (type locality: stream Tschornaïa Retschka near Inkerman, near Sevastopol, Crimea, Ukraine; syntypes: LU)

Abramis frivaldszkyi Heckel, 1843: 1032 (type locality: Brussa, in Notolien [Anatolia], Turkey; syntypes: NMW [not yet located; B. Herzig, pers. comm., 1995])

Leuciscus parvulus Valenciennes, in Cuvier \& Valenciennes, 1844: 64, pl. 487 (type locality: Crimea, Ukraine; holotype: MNHN 3954, Bertin \& Estève, 1948: 78)

Abramis Nordmannii Dybowski, 1862: 179 (type locality: Crimea, Ukraine; available by short diagnosis and indication to A. melanops of Nordmann, 1842: pl. 22 fig. 2; syntypes: LU)

Abramis parsa [sic] var. pontica Yashchenko, 1895: 90 (nomen nudum; locality: river Salgir where it enters the Sivash, [Biyuk-Karasu basin], southern Crimea, Ukraine) from Berg, 1949: 796 and N. da Costa Pereira, pers. comm.

Vimba vimba tenella natio karasuensis Tseeb \& Delyamure, 1938: 145 (infrasubspecific, name not available; river Biyuk-Karasu, Black Sea basin) from Berg, 1949: 796

Vimba vimba bergi Velikokhatko, 1940: 273 (river Bug upstream as far as Aleksandrovskaya Hydroelectric Station, Ukraine) from Berg, 1949: 795

Vimba vimba vimba infranatio borysthenica Velikokhatko, 1940: 272 (infrasubspecific, name not available; lower river Dnieper to river Konka, Ukraine) from Berg, 1949: 791

Vimba vimba tenella natio istanbulensis Battalgil, 1941: 181 (infrasubspecific, name not available; locality: stream Kâathane, draining to the Bosphorus near Istanbul, Turkey [European side])

Vimba vimba tenella natio sapancae Battalgil, 1941: 181 (infrasubspecific, name not available; locality: lake Sapanca, Turkey)

Vimba vimba tenella natio nicaeensis Battalgil, 1941: 181 (infrasubspecific, name not available; locality: lake Iznik, Turkey)

Vimba vimba tenella natio aphnitis Battalgil, 1941: 181 (infrasubspecific, name not available; locality: lake Manyas, Turkey)

Vimba vimba tenella natio abulyontis Battalgil, 1941: 181 (infrasubspecific, name not available; locality: lake Abulyont, Turkey)

Acanthobrama mirabilis Ladiges, 1960: 132, fig. 4 (type locality: river Menderes near Saraköy, Denizli Prov., Turkey; holotype: ZMH 1084, Wilkens, 1977: 157) 
Systematic notes. Vimba vimba carinata is considered as a distinct subspecies by Balon et al. (1987: 241) who do not provide explicit evidence, while Bănărescu et al. (1970: 69) could not distinguish subspecies within $V$. vimba. Bănărescu et al. (1963: 398, 1970: 69) considered that two (possibly three) forms differing by body shape and spawning season coexist over most of the range of the species which actually may be reproductively isolated; Balon et al. (1987: 245) disagree.

Nomenclatural notes. When creating the name Cyprinus persa, Gmelin (1774: 233, 241) wrote that the species is already described in "Comment. Acad. Petersb." without providing exact reference. I could find no later reference to such a paper by
Gmelin. As it is not accompanied by a description or an indication (sensu ICZN art. 12(b)), the name is a nomen nudum. The same name has been used again by Pallas (1814: 310) who based his description on Gmelin's manuscript, implying that the description has not been published. Pallas is author of the name as he is author of the conditions making it available.

Bănărescu et al. (1970: 47) indicate the author of Abramis persa pontica as Kessler (1877: 192), but I could not find it in this publication. Berg (1949: 796) states that this is an unpublished museum name of Kessler, first published in Yashchenko (1895: 90).

\section{Cobitidae}

The family name Cobitidae has sometime been spelt Cobitididae. The spelling Cobitidae has been confirmed by a ruling of the International Commission on Zoological Nomenclature (1988; see Kottelat, 1986 for history).

\subsubsection{Cobitis bilineata Canestrini, 1866}

Cobitis taenia var. bilineata Canestrini, 1866: 148 (type locality: Modenese and Treviso, Italy; syntypes: LU)

Cobitis taenia var. puta Cantoni, 1882: 362, pl. 1 fig. 1 (type locality: Torino and Vercellese, Italy; syntypes: LU)

Cobitis taenia var. septa Cantoni, 1882: 363, pl. 1 fig. 2 (type locality: Italy; type material: NT ?) Cobitis taenia zanandreai Cavicchioli, 1965: 1152, fig. 1 (type locality: river Volturno at Ponte Annibale, Capua, Caserta, Italy; holotype: Istituto di Anatomia Comparata, Università di Ferrara) Systematic notes. As implied by Vasiljev \& Vasiljeva (1994: 67), a comparison of their karyological data of Russian Cobitis with those of the Italian one by Cataudella et al. (1977: 163) indicates that the Italian material is not conspecific with any of the species known from the former USSR. I hypothesise that the species is restricted to the Italian peninsula and material from central Europe is conspecific with the Scandinavian C. taenia; both hypotheses need confirmation. See C. taenia for further comments.

Nalbant (1993: 108) lists C. zanandreai as a valid species, without supporting discussion or data.

Nomenclatural notes. I tentatively hypothesise that the species is restricted to the Italian peninsula and use the older available name for it. Among the many taxa considered to be synonyms of C. taenia, only C. spilura Holandre, 1837 was published before C. bilineata.

\subsection{2 'Cobitis' calderoni Bacescu, 1962}

Cobitis Calderóni Bacescu, 1962a: 440, pl. 1 figs. 2-3, pl. 2 figs. 6-7 (type locality: stream Arlanzón, tributary of river Duero at CardenaJimeno, Charcas de San Medel and La Gravera, Burgos, Spain; holotype: MGAB 7 [now 49415, P. Bacalu, pers. comm.])

Systematic notes. As indicated by Vasiljeva et al. (1992: 82 [translation]), this species does not belong to Cobitis and it should probably be placed in a genus of its own once the relationships within Cobitis sensu lato are understood.

Nomenclatural notes. The "type locality" given by Bacescu (1962a: 440) is obviously the combination of three localities. Exact locality data of the holotype are apparently lost; according to P. Bacalu (pers. comm.), data associated with the specimen in MGAB are 'Arlanzon river'. All localities listed by Bacescu are within a radius of ca. $2 \mathrm{~km}$ (B. Elvira, pers. comm. 1995). The actual publication date is 1962 and not 1961 as is usually indicated. 
6.1.3 Cobitis elongata Heckel \& Kner, 1858

Cobitis elongata Heckel \& Kner, 1858: 305, fig. 164 (type locality: river Sala near Idria, Krain, Slovenia; syntypes: NMW 48609-610 [5])

Systematic notes. See Bănărescu \& Nalbant (1957).

\subsubsection{Cobitis elongatoides Bacescu \& Maier, 1969}

Cobitis taenia var. elongatoïdes Bacescu, 1962b: 294 (infrasubspecific, name not available; locality: rivers Neajlov, Jiu and Sîi, southwestern Romania)

Cobitis taenia elongatoides Bacescu \& Maier, 1969: 39 [of translation], figs. 3a, 7-1 (type locality: river Argesel, Danube basin, Romania; syntypes: LU)

Cobitis taenia danubialis Nalbant, 1993: 109 (type locality: river Mures at Sarmas, Harghita, Transilvania, Romania; holotype: MGAB 49923)

Systematic notes. Nalbant (1993) treated C. elongatoides (as C. t. danubialis) as a subspecies of C. taenia but he did not explain this status and did not indicate the characters distinguishing the two taxa. Cobitis elongatoides is tentatively accepted as valid species, pending a critical revision of the genus.

Nomenclatural notes. In the original description of C. danubialis, Nalbant (1993: 109) listed Bacescu as author of the name. The text, however, makes it clear that Nalbant is author of both the name and for satisfying the criteria of availability and he is thus to be treated as author of the name (ICZN, art. 50(a)).

In Bacescu \& Maier (1969), the name C. taenia elongatoides is used. The mention in the text of characters distinguishing it makes the name available, although it is nowhere clearly stated on which material it is based, but there is an explicit mention of a locality, which becomes type locality.

Nalbant proposed $C$. danubialis as a valid name for the taxon named C. taenia var. elongatoides by Bacescu (1962b: 294) as this name is not available. He overlooked that the name C. t. elongatoides is available from Bacescu \& Maier (1969: 39) for apparently the same species and clearly has priority over C. danubialis.

\subsubsection{Cobitis megaspila Nalbant, 1993}

Cobitis megaspila Nalbant, 1993: 108, figs. 24-28

(type locality: delta of river Danube at Caraorman, Romania; holotype: ISBB 4497)

\subsubsection{Cobitis meridionalis Karaman, 1924}

Cobitis taenia meridionalis Karaman, 1924b: 75 (type locality: lake Prespa and its small tributaries, FYROM and Greece; syntypes: LU)

Systematic notes. Considered as valid by Economidis (1991: 29, 41, fig. 3) and Economidis \& Nalbant (in press).

\subsubsection{Cobitis paludica (de Buen, 1930)}

Acanthopsis taenia forma paludica de Buen, 1930: 33, fig. 48 (type locality: Fuente del Roble, Talayuela, Cáceres, Spain; holotype: LU; available by indication [ICZN art. 12(b)(7)])

Cobitis taenia paludicola Berg, 1932a: 155 (unjustified emendation of C. taenia paludica de Buen, 1930)

Cobitis taenia haasi Klausewitz, 1955: 42, fig. 2 (type locality: Albufera near Valencia, Spain; holotype: SMF 3232)

Systematic notes. Cobitis paludica was considered a synonym of C. maroccana Pellegrin, 1929 (p. 525, fig. 1; type locality: Oued Tiflet at Tiflet and Sidi Yahia, Morocco) by Doadrio et al. (1988: 156) and Vasiljeva et al. (1992). Doadrio (1994: 26) considers them as specifically distinct, a point of view apparently supported by data in Perdices et al. (1995). A detailed morphological comparison of the African and Iberian stocks would be desirable.

\subsubsection{Cobitis stephanidisi Economidis, 1992}

Cobitis stephanidisi Economidis, 1991: 27, 41 (fig.

7) (nomen nudum; locality: Kefalovrissos spring in Velestino village (Feres), Thessaly, Greece)

Cobitis stephanidisi Economidis, 1992: 60 (type locality: lake Karlas and Kefalovrissos spring in Velestino village (Feres), Thessaly, Greece; syntypes [material listed by Economidis \& Nalbant, in press]: DZAUT 1992-40 [1], 1988-30 [9], 198913 [56], 1989-35 [26], 1992-37 [23], ISBB 4572 [33], USNM 330137 [1], SMF [4], MNHN 1994516 [2], NMW 92822 [3])

Nomenclatural notes. The complete description Cobitis stephanidisi is in Economidis \& Nalbant (in press); however, the use of the name accompanied by a diagnosis and a figure in Economidis (1992) makes the name available from this publication and with Economidis as author.

\subsubsection{Cobitis strumicae Karaman, 1955}

Cobitis taenia strumicae Karaman, 1955: 190, fig. 4 (type locality: Monospitovo swamp and river Strumica, FYROM; syntypes: LU)

Cobitis peschevi Sivkov \& Dobrovolov, 1984: 1673. fig. 1 (type locality: river Eleshnitsa, a tributary of river Kamchiya, Bulgaria; holotype: MNHV 1627)

Systematic notes. Considered valid by Econo- 
midis (1991: 27) and Economidis \& Nalbant (in press). Synonymy follows Economidis \& Nalbant (in press).

\subsubsection{Cobitis taenia Linnaeus, 1758}

Cobitis Taenia Linnaeus, 1758: 303 (based on Artedi [1738: gen. 2 [4], syn. 3, spec. 4 [2], Cobitis aculeo bifurco ...], Linnaeus [1746: 126, n. 333, idem] and Gronovius [1754: 2, n. 5, idem]; type locality: lake Mälaren, Sweden [by present restriction; see Nomenclatural notes]; syntype: ? UUMZ 205, Wheeler, 1991: 163)

Cobitis spilura Holandre, 1837b: 253 (type locality: Liège, Belgium / river Moselle, stream Nied, below Malroy, river Meuse below Saint Mihiel, France; holotype: LU [description explicitly based on a single specimen; "syntypes": Mus. Metz, Blanchard, 1866: 288]; author stated as Carlier, but Holandre is responsible for conditions making name available and thus is author)

? Cobitinula anatoliae Hanko, 1924: pl. 3 fig. 6 (type locality: lake Ak-Göl, Turkey; syntypes: MNH [2]); synonymy according to Coad \& Sarieyyüpoglu, 1988: 426

Cobitis taenia ohridana Karaman, 1928: 162, figs. 4a, 5 (type locality: lake Ohrid, FYROM; syntypes: LU); repeated in 1929: 173

Cobitis taenia narentana Karaman, 1928: 163 (type locality: river Neretva, Dalmatia (Croatia and Bosnia); syntypes: LU); repeated in 1929: 173

Cobitis taenia dalmatina Karaman, 1928: 163 (type locality: river Cetina, Dalmatia; syntypes: LU); repeated in 1929: 173

? Cobitis taenia tanaitica Bacescu \& Maier, 1969: 39 [of translation], fig. 1 (type locality: river Don below Rostov, Ukraine; syntypes: LU [6])

Cobitis taenioides Bacescu \& Maier, 1969: 39 [of translation] (type locality: western Europe and Danube basin; syntypes: LU)

Systematic notes. Vasiljev \& Vasiljeva (1994: 67) report that ' $C$. taenia' of the former USSR actually includes at least 3 and probably up to 5 species. The identification of one of the Russian species as C. taenia sensu stricto is tentatively accepted, but comparison with and karyological data of material from the type locality of $C$. taenia is needed to confirm this hypothesis. Data on material of the Rhine basin is needed to confirm the conspecificity of C. spilura and C. taenia. The Italian material is not conspecific with any of the Russian species and is treated as a distinct species, C. bilineata. It seems likely that several populations from south-eastern Europe will turn out to be distinct species once examined in detail. Cobitis meridionalis and C. strumicae are considered as valid species by Economidis (1991) and Bănărescu (1992b: 753, 755).

Ráb \& Slavík (1996) report a diploid-triploidtetraploid complex involving C. taenia and an unidentified species in the Elbe basin in the Czech Republic. Again the identification of C. taenia will requires a comparison with material from the type locality. The unidentified species (fig. 4b) shows some superficial affinities with $C$. elongatoides as illustrated by Nalbant (1993: 104, as C. t. danubialis), but this should be confirmed by examination of well preserved material of both. The existence of triploids in European waters is also reported by Boron (1992) from the Vistula basin in Poland and by Sofradžija \& Berberovič (1978 cited by Ráb \& Slavík, 1996: 203) from the Morova basin in Bosnia. See Ráb \& Slavík (1996) for a review of chromosome numbers in the genus Cobitis. Boron (1992: 205) identified C. granoei as one of the parent of the triploid she described, a conclusion not supported by Ráb \& Slavík (1996: 209). [Note that C. granoei Rendahl, 1935 is a synonym of C. melanoleuca Nichols, 1925 according to Nalbant (1993: 108).]

Nomenclatural notes. The type locality of $C$. taenia presently encompasses most of Europe. Linnaeus's (1758) description is based on three earlier literature accounts and the specimens on which these accounts are based are syntypes and their localities together constitute the type locality. These accounts are in Artedi (1738), Linnaeus (1746) and Gronovius (1754.). Artedi's account is based on specimens from lake Mälaren (west of Stockholm), Sweden and literature accounts by Rondelet (1555: 204), Aldrovandri (1613: 617), Gesner (edition not stated, pp. 404, 482; 1563: 163), Charleton (1668: 157), Jonston (1649: pl. 26 fig. 21, pl. 46 fig. 1), Willughby (1686: 265, 266), Ray (1710: 124), Schonevelde (1624: 74, pl. 4 fig. 12), and Hildegarde de Pinguia (1533: 92). Linnaeus's (1746) account is possibly based on material from lake Mälaren too, on the above-mentionned account by Artedi, on the same accounts by Rondelet, Gesner, Jonston, Charleton, Schonevelde, Willughby, and Ray, and on Marsili (1726: part 3, pl. 1 fig. 2). Gronovius's account is based on specimens from Bruxelles (Belgium) and the same accounts by Artedi and Marsili. I have not checked all these secondary references, but some of them probably refer to earlier ones too. In summary, the type locality of Cobitis taenia includes a collection of localities probably including Sweden, France, Germany, England, Italy and the middle Danube, that is several of the species recognised 
here. As first reviser, I retain lake Mälaren (the only locality explicitly mentionned by Linnaeus and Artedi) as type locality. A possibly syntype survives in Linnaeus's collection (Wheeler, 1991: 163).

In Bacescu \& Maier (1969), the name C. taenioides (also misspelt C. taenoides) is used. The mention of characters distinguishing it in the text makes this name available, although it is nowhere clearly stated on which material it is based. The type locality is ambiguous, C. taenioides is once recorded from western Europe, once from the Danube basin, once from the "rest of Europe" [p. 39].

6.1.11 Cobitis trichonica Stephanidis, 1974 Cobitis trichonica Stephanidis, 1974a: 227, figs. 1-3 (type locality: lake Trichonis, Greece [by present restriction]; syntypes: lost ?)

Nomenclatural notes. Stephanidis (1974a: 227) designated two "holotypes", a male and a female. As there should be a single specimen designated as holotype, this designation is not valid and all the 34 specimens he examined are syntypes. As there is no holotype, there is no clear type locality and as first reviser, I restrict the type locality to lake Trichonis because this is the locality where Stephanidis's "holotypes" have been collected. Stephanidis's material is apparently lost (Economidis, pers. comm., Feb. 1996), so that a lectotype designation is not possible.

\subsubsection{Cobitis vardarensis Karaman, 1928}

Cobitis taenia vardarensis Karaman, 1928: 163 (type locality: basin of river Vardar, FYROM; syntypes: LU); repeated in 1929: 172

Systematic notes. Treated as valid species by Economidis (1991: 27) and Nalbant (1993: 107).

\subsubsection{Cobitis sp. 1}

Cobitis punctilineata Economidis, 1991: 28, 41 (fig. 4), 1992: 75 (nomen nudum; locality: stream Aggitis, tributary of river Strymon, Philippi plain, Greece)

Nomenclatural notes. This species is described and formally named by Economidis \& Nalbant (in press).

\subsubsection{Cobitis sp. 2}

Cobitis hellenica Economidis, 1991: 28, 41 (figs. 1-

2), 1992: 75 (nomen nudum; locality not stated)

Nomenclatural notes. This species is described and formally named by Econimodis \& Nalbant (in press).

\subsubsection{Cobitis sp. 3}

Cobitis hellenica arahthosensis Economidis, 1991:

28, 1992: 75 (nomen nudum; locality: rivers

Louros and Arachthos, Epirus, Greece)

Nomenclatural notes. This species is described and formally named by Economidis \& Nalbant (in press).

\subsubsection{Misgurnus anguillicaudatus (Cantor, 1842) introduced}

Nomenclatural notes. This species has been reported as introduced in Germany, as M. mizolepis Günther, 1888 (Riffel et al., 1994). Reasons for identifying this population as $M$. mizolepis are not stated. The latest systematic revision of the Chinese species of the genus Misgurnus treats $M$. mizolepis as a synonym of $M$. anguillicaudatus (Chen, 1981) and this is tentatively followed here. Considering the morphological and karyological variability observed in the species, it would not be surprising if a more detailed study shows that several species are confused under the name $M$. anguillicaudatus. Whatever its identity, the species is likely to evolve as a threat to other aquatic organisms.

\subsubsection{Misgurnus fossilis (Linnaeus, 1758)}

Cobitis fossilis Linnaeus, 1758: 303 (based on

J. F. Gronovius [1748: 79, pl. 3, Cobitis aculeo bifurco ...], Artedi [1738: gen. [spec.] 2, syn. 3, Cobitis caerulescens ...] and Linnaeus [1754: 76, idem]; type locality: "in Europa"; syntypes: BMNH 1853.11.12:121 [1], NRM 69 [1], Wheeler, 1958: 213, Fernholm \& Wheeler, 1983: 218)

? Petromizon [sic] variegatus Wulff, 1765: 16 (type locality: Borussia [Prussia]; type material: NT)

Nomenclatural notes. The original description of Petromizon variegatus Wulff (1765: 16) is ambiguous. The species is placed in the genus 'Petromizon' diagnosed by the presence of "7 spiracles", but the synonymy and description are indicative that at least part of the article is based on a loach and most likely M. fossilis; it is restricted here to this meaning.

\subsubsection{Sabanejewia balcanica (Karaman, 1922)}

? Cobitis aurata Filippi, 1863: 391 (type locality: Sartschem [apparently Sarcham-e Sofla, $39^{\circ} 07^{\prime} \mathrm{N} 47^{\circ} 54^{\prime} \mathrm{E}, \mathrm{B}$. W. Coad, pers. comm; near the falling of river Zanjan into Qezel Owzan; Berg, 1949], Iran; lectotype: MZUT 674, designated by Tortonese, 1961: 188) 
? Cobitis hohenackeri Kessler, 1877a: 177 (type locality: river Kura, Caspian Sea basin, Azerbaidjan [Berg, 1949: 894]; syntypes: LU; author indicated as Brandt, but Kessler is actual author)

? Cobitis aralensis Kessler, 1877a: 184 (type locality: mouth of rivers Syr-Darya and Amu-Darya, Aral Sea [Berg, 1949: 895]; syntypes: LU)

Cobitis balcanica Karaman, 1922: 307 (type locality: river Vardar [and its tributaries near Skopje (Ưskub) and Veles], FYROM, restricted by Bănărescu et al., 1972: 1; syntypes: LU, ?MNHN 28-222 [1], Bertin \& Estève, 1948: 93)

Cobitis montana Vladykov, 1925b: 320 (type locality: streams Apšica, Terešovka, Teresulka, Luzanka and Terebla, tributaries of river Theiss [Tisza]; and river Theiss between villages AknaSlatina and Buštino, Ukraine; syntypes: LU [219])

Cobitis aurata balcanica natio radnensis Jaszfalusi, 1951: 116, pl. 1 fig. 1 (infrasubspecific, name not available; locality: river Zebrak, a tributary of the Szamos [Somesul ?] / river Maros [Mures] near Gödemesterháza and creek Göde, Romania)

Cobitis aurata vallachica Nalbant, 1957: 209, figs. 1-3 (type locality: river Ialomitza near Crivina, north-east Vallachia, Romania; holotype: SMF 4087)

Cobitis aurata radnensis Bănărescu, Müller \& Nalbant, 1960: 124 (short diagnosis; locality: upper rivers Mures and Tirnava, Romania; syntypes: LU)

Cobitis aurata bosniaca Karaman, 1963: 629, fig. 1 (type locality: creeks Saturlija and Siroka Rijeka near Banja Luka, Vrba basin, Bosnia; syntypes: LU [144])

? Sabanejewia aurata kubanica Vasiljeva \& Vasiljev, 1988: 210 [p. 33 of translation], fig. 2 (type locality: river Nevinka, Kuban basin, Russia; holotype; ZMMSU P-16384)

Sabanejewia aurata baltica Witkowski, 1994: 44. fig. 10 (type locality: river Widawa near village Kielczopwek, $4 \mathrm{~km}$ from Wroclaw, river Oder basin, Poland, $51^{\circ} 18^{\prime} \mathrm{N} 17^{\circ} 12^{\prime} \mathrm{E}$ ); holotype: NMHW 638)

? Sabanejewia aurata doiranica Economidis, 1995: 211 (nomen nudum)

? Sabanejewia aurata thrakica Economidis, 1995: 211 (nomen nudum)

Systematic notes. Bănărescu et al. (1972: 3) consider S. balcanica, S. bulgarica, S. radnensis and $S$. vallachica as valid subspecies of $S$. aurata in Romania. The sympatric occurrence (Bănărescu et al., 1972: p. 38) of S. balcanica (or "inter- grades" balcanica-vallachica) and S. bulgarica indicates that they represent two species, as already concluded by Vasiljeva \& Vasiljev (1988). Sabanejewia vallachica and $S$. radnensis are tentatively considered conspecific with S. balcanica. The data in Bănărescu et al. (1972: 41) could suggest that $S$. balcanica and $S$. vallachica are distinct, but as the range of $S$. vallachica is quite restricted with a larger area occupied by balcanica-vallachica "intergrades", I treat them as a clinal variation of a single species.

Sabanejewia aurata has been described from Iran and subsequently several nominal species have been referred to it, as synonyms or as subspecies, resulting in a complex polytypic species extending from the Aral Sea to Iran South and West of the Caspian Sea, a narrow corridor across Russia and Ukraine, and the Danube basin (Bănărescu, 1992b: 688, fig. 11/10). This distribution range is quite tortuous and apparently unique. In Iran, S. aurata is known from the basins of the Safid Rud (where the type locality is situated; Caspian Sea drainage) and Tedzhen (a small endorheic basin in north-eastern Iran) (Coad, 1980: 96). It is not known from Turkey according to Bănărescu \& Nalbant (1964: 182); subsequent records from eastern Turkey (Kuru, 1971; Erkakan \& Kuru, 1982) are likely but need confirmation. As discussed above, in the Danube basin, especially Romania where detailed data are available (Bănărescu et al., 1972), what was treated as a single polytypic species under the BSC actually is at least two species under the PSC. Similar data are badly missing for the rest of the range of $S$. 'aurata' and at present it is not possible to know if one of the Danube species is possibly conspecific with the Iranian S. aurata. To consider one of them rather than another to be conspecific with $S$. aurata would be arbitrary given our present knowledge; and there would be no advantage in such an arbitrary treatment. I therefore consider tentatively that the real $S$. aurata is restricted to Iran and possibly adjacent areas, but does not occur in European waters. Vasiljeva \& Vasiljev (1988) considered the populations of the Caspian Sea and the river Don basin as representing a single subspecies, while they could not conclude on the status of the populations of the Danube basin. They distinguish two groups of populations on the basis of details of colour pattern in the Danube basin. Vasiljeva \& Ráb (1992) report that the karyotype of the S. balcanica from middle Danube basin differs from $S$. aurata (and S. kubanica).

The type locality of $S$. balcanica is in the 
Vardar basin. Confirmation of the identification of the Danube $S$. balcanica populations by direct comparison with Vardar material is needed.

Sabanejewia 'aurata' is present in the Vistula and Njemen basins (Rolik, 1960) and the Oder basin (Frankiewicz, 1985). Frankiewicz commented that the Oder (Baltic Sea drainage) population could be distinguished as a subspecies as there were "considerable differences" between it and Dniester (Black Sea) specimens; he referred to his table 3 which only shows differences in body depth, pectoral-pelvic distance and eye diameter (the data of the Dniester population are from Rolik, 1967: 143, and it is not possible to know if the methods are the same).

The Baltic populations have been treated as a distinct subspecies by Witkowski (1994: 44). The character states used in the diagnosis apparently are indicative of interpopulation variation but most statements of the diagnosis are not supported by the data: more lateral spots (not supported by figure 2 which shows 8-19 in Baltic populations [numbers $1-7$ ], vs. $8-18$ in other populations of $S$. balcanica [numbers 9-27]), more dorsal spots (not supported by figure 4: 9-18, vs. 8-20), presence of large caudal spots (possibly uninformative; the range of variation illustrated in figure 8 is commonly observed in numerous species of the suborder Cobitoidei and may also be influenced by stress and fixative), shape and size of lower lip (not supported by figure 6 and text p. 33), shallow body (not supported by graphs in figures 5: maximum depth $12-18 \%$ SL, vs. $13-20$; "minimum depth" [?] 6.5-9.5\% SL, vs. 5.5-10.0), longer caudal fin (not supported by graphs in figure 5: $18.5-23.0$, vs. $17-22$ [I ignore specimens apparently aberrant]). The caudal peduncle seems longer (supported by graphs in figure 5: 18-25, vs. 15-23) and the shape of the suborbital spine seems to be slightly different). Here again, a problem is that the tabulated data includes original measurements and values compiled from 5 additional sources and we cannot judge of their compatibility.

Nomenclatural notes. Cobitis aurata balcanica natio radnensis Jaszfalusi, 1951 is not available because it is an infrasubspecific name. The use of this name as a subspecies with a short diagnosis in Bănărescu et al. (1960: 24) also makes it available, with Bănărescu, Müller \& Nalbant as authors. The specimens then examined by these authors (not listed) are the syntypes. Its use as a subspecies in Bănărescu (1960) would also make it available by indication to Jaszfalusi (1951), with Jaszfalusi's material as syntypes. As the two publications appeared in 1960, their respective priority is determinant. Bănărescu (1960) is dated November 1960, Bănărescu et al. appeared in a volume for 1957-59 published in 1960; the exact publication date is unknown, but P. Bănărescu informed me that it appeared before his November 1960 paper and it thus has priority.

6.3.2 Sabanejewia bulgarica (Drensky, 1928) Cobitis bulgarica Drensky, 1928: 171 fig. 5-2 (type locality: small tributaries of the Danube in Widdin [Vidin] and Danube mainstream at Krai Panaira, $3 \mathrm{~km}$ downriver of Widdin [Vidin], Bulgaria; syntypes: ? NRM 10432 [2])

Cobitis albicoloris Chichkoff, 1932: 368, pl. 1 fig. 3 (type locality: creek Provadiiska Rieca, Bulgaria; holotype: LU)

Cobitis taenia tesselatus Pietschmann, 1937: 29 (type locality: Widin [Vidin] on the lower Danube, Bulgaria; syntypes: NMW 48624 [2], EAWAG 996 [1])

Systematic notes. See Sabanejewia balcanica for comments. Vasiljeva \& Vasiljev (1988) considered $S$. bulgarica as a valid species.

\subsubsection{Sabanejewia larvata (Filippi, 1859)}

Cobitis larvata Filippi, 1859: 50 (type locality: creeks near Settimo Torinese [North East of Torino], Italy; syntypes: MZUT 510 [4], Tortonese, 1940: 141, Mus. Zool. Univ. Pavia [1], Cantoni 1882: 366, ZMUC 439-441 [3], Nielsen, 1974: 49)

Cobitis taenia var. conspersa Cantoni, 1882: 364, pl. 1 fig. 3 (type locality: based on a specimen of unstated locality and reference to 2 specimens from Pavia, Italy, identified by B. Crivelli as $C$. larvata, cited by Pavesi, 1877: 499 [as p. 87]; syntypes [total 3]: Mus. Zool. Univ. Pavia [2], not listed by Cantoni, 1882)

Nomenclatural notes. Note that publication date of C. larvata is 1859. The often-cited paper (Filippi, 1860: lxxi) appeared after an earlier use of the name by Filippi (1859: 50).

6.3.4 Sabanejewia romanica (Bacesco, 1943) Cobitis caspia romanica Bacesco, 1943: 137, figs. 4-6, 7G (type locality: river Bratia, a tributary of the Arges, at Vladesti, Muscel, Romania; lectotype: MGAB 49916, present designation)

Nomenclatural notes. Mihai-Bardan (1984: 458) lists a specimen as holotype. Bacesco (1943: 137) stated that he examined 185 specimens but nowhere designated a type or a holotype; so all 
specimens are syntypes. He stated a type locality (p. 137), but as there is no holotype, all the localities he listed (p. 140) are part of the type locality. The specimen considered as holotype by Mihai-Bardan, from the "terra typica" of Bacesco, is here designated as lectotype.

\section{Balitoridae}

Loaches of the subfamily Nemacheilinae (about 400 valid species, mainly in tropical and subtropical Asia) where long classified in the family Cobitidae. Anatomical and phylogenetic studies have shown that they actually belong to the same lineage as the hill-stream loaches of Southeast Asia (Sawada, 1982). This family had been classically called Homalopteridae, but the valid name is Balitoridae (Kottelat, 1988b); this has been confirmed by a ruling of the International Commission on Zoological Nomenclature (1993).

\subsubsection{Barbatula barbatula (Linnaeus, 1758)} Cobitis barbatula Linnaeus, 1758: 303 (based on Artedi [1738: gen. [spec.] 2, syn. 2, Cobitis tota glabra...] and Linnaeus [1746: 125, n. 332, idem]; type locality: "in Europae, Asiae aquis dulcibus"; type material: NT)

Cobitis variabilis Günther, 1868: 355 (as a label in collection, name not available; locality: Moravia, Czech Republic)

Cobitis Barbatula Parisiensis De la Pylaie, 1835: 534 (nomen nudum; locality: Paris, France)

Cobitis Barbatula Pictava De la Pylaie, 1835: 534 (nomen nudum; locality: France)

Cobitis furstenbergii Bonaparte, 1846: 26 (nomen nudum; locality: eastern Germany [probably now Poland])

Cobitis Fürstenbergii Heckel \& Kner, 1858: 301 (cited in synonymy, name not available; author indicated as Fitzinger [Prodr. Faun. Austr.] which is apparently Fitzinger [1832], but the name does not appear in this work)

Cobitis barbatula var. pironae Nardo, 1866: 143 (type locality: Basso Friuli, Italy; type material NT ?)

Cobitis toni Dybowski, 1869: 957 (type locality: "common in both river systems" [rivers Onon and Ingoda, Amur basin, Siberia]; syntypes: ZISP ?, MNHN B-738 [8], MNHN B-739 [4])

Nemachilus Sturanyi Steindachner, 1892: 378, pl. 2 fig. 3 (type locality: lake Ohrid at Pestani, between Ohrid City and Naum monastery, FYROM; holotype: NMW 48440)

Nemachilus barbatulus vardarensis Karaman 1928: 164 (type locality: basin of river Vardar, FYROM; syntypes: LU); repeated in 1929: 173 Nemacheilus barbatulus aberr. erythrinna Berg, 1933: 550 (infrasubspecific, name not available)

Noemacheilus barbatulus quignardi Bacescu-Mester, 1967: 359, figs. 1, 5c-d (type locality: river Le Lez near Montpellier, France; holotype: MGAB 77)

Nemacheilus barbatulus forma anglicana BacescuMester, 1967: 369 (infrasubspecific, name not available; locality: river Cambridge, England)

Nemacheilus barbatulus forma blackiana BacescuMester, 1967: 369 (infrasubspecific name not available; locality: river Black Beck, England)

Nemacheilus barbatulus forma hispanica BacescuMester, 1967: 369, fig. 6a-b (infrasubspecific, name not available; locality: river Nervion at Durango and river Tajo, Spain)

Nemacheilus barbatulus hispanica Lelek, 1987: 256 (available by indication to Bacescu-Mester, 1967; type locality: river Nervion at Durango and river Tajo, Spain; syntypes: MGAB [11, material used by Bacescu-Mester, 1967])

Systematic notes. The status of several populations, especially in southern Europe (e.g., N. b. pironae, $N$. b. sturanyi, $N$. b. vardarensis, $N$. b. quignardi), needs a critical examination.

Nomenclatural notes. The common stone loach classically has been placed in the genus Nemacheilus Bleeker, 1863 whose type species is N. fasciatus (Valenciennes, in Cuvier \& Valenciennes, 1846) from Java and Sumatra (Kottelat, 1987, 1990a: 43). Nemacheilus includes only species from Southeast Asia. The European species has more recently been called Orthrias Jordan \& Fowler, 1903 (Bănărescu et al., 1978), but this name is a synonym of Barbatula Linck, 1789 (Kottelat, 1990a: 18) 


\subsubsection{Barbatula bureschi (Drensky, 1928)}

Nemacheilus bureschi Drensky, 1928: 160, 179, fig. 1 (type locality: river Struma, near Semen railway station, Radomir District, Bulgaria; syntypes: NMNHS [2])

Systematic notes. Treated as one of four subspecies of B. brandtii (Kessler, 1877a: 174, pl. 6 fig. 23) by Bănărescu et al. (1978: 261). Awaiting a revision of this 'polytypic' species, I tentatively recognise $B$. bureschi as a valid species. Other 'subspecies' are: B. b. brandtii from the
Arax basin in eastern Turkey and Azerbaidjan, B. b. simavika Balik \& Bănărescu (in Bănărescu et al., 1978: 261) from stream Simav near Izmir, western Turkey, and $B$. b. samantica Bănărescu \& Nalbant (in Bănărescu et al., 1978: 263) from Seyhan basin, southern central Turkey.

\subsubsection{Barbatula sp.}

Orthrias pindus Economidis, 1991: 26, p. 42 (fig. 4), 1992: 75 (nomen nudum; locality: river Aoos basin, Epirus, Greece)

\section{Ictaluridae}

\section{Ameiurus melas (Rafinesque, 1820) intro- duced}

\subsubsection{Ameiurus nebulosus (Lesueur, 1819) introduced}

Ictalurus nebulosus pannonicus Harka \& Pintér, 1990: 69 (type locality: river Tisza at Poroszló, Hungary; holotype: Mus. Nat. Sci. Budapest 87.1.1)

Systematic notes. The Hungarian introduced stock of the North American catfish Ameiurus nebulosus has been described as a distinct 'subspecies' on the claim that it is different from other introduced stocks in Europe or native ones in North America and the assumption that it might have had an hybrid origin. Beside the biological non sense of naming new taxa on the basis of introduced stocks of unknown origin, one may note that all the data on non-Hungarian stocks were based on the literature (without indication that terminology and method may be consistent), that no data are provided to support the hybrid hypothesis, and that recent North American literature on ictalurids has been largely ignored. Supposing that giving new names to introduced or captive stocks might make sense (to most systematists it certainly does not!), this should at least be based on direct examination of material of the already named, related taxa and should follow a study of the relevant literature on the wild populations of the species. For an introduction to recent literature on Ictaluridae, see, e.g., Jenkins \& Burkhead (1993: 528-569) and Lundberg (1992).

Ictalurus punctatus (Rafinesque, 1818) introduced

\section{Siluridae}

\subsubsection{Silurus aristotelis Garman, 1890}

Glanis Aristotelis Agassiz, 1856: 333 (nomen nudum; locality: river Achelous, Acarnania, Greece)

Silurus aristotelis Garman, 1890: 8 (type locality: river Achelous, Acarnania, Greece; syntypes: $\mathrm{MCZ})$

Nomenclatural notes. Glanis aristotelis Agassiz, 1856 is not available because Agassiz provided no description. Agassiz mainly said that the Greek silurid belong to a different genus than the northern European one and that he would describe it later, but he did not mention how they differ. The references (p. 332) to Aristotle cannot be considered as an indication because Aristotle did not describe the fish. Aristotle described its spawning behaviour, commented that Cordylus "has a tail like the Glanis", commented on the gill-arches 
("other [fishes] have four [gills] but in two lines, except the last, as the kichle, the perke, the glanis, the cyprinos"), that the gall-bladder is on the liver (shared with "galeodes, rhine, leiobatos, narke"), but there is no information allowing recognition of the species.

The identity of Aristotle's glanis, cordylus, kichle, perke, cyprinos, etc. is in limbo. Despite the similarity of the names with names used in modern nomenclature, none of them can be unambiguously identified. Later authors in other countries identified their local fauna as the same species as mentionned by Aristotle, but without comparison with the Greek fauna. While there is an historical and linguistic interest in identifying these animals, I doubt it serves any purpose to base today's nomenclature on Aristotle's description.

\subsubsection{Silurus glanis Linnaeus, 1758}

Silurus Glanis Linnaeus, 1758: 304 (based on Linnaeus [1751: 61, Mal], Osbeck [1756: 34, pl. 3, Silurus], Artedi [1738: gen. [spec.] 82, syn. 110, Silurus cirris 4 in mento] and Gronovius [1754: 6, n. 25, Silurus cirris duobus ...]; type locality: "in Oriente, minus frequens in Europae lacubus"; syntype: BMNH 1853.11.12:168 [1], Wheeler, 1958: 214).

Silurus silurus Wulff, 1765: 33 (type locality: "in lacu Curonico ac Bandeburgico, ... in fluviis Pregelae, Memelae \& ejusdem ostiis capiuntur", Germany; type material: NT ?)

Silurus glanis var. aralensis Kessler, 1872: 48 (type locality: Syr-Darya, Zeravshan, AmuDarya, Aral Sea basin [Berg, 1949: 904]; syntypes: ZISP ?)

\section{Esocidae}

10.1.1 Esox lucius Linnaeus, 1758

Esox Lucius Linnaeus, 1758: 314 (based on Artedi [1738: gen. 10 [53], syn. 26, spec. 52 [14], Esox rostro plagioplateo], Linnaeus [1746: 114, n. 304, idem], and Gronovius [1754: 9, n. 28, idem]; type locality: "in Europa"; syntype: BMNH 1853.11.12:114 [1], Wheeler, 1958: 209)

Luccius Vorax Rafinesque Schmaltz, 1810b: 68 (unnecessary replacement name for Esox lucius Linnaeus, 1758)

Esox Lucius var. variegatus Fitzinger, 1832: 339 (infrasubspecific, name not available; localities; river Danube and lakes Erlaph and Schwarzensee, Austria)
Esox Reichertii var. baicalensis Dybowski, 1874: 392 (type locality: all lakes and ponds of lake Baikal basin [in lake Baikal only at the mouth of the tributaries], Siberia; syntypes: IZPAN ?) Trematina foveolata Trautschold, 1884 (fossil) from Berg, 1948: 457

Esox lucius var. atrox Anikin, 1902: 109 (river Ob, Siberia) from Berg, 1948: 458

Esox lucius bergi Kaganovskii, 1933: 4 (river Anadyr, Siberia) from Berg, 1948: 458

Esox lucius lucius natio wiliunensis Kirillov, 1962: 37 (infrasubspecific, name not available; locality: river Vilyui basin, Yakutia, Siberia)

\section{Umbridae}

\subsubsection{Umbra krameri Walbaum, 1792}

Umbra Krameri Walbaum, 1792: 657 (type locality: Danube; syntypes: LU)

Aphyra lacustris Grossinger, 1794: 195 (not binominal, name not available [repeated in Hanko, 1923: 89; locality: Komitat Zemplén [Zemplin], now Slovakia [Hanko, 1923: 90])

Cyprinodon umbra Cuvier, 1829: 281 (type local- ity: "lakes of Austria, especially subterranean waters"; also available by indication to Kramer [1756: 396, Umbra]; syntypes: MNHN)

Umbra Krrameri Fitzinger, 1832: 333 (unnecessary replacement name for Cyprinodon umbra $\mathrm{Cu}-$ vier, 1829; junior homonym of Umbra krameri Walbaum, 1792)

Umbra lucifuga Gronow, 1854: 143 (available by 
indication to Kramer [1756: 396, Umbra], Marsili [1726: pl. 13, Gobius caninus] and Gronovius [1763: 114]; type locality: "in cryptis Danubii" [river Danube]; holotype: BMNH 1853.11.12:98, Wheeler, 1958: 209)

Umbra canina Károli, 1882: 188 (type locality: lake Fertö, Pest, lake Balaton, Komadi, Bodrogköz, Hungary, and Wien, Austria [from translation in Berg, 1948: 456]; syntypes: LU)
Umbra krameri pavlovi Kux \& Libosvárský, 1957: 219 (type locality: lake Kugurlui, Danube delta; syntypes: the 31 specimens measured by Pavlov, 1953)

Systematic notes. Umbra krameri pavlovi is not considered valid by Baruš \& Libosvárský (1983).

Umbra pygmaea (DeKay, 1842) introduced

\section{Osmeridae}

12.1.1 Osmerus eperlanus (Linnaeus, 1758) Salmo Eperlanus Linnaeus, 1758: 310 (based on Artedi [1738: gen. 10 [45], syn. 21, spec. 45 [10], Osmerus radiis pinnae ani 17 ...] and Linnaeus [1746: 118, n. 311, Osmerus radiis pinnae ...]; type locality: "in Europa, fluviis primo vere descendens"; syntype: LSL 45 [1], ? NRM 78 [1], Wheeler, 1985: 29)

Eperlanus Schonfoldii Rutty, 1772: 358 (work not consistently binominal, name not available (ICZN art. 11(c)); locality: Dublin, Ireland)

Salmo eperlano-marinus Bloch, 1782: 182, pl. 28 (type locality: North Sea and Baltic Sea; syntypes: ZMB 3736 [2])

Salmo Spirinchus Pallas, 1814: 387 (type locality: rivers and lakes of Germany, Russia, Ingria [now Russia], Livonia [now Estonia and Latvia], lakes Beloozero and Peipus [Chud], Russia; syntypes: LU)

Eperlanus vulgaris Gaimard, 1842: pl. 18 fig. 2 (type locality: Iceland; holotype: MNHN ?)

Osmerus eperlanus eperlanus natio ladogensis
Berg, 1932a: 133 (nomen nudum; locality: lake Ladoga, Russia)

Osmerus eperlanus eperlanus natio ladogensis Berg, 1932c: 281 (infrasubspecific, name not available; locality: lake Ladoga, Russia [Berg, 1948: 443])

Eperlanus Schonfieldii Kljukanov \& McAllister, in Hureau \& Monod, 1973: 158 (published in synonymy, name not available)

Osmerus eperlanus schonfoldi McAllister, in

Whitehead et al., 1984: 402 (type locality:

Poland westward to British Isles; syntypes: LU) Nomenclatural notes. The name Osmerus eperlanus schonfoldi is first available from McAllister (in Whitehead et al., 1984) since earlier uses of the name do not make it available. McAllister note that the two "subspecies" of $O$. eperlanus are apparently sympatric in parts of Poland, Denmark and the Baltic. If this is confirmed and if the two stocks are morphologically distinct, it seems doubtful that they should be treated as a single species.

\section{Coregonidae}

Recognition of the family Coregonidae as distinct from the Salmonidae follows Sanford (1990: 149). See Nelson (1994: 189) for alternative classifications.

\section{Coregonid systematics}

History. The history and development of coregonid systematics have been described and commented on at length in many earlier publications (e.g., Wagler, 1941, 1950; Steinmann, 1950a) and I shall not review that history here. Many data from these earlier works are valuable as they provide us with information about stocks as they were before man started to alter radically the habitats and their fish populations. Their taxonomic conclusions, however, are strongly biased by both personal and contemporary idiosyncrasy, if not by polemics (e.g., the comments by Nüsslin, 1903 on Fatio's considering as subspecies his 'good' $C$. 
macrophthalmus [this also points to a frequent semantic and psychological problem of the reductive or degrading perception of the word subspecies]).

To summarise (and simplify), the following trends or schools can be recognised in coregonid systematics during the last hundred years. Noteworthy is that no significant new alternative has been proposed in the last 50 years, with the notable exception of Svärdson (1979) for the Scandinavian species.

In central Europe, Fatio (1890) had recognised two main groups which he divided into eight species and a number of subspecies; he concentrated on the species of Switzerland and marginally dealt with those of adjacent areas. Although his classification was probably not very natural, this was the first attempt at reporting the whole diversity of the group in many different lakes ... and this was the last descriptive account with general information on the morphology of the species not restricted to a few (or a single) characters. Fatio's nomenclature is cumbersome and does not follow modern (present) rules. In addition, some confusion results from his using different names for the same species or the same name for different species in two subsequent versions of his system $(1885,1890)$ (he changed some of the earlier names which he found were not appropriate descriptors).

Berg (1932a, 1948) recognised a single species in central Europe (C. lavaretus), with several subspecies and an endless enumeration of natios and other infrasubspecific categories. His nomenclature is cumbersome and the natio concept was never really accepted outside eastern Europe (which does not mean that the importance of infraspecific variation is denied, just that it is not worth the trouble of formally naming every single population). Similarly Reshetnikov (1980: 219, 221; Dorofeyeva et al., 1985) implicitly considers that all European whitefishes belong to four species, C. albula, C. peled, C. pollan, and C. lavaretus, the latter with 6 subspecies and many "ecological forms" which can live sympatrically in the same water body. Under any species concept, two non-interbreeding, sympatric stocks cannot be conspecific.

Wagler $(1941,1950)$ concluded that there were only four species in Central Europe and he tried to have all European stocks fitting his divisions (1950: 9-10); he assumed that four species had spread across all Europe, occupying different combinations in different European lakes. It seems that he came to this conclusion quite early in his researches and I suspect that his concept of four catch-all species is strongly biased by his having started his coregonid work with lake Konstanz which hosts (hosted) four species. The main character recognised by Wagler was growth rate; stocks with similar growth rates from different lakes were squeezed in a single species, notwithstanding important morphological differences. Steinmann (1950a: 126) already commented that Wagler's four species are those known in lake Konstanz and applies poorly or not at all to other lakes. Wagler (1950: 9-10) commented: "I have called these units 'species'. One could as well choose another terminology; one could speak of species-groups, formgroups, etc. This is strictly a matter of taste and depends of the systematic value one gives to the separating properties, if one considers the small differences in the development of the characters (gill-rakers, snout shape, eye size, etc.) as more important than the general habitus and the biological behaviour (location in lake, feeding, growth, spawning habits, etc.)" [my translation]. I do not think that the names given to units is a matter of taste, but that in order to have a universal classification, concepts like species should follow a precise definition. The hierarchy of values of characters is reminiscent of the Aristotelian Logical Division mentioned in the introduction (p. 12). It is not clear also how detailed Wagler's studies of several of the stocks were: for instance, the palée of lake Neuchâtel (here C. palaea) appears under both his C. wartmanni and C. maraena (1941: 386, 394). Dottrens (1959:4) also criticised Wagler's concept; overemphasising growth patterns resulted in unnatural groupings of allopatric stocks.

Steinmann (1950a-b) also recognised a single species. In his view, its ancestors had invaded all central European lakes and had given rise within each lake or group of lakes (which he called natios; not equivalent to Berg's natios) to up to five "oecotypus". In each natio he recognised the same one to five "oecotypus" to which he consistently gave the same five names. Despite the obvious teleological bias, this was implicitly supposed to be a systematic discussion based on the BSC (Steinmann, 1951: 80); clearly, it was not. Again, the nomenclature is very cumbersome and pays no attention to the many non-interbreeding sympatric stocks which cannot be treated as conspecific.

One of the great weaknesses of most of these earlier works is that they tried to explain and describe coregonid evolution with a single and rigid pattern (Mayr's 1952 comments are still valid): Wagler with four species spreading all over Europe and diverging in the different lakes or Steinmann with a single species colonising each lake 
and evolving in situ in the same five morphs are the two extremes. The 'truth' is likely to be much more complex and the genesis of each lake community might be distinct and unique. I see no theoretical reason to exclude the possibility that sympatric speciation occurred in some lakes while allopatric speciation happened in others. Some lakes might have been colonised by the same original 2, $3, \ldots, n$ species, while some other could have been colonised (at the same or different times) by both some more ancestral stocks and some that had evolved recently in other, adjacent lakes. There is also no reason to hypothesise dispersal only from the east to the west (the collective unconscious of western Europe).

Also, it seems that Wagler and Steinmann's theories are not exempt from circular reasoning. For example, Wagler (1950: 47) discusses populations from Finland and comments on Järvi's (1943: 15) distinction of three forms based on gill-raker counts. He argues that it is known that within a single species this figure shows great variation from lake to lake, and that if one were to follow Järvi, the [various, Alpine] species should be dismantled. Then he concludes (p. 54-55): "It becomes more than likely that everywhere, in [northern Europe] as well as in the Alps, the same types $\ldots$ are present. Thus, .... the forms from the northern and from the southern distribution areas have to be put together." Järvi (1943) (as interpreted by Wagler, 1950: 54; I could not find such a statement in Järvi) followed Berg (1932) in seeing in the present distribution of the north European and Baltic maräne a reason against lumping them with the sub-Alpine forms and in considering the existing similarities of shapes as irrelevant. Wagler objected that gill-rakers are not the only character which can be used, but he actually used nothing but gill-rakers and growth in his whole discussion. Nowhere does he state clearly that he recognises confidently a sub-Alpine species in the Scandinavian material. Also revealing of the confusion between systematics (how many species ?) and nomenclatural issues (which name?) are Wagler's (1941: 382) arguments for considering a northern European C. maraena and the sub-Alpine C. fera as conspecific: the argument is not a biological one, but merely the desire for not using the name $C$. fera because he was not sure what exactly that species was.

Dottrens (1959) recognised six species, but most of his discussion is based on a single character, the number of gill-rakers. Stocks which had intermediate values were considered the result of hybridisation or introgression. Although this is cer- tainly true for some stocks, there is no reason why intermediate values should ipso facto be indicative of hybridisation; it could also be evidence of the existence of additional species. Stocks considered as conspecific on the basis of similar gill-raker counts by Dottrens can actually belong to species differing by other morphological characters (see Gasowska, 1960: 479, 1964). Finally, Dottrens's nomenclature does not follow the Code.

Wagler's nomenclature is so reductive that much information is lost while Fatio's, Berg's, Steinmann's and Dottrens's classifications are cumbersome and/or do not follow nomenclatural rules. As a result most workers preferred not to decide for one or the other and just give the name of their fish in the different systems, usually adding the name of the lake and the local name. For example the kropfer of lake Thun is called:

by Fatio (1890): Coregonus schinzii helveticus var thunensis,

by Wagler (1941): Coregonus acronius,

by Berg (1932): Coregonus lavaretus fera natio alpinus (notwithstanding misidentification),

by Steinmann (1950b): Coregonus lavaretus natio arurensis oecotypus profundus.

It is thus no wonder that most workers find more convenient to call it just by its local name or as Coregonus sp. Kropfer, lake Thun. If this is the end-result of 100 years of polemics on coregonid systematics, I doubt that it indicates significant progress.

Pragmatic approach. As previous coregonid classification do not follow the International Code of Zoological Nomenclature, express strong conceptual or teleological bias and result in ambiguous and cumbersome terminologies, they cannot be accepted and I introduce here a neutral classification which follows nomenclatural rules, does not require a priori assumptions on evolution or phylogeny and is based on intrinsic (objective) characters of the species.

As mentioned in the Introduction, one of the goals of systematics is to describe the phylogeny of a group, and this phylogeny is then reflected by the nomenclature. The proposed scenarios are very conflicting, ad hoc explanations (theories created for that particular purpose), without a strong base and the nomenclatural conclusions are often unnatural. But the present situation is so complex (and the image is definitely blurred by many introductions, transplantations, hybridisations, and extinctions) and the published data of such limited use in clarifying the phylogeny of the group, that even with proper support it would still need 
some time to present a natural phylogeny and a natural classification. As there is an obvious need for a workable nomenclature (and a set of testable hypotheses), I am thus left with no choice but to propose a pragmatic approach which temporarily conforms with the little we know of the phylogeny, the quite large amount of data we have about biology, the very poor morphological descriptions, as well as the apparent and reported uniqueness of many of the stocks.

Many workers have tried to group allopatric populations into species, often on the basis of a single character, e.g., gill-rakers (Dottrens, Svärdson), growth rate (Wagler) and stocks not fitting with their divisions were somehow forced into these species, assuming undemonstrated plasticity and environmental influence. The results are species supposedly homogenous on one character but displaying a wide variability for any other character. For a systematist this is usually a strong indication, if not evidence, of an artificial, thus unacceptable, classification. This was in part governed by the desire to avoid using "too many" names. This argument is not acceptable; if there are, let us say, 300 species, they should be recognised as such, and there is no advantage in artificially (and arbitrarily) recognising only a limited number of them. Why four, six or seven species ? Why not five, eight, seventeen or eighty-two ? As discussed p. 15, it is not the number of species which is the cause of chaotic systematics, but the absence of clear concepts.

The pragmatic way to handle the problem is not to consider the global coregonid population and wonder how to (subjectively) split it in a convenient number of species, but to (objectively) describe the diversity in using precise and workable definitions. The problem of the number of species is relatively easily solved within a single lake or group of water bodies where noninterbreeding stocks occur in sympatry (thus are distinct species). The difficulties arise in deciding whether allopatric populations are conspecific or not. I recognise as distinct those which were earlier shown to differ on the basis of morphological, life history or other characters, unless it has been demonstrated that they cannot be distinguished. There are actually very few inter-basin comparisons providing such data.

For Central Europe, within each lake oi group of lakes, I recognise each diagnosable and sympatric stock as a distinct species. Compared to earlier classifications, the result is an increase of the number of species, but this increase is compensated by abandoning the cumbersome subspecies, natios, etc. of earlier authors. One could argue that the use of subspecies associated, e.g., with Wagler's four-species system could result in a simpler system showing some affinities between the stocks of different lakes. The problems with subspecies have been discussed in the Introduction (p. 17); in addition, to group different stocks as subspecies of a single species implies that they are each other's closest relatives and derived from a common ancestor not shared by the other species. Without objective data supporting such an assumption, there is no advantage in hypothesising that a taxon $\mathrm{A}$ is more related to B than to any other. Such assumptions must be avoided as long as no objective data support them. Also, some stocks definitely do not fit in a four-species system.

It is possible (or likely) that some of the species actually occur in more than one lake or group of lakes, but I could not find an unambiguous demonstration of such a case. Therefore, at this stage, I prefer to retain geographically isolated stocks as different species because I see no advantage in postulating phylogenetic relationships between stocks [i.e. conspecificity of geographically isolated stocks] without evidence of the relationships.

This system may appear as a too fine tuning of the nomenclature, but within a given geographic area, it results in fewer entities than Berg's or Steinmann's systems, it escapes Wagler and Steinmann's teleological bias, it respects nomenclatural rules (contrary to Fatio's, Berg's, Steinmann's and Dottrens's), and it recognises sympatric, non-interbreeding and diagnosable stocks for what they are, species (as defined in Introduction, p. 15, PSC). The system escapes an implicit teleological assumption of most of these authors (except Fatio) that speciation is paralleled by morphological and adaptive divergence; we know that the two are not necessarily linked. With just binominal names, this system is more convenient to handle than the Fatio, Berg or Steinmann systems; it allows a more accurate and explicit designation of the different stocks than Wagler's system; it does not pretend to have resolved relationships: and, although not really relevant in a systematic discussion, it allows an unambiguous administrative handling which is vital for sound conservation management (e.g., Coregonus lavaretus fera natio alpinus has a great tendency in being abbreviated to C. lavaretus and become just the same 'thing' as any other ' $C$. lavaretus' subdivision; see example for lake Genève, p. 104).

I could apply these principles to almost all natural coregonid stocks known from central Europe and the British Isles; the situation in the differ- 
ent lakes will be briefly summarised under the respective headings. For Scandinavia and the Baltic Sea basin, I follow Svärdson's (1979) system as it satisfies my requirements in recognising noninterbreeding sympatric stocks as different species. Not having contradictory data, I follow his pooling of different allopatric stocks in single species, with the reservation that, as with most authors, the morphological base is very limited and somewhat restricted to gill-rakers counts. His evolutionary scenarios are ad hoc theories and they do not seem to have a phylogenetic base. Svärdson's nomenclature has been adjusted to correct some errors and for the disappearance of subspecies under the PSC. I suspect that many of his species actually include unrelated stocks and that, once usable descriptions become available, we may have to recognise more species (see Gasowska, 1960: 479, 481 for some examples, based on an earlier version of Svärdson's system).

The system used here does not aim at demonstrating any given theory of the evolution or speciation of coregonids (there is probably not a single, unifying theory, but an array of explanations which have to be found case by case for each lake or area) by uniting populations that authors think are a single species on the basis of a speculative evolutionary scenario; on the contrary it aims at recognising facts, and the only objective facts we have are species which are demonstrably different (diagnosed by their intrinsic characters).

The classification used here does not actually differ much from standard fisheries practice, where most species are often called 'Coregonus sp.' followed by the local name and the name of the lake. In any case, the present nomenclatural chaos is so confusing that it is desirable to replace the old terminology (and the implied philosophy) with a new set of names with precise and neutral meanings.

The argument that the introduction of a new system will represent a complete change and destabilisation of nomenclature is not defendable. Presently, coregonid nomenclature is all but stable. The present nomenclature is chaotic and it varies more or less randomly from one country to another. Most people using one or the other nomenclature cannot put forward arguments (relevant in a systematic context) to explain why they favour one system to another. The only objective argument is that in the absence of arguments they just follow local 'traditions'. Behnke (1972: 657 ) while recognising the distinctness of sympatric stocks commented that "for any other than salmonid fishes, full species recognition would be advised in such situation"; I do not perceive why there should be a all-but-salmonid species concept on the one hand, and a salmonid one on the other hand.

The complicated nomenclatures sometimes used actually reflect more the interest of the individual workers. Earlier researchers were often convinced that coregonids were some special animals just beginning to speciate [what is the beginning of speciation? what is the end ?], and, implicitly or explicitly, they were trying to show species appearing or disappearing in the populations they studied. Therefore some sophisticated, ad hoc theories and complicated nomenclatures appeared. These a priori conceptions made most of them unwilling or unable to realise that coregonids are just fish as other fish, whose evolution is ruled by the same principles as other groups; simply, their habitat (midwater in cold lakes) and absence of colour marks make it more difficult for the human eye and brain immediately to identify them than, e.g., colourful, benthic, territorial fishes in coral reefs or tropical lakes. But the important criteria in recognising species is not our ability to recognise them, but the fish's ability to do so. We do not know how they do, but the species have efficient mechanisms to avoid hybridisation, and unless the habitat is disturbed, they certainly do not hybridise. Actually silvery or plain-coloured (usually mid-water) fishes of lakes world-wide present the same type of systematic problems [or human difficulty to perceive species] (see, e.g., Chen, 1986; Yang \& Chu, 1987; Nagelkerke et al., 1994; Myers, 1960; Parenti, 1984; Lauzanne, 1982).

That different species are not mere ecological morphs of a single species is evinced by the extinction of single stocks by overfishing. If they were ecological morphs, there should be recruitment to compensate for the removed fishes. Similarly, after extinction of the two native stocks of lake Genève, the introduction of one species from lake Neuchâtel should have resulted in the reappearance of the two original 'ecological morphs'; this did not happen (Dottrens, 1950).

Molecular data. Published genetic data are diverse and usually relate to sympatric stocks. Most of them are presented as genetic distances based on electrophoretic data, and, as discussed in the Introduction (p. 19), are of very limited use in the present context. An outstanding exception is Bernatchez \& Dodson's (1994) analysis of mtDNA of 63 different stocks from Europe, northern America and Siberia. For the eight sub-Alpine and ten Scandinavian and Polish stocks analysed, the fol- 
lowing conclusions are of interest to systematics (see also Fig. 1). The sub-Alpine and the Scandinavian (together with some North American) stocks constitute two independent monophyletic lineages (this is especially clear from their fig. 3 which shows phenetic distances [see pp. 18, 19 for reservation on the significance of phenetic distances]). This disagrees with Svärdson's and Wagler's views that some of the sub-Alpine and Scandinavian stocks are conspecific.

Sympatric stocks from lakes Brienz and Konstanz do not cluster together, contradicting Steinmann's theory of parallel sympatric speciation. In Wagler's (1941: 390) system, the 'fall spawning' coregonid of lake Brienz (brienzlig) is conspecific with the gangfisch of lake Konstanz (C. macrophthalmus) and Bernatchez \& Dodson's data (fig. 3 , but not fig. 2) could be used to corroborate at least part of Wagler's conclusion. On the reverse, the 'winter spawning' of lake Brienz (= albock of lake Thun) and the blaufelchen of lake Konstanz which are considered as conspecific by Wagler (1941: 386, C. wartmanni) are not closely related in Bernatchez \& Dodson's clustering (but Wagler [1937: 442] considered the albock to be a 'sandfelchen', that is a C. maraena in his 1941 system). The 'spring spawning' of lake Brienz is reported as morphologically indistinguishable from the 'fall spawning' (Steinmann, 1951: 373; Kirchhofer, 1990: 57-64); among the central European stocks examined by Bernatchez \& Dodson, it does not cluster closely with any of the other stocks.

The analysed stock from lake Genève (Léman) is the introduced palée from lake Neuchâtel which Wagler (1941: 386) listed as being in part his $C$. wartmanni and again (p. 394) as his C. maraena; in 1950 (p. 12), he only recorded definitely C. macrophthalmus (bondelle) and C. maraena from lake Neuchâtel. In Bernatchez \& Dodson's figure 3 , the palée clusters with $C$. wartmanni from lake Konstanz.

Dottrens (1959) had recognised five species of Coregonus in Central Europe (conspecific with Scandinavian stocks). He considered the lavaret of lake Aiguebelette and the gangfish of lake Konstanz as conspecific; Bernatchez \& Dodson's data do not show them as each other's closest relatives; this also applies to the powan of Loch Lomond and the blaufelchen of lake Konstanz, also considered conspecific by Dottrens. In Dottrens's system, the palée is not conspecific with any of the four species of lake Konstanz. Finally, Bernatchez \& Dodson show that nucleotide variability, on average, is higher for European than for North American stocks.
Bernatchez \& Dodson suggested that all the stocks they examined should be considered as a single variable and circumpolar species. At least for the European stocks, this is definitely not acceptable because the many sympatric noninterbreeding stocks known from many lakes are different species and not just different morphs or any subdivision of a species. To use the argument that "levels of mtDNA variation ... is more representative of intraspecific variation" to conclude that a single species is involved is not acceptable because there is no theory to require that a given level equals a given taxonomic differentiation (see p. 18) (this might be a semantic problem linked with the use of words like hierarchy, level, rank and especially subspecies under the polytypic species of the $\mathrm{BSC}$; also there is a risk that this kind of reasoning quickly becomes circular). Bernatchez \& Dodson stated that "Such a genetic structure contrasts sharply with the general observation that most freshwater fishes currently exhibit the highest levels of interpopulation gene diversity reported in any animal group ... The broad scale continental and intercontinental geographic distribution of slightly differentiated phylogenetic assemblages observed in whitefish approaches the type of phylogenetic structure usually documented in species with life history conducive to dispersal and/or that occupy a range free of firm barriers to gene flow such as marine organisms, bird species and humans". In my point of view, this is an indication (if not actual evidence) that there is not a uniform tuning of 'genetic' distance and taxonomic differentiation; systematists have known for long that there is not a uniform tuning between phenetic distance and taxonomic level and a priori there is no reason that the same should not apply to any other kind of computed distance. In some groups of coregonids, obviously a quite fine tuning is needed.

Bernatchez \& Dodson concluded "This cast doubts on the validity and utility of different taxonomic designations ... based solely on morphological patterns". This may be true, but one must admit that most morphological descriptions and analysis of European coregonids are poor and too often based on a single or few characters and not acceptable for systematists as well. To me, data in Bernatchez \& Dodson is one more argument for recognising non-interbreeding sympatric stocks as distinct species, but also for recognising diagnosable stocks in different basins as distinct species. Such a classification may be artificial and temporary but it fits better with current knowledge and especially with management and conservation 


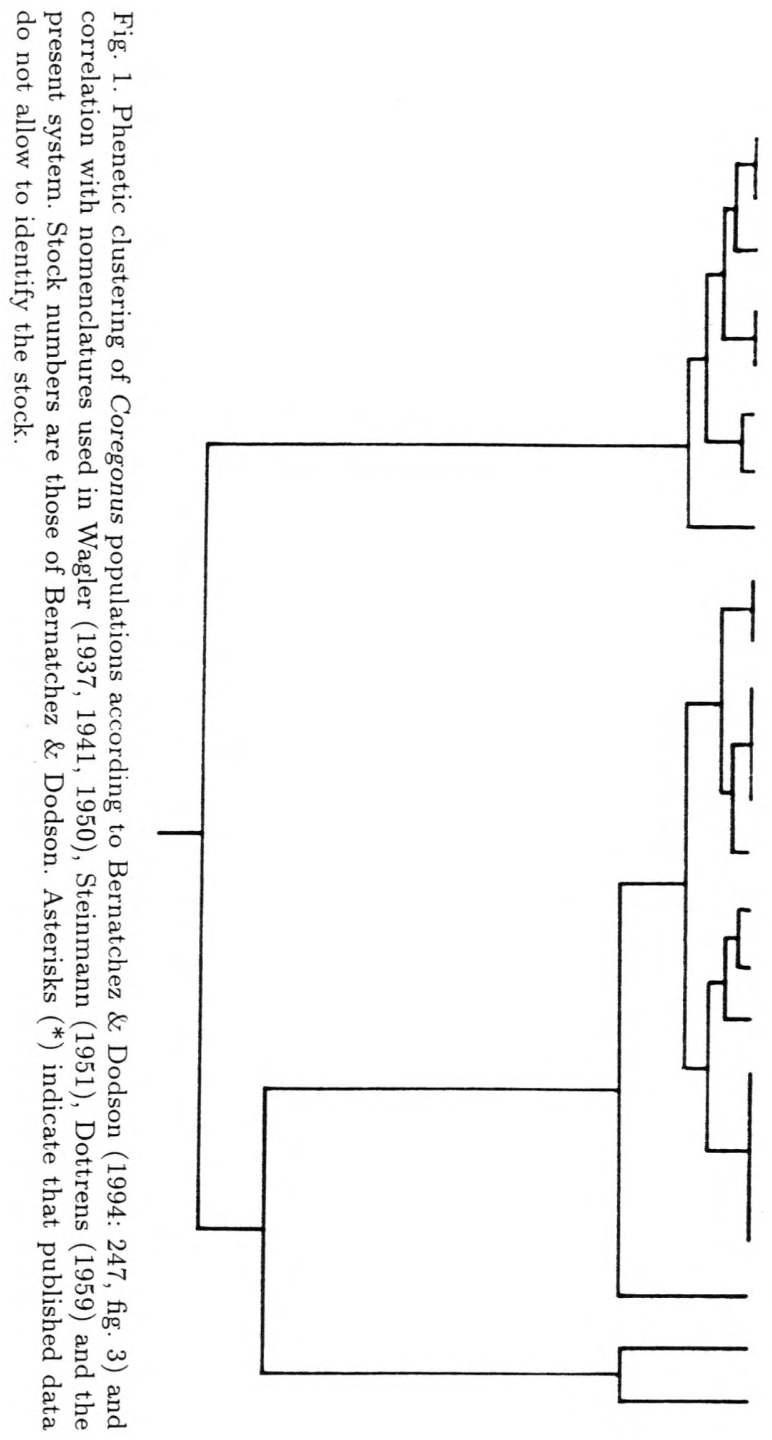

lake

Wagler (1937, 1941, 1950) Steinmenn (1951)

Dottrens (1959)

present

53 Konstanz

Konstanz
(gangtisch)

macrophthalmus

lavaretus natio bodensis

macrophthalmus

macrophtalmus

Brienz
(brienzlig)

macrophthalmus

laveretus natio arurensis

macrophthalmus

albellus

Brienz

wartmanni (1941)

fatioi

58 Aiguebelette

maraena (1937)

gvaretus natio arurensis

(lavaret)

lavaretus natio thodo

macrophthalmus

lavaretus

59 Annecy

(")

52 Konstanz

(blaufeichen)

Geneva

(palée)

oekot. pelagicus

wartmanni (1941)

wartmanni (1941: 386

maraena (1950: 394)

wartmann

lever. pologicus

oekot. primigenius

laveretus natio arurensis macrophthalmus

(spring spawning)

oekot. nanus

Kymijoki R., Finland

wartmann

('lavaretus')

Lomention

Malaxa R., Finland

('lavaretus')

62 Lapin R., Siberia

('pidschian')

63 Sos'va R. Siberia

('pidschian')

61 Kola Peninsula, K.

('lavaretus')

42 Kiiminkijoki R., Finland

Kiiminkijoki

3 Mickelsörarna, Finland

('widegreni')

44 Öja, Dinland

Kyrönjoki R., Finiand

('lavaretus')

46 ('lavaretus')

('lavaretus')

48 Dyhajarvi, Finland

('muksun')

50 Niewleno, Poland

('lavaretus')

51 Sperillen, Norway

('lavaretus')

36 Squanga, Canada

('clupeaformis')

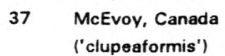


needs; it has a greater heuristic value as, at least, it provides a series of hypotheses which are explicitly stated and can be refuted or confirmed by future research.

To lump all these species into a single catchall species would not be an act of progress. The need for accurate and detailed taxonomy is best summarised by Kevan (1973: 1212): "One assumes that when [experimental biologists] state that they used $5 \mathrm{ml}$ ethanol, they were not using $6 \mathrm{ml}$ of methanol; and yet, if the experimental animal is wrongly identified, what are the grounds for such an assumption?"

Morphological changes ? Morphological changes of coregonid stocks in recent times is often alluded to in discussions and meetings, but I could not find any unambiguously documented case that this happened (it is also not clear which lakes and species are involved, or whether these were supposed to be pure, native, hybrid or introduced stocks). But as the topic shows up again and again, it seems justified to discuss it.

To be able to demonstrate that a stock has changed morphology, one would need detailed descriptions of the original stocks and well preserved voucher samples, properly labelled and curated in a suitable collection. Neither is available for most stocks.

Few or no hybrids are known from natural, undisturbed waters. On the contrary hybrids are known to be indicators of disturbed habitats (Hubbs, 1955) and the presence of Coregonus hybrids would merely indicate environmental stress (change of habitat topography, overfishing, introductions, etc.). Additionally the existence of hybrids has no value for a phylogenetic discussion, as it merely is a symplesiomorphy (see Introduction, p. 10). If the stocks are originally distinct, morphological shifts due to environmental changes (especially changes in growth rates consequent to eutrophication or different fisheries pressure) do not change the fact that the stocks are distinct. An overfed fat dog is still a dog.

Similarly, morphological changes of Coregonus stocks following transplantation in other lakes is often mentioned. Data in Dottrens (1950) and Svärdson (1979: 16-17) actually seem to show that the morphology of transplanted Coregonus populations does not change. When changes have been documented, they are apparently due to hybridisation with other (local or transplanted) species. There is apparently no published documentation of such changes; the closest seems to be Svärdson (1970: 38-41) which does not show important changes (and a description of methods and conditions of the experiment is missing). A 'classical' case of differentiation of transplanted Coregonus was reported by Thienemann (1912) from lake Laacher. The local population resulted from introductions and Thienemann assumed that it resulted from the introduction either of madümaräne from lake Madii [lake Miedwie, Poland] or fera [sandfelchen] of lake Konstanz, which had evolved into a new species since 1866-1872. Wagler (1937: 444-445; 1941: 460-462) reviewed the case and concluded that the fish if compared to the species known from lake Konstanz was more reminiscent of the gangfisch than any other species and that the introduced stock apparently was not from lake Konstanz but from some other Swiss lakes; he favoured C. candidus, the bondelle of lakes Neuchâtel and Bienne as the likely introduced stock.

Transplanted to another habitat, any species is likely to have to face somewhat different conditions and this may influence its morphological appearance. Of course these transplanted animals exist, but they should not be the basis for a discussion on systematics and phylogeny; neither should these populations be used for describing species. Systematics should be based on natural populations. Carp, goldfish and dog systematics are based on natural populations, not on transplanted or manipulated stocks. Some lake Victoria and neotropical cichlids fed different diets can develop different jaw and teeth morphology (Witte, 1984; Meyer, 1987; Wimberger, 1992), even showing close similarity to other species, but this does not affect or negate their identity as species because they still have they own separate phylogeny. Here again, what is demonstrated for one species cannot automatically be extrapolated to be valid for another species or for all fishes. Polymorphism and alternative life-history styles certainly exist but their existence can only be accepted as a fact once it is demonstrated; in systematic studies, polymorphism in one species cannot be guessed or assumed from what is known from another, related species. Also I suspect that several assumed cases of polymorphism (see Wimberger, 1994 for a review) actually involve different species.

Unless there is a serious effort by experienced systematists to investigate coregonid systematics, I predict that introductions, transplantations, hybridisations, and extinctions might quickly contribute to simplify the systematics into an ubiquitous hybrid-swarm. Although they may seem obvious, I wish to end here by repeating and stressing 
the concluding comments of Kirchhofer's (1990: 86) study of coregonids of lake Brienz. They apply to all lakes: only obviously identifiable specimens should be used for captive breeding and stocking; in any lake, no stocking should be done with stocks from other lakes. This is the only way to preserve what is left of the coregonid diversity.

\section{LAKes Genève, Bourget AND ANNeCy}

At least three species were originally present in lake Genève [river Rhône basin] and they are now assumed to be extinct (Dottrens, 1950; Spillmann, 1958). The only species now present in the lake is the introduced palée, C. palaea, first introduced from lake Neuchâtel in 1923 (Dottrens, 1950: 803). Other species have accidentally been introduced, including C. peled (?) (Gerdeaux, 1993: 21), perhaps an obvious example of management problems which can result from confusing all stocks under a single name like $C$. lavaretus.

River-spawning individuals are known to ascend the river Rhône upriver of lake Genève for a few kilometres (Büttiker, in Ruhlé \& Kindle, 1992: 407), and they would be worth a detailed study and comparison with the palée and the known museum specimens of the extinct species.

\subsubsection{Coregonus bezola Fatio, 1888} [bezoule (lake Bourget)]

Coregonus Bezola Fatio, 1888a: 1541 or 1888b: 185 (type locality: lake Bourget, France; type material: NT)

\subsubsection{Coregonus fera Jurine, 1825}

[féra (lake Genève)]

Corregonus [sic] Fera Jurine, 1825: 190, pl. 7 (type locality: lake Genève, Switzerland and France; type material: NT)

Coregonus Schinzii Fatio, 1885: 664, pl. 22 II.B (unnecessary replacement name for Coregonus fera Jurine, 1825)

Coregonus lavaretus natio rhodanensis Steinmann, 1950b: 383 (infrasubspecific, name not available; locality: lake Genève, Switzerland and France; originally included two stocks)

Systematic notes. Apparently extinct.

Nomenclatural notes. Coregonus fera Jurine, 1825 differs from Salmo ferra Walbaum, 1792 and S. muraena ferra Gmelin, 1818 (see C. wartmanni) by one letter. The names are not homonyms and C. fera is available (ICZN art. $57($ f)) .

When Fatio (1885) created C. schinzii, he divided it into three subspecies (C. s. alpinus Fatio, 1885, C. s. palaea Cuvier, 1829, C. s. fera Jurine,
1825), without using one of them as nominotypical subspecies (that is, without using for it the same name as for the species). As C. fera is the oldest name, it has priority as the name for the species (in Fatio's sense) and has to be regarded as nominotypical subspecies, making $C$. schinzii an objective junior synonym and an unnecessary replacement name.

\subsubsection{Coregonus hiemalis Jurine, 1825}

\section{[gravenche (lake Genève)]}

Corregonus [sic] hiemalis Jurine, 1825: 200, pl. 8 (type locality: lake Genève, Switzerland and France; type material: NT)

Systematic notes. Apparently extinct.

\subsubsection{Coregonus lavaretus (Linnaeus, 1758)} [lavaret (lakes Genève, Bourget and Aiguebelette)]

Salmo Lavaretus Linnaeus, 1758: 310 (based on Artedi [1738: gen. 10 [37], syn. 19, spec. 37[10], Coregonus maxilla superiore longiore pinna ...] and Gisler [1753: 195]; type locality: "in Europa"; restricted here by neotype designation to lake Bourget, France; neotype: MHNG 2583.51, present designation)

Coregonus Rondeletii Valenciennes, in Cuvier \& Valenciennes, 1848: 470 (unnecessary replacement name for Salmo lavaretus Linnaeus, 1758; name available, proposed conditionally before 1961, for the species called Lavaret by Rondelet [1555: 162, 163] and Bélon [1553: 186])

Coregonus dispersus cognatus Fatio, 1885: 660

(type locality: lake Bourget, Savoie, France; type material: NT)

Coregonus lavaretus wartmanni natio cuvieri Berg, 1932a: 123 (infrasubspecific, name not available; indication to lake Bourget specimens of $C$. lavaretus sensu Valenciennes, in Cuvier \& Valenciennes, 1848, and C. wartmanni lavaretus sensu Fatio, 1890)

Systematic notes. Spillmann (1958) reports two specimens of this species collected last century in lake Genève and preserved in MNHN. The species is now extinct in lake Genève.

Nomenclatural notes. The name lavaretus (or its variant spellings) dates back at least to Rondelet (1555: 162, Lauareto ... lacuum Allobrogum proprius est ut Burgetii \& Aequebeletii [the lavaret ... of the lakes of Savoie at Bourget and Aiguebelette]) and Bélon (1553: 186, 1555: 278). It is derived from the local name of the present species. The name formally entered the zoological nomenclature as Coregonus lavaretus Linnaeus, 1758. Linnaeus's species is based on Gisler's (1753) 
and Artedi's (1738) accounts. Artedi's species was composite, based on a description of Swedish material (1738: descr. 10) and on a long list of synonyms (1738: syn. 19), including material from Sweden, Germany (lake Ammersee), U.K. (the schelly), lakes Konstanz, Genève and Bourget (accounts by Rondelet and Bélon). None of Linnaeus or Artedi's specimens is left.

The name C. lavaretus has at some time been applied to almost any Eurasian coregonid stock and often without much respect to nomenclature rules, resulting in a great confusion. Several authors restricted its use to one or the other stock, without paying attention to or without knowledge of earlier restrictions of the type locality. In order to stabilise the nomenclature, it is important to formally link the name with a precise stock by the designation of a neotype.

It could be argued that Cuvier (1816: 163), referring to the "true lavaret of lake Bourget or of Rondelet and Bélon", restricted the name $S$. lavaretus to the species from lake Bourget, but this is not explicit because there is no reference to the composite species of Linnaeus. Valenciennes (in Cuvier \& Valenciennes, 1848: 456-461) reviewed the basis of Linnaeus's C. lavaretus, and restricted (p. 466-471) the name to a species which he considered to be the one recognised by Rondelet and Bélon under the name lavaret. To stabilise this usage, specimen MHNG 2583.51 (Fig. 2), collected on 12-13 September 1950 in lake Bourget is designated as neotype. This is part of the material described by Dottrens (1959). The neotype is 291 mm SL; head length $20.6 \%$ SL, snout length $5.6 \%$ SL, eye diameter $4.3 \%$ SL (adipose eyelid not taken into consideration), $84+5$ scales along lateral line, 13 scale rows between lateral line and dorsal-fin origin, 9 between lateral line and pelvicfin origin, $13 \frac{1}{2}$ between lateral line and ventral mid-line in front of pelvic-fin origin, $13+24=37$ gill-rakers on right anterior gill arch.

Smitt (1886: 249) had selected a marine stock from the Baltic as the 'typical form' of C. lavaretus, but this restriction was made after Valenciennes's (1848) and is not valid.

\section{Lakes Neuchâtel, Bienne, AND Morat}

Two species are presently recorded from these lakes of the Rhine basin (Dottrens \& Quartier, 1949; Rufli, 1978) but the data in Fatio (1890) seem to indicate that two more species were present in lake Morat [or Murten] which are now extinct. Fatio's material still in MHNG does not include specimens of these extinct stocks; material of this epoch exists in the collection of the Naturhistorishes Museum in Bern (K. Grossenbacher, pers. comm., 1996), but was not available at the time of writing due to ongoing building modifications; this material could allow to confirm or refute Fatio's identifications. Other Swiss museums do not have material from lake Morat in their collections.

The original hydrography and environment of the lakes has been much altered by the extensive so-called 'Jura water corrections' in 1868-78 and 1962-70 (Winistörfer, 1990: 69; Hantke, 1980: $579)$; these were large-scale hydrographic modifications in order to drain marshes and regulate water levels in lakes and rivers. The level of the lakes has been lowered by 2.4 meters and their area decreased by $30 \mathrm{~km}^{2}$.

\subsubsection{Coregonus candidus Goll, 1883} [bondelle (lakes Neuchâtel and Bienne)] Coregonus candidus Goll, 1883: 341 (type locality: lake Neuchâtel, Switzerland; neotype: MHNG 656.36, present designation)

Coregonus restrictus Bondella Fatio, 1885: 662, pl. 22 I.D.9 (type locality: lake Neuchâtel, Switzerland, by lectotype designation [originally: lakes Neuchâtel and Bienne, Switzerland]; lectotype: MHNG 656.36, present designation)

? Coregonus fera var. sancti benedicti Thienemann, 1912: 176, pl. 2 figs. 1-3, pl. 3 fig. 11 (type locality: lake Laacher, west of Andernach, Eiffel, Germany [apparently introduced from lakes Neuchâtel or Bienne; Wagler, 1937: 445]; syntypes: LU ["about 30"])

Nomenclatural notes. Goll's (1883) description of Coregonus candidus is very rudimentary; none of Goll's specimen is known to have been preserved (M. Sartori, Musée Cantonal de Zoologie, Lausanne, pers. comm., Feb. 1996) and in order to stabilise nomenclature, specimen MHNG 656.36 is designated as neotype. This specimen is also the lectotype of Coregonus restrictus bondella Fatio, 1885 and the two names are thus objective synonyms.

\subsubsection{Coregonus confusus Fatio, 1885 [pfärrig (lake Morat)]}

Coregonus annectus confusus Fatio, 1885: 661, pl. 22 I.C.5 (type locality: lakes Morat, Neuchâtel and Bienne, Switzerland; syntypes: MHNG, lost in 1902)

Systematic notes. Fatio (1885: 661; 1890: 132) recognised the pfärrig as a valid species. Steinmann (1950b: 394) considered it as the same 'eco- 

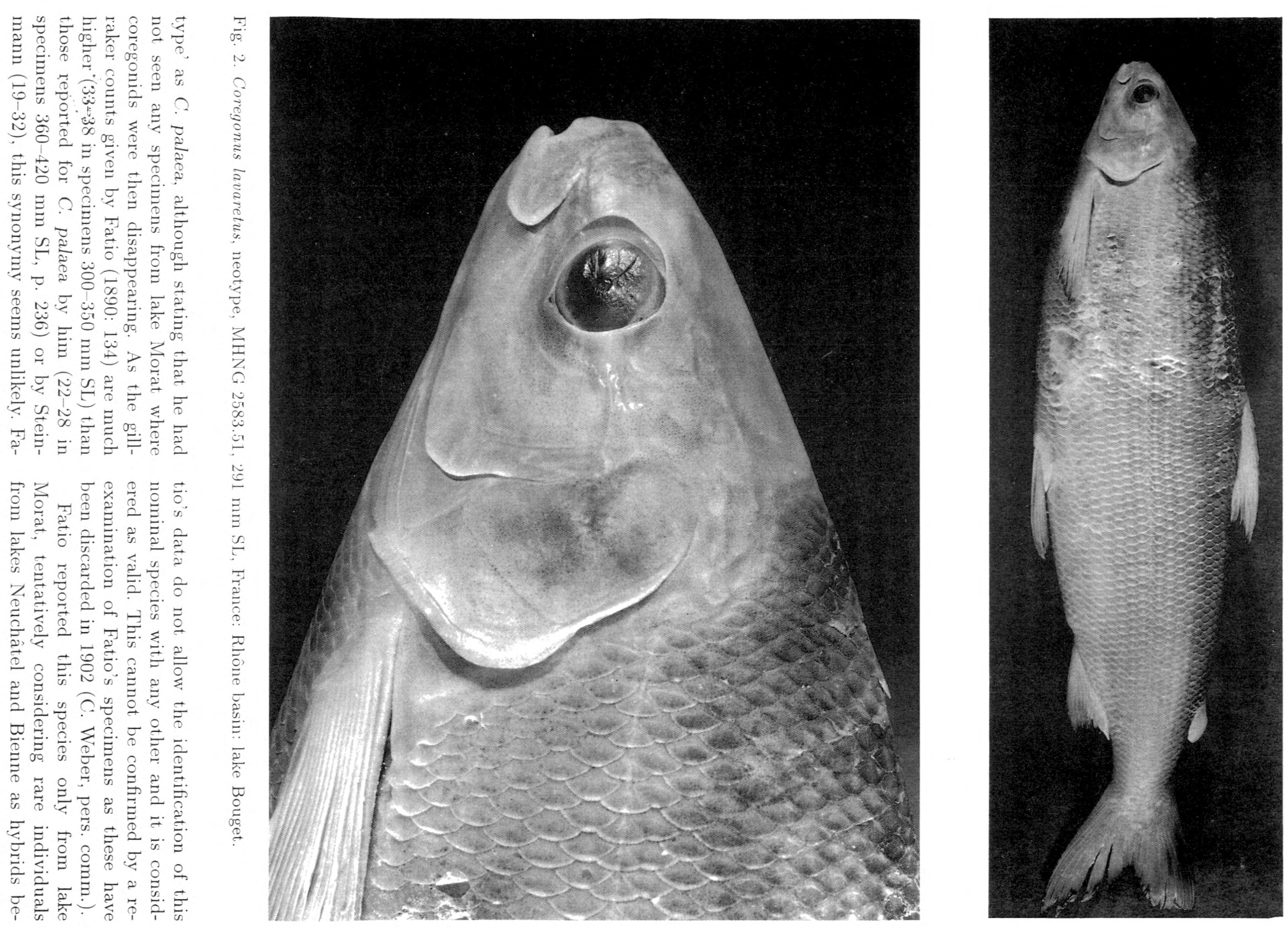
tween it and the local species. The appearance at that time of such hybrids could have resulted from the then recent (1868-78) completion of the 'correction' of the waters of lakes Neuchâtel, Bienne and Morat and associated rivers (see above). If the species is really distinct, it seems to be extinct as no coregonid survives in lake Morat (A. Kirchhofer, pers. comm.).

\subsubsection{Coregonus palaea Cuvier, 1829} [palée (lakes Neuchâtel, Bienne and Morat)]

Coregonus palaea Cuvier, 1829: 308 (type locality: lake Neuchâtel, Switzerland; syntypes: MNHN)

Coregonus lavaretus natio jurassica Steinmann, 1950b: 394 (infrasubspecific, name not available; locality: lakes Neuchâtel, Bienne and Morat, Switzerland; originally included two stocks)

Nomenclatural notes. Note correct original spelling and authorship of C. palaea.

\subsubsection{Coregonus restrictus Fatio, 1885} [férit, kropfer (lake Morat)]

Coregonus restrictus Fatio, 1885: 662, pl. 22 I.D

(type locality: lake Morat, Switzerland; type material: NT)

Coregonus dispersus Fatio, 1885: 660 (type locality: lake Morat, Switzerland; type material: NT) Coregonus restrictus Feritus Fatio, 1885: 662, pl. 22 I.D.7 (type locality: lake Morat, Switzerland; syntypes: MHNG 656.64 [2], lost in 1901)

Nomenclatural notes. Fatio (1885: 662, 1890: 185) recognised the férit as a valid subspecies. Steinmann (1950b: 394) considered it as the same 'ecotype' as bondelle, without examining specimens from lake Morat in which he stated that coregonids had almost disappeared. A re-examination of Fatio's material would be needed to confirm the existence of the morphological differences which he reported, but this material is not among Fatio's specimens preserved in MHNG and no material of this species has yet been found in other museums. There is no real argument showing that the bondelle and the férit could be conspecific and the férit is tentatively considered as a distinct species. It seems to be extinct as no coregonid survives in lake Morat (A. Kirchhofer, pers. comm.).

Nomenclatural notes. When he created the name C. dispersus, Fatio (1885) divided it into four species (C. wartmanni Bloch, 1784, C. crassirostris Fatio, 1885, C. annectus Fatio, 1885, C. restrictus Fatio, 1885) and nine subspecies. As first reviser, I restrict the name to C. restrictus. As C. restrictus and $C$. dispersus are thus simultaneous synonyms, as first reviser I retain $S$. restrictus as having priority.

When Fatio (1885) created C. restrictus, he divided it into three subspecies (C. r. feritus Fatio, 1885, C. r. nuesslini Fatio, 1885, C. r. bondella Fatio, 1885), without treating one of them as the nominotypical subspecies (that is without using for one of them the name of the species). Berg (1932a: 124), as first reviser, retained C. r. feritus has being the nominotypical subspecies, making $C$. restrictus and C.r. feritus objective simultaneous synonyms, and retaining $C$. restrictus as having priority.

\section{LAKES THUN AND BRIENZ}

Fatio (1890) recognised three species in these lakes (Rufli, 1978: 9, has it that Fatio recorded four, but I can find only three; Rufli lists different Fatio names for brienzlig and kropfer but, as shown below, Fatio (1890) used a single name for them [but obviously he had seen only one of them]). Wagler (1941) reports four species from lake Thun, but is not very explicit about them. Steinmann (1950b: 365) recognised three 'ecotypes' (primigenius, nanus, profundus) and mentioned occasional specimens which he considered as two additional 'ecotypes' (litoralis, pelagicus); it seems he was not very sure of their status, for when giving the names he added "in case it is necessary to give a name".

Rufli (1978) seems to admit that there were originally four species of coregonids in lake Thun and that two of them (bodenalbock and schwebalbock) hybridised resulting in today's 'albock'. The assumption of the earlier existence of these two 'parent' species seems to be based on Wagler (1937) and Steinmann. Steinmann (1950b) was not very clear and consistent with his terminology, even within a single paragraph; for example, p. 352 (figure 29) he lists "kropfer = bodenalböcke" and "balchen = uferalböcke", and he regularly refers to brienzlig as schwebenalbock. While p. 365-367 he named five "ecotypes", in his summary of lake Thun coregonids (p. 367-369), he recognised only three. Rufli assumed that the two kinds of albock differed by average gill-raker counts (but not range), spawning season and site. I cannot find any of these data in Steinmann's account of lake Thun (1950b: 340-369) or in Wagler (1937).

In an earlier part of the same work, Steinmann (1950a: 135) recognised three species from lake Thun (balchen, albock, kropfer) but he does not mention the brienzlig. He mentions different spawning times and places for balchen and albock. The balchen of the 1950a paper became the albock 
in the 1950b (p. 367) paper, and the albock became brienzlig, which he also called zwergalbock and schwebalbock (1950b: 366). I assume that Rufli has been confused by Steinmann's terminological inconsistency. Clear concepts, use of scientific names instead of labile and colloquial terminology and observance of the nomenclature rules would certainly have made Steinmann's discussion clearer.

It seems therefore that there have never been more than three species of Coregonus in lake Thun and that Rufli had collected all of them.

Three species are recognised from lake Brienz (Kirchhofer, 1990). They are recognised by their spawning season: winter, spring and fall. The winter spawning stock, locally known as fällchen, is morphologically distinct and is apparently conspecific with the albock of lake Thun. The spring and fall spawning stocks are morphologically indistinguishable (Kirchhofer, 1990: 57-64) but are genetically distinct (Bernatchez \& Dodson, 1994: 246,247 ). The fall spawning stock (brienzlig) apparently also occurs in lake Thun (zwergalbock of Steinmann, 1950b: 366). The spring spawning stock is apparently unique to lake Brienz; there is no name available for it.

\subsubsection{Coregonus albellus Fatio, 1890 [brienzlig (lakes Thun and Brienz)]}

Coregonus exiguus albellus Fatio, 1890: 179 (type locality: lake Thun, Switzerland, by lectotype designation [originally: lakes Thun, Brienz and Vierwaldstätter, Switzerland]; lectotype: MHNG 816.22, present designation [MHNG 676.1 , branchial arch of specimen $17 \mathrm{~cm}$, probably the lectotype])

Systematic notes. Fatio (1890: 179) is somewhat confusing about this species, because he considered as conspecific the kropfer of lake Thun and the brienzlig of lake Brienz. He also includes in it the 'weissfisch' from lake Vierwaldstätter which is assumed here to be a different species (see below, C. zugensis). Obviously his description and discussion are based mostly on material of brienzlig from lake Thun which he characterised by having 37-43 gill-rakers on the first gill arch. This agrees with the brienzlig of Steinmann which has 32-42 gill-rakers while the kropfer has 13-25. This is the albock of Dottrens (1959: 15).

\subsubsection{Coregonus alpinus Fatio, 1885} [kropfer (lake Thun)]

Coregonus schinzii alpinus Fatio, 1885: 664, pl. 22 II.B.4 (type locality: lake Thun, Switzerland; lectotype: MHNG 717.45, present designation)
Coregonus balleus Fatio, 1885: 663 (type locality: lake Thun, Switzerland; lectotype: MHNG 717.45, present designation)

Coregonus Schinzii helveticus Fatio, 1890: 222 (unnecessary replacement name for C. s. alpinus Fatio, 1890)

Coregonus Schinzii helveticus var. Thunensis Fatio, 1890: 228 (infrasubspecific, name not available; locality: lakes Thun and Brienz, Switzerland)

Coregonus lavaretus natio arurensis Steinmann, 1950b: 365 (infrasubspecific, name not available; locality: lakes Thun and Brienz, Switzerland; originally included five stocks)

Systematic notes. Fatio (1890: 227) recognised four "varieties" in his C. s. helveticus: one from lakes Thun and Brienz, one from lake Zug, one from lake Vierwaldstätter and one from lake Konstanz. His general description pools together data of the four 'varieties' and it is often difficult to sort out which are the characteristics of the individual ones. However, his variety thunensis is characterised by: mouth subterminal, eye $25 \%$ of head length (lectotype 22), 23-27 gill-rakers on first gill arch (lectotype 28), most individuals around 30$38 \mathrm{~cm}$, spawning in December, deep water.

The total length would be indicative of albock, while the eye size and gill-raker counts are more indicative of kropfer, as defined by Rufli (1978). It seems likely that either Fatio's material includes more than one species or that he partly confused the life history data, local names and specimens of some species.

Rufli (1978: 23) commented on slight differences in his gill-raker counts of the kropfer, compared to those of Steinmann (20-32, vs. 13-25) and assumed that this might be due to hybridisation. An alternative explanation is that either Steinmann consistently miscounted or that he used a different method. Steinmann's averages are four units less than Rufli's for both kropfer and brienzlig (see Rufli, pp. 23, 26) and Kirchhofer (1990: 78) also reports that Steinmann's maxima are 4 units less than his for brienzlig of lake Brienz. As a possible explanation, Rufli (p. 26) also suggested that small protuberances become 'real' rakers now that fish grow much larger than before.

Nomenclatural notes. When Fatio (1885) created $C$. balleus, he included in it three species $(C$. asperi Fatio, 1885, C. schinzii Fatio, 1885, C. acronius Rapp, 1854) and six subspecies. Coregonus schinzii is an unnecessary replacement name for C. fera Jurine, 1825 (see above). As first reviser, I designate here specimen MHNG 717.45 as lectotype of $C$. balleus Fatio, 1885. This specimen is 
also the lectotype of $C$. alpinus Fatio, 1885. They are simultaneous objective synonyms and as first reviser I retain $C$. alpinus as having priority.

\subsubsection{Coregonus fatioi Kottelat, 1997, new name}

[albock (lakes Thun and Brienz)]

Coregonus wartmanni alpinus Fatio, 1890: 140

(type locality: lake Thun, Switzerland; lectotype: MHNG 809.59, present designation [MHNG 675.96, branchial arches of a $160 \mathrm{~mm}$ specimen, probably lectotype]; junior homonym of C. schinzii alpinus Fatio, 1885)

Coregonus lavaretus wartmanni natio fatioi Berg, 1932a: 124 (replacement name for C. crassirostris nobilis d Fatio, 1885 and $C$. wartmanni alpinus Fatio, 1890; infrasubspecific, name not available)

Nomenclatural notes. This species has been described by Fatio (1890: 140) as Coregonus wartmanni alpinus. The name is not available because the name alpinus is preoccupied in the genus Coregonus by C. schinzii alpinus Fatio, 1885. Berg (1932a: 124) proposed a replacement name, but it is not an available name as it is infrasubspecific. I propose here the name $C$. fatioi as a replacement name for C. w. alpinus Fatio, 1890. The only extant syntype of C. fatioi (MHNG 809.59) is designated as lectotype.

\subsubsection{Coregonus sp. spring spawning}

Systematic notes. See general discussion of lakes Thun and Brienz above.

\section{LAKES VieRWALDSTÄtTER, Zug, SEMPACH, ETC.}

Available data do not allow to decide whether some or all of the species from this group of lakes are conspecific or not from those of lakes Thun and Brienz. Therefore they are conservatively considered as distinct. Alternatively, some of the species might be shared with lakes Zürich and Walenstadt. Three species are recognised and C. wartmanni from lake Konstanz has been introduced (Steinmann, 1950b; Svarvar \& Müller, 1982: 302).

\subsubsection{Coregonus nobilis Haack, 1882} [edelfisch (lake Vierwaldstätter)]

Coregonus nobilis Haack, 1882: 128 (type locality: lake Vierwaldstätter, Switzerland; neotype: MHNG 656.56, present designation)

Coregonus crassirostris Fatio, 1885: 661, pl. 22 I.B (unnecessary replacement name for Coregonus nobilis Haack, 1882)
Nomenclatural notes. The first use of the name C. nobilis by Haack, 1882 was a conditional ("I intended to name this fish C. nobilis, but Professor Siebold to whom I had sent a few of these fishes called them C. wartmanni, and I do not believe that I should go igainst such an authority"). Additionally the only information that we are given is that the local name of the fish is edelfisch; that it occurs in lake Vierwaldstätter and that it spawns in July and August. In the case of coregonids, the spawning season is a useful taxonomic character (as defined in the Glossary of ICZN) (if one considers that the spawning season is neither a description nor a definition in the sense of ICZN art. 12(a), Haack's C. nobilis is a nomen nudum and the next available name for this fish is $C$. nobilis Fatio, 1885; 4 of Fatio's specimens are still preserved [MHNG 807.26] and should then be treated as syntypes).

In order to stabilise nomenclature specimen MHNG 656.56 (out of MHNG 807.26) is designated as neotype of $C$. nobilis Haack, 1882. I could not find traces of Haack's specimen. He mentioned that some had been sent to Siebold; they may have been retained in ZSM and lost with the whole fish collection in 1945 (Kottelat, 1988a and unpublished data).

When Fatio (1885) created the name C. crassirostris, he divided it into two subspecies, (C. $\mathrm{cr}$. nobilis Haack, 1882, C. cr. compactus Fatio, 1885), without using one of them as nominotypical subspecies (that is without giving to one of them the name of the species). As C. nobilis is older than C. crassirostris, it has priority as the name for the species (in Fatio's sense) and has to be regarded as nominotypical subspecies, making $C$. crassirostris an objective junior synonym and an unnecessary replacement name.

Work is needed to show if this taxon is really distinct from $C$. albellus of lake Thun; if they are conspecific, the name $C$. nobilis has priority.

\subsubsection{Coregonus suidteri Fatio, 1885}

[ballen, balchen]

Coregonus annectus Fatio, 1885: 661, pl. 22 I.C (type locality: lakes Hallwyl and Baldegg, Switzerland, as restricted by Berg, 1932a: 123; type material: NT)

Coregonus annectus balleoides Fatio, 1885: 661, pl. 22 I.C.6 (type locality: lakes Hallwyl and Baldegg, Switzerland; type material: NT)

Coregonus Suidteri Fatio, 1885: 662 (type locality: lake Sempach, Switzerland; syntypes: MHNG 715.89 [1, MHNG 676.07, branchial arches of a 
specimen $330 \mathrm{~mm} \mathrm{SL}$, probably syntype 715.89], 816.26 [1])

Coregonus Schinzii helveticus var. Zugensis Fatio, 1890: 228 (infrasubspecific, name not available; locality: lake Zug, Switzerland; junior primary homonym of C. macrophthalmus zugensis Nüsslin, 1882)

Coregonus Schinzii helveticus var. Lucernensis Fatio, 1890: 229 (infrasubspecific, name not available; locality: lake Vierwaldstätter, Switzerland)

Coregonus lavaretus fera natio vogti Berg, 1932a:

129 (infrasubspecific, name not available; local-

ity: lake Zug, Switzerland; proposed as a replacement name for C. schinzii alpinus e Fatio, 1885 and C. s. helveticus var. zugensis Fatio, 1890)

Coregonus lavaretus natio riusensis Steinmann, 1950b: 397, 416 (infrasubspecific, name not available; locality: lakes Vierwaldstätter, Sarnen, Lungern, Zug, Lowerz and Aegeri, Switzerland; originally includes five stocks from six lakes)

Nomenclatural notes. Fatio (1885) described C. annectus, dividing it into two subspecies $(C$ a. confusus Fatio, 1885, C. a. balleoides Fatio, 1885), but without using one as nominotypical subspecies (that is, without giving to one of them the name of the species). He did not designate a holotype and the species is obviously composite. Berg (1932a: 123), as first reviser, restricted the name annectus to the ballen of lakes Hallwil and Baldegg, that is Fatio's C. a. balleoides. Thus C. balleoides and C. annectus are objective simultaneous synonyms; Berg (1932a: 123), as first reviser, retained C. annectus as having priority.

As the balchen of lakes Vierwaldstätter, Zug, Sempach, Baldegg, and Hallwyl are considered conspecific, then C. annectus and C. suidteri are simultaneous synonyms; as first reviser I retain $C$. suidteri as having priority.

\subsubsection{Coregonus zugensis Nüsslin, 1882} [albeli, weissfisch (lakes Vierwaldstätter and Zug)]

Coregonus macrophthalmus var. zugensis Nüsslin, 1882b: 211 (type locality: lake Zug, Switzerland; syntypes: LU)

Coregonus crassirostris compactus Fatio, 1885: 661, pl. 22 I.B.4 (type locality: lake Zug, Switzerland; syntypes: MHNG 715.93 [2])

Systematic notes. Concerning the original population of lake Zug, Steinmann (1950b: 426) commented that "Due to increased pollution, the spawning places [deeper parts of the lake] have been transformed into an oxygen-poor 'hydrogen sulphide zone' and can no longer be used. It is difficult to decide if this resulted, as the fishermen believe, in the extinction of the albeli, or if after appropriate transformation of its life style and adjusting to other spawning and habitat conditions it returned to the balchen type" [my translation]. Someway, this statement illustrates Steinmann's conception of coregonid evolution.

\section{LAKES ZÜRICH, WALENSTADT, ETC.}

In his discussion of the coregonids of lake Zürich, Steinmann (1950b: 442-448) is quite confusing, recognising two and four "ecotypes". In his discussion of the adjacent lake Walenstadt, he recognised four 'types' (one of them divided into two or three subunits; pp. 455-460) which he considered similar to the four types of lake Zürich. Formal and valid names exist only for two species, apparently present in both lakes. There are no valid names for the remaining stocks; one is Steinmann's grunder (lake Walenstadt) or blaalig (lake Zürich), the other is schwebblaalig (both lakes); I do not propose new names for them here, as their status is far from clear.

The status of these stocks, following Steinmann's identifications, has to be clarified. Steinmann's system for lake Walenstadt is reminiscent of Fatio's system (1890: 253) for lake Zürich [which Steinmann criticised vividly (1950b: 444)]. If more species actually occur in lake Zürich, some of Fatio's names listed here in the synonymy of $C$. zuerichensis might apply to them. This can only be clarified by the re-examination of Fatio's material (when extant), once the number of species and their characters are known. The hydrographic system has been greatly altered by the so-called 'correction of the Linth' waterworks between 1807 and 1822; see Steinmann (1950b: 451-454) for a description of their possible impact on fish communities and ecology of lakes Zürich and Walenstadt.

Two of the stocks recognised by Steinmann in lake Walenstadt were the sommeralbeli (spawning from July to December) and winteralbeli (spawning from December to February). Ruhlé (1986: 145) seems to imply that the winteralbeli appeared during this century; judging from Steinmann's (1951: 453, 458) account, an alternative explanation could be that it had been overlooked because of its small size, deep-water habitat, low intensity of fishing activity [especially in winter], gear selectivity and lack of scientific interest. A single stock (albeli) now exists in lake Walen- 
stadt, with a peak breeding period in OctoberNovember; some mature individuals may be found throughout the year (Ruhlé, 1988: 177). Ruhlé (1986: 151) concluded that the reproductive barriers between the two species have disappeared and that the present stock results from the introgression of the two earlier stocks. This may be true; but considering that the previous data on the stocks of lake Walenstadt are scanty and quite vague (Fatio [1890] lists a single stock in this lake; I could not find earlier accounts) and that Steinmann's (1951) data on their reproduction are apparently second-hand or anecdotal, I would not exclude the possibility that there was only one original stock.

13.1.16 Coregonus heglingus Schinz, in Cuvier, 1822

[hägling (lake Zürich), albeli, sommeralbeli, ? winteralbeli (lake Walenstadt)]

Coregonus Heglingus Schinz, in Cuvier, 1822: 275

(type locality: lake Ziirich, Switzerland; type material: NT)

\subsubsection{Coregonus zuerichensis Nüsslin,} 1882

[blaalig, bratfish (lakes Zürich and Walenstadt), albeli (lake Pfäffikon)]

Coregonus macrophthalmus var. Zürichensis Nuisslin, 1882b: 209 (type locality: lake Zürich, Switzerland; syntypes: LU)

Coregonus Sulzeri Nuisslin, 1882c: 253 (type locality: lake Pfäffikon, Switzerland; syntypes: SMNS 3125 [3], R. Fricke, in litt., NMW 78751 [1])

Coregonus Wartmanni dolosus Fatio, 1885: 660, pl. 22 I.A.2 (type locality: lakes Zürich and Walenstadt, Switzerland; syntypes: MHNG 656.53 [1], $715.92[6])$

Coregonus asperi Fatio, 1885: 663, pl. 22 II.A (unnecessary replacement name for Coregonus zuerichensis Nüsslin, 1882)

Coregonus asperi maraenoides Fatio, 1885: 663, pl. 22 II.A.1 (type locality: lake Zürich, Switzerland; syntypes: MHNG 816.42 [2])

Coregonus asperi dispar Fatio, 1885: 664, pl. 22 II.A.3 (type locality: lake Greifen, Switzerland; syntypes: MHNG 807.48 [3])

Coregonus tigurinus Fatio, 1890: 200 (listed in synonymy, name not available)

Coregonus Schinzii duplex Fatio, 1890: 252 (type locality: lake Zuirich, Switzerland; type material: NT)

Coregonus lavaretus sulzeri natio sieboldi Berg, 1932a: 130 (infrasubspecific, name not avail- able; unnecessary replacement name for C. asperi maraenoides Fatio, 1885)

Coregonus lavaretus natio intermedia Steinmann, 1950b: 438 (infrasubspecific, name not available; locality: lakes Sempach, Hallwil, Baldegg, Greifen and Pfäffikon, Switzerland; originally included one stock from five lakes)

Coregonus lavaretus natio lindimacensis Steinmann, 1950b: 448 (infrasubspecific, name not available; locality: lakes Zürich, Walenstadt and Klöntal, Switzerland; originally included five stocks from three lakes)

Systematic notes. The original populations from lakes Pfäffikon and Greiffensee are extinct (Steinmann, 1950b: 450).

Nomenclatural notes. The descriptions of $C$. $m$. zuerichensis and C. sulzeri appeared in consecutive issues of the same journal and the priority of C. $m$. zuerichensis is obvious; $C$. Zürichensis has to be emended into C. zuerichensis (ICZN art. $32(\mathrm{~d})(\mathrm{i})(2))$.

When Fatio (1885) created C. asperi, he divided it into three subspecies (C. a. sulzeri Nüsslin, 1882, C. a. maraenoides Fatio, 1885, C. a. dispar Fatio, 1885), without using one of them as nominotypical subspecies (that is, without giving to one of them the name of the species). As C. sulzeri is the oldest name, it has priority as the name for the species (in Fatio's sense) and has to be regarded as nominotypical subspecies, making $C$. asperi an objective junior synonym and an unnecessary replacement name.

\section{LAKE KONSTANZ}

Four species have classically been recognised in lake Konstanz (Wagler, 1941, 1950; Steinmann, 1950b). Nümann (1978: 519) recognises two species each with two subspecies in lake Konstanz; under any species concept, two subspecies of the same species cannot occur in sympatry. A fifth stock has recently been reported which is morphologically diagnosable from these four species and which has a distinctive biology. It undertakes spawning migrations in the Rhine in SeptemberNovember, reaching at least $45 \mathrm{~km}$ upriver of lake Konstanz (Ruhlé \& Kindle, 1992). It is tentatively recognised as a distinct (unnamed) species.

13.1.18 Coregonus arenicolus Kottelat, 1997, new species

[sandfelchen]

Coregonus Schinzii helveticus var Bodensis Fatio, 1890: 229 (infrasubspecific, name not available; 
locality: lake Konstanz, Switzerland [-GermanyAustria])

Diagnosis. Coregonus arenicolus is distinguished from the other species of Coregonus in lake Konstanz in having 18-27 gill-rakers (mean 22.3), an inferior mouth, and in inhabiting and spawning in shallow coastal areas (data from Steinmann, 1950b: 483-485, 490-491).

Holotype. EAWAG 239, 298 mm SL; Switzerland: lake Konstanz [Bodensee]; P. Steinmann, Dec. 1950.

Paratypes. EAWAG 239-B, 4, 286-345 mm SL; same data.

Description of holotype. Morphometric data (in percents of standard length): total length 119.1; head length 20.7; head depth 14.1; body depth at dorsal-fin origin 25.4; depth of caudal peduncle 8.0; length of caudal peduncle (from base of last anal-fin ray to posterior extremity of hypural complex) 12.9; eye diameter 5.6; interorbital width 6.3 ; predorsal length 48.7 ; prepelvic length 49.7; preanal length 75.5 ; length of pelvic fin 16.6; length of pectoral fin 15.2.

Dorsal fin with 3 simple and 10 branched rays, last one split to the base; anal fin with 2 simple and 10 branched rays, last one split to the base; 13 pectoral rays; 12 pelvic rays; caudal fin possibly misformed, with $6+8$ branched rays. $85+5$ scales in longitudinal row; $1 / 2261 / 2$ scales in transverse row from dorsal-fin origin to about 5 scales in front of pelvic-fin insertion; $1 / 220$ scales between dorsalfin origin and pelvic-fin origin. $10+1+15$ gill-rakers on anterior, right gill-arch. Method for counts and measurements follows Hubbs \& Lagler (1947).

Systematic notes. This is the species called sandfelchen by Steinmann (1950b: 483, 490), Wagler $(1941,1950)$ and Nümann (1978). The species is commonly referred to in the literature and appears to have no valid name; the purpose of the present action is to create a valid scientific name for it, linked with a correct holotype designation, in order to ease unambiguous discussion of the species. This does not constitute a taxonomic revision as advocated p. 20 which is still badly needed.

Nomenclatural notes. Although Fatio (1890) created two taxa with the name bodensis, no first reviser action is needed to establish priority as $C$. $s . h$. var. bodensis is not available. This is the species called sandfelchen, C. maraena or C. fera by Wagler (1941: 394). As understood here, it is restricted to lake Konstanz and as there is no available name for it, it is formally named here. The species is described in Wagler (1941: 394; 1951) and Steinmann (1950b: 483). Nümann (1978: 507) wrote that the species was becoming rare. It has sometime been considered extinct; actually it is still extant but for various reasons (mainly fishing grounds and fishing gears) it is usually not appearing in fishermen's catches (M. Douglas, pers. comm.).

Etymology. From the Latin adjective arenicolus, meaning living on or in the sand, a reference to the local name.

\subsubsection{Coregonus gutturosus (Gmelin, 1818)} [kilch]

Salmo Muraena [sic] gutturosa Gmelin, 1818: 305 (available by description and by indication to Wartmann, 1783: 431 [Kropffelche, Sandfelche] and Bloch [1784: 164, end of description of S. wartmanni]; restricted here to Kropffelche of Wartmann; type locality: lake Konstanz, Switzerland - Germany - Austria; type material: NT ?)

Salmo maraena media Hartmann, 1827: 145 (type locality: lake Konstanz at Ueberlingen, Langenargen and Konstanz, Germany; type material: NT?)

Caregonus [sic] Hartmannianus Gistel, in Gistel \& Bromme, 1850: 344 (nomen nudum).

Coregonus acronius Rapp, 1853: 158 (type locality: Langenargen and Ueberlingen, lake Konstanz, Germany; syntypes: LU)

Systematic notes. Extinct (Ruhlé \& Kindle, 1992: 403; C. Ruhlé, pers. comm. 1994; J. Hartmann, pers. comm., 1995).

Nomenclatural notes. Salmo maraena gutturosa Gmelin (1818) is available by indication to the accounts of kropffelche and sandfelche by Wartmann (1783: 431); Gmelin's account, by the mention of the small size and of the goitre clearly refers to the kropffelche, latter known as kilch. I restrict here C. gutturosus to the kropffelche of Wartmann. This is the earliest available name for the kilch.

\subsubsection{Coregonus macrophthalmus Nüsslin, 1882}

[gangfisch]

Coregonus macrophthalmus Nüsslin, 1882a: 164, fig. 2 (type locality: lake Konstanz, especially in Untersee, Germany [-Austria-Switzerland]; syntypes: SMNS 3123 [3], R. Fricke, in litt., NMW $75065[1])$

Coregonus macrophthalmus var. Steckbornensis Nüsslin, 1882b: 208 (type locality: outlet of Untersee near Steckborn, lake Konstanz, Germany; syntypes: LU)

Coregonus exiguus Klunzinger, 1884: 110 (unnec- 
essary replacement name for Coregonus macrophthalmus Nüsslin, 1882)

Coregonus restrictus Nüsslini Fatio, 1885: 662, pl. 22 I.D.8 (type locality: lake Konstanz; Switzerland [-Germany-Austria]; syntypes: MHNG $715.94[2])$

Coregonus exiguus Nusslinii var. Bodensis Fatio, 1890: 173 (infrasubspecific, name not available; locality: lake Konstanz, Switzerland [-GermanyAustria])

\subsubsection{Coregonus wartmanni (Bloch, 1784)} [blaufelchen]

Salmo Wartmanni Bloch, 1784: 161, pl. 105 (type locality: lake Konstanz, Switzerland - Germany - Austria; holotype: ZMB lost, H.-J. Paepke, in litt., 1995)

Salmo Ferra Walbaum, 1792: 76, 714 (binominal as from index, p. 714; available by description and indication to Wartmann [1777: 210, 1783: 431, Weisfelche \& Adelfisch], Bloch [1784: 164; end of description of Salmo wartmanni], Journal Helvétique A[nno ?] 1741, Gesner [1560: 341, "ein Grosse Art der Felchen ..."; includes "ferra, lavaret, bizola, wyssfisch, blawlingen"]; type locality: lake Konstanz, Switzerland - Germany Austria; lectotype [by present designation]: the holotype of Salmo wartmanni Bloch, 1784, then in ZMB, now lost, H.-J. Paepke, in litt., 1995)

Salmo Muraena [sic] Ferra Gmelin, 1818: 305 (available by description and by indication to Wartmann [1777: 210, Adelfisch, Weissfisch, Weissfelchen] and Bloch [1784: 164, end of description of $S$. wartmanni]; type locality: lake Konstanz, Switzerland - Germany - Austria; lectotype [by present designation]: the holotype of Salmo wartmanni Bloch, 1784, then in ZMB, now lost, H.-J. Paepke, in litt., 1995; a primary junior homonym of Salmo ferra Walbaum, 1792)

Coregonus Wartmanni acutirostris Fatio, 1885: 660, pl. 22 I.A.1 (type locality: lake Konstanz, Switzerland [-Germany-Austria]; type material: NT)

Coregonus Wartmanni coeruleus Fatio, 1890: 116 (unnecessary replacement name for C. w. acutirostris Fatio, 1885)

Coregonus lavaretus natio bodanensis Steinmann, 1950b: 490 (infrasubspecific, name not available; locality: lake Konstanz, Switzerland, Germany and Austria; originally included five stocks)

Nomenclatural notes. Salmo ferra Walbaum (1792) is available from the Index, p. 714. It is based on a collection of earlier literature references, most of them themselves based on earlier works. The name is potentially a senior synonym of most coregonid stocks then known. As part of the description of $S$. wartmanni by Bloch (1784) is (ambiguously ?) included in Walbaum's account I designate the holotype of $S$. wartmanni as lectotype of $S$. ferra and the two names are objective synonyms.

Salmo maraena ferra Gmelin (1818) is not available as it is a junior homonym of $S$. ferra Walbaum (1792). It is based in part on the same indication, including part of the description of $S$. wartmanni by Bloch (1794) and the holotype of $S$. wartmanni is designated as lectotype of $S$. maraena ferra and the two names are objective synonyms. The purpose of these two lectotype designations is to eliminate the risk that the overlooked name $S$. ferra becomes the valid name for another species and leads to confusion with or threatens the stability of C. fera Jurine (1825), a species from lake Genève whose name has been consistently used since. The two names differ by one letter and are therefore not homonyms [ICZN art. $57(\mathrm{f})]$.

\subsubsection{2 ? Coregonus sp. [migratory stock]} Systematic notes. Ruhlé \& Kindle (1992) report a morphologically distinct migratory species from lake Konstanz entering the Rhine for spawning. It is tentatively considered as a distinct, unnamed species. A formal description is not possible as I have not been able to obtain material It also remains to be demonstrated that no introduced stock is involved; C. maraena had been introduced in lake Konstanz at the end of the 19th century (Niimann, 1972: 835).

An other riverine stock may have existed in the Rhine near Strasbourg and Basel (Valenciennes, in Cuvier \& Valenciennes, 1848: 471), possibly the rheinschnäppel, C. nilssoni. There is apparently no recent record of the species in this area (Köhler \& Lelek, 1992: 73, 75). The Rhine coregonids cited by Fehlmann (1936) result from an introduction in Eglisau reservoir of "blaufelchen" from Rapperswil (lake Ziirich).

\section{DANUBE BASIN IN GERMANY AND AUstria}

I am treating the lakes of the upper Danube basin as a single unit, not because I am convinced or because it is demonstrated that they share the same stocks, but because they share the same lack of sound comparative data. One would expect that Wagler, who was based in München, would have reasonably treated them, but the stocks of this area are possibly those for which his data are the 
least detailed. He recognised there his 'standard' four species occurring in various combinations in the different lakes (e.g., Wagler, 1950: 18, table). Dottrens (1959: 21) noted that the samples he examined from different lakes did not exhibit the diversity reported by Wagler and he commented that some of Wagler's stocks could not be objectively recognised. Dottrens recognised four species in the material he examined, plus one species $(C$. acronius of Wagler) of which he had no material.

In lake Chiemsee, Wagler (1937: 381, 1950: 12) records three species in his table, although in the text (1950: 16) he mentions four; Dottrens (1959: 21) obtained a single species corresponding to the C. maraena of Wagler (1941: 394). He commented that the $C$. acronius of Wagler might be present but had not been obtained. A comparison of Wagler's monograph (1941) and his paper on Chiemsee coregonids (1932: 282) reveals that he did not report any data indicative that he actually had examined three species in this lake. In 1932, he mentioned that earlier authors (Vogt \& Hofer, 1909) reported only 2 species from Chiemsee (schwebrenke and bodenrenke) and that a third one (kilch) had been confused with young of schwebrenke and the paper provides data on both stocks. Data on the bodenrenke ( $C$. maraena sensu Wagler, 1941) are not provided in this paper and I could not even find an indication that Wagler had seen material of it. Actually, Vogt \& Hofer (1909: 335) wrote that bodenrenke had been introduced in 1876 but had not developed well and was rare. In 1941, Wagler did not include any information on Chiemsee kilch in his $C$. acronius account but all the data for Chiemsee C. macrophthalmus in the tables (pp. 391-394) are the same as for his 1932 (table 2) kilch. This change is apparently nowhere explained. Part of the 1941 data for the Chiemsee 'C. macrophthalmus' and ' $C$. wartmanni' in the tables (pp. 386-394) disagree with those in the key (pp. 380-382) which I suspect is based only on data of lake Konstanz stocks. The whole pattern is quite confusing; two species may be present, but I cannot find unambiguous data supporting this hypothesis and this deserves further study. Meanwhile, I am left with no choice but to follow Dottrens and tentatively recognise a single species in Chiemsee, C. hoferi. Wagler's views have also been doubted by Steinmann (1951: 64).

The same comments apply to Ammersee, except for the presence of Wagler's C. acronius. Wagler (1950: 16) recognises three species from Tegernsee and Würmsee [Starnbergersee], where Dottrens (1959: 21) sees only one, and Vogt \& Hofer (1909) two. Two species occur in both Traunsee and At- tersee (Dottrens, 1959: 20; Steinmann, 1951: 65, 66; Heckel, 1852a: 376). Altogether, I tentatively recognise five species in this area.

\subsubsection{Coregonus atterensis Kottelat, 1997, new species}

[reinanke (lakes Attersee, Mondsee, etc.)]

Coregonus lavaretus fera natio atterensis Berg, 1932a: 129 (infrasubspecific, name not available; locality: Attersee, Austria; based on Lechler, 1929a)

? Coregonus lavaretus fera natio lacus-lunae Berg, 1932a: 129 (infrasubspecific, name not available; locality: Mondsee, Austria; based on Lechler, 1929b [see also Lechler, 1930b])

Diagnosis. Coregonus atterensis occurs sympatrically in lake Attersee with C. renke (kröpfling). They are distinguished by number of gill-rakers on the first arch (21-31, mean 25.5 in C. atterensis, vs. 30-38, mean 34.6 in C. renke), spawning season and depth (from late January to mid-March, 20-40 $\mathrm{m}$ in $C$. atterensis, vs. from October to December, 80-100 $\mathrm{m}$ in C. renke), and apparently maximum size (up to $400 \mathrm{~mm}$ total length in C. atterensis, vs. 320 in C. renke) (Lechler, 1929a, 1930a).

Syntypes. 53 specimens on which Lechler's (1929a) account of reinanke is based. This material is not known to have been preserved.

Systematic notes. This is the species called reinanke by Lechler (1929a) and Vogt \& Hofer (1909: 328).

Nomenclatural notes. The two names proposed by Berg (1932a) are unavailable because infrasubspecific and a new name is proposed for these stocks which apparently represent a distinct species.

Berg's atterensis was based on the account by Lechler (1929a), whose material apparently has not been preserved. The coregonid material in NMW from lake Attersee which I have examined did not include this species and I have not been able to locate museum material of it. Recent material would be available, but considering that the lake has been stocked with fishes from other lakes, I consider that such material is not suitable for a formal description of the original stock. It has thus not been possible to examine material of the species and to write a diagnosis on the basis of this material.

The species is occasionally referred to in the literature and appears to have no valid name; the purpose of the present action is to create a valid scientific name for it in order to ease unambiguous discussion of the species. This does not constitute a taxonomic revision as advocated p. 20 which is 
still badly needed. Although not elegant, this procedure respects the provisions of the ICZN and to me is justified by the existence of an earlier, invalid name.

\subsubsection{Coregonus bavaricus Hofer, 1909} [kilch (lake Ammersee)]

Coregonus acronius var. bavarica Hofer, in Vogt \& Hofer, 1909: 332 (type locality: lake Ammersee, Germany; type material: NT)

\subsubsection{Coregonus danneri Vogt, 1908} [riedling (lake Traunsee)]

Coregonus exiguus var. danneri Vogt, in Vogt \& Hofer, 1908: pl. 14 fig. 4 (available by indication, ICZN art. 12(b)(7) [plates published in 1908, text pages 332-333 in 1909]; type locality: lake Traunsee, Austria; holotype: NT)

Systematic notes. Riedling and reinanke are sympatric in lake Traunsee. They do not seem to differ much by gill-raker counts (34-36, vs. 35-39, according to Berg, 1932a: 123), but riedling is a dwarf stock.

\subsubsection{Coregonus hoferi Berg, 1932} [schwebrenke (lake Chiemsee)]

Coregonus lavaretus hoferi Berg, 1932a: 130 (type locality: Chiemsee, Germany; available by indication to Schwebrenke, C. wartmanni var. of Vogt \& Hofer, 1909: 333; syntypes [material of Vogt \& Hofer]: LU, probably lost)

\subsubsection{Coregonus renke (Paula Schrank, $1783)$}

[kröpfling (lake Attersee), renke, reinanke (lakes Starnbergersee, Tegernsee, Traunsee, etc.)]

Salmo Renke Paula Schrank, 1783: 427 (type locality: lake Starnberg, Germany; syntypes: ZMB? )

Coregonus Reisingeri Valenciennes, in Cuvier \& Valenciennes, 1848: 496 (type locality: river Danube in Austria [mention of Wien does not mean that the specimen has been collected in Wien but that it has been sent from the museum in Wien]; actually lake Traunsee, Austria, according to Heckel, 1852a: 376; holotype: MNHN)

Coregonus steindachneri Nïsslin, 1882d: 279, fig. 7 (type locality: "lake Traunsee in Salzburg", Austria; syntypes: NMW 64818 [1])

Coregonus hiemalis var. austriaca Vogt, in Vogt \& Hofer, 1909: 330 (type locality: lake Attersee, Austria; type material: NT)

Coregonus lavaretus wartmanni natio wagleri Berg, 1932a: 124 (infrasubspecific, name not available; locality: Tegernsee, Germany; based on Wagler, 1930a)

Coregonus lavaretus wartmanni natio ammerensis Berg, 1932a: 125 (infrasubspecific, name not available; locality: Ammersee, Germany; based on Wagler, 1930b)

Systematic notes. Data on the Traunsee stock called reinanke is needed to determine its identity. Steinmann (1951: 67) counted 28-36 gill-rakers on first gill arch, but his figure are often 4 units less than those obtained by other authors (see discussion for C. alpinus, 13.1.10) and could agree with the 31-39 reported by Wagler (1930a-b) for the Ammersee and Tegernsee stocks and the 3145 by Dottrens (1959: 24) for the Starnbergersee stock (as Würmsee). Nüsslin (1882d: 280) also gave counts of 35-39 for the Traunsee stock (as C. steindachneri).

The species also called reinanke from Attersee and Mondsee, with 21-32 gill-rakers on the first gill-arch, seems to be a distinct species, called here C. atterensis.

\section{SCANDINAVIA}

For Scandinavian stocks, I largely follow Svärdson's (1979) argument and recognise ten species (including C. pidschian which seems to be known in Europe only in the White Sea basin). A problem with Svärdson's system is that the discussion is often confused by the use of too many different (usually vernacular) names for the same species and ambiguous nomenclature. More important is that his system has to rely on too many complicated scenarios which are not supported by a phylogenetic study and that he hypothesised a few ancestral species dispersing from the east and 'evolving' into the present stocks through introgressions. The status of all stocks should be revised on the basis of their intrinsic characters, not on the basis of a speculated evolution. Phylogeny has to be derived from a study of the species, not the species limits derived from a postulated phylogeny.

As stated in the family introduction (p. 100), the taxonomic resolution of Scandinavian coregonids is not as fine as for other areas, as a result of the non-availability of the needed information.

Three Scandinavian species (C. maraena, C. widegreni, C. nilssoni) also occur in eastern Europe; for clarity, their complete synonymy is listed under the present heading. 
13.1.28 Coregonus albula (Linnaeus, 1758) Salmo Albula Linnaeus, 1758: 310 (based on Artedi [1738: gen. 9 [40], syn. 13 [18], spec. 40 [9], Coregonus edentulus ...; actual material from Sweden] and Linnaeus [1746: 119, n. 313]; type locality: "in Europa"; type material: NT)

Salmo Vimba Linnaeus, 1758: 311 (based on Linnaeus [1747: 231, Wimba]; type locality: "in Waenero Sveciae"; type material: NT)

? Cyprinus muraenula Wulff, 1765: 48 (type locality: "in lacubus stagnantibus Poloniae Borussicae" [lakes of Poland and Prussia]; type material: NT)

Salmo Maraenula Bloch, 1779: 84, pl. 4 fig. 2 (type locality: Mark Brandenburg [lakes Grundlar and Wiirz near Lindow] / lake near Wandliz, Mühlenbeck / lake Liepeniz, Bernauschen Heide / Altenhof, Biesenthal / lake Werbelin, Merzischen Forst, Gute Zerlanck / lakes Dabelow and Brückentin, Badingen / near Rutenberg and Reinsberg / lake near Draz, Ruppin / lake near Mulkow, Wusterhausen / Soldin and Lippehne, Wusterhausen, Pommern [lake Lagower, Murin and Madui], Germany and Poland]; syntypes: ZMB 3753 [2])

Coregonus albula var. norvegica Günther, 1866: 193 (type locality: Norway; syntypes: BMNH [4])

Coregonus albula var. finnica Günther, 1866: 193 (type locality: Gulf of Finland; syntypes: BMNH [2])

Coregonus brevis Mäklin, 1869: 20 (type locality: Ekenäs village of Nyland [district ?] on the coast of Pojo-viken inlet of the Gulf of Finland [from Berg, 1948: 322]; syntypes: LU [2])

? Coregonus albula var. perejaslawica Polyakov, 1874: xxxi (nomen nudum; lake Pereslavskoe, Russia) from Berg, 1948: 326

? Coregonus albula natio pereslawicus Borisov, 1924: 64 (infrasubspecific, name not available; lake Pereslavskoe, Russia) from Berg, 1948: 326

? Coregonus albula olonensis Borisov, 1924: 66 (lakes Segozero and Vygozero, river Vyg basin, Karelia, Russia) from Berg, 1948: 318

? Coregonus sardinella vessicus Drjagin, 1932: 23 (lake Belozero, Karelia, Russia) from Berg, 1948: 333

? Coregonus albula pereslavicus Berg, 1932a:120 (lake Pereslavskoe, Russia) from Costa Pereira, in litt.

? Coregonus albula infrasp. ladogae Pravdin, Golubev \& Belyaeva, 1938: 216 (infrasubspecific, name not available; southern part of lake Ladoga, Russia) from Berg, 1948: 324

? Coregonus albula vodlosericus Lukash, 1939:
130 (lake Vodlozero, Karelia, Russia) from Berg, 1948: 326

? Coregonus albula infraspecies ladogensis Berg, 1948: 323 (infrasubspecific, name not available; replacement name for Coregonus albula infrasp. ladogae Pravdin et al., 1938 considered to be junior homonym of the [unavailable] Coregonus lavaretus baeri natio ladogae Pravdin, 1931)

? Coregonus albula natio topozeri Novikov, 1947: 60 (infrasubspecific, name not available; locality: lake Topozero, Karelia, Russia) from Berg, 1948: 318

Systematic notes. See Svärdson (1979: 87-88) for discussion.

\subsubsection{Coregonus maraena (Bloch, 1779)} [älvsik]

Salmo Maraena Bloch, 1779: 62, pl. 4 fig. 1 (type locality: lake Madii, Pommern [now lake Miedwie, Poland]; holotype: ZMB 3764)

Coregonus lloydii Günther, 1866: 174 (type locality: lake Wenern [Vänern], Sweden; syntypes: BMNH [10])

Coregonus lapponicus Günther, 1866: 181 (type locality: river Munio, Lapland, Sweden and Finland; syntypes: BMNH [4])

Coregonus maraena pommerana Thienemann, 1916: 98 (unnecessary replacement name for Coregonus maraena Bloch, 1782 which was split into two subspecies; C. m. pommerana is equivalent to what would now be recognised as a nominotypical subspecies, but Thienemann was avoiding tautonymies [e.g., C. maraena maraena])

Coregonus lavaretus vaetterensis Thienemann, 1921: 183, 188 (type locality: lake Vättern, Sweden; syntypes: LU)

? Coregonus lavaretus forma baltica Thienemann, 1922: 455 (type locality: river Schlei, Germany; syntypes: LU)

Coregonus holsatus forma vigrensis Lityński, 1923: 97 , pl. 6 figs. 1, 3 (type locality: lake Wigry near Suwalki, Poland; syntypes: LU)

Coregonus amnipetens Freidenfeldt, 1928: 92, fig. 2 (type locality: lake Vänern, Sweden; syntypes: LU)

Coregonus lavaretus mediospinatus Pravdin, 1931a: 189 (Neva, Gulf of Finland) from Berg, 1948: 364

Coregonus lavaretus bergi Pravdin, 1931b: 42, fig. 18 (Neva, Gulf of Finland) from Berg, 1948: 364

? Coregonus lavaretus vygensis Pravdin, 1948b (lake Vygozero, Karelia, Russia) from Berg, 1948: 1324 
? Coregonus lavaretus vygensis natio telekinae Pravdin, 1948b (infrasubspecific, name not available; lake Vygozero and river Telekina, Karelia, Russia) from Berg, 1948: 1324

Systematic notes. See (Svärdson, 1979: 86-87, as C. lavaretus) for discussion. The species is now extinct in lake Miedwie, the type locality (Heese, 1992: 493).

Nomenclatural notes. "C. aregonus Maraena" in Gistel \& Bromme (1850: 344) is a misprint for "Caregonus Maraena".

\subsubsection{Coregonus maxillaris Günther, 1866} [storsik]

Coregonus maxillaris Günther, 1866: 189 (type locality: lake Wenern [Vänern], Sweden; syntypes: BMNH [2])

Systematic notes. See Svärdson (1979: 85) for discussion.

\subsubsection{Coregonus megalops Widegren, 1863}

[blåsik]

Coregonus megalops Widegren, 1863: 589, pl. 11 fig. 15 (type locality: lake Storsjön, Indalsäven river system, Sweden; syntypes: NRM)

Coregonus pachycephalus Malmgren, 1864: 350 (type locality: lake Vänern, Sweden; available by indication to Coregonus fera from lake Vänern of Widegren, 1863: 580; syntypes: NRM ?)

Coregonus humilis Günther, 1866: 190 (type locality: lake Wenern [Vänern], Sweden; syntypes: BMNH [4])

Coregonus lavaretus lavaretoides Polyakov, 1874: xxxi (nomen nudum; lake Onega, Russia) from Berg, 1948: 377

Coregonus lavaretus forma bullarensis Malm, 1877: 547 (type locality: Bullarsjön, Sweden; syntypes: GNM)

Coregonus lavaretus forma bolmensis Malm, 1877: 546 (available by indication to Coregonus lavaretus of Nilsson, 1855: 458; type locality: lake Bolmen, Sweden; syntypes: LU)

Coregonus lavaretus forma ringsjöensis Malm, 1877: 546 (available by indication to Coregonus nilssonii of Nilsson, 1855: 460; type locality: Ringsjön, Sweden; syntypes: LU)

? Coregonus bolmeniensis Sniitt, 1883: 38 or Smitt, in Anonym, 1883: 190 (type locality: Ljusdal in Helsingland, Hammerdal in Jemtland, Bolmen, Åsnen, Yxkullsund, Willingsberg and Wenern [Vänern], Sweden; syntypes: NRM $35656[1])$

Coregonus lavaretus lavaretoides Berg, 1916: 91, fig. 84 (lake Onega, Russia) from Costa
Pereira, in litt.

Coregonus lavaretus chibinae Krogius, in Berg, 1932: 847 (type locality: lake Imandra, Russia [Berg, 1948: 412]; syntypes: LU)

Coregonus wartmanni forma borealis Järvi, 1928: 29, pl. 3 figs. $16-18$ (type locality: lake Inari at Palttoniemi and Virtaniemi, Finland; syntypes: LU [36])

Coregonus lavaretus lavaretoides natio sunensis Pravdin, 1929: 12, pls. 1-6 (infrasubspecific, name not available; river Suna, lake Onega, Russia) from Berg, 1948: 379

Coregonus lavaretus lavaretoides natio lacustris Pravdin, 1931a: 214, 227 (infrasubspecific, name not available; lakes Logmozero and Ukshezero, Surguba Bay, Russia) from Berg, 1948: 383

? Coregonus lavaretus mediospinatus natio mustasiiska Pravdin, 1948a: 76 (infrasubspecific, name not available; lake Ladoga, Russia) from Berg, 1949: 1323

Coregonus lavaretus arnoldi Pravdin, 1948b: 64, fig. 2 (lake Vygozero, Karelia, Russia) from Berg, 1949: 1324

Coregonus lavaretus lavaretoides natio schuensis Pravdin, 1949: 42 (infrasubspecific, name not available; river Shuya, lake Onega basin, Russia) from Berg, 1949: 1323

Coregonus lavaretus olonensis Pravdin, 1949: 43 (rivers Suna and Shuya, lake Onega basin, Russia; junior prinary homonym of Coregonus albula olonensis Borisov, 1924) from Berg, 1949: 1324

Coregonus lavaretus oronensis Kalashnikov, 1968: 516 [of translation] (type locality: lake Oron, right bank of river Vitim, about $554 \mathrm{~km}$ from the mouth, Siberia; syntypes: LU [46])

Systematic notes. See Svärdson (1979: 83-84) for discussion.

\subsubsection{Coregonus nilssoni Valenciennes, 1848 [planktonsik]}

Coregonus Nilssoni Valenciennes, in Cuvier \& Valenciennes, 1848: 497, pl. 631 (type locality: lake Bolmen, Småland, Sweden; Norway; syntypes: MNHN)

Coregonus generosus Peters, 1875: 791, fig. (type locality: lake Puls, near Bernstein, Neumark, Poland; syntypes: ZMB 8704 [1], 8706 [3], 8707 [3], 8709 [1])

Coregonus nilsonii manocentrus Berg, 1948: 378 (listed in synonymy, name not available)

Coregonus nilsonii pycnocentrus Berg, 1948: 378

(listed in synonymy, name not available) 
Systematic notes. See Svärdson (1979: 81-82) for discussion of part of synonymy.

Nomenclatural notes. Coregonus nilssonii manocentrus Smitt, 1886 and C. n. pycnocentrus Smitt, 1886 have occasionally appeared in the literature (e.g., Berg, 1948: 378; Fowler, 1974: 79, $81)$; manocentrus and pycnocentrus were actually intended as collective descriptors and are not available species-group names from this publication.

\subsubsection{Coregonus pallasii Valenciennes, 1848}

[aspsik]

Coregonus Pallasii Valenciennes, in Cuvier \& Valenciennes, 1848: 483 (type locality: specimens received from St. Pstersburg, Russia; syntypes: MNHN)

Coregonus aspius Smitt, 1883: 38 (type locality: Piteå Lappmark, Sweden; syntypes: NRM)

? Coregonus lavaretus multispinatus Pravdin, 1948b: fig. 3 (lake Vygozero, Karelia, Russia) from Berg, 1949: 1324

? Coregonus lavaretus multispinatus natio longiusculus Pravdin, 1948b (infrasubspecific, name not available; lake Vygozero, Karelia, Russia) from Berg, 1949: 1324

? Coregonus lavaretus multispinatus natio nasutus Pravdin, 1948b (infrasubspecific, name not available; lake Vygozero, Karelia, Russia) from Berg, 1949: 1324

? Coregonus lavaretus exiguus Pravdin, 1949: 42 (lake Syamozero and other lakes of the Shuya basin, Russia; junior primary homonym of Coregonus exiguus Klunzinger, 1884) from Berg, 1949: 1325

? Coregonus lavaretus pallasi natio exilis Pravdin, 1954: 105 (infrasubspecific, name not available; type locality: lake Onega, Russia; syntypes: LU)

Systematic notes. See Svärdson (1979: 79-81) for discussion of part of synonymy.

\subsubsection{Coregonus peled (Gmelin, 1788)}

Salmo Peled Gmelin, 1788: 1379 (based on Lepechin, 1780: 226, pl. 12; type locality: "Russia boreali"; syntype: ZMB 23555 [1], Svetovidov, 1978: 22, fig. 40)

Salmo cyprinoides Pallas, 1814: 412 (type locality: river Lena at Tungusis, Siberia; syntypes: LU)

Salmo Pelet Pallas, 1814: 412 (type locality: estuary of river Ienissei, Siberia; syntype: ZMB 23555, Svetovidov, 1978: 22, fig. 40)

Coregonus rudolphianus Valenciennes, in Cuvier \& Valenciennes, 1848: 531 (based on Coregonus pelet of Pallas, 1814: 412; type locality not stated, but river Ienissei, Siberia; holotype: ZMB 23555, Svetovidov, 1978: 22, fig. 40)

Systematic notes. The stock from lake Storvindeln is reportedly the only known natural, relict population of $C$. peled in the area covered here (Svärdson \& Filipsson, 1994). This stock was reported as C. pallasi in Svärdson (1979: 79-81). The terminology used in these papers is confusing and it is sometimes difficult to understand which is which. Awaiting a clear description and explicit statements, I tentatively follow Svärdson \& Filipsson.

Coregonus peled also occurs in European Russia in rivers Mezen and Pechora (Reshetnikov, 1980).

13.1.35 Coregonus pidschian (Gmelin, 1788) Salmo Pidschian Gmelin, 1788: 1377 (available by indication to Pallas, 1776: 705; type locality: not clearly stated, but river Ob, Siberia, is implied by statement in the description of Salmo nasus in Pallas, 1776: 705; syntypes: ZISP ?)

Salmo Polcur Pallas, 1814: 400 (type locality: from the Arctic Ocean migrates to the river Ob somewhat above Berezov, Siberia; syntypes: LU)

Coregonus sikus Cuvier, 1829: 308 (type locality: rivers of Norway; syntypes: MNHN)

? Coregonus smitti Warpachovski, 1900: 414, pl. 13 fig. 1 (type locality: lake Teletskoe, Ob basin, Siberia; syntypes: ZISP 11486 [3], 11487 [4], 11488 [6], 11489 [6])

Coregonus fera forma inarensis Järvi, 1928: 29, pl. 4 figs. 19-20 (type locality: lake Inari at mouth of rivers Juutuan and Niipi and at Virtaniemi, lake Muddus, Finland; syntypes: LU)

? Coregonus lavaretus pidschianoides Pravdin, 1931a: 232 (rivers Vyg and Kem, Karelia, Russia) from Berg, 1948: 395

? Coregonus lavaretus pidschian natio bergiellus Svetovidov, 1934: 344 (infrasubspecific, name not available; river Kara, Kara Bay, Russia)

? Coregonus lavaretus pidschianoides natio soldatovi Pravdin, in Pravdin \& Berg, 1948: 15, fig. 14 (infrasubspecific, name not available; lake Kildin, Kola basin, Russia) from Berg, 1948: 398 Nomenclatural notes. The author of C. pidschian is sometimes erroneously indicated as Pallas (1776: 705). In that publication, Pallas consistently indicated in the headings both the Latin and local names. The local names were all preceded by the name of the populations using them. Thus the heading for pidschian reads "Salmo an Lavareti varietas ? Ostiacis Pidschian. Samoiedis Polcur" [a Salmo variety related to lavaretus ? Pidschian in Ostiac language, Polcur in Samoyed language]. The name 
C. pidschian clearly is not available from Pallas (1776).

13.1.36 Coregonus trybomi Svärdson, 1979 Coregonus trybomi Svärdson, 1979: 71, figs. 3031 (type locality: lake Ören, Sweden; holotype NRM 35748)

Systematic notes. This is the C. baunti of Svärdson (1957). Coregonus sardinella baunti Mukhomediyarov (1948) is known from the lakes of the upper course of river Tsipikan, Vitim basin, Baunt, Siberia. Genetic data of Finnish stocks show that spring-spawning coregonid stocks are probably not each-other closest relative (Vuorinen et al., 1981), that is, spring spawning possibly evolved independently in different lineages.

Nomenclatural notes. Coregonus baunti Karasev, 1987 (p. 54) is an invalid primary homonym of C. sardinella baunti Mukhomediyarov, 1948.

\subsubsection{Coregonus widegreni Malmgren, 1863} [sandsik]

Coregonus Widegreni Malmgren, 1863: 52 [German translation: 1864: 325] (type locality: northern part of lake Ladoga, Finland [now in Russia]; syntypes: MZH ?)

Coregonus Baerii Kessler, 1864: 138, pl. 2 (type locality: Volkhov, Ilmen, Msta, Syas, Russia [from Berg, 1948: 365]; syntipes: ZISP ?)

Coregonus gracilis Günther, 1866: 182 (type locality: Gestrickland, Sweden; syntypes: BMNH [4])

? Coregonus tscholmugensis Danilevskii, 1873: 69 (Cholmuga Bay, lake Onega, Russia) from Berg, 1948: 377

? Coregonus ludoga Poljakov, 1874: xxx (nomen nudum; lake Ladoga, Russia) from Berg, 1948: 372

? Coregonus ludoga var. swirensis Polyakov, 1874: xxx (nomen nudum; river Svir, lake Ladoga basin, Russia) from Berg, 1948: 368

? Coregonus ludoga var. verchoswirka Polyakov, 1874: xxx (nomen nudum; lake Onega near source of river Svir and upper Svir, Russia) from Berg, 1948: 370

Coregonus ludoga var. kessleri Polyakov, 1874: xxx (unnecessary replacement name for Coregonus widegreni Malmgren, 1863) from Berg, 1948: 374

? Coregonus widegreni ludoga Berg, 1916a: 97 (in part; type locality: lake Ladoga, Russia [Berg, 1948: 372]; syntypes: LU)

Coregonus maraena holsata Thienemann, 1916: 99 (type locality: lake Selenter near Luitjenburg, Holstein, Germany; syntypes: LU)

Coregonus holsatus forma suecica Thienemann, 1921: 177 (type locality: northern part of lake Vättern near Motala, Sweden; syntypes: LU [10 heads])

Coregonus holsatus forma scallensis Thienemann, 1921: 177 (type locality: lake Schaal, Mecklenburg, Germany; syntypes: LU)

Coregonus lavaretus forma okoniensis Kulmatycki, 1927: 288, 325 (type locality: lake WielkieOkoninskie, Poland; syntypes: ? IZPAN [22])

Coregonus lavaretus forma polonica Kulmatycki, 1927: 295, 328 (type locality: Putziger Wiek [Zatoka Pucka], western part of Gulf of Danzig, Poland; syntypes: ? IZPAN [18], Gasowska, 1960: 483)

Coregonus holsatus forma anarensis Järvi, 1928: 29, pl. 4 fig. 21-23 (type locality: lake Inari at Palttoniemi, lakes Tiais and Tshuolis, Finland; syntypes: LU)

? Coregonus lavaretus baeri natio ladogae Pravdin, 1931a: 195 (infrasubspecific, name not available; lake Ladoga, Volkhov Bay, Russia) from Berg, 1948: 370

? Coregonus lavaretus baeri natio swirensis Pravdin, 1931a: 201, 204 (infrasubspecific, name not available; river Svir, lake Ladoga basin, Russia) from Berg, 1948: 368

? Coregonus lavaretus poljakovi Pravdin, 1931a: 208, 227 (lake Onega, opposite source of river Svir, Russia) from Berg, 1948: 370

? Coregonus lavaretus ludoga natio onegi Pravdin, 1931a: 208, 227 (infrasubspecific, name not available; lake Onega, Russia) from Berg, 1948: 373

? Coregonus lavaretus poljakovi natio werchoswirka Pravdin, 1931a: 212, 227, pl. 11 (infrasubspecific, name not available; lake Onega, river Svir, Russia) from Berg, 1948: 370

Coregonus lavaretus widegreni natio onegi Pravdin, 1931a: pl. 10 (infrasubspecific, name not available; lake Onega, Russia) from Berg, 1948: 376

Systematic notes. See Svärdson (1979: 85-86) for discussion.

Nomenclatural notes. The specimen considered the holotype of $C$. widegreni Malmgren, 1863 by Berg (1948: 374) cannot be the holotype as Malmgren did not explicitly state that he had a single specimen. From his text, it seems that he examined more than one specimen and they are thus syntypes. 


\section{Poland, Northern Germany, Denmark, Netherlands, Belgium}

Six species occur in this area. The synonymy of C. maraena, C. widegreni, C. nilssoni and C. albula have already been listed under Scandinavia, where these species also occur. The synonymies follow mostly discussion in Svärdson (1979) which is sometime confusing (e.g., p. 51, 82, his discussion of $C$. lavaretus baltica is ambiguous and it is not clear if he means it is the same as his $C$. nilssoni, C. lavaretus, C. oxyrinchus or a distinct species).

Coregonus albula (Linnaeus, 1758)

[kleine maräne]

See above under Scandinavia, 13.1.28

\subsubsection{Coregonus lucinensis Thienemann, 1933}

[quietschbiikers (lake Lucin)]

Coregonus albula lucinensis Thienemann, 1933:

655, figs. 1-2 (type locality: lake Breiten Lu-

cin, Mecklenburg-Strelitz. Germany; syntypes: LU [72])

Systematic notes. Thienemann (1933) described two sympatric stocks from lake Breiten Lucin which are morphologically distinct and do not interbreed; they are thus two distinct species. One is the widespread C. albula and the other, C. lucinensis, a small deep-water and big-eyed species apparently endemic to three small lakes of Mecklenburg, northern Germany. Svärdson (1979: 88) considered it as possibly conspecific with $C$. vandesius, but this is apparently based on zoogeographic or evolutionary speculations only as he provides no taxonomically relevant data.

\section{Coregonus maraena (Bloch, 1779)}

[maräne]

See above under Scandinavia, 13.1.29

\section{Coregonus nilssoni Valenciennes, 1848}

[rheinschnäppel, edelmaräne]

See above under Scandinavia, 13.1.32

\subsubsection{Coregonus oxyrinchus (Linnaeus, 1758)}

[houting, nordseeschnäppel]

Salmo Oxyrinchus Linnaeus, 1758: 311 (based on Artedi [1738: gen. [spec.] 10, syn. 21, Coregonus maxilla superiore longiore conica...; material from "Flandria, Batavia"], Gronovius [1754: 18, n. 48, idem; material from "Mare Septentrionale"]; type locality: "in Oceano Atlantico"; syntype: BMNH 1853.11.12:160 [1], Wheeler, 1958: 208)

Salmo Thymallus latus Bloch, 1782: 170, pl. 26 (type locality: Danzig, Pommern [Poland] / Hamburg, Germany / Denmark / Sweden; holotype: ZMB 33749 [although he lists many localities, Bloch apparently had a single specimen, without locality information; H.-J. Paepke, in litt., 1995])

Tripteronotus hautin La Cepède, 1803: 48 (type locality: Anvers, Belgium [based on Rondelet, 1555: 195, oxyrynchis piscibus]; type material: NT)

Salmo Rostratus Shaw, 1804: vol. 5 (1): 86 (unnecessary replacement name for Salmo thymallus latus Bloch, 1782)

\section{Coregonus widegreni Malmgren, 1863}

[Selenter-maräne, Schalsee-maräne]

See above under Scandinavia, 13.1.37

\section{BRITISH ISLES}

The latest revision based on direct examination of material of all stocks from the British Isles is by Regan (1908). Subsequent studies have been based on some of the populations only and are very contradictory (see Maitland, 1970 for a brief summary and distribution data; Beaumont et al., 1995, for genetic data of some species; species accounts below for some of the conclusions).

\subsubsection{Coregonus clupeoides La Cepède,} 1803

\section{[powan (Loch Lomond)]}

Coregonus clupeoïdes La Cepède, 1803: 698 (type locality: island Inchtonachon, Lochlomoud [Loch Lomond], Scotland; syntypes: LU)

Coregonus Lacepedei Parnell, 1838a: 162, pl. 6 (type locality: Loch Lomond, Scotland, U.K.; syntypes: LU)

Coregonus microcephalus Parnell, 1838a: 163 (type locality: Loch Lomond, Scotland, U.K.; syntypes: LU)

Coregonus Cepedii Valenciennes, in Cuvier \& Valenciennes, 1848: 503 (unjustified emendation of Coregonus lacepedei Parnell, 1838)

Systematic notes. Svärdson (1979: 83-84) considered the powan as a subspecies of the Scandinavian C. megalops on the basis of its assumed phylogeny (see general comment under introduction to Scandinavian coregonids, p. 115).

Gasowska (1965) examined the powan from Loch Lomond (C. clupeoides), the gwyniad of Llyn Tegid (C. pennantii) and the schelly of Haweswa- 
ter and Ullswater (C. stigmaticus). She concluded that they all belong to a polytypic Coregonus lavaretus sensu lato (sensu Berg, 1948; see Coregonus introduction, p. 97). She concluded that the powan and the gwyniad were morphologically distinct and should be recognised as subspecies, but did not reach any conclusion about the schelly. The gwyniad and the powan are morphologically distinct and satisfy the definition of the species as understood here. The status of the schelly is still not clear and I tentatively treat it as valid species, C. stigmaticus. Beaumont et al. (1995: 887) report that the gwyniad from Llyn Tegid is genetically "quite distinct ... because they exhibit unique alleles at quite high frequencies".

Ferguson (1974) considered C. clupeoides, C. pennantii and C. stigmaticus as conspecific on the base of their genetic similarity. As discussed in the Introduction (p. 19) and under Alosa alosa (4.1.3), there is no theory to demand that species have to differ electrophoretically or that stocks not distinguishable electrophoretically are conspecific.

\subsubsection{Coregonus pennantii Valenciennes, 1848}

\section{[gwyniad (Llyn Tegid, Bala)]}

Coregonus Pennantii Valenciennes, in Cuvier \& Valenciennes, 1848: 507 (based on Willughby [1686: 183, Guiniad, "in lacu Pimblemeer ... proprie oppidum Bala in comitatu Merionethensi Walliae, non autem in fluvio Dea qui eum perfluit"], Pennant [1769: vol. 3: 267, pl. 16) and Yarrell [1836: vol. 2: 85, Gwyniad, Llyn Tegid (Fair Lake) at Bala]; type locality: "lac de Fer" [Fair Lake], Llyn-Tegid, Wales, U.K.; type material: NT ? [material of these authors])

Systematic notes. Gasowska (1965) commented on Dottrens's (1959: 30) record of the presence of two species in Llyn Tegid that only one species is present in her material and that the other is only known from old museum specimens. She concluded that this second species might have been an introduced stock which became extinct since, but did not provide evidence for it; neither did she consider the possibility of a second native species which either is extinct or has not been seen by her. More information is needed on this before reaching a conclusion.

Svärdson (1979: 81) considered that C. pennantii "seems" to be the same as C. nilssoni "judging by the gill-raker counts, around 38". In the absence of more substantial information and comparison, they are considered distinct. If C. nilssoni and C. pennanti were considered conspecific, they would be simultaneous synonyms and $C$. nilssoni would have priority by decision of the first reviser (Svärdson, 1979: 82).

\subsubsection{Coregonus pollan Thompson, 1835 [pollan]}

Coregonus Pollan Thompson, 1835: 78 (type locality: Lough Neagh, Ireland; syntypes: BMNH ?)

Coregonus elegans Thompson, 1839: 422, pl. 16 fig. 5 (type locality: river Shannon near Killaloelakes, Ireland; syntypes [total 2]: BELUM [1], Regan, 1908b: 487)

Coregonus altior Regan, 1908b: 486 (type locality: Lough Erne, Fermanagh, Ireland; syntypes: BMNH [14], BELUM [2])

Systematic notes. Ferguson et al. (1978) and Svärdson $(1979: 6,81)$ considered this species as conspecific with the Arctic C. autumnalis (Pallas, 1776) (p. 705; type locality: rivers Petchora and Ienissei, river Angara and lake Baikal, river Tubam and lake Madsharem, Siberia; includes probably several species). Bodaly et al. (1991: 37) considered that $C$. autumnalis [from Siberia and Canada], C. artedii [from Canada] and C. pollan "appear to be conspecific on the basis of genetic distances" obtained by electrophoretic studies. The non-significance of such distances for deciding systematic status or species distinctness has been discussed above (pp. 18, 19, 101).

Ferguson et al. (1978) found no electrophoretic difference between the two stocks. They considered this to "confirm Behnke's (1972) view that [the two] are the same species and merit, at most, sub-specific separation" (p. 228). Behnke (1972: 664) effectively stated that he could find no difference, but I could not find actual data or information on the examined material. In this context, the statement "I would consider the Irish population as no more than a subspecies of C. autumnalis" is somewhat puzzling. Similarly, Ferguson et al. (1978: 230) considered that "since the name pollan is well established in the literature and since the Irish populations show a number of structural and behavioural differences from the Arctic populations it is probably advantageous to regard pollan as a subspecies of $C$. autumnalis". Either the two stocks are identical (then I see no point at distinguishing subspecies, especially on the ground that a name is established in the literature) or they can be diagnosed ["structural and behavioural differences" ?] and as they obviously are different lineages, they should be considered species under the PSC. In the absence of data demonstrating that a single species is involved, I see no advantage in considering the land-locked Irish stocks conspe- 
cific with the anadromous C. autumnalis of the Arctic Ocean in Siberia, Alaska and north-eastern Canada. Electrophoretic identity in some alleles is not in itself evidence of conspecificity. Zoogeographic hypotheses or speculations (Behnke, 1972: 664; Ferguson et al., 1978: 229) should follow a phylogenetic construction but cannot be used as argument for reaching a taxonomic conclusion.

There are two more reasons to consider that this synonymy is premature or unjustified with the available data. First the $C$. autumnalis stock used for comparison is a hatchery stock of unstated origin ["USSR"] said to be C. autumnalis, but the source or reference for identification is not stated. Second, "there is evidence of the existence of several partially isolated populations in Lough Neagh" (Ferguson, 1974: 314); the status of these should be elucidated first. It might be that several species are involved. The formal synonymy of $C$. pollan and $C$. autumnalis requires first an unambiguous identification of both stocks.

Regan (1911b: 120) considers that C. pollan, C. altior and $C$. elegans are distinct; Dottrens (1959: 57 ) considered the stocks of Lough Neagh (C. pollan) and Lough Erne (C. altior) as representing two different species. See also Gasowska (1964).

\subsubsection{Coregonus stigmaticus Regan, 1908} [schelly (Haweswater and Ullswater)]

Coregonus stigmaticus Regan, 1908b: 489 (type locality: Haweswater, Cumberland, and Ullswater, Westmoreland, U.K.; syntypes: BMNH [12])

\subsubsection{Coregonus vandesius Richardson, 1836}

\section{[vendace]}

Coregonus vandesius Richardson, 1836: 213 (type locality: Salway Firth, Lochmaben and Castle Loch, Scotland, U.K.; syntypes: LU)

Coregonus Willughbeii Jardine, 1840: pl. 6 (type locality: Castle Loch, Lochmaben, Dumfrieshire, Scotland, U.K.]; syntypes: ? BMNH, Günther, 1866: 194)

Coregonus gracilior Regan, 1906: 181, pl. 7 (type locality: lake Derwentwater, Cumbria, U.K.; syntypes: BMNH [1], Keswick Museum [4])

Systematic notes. Ferguson et al. (1978) considered C. vandesius as conspecific with $C$. albula, a point of view not shared by Svärdson (1979: 88). Bodaly et al. (1991: 37) considered that C. albula (including C. vandesius) and C. sardinella [from Siberia, Alaska and Canada] "appears to be conspecific" on the basis of genetic distances obtained by electrophoretic studies. The non-significance of such distances for deciding systematic status or species distinctness has been discussed above (pp. 18, 19, 101).

The author of $C$. willughbii is often erroneously indicated as Jardine (1831). Regan (1911b: 117) considers C. vandesius and C. gracilior as distinct species.

\subsubsection{Names of unclear status:}

The status of the following names which appear in the literature cannot be elucidated at present:

Coregonus lavaretus microps Smitt, 1883: 39 or Smitt, in Anonym, 1883: 191 (type locality: lake Wenern [Vänern] [restricted by Berg, 1932a: 127]; syntypes: NRM 35647-35652 [6], 35662 [2], 35687 [1], 35688 [2])

Coregonus lavaretus microcephalus Smitt, 1883: 39 or Smitt, in Anonym, 1883: 192 (type locality: Ångermanelfven, Sweden; syntypes: NRM 35659 [1], 35660 [1]; junior homonym of C. microcephalus Parnell, 1838)

Coregonus maraena maraenoides Polyakov, 1874: xxxi (nomen nudum) from Berg, 1948: 385

Coregonus nordmanni Mela, 1882: 350, fig. 200 (type locality: northern part of lake Ladoga, Finland [now Russia]; holotype: LU ?)

Coregonus maraena maraenoides Berg, 1916a: 94, fig. 86 (type locality: lakes Chud [Peipus] and Pskov, Estonia and Russia [Berg, 1948: 385]; syntypes: ZISP ?; junior primary homonym of C. asperi maraenoides Fatio, 1885)

Coregonus lavaretus forma norvegica Thienemann, 1922: 458 (type locality: lake Mjören and its outlet, stream Vormen, Norway; syntypes: LU; restricted to the form spawning in December and January by Berg, 1932a: 128)

Coregonus lavaretus forma lapponica Järvi, 1928: 37, pl. 3 fig. 15 (type locality: lake Vuontis, Finland; syntypes: LU; junior homonym of C. lapponicus Günther, 1866)

Coregonus lavaretus gruppe orientalis Kulmatycki, 1928: 44 (infrasubspecific, name not available; locality: lakes Wielkie-Gluche, WielkieTuczno and Gorynskie, Poland)

Coregonus lavaretus gruppe pulsensis Kulmatycki, 1928: 44 (infrasubspecific, name not available; locality: lakes Puls and Raakow, Poland; based on data in Thienemann, 1928])

Coregonus lavaretus gruppe danica Kulmatycki, 1928: 44 (infrasubspecific, name not available; locality: lakes: Tjele Langsö, Glenstrup Sö, Klejtrup Sö and Nipsaa, Denmark; based on Otterstrøm, 1922])

Coregonus lavaretus holsatus forma karelica Pravdin, 1931a: 221, 226, pl. 12 (infrasubspecific, name not available; lakes Pyalozero and 
Syamozero, Karelia, Russia) from Berg, 1948 383

Coregonus lavaretus prawdini Berg, 1932a: 125 (replacement name for Coregonus lavaretus forma lapponica Järvi, 1928; type locality: lake Vuontis, Finland; syntypes: LU)

Coregonus lavaretus järvii Berg, 1932a: 126 (replacement name for C. wartmanni sensu Järvi, 1928; type locality: lakes Leppävesi, Lievestuore and Keitele, Finland; syntypes: LU)

Coregonus lavaretus angermanensis Berg, 1932a: 127 (replacement name for C. microcephalus Smitt, 1883; type locality: Ångermanelfven, Sweden; syntypes: NRM)

Coregonus lavaretus norvegicus natio thienemanni Berg, 1932a: 129 (infrasubspecific, name not available; locality: lake Mjören and river Vormen, Norway; reference to the SeptemberNovember spawning form of C. lavaretus norvegicus Thienemann, 1922)

Coregonus lavaretus imandrae Krogius, in Berg, 1933: 847 (type locality: lake Imandra, Murmansk, Russia [Berg, 1948: 389]; syntypes: LU)

Coregonus lavaretus imandrae infrasubspecies pelagicus Krogius, in Berg, 1933: 847 (infrasubspecific, name not available; lake Imandra, Murmansk, Russia [Berg, 1948: 389])

Coregonus lavaretus imandrae infrasubspecies knipowitschi Krogius, in Berg, 1933: 847 (infrasubspecific, name not available; lake Imandra, Murmansk, Russia [Berg, 1948: 389])

Coregonus lavaretus imandrae natio umbrae Krogius, in Berg, 1933: 847 (infrasubspecific, name not available; lake Umbozero, Murmansk, Russia [Berg, 1948: 390])

Coregonus lavaretus imandrae natio umbrae infranatio pelagicola Krogius, in Berg, 1933: 847 (infrasubspecific, name not available; lake Umbozero, Murmansk, Russia [Berg, 1948: 390])

Coregonus lavaretus vimbaeformis Pravdin, in Pravdin \& Berg, 1948: 11, fig. 8 (lake Vuljavr, Ponoi river basin, Kola, Russia) from Berg, 1948: 390

Coregonus lavaretus pidschian natio lovensis Pravdin, in Pravdin \& Berg, 1948: 12, fig. 9 (infra- subspecific, name not available; lake Lovozero, Kola, Russia) from Berg, 1948: 391 and Costa Pereira, in litt.

Coregonus lavaretus lavaretoides natio voronjensis Pravdin, in Pravdin \& Berg, 1948: 12, fig. 10 (infrasubspecific, name not available; basin of lake Lovozero, Kola, Russia) from Berg, 1948: 392 and Costa Pereira, in litt.

Coregonus lavaretus bergianus Pravdin, in Pravdin \& Berg, 1948: 12, fig. 11 (lake Ondomozero, Kola, Russia) from Berg, 1948: 390

Coregonus lavaretus lovensis Berg, 1948: 391, fig. 229 (type locality: lake Lovozero, Kola, Russia; syntypes: ZISP ?)

Coregonus lavaretus voronjensis Berg, 1948: 392, fig. 230 (type locality: basin of lake Lovozero, Kola, Russia; syntypes: ZISP)

Coregonus lavaretus neschka Pravdin, 1950: 74 (lake Segozero, Russia) from Costa Pereira, in litt.

Coregonus lavaretus palloni Pravdin, 1950: 75 (lake Segozero, Russia) from Costa Pereira, in litt.

Coregonus lavaretus kilone Pravdin, 1950: 77 (lake Segozero, Russia) from Costa Pereira, in litt.

Coregonus lavaretus melga Pravdin, 1950: 77 (lake Segozero, Russia) from Costa Pereira, in litt.

Coregonus tapinorhynchus Fowler, 1974: 79 (listed in synonymy, name not available; refers to tapinorhynchi Smitt, 1895: 900 which was not intended as a specific name)

Coregonus hypselorhynchus Fowler, 1974: 79 (listed in synonymy, name not available; refers to hypselorhynchi Smitt, 1895: 902 which was not intended as a specific name)

Coregonus stenorhynchus Fowler, 1974: 79 (listed in synonymy, name not available; refers to stenorhynchi Smitt, 1895: 902 which was not intended as a specific name)

Coregonus euryrhynchus Fowler, 1974: 79 (listed in synonymy, name not available; refers to euryrhynchi Smitt, 1895: 902 which was not intended as a specific name) 


\section{Salmonidae}

14.1.1 Acantholingua ohridana (Steindachner, 1892)

Salmo ohridanus Steindachner, 1892: 379, pl. 3 figs. 1-3 (type locality: lake Ohrid, FYROM; syntypes: NMW 58154 [1], 65592-603 [31], 65611 [1], 66021 [1], 66447 [2])

Systematic notes. Systematic position follows Stearley \& Smith (1993: 14). Eschmeyer (1990: 10) considered that Acantholingua is not available from Hadzisce (1960: 47, 1961: 790) because two species are included and none is designated as type species (ICZN art. 13(b)). Actually, in both papers, Hadzisce explicitly included a single species (A. ohridana) in Acantholingua; it is type species by monotypy (ICZN art. 68(d)) and the name is available from Hadzisce (1960).

\subsubsection{Hucho hucho (Linnaeus, 1758)}

Salmo Hucho Linnaeus, 1758: 309 (based on Artedi [1738: gen. [spec.] 12, syn. 25, Salmo oblongus dentium ...; based on Gesner, 1556: 1075 [1604: 1015; 1558: 1213; Bavaria, Carinthia], 1563 [and 1575]: 173, and Willughby, 1686: 199; "Viennae, Austriae"]; type locality: “.."; type material: NT)

Hucho germanorum Günther, 1866: 140 (listed in synonymy, name not available)

Systematic notes. Hensel \& Holčík (1983) recognise two subspecies, $H$. h. hucho from the Danube basin and H. h. taimen (Pallas, 1773) in Russia, whose range extends from the Kama and Pechora rivers eastwards to the Jana and Amur basins. Figures 6-7 in Holčík et al. (1988) show distinct cranial osteology and their figure 6 shows a cladogram where taimen forms a monophyletic lineage with $H$. perryi and $H$. ishikawai (two East Asian species), this lineage forming an unresolved trichotomy with $H$. hucho and $H$. bleekeri (a species from the Yangtze); H. hucho as understood by these authors would then be polyphyletic. Considering that each taxon is diagnosable, that they occupy two distinct and not contiguous areas, they are regarded as two species.

Nomenclatural notes. Linnaeus (1758) did not indicate a type locality for his Salmo hucho. Linnaeus's diagnosis is actually based on Artedi (1738), itself based on Gesner and Willughby. Artedi gives the locality as "Viennae, Austriae"; this is the locality given by Willughby. Gesner's (1558) observations are based on material from Bavaria and Carinthia.
Berg (1948) included Salmo lossos Günther, 1866 (p. 140; type locality: Baltic Sea, rivers Kama, Kolva, Wolga, Petschow, Vitchegda, and Muilwa, Caspian Sea; syntypes: lost ? [naterial of Salmo hucho in Pallas, 1814: 344]) and S. fluviatilis Pallas, 1814 (p. 359; type locality: tributaries of rivers $\mathrm{Ob}$ and Irtis, river Lena and tributaries, lake Baikal, Siberia; holotype: ZMB 23561) in the synonymy of $H$. hucho. It does not seem that any of the mentioned localities is within the known distribution range of $H$. hucho and these nominal species are considered to be synonyms of H. taimen. See also Svetovidov (1978: 21, figs. 34$35)$.

Oncorhynchus mykiss (Walbaum, 1792) introduced

Systematic notes. This species was formerly known as Salmo gairdneri Richardson, 1836. Smith \& Stearley (1989) showed that the relationships of this species lies with the Pacific salmons (genus Oncorhynchus) and not with the trouts and Atlantic salmon (genus Salmo). This species naturally occurs in North America and Kamchatka and the earliest available name for it is $O$. mykiss, based on material from Kamchatka.

\section{Salmo}

The existence of ecological morphs of the trouts have been much discussed, usually focusing on whether the marine, lacustrine and brook stocks were different species, subspecies or morphs. Most authors were only concerned with their national waters and paid little or no attention to inter-basin variability. The earlier authors had recognised the marine, lacustrine and brook stocks as different species (e.g., Linnaeus, 1758; Günther, 1866), but as early as 1825, Jurine already considered lake and brook trouts to be a single species. In this century, the tendency has been to consider them as subspecies of a single species or as morphs. In my point of view, these discussions suffer from several weaknesses, the main one being that the authors implicitly considered the trout (or the three species or subspecies) as an homogenous entity all over its (their) range, neglecting the possibility that the situation of a population in a given basin a priori could not be extrapolated to other areas. Nümann (1967) and Balon (1968) already pointed out that a classification of trouts into three species, 
subspecies or forms trutta, lacustris and fario was unlikely to be a correct phylogenetic interpretation of the evolution of European trouts as, for example, each lake stock is most likely independently derived from different riverine stocks; thus a 'S. lacustris' including all European lake trouts would be polyphyletic, thus not acceptable. The data now becoming available confirm this view (see below).

Ecological morphs or species ? There is evidence that in some basins different morphs constitute a single genetic pool while in other basins different morphs are genetically distinct. We must consider, population by population, three alternatives:

1) the different morphs are genetically distinct and reproductively isolated; they obviously are different species;

2) the different morphs are not distinguishable genetically [or more objectively, the techniques used did not lead to differences being observed] but are reproductively isolated; if they can be diagnosed, they conform to the definition of species and are distinct species;

3 ) the different morphs are not distinguishable genetically and are not reproductively isolated; they constitute a single species.

Sympatric stocks which are genetically distinct and reproductively isolated (alternative 1) are known from lake Bunnersjöarna, Sweden (Ryman, 1979), Lough Melvin, Ireland (Ferguson \& Mason, 1981: Ferguson \& Fleming, 1983; Ferguson, 1986; Ferguson \& Taggart, 1991; McVeigh et al., 1995), Lough Neagh, Ireland (Crozier \& Ferguson, 1986), and lake Sevan, Armenia (Dorofeyeva \& Rukhyan, 1982). There are no data on morphological characteristics of the two stocks of lake Bunnersjöarna (as well as most of Scandinavia). In Lough Melvin, three morphologically distinct stocks (known as sonaghen, gillaroo and ferox) occur together and have been documented for over 200 years; beside morphological differences, they differ in allele frequencies, spawning grounds and seasons, intrabasin distribution and feeding habits (Ferguson \& Mason, 1981; Ferguson, 1986; Cawdery \& Ferguson, 1988; Ferguson \& Taggart, 1991; McVeigh et al., 1995). In the Lough Neagh river system, two to four stocks are recognised (ferox, gillaroo, dollaghan and salmon-trout) of which only the last two appear to be common and well documented (Crozier \& Ferguson, 1986: 461). Nyman (1967: 8, 19) reports electrophoretic differences between anadromous and "brook locked" trouts in river Indalsälven basin. Sweden. Skaala \& Nae- val (1989) found significant genetic differences between anadromous and sedentary trouts in three adjacent small river basins in Norway, as well as significant differences between the three basins. Skaala \& Jørstad (1987, 1988; Skaala et al., 1991) found two genetically distinct stocks identifiable by colour pattern in lake Svartavasstjønn on the Hardangervidda plateau, southern Norway; except for differences of colour pattern, no morphological character has been analysed; the fine spotted trout is probably a distinct species under the PSC; its whole spawning area is limited to less than 20 $\mathrm{m}^{2}$ and it is badly threatened by acid rains and stocking with allochtonous stocks. Two genetically distinct stocks (anadromous and non-migratory) have been found in an unspecified stream of the British Isles (Ferguson, 1989: 42).

In the case of genetically indistinguishable populations, very few unequivocal data on reproductive isolation (or its absence) are available. Noteworthy is Guyomard et al. (1984) who report that individuals resulting from a single introduction to the (fishless) freshwaters of the Kerguelen Islands rapidly developed a sedentary and a migratory morphs. The same had been reported from Chile (Riegel, in Nümann, 1967: 281) and New Zealand (Day, 1887: 183), but this last case is not very clear as the original stock might have been a mixture from different localities. Cligny (1907) discussed a population of sea trout which cannot return to freshwaters and where recruitment apparently results only from juveniles drifted from upstream.

Guyomard (1989: 129) commented that the coexistence of sedentary and migratory individuals in a single basin likely does not result from the sympatry of two species or subspecies but from a phenotypic variability within a population. While this is certainly correct in many cases, it should not be considered as a universal rule. Three points should be noted. First, even under the BSC, two subspecies cannot be sympatric [but this is not really relevant as with the PSC used here, subspecies are not recognised]. Second, there is no reason to think that morphological differentiation (and speciation) is always reflected by genetic differentiation (or more accurately by differentiation in that little part of the genome which we are able to analyse; see pp. 16, 19; see also Mayden \& Wood, 1995: 85). The species flocks of African cichlids are known to exhibit very little genetic differentiation (Meyer et al., 1990; Moran et al., 1994), despite the fact that within each lake several hundreds of morphologically, ecologically, and ethologically distinct species are involved. Species flocks, or explosive speciation, are known to have 
occurred in a variety of tropical lakes (see Echelle \& Kornfield, 1984 for general discussion; several additional cases have been documented since, e.g., Stiassny et al., 1992; Nagelkerke et al., 1994; Kottelat \& Chu, 1988; Kottelat, 1990b, 1991) within a relatively short time span and there is no reason to think that this could not happen in temperate or Nordic waters (admittedly the pace could be lower as the concerned species mature later). Third, there are cases where it is definitely known that two or more species are involved (see above).

Skrochowska (1969) studied migrations of sea and brook trouts. Out of the recovered offspring of brook trouts, $6.4 \%$ were migrating, vs. 65.7 , $55.1,15.9$ and $31.5 \%$ of the F1 to F4 offspring of sea trouts (p. 130). The variation of the recapture rates of the sea trout is not discussed, but may have depended of season of release, water temperature (p. 183) and intensity of other fisheries (p. 160). Sea trouts which did not migrate were those with a lower growth rate. Males which were already mature at the time of release did not migrate (p. 184). The paper is not very clear on this, but it seems that most females migrated; it is not stated whether females which did not migrate are able to successfully reproduce in nature (pond-kept individuals are successfully propagated by aquaculture techniques). These figures may support a hypothesis that, at least in the studied population, the migratory behaviour might be an hereditary character. Unfortunately Skrochowska was mainly interested in the demography and timing of migrations, and did not discuss them in relation to ancestor stock and did not document possible morphological changes (except a very laconic statement p. 148).

The case of lake trouts is more difficult to handle. Possibly, one should first distinguish between stocks inhabiting large, old, natural lakes and those appearing in recent and smaller lakes. It seems that 'lake trout' is a concept understood in different ways by different authors and it remains to be demonstrated that 'lake trouts' from a minor and recent reservoir can be handled in the same way as lake trouts from major lakes. Until this is demonstrated, I am treating them as two distinct cases. There are numerous reported cases where brook trout stocks developed into 'lake trouts' after damming streams or rivers (e.g., Sihlsee, Switzerland [Steinmann, 1944: 168]; Hnilec and Orava Valleys, Slovakia [Holčík et al., 1965: 106, 137; Miš́́k, 1959; Balon, 1968: 8]; and possibly Mavrovo, Radika basin, FYROM [S. Karaman, 1957; M. Karaman, 1966: 24]). The first two cases seem genuine; but problems with many similar cases of reported evolution from brook trouts into 'lake trouts' include: 1) the difficulty of being sure that there has really not been any stocking after damming; 2) the lack of comparison with naturally-occurring lake form(s) from natural lakes in the same basin; and 3) the possibly plesiomorphic nature of the 'lake trout' phenotype (see below).

For the present discussion, I shall restrict myself to those stocks inhabiting natural lakes; they usually have obvious spawning migrations, but within a lake, different sub-populations can be recognised (see, e.g., Steinmann, 1944; Ruhlé et al., 1984: 273; Büttiker \& Matthey, 1986). Typical examples are the stocks of the large sub-Alpine lakes (e.g., lake Konstanz, lake Genève). To resolve their taxonomy the two main questions are: 1) are the lake and brook trouts stocks within a single basin conspecific; 2) are the stocks of the different lakes conspecific ? From the published evidence there is no conclusive answer to question 1 (both yes and no are still reasonable answers). The second question has apparently never been really addressed. Here, as in other cases, one should avoid generalisation because the situation in one lake is not necessarily representative of other lakes. Finally, we know several lakes where two or more sympatric, diagnosable and evolutionarily distinct stocks co-occur.

There have been experiments in which brook trouts transplanted into the sea developed the sea trout morph (see Neresheimer, 1941) and brook trouts transplanted into lakes developed the lake trout morph (Nümann, 1967). Although they seem to yield obvious results, these experiments actually are difficult to interpret; the results of many of them cannot be doubted, but results from one population should not hastily be considered as a rule applying to all trout populations or species. One of the problems is to know how homogeneous the brook trout stock used is. This is particularly important when dealing with large river basins. The material used for Nümann's experiments originated from a pond stock (whose precise origin is not stated), probably from an area immediately adjacent to the lake in which the brook trouts have been transplanted (that is, within the reach of migratory stocks). Would the results be the same if brook trouts from further upstream were used, where no lake trout ever migrated ? Which proportion of the riverine population in these localities migrates, or is it definitely known that only a part of the population migrates while the rest (both sexes) is permanently sedentary? To be meaningful, this experiment should have included at least 
two different samples with precise origin, one from parents who spent all their life in brooks and one from migratory parents. The tags used could have a negative effects on recovery rate in streams and this should be estimated (while the tag is unlikely to cause problems to fish in lake midwaters, in streams the fishes can easily get can hit in obstacles; also the used string type can get more easily abraded and torn in streams). And the different stocks (and controls) should be submitted to a detailed morphological analysis (Nümann basically discusses only the presence of red spots and this is too superficial to be conclusive - and to be called a morphological analysis).

The result of this kind of transplantation experiment is also difficult to interpret and incorporate in a systematic analysis: anadromy and the silvery lake (or sea) morphs could be plesiomorphic character states for the whole family Salmonidae (or even the suborder Salmonoidei) or an atavism, if one follows Stearley (1992: 627) who considers that the plesiomorphic salmonid habitat type is freshwater. On the reverse, different colour marks seem to be reliable apomorphies (which even allow some sub-basins to be distinguished). [While plesiomorphic character states may be valuable for identification, they are useless for phylogenetic analysis; p. 11]. While coloration can be a specific diagnostic character, it is also a common experience that any colourful fish from clear waters under stress or kept in lake or murky waters for a long period will loose part or all of its coloration and become more or less silvery [the way specimens are fixed, preserved and stored may influence coloration and colour pattern; material used for fisheries and genetic work is rarely fixed in a way allowing optimal use for systematic analysis]. While growth, maximum size and size at maturity can also be diagnostic characters, it must be kept in mind that in many instances they are at best indicative because growth and size at maturity depend on available food and space and maximum size depends on available space and the presence of competitors.

The reverse, that is a demonstration that any lake or sea trout can develop into a brook trout, would be much more significant because of the apomorphic nature of the details of the colour patterns. Obviously, this demonstration cannot be the result of simple transplantation experiments as both sea and lake trouts spawn in rivers where they spend their first months or years (various authors disagree about the duration but again, their experience is gained from different basins), then move downriver. If re-transported upriver, they are likely to move downriver again and such an experiment would necessarily be inconclusive too. But the release of marked fry of lake trouts in the normal spawning grounds of lake trouts as well as upriver could show whether or not the lake trout stock has the potential to stay permanently in head-waters with a brook trout phenotype throughout its life. While it seems likely that the lake (or sea) trouts and those from nearby rivers constitute a single stock (entirely or partially migrating), it also seems likely that those from further upriver do not migrate at all, and the potential for different species to inhabit a single basin actually exists. Some of the data in Champigneulle et al. (1993) seem to indicate that the sedentary and anadromous trouts in some tributaries of lake Genève are distinct stocks, while others of their data (p. 42) indicate that some of the offspring of the lake stock can reproduce without going through a lacustrine phase. Büttiker et al. (1987: 3325) report that lake and brook trout occur in sympatry in tributaries of lake Genève. In the mtDNA analysis of Bernatchez et al. (1992), specimens of lake trout from the stream Aubonne (Swiss slope of lake Genève) belong to the Atlantic group (see below) while the brook trout from the stream Dranse (French slope; several dams on this stream prevent upstream migration of lake trouts, but the exact location of collection is not stated) belong to the Adriatic group (and not to the Mediterranean one as other stocks of the Rhône basin); Guyomard's (1989) electrophoretic study of apparently the same populations does not show differences; transplantations or dispersal along man-made canals could be an explanation, but at this stage it cannot be ruled out that migratory and sedentary trouts are distinct species in lake Genève. Steinmann (1952) was considering lake and brook trouts [of unspecified lakes] as conspecific, but at the same time was implying that they represent different phyletic lines.

At least some stocks of lake trouts in Scandinavia (e.g., Lake Vättern) are morphologically distinguishable from brook trouts, mature later, grow larger, etc.; these character states are inherited (Alm, 1939, 1949). Alm (1939: 81) reports that in Sweden eggs and juveniles of lake trouts are larger than those of brook trouts. So, in Scandinavia too, it still cannot be excluded that lake and brook trouts are different species under the PSC. Schnakenbeck (1942) reports that brook, lake and sea trouts have different pigmentation ontogenies (the origin of the stocks is not stated).

Sea trouts introduced in Swiss rivers in 1933 and 1934 grew faster than local trouts for 3 years, 
but without showing signs of gonad development, then disappeared (Geiger, 1961: 302).

Some stocks from high altitude lakes in the Swiss and Austrian Alps have been called lake trouts, but clearly they are not related to the large lowland lake trouts. They only reach a small size, grow slowly and reportedly are morphologically distinguishable from sympatric brook trouts (e.g., lake Silsee, Switzerland; Steinmann, 1944: 286). If one follows Steinmann and considers that they are reproductively isolated, then they are distinct species under the PSC. This remains to be demonstrated.

Heuristic conclusions. At least in the trout species of the Atlantic drainage (S. trutta) the existence of several ecological morphs in some populations is demonstrated. This, however, should not be taken as a demonstration applying to all trout stocks. There are strong indications that in some populations this is not true and there are instances where the alternative hypothesis (that the morphs indeed are species) is at least as likely. More research is needed to solve the status of several stocks, keeping in mind that no result from one population can be automatically extrapolated to other populations without demonstration. What is true for one population may not be true for others. While the potential to develop a lacustrine morph in dammed rivers seems well corroborated, work is needed to unambiguously demonstrate whether offspring of brook trout, lake trout and sea trouts really have the potential to develop into any other form. The migratory lake trouts of large lakes are not equivalent to 'lake trouts' of recent, man-made lakes.

This may apply to some other species (e.g., $S$. cenerinus) but definitively does not apply ipso facto to all.

Geographic variation. Once the problem of the number of species in a given (sub-) basin is answered, the major problem is to relate them to what is known from other basins and there are too few studies addressing this topic. Let us imagine that there are two species in basin $\mathrm{A}$, one migratory species in basin $\mathrm{B}$, one species with two morphs in basin $\mathrm{C}$, two species in basin D, etc. What is the total number of species. Are species 1 and 2 in basins $A$ and D the same? Is the monomorphic species in basin B conspecific with the bimorphic one in basin $\mathrm{C}$ ? Is one of the species in basin $\mathrm{A}$ the same as the single species in basin B ?

These questions are significant not only for sys- tematics but they are also relevant to effective resource management and conservation. Krieg \& Guyomard (1985: 239) and Guyomard (1989: 132) report the case of the river Orne (France). The original population apparently consisted of a single, sedentary morph. Two stocks have been introduced: a migratory one from Dunajec (Vistula basin, Poland, occasionally stocked in the 1960s) and a migratory hatchery one from Etrun (Pas-deCalais, France) which is regularly stocked (it is not stated if a sedentary morph is also present in this last stock). Sea trouts returning to freshwaters for spawning have been genetically identified as the Etrun stock. If the two stocks do not or cannot interbreed, we could theoretically be dealing with distinct species (unfortunately, the reverse situation, interbreeding, is an inconclusive observation in the present context; see Introduction, p. 15). The fact that introduced stocks are involved makes the case more complicated and other factors could play a role in determining the anadromous or nonmigratory behaviours, like early environment of the stocked trouts, artificial conditions, etc. (Krieg \& Guyomard, 1985; Guyomard, 1989).

The genetics of trout populations in France have been discussed by Guyomard (1989) and Presa et al. (1994: 197) on the basis of electrophoretic data. They recognised two genetically distinct subspecies on the mainland, one in basins draining to the Atlantic Ocean and one in those draining to the Mediterranean. Atlantic stocks exhibit inter-population variability, possibly suggesting interbasin differentiation, while the Mediterranean stocks constitute a homogeneous group. Populations from Corsica are derived from two ancestral stocks, the Mediterranean one (in the North) and another stock (in the South) not yet known from other areas, but comparable data are missing for Sardegna, Sicily and southern Italy (Krieg \& Guyomard, 1983: 1094, 1985: 238; Berrebi, 1994b). The Atlantic, North Sea and Baltic Sea stocks seem to constitute a monophyletic lineage distinct from the Mediterranean one.

These results are congruent with mtDNA data of Bernatchez et al. (1992) for trouts of 24 European localities and Giuffra et al. (1994) for trouts of the Po basin, northern Italy. Bernatchez et al. found a well characterised Atlantic group. This group has also been observed in the Mediterranean and the Danube basins. For the Mediterranean, Bernatchez et al. concluded that it is due to transplanted Atlantic stocks. This might be true for several stocks, but unlikely for their population from stream Aubonne, lake Genève basin. Allozyms data of Largiadèr et al. (1996) also show the great 
affinity of lake Genève trouts with Atlantic stocks and they considered stocking as a possible explanation; they point, however, to the fact that the upper Rhône was completely covered by glaciers during the Würmian glaciations and that recolonisation of the basin by trouts of the Atlantic stocks is a likely alternative. Lake Genève is separated from the middle Rhone by the Bellegarde falls (Perte du Rhône) which apparently inhibited upstream colonisation of many species known from the rest of the Rhône basin; the complex evolution of the many glaciers meeting in this area in late Wuirmian (Hantke, 1980: 495-543, 1993: 117-123, 349) probably resulted in many headwater captures, stream reversals, lake formations and disparitions, occasionally connecting the Rhine basin with the upper Rhône. Such connections could have been used by fish colonists, including coregonids and Salvelinus which are not known from the rest of the Rhône basin (except lake Bourget) (Steinmann, 1951: 76). Museum specimens collected at the beginning of the 19th century (supposedly before introductions started), could be included in future analysis to confirm or refute the natural occurrence of Atlantic group trouts in lake Genève.

Similarly, the presence of Atlantic group trouts in the upper Danube (two populations in Germany and Austria examined by Bernatchez et al.) might reflect stocking and/or earlier connections and river captures between the Rhine and the upper Danube (Hantke, 1993: 112-115, 226). (Noteworthy is that an underground connection between the upper Danube and tributaries of lake Konstanz still exists near Aach, Germany; see, e.g., Balon et al., 1992: 3).

Hamilton et al. (1989) hypothesise that present Atlantic group populations are the result of colonisation by two races at different periods and that the earlier race is still present in a few basins, usually isolated from the sea (from where the second colonist arrived) by waterfalls. They also report that populations identifiable as this earlier race tend to live longer and reach larger sizes than those of the recent race. Trouts of the Rhine basin should be examined in this context; a testable hypothesis is that the lake trout of lake Konstanz belong to this earlier race. Hamilton et al. called them "ancestral" and "modern" races; I do not use this terminology, because it implies an as yet undemonstrated ancestor-descendant relationship; the two 'races' could as well have no immediate phylogenetic relationships. Data in Hynes et al. (1996) suggest that the colonisation history of northwestern European waters was more complex.
Data in Bernatchez et al. (1992), Giuffra et al. (1994) and Berrebi (1994) confirm that S. marmoratus is a distinct lineage; as it is diagnosable, it is considered here as a distinct species (Giuffra et al. and Berrebi call it a subspecies of S. trutta), as had been recognised, e.g., by Gandolfi et al. (1991). The remaining northern Italian stocks studied belong to an Adriatic group, also including trouts from Corsica and lake Ohrid (AlbaniaFYROM); the northern Italian S. 'trutta' seems to belong to two different lineages. Unfortunately, trouts from the rest of Italy have not been investigated in this study. Known past connections between both sides of the Adriatic (Bianco, 1990a: 178, 1992: 183; Bianco \& Miller, 1990: 1298; van Straaten, 1971) lead to the prediction that the same group could occur at least on the Adriatic slope of Italy. The topography of the Mediterranean basins during the Mediterranean Salinity Crisis (some 6 MYA; Hsü et al., 1973, 1977) suggests that Corsican freshwaters were draining to a western Mediterranean sub-basin to the west (also draining the Rhône basin) and a Thyrrenian subbasin to the east (also draining the western slope of Italy). Bernatchez et al. and Giuffra et al. found no differences between $S$. carpio, an endemic of lake Garda, and other Po basin 'S. trutta'; it would have been interesting if their material had included the original sympatric lake Garda 'S. trutta' as well, but it is now extinct.

Patarnello et al. (1994) also analysed mtDNA sequence variation in Italian trouts; they more or less analysed the same Po basin stocks as Giuffra et al. (1994), but also S. 'macrostigma' from Sardegna and $S$. fibreni. The number of examined specimens is low (1-3 per locality). The differences between the different stocks are less contrasting and there is apparently no difference between $S$. carpio, S. fibreni and S. 'macrostigma'. Differences between these and $S$. marmoratus and some $S$. 'trutta' are slight. The dichotomy in northern Italian S. 'trutta' observed by Giuffra et al. (1994) is apparently also present (but the sample includes one population of each lineage). They found no difference between southern Italian and Sardinian S. 'macrostigma' and no differences between the sympatric S. 'macrostigma' and S. fibreni. The low molecular differentiation between the different stocks is uninformative in a systematic framework (see Introduction, p. 19) and does not affect previous conclusions on the specific distinctness of S. carpio, S. 'trutta' and S. marmoratus. Salmo 'macrostigma' and S. fibreni occur in the same basin (but not in syntopy), are (as far as known) non-interbreeding, are morphologically di- 
agnosable and are thus considered here as distinct species; the lack of differentiation by any given technique does not indicate that they are conspecific. Alternative explanations are that the technique is useless or that the examined characters are uninformative; in the case of this pair of stocks, morphological analysis and field observations (ecology and ethology) are apparently more informative (see p. 20).

Information on Greek stocks include electrophoretic data (Karakousis \& Triantaphyllidis, 1989; Apostolidis et al., 1996), karyotypes (Karakousis et al., 1992) and unpublished mtDNA data (Apostolidis, pers. comm.). These studies show that $S$. peristericus from endorheic lake Prespa is conspicuously distinct. Besides, two groups of populations are recognised. One includes stocks from western Greece and the western Mediterranean basin (Spain and France), the other one includes stocks from northern and southern Greece and lake Ohrid (a single stock examined). The morphological analysis by Karakousis et al. (1991) is not very informative.

Electrophoretic and mtDNA analyses of stocks from the former USSR, the Danube, the Black Sea, Caspian Sea and Aral Sea basins also showed that the Atlantic (Baltic) stocks and the southeastern European and west Asian ones represent distinct lineages (Osinov, 1984; Bernatchez \& Osinov, 1995; Largiadèr \& Scholl, 1995; Riffel et al., 1995). Genotypes of both lineages occur in the upper Danube basin. Electrophoretic data for two stocks in Turkey show that they are conspicuously distinct (Togan et al., 1995).

There is one point on which I disagree with several of these genetic analyses, however brilliant their results may be. They often report e.g., "the utility of morphological criteria ... is hampered by their phenotypic plasticity" (Bernatchez et al., 1992: 170), "phenotypic plasticity ... limits the usefulness of morphological characters" (p. 161), "this could eliminate a purely morphological approach" (Patarnello et al., 1994: 73), etc. This might be true, but the published data do not support this conclusion because there have been so few detailed morphological descriptions (or comparisons) of stocks (and especially nonAtlantic stocks) during this century (see also p. 20). As stressed in the Introduction, 'taxonomic' research on western European fishes (and especially salmonids and coregonids) has been almost exclusively done by fisheries biologists without a theoretical and practical background in systematics; systematic concepts have been misunderstood (e.g., Steinmann's [1951, 1952] version of BSC) and morphological analyses were generally superficial, rudimentary and non-standardised, often restricted to very few characters of limited information content or irrelevant for systematic analysis (but of fisheries interest, like growth patterns, circumference or fork length); the overemphasis on meristic characters (i.e. countable characters like fin rays, gill-rakers, etc.) over morphometry, descriptive morphology, anatomy and osteology is diagnostic of a situation where lay systematists prefer characters which are counted because they seem more reliable than those which have to be described (it is easier to count 23 gill-rakers than to describe the shape of a bone, the structure of the lips, the coiling of the intestine or subtleties of the colour pattern, especially in a foreign language).

The dramatic dearth of decent and critical morphological analysis should be kept in mind before commenting about the supposed limitations of morphological criteria, especially if this cannot be supported by real data as is often evidenced by implicit or explicit statements that all that has been seen of the fish are tissue samples shipped by collaborators. Beside showing that such statements on morphological characters are premature, I want to point to the need for voucher specimens on which to check identifications. To contribute significantly to systematics, genetic studies should be linked with original morphological work or at least with accessible voucher material. The voucher material also should be prepared in a way that allows morphological analysis; it is a too common experience that voucher material is in such a state that it precludes any reasonably reliable identification, not to mention that it is useless for a morphological definition of the stock. To me, a lack of congruence between genetic and taxonomic data is more often due to the lack of recent, competent systematic revision or to misidentified material than to any other reasons. Genetic analysis and classical morphological examination of well-preserved (or live) specimens of fish previously not distinguished or considered as a wide-ranging and very variable species by fisheries biologists and earlier systematists yielded almost simultaneously the same results in the south-east Asian catfish Hemibagrus nemurus (Lim et al., 1990; Ng \& Ng, 1995; Dodson et al., 1995; Kottelat \& Lim, 1995).

But I endorse the conclusion of these genetic studies when conservation and management of trout stocks or species are concerned. Actual stocking programs all over Europe are threatening genetic integrity of most local populations and may push several overlooked species towards extinction. The conclusion of M. Karaman's (1966: 
36) would-be systematic analysis of 'southern European' salmonids (actually just trouts of a small part of former Yugoslavia) is a good demonstration of the irremediable damage which can result from poor concepts: "According to taxonomic and historico-evolutive facts clarified in this study, it is possible, without reservation, to transplant new forms of the genus Salmo in this basin [apparently lake Ohrid basin], without fearing possible undesired results of hybridisation. Similarly, it is possible to transplant these forms in other basins, because all European trouts belong to the superspecies S. trutta [apparently meaning that they are all equivalent]". (S. Karaman's [1938: 138-139] conclusion was opposite to M. Karaman's above quoted one !).

Available data actually show that many stocks are potentially distinct species and that stocking should be done exclusively with parents from the same basin; individuals which cannot be unambiguously identified as belonging to the local stock should not be used for stocking programs.

Systematic and taxonomic hypotheses. Considering the available data (and often the lack of detailed and relevant data), I am presenting alternative nomenclatures which correspond to different, alternative (and admittedly simplified) situations.

The first hypothesis, which I would qualify as the Simplistic Trout Concept, is to consider tentatively all the brook, lake and sea trouts of northern and western Europe as constituting a single species. There is strong evidence that at least in some small coastal basins sea and brook trouts belong to the same breeding pool or that in interior basins lake and brook trouts are conspecific (see above). Under this hypothesis, S. trutta is the valid name of the trout. I do not favour this hypothesis because it hides much of what is now known of trout diversity.

The second hypothesis, a Multispecific Trout Concept, recognises the Atlantic, Baltic and North Sea stocks as one species, including sea trouts and brook trouts. The western Euro-Mediterranean stocks would constitute a second species. Noteworthy, sea trouts have long been virtually unknown in the Mediterranean and their recent increased frequency (Allardi \& Keith, 1991: 50) might actually be the result of introduced anadromous stocks (all cultivated stocks in France are of Atlantic origin; Presa et al., 1994); alternative explanations do exists (e.g., high Mediterranean salinity and temperature, effect of competitors). In the Atlantic trouts it remains to be demonstrated that a sin- gle species occurs throughout large river basins, i.e. that the populations in the coastal areas belong to the same species as those from the interior. For example, in the Rhine basin, it is known that the migratory sea trout reaches (reached ?) upriver only to the rivers Main and Moselle (Fatio, 1890: 369). Are the interior, sub-Alpine and Alpine non-migratory stocks conspecific with the coastal ones? Is the lacustrine stock resident in lake Konstanz (and migrating upriver in the Rhine; Ruhlé et al., 1984) conspecific with the lower Rhine one and with those from other pre-Alpine lakes which, although situated in the Rhine basin, are separated from lake Konstanz by the Rhine falls in Schaffhausen? The same questions also apply to the Rhône basin above Bellegarde falls (including lake Genève; see above).

Under this second hypothesis, the name $S$. trutta is to be restricted to the Atlantic species. The name of the western Euro-Mediterranean species depends of the status of the trout of lake Genève. If considered as conspecific with the western Euro-Mediterranean species, the first valid name for it would be $S$. lemanus Cuvier, 1829 as this species was originally based on material from lake Genève. But, as mentioned above (p. 129), this stock does not seem to belong to the same lineage as the lower Rhône ones and the name does not therefore come into consideration. The name S. punctatus Cuvier, 1829, is simultaneous to $S$. lemanus but Cuvier's description is very short ("dotted with black and red spots") and hardly qualifies as a description (but, formally speaking, under the ICZN, it is a description); the locality data are vague ("all around the Alps") and obviously include stocks other than the Mediterranean one; fortunately Cuvier also includes the S. alpinus of Bloch (1784: 158, pl. 104) in his S. punctatus: Bloch's account is based on a drawing and on various literature accounts, including Linnaeus's original description of Salvelinus alpinus. Salmo punctatus is here formally restricted to Linnaeus's account of Salvelinus alpinus and can thus be eliminated from the synonymy of $S$. trutta sensu lato [this conforms to earlier practice, e.g., Gistel, in Gistel \& Bromme, 1850: 344]. Salmo rhodanensis Fowler, 1974 is the earliest available name for the western Euro-Mediterranean species.

The brook trout of the Adriatic basin should be called $S$. cenerinus Chiereghini, 1847, which is the oldest available name. From the available data, $S$. 'macrostigma' still can be recognised as a distinct and diagnosable lineage, and is thus treated as a valid species; its correct name however is $S$. cettii Rafinesque Schmaltz, 1810; it remains to be 
demonstrated whether the stocks from southern Italy, Sardegna, Sicilia and Corsica constitute a single species and whether they are conspecific with the North African S. macrostigma (it also remains to be demonstrated whether the different North African nominal species constitute a single species; I hypothesise they are [were] not). I doubt that the Balkan stocks referred to as S. macrostigma are conspecific with the western Mediterranean ones.

The migratory trouts of the Black Sea and lower Danube basins have classically been distinguished as a distinct species or subspecies $S$. labrax (e.g., Berg, 1948; Bănărescu, 1964; Lelek, 1988) and I tentatively recognise it here as a distinct species. It migrates from the Black Sea only as far upriver as Calarasi, Romania (about 400 river-km from the sea; Bănărescu, 1964: 264). Balon (1968) considers $S$. labrax as conspecific with $S$. trutta, but he mentions only two characters (vertebrae and gill-raker counts) and the argument is very speculative and sometime confusing. The material he refers to as $S$. $t$. labrax originates from the Danube basin in Slovakia and it remains to be demonstrated that it is conspecific with the migratory Black Sea species. On the basis of the data in Bernatchez et al. (1992) and Bernatchez \& Osinov (1995), it seems that $S$. trutta and a second species (which might be $S$. labrax) occur in the upper Danube headwaters. I tentatively list the nominal taxa described from the middle and upper Danube populations as conspecific with the Black Sea S. labrax, following Holčík (1969, S. t. labrax); the primary types of all these taxa should be examined to confirm their identification. Clearly more populations should be examined from the upper Danube, where it cannot a priori be excluded that more than one species cooccur. The status of the stocks of the high Alpine lakes of the Danube basin in Austria and Switzerland should also be investigated (the name $S$. excelsus Fatio, 1890 is available for them should they deserve a distinct taxonomic treatment), but it might be too late to find pure stocks (see Largiadèr \& Scholl, 1995). The presence of 'real' S. trutta in the upper Danube may result from stocking (Largiadèr \& Scholl, 1995; Riffel et al., 1995).

Unfortunately, the available data on Balkan trouts are limited. Modern morphological work has been scanty and genetic data are available only for some Greek stocks (see above). Sound and compatible data on several key populations from former Yugoslavia are badly missing. The status of some nominal species seems clear (e.g., S. peristericus), but for others, unless they have been demonstrated to be synonyms by earlier authors, I have no other objective way to treat them than to accept them at face value as valid. Brief morphological examination of several Greek populations suggests that several species occur in the country, some possibly unnamed. Future studies should include comparative material from adjacent areas; material from eastern Macedonia and Thrace should be compared with $S$. labrax from the Black Sea basin. The northeastern group of population recognized by Apostolidis et al. (1996: 554) is tentatively recognised as $S$. macedonicus. The western Greece stocks have sometime been called $S$. macrostigma (here $S$. cettii), but there is presently no evidence that they are conspecific with it or that they represent only one species; they might be conspecific with one or several of the trouts described from Albania and former Yougoslavia.

Finally, I doubt that any of the Caucasian and western Asian stocks (as far East as Afghanistan) referred to as S. trutta would be conspecific with the Atlantic species. Much more work is needed to assess the number and identity of the involved species.

In the cases of sympatric occurrence of two noninterbreeding stocks, they are considered as distinct species. This applies to the following documented cases: lake Garda (?S. cenerinus, S. carpio; Malfer, 1927; Nümann, 1953: 307-309), lake Posta Fibreno (S. cettii, S. fibreni; Zerunian \& Gandolfi, 1990), Lough Melvin (S. stomachicus, S. nigripinnis, S. ferox; Ferguson, 1986; Ferguson \& Taggart, 1991), Lough Neagh (S. sp. 'dollaghan', S. sp. 'salmon-trout'; Crozier \& Ferguson, 1986) and lake Ohrid (S. letnica, S. aphelios, S. balcanicus, S. lumi). Outside Europe, this probably also applies to the trouts of lake Sevan, Armenia (Dorofeyeva \& Rukhkyan, 1982; Osinov, 1989 [p. 1213 of translation]; Savvaitova et al., 1989). In Lough Melvin and Lough Neagh, one of the sympatric species is possibly $S$. trutta, but the available data neither allow to confirm this hypothesis nor to decide which of the sympatric species could be S. trutta; thus they are all treated as distinct species awaiting data or material to solve this question. In the case of lake Garda, the larger species which spawned in winter in the tributaries and outlet of the lake possibly belonged to $S$. cenerinus of the Po basin and the smaller one which spawns in the lake twice a year (December-January, July-August; Gandolfi et al., 1991: 314) is the endemic $S$. carpio. The present large lake trout of lake Garda is apparently an introduced stock as all the original lake trout stocks of northern Italian lakes went extinct more than 30 years ago (Schöffmann, 1994: 98); the lakes have 
been re-populated with stocks from northern Europe.

Balon (1968: 15-16) recognises a deep-water stock from lakes Attersee, Traunsee and Fuschlersee, Austria, as a distinct infraspecies schiefermuelleri, locally named mayforelle (May trout) because it is only caught in May when it comes into shallower waters (Heckel \& Kner, 1858: 264; apparently, no original data have been published since), possibly for spawning, while sympatric lake trouts reproduce in November-December (Heckel, 1851b: 287) as is known for most other trouts (mostly November; Ruhlé et al., 1984: 276). Heckel (1851b: 286-287) further reports that it differs from the sympatric lake trout in lake Attersee by head shape, coloration, white eggs (vs. yellow) "never larger than millet seeds" (vs. "pea size"), more deciduous scales and greater difficulty to keep it alive. On the basis of Heckel's data and in the absence of any published contrary information, I tentatively accept $S$. schiefermuelleri as specifically distinct.

The common classification of the trouts into three subspecies is incorrect under any species concept. The ESC and PSC do not recognise subspecies by definition (see Introduction) and, under the BSC, two subspecies cannot occur sympatrically except in the exceptional case of a contact zone; in the present case, there is no contact zone but a broad or complete overlap. In addition, we know that in several cases these are not subspecies but variants of a single population.

The alternative classical classification as a single species with three morphs is not retained because it artificially oversimplifies, it overlooks well documented cases which prove it wrong and it arbitrarily generalises a few peculiar cases. Unquestionably, in several instances there are different morphs and these can be recognised if one wishes to. But these morphs should be recognised by names other than names used in the formal scientific nomenclature: the three classicallyrecognised morphs (fario, lacustris, trutta) are derived from species-group names which have different type localities. As discussed above, the classical trout might have to be divided into different species and there is a great likelihood that one or the other of these names might have to be re-used for one of these species. The use of the same name for a species and for a morph of another species can only lead to confusion (e.g., if lake Konstanz trout is considered as a distinct species, it must be called $S$. lacustris and it would be confusing if a lake morph from any reservoir in
Europe is called S. trutta m. lacustris). Additionally, as morph names have (technically speaking) no value in formal nomenclature, infrasubspecific names should not mimic species names, should not be latinised and should not be printed in italics in order to avoid confusion. I find that descriptors like sea trout, lake trout or brook trout or their translation in any language are self-explanatory, much more informative and less ambiguous than fario, lacustris and trutta. Finally, I find it misleading to use the same three morph names (especially if they have the formalised appearance of Latin names) all over Europe for trouts which have nothing in common and belong to different lineages (species).

\subsubsection{Salmo aphelios Kottelat, 1997, new name}

Salmo letnica aestivalis Stefanovic,1948: 190, 2 figs. (type locality: lake Ohrid; syntypes: LU; junior primary homonym of Salmo ischchan aestivalis Fortunatov, 1927)

Systematic notes. See comments under Salmo letnica.

Nomenclatural notes. Salmo is a masculine name (Eschmeyer, 1990) and species names, if adjectives, have to agree in gender.

Salmo letnica aestivalis Stefanovic, 1948 is preoccupied by $S$. ischchan aestivalis Fortunatov (1927: 114, pl.; type locality: lake Sevan, Armenia: syntypes: LU [111]; possibly already available from Fortunatov, 1926: 243, cited by Berg, 1948: 261) and the name must be replaced.

Etymology. From the Greek apo- (far, remote) and helios (sun). The aphelion is the astronomic term for the point of the orbit where a planet is at its greatest distance from the sun (early July for the Earth).

14.3.2 Salmo balcanicus (Karaman, 1927)

Trutta balcanica Karaman, 1927: 253, fig. 1 (type locality: lake Ohrid, FYROM; syntypes: LU)

Systematic notes. See comments under Salmo letnica.

\subsubsection{Salmo carpio Linnaeus, 1758}

Salmo Carpio Linnaeus, 1758: 309 (based on Artedi [1738: gen. [spec.] 13, syn. 27 [24], Salmo pede minor ...; Benaco, Cambria]; type locality: lake Garda, Italy, by neotype designation [originally: "in Angliae, Vallesiae fluviis"]; neotype: NRM 28000, present designation)

Salmo lacustris var. Benaci Malfer, 1927: 130 (type locality: lake Garda, Italy; type material: NT)

Systematic notes. Stearley \& Smith (1993: 14) 
suggest that future research might show that $S$. carpio belongs to Salmothymus, which seems contradicted by the study of Giuffra et al. (1994) and Patarnello et al. (1994) cited above (p. 129). Giuffra et al. (1996: 217) hypothesised that S. carpio might have resulted from the hybridisation between $S$. marmoratus and $S$. cenerinus, but did not provide unambiguous evidences. Their data apparently could as well be interpreted as an unresolved trichotomy in a study involving 3 lineages only. To lead to results usable in a phylogenetic analysis, such a study has to include more lineages (especially representatives of the many nominal species described from the eastern slope of the Adriatic basin) and the conceptual basis needs to be clarified.

Admitting that $S$. carpio might have an hybrid origin, this does not negate its recognition as a species under the PSC as it is an independent and diagnosable lineage. Several such cases are well documented and supported; see Stauffer et al. (in press) for a brief review.

Giuffra et al. (1996) also discuss whether there was reproductive isolation between $S$. carpio and the migratory trout stock of lake Garda ( $S$. cenerinus?, S. marmoratus?). While the discussion may make sense under the BSC, it is not really relevant here under the PSC. Anyway, there is apparently no evidence that they were not isolated. Giuffra et al. compared the lake Garda trout stocks with Scandinavian Salvelinus stocks. Although such a reference is common in the literature, it is a gross oversimplification of a diversity of non-homologous cases and this partly results from poorly defined concepts, as discussed below (p. 144), and the comparison is irrelevant here.

Nomenclatural notes. Linnaeus (1758) apparently had no material of $S$. carpio and based his description on Artedi who apparently had not examined the species either but based it on earlier accounts. He obviously included two species and gave (1738; also in Walbaum, 1792: 62) the localities as "Carpio lacus Benaci Auctorum. Gilt Charre Westmorlandorum in Anglia". Lacus Benacus is lake Garda (Italy). Although Linnaeus mentioned only one locality ("Angliae, Vallesiae fluviis"), as the name is available by indication, it also includes the lake Garda stock. The first reviser apparently is Heckel (1852a: 361-367) who clearly restricted the name $S$. carpio to the lake Garda species. Mohr (1786: 80) redescribed the species from Iceland, but did not act as first reviser. Specimen NRM 28000, $143 \mathrm{~mm}$ SL, is here designated as neotype; it is from a wild-caught stock grown up by Istituto Sperimentale San Michele al Adige in order to establish a captive breeding stock of the species.

\subsection{4 ? Salmo cenerinus Chiereghini, 1847}

Salmo Cenerinus Chiereghini, in Nardo, 1847: 125

(type locality: not far from the sea in rivers [draining to the lagoon of Venezia, Italy]; syntypes: LU); see also Ninni, 1872: 40

Salmo lacustris $\mathrm{A}, b$, fécond, var. meridionalis Fatio, 1890: 351, 354 (infrasubspecific, name not available; locality: lakes Lugano, Maggiore and Como, Switzerland and Italy, lake Poschiavo and stream Poschiavine, creek Soveglia, Switzerland)

Nomenclatural notes. The name of this species is still uncertain as the types of all nominal species described from northern Italy are missing. I tentatively retain $S$. cenerinus as the valid name for the native species usually called $S$. trutta or $S$. fario in northern Italy (see pp. 129, 131). The original description is very vague and could apply to about any salmonid. A problem is that the type locality ("not far from the sea in rivers") could indicate that Chiereghini possibly had S. marmoratus (see next paragraph); it may as well be drifted individuals of the present species. In the absence of evidence for one or the other hypothesis, as first reviser I retain the name for the 'northern Italian fario'. The alternative is to treat $S$. cenerinus as a synonym of S. marmoratus; there would then be no available name for the present species and it should be either listed as Salmo sp. or a new name should be created for it. For clarity, I feel it is straightforward to re-introduce here the name $S$. cenerinus with a more precise meaning. A neotype designation should definitively fix the name.

According to Sommani (1948: 144; 1960: 45) and Gandolfi et al. (1991: 281, 295), in northern Italy, ' $S$. trutta' is sedentary and lives in Alpine brooks, whereas $S$. marmoratus has a more migratory life-style and is found further downstream. In this, Chiereghini's account of S. cenerinus could fit better with $S$. marmoratus. Beside the above-listed names, Trutta adriatica may also come into consideration, but its original description is uninformative. It was described from the sea (Kolombatovic, 1890: 165), and it seems thus to be based on S. marmoratus and is listed under this species.

Trutta fario marmoratus Siebold, 1863 apparently also applies to $S$. marmoratus; anyway, the name is preoccupied in Salmo by $S$. marmoratus Cuvier, 1829, and thus is not available. Salmo lacustris var. meridionalis Fatio, 1890 is infrasubspecific, thus not available; the name probably is 
based on a mixture of the present species and $S$. marmoratus.

Some of the poorly described nominal species from Croatia may belong to this species.

\subsection{5 ? Salmo cettii Rafinesque Schmaltz, 1810}

Salmo cettii Rafinesque Schmaltz, 1810b: 32, 55 (type locality: Val Demone and Val di Noto, Sicilia; type material: NT ?)

? Salmo macrostigma Duméril, 1858a: 161 (type locality: Oued-el-Abaïch, Kabylie, $40 \mathrm{~km} \mathrm{~W}$ of Collo, Algeria; syntypes [total 2]: MNHN A7285 [1]); description also appeared in Duméril, 1858b: 398, pl. 10 (reproduced in Pomini, 1940: $35)$ and $1858 \mathrm{c}: 445$

? Salmo Lapasseti Zill, 1858: 127 (type locality: El-Abaïch, a tributary of Oued-Z'hour entering the Mediterranean about $30 \mathrm{~km}$ west of Collo, Algeria; syntypes: LU)

? Salmo Pallaryi Pellegrin, 1924a: 971 (type locality: lake (Aguelman) Sidi Ali or Mohamed, about $30 \mathrm{~km}$ north-west of Itzer, Middle Atlas, Morocco; syntypes: MNHN 1923-66 [2]); description also appeared in 1924b: 181, fig. 1

? Salmo Trutta var. Pellegrini Werner, 1931: 262, pl. 1 fig. 1 (type locality: stream Ourika near Timishi, Grand Atlas, Morocco; syntypes: NMW 19546 [1])

Salmo ghigii Pomini, 1941: 39, figs. 4-8 (type locality: stream Sagittario [Aterno-Pescara basin], Abruzzo, Italy; syntypes: LU)

Systematic notes. As discussed above, this species is tentatively considered as distinct. Bianco (1994b: 440) treats S. cenerinus (as S. trutta), S. cettii and $S$. fibreni as a single species.

This species is usually referred to as S. macrostigma, originally described from Algeria; various populations from Corsica, Sardegna, Sicilia, Italy, the Balkan peninsula and Turkey have later been referred to this taxon. The real identity of all these populations is to be considered as doubtful: none of them has been compared directly with Algerian material or with material from any of the other populations. It seems unlikely that all these disjunct populations would be conspecific. Karaman (1966: 5) claims to have compared some and concluded that they differ, but he did not provide supporting data. Schöffmann (1993: 166) and Doadrio (1994: 26) report that the Algerian populations are now extinct. Data on Moroccan populations in Bernatchez (1995) support the view that they do not belong to the same lineage as the Italian ones; but assuming that these are indigenous stocks, it still cannot be excluded that at least some of the
Moroccan trouts are not conspecific with the $S$. macrostigma from north-eastern Algeria. The figures of Moroccan trouts in Schöffmann (1993: 146) seem to represent a $S$. macrostigma-like individual (fig. 2) and a S. pellegrini-like one (fig. 3). These stocks are threatened by habitat alteration, introduction and hybridisation and restocking by local hatcheries.

Nomenclatural notes. The oldest available name for the Corsican, Sardinian, Sicilian and southern Italian group of populations is $S$. cettii Rafinesque Schmaltz, 1810 (see also Bianco, 1994b: 442; Zava et al., 1996: 416). Rafinesque used the spelling cetti on p. 32 and cettii on p. 55 and as first reviser I retain cettii.

Duméril's description of $S$. macrostigma appeared three times in 1858 and I have not tried to find out which one has priority. The $1858 \mathrm{a}$ is the only one mentioned in Zill (1858) and is possibly the oldest one. Behnke (in Daget et al., 1984: 125) indicated the publication date as 26 July 1858 but this is the date at which the paper has been read at the academy, not the publication date (which is not stated). Behnke lists a specimen as holotype, but it is a syntype as Duméril clearly stated that he had two specimens; the second specimen was in the collection of the Société d'Acclimatation (Duméril, 1858b: 398).

Salmo macrostigma and $S$. lapasseti have been published in 1858 and $S$. macrostigma has priority because it appeared first as indicated in a footnote by the editor in the description of $S$. lapasseti.

Salmo pallaryi has been considered a synonym of S. macrostigma, but a comparison of both descriptions suggests that they are very distinct species; this should be confirmed by a detailed examination of Pellegrin's material. They are reminiscent of Salvelinus, as already suggested by Pellegrin (1924b: 183). This species is now extinct (Schöffmann, 1993: 164).

Salmo ghigii apparently is a synonym of $S$. cettii. Pomini (1941) reported that 3 'forms' are present at the type locality, not distinct from those from Sardegna (Bianco, 1994b: 442; pers. comm.).

\subsection{6 ? Salmo dentex Heckel, 1852}

Salar dentex Heckel, 1852a: 371, pl. 10 (type locality: stream Kerka [Krka] near Knin, stream Cettina [Cetina] near Sign [Sinj] and stream Narenta [Neretva], Dalmatia; syntypes: NMW 16505 [1], 65887 [9], 65888 [4], 65894 [4], 92806 [1], NRM 25000 [1])

14.3.7 ? Salmo farioides Karaman, 1938 Salmo farioides Karaman, 1938: 136, fig. 3 (type 
locality: river Krka near Knin and Skradin, river Neretva near Prenj, Obod, river Radika near Rostusa, river Bistrica near Pec, small tributaries of lake Ohrid, creeks Cijevna and Crnojevica Reka (tributaries of lake Scutari), FYROM; syntypes: LU)

? Salmo trutta macrostigma morpha lacustris Poljakov, Filipi \& Basho, 1958: 68 (infrasubspecific, name not available; locality: Shkodrës, Albania)

Systematic notes. Karaman (1966: 15) noted that $S$. farioides differs from $S$. letnicus by the number of gill-rakers (among other characters). He concluded that this difference is caused by food differences. The conclusion (p. 24) that in reservoir Mavrovo, shortly after dam completion, the fish showed a great morphological change is not supported by the data in his table 3 which compares two populations but not the same population before and after completion of the dam. Additionally when one reads how many populations have been transplanted (and certainly more transplantations are involved which are not documented) in lakes situated on international borders (with no information about what other countries are doing) one is sceptical about how the results could be interpreted. Much good faith is needed to believe that such variability exists in the absence of hard data. Awaiting more conclusive data, I am left with no alternative but to treat $S$. farioides as a valid species.

\subsubsection{Salmo ferox Jardine, 1835}

Salmo ferox Jardine, 1835a: 55 (type locality: Loch Awe, Loch Laggan, upper end of Loch Shin, Loch Loyal and Loch Assynt, Scotland, U.K.; syntypes: LU; author indicated as Jardine \& Selby, but Jardine alone is actual author)

Systematic notes. This name has been used for the ferox, a large trout from Scotland and Ireland. It is one of the three sympatric Salmo species of Lough Melvin and one of the four of Lough Neagh, Ireland. It is treated as subspecies S. trutta ferox by Ferguson \& Taggart (1991) and as species by Ferguson (1986: 1) and Cawdery \& Ferguson (1988: 276). See above for discussion. See also Hamilton et al. (1989: 658) and McVeigh et al. (1995) for additional data. It remains to be demonstrated that the Lough Melvin and Lough Neagh stocks are conspecific and, as the species has originally been described from various lakes in Scotland, that the Irish stocks are conspecific with those from Scotland. Cawdery \& Ferguson (1988: 274) report that genetically similar populations are found in many isolated mountain lakes in Scotland, but do not provide details. Data on some Irish and Scottish populations in Hynes et al. (1996: 62) do not suggest close similarity, at least not in the investigated populations.

\subsection{9 ? Salmo fibreni Zerunian \& Gandolfi, 1990}

Salmo fibreni Zerunian \& Gandolfi, 1990: 523, figs.

2-3 (type locality: lake Posta Fibreno, Italy; holotype: MSNM Pi 3723)

Systematic notes. Zerunian \& Gandolfi (1990) described $S$. fibreni from the lake Posta Fibreno basin, Italy. They report that $S$. cettii occurs in sympatry and that the two stocks differ by size at maturity $(11 \mathrm{~cm}$ in males $S$. fibreni, vs. 17 in $S$. cettii; 13 , vs. 28 in females), spawning season (December-January, vs. February-March [data actually not in original description; from Gandolfi et al., 1991: 302]) and number of parr marks (7-9, vs. $9-13)$ and spots (15-20, vs. 20-60) on the sides. Their table 3 shows an almost complete overlap for all meristic data and their table 2 comparing morphometric data seem to indicate differences in maximum body depth, length of anal fin and eye diameter but as only the mean and standard deviation are given, we have no real idea of the actual overlap and variability. Salmo fibreni is reported to spawn in deep waters, in springs in underwater caves (see also Chiappi, 1924: 4-5), while $S$. cettii spawns in the outlet of the lake (P. G. Bianco, pers. comm.). Bianco (1994b: 442) considers $S$. fibreni as a dwarfed population of $S$. cettii (which Bianco does not consider as distinct from S. trutta); this cannot yet be ruled out, but as the species is cultivated (P. G. Bianco, pers. comm.) this could be easily investigated.

\subsubsection{0 ? Salmo labrax Pallas, 1814}

? Salmo Fario varietas alba Meidinger, 1794: iv, pl. 46 (type locality: "in valle Breinina, circa novem Ecclesias, Scotto-Viennam, Neobergam, ad Waltersdorf \& Schwanendorf in Fischa", Austria; type material: NT ?; junior primary homonym of Salmo albus Bonnaterre, 1788)

Salmo labrax Pallas, 1814: 346 (type locality: Sevastopol and river Biyuk-ozen / Chersonesus / Ochakov, Crimea, Ukraine; syntypes: LU)

? Salmo Fario var. niger Fitzinger, 1832: 339 (available by indication to Marsili [1726: 78, pl. 26 fig. 2, Trutta nigra, "in Austriam nostram inferiorem"], Bloch [1782, "Salmo Fario sylvaticus", actually a Walbaum, 1792 name, based on Waldforelle in Bloch, 1782: 157, pl. 23] and Paula Schrank [1798: 320, Salmo saxatilis]; type locality: lower Austria [Mar- 
sili], Norway / Berlin, Germany / Danzig [Gdansk], Poland [Bloch], Bavaria, Germany [Paula Schrank], rivers Schwarza and Glocknitz, Austria [Fitzinger]; syntypes: NMW ?, ZMB ?)

? Fario Marsilii Heckel, 1851a: 146 (type locality: lakes of upper Austria [Oberösterreich]; syntypes: NMW [not yet located; B. Herzig, in litt., 1995]); predates Heckel, 1851b: 286

? Salmo microlepis Günther, 1866: 85 (type locality: Pohorella [Pohorelá], Hungary [now in Slovakia]; syntypes: BMNH [3])

Salmo pallasii Günther, 1866: 112 (type locality: Chersonesus [Kherson], Crimea, Ukraine; syntypes: LU [material of S. trutta in Pallas 1814: 347])

? Salmo lacustris var. excelsa Fatio, 1890: 355 (type locality: sources of river Inn, lakes Sils, Silvaplana, Campfer and St.-Moritz, Danube basin, Switzerland; syntypes: MHNG 816.09 [2])

Salmo trutta labrax morpha fario Berg, 1916a: 47 (infrasubspecific, name not available)

Salmo trutta labrax morpha lacustris Berg, 1932a: 113 (infrasubspecific, name not available)

? Trutta Ungeri Vásárhelyi, 1940: 187, fig. 2 (type locality: creek Várvölgy in Buikk Range, Kom. Borsod, Hungary; syntypes: LU [3])

? Salmo trutta labrax infraspecies danubicus Holč́k, 1969: 227 (infrasubspecific, name not available; locality: river Danube in Bratislava, Slovakia)

Systematic notes. As discussed above (pp. 129 , 132), the status of brook trouts from the upper Danube basin cannot be solved with the available data and names based on material from this area are tentatively listed as synonyms of S. labrax. Examination of the type material is needed.

Nomenclatural notes. The earliest available name for this species is apparently Salmo fario albus Meidinger (1794) but the name is preoccupied by $S$. albus Bonnaterre, 1788 and cannot be used. The next available name is possibly $S$. saxatilis Paula Schrank (1798); the name was based possibly on actual material from Bavaria (Danube basin) and the description of material from Atlantic basin localities by Bloch (1782: 157). I restrict here the usage of the name to Bloch's material from Berlin and the name $S$. saxatilis is thus a synonym of $S$. trutta and is not available for the Danube species. Bloch's material is lost (H.-J. Paepke, in litt., 1995). A neotype designation could be needed to definitively fix the synonymy. I decided not to designate a neotype here: in case future research shows that the upper Danube species is distinct from the lower Danube, migratory S. labrax, the name S. saxatilis could be used for it by a judicious neotype designation.

Although Heckel described Fario marsilii in 1852 a (p. 349, figs. 6-8), his 1851 a account makes the name available.

Vásárhelyi (1940: 188) reports differences in body and mouth shape and coloration between 'Salmo ungeri' and S. trutta [here S. labrax] from adjacent basins. He also indicates differences in egg size, incubation time and that hybridisation experiments failed. In 3 years of experimentation, fecundation was possible but the eggs always died after a few days. I could not find later reference on this species and I understand that subsequent authors have doubted these data. But there is no objective published data to confirm or refute them and this would be worth a critical study.

\subsubsection{Salmo letnica (Karaman, 1924)}

Trutta letnica Karaman, 1924b: 20 (type locality: lake Ohrid, FYROM; syntypes: LU)

Salmo letnica typicus Stefanovic, 1948: 181, 4 figs.

(unnecessary replacement name for Trutta letnica Karaman, 1924)

Systematic notes. Three or four stocks with different ecology and spawning places and seasons occur in lake Ohrid (for a brief summary, see Stankovic, 1953: 482, 1960: 202-205). The 'letnica' and 'aestivalis' stocks occur along the eastern shore and spawn in the lake near sub-lacustrine springs, 'letnica' in January-February, 'aestivalis' in June-July. The 'balcanica' stock occurs in the north-west of the lake and spawns at and in the outlet of the lake in December. The 'fluviatile' stock of Stankovic is probably the 'lumi' of Albanian authors and occurs along the northern and western shores, spawns in tributaries and at their mouths around February. The four stock reportedly differ morphologically and these character states are inherited (Stankovic, 1953: 482; Rakaj \& Flloko, 1995: 197). Osteological differences between three stocks are illustrated and discussed by Dorofeyeva et al. (1983). Their figures show salient differences (e.g., orbit region). Salmo balcanicus is treated as a valid species by Vukovic \& Ivanovic (1971). Available (published) data suggest that these four stocks are species under the PSC and they are here treated as such: S. letnica, $S$. aphelios (earlier $S$. aestivalis), S. balcanicus and S. Iumi.

\subsubsection{Salmo lumi Poljakov, Filipi \& Basho, 1958}

Salmo letnica lumi Poljakov, Filipi \& Basho, 1958:

67 (type locality: ? Tushemishtit, Volorekës, 
Linit, Piskupatit, Hudënishtit and Pogradec, Albania; syntypes: LU)

Systematic notes. See comments under Salmo letnica. Schöffmann (1994: 99) doubt that S. lumi is still extant in Albania.

\subsubsection{Salmo macedonicus (Karaman, 1924)}

Trutta fario var. macedonica Karaman, 1924b: 16 (type locality: estuary of river Treska, Vardar basin, FYROM; syntypes: LU)

? Trutta fario macroptera Chichkoff, 1939: 121, pl. 2 fig. 4 (type locality: lakes Gorno Ribno Esero [Ezero] and Dolno Ribno Esero [Ezero], river Struma basin, Rila range, Bulgaria; syntypes: LU)

\subsubsection{Salmo marmoratus Cuvier, 1829}

Salmo marmoratus Cuvier, 1829: 304 (type locality: lakes of Lombardia, Italy; syntypes: MNHN ?)

Salar genivittatus Heckel \& Kner, 1858: 260, fig. 144 (type locality: stream Sala, a tributary of the Isonzo, Dalmatia; holotype: NMW [not yet located; B. Herzig, pers. comm., 1995])

? Trutta Fario var. marmorata Siebold, 1863b: 187 (type locality: all brooks of the Alps draining to the south to rivers entering the Adriatic Sea; syntypes: ? ZSM, now lost [pers. obs.]; preoccupied in Salmo by $S$. marmoratus Cuvier, 1829)

? Trutta adriatica Kolombatovic, 1890: 165 (type locality: Adriatic Sea at Vranjic, Croatia; holotype: LU)

? Salmo trutta marmoratus morpha lacustris Poljakov, Filipi \& Basho, 1958: 69 (infrasubspecific, name not available; locality: Shkodrës, Albania)

Nomenclatural notes. The author of S. marmoratus is often given as Cuvier (1816). Actually it was first named by Cuvier (1829: 304). See S. cenerinus for discussion of Trutta adriatica and Trutta fario marmoratus.

\subsubsection{Salmo nigripinnis Günther, 1866}

Salmo nigripinnis Günther, 1866: 96 (type locality: Lough Melvin; Ireland, by lectotype designation; lectotype: BMNH 1865.9.18:11, present designation)

Systematic notes. This is the sonaghen, one of the three sympatric Salmo species of Lough Melvin, Ireland. Treated as subspecies S. trutta nigripinnis by Ferguson \& Taggart (1991) and as species by Ferguson (1986: 1) and Cawdery \& Ferguson (1988: 276). See Salmo discussion (pp. 125, 132).

Nomenclatural notes. Günther (1866: 96) based this species on material from four localities: Lough Melvin, Llyn Beguilin, Llyn Gadr and river Towey; it remains to be demonstrated that these four stocks are conspecific. Specimen BMNH 1865.9.18:11, $230 \mathrm{~mm}$ SL, from Lough Melvin, is designated as lectotype; thus, the name is definitively linked with the Lough Melvin sonaghen.

14.3.16 ? Salmo pelagonicus Karaman, 1938 Salmo pelagonicus Karaman, 1938: 133, fig. 1 (type locality: creek Bela Reka, Kajmakcalan range, east of Bitolj-Monastir, Vardar basin / creeks Zlokucanska Reka and Dihovska Reka, north-eastern Perister range, west of Bitolj, Vardar basin, FYROM; syntypes: LU [3])

14.3.17 Salmo peristericus Karaman, 1938

Salmo macedonicus peristericus Karaman, 1938: 133 (type locality: lake Prespa and creek Brajcinska Reka flowing from Perister range to lake Prespa, FYROM; syntypes: LU [5])

\subsubsection{Salmo rhodanensis Fowler, 1974}

Salno trutta forma major facies rhodanensis Roule, 1923: 291 (infrasubspecific, name not available; river Rhône [near Valence ?], France) Salmo eriox rhodanensis Fowler, 1974: 62 (available by indication to Salmo trutta forma major facies rhodanensis Roule, 1923: 291; type locality: river Rhône [near Valence ?], France; syntype: MNHN 1922-164 [1], discarded in 1947, J.-C. Hureau, pers. comm., 1996)

Systematic notes. See Salmo discussion (pp. 128, 131). Populations from the northern part of the Rhône basin have a colour pattern apparently met in no other trout, consisting of three broad, dark, transverse bands on the posterior part of the body. It remains to be demonstrated if these populations are conspecific with material from the lower Rhône on which Roule (1923) and Fowler (1974) based S. rhodanensis.

Populations of S. trutta and S. rhodanensis from streams on both sides of the water divide between the Doubs (upper Rhône basin) and the Rhine can be distinguished very easily on the basis of external characters, especially colour pattern.

\subsubsection{Salmo salar Linnaeus, 1758}

Salmo Salar Linnaeus, 1758: 308 (based on Artedi [1738: gen. 11 [48], syn. 22, spec. 48 [11], Salmo rostro ultra ...; based on various references but actual material described from Baltic Sea] and Linnaeus [1746: 115, n. 306]; type locality: "in Oceano Europae, parit in fluviis"; type material: NT) 
Salmo nobilis Olafsen, 1772: 65 [German translation 1774: 35] (available by indication to Artedi [1738: gen. 9 [actually spec. 11], Salmo nobilis]; type locality: Sweden [Artedi], HellerAa, Kortolfstade-Aa, Lar-Aa, Iceland [Olafsen]; type material: NT)

Salmo Goedenii Bloch, 1784: 155, pl. 102 (type locality: Baltic Sea in Ruigenwalde, Hinterpommern [now Darlowo, Poland]; holotype: ZMB 3710); synonymy follows H.-J. Paepke's examination of the holotype (in litt., 1995)

Salmo Salmulus Walbaum, 1792: 61, 64, 720 (based on Pennant [p. 303, pl. 59, edition not stated; 1769: vol. 3: 253; 1776: vol. 3: 265 n. 148, pl. 59; The Samlet] and Ray [1710: 63]; type locality: rivers Wye, upper Severn and tributaries, north of England and Wales, U.K.; type material: NT)

Salmo caerulescens Schmidt, 1795: 65 (nomen nudum; locality: river Moldau [Vltava], Czech Republic)

Salmo renatus La Cepède, 1803: 224 (type locality: river Moselle, France; holotype: MNHN ?)

Salmo rilla La Cepède, 1803: 224, pl. 5 fig. 3 (type locality: river Rille, a tributary of river Seine, France; type material: NT)

Salmo nobilis Pallas, 1814: 342 (type locality: Baltic, Arctic and White Seas, in streams of Livonia [now Estonia and Latvia], Estonia, northern Russia; syntypes: LU; junior homonym of Olafsen, 1772: 65)

Salmo hamatus Cuvier, 1829: 303 (type locality: "mouth of our rivers" [France]; also available by indication to Bloch, 1784: pl. 98; syntypes: MNHN?)

Salmo Ocla Nilsson, 1832: 4 (type locality: Baltic Sea, where it ascends river Dalelfven / Elfkarlby [Älvkarleby on river Dalälfven, north of Uppsala], Sweden; syntypes: LU)

Salmo Salmo Valenciennes, in Cuvier \& Valenciennes, 1848: 169, pl. 614 (type locality: numerous localities cited, but examined material from: Dieppe, Fécamp, Abbeville, France, markets of Belgium, Netherlands, England and Berlin; syntypes: MNHN)

Salmo Salar var. lacustris Hardin, 1862: 382 (type locality: lake Venern [Vänern], Sweden; syntypes: LU; junior homonym of Salmo lacustris Linnaeus, 1758)

Trutta relicta Malmgren, 1863: 59 [German translation: 1864: 333] (type locality: lake Ladoga. at Kexholm, Finland [now in Russia]; syntypes: $\mathrm{MZH} ?)$

Salmo gracilis Couch, 1865: 216, pl. 216 (type locality: harbour of Fowey, U.K.; holotype:
BMNH; primary junior homonym of Salmo gracilis Valenciennes, in Cuvier \& Valenciennes, 1848: 265)

Salmo hardinii Günther, 1866: 107 (type locality: lake Wenern [Vänern], Sweden; syntypes: BMNH [1])

Salmo brevipes Smitt, 1883: 32 or Smitt, in Anonym, 1883: 182 (type locality: Arkangel, Russia; syntypes: NRM)

Salmo salar brevipes morpha relictus Berg, 1932a: 112 (infrasubspecific, name not available; locality: landlocked in lakes Segosero and Wygosero, river Wyg basin, Russia; junior homonym of Trutta relicta Malmgren, 1863)

Salmo salar infraspecies biennis Berg, 1912: 137 (infrasubspecific, name not available) from Berg, 1934: 714, 1948: 206, 212

Salmo salar saimensis Seppovaara, 1962: 78 (type locality: Iso-Saimaa, Finland; syntypes: LU)

Salmo salar europaeus Payne, Child \& Forrest, 1971: 251 (type locality: Europe; type material: NT)

Systematic notes. European and North American salmons are distinguished by chromosome numbers, enzymatic patterns and DNA analyses (Guyomard, 1994: 147; Taggart et al., 1995) and they constitute distinct lineages. Payne et al (1971) concluded that there is no gene exchange between them and treated them as different subspecies. They can unambiguously be treated as two species under the PSC.

There are no known significant genetic differences between anadromous and land-locked salmons in Europe. However, anadromous and lacustrine stocks exist sympatrically in lakes Ladoga and Onega (Russia) (L. S. Berg, 1948: 233; O. K. Berg, 1985: 810). Permanent freshwater stocks also exist(ed) in lake Vänern (Sweden), lake Bygglandsfjord and rivers Namsen and Nidelva (Norway) and lakes Saimaa and Pielinen basins (Finland) (L. S. Berg, 1948: 233; O. K. Berg 1985) and as they have independent histories, their status should be investigated independently of the Ladoga stock. The stock from lake Saimaa reportedly has a different chromosome number (Seppovaara, 1962: 30). Actual descriptive (morphological and genetic) work on these stocks would be more informative than research on speculated evolution and ad hoc zoogeographic fancy. Sound evolutionary hypothesis have to rely first on hard facts of the animals before interpreting geological and paleogeographical data.

Nomenclatural notes. Salmo salar mas Bloch, 1784 (p. 146, pl. 98) is sometimes erroneously cited as a nominal species (e.g., Fowler, 1974: 
58). The original text reads "Salmo Salar.Mas" which means "Salmo salar, male". Bloch (1782: pl. 20) also labelled a plate "Salmo Salar.Foem[ina]" (Salmo salar, female). These were obviously not intended as scientific names.

Day (1887: 51) lists Salmo gracilis as available from Couch (1859), where this name is not mentioned. It is first available from Couch (1865).

The nomenclature of Payne et al. (1971) (S. s. europaeus and S. s. americanus) grossly violates the Code. If recognised distinct, the European species retains the name $S$. salar (a subspecies should be called S. s. salar). I have not tried to trace the oldest available name for the American species but there definitively are several available names. An early name apparently is $S$. omiscomaycus Walbaum (1792: 65, 714) which is available (as S. omiscomaycus) from the Index (p. 714) (the text, p. 65, does not make the name available because the species group name is published as separate words; ICZN art. 11(v)).

\subsubsection{0 ? Salmo schiefermuelleri Bloch, 1784}

Salmo schiefermülleri Bloch, 1784: 157, pl. 103 (type locality: various lakes of Austria, restricted by Valenciennes, in Cuvier \& Valenciennes, 1848: 287, 344 [other locality: Wipperstrohm and shores of Baltic Sea]; syntypes: ZMB, probably lost)

? Salmo schiefermülleri argenteus Reisinger, 1830: 39 (type locality: lakes of Hungary; type material: NT ?)

Salmo argenteus Fowler, 1974: 61 (listed in synonymy, name not available; reference to Bonnaterre, 1788: 160, pl. 67 [saumon argenté]; localities: Baltic Sea and lakes of Austria)

Systematic notes. See discussion under Salmo above (p. 133). Available (but somewhat outdated) data seem to indicate that it is a distinct species. Balon (1968) wondered if the original stock still survives; only the stocked $S$. trutta is now recorded from these lakes (B. Herzig, pers. comm., 1995). It is not mentioned for Attersee and Fuschlersee by Haempel (1930: 180-184) who records only 'normal' lake trout. For lake Traunsee, Haempel (1930: 191) considers the mayforelle to be a sterile form without further discussion; Heckel (1851b: 287) explicitly stated that his mayforelle had developed ova. Accurate and up-todate information is needed, but it is probably too late as the original stock seems to be extinct (B. Herzig, pers. comm., 1995), and it is not clear whether museum material is still extant.

Nomenclatural notes. The name to be at- tributed to Heckel's mayforelle is not very clear. Balon (1968: 15-16) called it S. schiefermuelleri. Bloch's (1784) original description of S. schiefermuelleri is based on two very dissimilar populations, one from Austria and one from the Baltic Sea. Valenciennes (in Cuvier \& Valenciennes, 1848) acted as first reviser and restricted the type locality to Austria: p. 287, he stated that Bloch mixed a silberlachs of the Baltic and a Danubian trout under S. schiefermuelleri and p. 344 he used that name only for the Austrian species.

Two specimens in ZMB $(3720,3721)$ are labelled as syntypes of S. schiefermuelleri (H.-J. Paepke, in litt.). The associated locality data say that they are from Switzerland. As Bloch explicitly based his description on the above-mentioned material from Austria and the Baltic Sea, these specimens cannot be syntypes (or erroneous data have subsequently been introduced on the labels). Bloch (1784: 158) mentions the rheinanken or illanken from Switzerland (lake Konstanz trout) which, on the basis of a drawing, he cannot distinguish from the common salmon. Clearly, Bloch had no material of this rheinancken and he was not considering it as conspecific with S. schiefermuelleri. In the absence of material of both Bloch and Heckel, I tentatively consider that Heckel's material is conspecific with Bloch's one.

\subsubsection{Salmo stomachicus Günther, 1866}

Salmo stomachicus Günther, 1866: 95 (type locality: Lough Melvin, Ireland; syntypes: BMNH $[[3])$

Systematic notes. This is the gillaroo, one of the three sympatric Salmo species of Lough Melvin. It is treated as subspecies S. trutta stomachius [sic] by Ferguson \& Taggart (1991) and as species by Ferguson (1985: 1) and Cawdery \& Ferguson (1988: 276). See above for discussion (p. 125, 132).

A stock from Lough Neagh, Ireland, has also been called gillaroo but it remains to be demonstrated if it is really conspecific. Data in Ferguson (1986: 7) and Crozier \& Ferguson (1986: 461) suggest that the presence of the gillaroo in Lough Neagh is not well documented; this might be misidentified individuals of other stocks.

\subsubsection{2 ? Salmo taleri (Karaman, 1932)}

Trutta taleri Karaman, 1932: 2 (type locality: upper river Zeta at Niksic, lake Skadar basin, Montenegro; syntypes: LU)

? Trutta likana Karaman, 1932: 3 (type locality: stream Jesenice in Lika, river Save basin, Croatia; syntypes: LU) 


\subsubsection{Salmo trutta Linnaeus, 1758}

Awaiting a satisfactory resolution of the relationships among the lake and brook trouts of the Atlantic basin, I tentatively consider them as conspecific. If the lake trout is considered different, or if it is demonstrated to be distinct, it should be called S. lacustris; its synonymy is listed separately. Similarly, the synonymy of the three main lake-trout stocks, lake Genève, lake Konstanz and lakes Vänern and Vättern, are listed separately. If considered valid, their respective names should be $S$. lemanus, $S$. lacustris and $S$. truttula.

14.3.23.1 Marine, riverine and brook stocks [Salmo trutta Linnaeus, 1758 if recognised as a distinct species]

Salmo Trutta Linnaeus, 1758: 308 (based on Linnaeus [1746: 116, n. 308, Salmo ocellis nigris ...], Artedi [1738: gen. 12 [51], syn. 14 [24], spec. 48 [12], Salmo latus, maculis rubris nigrisque, cauda aequali; based on Willughby, (1686: 193), Ray (1710: 63, Northumbria) and material from Sweden] and Gronovius [1756: 12, n. 164, Salmo latus, cauda ...; locality: river Rhine near Basel, Switzerland]; type locality: "in fluviis Europae"; type material: NT)

Salmo Eriox Linnaeus, 1758: 308 (based on Artedi [1738: gen. $12[-]$, syn. 23, spec. 50 [12], Salmo maculis cinereis ...; based on Ray [1710: 63, Grey], Willughby [1686: 193, Grey, "Septentrionalibus Angliae"] and material from Sweden [Grälax]] and Linnaeus [1746: 116, n. 307]; type locality: "in Oceano Europae, parit in fluviis"; type material: NT)

Salmo Fario Linnaeus, 1758: 309 (based on Artedi [1738: gen. 12 [51], syn. 23 [24], spec. 51 [12], Salmo maxilla inferiore paulo longiore, maculis rubris] and Linnaeus [1746: 117, n. 309, idem]; type locality: "in Sveciae, Helvetiae fluviis"; type material: NT)

Trutta marina Duhamel, 1771: 200 (not binominal, rejected, ICZN, Opinion 859, 1968: 92)

Trutta fluviatilis Duhamel, 1771: 202 (not binominal, rejected, ICZN, Opinion 859, 1968: 92)

? Trutta Salmonata Rutty, 1772: 357 (work not consistently binominal, name not available (ICZN art. 11(c)); locality: river Liffey, Dublin County, Ireland)

? Trutta salmanata Strøm, 1784: 123 (type locality: Eger, Buskerud district, Norway; type material: NT)

Salmo albus Bonnaterre, 1788: 161 (based on Pennant [British Zoology, edition not stated, vol. 3: 302, White and Whiting]; type locality: river
Esk, England, U.K. / Carolina, U.S.A.; type material: NT ?)

Salmo sylvaticus Gmelin, 1788: 1367 (based on Bloch, [1782: 157, pl. 23; Wald- oder Steinforelle]; type locality: Norway / Berlin, Germany / Danzig [Gdansk], Poland; syntypes: lost, H.-J. Paepke, in litt., 1995)

? Salmo Stroemii Gmelin, 1788: 1374 (available by indication to Strøm [1762: 292]; type locality: "Denmark" [actually Søndmør, now in Norway]; type material: NT ?)

Salmo Fario Loënsis Walbaum, 1792: 61 (type locality: "lacu Lo Cornubiensi" [lake Lo in Cornwall, U.K.]; based on Borlase [1758: 263, The Lo-Trout]; type material: NT ?)

Salmo cornubiensis Walbaum, 1792: 65 (type locality: "lacu Lo Cornubiensi" [lake Lo in Cornwall, U.K.]; based on Borlase [1758: 263, pl. 76 fig. 1, The Lo-Trout]; type material: NT ?) Salmo albus Walbaum, 1792: 76 (based on Pennant [British Zoology, edition not stated, vol. 3: 302, White and Whiting; 1784: suppl. 140]; type locality: river Esk, England, U.K. / Carolina, U.S.A.; type material: NT ?; junior primary homonym and objective synonym of Salmo albus Bonnaterre, 1788: 161)

Salmo saxatilis Paula Schrank, 1798: 320 (available by diagnosis and indication to Bloch, 1782: 157, pl. 23, Wald- oder Steinforelle]; type locality: Berlin, Germany, by present restriction [originally: Bavaria, Germany (Paula Schrank) / Norway / Berlin, Germany / Danzig (Gdansk), Poland (Bloch)]; syntypes: lost, H.-J. Paepke, in litt., 1995)

Salmo faris var. Forestensis Bloch, in Schneider, 1801: 400 (available by diagnosis and indication to Bloch, 1782: pl. 23 [Wald- oder Steinforelle]; type locality: Norway / Berlin, Germany / Danzig [Gdansk], Poland; syntypes lost, H.-J. Paepke, in litt., 1995)

Salmo gadoïdes La Cepède, 1803: 224, 228 (type locality: pond in Trouville near Rouen, France; type material: NT)

Salmo cumberland La Cepède, 1803: 696 (type locality: lakes of Cumberland and Scotland, U.K.; type material: NT)

Salmo cambricus Donovan, 1806: pl. 91 (type locality: Glamorganshire and Carmarthenshire, Wales, U.K.; type material: NT ?)

Salmo Phinoc Shaw, 1804: vol. 5 (1): 54 (based on Pennant [British Zoology, edition not stated, vol. 3: 302, White and Whiting]; type locality: river Esk, England, U.K.; type material: NT ?)

Salmo Levenensis Neill, 1808: 541 (nomen nudum; locality: Loch Leven, Scotland, U.K.; author- 
ship usually attributed to Walker, but Neill is actual author)

Salmo Levenensis Walker, 1812: 373 (nomen nudum; locality: Loch Leven, Scotland, U.K.)

Salmo Taurinus Walker, 1812: 373 (nomen nudum [but the indication "as red in the fish as salmon" could be interpreted as a description]; locality: Loch Leven, Scotland, U.K.)

Salmo Montana Walker, 1812: 374 (nomen nudum; locality: Loch Leven, Scotland, U.K.)

Salmo caecifer Parnell, 1838b: 306, pl. 30 (type locality: Loch Leven, Scotland, U.K.; syntypes: LU); also Parnell, 1839b: 154, pl. 8

Salmo Levenensis Yarrell, 1839: suppl. vol. 2: 9, fig. (type locality: Loch Leven, Scotland; syntypes: LU ?; author indicated as Walker, but this refers to Salmo levenensis Neill, 1808: 541 which is a nomen nudum)

Fario argenteus Valenciennes, in Cuvier \& Valenciennes, 1848: 294, pl. 616 (based on specimens of unstated locality and on several earlier accounts; here restricted to Atlantic drainage of France; syntypes: MNHN)

Salar Ausonii Valenciennes, in Cuvier \& Valenciennes, 1848: 319, pl. 618 (type locality: several streams of Normandie entering the sea near Dieppe and Caen, France; several other localities listed pp. 323-325; syntypes: MNHN)

Salar spectabilis Valenciennes, in Cuvier \& Valenciennes, 1848: 340 (type locality: Russia; syntypes: MNHN [3])

Salar Gaimardi Valenciennes, in Cuvier \& Valenciennes, 1848: 341 (type locality: Iceland; syntypes: MNHN)

Salar Bailloni Valenciennes, in Cuvier \& Valenciennes, 1848: 342, pl. 619 (type locality: river Somme at Abbeville, France; holotype: MNHN)

Salmo Estuarius Knox, 1854: 29 (type locality: mouth of the Tyne of East Lothian in North Britain / river Nith of Dumfriesshire, between the bridge and the Solway Frith, Scotland / near Bute among the Western Isles, U.K.; syntypes: LU) from A. Wheeler, pers. comm.; also Knox, 1855: 4662

Salar Ausonii var. semipunctata Heckel \& Kner, 1858: 251 (colour variety, not linked with any locality; infrasubspecific, name not available)

Salar Ausonii var. parcepunctata Heckel \& Kner, 1858: 251 (colour variety, not linked with any locality; infrasubspecific, name not available)

Salmo fario var. major Walecki, 1863: 342, 362 (infrasubspecific, name not available; nomen nudum; locality: Poland)

Salmo brachypoma Günther, 1866: 87 (type lo- cality: rivers Forth, Tweed and Ouse, England, U.K.; syntypes: BMNH [7])

Salmo gallivensis Günther, 1866: 88 (type locality: Ballinahibch fishery, Galway, Ireland; syntypes: BMNH [8])

Salmo orcadensis Günther, 1866: 91 (type locality: Loch Stennis, Orkney Islands, U.K.; syntypes: BMNH [2])

Salmo mistops Günther, 1866: 105 (type locality: river Eidfjord, Norway; syntypes: BMNH [4])

Salmo polyosteus Günther, 1866: 111 (type locality: Lapland; syntypes: BMNH [8])

Salmo Islayensis Thomson, 1873: 86, figs. 60-61 (type locality: Loch Na-Maorichen, Islay island, U.K.; syntypes: LU [at least 4])

Trutta marina Moreau, 1881: vol. 3: 537 (type locality: rivers Meuse, Seine and Loire, France; syntypes: MNHN)

Salmo lacustris forma fecunda, minor Fatio, 1890: 343 (infrasubspecific, name not available; locality: streams in Switzerland)

Salmo lacustris forma fecunda, major Fatio, 1890 347 (infrasubspecific, name not available; locality: lakes in Switzerland)

Salmo lacustris var. Rhenana Fatio, 1890: 347 (type locality: basin of river Rhine downriver of the falls [of Schaffhausen], Switzerland, Germany and Austria; syntypes: MHNG 806.96 [1])

Salmo lacustris var. septentrionalis Fatio, 1890 354 (unnecessary substitute name for $S$. 1 . var rhenana Fatio, 1890: 347)

Salmo trutta forma major Roule, 1925: 98 (infrasubspecific, name not available; locality: France)

Salmo trutta forma minor Roule, 1925: 98 (infrasubspecific, name not available; locality: France) Nomenclatural notes. See discussion of Salmo saxatilis under S. labrax.

Salmo punctatus Nilsson, 1832 (p. 6) sometimes incorrectly appears in the literature. The name is explicitly indicated as Cuvier's (1829) species by Nilsson.

Fowler (1974: 62) listed a "Salmo eriox pallayeri Hoyer \& Michalski, 1915: 212" from "Cracow, Austria" [Krakow is in Poland]. This name is nowhere used by Hoyer \& Michalski (1915). It is arguable whether the name $S$. pallayeri Fowler, 1974 might be available by indication to Hoyer \& Michalski (1915), but I treat it as an erroneous subsequent spelling of $S$. pallaryi Pellegrin, 1924. Four lines below, Fowler lists, as a synonym of his $S$. e. pellegrini, "S. pallayeri Moyer and Michalski: Pellegrin ... 1924 ... (misidentification)". Pellegrin's (1924a) S. pallaryi 
is definitively not a misidentification; it clearly is an original name, is unambiguously named for the collector Paul Pallary, and nowhere refers to any paper by Hoyer \& Michalski. Fowler apparently confused or mixed data from different sources.

14.3.23.2 Great European lakes [Salmo lacustris Linnaeus, 1758 if recognised as a distinct species]

\subsection{Lake Genève [Salmo lemanus} Cuvier, 1828 if recognised as a distinct species]

Salmo Lemanus Cuvier, 1829: 303 (type locality: lake Genève "and some neighbouring lakes", France and Switzerland; syntypes: MNHN)

Trutta variabilis Lunel, 1874: 146, pls. 16-18 (type locality: lake Genève and its tributary streams and outlet: Rhône, Versoix and London, Switzerland; syntypes: MHNG 816.06 [1], 816.07 [1], 807.35 [1])

Salmo lacustris var. Lemani facies arvensis Fatio, 1890: 350 (infrasubspecific, name not available; locality: river Arve, tributary of river Rhône in Genève, Switzerland)

Nomenclatural notes. "Salmo fario var. profundus Fuhrmann, 1903" listed by Fowler (1974: 64) does not exist. Fuhrmann (1903: 332) actually discussed Salvelinus salvelinus var. profundus.

14.3.23.2.2 Lake Konstanz [Salmo lacustris Linnaeus, 1758 if recognised as a distinct species]

Salmo lacustris Linnaeus, 1758: 309 (based on Artedi [1738: gen. $12[-]$ syn. 25 [12], Salmo cauda bifurca ...; based on Rondelet (1555: 161; lake Genève), Gesner (no year given; in 1575: 189, localities: "Sitten in Wallis" [Sion, Valais], lakes Genève, Zürich and Konstanz), Aldrovandri, 1613]; type locality: "in Helvetia", restricted here to lake Konstanz, a locality included in the account of Gesner, 1575: 189; type material: NT [Wheeler, 1989: 161])

Salmo Illanca Wartmann, 1783: 55 (type locality: lake Konstanz and river Rhine between lake Konstanz and Rheinwald, Switzerland; syntypes: ZMB ?)

Salmo rappii Günther, 1866: 82 (type locality: lake Konstanz, Germany, Switzerland and Austria; syntypes: BMNH [3])

Salmo lacustris var. Rhenana facies Bodensis Fatio, 1890: 348 (infrasubspecific, name not available; locality: lake Konstanz, Switzerland, Germany and Austria)
Salmo lacustris forma sterilis lacustris Fatio, 1890: 358 (infrasubspecific, name not available; locality: lakes Konstanz, Thun, Vierwaldstätter, Neuchâtel, Zürich and Genève, Switzerland)

14.3.23.2.3. Lakes Vänern and Vättern, Sweden [Salmo truttula Nilsson, 1832 if recognised as a distinct species]

Salmo Truttula Nilsson, 1832: 5 (type locality: sea around Göteborg and lake Vättern, Sweden; syntypes: LU)

Salmo microps Hardin, 1862: 383 (type locality: lake Venern [Vänern], Sweden; syntypes: LU)

Salmo venernensis Günther, 1866: 110 (type locality: lake Wenern [Vänern] and river Gotha [Göta älv], Sweden; syntypes: BMNH [7])

14.3.24? Salmo visovacensis Taler, 1950

Salmo visovacensis Taler, 1950: 118 (type locality: lake Visovac, lower river Krka, Dalmatia; syntypes: LU)

\subsubsection{5? Salmo zrmanjaensis Karaman, 1938}

Salmo farioides zrmanjaensis Karaman, 1938: 138 (type locality: river Zrmanja near Obrovac and Zegar, FYROM; syntypes: LU)

\subsubsection{Salmo sp. 'dollaghan'}

14.3.27 Salmo sp. 'salmon trout'

Systematic notes. These are two of the four sympatric species of Lough Neagh, Ireland, for which formal names are not yet available. See discussion, pp. 125, 132 .

14.3.28 Names commonly placed in the synonymy of Salmo trutta but representing the Caucasian and western Asian lineage (probably several species). List not exhaustive, several species usually recognised as valid not included

Salmo spurius Pallas, 1814: 343 (type locality: river Terek, Caspian Sea basin, Russia; restricted by Berg, 1948: 242, 247 [material from river Neva, Russia, possibly not conspecific; Berg, 1948: 205]; syntypes: LU)

Salmo orientalis M'Clelland, 1842: 585 (type locality: "northern declivities of the Hindoo Koosh, and river Bamean, one of the tributaries of the Oxus, Afghanistan"; types: LU; junior homonym of Salmo orientalis Pallas, 1814: 367) Salmo oxianus Kessler, 1874a: 35, pl. 5 fig. 1 (Alai, basin of the upper Amu-Darya [Tadzhikistan ?]) from Berg, 1948: 258

Salmo caspius Kessler, 1877a: 62 (type locality: 
lower river Kura near Bozhii Promysel fishing ground, Azerbaidjan) [Berg, 1948: 242]; syntypes: LU)

Salmo lacustris var. Romanovi Kawraisky, 1896 43,79 , pl. 8 figs. $1-2$ (type locality: lake Tabiszchuri, Transcaucasia; syntypes: ZMT ? [20])

Salmo trutta aralensis Berg, 1908b: 317 (type locality: Aral Sea at mouth of river Amu-Darja, Taldyk straight [near the Ulkun-Darya, one of the mouths of the Amu; Berg, 1948: 249], Kazakhstan; holotype: ZISP 14418)

Salmo trutta aralensis morpha fario Berg, 1916a: 47 (infrasubspecific, name not available; junior homonym of Salmo fario Linnaeus, 1758)

Salmo trutta caspius morpha fario Berg, 1932a: 113 (infrasubspecific, name not available; junior homonym of Salmo fario Linnaeus, 1758)

Salmo trutta caspius morpha lacustris Berg, 1932a: 113 (infrasubspecific, name not available; junior homonym of Salmo lacustris Linnaeus, 1758)

Salmo trutta ezenami Berg, 1948: 253 (type locality: lake Ezenam in Dagestan, Caucasus; holotype: ZISP 28356)

Salmo trutta abanticus Tortonese, 1954: 19, fig. 2 (type locality: lake Abant [Abant Gölü], Turkey; holotype: MSNM 5031, Conci \& Michelangeli, 1974: 222)

Salmo trutta ciscaucasicus Dorofeyeva, 1967: 15 (type locality: river Keyranchay, eastern Ciscaucasia, Russia; holotype: ZISP 26244)

\subsubsection{Salmothymus obtusirostris (Heckel, 1852)}

Salar obtusirostris Heckel, 1852a: 367, pl. 9 (type locality: streams Zermagna and Salona, Spalato [Split], stream Verlica near Imosky, Dalmatia [Croatia]; syntypes: NMW 66413 [1], 66427 [1], 66456 [2], 78817 [1], ZMB 3722 [1])

Thymallus Microlepis Steindachner, 1874: 172 (type locality: hill stream near Vergoraz, Dalmatia; syntypes: NMW [not yet located; B. Herzig, pers. comm., 1995]; junior secondary homonym of Salmo microlepis Günther, 1866 when placed in genus Salmo); also Steindachner, 1875: 367 , pl. 2

Salmo obtusirostris var. oxyrhynchus Steindachner, 1882a: 75, pl. 4 (type locality: rivers Narenta [Neretva] and Grupa near Metcovich [Metković], Croatia; syntypes: NMW 82297 [2]; junior homonym of Salmo oxyrinchus Linnaeus, 1758 [ICZN art. 58])

Trutta obtusirostris salonitana Karaman, 1927: 256 (type locality: river Jadro near Split, river Krka near Knin, Croatia; syntypes: LU)

Trutta obtusirostris krkensis Karaman, 1927: 259, fig. 4 (type locality: river Krka near Knin, Croatia; syntypes: LU)

Trutta montenigrina Karaman, 1933: 104 (type locality: river Morace, Montenegro; syntypes: LU) Salmo zetensis Hadzisce, 1960: 49 (type locality: river Zeta near Danilovgrad, a tributary of Skadar lake, Montenegro; syntypes: MMNHS [3])

Systematic notes. According to Behnke (1968: 8) Trutta montenigrina could be a valid species of Salmothymus, while Karaman (1966: 31) seems to consider it a synonym of Salmo farioides or $S$. taleri (unless he means that some specimens are misidentified ?). Vukovic \& Ivanovic (1971) treat it as a valid species of Salmo.

Karaman (1927) described two subspecies ( $S$. o. salonitana, S. o. krkensis) with the same locality (river Krka near Knin). If this were real, it might be indicative that more than one species is involved.

Nomenclatural notes. Hadzisce (1960: 49) proposed Salmo zetensis as a replacement name for Trutta obtusirostris letnica of Karaman (1932: 2) which he considered preoccupied by Salmo letnica (Karaman, 1924). Karaman (1932) actually did not propose a new name but used a new combination of his 1924 name. Hadzisce considered that the two uses of the name applied to different species. I treat Hadzisce's action as the description of a new species.

\section{Salvelinus}

The main problem with the systematics of the genus Salvelinus is the existence of a diversity of stocks which have been variously called species, subspecies, forms, ecomorphs, etc. The classification adopted here roughly follows Behnke's (1972, 1980) ideas, but with many important modifications, in order to adjust to the PSC. A problem with Behnke's review is that it seems to be based on recognising the past existence of several distinct stocks which repeatedly colonised the area; this necessitates numerous elaborate, ad hoc zoogeographic explanations. In my view, species (diagnosable entities) should first be recognised before working out the evolution, phylogeny and zoogeography of the group. Another problem is that Behnke used the BSC (he pointed several times to problems resulting from a strict adherence to the BSC; e.g., 1972: 644) and this leads to some systematic decisions which cannot be followed, like accepting two sympatric and reproductively isolated stocks as conspecific. Behnke (1972: 644) was concerned that "each of the numerous sym- 
patric sibling species could be recognised as a full species with a unique [name], resulting in a proliferation of names until they [become] meaningless" [note the semantic problem of using the word species with two potentially different meanings]. Similar concerns for keeping low the number of species have already been addressed above (see Subspecies, p. 17) and this argument is not objectively acceptable; if systematics aims at describing nature's diversity, it has to describe diversity regardless of how inconvenient or complex this diversity may be and should not artificially reduce it. If, using one or another species concept, one considers that there are, let us say 60 species in a genus, one has to handle 60 species and not to find ways of reducing their number. Some genera are speciose or have lineages quickly exhibiting striking differences once isolated, and we have to adjust our thinking to this and not to squeeze the fishes into what we want them to be. (Or why not suppress the recognition of hundreds of vintages because an untrained palate does not perceive differences?).

A few lines further, Behnke (1972: 644) stated that "certainly from a fisheries management point of view, sympatric populations with ecological and life history distinctions should be treated as distinct species". To me, it does not make sense to recognise different stocks as biological species and to manage them as species, but not to call them species; the coherent way to handle the problem is that an entity recognised as species using one or the other species concept be called species and be handled as such in systematics, ecology and resources management.

The internal consistency of systematics requires that the basic principles be applied universally; to introduce ad hoc principles for the sake of the management of a single group may make sense in the context of national legislation, etc., but certainly not for global systematics. It is not to fisheries management rites and administrative rules to decide what a species is, but to taxonomy and biology (or, ideally, to the fish !). If fisheries management has a biological base (is not only an administrative exercise), undoubtedly it will be able to adapt.

Central Europe. Behnke (1980: 462) recognises the Alpine and Scandinavian groups of populations as distinct (diagnosable) lineages with independent histories. This corresponds to the present species concept and they are accepted here as distinct species.

In sub-Alpine and Alpine lakes, various stocks have traditionally been recognised. Up to three have been reported from a single lake. For example, wildfangsaibling, schwarzreuter and tiefseesaibling were originally recognised in lake Königssee. Schindler (1940) has shown that wildfangsaibling and schwarzreuter are most likely to be just different year classes of the same species (Heckel \& Kner, 1858: 283, had already reached this conclusion). The deepwater stock (tiefseesaibling) has not been discussed by Schindler, but he considered it a distinct race without further discussion. Three stocks were also recognised in lake Attersee (Buresch, 1925: 108; Haempel, 1924: 130) of which a deepwater one survives now (Brenner, 1980). The Ammersee community has been considered similar to the Königssee one, but actually it does not seem to be. Haempel distinguishes a schwarzreuter and a wildfangsaibling, itself divided into a 'sterile' [pre-reproductive] midwater and a reproducing bottom forms and it seems that altogether a single stock was involved.

In cases of sympatry, this often involves a normal and a deepwater stock. Such cases have been reported at least from lakes Konstanz, Neuchâtel, Walchensee, Ammersee, Königssee, Tegernsee, Achensee, Plansee, Attersee and Traunsee (Buresch, 1925; Haempel, 1930: 82; Schindler, 1951; Behnke, 1972: 641), but some are dubious (see above comment on Attersee). In lake Konstanz, the two stocks differ in habitat, life history, external morphology (Schillinger, 1901; Dörfel, 1974) and osteology (Cavender, 1980; Behnke, 1980: 464), and Behnke (1980) recognised them as distinct species. While it seems possible that in several lakes deepwater and normal stocks are distinct species, it has never been demonstrated for any lake other than lake Konstanz and it has never been demonstrated that all the deepwater stocks belong to the same species. The likelihood that different deepwater species exist in different lakes cannot be excluded and this should be investigated as soon as possible. Actually, only few have been described with some detail (e.g., lake Neuchâtel; Quartier, 1951). Several of the deepwater stocks apparently are already extinct (e.g., lake Neuchâtel; Rubin \& Buttiker, 1987; Buttiker, pers. comm., Feb. 1996) or seriously threatened. The way Haempel (1930: 184) suggested they be treated is typical of the fisheries practices of the time: "because of its small size (stunted form) it has no fisheries value and should be replaced by a fast-growing form". In lake Konstanz, it is the 'normal' stock which is becoming rare (Hartmann, 1994).

Some authors have assumed that the normal 
and deepwater species actually are merely "local forms [of a single species] created in response to different environmental and feeding conditions" (Brenner, 1980: 767, for the three forms of lake Attersee). I shall not discuss the semantic problem in the use of the word 'created'. If the two species were mere forms of a single species, any individual should have the potential to develop randomly into any stock. The fact that in lake Attersee two stocks disappeared and only the deepwater stock survived "as a result of overfishing" (Brenner, 1980: 770 ) could indicate that stocks can be fished to extinction (that is selectively removed) without recruitment from the offspring of the remaining (deepwater) stock. This could demonstrates that there were at least two stocks (deepwater and 'normal') which were diagnosably distinct and with a parental pattern of ancestry and descent; that is, two distinct species. The proof that extinction is effectively a consequence of overfishing should also be given; without monitoring of other possible factors, the conclusion is always open to question. An other explanation is the existence of alternative life-history styles, whose expression depends of environmental conditions; environmental changes might result in the expression of a single of the alternative life-history styles (see Balon, 1989; Bruton, 1989). To be acceptable, this explanation requires a demonstration, case by case, that the 'forms' represent alternative life-history trajectories of the same species; it certainly cannot be a theory automatically valid for all possible species. This has not (yet) been done for a single central European stock.

The status of populations of high-altitude lakes like lake Dösener (see Balon \& Penczak, 1980) and other Alpine lakes is more difficult to evaluate, especially as it is likely that most of them are the result of stocking. [Contrary to Balon \& Penczak (1980: 792), I do not see problems at accepting stocking as the origin of such populations. Balon \& Penczak assume that "to reach a high altitude Alpine lake in the 16th century, with a heavy load [...], would have been even more difficult and adventurous than nowadays, for roads barely extended into the lower central valleys". I think that what might be considered adventurous for a contemporary tourist or untrained person was certainly not for a population which was living in and from the mountains, which was earning its livelihood from guarding herds in the mountains, from hunting or from carrying goods across passes (even nowadays, how much of the 'adventures' of modern, western travellers are just the normal life [often without alternative] of most of the world's population ?). They certainly were accustomed to using pathways probably more diverse and better maintained than nowadays, they used to carry heavy loads with unsophisticated equipment, as porters still do in mountainous parts of the world. The absence of records [accepting the 16th century as a possible stocking date, although later dates and repeated stockings cannot be excluded in many lakes; until the 16th century, climate was warmer and forest limit higher (Hantke, 1978: 382); Alpine glaciers reached a maximum historical extent in the period 1600-1650 (Holzhauser, 1982: 123)] is not only not surprising but also expected, as the decision of stocking is unlikely to have been taken by an administration or a centralised body but by individuals or small local communities; except for the possible clergy and officers (garrison and customs), these populations generally were illiterate. Today, stocking and introductions are going on all over the world for a variety of reasons by a variety of agencies and individuals and it is still difficult or impossible to find accurate records of these operations. People spending the summer guarding herds at high altitudes could certainly perceive the advantage of stocking fish to diversify their diet. Admittedly, this was all but an easy job. In order to accept a theory of natural colonisation of high altitude lakes, one would need to have a reasonable demonstration that a fish basically occurring in lakes would enter high gradient mountain streams and torrents]. Several of these high-altitude lakes are located at the edges of glaciers and are of very recent age. Longitudinal movements of several Alpine glaciers are quite well documented in historical ages. Alpine glaciers had a maximum extension in the 17th century and have significantly regressed in the last 150 years (see Hantke, 1978: 382-386 for general discussion, p. 64 for details of the $1480 \mathrm{~m}$ regression of Trient glacier between 1845 and 1956 ; 1980: 348-349, 640-642 for details of the $2200 \mathrm{~m}$ regression of Rhône glacier between 1818 and 1980; for further data see Maisch, 1982; Gamper \& Suter, 1982; Holzhauser, 1982), so that one has to demonstrate that natural colonisation could happen given today's climates and topography (admittedly, stream beds have been profoundly altered by man during the last 100 years). Whichever theory is accepted, the key problem is to determine the origin of the transplanted or colonising stocks before arguing on their taxonomic status.

Scandinavia. In Scandinavia, normal, dwarf and anadromous stocks exist, in several cases in sym- 
patry (see Johnson, 1980: 26-32, for an overview). They have been considered as either ecological polymorphism (Reisinger, 1953; Savvaitova, 1980: 291, 1983) or different species, evolved in sympatry (Savvaitova, 1983: 7), in sympatry from slightly different succeeding invaders (Behnke, 1972: 643) or allopatry (Nyman, 1972; Nyman et al, 1981). Behnke (1980: 459) stated that there are "numerous examples of sympatric and reproductively isolated forms [...] in many lakes". He considered that "sympatric occurrence with reproductive isolation is not sufficient evidence by itself for species recognition. Innate reproductive homing behaviour in salmonid fish can maintain reproductive isolation between two or more stocks with little genetic divergence" (p. 450). No species concept would recognise two sympatric and reproductively isolated stocks as conspecific. Furthermore, I disagree with the use of arguments like 'minor divergence' or 'major difference' because they are subjective. Stocks are either diagnosable or not; distinct lineages or not. But degrees, levels, ratios or distances are not acceptable arguments to decide if two stocks are conspecific or not, because "there is no theory to suggest that a trait must be of a certain quality or magnitude to provide historical information or to delimit species" (McKitrick \& Zink, 1988: 10). "The only rule [for the 'systematic value' of characters] is that there is no rule or only rules with many exceptions" (Wagenitz, 1953: 94). There are no characters which are 'family characters' or 'species characters'. Morphological characters used to distinguish different dog races are of the magnitude of those characters used to distinguish genera or families in other animal lineages. (See also p. 12). Similarly, there is no theory to suggest that a species must have a minimum distribution area.

In this context, it is important to compare categories which can be compared. Two cases should be distinguished. Firstly, there are populations with two or more discrete stocks in which individuals permanently (that is as early as they can be recognised; we have no data on detailed morphology of fry and early juveniles) exhibit the same phenotype. Secondly, there are populations in which individuals of one of the morph (usually large piscivorous ['charr-ivorous'] individuals) first go through the normal stages of another morph (usually small planktivorous individuals); the Jan Mayen stock (Skreslet, 1973), the Salangen stock, northern Norway (Nordeng, 1983), the Königsee's schwarzreuter and wildfangsaibling (Schindler, 1940), and possibly the lake Tasersuaq, Greenland, stock (Riget et al., 1986) belong to this category. Obviously, these two categories of populations are not equivalent in the framework of a systematic discussion. [Clearly there are semantic problems resulting from the use of the word polymorphism for a variety of phenomena which may have very little in common. Clear concepts are needed and the discussion and analysis of 'polymorphism' could only benefit from introducing different words to designate different phenomena, e.g., 1) alternative appearances of a single species which a given individual will exhibit during all its life [genetic polymorphism apparently belongs here]; 2) the successive appearances which a given individual will obligatorily or facultatively displey durings its life, 3) sexual polymorphism, 4) colonial polymorphism, etc. I use here polymorphism for the first category only. Another problem is when authors compare polymorphism in different groups in which different species concepts are used; e.g., the comparison of polymorphism in some cichlids with polymorphism in some salmonids is questionable as it actually seems to be a comparison of morphs of a single species on the one hand with different, successive phases of a species or with several independent (possibly not each other closest related) species on the other hand].

Sympatric and supposedly genetically distinct stocks are reported from Batsvatn reservoir, Norway (Klemetsen \& Grotnes, 1980), lakes Sirdalsvatnet and Selura, Norway (Hindar et al., 1986), lakes Björkvattnet, Stora Rösjön and Yraf, Sweden (Nyman, 1972: 116-120; Nilsson \& Filipsson, 1971; Svedäng, 1990).

Hindar et al. (1986: 271) refer to rearing experiments by Nordeng (1983) and life history studies by Skreslet (1973), Hindar \& Jonsson (1982) and Jonsson \& Hindar (1982) as "indicating that all of the naturally occurring morphotypes may be found in the offspring $[\mathrm{s}]$ from each of the parental types". Actually, Skreslet (1973) is uninformative in the present context, his limited data merely suggesting that a single stock exists in Jan Mayen Islands with some larger-growing individuals, and Hindar \& Jonsson (1982) and Jonsson \& Hindar's (1982) life history data do not contribute to this argument. Hindar \& Jonsson (1982: 1043) merely concluded their study by referring to breeding experiments of Nordeng (1983) and by the hypothesis that "the occurrence of different charr morphs depends on the number of available niches during the growth season". Nordeng (1983) is usually quoted as having demonstrated through "rearing and transplantation experiments [that] three coexisting stocks belong to the same gene pool" and 
that the offspring of each stock can develop into any of the three. Even if this were the case, one should be cautious before extrapolating his results to all other cases of 'sympatric stocks'. Nordeng studied a stock from the Salangen basin, northern Norway (about $69^{\circ} \mathrm{N}$ ) where three morphs were recognised (small resident, large resident, anadromous); they do not appear to be morphologically distinct. I think that Nordeng conclusively showed that in this population (and possibly others) the morphs are development stages of the same stock. But in the case of the Salangen population it was already known before the study that "some resident char transform into anadromous char after spawning 1-3 times (Nordeng, 1961)" (Nordeng, 1983: 1375, 1382) and this shed a different light on this local case. (Admittedly this is supposed to concern only $9 \%$ of the anadromous charrs). We are not told if and how the three 'morphs' differ morphologically and how the parents were classified. If the offsprings were mature in NovemberDecember and in the size range $10.5-21.9 \mathrm{~cm}$ they were called small resident and between 22.0 and 28.0 large resident; mature specimen were removed and the non-mature ones and smolts kept for one more year and checked again; we are not informed how they were classified as anadromous [probably smolt]. Table 3 is supposed to show that out of 2705 offsprings of two small resident parents $67.9 \%$ were small residents, $10.8 \%$ large resident and $21.3 \%$ anadromous; to me, this table means that out of two small size parents, in NovemberDecember $67.9 \%$ of the offsprings were mature at a size of $10.5-21.9 \mathrm{~cm}, 10.8 \%$ at $22.0-28.0 \mathrm{~cm}$ and $21.3 \%$ belong to a category which is probably smolt; nothing more. Clearly, extrapolation of these results to areas with morphologically different, sympatric stocks is not defendable.

From somewhat similar breeding experiments with lake Stora Rösjön (Sweden) stocks, Svedäng (1990) concluded that dwarf and 'normal' charr forms "separate in several, partly inherited lifehistory traits". In this case too, the author is vague on the morphological definition of the stocks. These results, however, tend to support the hypothesis that what is true for the stocks of one lake cannot be automatically extrapolated to other stocks. Laboratory-rearing experiments of the lake Thingvalla (Iceland) 'morphs' indicate that morphological differences have a genetic basis (Skúlason et al., 1989: 296).

Hindar \& Jonsson (1993) also concluded that 'dwarf' and 'normal' charr in lake Vangsvatnet (Norway) belong to a single population, but at the same time they refer to differences in mortality, growth rates and mouth shape. The morphological descriptors (presence/absence of parr marks and jaw lengths) are too few and presented in an inconclusive way (especially when raw jaw measurements in $\mathrm{mm}$ are compared [instead of standardised ratios], without precise anatomical definitions). Despite this reservation, I agree with most of their discussion, except their taxonomic conclusion which relies on "amount of genetic differentiation" (see below; also p. 19). Joensen \& Tåning (1970: 71) mention that specimens from lake Leynavatn, Faroes, transferred to a reservoir changed "their growth and appearance", but did not provide details. In conclusion, available data indicate that a single polymorphic species is present in some lakes, but there is no argument to justify the extrapolation that a single (and the same) species is involved in all water bodies with several sympatric stocks.

On the basis of the allele frequency distribution at a single esterase locus, Nyman (1972) and Nyman et al. (1981) hypothesised that three species are involved which invaded Scandinavia after the last glaciation, some or all of them occasionally occurring in sympatry in some lakes while there has been introgression in other lakes. Behnke (1980) questioned this interpretation and pointed to the fact that each stock (in a pair of sympatric stocks) seems more closely related to the sympatric stock than to any allopatric stock. I doubt that allele frequencies at a single locus can be used alone to define species. Nyman's three species are not defined by any other character and his nomenclatural choices are weakly supported (we are not provided with sound argument to justify using the names S. salvelinus Linnaeus, 1758 [type locality Austria] and S. stagnalis Fabricius, 1780 [p. 175; type locality: Greenland "in aquis montanis remotioribus"] for Swedish material.

From an electrophoretic study of stocks from 15 Norwegian lakes, Hindar et al. (1986) conclude that a single species is involved. The methods and concepts used, and the presented data however, are very difficult to include in a systematic analysis. First of all, their species concept (if any) is not clear (they seem to accept that sympatric stocks with "restricted gene flow" are conspecific, p. 281). I already mentioned in the Introduction (p. 19) a general problem with genetic data presented as genetic distances: admittedly, they are sometimes informative in terms of chronology or relative dating of lineages, but they are uninformative in terms of lineage recognition as long as they cannot be analysed together with any other characters. Electrophoretic data of 10 populations 
from Sweden are reported by Andersson et al. (1983); they have examined material from a locality were two sympatric (but not morphologically distinct !) stocks had been reported and found a single population (p. 90). Their data do not support Nyman et al. (1981) three species theory (but their material came from different localities).

I think that, as for some other groups, one problem with Salvelinus is that some researchers have tried to find a global theory to explain all individual cases. Here again, what seems to be the same situation might have independent and different histories. It is possible that in lake Vangsvatnet normal and dwarf Salvelinus are 'morphotypes' of a single species which evolved in situ (as suggested by data in Hindar et al., 1986) or alternative lifehistory styles. But the electrophoretic data in Hindar et al. (1986) seem to indicate that the dwarf stock of lake Sirdalsvatnet is closely related to the normal stock of lake Selura, and the normal stock of lake Sirdalsvatnet is closely related to the dwarf stock of lake Selura. If this is confirmed, it means that in both lakes, the two stocks (which apparently are diagnosable) belong to two independent lineages which maintain genetic identity; that is, there is no interbreeding. With any species concept these have to be called two species. The key problem is to know if a given species is restricted to one basin or if it occurs outside. If the relationships among the stocks in lakes Sirdalsvatnet and Selura are confirmed, there are three alternatives: they can be considered as four, three, or two species. The situation where four species are recognised is trivial and does not need further discussion.

If three species are recognised, this would mean that one species would include the normal stock of one lake and the dwarf stock of the second lake, the two remaining stocks being two species. If two species are recognised, it would mean that the normal stock of one lake is conspecific with the dwarf stock of the second lake while the dwarf stock of the first lake is conspecific with the normal stock of the second lake. While these alternatives cannot be excluded on purely biological grounds, they are quite confusing for practical purposes especially as each stock is in any case diagnosable. In such a case, they should be considered as four species.

With the data now available, it could as well be hypothesised that the present Salvelinus species originate from several (at least two, put possibly more) ancestral stocks. They had (and most probably still have) the potential to develop two (or more ?) 'morphotypes' or life-history styles and in some cases they actually developed them in sympatry but in other cases (e.g., in presence of another species) a single 'morphotype' is developed. Under this 'model', one can understand (or predict) a great number of combinations, including monospecific monomorphic, monospecific dimorphic (one species with two morphs), bispecific monomorphic (two species with the same morph), bispecific dimorphic (two species, each with a distinct morph), bispecific trimorphic (two species, one with two morph, one with a single morph), bispecific quadrimorphic, trispecific polymorphic, etc. populations. On top of this, hybridisation might in some instances have made the pattern obscure. Under this 'model', the same two species could occur in sympatry possibly with each always developing the same 'morphotypes', although there is no theoretical reason why the 'morphotypes' could not be developed in a random or opportunistic way, lake by lake. Genetically, this hypothesis probably cannot be tested in terms of genetic distance but only with methods resulting in data compatible with standard systematic analysis, that is in terms of derived and primitive states of a given character (see p. 10).

I would imagine that it is possible (but expensive and labour intensive) to obtain this kind of data. With this data and individual biological information for the population of each water body, it should be possible, if not to understand the evolution of the whole group, at least to recognise the main lines. At this stage, I predict that a detailed morphological examination will allow a reasonable identification of the different species. Finally, I should note that despite the implicit acceptance by various authors that there have been detailed morphological or morphometric work done on fishes of this genus, I could find only very few which can compare with the kind of work needed for even the rawest systematic work (notable exceptions are: Cavender, 1980; Balon \& Penczak, 1980; Smith \& Stearley, 1993). Most discussion of 'morphology' rarely discuss anything but gill-raker and pyloric caeca counts presented in a more or less confusing way.

Iceland. Stocks from Iceland have usually been considered as conspecific with those from Europe, especially Scandinavia, but I could not find a real comparison. Lake Thingvalla is noteworthy for the sympatric occurrence of four stocks; most aspects of their biology have been the subject of detailed studies summarised by Sandlund et al. (1992), except their taxonomy which received only a simplistic treatment. The stocks differ in 'morphology', coloration, colour pattern (Skúlason et al., 
1989; Snorrason et al., 1994), habitat (Sandlund et al., 1987), life history (Jonsson et al., 1988), food (Malmquist et al., 1992), feeding behaviour (Skulason et al., 1993), spawning season (Sigurjónsdóttir \& Gunnarsson, 1989: 171), etc. Sandlund et al. (1992: 346) concluded that "the pronounced phenotypic and ecological differences and the fact that we have found no signs of interbreeding ... suggest reproductive isolation". Under any species concept this indicates that several species are involved. Despite this, they concluded (p. 345) that the small genetic distance indicates that the four stocks are conspecific (see also Magnusson \& Ferguson, 1987). I have discussed above (pp. 19, 148) the non-significance of 'small' genetic distances in making taxonomic decisions. The data of Sandlund et al. (1992: 343) actually show that some alleles are unique to some stocks (e.g., Gpi3(92) is unique to SB stock, $\operatorname{Pgm} 2(114)$ to $\mathrm{SB}$ and LB, etc.) and could be considered as apomorphies or synapomorphies if outgroup information were available.

A problem with most discussions on sympatric salmonid and coregonid stocks seems to be that some questions are not asked in the appropriate sequence, and this probably results from a lack of familiarity with the practice of systematics and its procedures. For some reasons (maybe because it is considered intellectually more rewarding) there seems to be a need to discuss sympatric, vs. allopatric speciation, and this is often done without the appropriate data. To schematise, the following questions have to be answered in the following sequence: 1) how many stocks are involved (corollary: how are they distinguished) ? 2) are they conspecific or not? 3) for each of the recognised species, which is the closest related species or group of species (or sister-group)?

The answer to question 1 provides data to satisfy the first part of the condition to recognise species (an irreductible cluster of organisms diagnosably distinct from other such clusters). The answer to question 2 has to deal with the second part of the definition (there is a parental pattern of ancestry and descent). To answer question 3 implies to answer corollary questions: 3.1 ) is each of the species endemic to the lake or does it occur outside ? 3.2) for each species, is the sister-group in or outside the lake? And finally, facultative, 3.3) what is the speciation mechanism?

Without answering questions 3.1 and 3.2 , a discussion of 3.3 does not make sense as the needed information is missing. To answer these, one of course needs to have reliable data on the other, related species.
In the case of lake Thingvalla, I think that Sandlund et al. (1992) clearly answered question 1 (judging from figure 5 , there is a potential for several useful morphological characters like respective position of fins, body shape, caudal peduncle shape, distance between dorsal and adipose fins, etc.; none of these characters is discussed; also standard length should be used for such an analysis instead of fork length, especially as fin lengths are potentially informative characters). They answered question 2 (but I disagree with their answer, see below) and they tried (see also Snorrason et al., 1994: 14) to discuss 3.3 without answering questions 3.1 and 3.2 ; thus, a conclusion is not possible.

Although admitting that the four stocks are conspecific, Sandlund et al. (1992: 347) considered that the two benthic ones (SB, LB) belong to two different breeding populations that "may be reproductively isolated through differences in spawning time and assortative mating behaviours" and that the two stocks of the "pelagic morphotype" (PL, PI) are "two trophically specialized morphs [which] may develop from each of the two parental types" (apparently similar to the case postulated by Skeslet [1973] for Jan Mayen Is. and Nordeng [1983] for river Salangen, Norway). As the stocks seem to have assortative mating (Sigurjónsdóttir \& Gunnarson, 1989: 174) and as they seem to consistently differ in breeding colours, the SB and LB stocks should be treated as valid species under the PSC. Available data do not allow to reach a conclusion concerning the PL and PI stocks, and I tentatively treat them as conspecific (although the PL stock might be unique in having striking colour sexual dimorphism; Sandlund et al., 1992: 316).

Contrary to Sandlund et al. (1992), I conclude that their data demonstrate that three stocks are diagnosable and constitute separate lineages (are reproductively isolated) and I do not see reasons for not calling them species. Available data do not allow me to know whether or not they are restricted to lake Thingvalla and whether one of them could be exactly the same as the 'S. alpinus' outside the lake. At this stage there is no advantage in arbitrarily equating one of the lacustrine species with ' $S$. alpinus' from the rest of Iceland and I decided to recognise them as three species endemic to the lake. I could not find published data allowing to compare non-lacustrine Icelandic Salvelinus with other European stocks.

Sympatric stocks are also reported from lakes My [Myvatn] (Lamby, 1941: 772), but the available data no not allow a discussion. 
There is a variety of works on Russian stocks of Salvelinus, with a variety of contradictory opinions (see, for example, Chereshnev, 1982; Savvaitova, 1983). As they concern mainly northern and eastern Asian stocks (and also because of my inability to read them in original, without translation bias), I have decided not to comment on them. As for other areas, I cannot follow Savvaitova's model because she considers sympatric non-interbreeding stocks as conspecific. I do not perceive it as a problem if a great number of species have to be recognised and called by their names. If this diversity exists, we want it to be displayed, not hidden.

Nomenclature. I consider here that the Alpine and Scandinavian groups of populations are distinct species, S. salvelinus and $S$. alpinus respectively. The deepwater stock of lake Konstanz is a distinct species, $S$. profundus; it should urgently be investigated if the deepwater stocks of other Alpine lakes are conspecific or not (see below discussion of $S$. profundus) as several are potentially under serious threat of extinction. High altitude forms are tentatively referred to $S$. salvelinus, although, if naturally occurring populations really exist, their status might have to be re-evaluated.

For Scandinavian stocks, awaiting more data allowing resolution of their phylogeny and taxonomy, a pragmatic way to handle their nomenclature is needed. It would not be logical to treat as conspecific sympatric stocks known to be reproductively isolated. Stocks with the 'normal' $S$. alpinus habitus are tentatively (and somewhat arbitrarily) considered as conspecific. In cases where sympatric stocks shows reproductive isolation and are diagnosable, they should be considered as species. The problem is that the 'morphological' data presented up to now are of very limited or no use to decide whether or not stocks are diagnosable and to link them with the available names. As a result, a single Scandinavian species (S. alpinus) appears in the list of valid species, but there is a potential that more species can be diagnosed and recognised.

Sixteen species had been recognised from the British Isles and one from the Faroes. Their status should be re-examined and if they really are independent and diagnosable lineages, they should be recognised as valid species (this applies to populations from Spitzbergen and Jan Mayen islands too). Available data (Regan, 1908a, 1909a, 1911b; Friend, 1959) are strongly indicative that lineages are present that deserve recognition as species under the PSC, but the same data do not allow a determination of the exact number of involved species. Also, more recent data indicate that here too, two species occur in sympatry in some lakes but it is not known if they are identical or not with stocks in other lakes. Under these conditions, considering that presently all are diagnosable and seem to constitute independent lineages, I am left with no choice but to accept them as valid species, pending confirmation (or rejection). Ferguson (1981) analysed tissue proteins of Irish charr and found a high degree of similarity (for comments on similar studies, see p. 19). Some authors refer to Friend (1959) for a review of British species. Although this publication includes some interesting data, it does not contain any concrete result or conclusion and was not intended as a revision. Friend was considering the British stocks as different subspecies under the BSC (see Introduction, p. 17). He points to the existence of a great number of still unreported, geographically isolated stocks which "can be identified, in most cases at a glance, because of the varied yet racially constant characters they show" (p. 128). It seems that the two criteria for identifying species (diagnosability and monophyly) are met. This was in 1959 and Friend's researches have not resulted in any other publication. (How many of these stocks are now lost, in part because of failure to recognise them as distinct or unwillingness to handle too many names?)

For lake Thingvalla, names are available for two of the three species. The SB stock should be called $S$. thingvallensis and the PI and PL stocks $S$. murta. There is no available name for the LB stock and it was my intention to provide one. This has not been possible, as I have been unable to personally examine specimens at the time of completing this manuscript. Hopefully it can be done soon.

A potential nomenclatural problem results from Linnaeus's (1758) making four names simultaneously available (S. salvelinus, S. alpinus, S. salmarinus, S. umbla) which have been treated as synonyms by subsequent authors. The first reviser's action is decisive to determine respective priority among these four names. Table 3 shows the earliest decisions I could find for each pair of names.

The earliest synonymy apparently is by Paula Schrank (1798: 322) who considered S. salvelinus, S. alpinus and S. salmarinus as conspecific but he did not explicitly gave priority to one of the names. Fitzinger (1832: 338) treated S. alpinus as subspecies of $S$. salvelinus, thus explicitly giving priority to $S$. salvelinus over S. alpinus. Agassiz (1835a: 622 ) considered the four of them as synonyms and retained S. umbla as the valid name, thus giving priority to this name over the three others (Agassiz 
Table 3. Respective priority between Salmo alpinus Linnaeus, 1758, S. salvelinus Linnaeus, 1758, S. umbla Linnaeus, 1758 and S. salmarinus Linnaeus, 1758 as determined by the first subsequent reviser's action for each pair of names.

\begin{tabular}{llll}
\hline & S. salvelinus & S. alpinus & S. umbla \\
$\begin{array}{l}\text { and } \\
\text { S. alpinus }\end{array}$ & S. salvelinus & & \\
by & Fitzinger, 1832 & \\
S. umbla & S. umbla & S. umbla & \\
by & Agassiz, 1835a & Agassiz, 1835a & \\
S. salmarinus & S. salvelinus & S. alpinus & S. umbla \\
by & Bloch, in Schneider, 1801: 401 & present decision & Agassiz, 1835a \\
\hline
\end{tabular}

has been followed, e.g., by Siebold, 1863a; Smitt, 1895: 842; Roule, 1925). According to Günther (1862a: 43), Jardine (1835b: 614), same volume, adopted Agassiz's views but retained S. alpinus; formally speaking this is not the case as Jardine indicated the author of S. umbla as Agassiz and did not indicate the author of S. alpinus. Therefore I consider that Agassiz (1835a) is the first reviser. Should one consider that Jardine (1835b) acted as first reviser, then it has been published in the same volume, at the same date as Agassiz's (1835a) action and the first reviser's action is decisive to decide which of these two first revisers has priority (ICZN art. 24). Agassiz's action being much more detailed and explicit, as first reviser I retain Agassiz's act as having priority over Jardine's. Day (1887: 231) states that Richardson (1835) considered S. umbla, S. alpinus and $S$. salvelinus as conspecific, but I could not find such a statement in Richardson (1835).

This means that if S. umbla, S. salmarinus and $S$. salvelinus are considered synonyms, the valid name of the species has to be $S$. umbla. If $S$. alpinus and S. umbla are considered as constituting a single species (as concluded, e.g., by Behnke, 1980), the valid name of the Arctic charr is S. umbla, and not $S$. alpinus (unless it can be demonstrated that before 1835 an author explicitly designated $S$. alpinus as having priority). Earlier authors have apparently not paid much attention to priority and the use of one name or another often seems to have depended mainly on local traditions. Scandinavian authors tended to prefer $S$. alpinus (maybe because it had been described from Scandinavian material), central European authors $S$. salvelinus (maybe because the name is derived from the German saibling) and south-western European authors $S$. umbla (maybe because the name is derived from the French omble).

\subsubsection{Salvelinus alpinus (Linnaeus, 1758)}

Salmo alpinus Linnaeus, 1758: 309 (based on Linnaeus [1746: 117, n. 310, Salmo dorso nigro ...; 1747: 257, Roeding] and Artedi [1738: gen. 13 [52], syn. 25, spec. 52 [13], Salmo vix pedalis ...; based on "Salmo Lapponicus Alpinus Linnaei", probably described in Linnaeus, 1732]; type locality: "in Laponniae, Angliae alpibus, solus"; type material: NT)

Salmo carbonarius Strøm, 1784: 122 (type locality: Modum [in Buskerud District, southwest of Oslo], Norway; type material: NT)

Salmo levis Mohr, 1786: 80 (type locality: Iceland; type material: NT ?; refers to Olafsen, 1732 [sic 1772]: 595)

Salmo Lepechini Gmelin, 1788: 1374 (available by indication to Lepechin, 1780: 229, pl. 14 fig. 2 [according to Gmelin; addendum p. 19, pl. 15 fig. 2 according to Berg, 1948: 275]; type locality: erroneously given as rivers of Olonets district by Lepechin, 1780, but should be lake Ladoga, according to Berg, 1948: 275; syntypes: LU)

Salmo laevis Walbaum, 1792: 60 (based on Hammer [1775: 85, number 381, Salmo laevis pinnis maximis, Iceland, itself based on Olafsen, 1772: 595], Olafsen [1772: 595, Reydus, Bleikia] and Mohr [1786: 80, Salmo levis]; type material: NT ?; either an unnecessary replacement name for, an unjustified emendation or a junior homonym of Salmo levis Mohr, 1786: 80 [ICZN art. 58(1)]); synonymy follows Faber, 1829: 168

Salmo palja Walbaum, 1792: 67 (based on Lepechin, 1780: 229 [according to Walbaum; addendum p. 19, pl. 15 fig. 2 according to Berg, 1948: 275] and S. lepechini Gmelin, 1788: 1374; type locality: erroneously given as rivers of Olonets district by Lepechin, 1780, but should 
be lake Ladoga, Russia, according to Berg, 1948 275; syntypes: LU)

Salmo punctatus Cuvier, 1829: 304 (type locality: Lapland and British Isles, by present restriction [originally: "all around the Alps"]; available by description and indication to $S$. alpinus of Bloch, 1784: 158, pl. 104, itself based on a drawing of material from the [Swiss ?] Alps and earlier records, including original description by Linnaeus, 1758: 308; S. punctatus is here restricted to Linnaeus's (1758: 308) account of S. alpinus (see p. 131); type material: NT)

Salmo nivalis Faber, 1829: 170 (type locality: Greenland, Iceland, Faroe Islands, Norway; syntypes: LU)

Salmo ventricosus Nilsson, 1832: 7 (type locality: Sigdal, Norway; syntypes: LU)

Salmo rutilus Nilsson, 1832: 10 (type locality: "lakes in Hadeland territories", Norway; syntypes: LU)

Salmo Ascanii Valenciennes, in Cuvier \& Valenciennes, 1848: 256 (type locality: lakes of Christiansadvis, Norway; based on the Röding, Roëding or Roetelet of Ascanius, 1775: 4, pl. 32; type material: NT)

Salmo salvelino-stagnalis Smitt, 1886: 111, 163 (type locality: Teriberka, mouth of river Murmansk, Kola Peninsula, Russia; syntypes: NRM 12050-12051 [2, \#414-415])

Salmo alpino-stagnalis Smitt, 1886: 112, 163 (type locality: Godthaab and Godhavn, Greenland; syntypes: NRM [12, \# 402-413])

Salmo hybridus Smitt, 1886: 112 (type locality: lake Yngern $\left[59^{\circ} 45^{\prime} \mathrm{N} 14^{\circ} 15^{\prime} \mathrm{E}\right.$, near Persberg], Sweden; syntypes: NRM)

Salvelinus lepechini melanostomus Berg, 1932a: 116 (nomen nudum; locality: lake Segozero, White Sea drainage)

Salvelinus lepechini infraspecies profundicola Berg, 1932c: 174 (infrasubspecific, name not available; locality: lake Ladoga, Russia [Berg, 1948: 278])

Salvelinus lepechini melanostomus Berg, 1932c: 177 (type locality: Segozero, basin of river Vyg [a tributary of lake Onega], Russia [Berg, 1948: 281]; syntypes: ZISP ?)

Nomenclatural notes. Nyman et al. (1981: 133) discussed the type locality of Salmo alpinus on the assumption that Linnaeus (1758: 309) based it on Artedi (1738) and his own Iter Lapponicum (1732). Actually Linnaeus based his account on his Fauna Svecica (1746) and Wästgöta-resa (1747) and on Artedi (1738) [who himself refers to Linnaeus (1732), but there is no unambigu- ous statement of this]. A type locality restriction should be based on these accounts and is best linked to a neotype designation.

The author of Salmo laevis is sometime indicated as Olafsen (1772: 595). Olafsen's use of the name $S$. laevis is obviously not binominal, as "Salmo laevis, pinnis maximis, corpore subtereti pallide fusco". The first use making the name available is as S. levis by Mohr (1786). The spellings laevis and levis are homonyms (ICZN art. $58(1))$

If the Scandinavian charr is considered as conspecific with the Alpine one, the valid name is $S$. umbla (see above, p. 151).

\subsubsection{Salvelinus colii (Günther, 1863)}

Salmo colii Günther, 1863: 12, pl. 2 (type locality: Lough Eske, county of Donegal, Ireland / Lough Dan, Wicklow county, Ireland; syntypes: $\mathrm{BMNH})$

\subsubsection{Salvelinus faroensis Joensen \& Tåning, 1970}

Salvelinus alpinus faroensis Joensen \& Tåning, 1970: 68 (type locality: Leynavatn, Faroe Islands; holotype: ZMUC, missing according to Nielsen, 1974: 12)

Systematic notes. The status of this taxon is unclear because the diagnosis is vague and not very explicit. The characters distinguishing it from $S$. gracillimus from the 'nearby' Shetlands are not mentioned.

\subsubsection{Salvelinus fimbriatus Regan, 1908}

Salvelinus fimbriatus Regan, 1908a: 232, fig. 5 (type locality: Lough Coomasaharn in Kerry. Ireland; holotype: BMNH or NMI)

\section{Salvelinus fontinalis (Mitchill, 1814) intro- duced}

\subsubsection{Salvelinus grayi (Günther, 1862)}

Salmo grayi Günther, 1862a: 51, pl. 7 (type locality: Lough Melvin in Fermanagh, Ireland; syntypes: BMNH [3])

\subsubsection{Salvelinus gracillimus Regan, 1909}

Salvelinus gracillimus Regan, 1909a: 115, fig. 2a (type locality: Loch of Girlsta, Tingwall, Shetland islands, U.K.; syntypes: BMNH [4])

14.5.7 Salvelinus inframundus Regan, 1909 Salvelinus inframundus Regan, 1909a: 116 (type locality: lake Hellyal, Hoy Island, Orkney islands, U.K.; syntypes: BMNH [2]) 
Systematic notes. Extinct according to Regan (1909a: 116; 1911b: 101) and Joensen \& Tåning (1970: 70); recent data would be desirable.

14.5.8 Salvelinus killinensis (Günther, 1865)

Salmo killinensis Günther, 1865a: 699, pl. 40 (type locality: Loch Killin, Inverness-shire, U.K.; syntypes: BMNH, ZMB 8330)

\subsubsection{Salvelinus lonsdalii Regan, 1909}

Salvelinus lonsdalii Regan, 1909a: 119, fig. 2b (type locality: Haweswater, Cumberland, U.K.; syntypes: BMNH [2])

\subsubsection{Salvelinus mallochi Regan, 1909}

Salvelinus mallochi Regan, 1909a: fig. 4 (type locality: Loch Scourie, Sutherlandshire, U.K.; syntypes: BMNH [4])

\subsubsection{Salvelinus maxillaris Regan, 1909}

Salvelinus maxillaris Regan, 1909a: 117, fig. 3 (type locality: Loch near Ben Hope, Sutherlandshire, U.K.; syntypes: BMNH [11])

\subsubsection{Salvelinus murta (Saemundsson, 1909)}

Salmo alpinus var. murta Saemundsson, 1909: 93 (type locality: lake Thingvalla, Iceland; syntypes: IMNH uncat.)

\subsubsection{Salvelinus obtusus Regan, 1908}

Salvelinus obtusus Regan, 1908a: 233 fig. 6 (type locality: Lough Luggala and Lough Dan in Wicklow, Killarney and Lough Acoose in Kerry, Ireland; syntypes: BMNH or NMI [17])

\subsubsection{Salvelinus perisii (Günther, 1865)}

Salmo cambricus Günther, 1862a: 49, pl. 6 (type locality: lakes of Llanberis, Carnarvonshire, North Wales, U.K. / lake Coss-y-gedawl, near Barmouth in Merionethshire, U.K.; syntypes: BMNH [ca. 24]; junior primary homonym of $S$. cambricus Donovan, 1806)

Salmo Perisii Günther, 1865b: 75 (replacement name for Salmo cambricus Günther, 1862)

\subsubsection{Salvelinus profundus Schillinger, 1901}

Salmo salvelinus var. profundus Schillinger, 1901: 149, fig. (type locality: lakes Konstanz and Ammersee, Germany restricted here to lake Konstanz; type material: NT)

? Salvelinus salvelinus var. profundus Fuhrmann, 1903: 332 (type locality: lake Neuchâtel, Switzerland; syntypes: MHNN 837 [2], MHNG 809.61
[3, 2 now lost]; junior secondary homonym of Salmo salvelinus var. profundus Schillinger, 1901)

Systematic notes. Although formally named in 1901, a detailed description of this species has never been published. It is definitely reported from lake Konstanz only. Deep water forms from other sub-Alpine lakes have occasionally been referred to it, but evidence that they are conspecific is missing. Descriptive data of other stocks are only available for lake Neuchâtel, Switzerland (Fuhrmann, 1903; Quartier, 1951), but without comparison with the material of lake Konstanz.

The identity of material presently referred to as deepwater charr in lake Konstanz is open to question. Behnke's (1972: 641) and Cavender's (1980: 298) examination of ancient material indicates that $S$. profundus differs from the 'normal' charr S. umbla. This is supported by data in Dörfel (1974). The normal charr used to be the dominant species, but since about 1980 the deep-water one became dominant (Hartmann, 1994). Hartmann also reports that today's deep water charr in lake Konstanz does not agree with descriptions of earlier workers; this is confirmed by my examination of material caught in 1995 (provided by J. Hartmann). It has not yet been possible to compare this material with ancient museum specimens of $S$. profundus, but preliminary observations suggest that the present deepwater charr is not $S$. profundus but possibly small individuals of $S$. umbla. If this is confirmed, it should be investigated if the real $S$. profundus possibly survives in deeper areas where commercial fishermen do not operate. It may already be too late and the species might be extinct.

The large charr of lake Konstanz reportedly is (was) not identical with the 'normal' charrs of other alpine lakes (Nümann, 1972: 835).

Pollution, eutrophication and decreased oxygen concentration in deep-water layers (Nümann, 1972: 842) may be responsible for the extinction of $S$. profundus which was apparently restricted to this habitat. Lake Konstanz has been regularly stocked with allochtonous material since 1978 (from lake Vierwaldstätter; Ruhlé, 1991) and it also cannot be ruled out that hybridisation might be partly responsible for this change.

Nomenclatural notes. Fuhrmann (1903: 332) described Salvelinus salvelinus var. profundus from lake Neuchâtel. The name is a junior secondary homonym of Salmo salvelinus var. profundus Schillinger, 1901. As there is no indication that Fuhrmann was aware of Schillinger's work and of the lake Konstanz species, it is not possible to con- 
sider that he was simply using the name created by Schillinger and I treat it as an independent (but unavailable) name.

\subsubsection{Salvelinus scharffi Regan, 1908}

Salvelinus scharffi Regan, 1908a: 228, fig. 2 (type locality: Lough Owel in Westmeath, Ireland; holotype: BMNH or NMI)

Systematic notes. Charrs have become extinct in Lough Owell (Quigley \& Flannery, 1996: 31). It is not known whether this species possibly survives in other lakes.

\subsubsection{Salvelinus struanensis (Maitland, 1881)}

Salmo struanensis Maitland, 1881: 516 (type locality: Loch Rannoch, Scotland, U.K.; syntypes: LU)

Systematic notes. Walker et al. (1988) reports that two stocks are present in Loch Rannoch, with different morphology, habitat, spawning places and feeding habits, which would apparently justify them being recognised as two species under the PSC. One of them is S. struanensis. The identity of the second one can only be determined after careful comparisons with stocks from other lakes as it might be identical with one of them. On the other hand, it might represent an unnamed species.

Salvelinus struanensis in Regan (1911: 94, pl. 10 fig. 1) is apparently not Maitland's species but the unnamed benthic species of Walker et al.

\subsubsection{Salvelinus thingvallensis (Saemund- sson, 1909)}

Salmo alpinus var. Thingvallensis Saemundsson, 1909: 93 (type locality; lake Thingvalla, Iceland; syntypes: IMNH uncat.)

Salmo alpinus forma niger Fridriksson, 1939: 24 (subspecific because treated as valid prior to 1985, e.g., Fowler, 1974: 70 (ICZN art. 45(g)(ii)(1)); type locality: lake Thingvalla, Iceland; syntypes: LU)

Systematic notes. According to S. Snorrason (pers. comm., 1995), the S. a. niger of Fridriksson (1939) can hardly be anything else than the SB stock of Sandlund et al. (1992); it cannot be excluded that some of Fridriksson's specimens belonged to the LB stock, but this cannot be demonstrated as none of his material is extant.

\subsubsection{Salvelinus trevelyani Regan, 1908}

Salvelinus trevelyani Regan, 1908a: 229, fig. 3 (type locality: Lough Finn in Donegal, Ireland; holotype: $\mathrm{BMNH}$ )
14.5.20 Salvelinus umbla (Linnaeus, 1758)

Salmo Salvelinus Linnaeus, 1758: 309 (based on Artedi [1738: gen. [spec.] 13, syn. 26, Salmo pedalis maxilla ...; based on Ray [1710: 64, Linz, Austria]]; type locality: "in Austria ad Lintz"; type material: NT)

Salmo Salmarinus Linnaeus, 1758: 310 (based on Artedi [1738: syn. 24, Salmo dorso fulvo ...; based on Salviano (1554-58: fol. 102, pl. 27, "Tridento ... alio fluuio apud Brissanam [Bressanone?, Alto Adige, Italy] quadra ginta millia passum a Tridento")]; type locality: "Tridenti in fluviis frigidis saxosis"; type material: NT)

Salmo Umbla Linnaeus, 1758: 310 (based on Artedi [1738: gen. [spec.] 13, syn. 25, Salmo lineis lateralibus ...; based on Rondelet [1555: 160, lake Genève] and Gesner [no year, 1775: 190, 191, lakes Genève, Zürich, Luzern and Konstanz]; type locality: "in Helvetiae, Italiae lacubus"; type material: NT)

Salmo Salvelinus var. Marsilii Fitzinger, 1832: 338 (available by indication to Marsili [1726: 83, pl. 29 fig. 1, Umbla II, "Männ-Seer"]; type locality: "Männ-See" [Marsili], lake Mondsee, Austria; syntypes: NMW [not yet located; B. Herzig, pers. comm., 1995])

Salmo Salvelinus var carneus Fitzinger, 1832: 338 (nomen nudum; locality: lake Traunsee, Austria)

Salmo pallidus Nilsson, 1832: 9 (type locality: lake Vättern, Sweden; syntypes: LU)

Salmo monostichus Heckel, 1851a: 146 (type locality: lake Königssee, Germany; syntypes: NMW 65458 [1], 66063 [2]); predates Heckel, 1852a: 299

Salmo distichus Heckel, 1852a: 359, 361 (type locality: lakes of Salzkammergut, Austria; syntypes: NMW [not yet located; B. Herzig, pers. comm., 1995])

Salmo costatus Gronow, 1854: 152 (type locality: Switzerland; type material: NT)

Salmo Schrankii Heckel \& Kner, 1858: 280 (listed in synonymy, name not available; author stated to be Fitzinger, but Heckel \& Kner are actual authors)

Systematic notes. This species includes all the 'normal' stocks of the Alpine and sub-Alpine lakes, and, according to Behnke $(1972,1980)$, the stocks from lake Vättern, Sweden (for which the name S. pallidus would be available if further research proves it distinct) and lake Coomarsaharn, Ireland (here treated as $S$. fimbriatus). 


\subsubsection{Salvelinus willoughbii (Günther, 1862}

Salmo willoughbii Günther, 1862a: 46, pl. 5 (type locality: lake Windermere, Westmorland, U.K.; syntypes: BMNH [2])

Salvelinus willughbii forma autumnalis Frost, 1966: 260 (infrasubspecific, name not available; locality: lake Windermere, Cumbria, U.K.; autumn spawning form)

Salvelinus willughbii forma vernalis Frost, 1966: 260 (infrasubspecific, name not available; locality: lake Windermere, Cumbria, U.K.; spring spawning form)

Systematic notes. Frost (1966) documented the existence of a spring and an autumn spawning stocks in lake Windermere; they do not interbreed in nature (but can be crossed under laboratory conditions) and are apparently not distinguishable morphologically (but we are not given much information on characters other than gill-rakers). Child (1984: 249) obtained "evidence ... supporting the genetic isolation" of the two stocks.

Nomenclatural notes. The original spelling willoughbii has been emended to willughbii by Giinther (1863: 11). As both spellings Willughby and Willoughby appeared on the title pages of his works, I consider that willoughbii (based on a latinised form of Willoughby) is a correct original spelling which should not be emended.

\subsubsection{2 ? Salvelinus youngeri Friend, 1956}

Salvelinus alpinus youngeri Friend, 1956: 220 (type locality: Loch Eck, Cowal, Argyllshire, Scotland, U.K.; syntypes: ? Zoology Dept., Edinburgh Univ. [upwards of 50])

Systematic notes. The data provided by Friend (1956) do not allow to identify this stock with any of the named ones and it is tentatively treated as a valid species.

\subsubsection{Salvelinus sp. Lake Thingvalla LB stock}

Systematic notes. This is the LB stock of Sandlund et al. (1992) which is considered here as a distinct species. No name is available for it.
14.6.1 Thymallus thymallus (Linnaeus, 1758)

Salmo Thymallus Linnaeus, 1758: 311 (based on Artedi [1738: gen. 10 [41], syn. 20, spec. 41 [10], Coregonus maxilla superiore ...], Linnaeus [1746: 119, n. 314, idem], and Gronovius [1756: 13, n. 165 (actually p. 12, n. 162), idem; river Rhine near Basel, Switzerland]; type locality: "in Europae fluviis maritimis"; syntype: BMNH 1853.11.12:159, Wheeler, 1958: 207)

Salmo thymus Bonnaterre, 1788: 167, pl. 53 fig. 202 (type locality: streams of England and Germany; type material: NT [apparently based on Willughby (1686: 187) and Salviano (1554-58: fol. 81, pl. 16, thymallo, Ticino, Athesi, Addua, Pado, Transalpinae Galliae)

Salmo striatus Reisinger, 1830: 44 (type locality: 'in rivis frigidis Liptoviae' [Liptau = Liptov], Hungary [now Slovakia]; type material: NT)

Thymallus vulgaris Nilsson, 1832: 13 (unnecessary replacement name for Salmo thymallus Linnaeus, 1758)

Thymallus vexillifer Perty, 1832: 718 (unnecessary replacement name for Salmo thymallus Linnaeus, 1758)

Thymallus decorus Koch, in Koch, Herrich-Schäffer \& Forster, 1840: 42 (unnecessary replacement name for Salmo thymallus Linnaeus, 1758)

Thymallus gymnothorax Valenciennes, in Cuvier \& Valenciennes, 1848: 445, pl. 625 (type locality: Berlin, Germany, and Russia; syntypes: MNHN)

Thymallus gymnogaster Valenciennes, in Cuvier \& Valenciennes, 1848: 446, pl. 626 (type locality: river Neva, Russia; syntypes: MNHN [3])

Thymallus Æliani Valenciennes, in Cuvier \& Valenciennes, 1848: 447 (type locality: lake Maggiore, Italy; syntypes: MNHN [3])

Thymallus umbrosa Gistel, in Gistel \& Bromme, 1850: 344 (no locality data; type material: NT)

Salmo punctatus Gronow, 1854: 153 (type locality: Germany; type material: NT; preoccupied by Salmo punctatus Cuvier, 1829)

Thymallus thymallus kamensis Lukash, 1929: 7 (upper reaches of river Kama [a tributary of river Volga], Russia) from Berg, 1948: 432 and Svetovidov, 1936: 218

Thymallus thymallus morpha lacustris Balon, 1962: 152 (infrasubspecific, name not available; locality: reservoir on river Hnilec, Slovakia) 


\section{Gadidae}

15.1.1 Lota lota (Linnaeus, 1758)

Gadus Lota Linnaeus, 1758: 255 (based on Linnaeus [1746: 109, n. 292, Gadus dipterygius ... aequalibus], Artedi [1738: gen. [spec.] 22, syn. 38 , Gadus dorso ... ore cirrato, spec. 107, Silurus ciro in mento unico]; type locality: "in lacubus Europaeis"; syntypes: LSL 37, 38 [2], Wheeler, 1985: 37, UUMZ 178, Wheeler, 1991: 165)

Lota vulgaris Fitzinger, 1832: 331 (unnecessary replacement name for Gadus lota Linnaeus, 1758)

Lota fluviatilis Perty, 1832: 717 (unnecessary replacement name for Gadus lota Linnaeus, 1758)

Lota marmorata Koch, in Koch, Herrich-Schäffer \& Forster, 1840: 42 (unnecessary replacement name for Gadus lota Linnaeus, 1758)

Lota communis Rapp, 1854: 172 (type locality: lake Konstanz, Germany-Switzerland-Austria; syntypes: LU; author indicated as Cuvier, but Rapp is actual author)

Lota Linnéi Malm, 1877: 491 (unnecessary replacement name for Gadus lota Linnaeus, 1758)

Lota vulgaris var. obensis Anikin, 1902: 108 (river Ob, Siberia) from Berg, 1949: 943

Lota lota kamensis Markun, 1936: 211 (river Kama near Okhansk, Russia) from Berg, 1949: 944

Lota lota leptura Hubbs \& Schultz, 1941: 17 (type locality: Kotlik, one of the mouth of river Yukon on Norton Sound, Alaska; holotype: USNM 29916)

Lota lota infrasp. onegensis Melyantsev, 1948: 96 (infrasubspecific, name not available; locality: lake Onega, Russia) from Berg, 1949: 944

Lota lota asiatica Kirillov, 1972: 279 (type locality: Yakutia, Siberia; syntypes: LU)

Systematic notes. Kirillov (1972) distinguished two subspecies on the basis of pyloric caeca, but according to Holčík \& Nagy (1987) the number of pyloric caeca shows clinal variation and increases with size.

Nomenclatural notes. The author of Lota vulgaris is often cited as Cuvier (1829: 215) but Cuvier only used a French name and no Latin name. The first use making the name available seems to be by Fitzinger (1832: 331).

\section{Mugilidae}

\subsubsection{Chelon labrosus Risso, 1826}

Mugil labrosus Risso, 1826: 389 (type locality: not stated, probably around Nice, France; syntypes: apparently lost)

Mugil chelo Cuvier, 1829: 232 (type locality: Brest, France; lectotype: MNHN 6400, designated by Blanc \& Hureau, 1971: 686)

? Mugil curtus Yarrell, 1836: 210, fig. (type locality: between Brownsey Island and South Haven, at the mouth of Poole Harbour, England; holotype: LU)

Mugil corrugatus Lowe, 1838: 184 (type locality: Madeira; syntypes: LU) from Trewavas, in Hureau \& Monod, 1973: 569

Mugil Buosega Nardo, 1847: 127 (cited in synonymy, name not available)

Mugil septentrionalis Günther, 1861b: 349, fig. (type locality: British coasts; syntypes: BMNH, Trewavas, in Hureau \& Monod, 1973: 569)

16.2.1 Liza aurata (Risso, 1810)

Mugil Auratus Risso, 1810: 344 (type locality:
Nice, France; syntypes: apparently lost)

Mugil cryptocheilos Valenciennes, in Cuvier \& Valenciennes, 1836: 61 (type locality: river Nile [Egypt]; holotype: MNHN A.3665, Thomson, in Daget et al., 1986: 345)

Mugil breviceps Valenciennes, in Cuvier \& Valenciennes, 1836: 106 (type locality: Gorée [near Dakar, Senegal]; holotype: MNHN A.4677, Thomson, in Daget et al., 1986: 345)

Mugil maderensis Lowe, 1839: 82, 83 (type locality: Lanzarote, Canary Islands; holotype: BMNH 1860.5.3:22) from Thomson, in Daget et al., 1986: 345 and Costa Pereira, in litt.

Mugil Lotreganus Nardo, 1847: 127 (cited in synonymy, name not available)

Mugil octo-radiatus Günther, 1861b: 347, fig. (type locality: England; holotype: BMNH)

\subsubsection{Liza ramada (Risso, 1826)}

Mugil ramada Risso, 1826: 390 (type locality: not stated, probably around Nice, France; syntypes: apparently lost) 
Mugil capito Cuvier, 1829: 232 (type locality: La Rochelle, France; lectotype: MNHN A.3587, designated by Blanc \& Hureau, 1971: 682)

? Mugil britannicus Hancock, 1830: 137 (type locality: London market, U.K.; type material: NT) from Trewavas, in Hureau \& Monod, 1973: 570

Mugil Dubahra Valenciennes, in Cuvier \& Valenciennes, 1836: 60 (type locality: river Nile [Egypt]; syntypes: MNHN A. 3730-3732 [5], Thomson, in Daget et al., 1986: 346)

Mugil Caustelus Nardo, 1847: 127 (cited in synonymy, name not available)

Mugil petherici Günther, 1861a: 441 (type locality: river Nile at Cairo, Egypt; holotype: BMNH 1861.9.9:5, Trewavas, in Hureau \& Monod, 1973: 570)

Myxus maroccensis Mohr, 1927: 191, fig. 13 (type locality: Morocco; holotype: ZMH 75, Ladiges et al., 1958: 162)

\subsubsection{Liza saliens (Risso, 1810)}

Mugil Saliens Risso, 1810: 345 (type locality: Nice, France; syntypes: apparently lost)

Mugil Verselata Nardo, 1847: 127 (cited in synonymy, name not available)

Liza saliens furcata Popov, 1930: 72, 122 (Black Sea) from Trewavas, in Hureau \& Monod, 1973: 573

\subsubsection{Mugil cephalus Linnaeus, 1758}

Mugil Cephalus Linnaeus, 1758: 316 (based on Artedi [1738: gen. 32 [71], syn. 52, spec. 71
[32], Mugil] and Hasselqvist [1757: 385, Mugil cephalus]; type locality: "in Oceano Europaeo, fluvios subiens"; potential syntypes: NRM LP 43 [1], 44 [1], 143 [2], Fernholm \& Wheeler, 1983: 257)

Mugil Öur Forsskål, 1775: 74 (type locality: Red Sea; holotype: ZMUC 67, Klausewitz \& Nielsen, 1965: 26, pl. 36 fig. 67)

? Mugil Tang Bloch, 1794: 171, pl. 395 (type locality: Acara [Accra], Ghana; syntypes: ZMB 1785 [1])

Mugil Provensalis Risso, 1810: 346 (type locality: river Var, near Nice, France; syntypes: apparently lost)

Mugil cephalotus Valenciennes, in Cuvier \& Valenciennes, 1836: 110 (type locality: Pondichery, India; syntypes: MNHN 8102 [1], A.4645 [1], A.4698 [1], Thomson, in Daget et al., 1986: 348)

Mugil Vulpinus Nardo, 1847: 127 (cited in synonymy, name not available)

Mugil ashanteënsis Bleeker, 1863a: 91, pl. 19 fig. 2 (type locality: Ashanti, Guinea [now Ghana]; holotype: RMNH 1631)

Myxus caecutiens Günther, 1876: 397 (type locality: Rodriguez Island, Indian Ocean; syntypes: BMNH 1877.12.10:37-38 [2], Thomson, in Daget et al., 1986: 348)

Nomenclatural notes. Fernholm \& Wheeler (1983: 257) comment that the potential type material of Mugil cephalus includes at least three species and that a lectotype designation is needed.

\section{Atherinidae}

\subsubsection{Atherina boyeri Risso, 1810}

Atherina Boyeri Risso, 1810: 338 (type locality: sea shore and lower course of rivers in Département du Var, France; syntypes: MNHN A.4342 [2], B.860 [1], Maugé, in Daget et al., 1986: 277)

Atherina presbyter var. pontica Eichwald, 1831: 72 (type locality: Odessa, Ukraine; syntypes: LU)

Atherina presbyter var. caspia Eichwald, 1831: 72 (type locality: "sinu Balchanensis" [Balkhan Bay, Caspian Sea; Berg, 1949: 1003]; syntypes: LU)

Atherina Risso Valenciennes, in Cuvier \& Valenciennes, 1835: 435 (type locality: Nice, France; syntype: MNHN A.4350 [listed as holotype by Kiener \& Spillmann, in Hureau \& Monod, 1973: 576 , but Valenciennes had at least one specimen and a skeleton])

Atherina sarda Valenciennes, in Cuvier \& Valenciennes, 1835: 435 (type locality: salt marshes of Sardegna, Italy; holotype: MNHN A.4354, Kiener \& Spillmann, in Hureau \& Monod, 1973: 576)

Atherina Lacustris Bonaparte, 1836: [fasc. 17-18, p. 279], pl. 118 fig. 3 (type locality: lakes Albano, Castel Gandolfo and Nemi, Italy; syntypes: ANSP 9953-9974 [22], Böhlke, 1984: 23) 
Atherina anterina Nardo, 1847: 127 (listed in synonymy, name not available)

? Atherina hyalosoma Cocco, 1885: 239 (type locality: Sea of Messina, Sicilia; syntypes: LU)

Atherina Riqueti Roule, 1902: 263, fig. 1 (type locality: Canal du Midi, France; syntypes: MNHN 1908.88-89, Kiener \& Spillmann, in Hureau \& Monod, 1973: 577)

Atherina mochon var. aegyptia Boulenger, 1907: 424, fig. 31 (type locality: Mediterranean coast of Egypt / lakes Temsah and Menzaleh, Egypt; syntypes: BMNH, MNHN 1907-238, Maugé, in Daget et al., 1986: 277)

? Atherina Bonapartii Boulenger, 1907: 426 (type locality: Gulf of Cagliari, Italy; syntypes: BMNH)

Systematic notes. The existence of different 'populations' of $A$. boyeri inhabiting different habitats is quite well documented (see, e.g., Kiener \& Spillmann, 1969: 53) and various authors have studied this variability around the Mediterranean.

Kartas \& Trabelsi (1990) analysed the variability of $A$. boyeri in Tunisia. They recognise marine, lacustrine and insular 'populations'. From their data (pp. 301-304), while the insular stock can be distinguished from the marine one, there do not seem to be striking differences between the insular and the mainland, lacustrine ones. The morphological distinctness (diagnosability) is complete between the marine and the lacustrineinsular populations and, unless it is demonstrated that there is complete intergradation between the stocks, it is more parsimonious to consider that we have to deal with two species with different habitat requirements than with a single polymorphic species developing different adaptations in different habitats under the supposed pressure of some hypothetical external factors. One is surprised to see that the difference in gill-raker counts (23-31, vs. 33-41) between the two stocks of $A$. boyeri coincides with the histograms of Kiener \& Spillmann (1969: 46) for $A$. boyeri and $A$. hepsetus, but as the range of values for vertebrae and lateral line scales (among the most useful characters to distinguish the two species) for the two stocks agree with those of $A$. boyeri of Kiener \& Spillmann, there is apparently no doubt about the identification as 'A. boyeri'.

Marfin (1982) also confirmed the existence of two 'morphological types' in southern France: a marine and a lagoon stocks. Marfin reports the same type of differences, plus differences in teeth position, premaxillary shape and scale shape. The two stocks occur in different but overlapping habitats. Marfin considers that the two stocks have dis- tinct spawning areas, do not $\cdot$ form mixed schools while spawning (but no details are provided) and maintain their morphological characteristics. Thus, under the PSC, they are different species. Marfin (1982: 25) considered the fact that they co-occur in part of their range as indicative of the possibility of gene flow, but the stability of the diagnostic characters over 200 years (the age of the oldest museum samples) and the lack of intergrades seem to be evidence that there is no gene flow. I do not think that the size of the mouth and the number of gill-rakers are individual adaptations to different food types (a Lamarkian interpretation ?) but species characteristics. Marfin stated that when the whole range of variability within 'A. boyeri' is considered it is not possible to distinguish several species (referring to Kiener \& Spillmann, 1969). This makes little biological sense and it is obvious that in order to reach a conclusion on how many species are involved in, let us say, southern France, one should consider the local stocks only. The variability observed in, let us say, the eastern Mediterranean is uninformative and possibly irrelevant (but it might provide information on parallelism).

With the available data, it seems well documented that at least in some parts of the Mediterranean, there are two diagnosable lineages of 'A boyeri' which satisfy the definition of two species under the PSC, a marine (here A. mochon) and a lacustrine one (here A. boyeri, see Nomenclatural notes, below). Results of electrophoretic study by Focant et al. (1992) also show this dichotomy.

Trabelsi et al. (1994) report that within the marine 'A. boyeri' from Tunisia and Corsica (here $A$. mochon) they also distinguish two groups differing, among other characters, in coloration, premaxillary shape and gill-raker counts. Although they claim that all belong to the marine 'population' (here A. mochon) (referring among others to Kartas \& Trabelsi, 1990), their Y1 population seems to fall within the lacustrine 'populations' of these authors (here A. boyeri): they give mean values for total gill-raker counts of 29.55 and 28.57 for the Corsican, respectively Tunisian Y1 specimens (they do not indicate range, which would be a more interesting and biologically meaningful information), while Kartas \& Trabelsi reported a range of 33-41 in the marine species (but 23-31 in the lacustrine species). The same remark applies for the counts of lower and upper gill-rakers, lateral row scales and vertebrae. Other characters were not examined by Kartas \& Trabelsi (1990). Trabelsi et al. (1994) do not state how their Y1 stock differs from the lacustrine species (A. boyeri) 
and unless clarification about the noted apparent contradiction is provided, it does not seem necessary to comment further on marine populations.

Svetovidov (1964: 226-229) considers that the Black Sea stock (called A. mochon pontica) differs from a Mediterranean one (called A. m. mochon) in number of gill-rakers (26-31, vs. 22-26) and scale rows $(44-51$, vs. $45-46)$. It seems possible that his concept of $A . \mathrm{m}$. pontica may have included both $A$. boyeri and $A$. mochon as recognised here, but this should be confirmed by direct examination of specimens. His concept of $A . m$. mochon includes most of the species listed above in the synonymy of $A$. boyeri and it is not clear to me whether he examined material from the Mediterranean. A comparison of the values he reports for the Black Sea stock with those reported by the above mentioned authors for the Mediterranean stock does not allow to distinguish the Black Sea stock from A. boyeri. Svetovidov (1964: 229) also recognises $A$. bonapartei as valid species, but I cannot discuss its status; it might be $A$. mochon as understood here.

Bamber \& Henderson (1985) considered that $A$. boyeri and $A$. presbyter represent a single species, on the basis of material from the British Isles. They considered that the morphological differences reported by earlier authors was due to environmental factors (but it is not clear whether their material actually included both species). An electrophoretic study showed that they are distinct species (Creech, 1991). There is no data in Creech to determine whether his $A$. boyeri is the real $A$. boyeri or A. mochon.

Nomenclatural notes. I have examined the syntypes of $A$. boyeri. MNHN B. 860 is a dry, mounted specimen ca. $74.0 \mathrm{~mm} \mathrm{SL}$, with 42 rows of scales; gill-rakers cannot be counted; MNHN 4342 includes 2 alcohol preserved specimens in poor state, one $70.6 \mathrm{~mm}$ SL with about 46 rows of scales and 24 distinct gill-rakers (some may be missing) and one $67.0 \mathrm{~mm}$ SL with 30 gill-rakers. They seem to agree with the lacustrine stock of Kartas \& Trabelsi and I restrict the use of the name $A$. boyeri to this species. The holotype of A. risso (MNHN A.4350, $75.5 \mathrm{~mm} \mathrm{SL}, 25$ gill-rakers), the holotype of A. sarda (MNHN A.4354, $47.9 \mathrm{~mm} \mathrm{SL}$, ca. 25 gill-rakers) and one syntype of $A$. riqueti (MNHN 1908-88, $41.0 \mathrm{~mm} \mathrm{SL}, 23$ gill-rakers) also clearly belong to this species. The lectotype of A. mochon (MNHN A.4355, 57.9 mm SL, 32 gill-rakers) apparently represent the marine species and this is the earliest available name for it. As most of these specimens are in quite poor state and many had been damaged by earlier researchers, it might be desirable that these identifications be confirmed by a researcher more familiar with this family and in the context of a critical, taxonomic revision. As the marine species only sporadically and marginally occurs in freshwaters, I did not investigate further its identity and use here for this species the name A. mochon Cuvier, 1829 (p. 235; type locality: Iviça, Balearic islands; lectotype: MNHN A.4355, designated by Blanc \& Hureau, 1971: 707). Some of the names listed in the above synonymy might actually apply to this species.

\subsubsection{Atherina hepsetus Linnaeus, 1758} Atherina Hepsetus Linnaeus, 1758: 315 (based on Artedi [1738: syn. 117, Atherina], Gronovius [1754: 23, n. 66, Atherina] and Hasselquist [1757: 382, Atherina Hepsetus]; type locality: "in Mari Mediterraneo"; syntypes: BMNH 1853.11.12:79 [1], NRM LP 65 [5], ? UUMZ 209 [1], Wheeler, 1958: 236, 1991: 168, Fernholm \& Wheeler, 1983: 229)

Athaerina athaerina Nardo, 1827b: 33, 39 (available by indication to Rondelet [1554: 216, Athaerina] and "Ann. du Mus. V. 13 p. 288 var. 1 ?" [Delaroche, 1809: 357]; type locality: Marseilles, France [Rondelet] and Iviza, Balearic Island [Delaroche]; syntypes: ? MNHN)

Nomenclatural notes. Wheeler (1958: 236) and Fernholm \& Wheeler (1983: 229) indicate that the type series of Atherina hepsetus includes two species and that a lectotype should be designated. Inclusion of this species as occurring in freshwaters follows Blanc et al. (1971), Maitland (1977: 204) and others.

\subsubsection{Atherina presbyter Cuvier, 1829} Atherina presbyter Cuvier, 1829: 235 (type locality: La Rochelle, France; lectotype: MNHN 4377, designated by Blanc \& Hureau, 1971: 710) Systematic notes. See A. boyeri.

Odonthestes bonariensis (Valenciennes, 1835) introduced 


\section{Fundulidae}

18.1.1 Fundulus heteroclitus (Linné, 1766) introduced

Valencia lozanoi Gomez, Peiro \& Sanchez, 1984: 67, fig. 3 (type locality: Trebujena, Cádiz, Spain; holotype: Estación Ornitológica y Piscicola Experimental "Mata del Fang", Albufera de Valencia 840106)

Systematic notes. Synonymy follows FernándezDelgado et al. (1986) and Bianco \& Miller (1989).

While the identity of the introduced Spanish population seems beyond doubts, Delgado et al. (1991) noted differences between the karyotype of ' $V$. lozanoi' and the one reported by Chen \& Ruddle (1970) for $F$. heteroclitus. They concluded that either 1) V. lozanoi is a European Fundulus which had been misplaced in the genus Valencia, or 2) V. lozanoi is an introduced Fundulus, distinct from $F$. heteroclitus. The hypothesis of a European, native Fundulus seems unlikely, especially with the absence of supporting evidences. There are additional alternative hypotheses to explain the differences between the karyotypes described by Delgado et al. and Chen \& Ruddle: 3) Chen \& Ruddle's material might have been misidentified; 4) F. heteroclitus having an extensive distribution, geographical chromosome polymorphism may occur (or many species may presently be lumped under that name); 5) $V$. lozanoi is a native species belonging to a still unrecognised genus superficially similar to Fundulus; 6) differences in methodology and/or terminology. Hypothesis 5 seems unlikely. Hypothesis 4 seems the most likely and is actually confirmed by data in Bernardi et al. (1995) and Fernández-Pedrosa et al. (1996). The karyotype of $F$. heteroclitus illustrated by Fisher \& Rachlin (1972) does not seem to differ from the one by Delgado et al. Fundulus heteroclitus has been revised by Relyea (1983).

One may only regret that so much attention is given to an introduced fish, and not to the native European species deserving urgent work.

\section{Valenciidae}

19.1.1 Valencia hispanica (Valenciennes, 1846)

Hydrargyra Hispanica Valenciennes, in Cuvier \& Valenciennes, 1846: 214, pl. 531 fig. 1 (type locality: freshwaters of Catalogna, Spain; syntypes: MNHN)
19.1.2 Valencia letourneuxi (Sauvage, 1880) Fundulus Letourneuxi Sauvage, 1880: 214 (type locality: sources near Cressida, Corfou [Kerkira island], Greece; syntypes: MNHN)

Systematic notes. See Villwock et al. (1982) and Bianco \& Miller (1989) for discussion.

\section{Poeciliidae}

Gambusia affinis (Baird \& Girard, 1853) introduced

Gambusia holbrooki Girard, 1859 introduced

Poecilia reticulata Peters, 1859 introduced

\section{Cyprinodontidae}

20.1.1 Aphanius fasciatus (Valenciennes, 1821)

Lebias fasciata Valenciennes, in Humboldt \& Valenciennes, 1821: 160, pl. 51 fig. 4 (type local- ity: not stated [Cagliari, Sardegna according to Valenciennes, in Cuvier \& Valenciennes, 1846: 156]; syntypes: MNHN)

? Ciprinoides [sic] Nanus Nardo, 1824: 259 [23 
of reprint] (nomen nudum; locality not stated [streams of Adriatic Sea basin, Italy])

? Cyprinoides Nanofasciatus Nardo, 1824: 259 [23 of reprint] (nomen nudum; locality not stated [streams of Adriatic Sea basin, Italy])

Aphanius Nanus Nardo, 1827a: 488 (type locality: Adriatic Sea; syntypes: then in Nardo's collection in Chioggia) or 1827b: 34, 40 (same data)

Lebias lineato-punctata Wagner, 1828: 1055, pl. 12 figs. 1-6 (type locality: fresh and marine waters around Cagliari, Sardegna; syntypes: probably ZSM, now lost)

Lebias Sarda Wagner, 1828: 1055, pl. 12 fig. 7 (type locality: marine waters around Cagliari, Sardegna; syntypes: probably ZSM, now lost)

Poecilia calaritana Cuvier, 1829: 280 (type locality: Sardegna; syntypes: MNHN 187 [3], Wildekamp et al., in Daget et al., 1986: 168)

Lebias flava Costa, 1838: fasc. 19: 35, pl. 17 fig. 1 (type locality: lake Varano, Italy; syntypes: LU)

Cyprinodon Hammonis Valenciennes, in Cuvier \& Valenciennes, 1846: 169 (type locality: Oasis Jupiter Ammon and other parts of Egypt and Syria; syntypes: MNHN 23346 [8], A-3822 and A-3979 [4], A-3961 and A-3980 [9], A-3981 and A-3982 [14], Wildekamp et al., in Daget et al., 1986: 168, ZMB 3479 [3], Paepke \& Seegers, 1986: 147)

Lebias nigropunctata Schinz, 1840: 334 (type locality: salty ponds of Sardegna; type material: NT ?)

Cyprinodon Cyanogaster Guichenot, 1859: 378 (type locality: Biskara, north-eastern Algeria; syntypes: MNHN 3218 [5], Wildekamp et al., in Daget et al., 1986: 168)

Cyprinodon doliatus Guichenot, 1859: 379 (type locality: Biskara, north-eastern Algeria; syntypes: MNHN 4392 [8], Wildekamp et al., in Daget et al., 1986: 168)

Micromugil timidus Gulia, 1861: 11 (type locality: Malta Island; type material: NT ?)

Micromugil macrogaster Gulia, 1861: 11 (type locality: Malta Island; type material: NT ?)

Cyprinodon Desioi Gianferrari, 1932: 214, fig. 1 (type locality: sulphurous sources in Ain elBraghi near Agheila, northern Libya; syntypes: MSNM 4546)

Nomenclatural notes. The type species of the genus Lebias Goldfuss, 1820 (p.16) is L. fasciata Valenciennes, 1821 as designated by Lazara (1995: 502). Thus Lebias is a subjective senior synonym of Aphanius Nardo, 1827, has priority and is the valid name. Lebias has been sometime used for this genus between 1820 and 1907 and it is only since 1948 that Aphanius has been used
(Lazara, 1995: 502). Lazara's type-species designation is irrevocable (except by petitioning the International Commission on Zoological Nomenclature), but it is arguable since he could as well have designated $L$. rhomboidalis Valenciennes, in Humboldt \& Valenciennes, 1821 [p. 160, pl. 51 fig. 3; type locality not stated] as type species, making Lebias a junior synonym of Cyprinodon La Cepède, 1803: 486 and thus avoiding nomenclatural changes. Some name changes are unavoidable, but this one not only could easily have been avoided but should have been avoided as it concerns a large number of species, most of which are endangered or threatened and listed on a variety of national and international legislations. An application has been submitted to the International Commission on Zoological Nomenclature to rule out Lazara's type species designation for Lebias (Kottelat \& Wheeler, in press a). The existing usage (Aphanius) is to be maintained until the Commission publishes its ruling (ICZN art. 80(a)).

The publication date of Lebias fasciata is somewhat confused. Valenciennes (in Cuvier \& Valenciennes, 1846: 156) wrote about Humboldt \& Valenciennes's paper as "our work of 1817". Valenciennes (in Humboldt \& Valenciennes, 1821: 193) stated that his work on poeciliids (p. 159; L. fasciata is on p. 160) was already printed when he received Lesueur (1821). It seems that the work was printed in 1817 but not distributed before 1821. Fascicle 10 appeared in 1817, fascicle 12 in 1821 (Kottelat, 1988: 88) and fascicle 11 in 1821 (Lazara, 1993). Humboldt \& Valenciennes appeared in part in fascicles 11 (pp. 145-176) and 12 (177-216). For nomenclatural purposes, the year of publication is the year the name is made available (that is the date on which the work has been distributed, and not the year in which it has been printed).

Aphanius nanus Nardo (1827b) has been declared a nomen oblitum by Tortonese (1970b); note that this explicitly applies to Nardo's $1827 \mathrm{~b}$ paper, but not to the 1827a one.

Paepke \& Seegers (1986: 147) list three ZMB specimens (ZMB 3479) as syntypes of Cyprinodon hammonis because they have the same collecting data as the material mentioned by Valenciennes and on the assumption that this material is a part of the type series which had been returned to Berlin. Although loans were not a common curatorial practice of the time, there is evidence that some happened between Berlin and Paris (H.-J. Paepke, in litt., 1995). Valenciennes visited Berlin 
at least twice before 1846 (1826 and 1829, H.-J. Paepke, in litt., 1995) and he might have based his account on both the specimens with him and those left in Berlin.
20.1.2 Aphanius iberus Valenciennes, 1846 Cyprinodon Iberus Valenciennes, in Cuvier \& Valenciennes, 1846: 160, pl. 528 fig. 1 (type locality: Spain; syntypes: MNHN 185 [8], Tortonese, in Hureau \& Monod, 1973: 271)

\section{Gasterosteidae}

\subsection{Gasterosteus}

Data on the distribution of the different forms of European three-spined sticklebacks are summarised by Münzing (1963) and Paepke (1982: 273-275, 317-320, 1983: 115-119). Briefly, pure low-plated ("gymnura") populations inhabit streams of the Mediterranean drainage, streams draining to the Atlantic coast south of the Channel and along the western slope of British Isles, and the Rhine basin, while pure completely plated ("trachura") populations inhabit the Atlantic coast of Scotland and Scandinavia, the south-eastern shore of the Baltic Sea and tributary streams, the shores of the Black Sea and tributary streams. In the intermediate area (Baltic Sea, drainage of the North Sea, basins of rivers Elbe and Oder), populations include a mixture of these two types plus an intermediate type ("semiarmata"). See Paepke (1982: 292) for a detailed study of the sticklebacks of the Elbe and Oder basins.

Münzing proposed a complicated hypothesis for the evolution of the various populations. It would probably be difficult to have most of the details of Münzing's hypothesis fitting to present zoogeographical philosophy and procedures, but the main lines are that at some time (during glaciations), two distinct stocks had a widely disjunct distribution: the low-plated ("gymnura") form in the Mediterranean and western Europe and the fullyplated ("trachura") form in northern and eastern Europe and the Black Sea. They came into contact (supposedly in interglacial but definitively in postglacial times) in the area of the North Sea and the Baltic Sea where they hybridised ("semiarmata"). The existence of landlocked populations in northern Scandinavia and Iceland was considered to result from dispersal (p. 331).

In other words, we have two independent and diagnosable lineages with a contact zone, and these two lineages are species under the PSC.
I found that many of the discussions on the systematics of Gasterosteus are strongly biased because most authors focused on a single character (plate development) and omit discussing any other. Data on some European populations as summarised by Miller \& Hubbs (1969: 65) seem to indicate that several geographically isolated populations are morphologically distinct, without known intergrades, and would be recognisable as species under the PSC (Miller \& Hubbs recognised them as subspecies under the BSC). The long-range northward dispersal of southern European low-plated individuals/stocks to colonise isolated water bodies in northern Europe (Iceland, northern Scandinavia) seems very unlikely, and these isolated northern stocks are hypothesised to result of onsite differentiation from the local fully plated stocks.

Accordingly, I recognise here two species in mainland Europe, the fully plated, eastern G. aculeatus and the low-plated, western G. gymnurus with a contact zone in the North Sea and Baltic Sea area (in the following synonymies, nominal species based on intermediate specimens are arbitrarily referred to $G$. aculeatus in the North Sea and Baltic Sea and to G. gymnurus in the Channel). Isolated populations with independent histories and morphologically diagnosable are treated as species. These include two partially plated species, in Iceland and Romania. The naked population from Roma, Italy, named G. hologymnus by Regan (1909b) is included in G. gymnurus because there is apparently a north-south clinal variation of the plate number in Italy (P. G. Bianco, pers. comm., 1995). The status of the low-plated populations from northern Scandinavia has not been investigated.

Outside Europe, noteworthy are G. algeriensis Sauvage (1874a: 17; type locality: Algeria; 
holotype: MNHN 5123 [Monod, in Hureau \& Monod, 1973: 281 list 4 syntypes, while Sauvage explicitly designated a holotype]), if not introduced (Bertin, 1925), the only African stickleback (possibly extinct; Münzing, 1963: 326), and stocks from Iznik Gölü, Turkey (Münzing, 1962) whose taxonomic status should be evaluated.

This discussion is only based on published data based on European stocks. Results of the numerous studies on genetics, ecology, etc. of North American and East Asian stocks are a priori not relevant for discussion on systematics of European ones, unless it is demonstrated that they are conspecific.

Haglund et al. (1992a) examined allozyme variation among 16 stocks of European, North American and Japanese sticklebacks referred to G. aculeatus. They included one stock from Italy, two from U.K. and one from Sweden. Their data show the Italian stock more closely related with one of the U.K. stocks and the Swedish stock more closely related with the other U.K. stock. The Italy-U.K. clade was more closely related to a New York stock than to the other European clade. A similar study involving more European stocks could be used as a test for the hypothesis that $G$. aculeatus and $G$. gymnurus are distinct species. The mitochondrial DNA study by Ortí et al. (1994) is inconclusive in the present context; it shows (p. 619) that sympatric and reproductively isolated stocks (= species) of Gasterosteus display "small or no genetic divergence" (thus that speciation [especially recent] is not necessarily linked with genetic divergence, see p. 19).

If one does not want to consider $G$. aculeatus and $G$. gymnurus as different species, one should at least refrain from using a terminology similar to formal nomenclature (leiurus, trachurus, etc.) for different forms or morphs; readers (and authors) without clear understanding of these concepts often confuse them with species and subspecies. Bakker \& Sevenster (1988) reviewed the confusing history of the uses and misuses of names like trachurus, leiurus and semiarmatus for plate morphs, ecological forms, etc. and I agree with their conclusions, except that if latinised names are used for morphs (leiura, semiarmata, trachura), they should in no case be italicised in order to avoid confusion with species names. Actually, I find that the use of vernacular descriptors (e.g., armored, semi-armored, etc.) has the advantage of explicitly describing what the author means.
The following synonymies include only taxa based on European specimens.

\subsubsection{Gasterosteus aculeatus Linnaeus, 1758}

Gasterosteus aculeatus Linnaeus, 1758: 295 (based on Artedi [1738: gen. 52 [96], syn. 80, spec. 26 [52], Gasterosteus aculeis in dorso tribus] and Linnaeus [1746: 103, n. 276, idem]; type locality: "in Europa"; syntypes: LSL 29-31 [3], Wheeler, 1985: 43)

Gasterosteus teraculeatus La Cepède, 1801: 295 (unnecessary replacement name for Gasterosteus aculeatus Linnaeus, 1758)

Gasterosteus trachurus Cuvier, 1829 [March]:170 (type locality: France; also available by indication to Bloch [1783: pl. 53 fig 3; Gasterosteus aculeatus; locality not stated but likely to be around Berlin, Germany]; syntypes: MNHN, ZMB, ? NMW 6918 [4])

? Gasterosteus spinulosus Jenyns, 1835a (nomen nudum); Yarrell, 1835: 83, fig. (type locality: near Edinburgh, Scotland; syntypes: LU ?; authors stated to be Jenyns \& Yarrell, but Yarrell is alone responsible for conditions making the name available, see below); Jenyns, 1835b: 350 (locality: Edinburgh, Scotland).

Gasterosteus ponticus Nordmann, 1840: 380 (type locality: Black Sea; syntypes [total 6]: MNHN 7098 [1], Monod, in Hureau \& Monod, 1973: 281, ? NMW 77244 [4])

Gasterosteus biarmatus Nordmann, 1840: 381

(type locality: lagoons near Tarkanckut, Crimea; type material: NT ?)

Systematic notes. It remains to be demonstrated that the Black Sea stocks are conspecific with the northern European ones.

Nomenclatural notes. The restriction of the name $G$. aculeatus to the armored species is discussed by Miller \& Hubbs (1969: 66). They pointed out correctly the composite nature of Linnaeus's $G$. aculeatus which apparently included material of three species ( $G$. aculeatus, G. gymnurus and G. hologymnus [included in G. gymnurus here]) and that a definitive restriction of the name is only possible by the designation of a lectotype or a neotype. Linnaeus syntypes are still extant (Wheeler, 1985: 43).

Yarrell (1835-36) and Jenyns (1835a-b) indicate the authorship of $G$. spinulosus as Yarrell \& Jenyns, but there is no joint paper by these authors. They published separately on this fish, but obviously shared information as they refer to each other, often with exact page numbers. Jenyns (1835a: 22) is the earliest use of the name (April 
1835 according to Sherborn, 1922: lxxi) but it is a nomen nudum. The first use making the name available is apparently by Yarrell (1835-36: 83); this work has been issued in 19 parts between April 1835 and September 1836, and I assume that p. 83 appeared before the next use by Jenyns (1835b) in November 1835 (Sherborn, 1922: lxxi, cxxx) Jenyns (1835b: 350) refer to Yarrell \& Jenyns, Edinburgh New Phil. J., April 1831, p. 386. This page refers to a paper by Stark (1831) which actually describe the same fish under the name $G$. aculeatus, but the paper has nothing to do with Jenyns or Yarrell.

The author of Gasterosteus biarmatus is often given as Krynicki or Krynicki in Nordmann (1840). Nordmann is actually responsible for the conditions which make the name available and is thus author of the name.

\subsubsection{Gasterosteus crenobiontus Bacescu \& Mayer, 1956}

Gasterosteus aculeatus forma crenobionta Bacescu \& Mayer, 1956: 24 (type locality: sources of Techirghiol, Romania; syntypes: MGAB 49912, Mihai-Bardan, 1984: 460)

Systematic notes. This species is extinct (Bănărescu, 1994: 8).

Nomenclatural notes. Mihai-Bardan (1984: 460) lists specimen MGAB 49912 as holotype of Gasterosteus crenobiontus. As Bacescu \& Mayer (1956) did not designate a holotype, all their specimens are syntypes.

\subsubsection{Gasterosteus gymnurus Cuvier, 1829}

Gasterosteus gymnurus Cuvier, 1829 [March]: 170 (type locality: France; also available by indication to Willughby, 1686: 341; syntypes: MNHN ?)

Gasterosteus leiurus Cuvier, in Cuvier \& Valenciennes, 1829b [November]: 481, 487, pl. 98 fig. 4 (type locality not clearly stated; on pp. 494-495, Cuvier lists a number of localities in Europe: coasts of Normandie, Caen, and Hable d'Ault near Le Tréport, France; Berlin, Germany; syntypes: MNHN 7088 [3], 7151 [7], Monod, in Hureau \& Monod, 1973: 281)

Gasterosteus semiarmatus Cuvier, in Cuvier \& Valenciennes, 1829b [November]: 493 (type locality: Le Havre and creek of Braie near Abbeville, France; syntypes: MNHN 2348 [1], 7078 [3], 2347 [1], Monod, in Hureau \& Monod, 1973: 281)

Gasterosteus semiloricatus Cuvier, in Cuvier \& Valenciennes, 1829b [November]: 494 (type locality: river Somme, France; syntypes: MNHN)
Gasterosteus argyropomus Cuvier, in Cuvier \& Valenciennes, 1829b [November]: 498 (type locality: Toscana [Firenze, Monod, in Hureau \& Monod, 1973: 281], Italy; syntypes: MNHN 677 [3], Monod, in Hureau \& Monod, 1973: 281)

Gasterosteus brachycentrus Cuvier, in Cuvier \& Valenciennes, 1829b [November]: 499, pl. 98 fig. 2 (type locality: Toscana [Firenze, Monod, in Hureau \& Monod, 1973: 281], Italy; syntypes: MNHN 694 [3], 7097 [2], Monod, in Hureau \& Monod, 1973: 281)

Gasterosteus tetracanthus Cuvier, in Cuvier \& Valenciennes, 1829b [November]: 499 (type locality: Toscana [Firenze, Monod, in Hureau \& Monod, 1973: 281], Italy; holotype: MNHN 1821, Monod, in Hureau \& Monod, 1973: 281)

Gasterosteus quadrispinosa Crespon, 1844: 283 (type locality: stream Vistre and ditches around Nîmes, France; syntypes: LU)

Gasterosteus nemausensis Crespon, 1844: 283 (type locality: standing waters of plain around Nîmes, France; syntypes: LU)

Gasterosteus neustrianus Blanchard, 1866: 220, fig. 28 (type locality: brooks in Harfleur and Gournay, Seine-Inférieure, France; syntypes: MNHN 7114 [1, listed as holotype by Monod, in Hureau \& Monod, 1973: 281])

Gasterosteus Bailloni Blanchard, 1866: 231, fig. 34 (type locality: Abbeville, France; syntypes: MNHN 7153 [12], Monod, in Hureau \& Monod, 1973: 281)

Gasterosteus argentatissimus Blanchard, 1866: 232, fig. 35 (type locality: brooks near Avignon, France; syntypes: MNHN 6675 [6], 6678 [18], Monod, in Hureau \& Monod, 1973: 281)

Gasterosteus elegans Blanchard, 1866: 234, fig. 37 (type locality: between Cadillac and Langon, Gironde, France; syntypes: MNHN 6673 [2], 7100 [1], Monod, in Hureau \& Monod, 1973: 281)

Gastrosteus [sic] hologymnus Regan, 1909b: 436 (type locality: Roma, Italy; syntypes: BMNH [5])

Gasterosteus aculeatus var. messinicus Stephanidis, 1971a: 202, fig. 10 (infrasubspecific, name not available; locality river Pamissos near Messini, Greece)

Nomenclatural notes. The description of $G$. gymnurus has priority over $G$. leiurus because it was published in March (Boeseman, 1962: 79), vs. November (Eschmeyer, 1990).

\subsubsection{Gasterosteus islandicus Sauvage, 1874}

Gasterosteus islandicus Sauvage, 1874a: 20, pl. 1 
fig. 8 (type locality: Iceland; holotype: MNHN 5594 [Monod, in Hureau \& Monod, 1973: 281 list 3 syntypes, while Sauvage explicitly mentioned a holotype])

Systematic notes. See Penczak (1964).

\subsubsection{Pungitius hellenicus Stephanidis, 1971}

Pungitius hellenicus Stephanidis, 1971b: 228, figs. 7-8 (type locality: Spercheios valley, Greece; syntypes: lost ? [40])

\subsubsection{Pungitius laevis (Cuvier, 1829)}

Gasterosteus laevis Cuvier, 1829: 170 (type locality: France; syntypes: MNHN 7102 [5, Somme], 7103 [3, Bobigny, near Paris], Monod, in Hureau \& Monod, 1973: 284)

Gasterosteus vulgaris Mauduyt, 1848: 19 (type locality: la Vergne near Poitiers, la Bergue near Gençay, les Aiffes near St-Maurice, la Séguinière near St-Julien-Lars, Vienne, France; syntypes: Musée de Poitiers ?)

Gasterosteus burgundianus Blanchard, 1866: 240 (type locality: Dijon, Côte d'Or, France; syntypes: MNHN 7103 [5], Monod, in Hureau \& Monod, 1973: 284)

Gasterosteus lotharingus Blanchard, 1866: 244, fig. 42 (type locality: river Meuse and adjacent brooks near Saint Mihiel, France; syntypes: MNHN 7090 [2], Monod, in Hureau \& Monod, 1973: 285)

Gasterosteus breviceps Blanchard, 1866: 245, fig. 43 (type locality: Caen, France; syntypes: MNHN 7110 [1, listed as holotype by Monod, in Hureau \& Monod, 1973: 285])

? Gasterosteus globiceps Sauvage, 1874a: 35, pl. 1 fig. 17 (type locality: North America [erroneous according to Berg, 1932a: 169, Münzing, 1969: 217]; syntypes: MNHN 4081 [2], Monod, in Hureau \& Monod, 1973: 284)

Pygosteus pungitius forma hologymna Bertin, 1925: 122 (infrasubspecific, name not available)

Systematic notes. Münzing (1969: 210) recognises two subspecies of $P$. pungitius in Europe. Pungitius $p$. laevis is distinguished from P. p. pungitius by the complete absence of lateral scutes (vs. presence of 3-18 scutes, generally 4-11). Although Münzing considers them as a cline, his data do not show a clear clinal variation (or if there is one, it is obscured by the way the data are presented), but a narrow fringe in northern France and Netherlands (and England according to his figure, but not his table 1) where some naked individual occur among populations usually with 38 plates. His figure 1 shows many more localities than cited in the text; these are probably from the literature. If a clear clinal variation were observable, I would conclude that a single species exists. But the published data seem to indicate more the existence of two species with a contact zone (see Warren 1992: 25-26 for brief discussion of contact or hybrid zones and further references) and so I recognise both $P$. pungitius and $P$. laevis as valid species. Münzing (1969: 226) considered that during the Pleistocene glaciations, Pungitius stocks survived in a western European, a Ponto-Caspian and an East Asian refugia. After the glaciations, $P$. pungitius would have recolonised eastern and northern Europe and P. laevis south-western Europe. This pattern is similar to the case discussed under Gasterosteus aculeatus. If this hypothesis is accepted, here too we have two independent and diagnosable lineages, which are species under the PSC.

It might be worth investigating if the $P$. laevislike stocks from England and Ireland are conspecific with those from France. In this context, it should be pointed out that these stocks are united by a shared reductive character (the loss of scutes) which can hardly be used as a synapomorphy if not corroborated by additional characters. It does not seem that many other characters have been looked at.

'Clinal' variation of meristic characters are often interpreted as resulting from different temperatures. This hasty generalisation to all fishes of the results of some experiments with a few populations of a few species is not acceptable; see Gross (1979: 410) or Spillmann (1962: 450) for examples where this theory does not apply.

\subsubsection{Pungitius platygaster (Kessler, 1859)}

Gasterosteus platygaster Kessler, 1859b: 202 (type locality: harbour of Odessa and side arm of the Dnieper in Aleschki, Ukraine; syntypes: [21] lost, H.-J. Paepke, in litt., 1995)

Gasterosteus platygaster var. Kessleri Yakovlev, 1870: 110 (Astrakhan, Russia) from Berg, 1949: 969

Gasterosteus platygaster var. niger Yakovlev, 1870: 110 (Astrakhan, Russia) from Berg, 1949: 969

Gasterosteus platygaster var. caucasicus Kessler, 1877a: 3 (type locality: Transcaucasia [Berg, 1949: 969]; syntypes: LU [12])

Gasterosteus platygaster var. danubica Steindachner, 1899: 542 (type locality: Danube swamps near Negotin, Serbia and river Save [Sava] near Belgrad, Serbia; syntypes: NMW $6823[6])$ 
Pygosteus platygaster var. nuda Berg, 1905: 218 (type locality: lake Charkhal, river Ural basin; syntypes: ZISP 10613; author given as Nikolsky, but Berg is actual author)

Systematic notes. Münzing (1969: 222) recognises two subspecies, P. p. platygaster and P. p. aralensis (Kessler, 1877a: 4). The presentation of his data does not allow a clear interpretation of the characters and their variability in both subspecies, but it seems that $P$. aralensis, restricted to the Aral Sea basin, is a distinct species under the PSC.

21.2.4 Pungitius pungitius (Linnaeus, 1758) Gasterosteus Pungitius Linnaeus, 1758: 296 (based on Artedi [1738: gen. 52 [97], syn. 80, spec. 97 [52], Gasterosteus aculeis in dorso decem] and Linnaeus [1746: 104, n. 277, idem]; type local- ity: "in Europa"; syntypes: LSL 34-35, Wheeler, 1985: 44)

Pygosteus pungitius forma trachura Bertin, 1925: 122 (infrasubspecific, name not available)

Pygosteus pungitius forma semiarmata Bertin, 1925: 122 (infrasubspecific, name not available) Pygosteus pungitius forma carinata Bertin, 1925: 122 (infrasubspecific, name not available)

Systematic notes. See Pungitius laevis for comments. Similarly, it seems unlikely that the East Asian and nearctic populations are conspecific with the European ones, and they have been omitted from the above synonymy. Allozyme studies by Haglund et al. (1992b) confirms this hypothesis, but do not contribute to understanding relationships among European stocks as too few have been examined.

\section{Syngnathidae}

22.1.1 Nerophis ophidion (Linnaeus, 1758) Syngnathus Ophidion Linnaeus, 1758: 337 (based on Artedi [1738: gen. 1, syn. 2, spec. 1, Syngnathus teres ...], Linnaeus [1746: 126, n. 334, idem] and Gronovius [1754: 1, n. 2, idem]; type locality: "in Europa"; syntype: BMNH 1853.11.12:185, Wheeler, 1958: 216)

Scyphius violaceus Risso, 1826: 187 (type locality: not stated, probably around Nice, France; type material: NT)

Scyphius littoralis Risso, 1826: 188 (type locality: not stated, probably around Nice, France; type material: NT)

Scyphicus teres Rathke, 1837: 319 (Sevastopol, Crimea, Ukraine) from Berg, 1949: 978

Nomenclatural notes. Wheeler (1958: 216) noted that Linnaeus's (1758) description of Syngnathus ophidion is based on different accounts which include two species. The only surviving syntype of this composite series is not conspecific with the species usually called $N$. ophidion.

\subsubsection{Syngnathus abaster Risso, 1826}

Syngnathus abaster Risso, 1826: 182 (type locality: not stated, probably around Nice, France; type material: NT)

Syngnathus ethon Risso, 1826: 182 (type locality: not stated, probably around Nice, France; type material: NT)

Syngnathus Agassiz Michahelles, 1829: 1013 (type locality: Triest, Italy; type material: NT ?)

Syngnathus nigrolineatus Eichwald, 1831: 61 (type locality: Black Sea near Odessa, Ukraine; syntypes: LU)

Syngnathus caspius Eichwald, 1831: 61 (type locality: "sinu balchanensi caspii maris"[Balkhan Bay, Caspian Sea; Berg, 1949: 985]; syntypes: LU)

Syngnathus bucculentus Rathke, 1837: 317 (Sevastopol, Feodosiya, Crimea) from Berg, 1949: 982

Syngnathus flavescens Kaup, 1856b: 35 (type locality: Tripoli, Libya; holotype: RMNH, lost according to Dawson, in Daget et al., 1986: 287)

Syngnathus cuvieri Kaup, 1856b: 38 (type locality: Catwyk, North Sea / Napoli, Italy; syntypes: RMNH [several], MNHN [1])

Syngnathus algeriensis Günther, 1870: 164 (type locality: freshwaters in Algiers [Alger], Algeria; syntypes: BMNH 1869.1.29:17 [5], Dawson, in Daget et al., 1986: 287)

Syngnathus Anguisigola Chiereghini, in Nardo, 1847: 113 (type locality: lagoon of Venezia, Italy; type material: LU) 
Syngnathus microchirus Moreau, 1891: 188 (type locality: pool of Pinetto, near Bastia, Corsica; syntypes: LU [22], lost according to Dawson, in Daget et al., 1986: 287)

Syngnathus abaster ganzirriensis d'Ancona, 1934: 53 (type locality: Lago grande di Ganzirri, Messina, Sicilia; syntypes: LU)

Syngnathus nigrolineatus maeoticus Slastenenko, 1938a: 174 (nomen nudum), 1938b: 138 (Azov Sea, Black Sea, lakes of Danube delta in Romania) from Berg, 1949: 982

Syngnathus ethon aeolicus Caporiacco, 1948: 91 (type locality: Napoli, Messina, Palermo, southern Tyrrhenian Sea, Italy; syntypes: LU [11])

\subsubsection{Syngnathus acus Linnaeus, 1758}

Syngnathus Acus Linnaeus, 1758: 337 (based on Artedi [1738: gen. 1 [2], syn. 2, spec. 2 [1], Syngnathus corpore medio heptagona ...], Linnaeus [1746: 126, n. 335, idem] and Gronovius [1754: 1, n. 3, idem]; type locality: "in Europa"; type material: NT)

Syngnathus Rubescens Risso, 1810: 66 (type locality: sea shore in Nice, France; type material: NT)

Syngnathus Temminckii Kaup, 1856b: 36 (type locality: Cape [of Good Hope, South Africa]; syntypes: RMNH [2, lost, Dawson, in Daget et al., 1986: 287])

Syngnathus brachyrhynchus Kaup, 1856b: 42 (type locality: "Isle of Bourbon" [Réunion Island]; holotype: MNHN 6133, Dawson, in Daget et al., 1986: 287)

Syngnathus Delalandi Kaup, 1856b: 45 (type locality: Cape of Good Hope and Cape Seas, South Africa; syntype: MNHN 6139 [1], Dawson, in Daget et al., 1986: 287, ZMB [2])

Syngnathus alternans Güther, 1870: 162 (type locality: Seychelles islands; holotype: BMNH 1855.9.19:1395, Dawson, in Daget et al., 1986: 287)

Nomenclatural notes. Dawson (in Daget et al., 1986: 287) lists MNHN as holotype of Syngnathus Delalandi Kaup, 1856. As Kaup clearly stated that he had examined three specimens, they all are syntypes.

\subsubsection{Syngnathus taenionotus Canestrini, 1871}

Syngnathus taenionotus Canestrini, 1871: 143 (type locality: Laguna Veneta, Italy; syntypes: MSNG 14038 [3], Tortonese, 1963a: 312)

\subsubsection{Syngnathus typhle Linnaeus, 1758}

Syngnathus Typhle Linnaeus, 1758: 337 (based on Artedi [1738: gen. 1 [3], syn. 1, spec. 3 [1], Syngnathus corpore medio hexagono [heptagono in gen. 1] ...], Linnaeus [1746: 126, n. 336, idem] and Gronovius [1754: 2, n. 4, idem]; type locality: "in Europa"; potential syntype: LSL 49, Wheeler, 1985: 42)

Syngnathus Rondeletii Delaroche, 1809: 324, pl. 21 fig. 5 (type locality: Iviça [Ibiza], Spain; syntypes: MNHN)

Tiphle hexagonus Rafinesque Schmaltz, 1810a: 18 (unnecessary replacement name for Syngnathus typhle Linnaeus, 1758)

Syngnathus pelagicus Risso, 1810: 63 (type locality: Nice, France; type material: NT; junior homonym of Syngnathus pelagicus Osbeck, 1765)

Syngnathus Viridis Risso, 1810: 65 (type locality: "mer de S. Hospice" / Villefranche, France; type material: NT)

Syngnathus ponticus Pallas, 1814: 118 (type locality: Black Sea, Azov Sea; syntypes: LU)

Syngnathus argentatus Pallas, 1814: 120 (type locality: Black Sea; syntypes: LU)

Syngnathus pyrois Risso, 1826: 180 (replacement name for Syngnathus pelagicus Risso, 1810)

Syngnathus rotundatus Michahelles, 1829: 1014 (type locality: Triest, Italy; syntypes: LU)

Nomenclatural notes. The only extant potential syntype of Syngnathus typhle is actually a specimen of $S$. acus Linnaeus, 1758 (Wheeler, 1985: 42). Linnaeus also based his diagnosis on the accounts of Artedi (1738) and Gronovius (1754) who apparently refer to the species presently called S. typhle. 


\section{Cottidae}

\subsubsection{Cottus ferrugineus Heckel \& Kner,} 1858

Cottus ferrugineus Bonaparte, 1846: 62 (nomen nudum)

Cottus ferrugineus Heckel \& Kner, 1858: 34, figs. 14-15 (type locality: lake Garda, Milano, Treviso, Italy, and Xegar, Dalmatia [Croatia]; syntypes: NMW 6036 [4], 6419 [5], 6562 [2], 6563 [3], 6673 [6], 78799 [4])

Systematic notes. Koli (1969: 385) treated C. ferrugineus as synonym of C. gobio. Bianco (1994: 472 ) lists Italian material as $C$. gobio without commenting on the nomenclature. With the present (lack of) data, the characters reported by Bacescu \& Bacescu-Mester (1964: 444) justify treating C. ferrugineus as a distinct species until this is confirmed or refuted.

\subsubsection{Cottus gobio Linnaeus, 1758}

Cottus Gobio Linnaeus, 1758: 265 (based on Artedi [1738: gen. 48 [82], syn. 76, spec. 82 [48], Cottus alepidotus glaber .....], Linnaeus [1746: 104, n. 279, idem] and Gronovius [1756: 14, a. 166, Uranoscopus capite utrinque ...; "Mare septentrionale"]; type locality: "in Europae fluviis"; type material: NT)

Cottus microstomus Heckel, 1837: 147, pl. 8 figs. 3-4 (type locality: vicinity of Krakow, Poland; holotype: NMW [not yet located; B. Herzig, pers. comm., 1995])

Cottus affinis Heckel, 1837: 130 (available by indication to Ekström [1835: 167, Cottus gobio (1832: 308 in original)]; type locality: Mörkö Island [near Stockholm], Sweden; syntypes: LU)

Cottus gobio var. macrostomus Jeitteles, 1863: 14 (infrasubspecific, name not available; locality: Baden in der March [Morava], near Neustift, near Olmuitz [Olomouc], Czech Republic; junior homonym of Cottus poecilopus var. macrostomus Jeitteles, 1862)

Cottus koshewnikowi Gratzianow, 1907a: 658 (type locality: river Goredwa [Goredva] (Oka system), river Kolomna, lake Selig and Malysch district, Wjatka [Vyatka], Russia; syntypes: ZMMSU)

? Cottus gobio jaxartensis Berg, 1916a: 437 (type locality: river Ugam, a tributary of river Chirchik, itself a tributary of the Syr-Darya, Uzbekistan; holotype: ZISP ?)

Cottus gobio milvensis Soldatov, 1924: 57 (river Pechora, near confluence with river Mylva, Russia) from Berg, 1949: 1147
Cottus gobio var. roseus Odenwall, 1927: 56 (type locality: Lappajärvi Sjö, Finland; syntypes: MZH, Koli, 1969: 384)

Cottus gobio natio pellegrini Vladykov, 1931: 353, fig. 28 (infrasubspecific, name not available; locality: "Russie sous-carpathique" [Carpathes range in Ukraine], Danube basin)

Cottus gobio pellegrini Bacescu \& Bacescu-Mester, 1964: 442 (available by indication to Vladykov, 1931: 353; type locality: "Russie souscarpathique" [Carpathes range in Ukraine], Danube basin; syntypes: LU)

Cottus gobio hispaniolensis Bacescu \& BacescuMester, 1964: 445 (type locality: river Garona at Viella, Eastern Pyreneans, Spain; syntypes: MGAB ?)

? Cottus gobio haemusi Marinov \& Dikov, 1986: 19 (type locality: upper river Beli Vit above village Ribarica, Danube basin, Bulgaria; holotype: BFSU 6039-1)

Systematic notes. A revision of this 'species' is needed. Material from the whole range of the species should be critically compared. It seems very likely that several species have been confused under a single name. From a study of Scandinavian populations, Koli (1969: 353) concluded that there are two distinct groups resulting of "the postglacial immigration of morphologically different strains from two different directions" with a zone of secondary intergradation (a case similar to the one reported above for Gasterosteus). Witkowski $(1979,1995)$ made the same observations with material from Poland. If this is confirmed by study of additional material from other areas, these two independent and diagnosable lineages may have to be treated as species under the PSC. Robins (1961: 310 ) considered $C$. gobio as a species-complex. The genetic data in Riffel \& Schreiber (1995) also suggest that several species may be confused.

A suggested reading on characters useful for distinguishing some North American species of Cottus is Jenkins \& Burkhead (1994: 625-674).

Cottus gobio haemusi Marinov \& Dikov (1986) is said to differ from all other populations of Cottus gobio in having a smaller head and less anal rays. Data from all other populations have been compiled from the literature and it remains to be demonstrated that all authors used the same methods, especially for measuring head length. If the differences are confirmed, this stock may have to be treated as a valid species and should be described in detail. 
23.1.3 Cottus petiti Bacescu \& BacescuMester, 1964

Cottus petiti Bacescu \& Bacescu-Mester, 1964: 432, fig. 1, pl. 1 (type locality: sources of river Le Lez, France; holotype: MGAB 50)

Systematic notes. Koli (1969: 386) treated C. petiti as conspecific with $C$. gobio because the characters used to recognise them are within the range of variation of his C. gobio. His data are based mostly on Scandinavian specimens, and it seems dubious that the similarity in some characters (extent of prickling, complete lateral line) between C. petiti from southern France and some specimens from northernmost Finland should justify considering them conspecific. The comparison with material from adjacent waters would be more meaningful and the data presented by Bacescu \& Bacescu-Mester (1964) presently justify recognising $C$. petiti as a distinct species. Persat et al. (1996: 325) report possible differences in reproduction biology of $C$. petiti and C. gobio.

To just rule out the existence of species on the ground that they "have been recorded ... by single authorities, but their occurrence has not been subsequently confirmed" or that "they have not been accepted" (Changeux \& Pont, 1995: 137) is definitively not acceptable in the case of $C$. petiti because there is no demonstration that it is not valid and the species has simply been overlooked in the literature. That a species fails to attract attention or that a paper is overlooked does not imply that a species does not exist.

\subsubsection{Cottus poecilopus Heckel, 1837}

Cottus poecilopus Heckel, 1837: 145, pl. 8 figs. 12 (type locality: a hill stream [probably Červený; J. Holčík, pers. comm.] of the Carpathes [Vysoké Tatry], near Grossschlagendorf [Velký Slavkov] near Käsmark [Kežmarok], Upper Hungary [now Slovakia], Vistula basin; syntypes: NMW 6424 [1], 6729 [3], 78816 [2])

Cottus poecilopus var. macrostomus Jeitteles, 1862: 292, pl. 2 fig. 2 (type locality: creeks near Koritnicza [Korytnica] in Liptau [Liptov], hill streams of Pohorella [Pohorelá] at the foot of Königsberg [Králova Hola], Hamor [Hámor] near Kaschau [Košice], Slovakia, Danube basin; syntypes: LU)

Cottus poecilopus var. microstomus Jeitteles, 1862: 292, pl. 2 fig. 3 (type locality: creek in Zips [area of Stará Lubovňa on upper Poprad], Slovakia; syntypes: LU; junior homonym of Cottus microstomus Heckel, 1837)
Cottus gobio var. microcephalus Kessler, 1868: 44 (Onega district, Russia) from Berg, 1949: 1145

Systematic notes. Cottus szanaga Dybowski, 1869 (p. 949, pl. 14 fig. 1; type locality: river Onon and its tributaries, Amur basin, Siberia; syntypes: IZPAN ?, ZMB 7107, 84 mm SL, Paepke \& Fricke, 1992: 284) treated as a synonym of $C$. poecilopus by Berg (1949: 1143) is a valid species (Holčík \& Pivnička, 1969: 18). According to these authors (p. 23), Cottus minutus volki Taranec, 1933 (p. 84; type locality: rivers of eastern slope of Sikhote-alin' from river Suchan [Peter the Great Bay] to river Takema or Takkoma $\left[45.5-46^{\circ} \mathrm{N}\right]$, Siberia; syntypes: LU) treated as a subspecies of C. poecilopus by Berg (1949) is also specifically distinct. Berg (1949: 1143) also lists C. kuznetzovi Berg, 1903 (p. 110; type locality: river Aunakit, river Lena basin [not Baikal, see Berg, 1949: 1143], Siberia, Russia; holotype: ZISP 12476) as a synonym of C. poecilopus; it most likely is not conspecific (J. Holčík, pers. comm.) and is not included in the above synonymy.

\subsubsection{Triglopsis quadricornis (Linnaeus, 1758)}

Cottus quadricornis Linnaeus, 1758: 264 (based on Linnaeus [1746: 104, n. 278, Cottus verrucis capitis ...; 1748: 47, pl. 4 fig. 3, idem; 1754: 70, pl. 32 fig. 4] and Artedi [1738: gen. 48 [84], syn. 84 [77] [spec. 48], Cottus scaber, tuberculis $4 \ldots$...; type locality: "in M. Balthico"; syntypes: NRM 2491, 2492 [2], Fernholm \& Wheeler, 1983: 235)

Cottus quadricornis var. relicta Lilljeborg, 1884: 146 (type locality: lake Vettern [Vättern], Sweden [restricted by Lönnberg, 1919: 164]; syntypes: LU)

Cottus latifrons Gatzianov, 1904: 195, pl. 11 (west coast of Kanin peninsula) from Berg, 1949: 1130

Myoxocephalus quadricornis morpha lönnbergi Berg, 1916a: 431, fig. 318 (infrasubspecific, name not available; localities: lake Ladoga, Russia and lake Mälaren, Sweden [restricted by Berg, in Lönnberg, 1932b: 5]) and 1916b: 1346, fig. 2 (same data)

Cottus quadricornis vaenernensis Lönnberg, 1919: 165 (type locality: lake Vänern, Sweden; syntypes: NRM 20336-20338 [6], 40338 [1])

Cottus quadricornis lönnbergi Lönnberg, 1919: 167 (type locality: lake Mälaren, Sweden; syntypes: NRM ?)

Cottus quadricornis frykenensis Lönnberg, 1932a: 14, fig. 5 (type locality: lake Fryken, Sweden; 
syntypes: NRM 30315 [1], museum of Karlstad's school [1], UUZM [1])

Cottus quadricornis oernensis Lönnberg, 1932a: 16, figs. 6a-b (type locality: lake Örn, Sweden; syntypes [total 9]: NRM 20323-20325 [7])

Cottus quadricornis asundensis Lönnberg, 1932a: 17, figs. 7a-b (type locality: lake Åsunden, Sweden; syntypes: NRM 20329-20332 [12])

Myoxocephalus quadricornis onegensis Berg \& Popov, 1932: 158 (type locality: lake Onega, Russia; syntypes: ZISP 3842 [1], 9026a [1])

Cottus quadricornis kallavesensis Lönnberg, 1932b: 7, fig. 1 (type locality: lake Kallavesi, Finland; syntypes: MZH [9])

Cottus quadricornis pygmaeus Lönnberg, 1932b: 9, fig. 2 (type locality: lake Puruvesi, Finland; syntypes: MZH)

Cottus quadricornis borkensis Lönnberg, 1939: 4

(type locality: lake Borken, parishes of Yxnerum and Björsäter, Osthrogothland Prov., Sweden; syntypes: NRM 20327 [2])

Cottus quadricornis vermelnensis Nybelin, 1940: 77, fig. 1 (type locality: lake Värmeln, Sweden syntypes: LU)

Systematic notes. Generally called Myoxocephalus quadricornis or Oncocottus quadricornis. Generic placement follows Neyelov (1979: 115, 131 ) and Fedorov, in Whitehead et al. (1986: 1259).

Several landlocked stocks are known from relatively deep lakes and many have been recognised as subspecies. It seems that some of these stocks should be treated as valid species under the PSC (they are diagnosable and constitute independent lineages), but a detailed revision is needed in order to determine the extent of their variability and distribution.

\section{Serranidae}

24.1.1 Dicentrarchus labrax (Linnaeus, 1758) Perca Labrax Linnaeus, 1758: 290 (based on J. F. Gronovius [1751: 39, pl. 4, Perca ...] and Artedi [1738: gen. [spec.] 41, syn. 69, Perca radiis pinnae ...]; type locality: "in Europa australiore"; type material: NT)

Sciaena diacantha Bloch, 1792: 58, pl. 302 (type locality: Mediterranean Sea; holotype: ZMB ?)

Centropomus mullus La Cepède, 1802: 251, 268, 271 (type locality: entering river Seine, France; type material: NT)

Perca elongata Geoffroy Saint-Hilaire, 1817: pl. 19

fig. 1 (type locality: Egypt; holotype: LU)

Centropomus lupus La Cepède, 1802: 267 (type locality: Adriatic Sea, Mediterranean Sea, European coasts of Atlantic Ocean, especially Golf of Gascogne, Channel and "Golfe Brittanique"; syntypes: LU)

Labrax Linnéi Malm, 1877: 379 (unnecessary replacement name for Perca labrax Linnaeus, 1758)

24.1.2 Dicentrarchus punctatus Bloch, 1792 Sciaena punctata Bloch, 1792: 64, pl. 305 (type locality: Mediterranean; holotype: ZMB, lost, H.J. Paepke, in litt., 1995)

Labrax orientalis Günther, 1863b: 175 (type locality: Alexandria, Egypt; syntypes: BMNH [2])

Systematic notes. Inclusion of this species as occurring in freshwaters follow Blanc et al. (1971), Maitland (1977: 206) and others.

\section{Centrarchidae}

\author{
Ambloplites rupestris (Rafinesque, 1817) \\ introduced \\ Lepomis auritus (Linnaeus, 1758) intro- \\ duced \\ Lepomis cyanellus Rafinesque, 1819 intro- \\ duced
}

Lepomis gibbosus (Linnaeus, 1758) introduced

Micropterus dolomieu La Cepède, 1802 introduced

Micropterus salmoides (La Cepède, 1802) introduced 


\section{Percidae}

\subsubsection{Gymnocephalus baloni Holčík \&} Hensel, 1974

Gymnocephalus baloni Holčík \& Hensel, 1974: 472, fig. 1a (type locality: river Danube near Kližská Nemá, Slovakia; holotype: SNM 2261) Systematic notes. Fricke (in Riehl \& Meinel, 1994: 29) considers G. baloni as an 'ecological morph' of G. cernuus. As long as no data or evidence are provided, it is difficult to follow this view. The wording of the brief argument is somewhat ambiguous: "while there are pure G. cernuus populations, there is no pure G. baloni populations. All individuals of $G$. baloni also exhibit characters of $G$. cernuus; there is always an overlap of character". If the 'absence of pure G. baloni populations' mean that the range of $G$. baloni is included within the range of $G$. cernuus, then this is not evidence of conspecificity in itself; similar cases involving closely related species abound (e.g., G. acerina and G. schraetser). 'Ecological morphs' of a single species usually do not occur mixed but in distinct habitats. Although not impossible, it seems strange that the G. baloni 'morph' would exist only in part of the range of $G$. cernua. Holčík \& Hensel's (1974) character analysis seems to indicate that some osteological character states are unique to G. baloni (e.g., shape of operculum and suboperculum); if the sentence on characters means that character states of G. cernuus are shared by $G$. baloni, this does not negate the existence of unique characters of $G$. baloni. Isozyme patterns further support the distinctness of G. baloni (Šlechtová \& Šlechta, 1996).

\subsubsection{Gymnocephalus cernuus (Linnaeus, 1758)}

Perca Cernua Linnaeus, 1758: 294 (based on Linnaeus [1746: 107, n. 286, Perca ... radiis 27], Artedi [1738: gen. 40 [80], syn. 68, spec. 77 [40], Perca dorso monopterygio capite...], Gronovius [1754: 41, n. 94, idem]; type locality: "in Europae lacubus"; syntypes: BMNH 1853.11.12:5 [1], 2 [1], Wheeler, 1958: 219, 1985: 51)

Holocentrus post La Cepède, 1802: 357 (unnecessary replacement name for Perca cernua Linnaeus, 1758)

Cernua fluviatilis Fleming, 1828: 212 (type locality: "rivers in England"; type material: NT ?)

Acerina vulgaris Cuvier, in Cuvier \& Valenciennes, 1829a: 4, pl. 41 (unnecessary replacement name for Perca cernua Linnaeus, 1758: 294)
Acerina fischeri Eichwald, 1871: 16, pl. 2 (lakes of the Tomsk Province, Siberia) from Berg, 1949: 1046

Acerina Czekanowskü Dybowski, 1874: 383 (type locality: middle and lower course of river Angara, Siberia; syntypes: IZPAN ?)

Acerina cernua essipovi Burmakin, 1941: 156, fig. 2 (Gyda Bay basin [Arctic Ocean], river Yuribei, Siberia) from Berg, 1949: 1047

Acerina cernua natio danubica Vladykov, 1931: 345 (infrasubspecific, name not available; locality: river Danube)

\subsubsection{Gymnocephalus schraetser (Linnaeus, 1758)}

Perca Schraetser Linnaeus, 1758: 294 (based on Artedi [1738: gen. [spec.] 40, syn. 68, Perca dorso monopterygio lineis ...]; type locality: "in Europa australis"; type material: NT)

Schraitser ratisbonensium Schaeffer, 1761: 48, pl. 2 fig. 4 (locality: Ratisbonum [Regensburg], Germany; name not available because work not consistently binominal; ICZN art. 11(c))

Perca danubiensis Gronow, 1854: 112 (type locality: river Danube; holotype: BMNH 1853.11.12: 41 [1], Wheeler, 1958: 219)

Nomenclatural notes. "Schraitser ratisbonensium Schaeffer" (1761: 48) is sometimes listed as a synonym of Gymnocephalus schraetser. This name is actually not an available name as it was used by Schaeffer in a work not consistently binominal and was meaning "Schraitser of the inhabitants of Regensburg".

"Holecentrus schraizer La Cepède" is sometime listed as a synonym of $G$. schraetser. La Cepède (1802: 347) clearly referred to Linnaeus's species and used the same spelling, and thus did not create a new name.

\subsubsection{Perca fluviatilis Linnaeus, 1758}

Perca fluviatilis Linnaeus, 1758: 289 (based on Linnaeus [1746: 106, n. 285 (sic; 284)], Artedi [1738: gen. 39 [74], syn. 66, spec. 74 [39], Perca lineis utrinque ...] and Gronovius [1754: 42, n. 96, idem]; type locality: "in Europae lacubus imprimis"; syntypes: BMNH 1853.11.12: 3 [1], LSL 1 [1], Wheeler, 1958: 219, 1985: 52)

Perca vulgaris Schäfer, 1761: 1, pl. 1 (locality: Ratisbonum [Regensburg], Germany; not available because work not consistently binominal, ICZN art 11(c)) 
Perca vulgaris Paula Schrank, 1792: 99 (type locality: Regensburg, Germany [by indication to Schäfer, 1761: 1, pl.1; type material: ZMB ?)

Perca italica Cuvier, in Cuvier \& Valenciennes, 1828b: 45 (type locality: Bologna, Italy; syntypes: MNHN)

Perca vulgaris Fitzinger, 1832: 331 (available by indication to Schäfer [1761: 1, Perca vulgaris] and Meidinger, 1785: pl. 5, Perca fluviatilis]; type locality: Regensburg, Germany [Schäfer], lakes and rivers of Austria [Meidinger], rivers Danube, Leytha and Schwarza, lake Traunsee, Austria; syntypes: NMW [not yet located; B. Herzig, pers. comm., 1995]; junior homonym of Perca vulgaris Paula Schrank, 1798)

Perca vulgaris var. aurata Fitzinger, 1832: 331 (nomen nudum; localities: rivers Danube, Leytha and Schwarza, lake Traunsee, Austria)

Perca fluviatilis var. nigrescens Heckel, 1837: 155 (nomen nudum; locality: river Marizza near Philippoli in Rumelia [southern Bulgaria])

Perca helvetica Gronow, 1854: 113 (type locality: Basel, Switzerland; holotype: BMNH 1853.11.12:2 [1], Wheeler, 1958: 219)

Perca fluviatilis var. gibba Smitt, 1892: figs. 3-4 (infrasubspecific, name not available [see p. 30])

Perca fluviatilis var. maculata Smitt, 1892: 30, fig. 5 (type locality: lake Tisaren, Nerike, Sweden; syntypes: ? NRM 35749; junior primary homonym of Perca maculata Bloch, 1792: 92, pl. 313 ; could possibly be treated as infrasubspecific, thus unavailable name)

Perca fluviatilis var. aurea Smitt, 1892: 30 (type locality: Eskilstuna, Sweden; holotype: ? NRM 35750 ; could possibly be treated as infrasubspecific, thus unavailable name)

Perca fluviatilis var. macedonica Karaman, 1924b: 79 (type locality: lake Dojran and river Vardar near Veles, FYROM; syntypes: LU)

Perca fluviatilis morpha phragmiteti Berg, 1933: 635 (infrasubspecific, name not available)

Perca fluviatilis infrasp. gracilis Pokrovsky, 1951 (infrasubspecific, name not available; junior homonym of Perca gracilis Cuvier, in Cuvier \& Valenciennes, 1828b: 50) from Oliva et al., 1989: 222

Perca fluviatilis zaissanica Dianov, 1955 (locality: lake Zaissan, Kazakhstan) from Svetovidov \& Dorofeyeva, 1963: 637

Perca fluviatilis intermedius Svetovidov \& Dorofeyeva, 1963: 639 (type locality: river Kolyma, Siberia; holotype: ZISP 23998)

Nomenclatural notes. "Bürstel bavarorum Schaeffer" (1761: 1) is sometimes listed as a synonym of Perca fluviatilis. This name is actually not available as it was used by Schaeffer under the heading "Perca vulgaris" and it means simply "Buirstel of the Bavarians".

\subsubsection{Romanichthys valsanicola Dumitres-} cu, Bănărescu \& Stoica, 1957

Romanichthys valsanicola Dumitrescu, Bănărescu \& Stoica, 1957: 230, figs. 1-4 (type locality: river Vilsan, a left side tributary of river Arges, near Gales village, Raion Curtea de Arges, Romania; holotype: MGAB 49900, Mihai-Bardan, 1984: 464)

\subsubsection{Sander lucioperca (Linnaeus, 1758)}

Perca Lucioperca Linnaeus, 1758: 289 (based on Linnaeus [1746: 106, n. 285, Perca ... radiis 23] and Artedi [1738: gen. 39 [76], syn. 62 [67], spec. 76 [39], Perca pallide maculosa ...]; type locality: "in Europae lacubus"; type material: NT)

Centropomus sandat La Cepède, 1802: 248, 255 (unnecessary replacement name for Perca lucioperca Linnaeus, 1758: 289)

Lucioperca sandra Cuvier, in Cuvier \& Valenciennes, 1828b: 110, pl. 15 (unnecessary replacement name for Perca lucioperca Linnaeus, 1758: 289)

Lucioperca Linnéi Malm, 1877: 381 (unnecessary replacement name for Perca lucioperca Linnaeus, 1758)

Nomenclatural names. Stizostedion Rafinesque (1820: 23), Lucioperca Schinz (in Cuvier, 1822: 475), Lucioperca Fleming (1822: 394), Lucioperca Cuvier (in Cuvier \& Valenciennes, 1828b: 110 ) and at least six other generic names (see Collette \& Bănărescu, 1977: 1457) are junior synonyms of Sander Oken (1817: 1782); see also Gill (1903), and Eschmeyer (1990: 365).

\subsubsection{Sander volgensis (Gmelin, 1788)}

Perca Volgensis Gmelin, 1788: 1309 (available by indication to Pallas [1771: 461, Perca asper]; type locality: rivers Volga and Rhymnus [Ural], Russia; syntypes: ZISP ?)

Schilus Pallasii Krynicki, 1832: 443, pl. 25 (type locality: rivers Volga, Rhymnus [Ural], Dnieper and Donets, Ukraine; syntypes: ZISP ?)

\subsubsection{Zingel asper (Linnaeus, 1758)}

Perca Asper Linnaeus, 1758: 290 (based on Artedi [1738: gen. [spec.] 40, syn. 67, Perca lineis utrinque ...]; type locality: "in Europa australiore"; type material: NT)

Aspro Apron Siebold, 1863a: 55 (type locality: river Rhône, France; holotype: probably ZSM, now lost) 
Aspro vulgaris Cuvier, in Cuvier \& Valenciennes, 1828b: 188, pl. 26 (unnecessary replacement for Perca asper Linnaeus, 1758: 290)

25.5.2 Zingel balcanicus (Karaman, 1936) Aspro streber balcanicus Karaman, 1936: 60 (type locality: river Vardar, FYROM; syntypes: LU) Systematic notes. See discussion under Zingel streber.

\subsubsection{Zingel streber (Siebold, 1863)}

Asper verus Schaeffer, 1761: 69, pl. 3 fig. 4 (locality: Ratisbonum [Regensburg], Germany; name not available because work not consistently using binominal nomenclature; ICZN art. 11(c))

Aspro streber Siebold, 1863a: 54 (type locality: rivers Danube and Amper, Germany; syntypes: [14, probably ZSM, now lost])

Zingel streber nerensis Bănărescu \& Nalbant, 1979: 353 (type locality: river Nera at Sasca Montana, Romania; holotype: ISBB 2534)

Systematic notes. Bănărescu \& Nalbant (1979) recognise three subspecies within $Z$. streber. The mosaic distribution of the characters between the three subspecies (as well as $Z$. asper and $Z$. zingel) and the apparent absence of intergrades seems to indicate that at least two species are involved. The naked cheeks, the shape of the nostrils, the presence of two opercular spines and other characters listed by Bănărescu \& Nalbant seem very diagnos- tic for the Vardar population and it is treated here as a distinct species, $Z$. balcanicus. It seems likely that $Z$. s. nerensis could be a valid species too, but I am unable to reach a conclusion from the published data.

Nomenclatural notes. "Streber ratisbonensium Schaeffer" (1761: 69) is sometimes listed as a synonym of Zingel streber. This name is actually not an available name as it was used by Schaeffer under the heading "Asper verus" and it means simply "Streber of the inhabitants of Regensburg".

\subsubsection{Zingel zingel (Linné, 1766)}

Perca zingel Linné, 1766: 482 (based on Gronovius [1763: 92, n. 303, Perca dorso dipterygio ...], Schaeffer [1761: 58, pl. 3 fig. 1, Zingel], and Kramer [1756: 386, Zingel]; type locality: river Danube at Regensburg [Schaeffer] and in Hungary [Kramer, Gronovius]; syntype: BMNH 1853.11.12:81 [1], Wheeler, 1958: 219)

Nomenclatural notes. "Asperulus ratisbonensium Schaeffer, 1761" and "Zindel ratisbonensium Schaeffer, 1761" are sometimes listed as synonyms of Zingel zingel. These names are actually not available; they were used by Schaeffer (1761: 58) as the heading "Asperulus" (an unavailable name, because not binominal), with the subheading "Zindel ratisbonensium", meaning "Zindel of the inhabitants of Regensburg".

\section{Cichlidae}

'Cichlasoma' facetum (Jenyns, 1842) introduced

Systematic notes. The identification of the ci- chlid introduced into Portuguese' waters (Almaça, 1995: 22) has been confirmed by S. O. Kullander (in litt., 1995).

\section{Blenniidae}

The generic nomenclature of Bath (1977) is followed here. Bock \& Zander (1986: 142) united the genera Lipophrys and Salaria and used Lipophrys Gill, 1896 as the valid name. Salaria Forsskål, 1775 being the oldest name is the one to be used. The argument of similarity of the generic names Salaria and Salarias Cuvier, 1816: 251 is irrele- vant to the discussion (ICZN art. 56(b) is explicit). Similarly, the argument for rejecting Ichthyocoris Bonaparte, 1840: [fasc. 28, p. 182], another name older than Lipophrys, is not relevant.

The argument of Bock \& Zander (1986: 142) for not recognising Paralipophrys and Salaria as distinct from Lipophrys ("which are to dissolve 
as these show no differences in ancient-relevantcharacters") is a semantic mystery to me and at this point I cannot see reasons for not following Bath (1977).

\subsubsection{Salaria fluviatilis (Asso, 1801)}

Blennius fluviatilis Asso, 1801: 31, pl. 33 (type locality: river Ebro in Zaragoza, Spain; type material: NT ?)

Blennius Frater Bloch, in Schneider, 1801: 171 (type locality: "Ibero amne prope Caesar-Augustam" [Zaragoza, Spain]; based on Asso, 1784: 95 , pl. 6 fig. 2; type material: NT)

Blennius fluviatilis Rafinesque Schmaltz, 1810a: 31 (type locality: Sicilia; type material: NT ?; junior homonym of Blennius fluviatilis Asso, 1801)

Blennius vulgaris Pollini, 1816: 20, pl. 1 fig. 1 (type locality: lake Garda, Italy; type material: NT)

Salarias varus Risso, 1826: 237 (type locality: river Var, France; type material: NT)

Blennius inaequalis Valenciennes, in Cuvier \& Valenciennes, 1836: 230 (type locality: Cette [Sète], France; holotype: MNHN A.1789, Bath, 1977: 209)

Blennius cagnota Valenciennes, in Cuvier \& Valenciennes, 1836: 249 (type locality: Toulon. France; lectotype: MNHN A.1667, present designation)

Blennius Lupulus Bonaparte, 1840: [fasc. 28, p. 185], pl. 106 fig. 5 (type locality: stream Fiora and others draining to the Mediterranean in Toscana and Romana, Italy; syntypes: LU)

Blennius Anticolus Bonaparte, 1840: [fasc. 28, p. 188], pl. 106 fig. 4 (type locality: lake Nemi and "ne'sudetti laghi suburbicarii" ["in these suburban lakes" = volcanic lakes surrounding Roma] [p. 280; see reference p. 188]; type material: NT) Ichthyocoris pollinii Bonaparte, 1846: 68 (unnecessary replacement name for Blennius vulgaris Pollini, 1816)

Blennius Petteri Heckel \& Kner, 1858: 44, 46 (nomen nudum; locality: creek near Salona [near Split], Croatia)

Blennius alpestris Blanchard, 1866: 261, fig. 49 (type locality: lake Bourget, France; syntypes: MNHN A.2368 [5], Bath, in Daget et al., 1986: 357)

Nomenclatural notes. Bath (in Daget et al., 1986: 356) indicates that Asso (1784: 95, pl. 6 fig. 2 ) is author of Blennius fluviatilis. The species is described on this page, but no binominal name is used. The first use of the name is by Asso (1801).

Bath (1977: 209) lists a specimen (MNHN A.1667) from Toulon as holotype of Blennius cagnota. Even if Valenciennes based his description on this specimen, as he referred additional specimens to the species and as none is explicitly designated as holotype or type, all are syntypes. I designate here specimen MNHN A.1667 as lectotype of B. cagnota Valenciennes, 1836.

Both Blennius fluviatilis Asso and B. frater Bloch have been published in 1801. Precise publication dates are not known and as first reviser, I retain B. fluviatilis Asso, 1801 as having priority.

See Costa Pereira (1995: 517) for a discussion of the status of Blennius sujefianus La Cepède, 1800 (p. 473; type locality: not stated; type material: NT) sometime tentatively listed as a synonym of Salaria fluviatilis.

\section{Gobiidae}

27.1.1 Benthophiloides brauneri Beling \& Iljin, 1927

Benthophiloides brauneri Beling \& Iljin, 1927: 309, figs. 1-2 (type locality: lower course of river Dnieper at Ossnowa [Osnova], Britany, Kachowka [Kakhovka], Berisslaw [Berislav] and Chersson [Kherson], southern river Bug at Novaya Odessa and its liman at Nikolaev, Ukraine; syntypes: LU [34])
27.2.1 Benthophilus stellatus (Sauvage, 1874)

Doliichthys stellatus Sauvage, 1874b: 336 (type locality: river Don at Woronesch [Voronezh], Russia; syntypes: MNHN 7341 [2], Bauchot et al., 1991: 21)

Benthophilus macrocephalus var. maeotica Kuznetsov, 1888: 207 (Azov Sea: Arabat Spit, mouths of river Don) from Berg, 1949: 1114 
Benthophilus monstrosus Kuznetsov, 1888: 208 (Azov Sea: lower reach of Berdyansk liman) from Berg, 1949: 1114

Benthophilus macrocephalus var. nudus Berg, 1898: 34 (river Dniester at Benderi, Ukraine) from Berg, 1949: 1114

Benthophilus macrocephalus ponticus Berg, 1916a: 427 (type locality: north-western Black Sea [Berg, 1949: 1114]; syntypes: ZISP ?)

Systematic notes. The above synonymy includes only nominal species based on material from the Black Sea basin. Ragimov (1978) recognises two subspecies from the Caspian Sea, B. s. casachicus Ragimov (1978: 705) and B. s. leobergi Ragimov (1978: 707; see below). The available data do not indicate if the two Caspian 'subspecies' are sympatric or not. The two 'subspecies' are reportedly diagnosable and it does not seem that they are conspecific with $B$. stellatus which I tentatively consider as restricted to the Black Sea basin.

Nomenclatural notes. Bentophilus stellatus leobergius Iljin, 1949 is recognised as a valid subspecies by Ragimov (1978: 707), but the name is a nomen nudum in Iljin (1949: 28) as it is not accompanied by a description or diagnostic characters. As the taxon does not occur in the area covered by the present work, I did not search intensively for subsequent uses of this name, but the earliest subsequent use I found is in Ragimov's (1978) description of B. s. casachicus which lists diagnostic characters of B. s. leobergius and makes the name available. If it is confirmed that this is the earliest use of making the name available, 100 specimens from the Caspian Sea (no precise locality data published) are syntypes (in Laboratory of Regeneration of Fish Stocks, Caspian Biological Station, Zoological Institute, Azerbaidjan Academy of Sciences). The name leobergius is based on a personal name and should be emended into leobergi [ICZN art. 31(a)(i), (iii), 32(c)(i), (d)]. If valid, this taxon should be called B. leobergi Ragimov, 1978.

\subsubsection{Economidichthys pygmaeus (Holly,} 1929)

Gobius pygmaeus Holly, 1929: 487, figs. 1-2 (type locality: near Zoodochos [Piggi] near Kaligoni, a small village on island Lafkas, Greece; syntypes: NMW 33930 [12])

Gobius martensii var. prevesicus Stephanidis, 1939a: 35, pl. 8 fig. 5 (type locality: stream Louros and lake Trichonidos, Greece; syntypes: lost ? [86])

Systematic notes. Generic position follows Bianco et al. (1987) and Economidis \& Miller (1990).
27.3.2 Economidichthys trichonis Economidis \& Miller, 1990

Economidichthys trichonis Economidis \& Miller, 1990: 141, figs. 7-9 (type locality: stream Mataranga, south-west lake Trichonis, Greece; holotype: BMNH 1989.3.15.1)

\subsubsection{Gobius cobitis Pallas, 1814}

Gobius cobitis Pallas, 1814: 160 (type locality: "Sinu Theodosiano Ponti" [Feodosiya, Crimea, Ukraine]; syntypes: LU)

Gobius guttatus Valenciennes, in Cuvier \& Valenciennes, 1837: 24 (type locality: Sicilia, Italy; syntypes: MNHN A.1144 [1], A.1147 [1], A.1148 [2], Bauchot et al., 1991: 33)

Gobius limbatus Valenciennes, in Cuvier \& Valenciennes, 1837: 26, pl. 345 (type locality: Nice, France; holotype: MNHN A.1191, Bauchot et al., 1991: 35)

Gobius algarbiensis Capello, 1880: 23 (available by indication to Capello, 1869: 224 [Gobius sp.]; type locality: seas of Lisboa, Setubal and Lagos, Portugal; syntypes: LU)

? Gobius spilogonurus Cocco, 1885: 88 (type locality: Sea of Messina, Sicilia; syntypes: LU)

Gobius exanthematosus var. gibbosus Ninni, 1938: 160, fig. 50, pl. 19 fig. a (type locality: Laguna di Venezia, Italy; syntypes: LU)

\subsubsection{Gobius niger Linnaeus, 1758}

Gobius niger Linnaeus, 1758: 262 (based on Artedi [1738: gen. [spec.] 28, syn. 46, Gobius ex nigricante ...], Linnaeus $[\mathbf{1 7 5 4}, \mathbf{7 4}$, idem] and Osbeck [1757: 131, Apocryptes cantonensis]; type locality: "in Europa, Asia"; syntypes: NRM 103-104 [2], Fernholm \& Wheeler, 1983: 267)

Gobius Jozo Linnaeus, 1758: 263 (based on Artedi [1738: gen. [spec.] 29, syn. 47, Gobius pinna ventrali ...]; syntypes: ? NRM 9453 [1], ? 9454 [1], Fernholm \& Wheeler, 1983: 268)

? Gobius gorgione Rafinesque Schmaltz, 1810a: 35 (type locality: harbour of Palermo, Sicilia; type material: NT ?)

? Gobius viridis Otto, 1821a: 1, 1821b:7 (type locality: Nice, France; type material: NT ?)

Gobius longiradiatus Risso, 1826: 286 (type locality: not stated, probably around Nice, France; type material: NT, Bauchot, 1991: 56)

Gobius Brittanicus Thompson, 1837: 61 (type locality: Galway Bay, western coast of Ireland / Corfou [Kerkira island], Greece; syntypes: BELUM ?)

Gobius fuliginosus M'Coy, 1841: 403 (type locality: Connamara, Ireland; holotype: NMI ?)

Gobius jozo var. pontica Kessler, 1859b: 255 
(type locality: Odessa and the coast of Crimea, Ukraine; syntypes: ZISP ?)

Gobius jozo var. albescens Canestrini, 1861: 129 (infrasubspecific, name not available; locality: Gulf of Genova, Italy)

Gobius jozo var. nigrescens Canestrini, 1861: 129 (infrasubspecific, name not available; locality: Gulf of Genova, Italy)

Gobius punctulatus Cocco, 1885: 87 (type locality: Sea of Messina, Sicilia; syntypes: LU ?)

Gobius jozo var. major Kolombatovic, 1891: 6 (infrasubspecific, name not available; locality: Spalato [Split], Dalmatia)

Gobius jozo var. minor Kolombatovic, 1891: 6 (infrasubspecific, name not available; locality: Spalato [Split], Dalmatia)

Gobius niger forma hispanicus de Buen, 1928: 16, fig. 6 (type locality: Malaga, Spain; syntypes: LU)

Gobius niger forma nigerrimus de Buen, 1928: 18, fig. 7 (type locality: Bahia de Porto-Pi, Mallorca, Balearic islands, Spain; holotype: LU)

Nomenclatural notes. Fernholm \& Wheeler (1983: 267) comment that the type series of Gobius niger includes several species, including one from China.

27.5.1 Knipowitschia caucasica (Berg, 1916) Gobius lenkoranicus Kessler, 1877a: 34 (type locality: Lenkoran, Azerbaidjan, Caspian Sea basin; holotype: LU; name suppressed by the International Commission on Zoological Nomenclature, 1968b; see also Svetovidov, 1966)

Gobius caucasicus Kawrajsky, in Radde, 1899: 309 (nomen nudum; localities: Batumi / Temirgoe [station south of Sulak mouth; Berg, 1949: 1065], Georgia)

Pomatoschistus caucasicus Berg, 1916a: 409 (type locality: swamp near Batum and lake Inkit near Pitzunda, Georgia [Berg, 1949: 1065; Svetovidov, 1966: 55]; syntypes: ZISP)

Bubyr caucasicus kosswigii Sözer, 1941: 159, fig. (type locality: lake Manyas, Turkey; syntypes: LU [7])

Systematic notes. See Economidis \& Miller (1990: 152) for redescription.

27.5.2 Knipowitschia croatica Mrakovcic, Kerovec, Misetic \& Schneider, 1994

Knipowitschia punctatissima croatica Mrakovcic, Kerovec, Misetic \& Schneider, 1994: 46 (type locality: freshwater spring in Polje Jezero, Dalmatia, Croatia, $43^{\circ} 10^{\prime} \mathrm{N} 17^{\circ} 25^{\prime} \mathrm{E}$; lectotype: HZM 9 VRG, designated by Mrakovcic et al., 1996: 313)

Nomenclatural notes. Mrakovcic et al. (1994) used the name $K . p$. croatica in the printed abstract of a symposium with a short description, making the name available (ICZN art. 13(a)(i)). As no holotype was designated, all specimens on which their original communication was based are syntypes. The taxon has been described by Mrakovcic et al. (1996: 313).

27.5.3 Knipowitschia goerneri Ahnelt, 1991 Knipowitschia goerneri Ahnelt, 1991: 266, figs. 12 (type locality: freshwater springs south-west of Korissias lagoon, Corfou [Kerkira island], Greece; holotype: NMW 90127)

\subsubsection{Knipowitschia longecaudata (Kessler,} 1877)

Gobius longecaudatus Kessler, 1877a: 35, pl. 3 fig. 8 (type locality: Caspian Sea [Berg, 1949: 1066]; syntypes: LU)

Pomatoschistus knipowitschi Beling, 1927: 351 (nomen nudum; lower reaches of river Bug to Belousovka, liman above Nikolaev, Ukraine [from Berg, 1949: 1066])

Knipowitschia georghievi Pinchuk, 1978: 798 (type locality: lake Gebedjinsky, Bulgaria; holotype: ZISP 42337)

\subsubsection{Knipowitschia milleri (Ahnelt \&} Bianco, 1990)

Orsinigobius milleri Ahnelt \& Bianco, 1990: 2, figs. 1-2 (type locality: delta of river Acheron, Epirus, western Greece; holotype: NMW 86065)

\subsubsection{Knipowitschia panizzae (Verga, 1841)} Gobius Panizzae Verga, 1841: 379 (type locality: lagoon of Comacchio, Italy; syntypes: NMW 29810 [5], from Miller, in Hureau \& Monod, 1973: 497)

Systematic note. See Miller (1972: 157) for redescription and discussion.

\subsubsection{Knipowitschia punctatissima (Canes-} trini, 1864)

Gobius punctatissimus Canestrini, 1864: 101 (type locality: Mantova, Modena and Castelfranco di Bologna, Italy; syntypes [total: at least 10]: MSNG 126547 [1], Tortonese, 1963b: 344])

Systematic notes. Redescribed by Gandolfi et al. (1985).

\subsubsection{Knipowitschia thessala (Vinciguerra,} 1921)

Gobius thessalus Vinciguerra, 1921: 323, pl. 4 fig. 1 (type locality: Kefalovriso spring on Mount Kissavo (earlier Ossa) near Chasabali village, Thessaly, Greece; syntypes: MSNG 
12606 [9], Tortonese, 1963b: 344)

Systematic notes. See Economidis \& Miller (1990: 152) for redescription.

\subsubsection{Mesogobius batrachocephalus (Pal-} las, 1814)

Gobius batrachocephalus Pallas, 1814: 149 (type locality: Black Sea near Chersonesus [Kherson] and Balaklava, Crimea, Ukraine; syntypes: LU)

\subsubsection{Neogobius eurycephalus (Kessler,} 1874)

Gobius eurycephalus Kessler, 1874: 281 (type locality: Yenikale near Kerch, Crimea; lectotype: ZISP 2234, designated by Pinchuk, 1977: 518)

Neogobius eurycephalus odessicus Pinchuk, 1977: 519 (type locality: 'M[are] Nigrum' [Black Sea, near Odessa ?, Ukraine]; holotype: ZISP 2090)

Systematic notes. Included on the basis of Romanian records by Bănărescu (1994b: 6).

27.7.2 Neogobius fluviatilis (Pallas, 1814) Gobius fluviatilis Pallas, 1814: 162 (type locality: near the mouths of the rivers entering the Black Sea, restricted by Berg, 1949: 1091; syntypes: LU)

? Gobius sordidus Bennett, 1835: 91 (type locality: Black Sea at Trebizond [Trabzon], Turkey; holotype: BMNH ?)

Gobius lacteus Nordmann, 1840: 430, pl. 10 fig. 3 (type locality: river Dniester and lagoon crossed by it near Ovidiopol and Akkerman, Ukraine; syntypes: MNHN A.1198 ? [3], Bauchot et al., 1991: 34, NMW 29505-506 ? [2])

Gobius stevenii Nordmann, 1840: 430, pl. 13 fig. 1 (type locality: near Kertch, Crimea; syntypes: $\mathrm{LU}[2])$

Gobius fluviatilis var. nigra Kessler, 1859b: 247 (type locality: mouth of rivers Bug and Dniester. Ukraine; syntypes: ZISP ?; junior primary homonym of Gobius niger Linnaeus, 1758)

\subsubsection{Neogobius gymnotrachelus (Kessler, 1857)}

Gobius gymnotrachelus Kessler, 1857: 464 (type locality: river Dniester and its tributaries, especially river Slutsch, Ukraine; syntypes: ZISP ?)

Gobius macropus Filippi, 1863: 391 (type locality: lake Palestrom near Poti, Georgia; syntypes: MZUT 675 [2], Tortonese, 1940: 144)

Gobius Burmeisteri Kessler, 1877a: 26, pl. 1 fig. 5 (type locality: Poti, Georgia [Berg, 1949: 1098]; syntypes: LU)

Mesogobius gymnotrachelus otschakovinus Zubo- vich, 1925: 192 (Ochakov, Ukraine) from Berg, 1949: 1098

27.7.4 Neogobius kessleri (Günther, 1861) Gobius platycephalus Kessler, 1857: 468 (type locality: river Dniester and tributaries, river Southern Bug upriver of Wossnesensk, Ukraine; lectotype: ZISP 2107 [? designated by Pinchuk, 1977: 520, comment not clear; junior primary homonym of G. platycephalus Richardson, 1846: 204 and G. platycephalus Peters, 1852: 681 )

Gobius kessleri Günther, 1861: 553 (replacement name for G. platycephalus Kessler, 1857: 468)

Systematic notes. This species is known from the north-western Black Sea (Miller, in Whitehead et al., 1986: 1062). The subspecies from the Caspian Sea is treated here as a valid species $N$. gorlap Iljin, in Berg, 1949. This conclusion has also been reached by Vasiljeva \& Vasiljev (1996).

Nomenclatural notes. Neogobius iljini Vasiljeva \& Vasiljev, 1996 (p. 264, fig. 1; type locality: Mangyshlak region, Caspian Sea; holotype: ZMMSU P-19726) has been proposed for the $N$. gorlap of Berg (1949) and subsequent authors because they feeled that $N$. gorlap was 'nonvalid unpublished' (p. 262). According to them, the name was used by Iljin in a manuscript which has never been published and is now lost ( $p$. 261). Berg (1949) obviously had access to the manuscript and included some of Iljin's data; his use of the name makes the name available, with Iljin in Berg as author. Vasiljeva \& Vasiljev list several subsequent authors who used the name (as a subspecies of $N$. kessleri) and mentionned morphological, osteological and karyological differences between $N$. gorlap and $N$. kessleri; if $N$. gorlap had not been available from Berg (1949) each of these papers using the name $N$. gorlap with descriptive material would be enough to make $N$. gorlap an available name. Neogobius iljini is thus a junior synonym of N. gorlap.

\subsubsection{Neogobius melanostomus (Pallas,} 1814)

Gobius melanostomus Pallas, 1814: 151 (type locality: "Chersonesus, Portu Symbolorum" [Sevastopol, Crimea / Balaklava, Ukraine; Berg, 1949: 1083]; syntypes: LU)

Gobius Cephalarges Pallas, 1814: 155 (type locality: Theodosianus [Feodosiya], Crimea, Ukraine; holotype: LU; see Pinchuk, 1976: 550 for discussion of unpublished figure)

Gobius Chilo Pallas, 1814: 156 (type locality: 
Theodosianus [Feodosiya], Crimea, Ukraine; holotype: LU)

Gobius Melanio Pallas, 1814: 157 (type locality: Black Sea; syntypes: LU [or holotype? see Berg, 1949: 1083])

Gobius virescens Pallas, 1814: 158 (type locality: Theodosianus [Feodosiya], Crimea, Ukraine; syntypes: LU)

Gobius exanthematosus Pallas, 1814: 160 (type locality: shores of Crimea, Ukraine; syntypes: LU)

Gobius affinis Eichwald, 1831: 75 (type locality: "in caspii maris sinu balchanensi" [Balkhan Bay, Caspian Sea; Berg, 1949: 1085]; syntypes: LU)

Gobius sulcatus Eichwald, 1831: 75 (type locality: "in caspii maris sinu balchanensi" [Balkhan Bay, Caspian Sea; Berg, 1949: 1085]; syntypes: LU)

Gobius lugens Nordmann, 1840: 414, pl. 9 fig. 1 (type locality: river Codor [Corax] near Drandarium, Abkhazia; syntypes: LU)

Gobius Grossholzii Steindachner, 1894: 447, pl. 2 fig. 1 (type locality: lake Küjük Cekmedze near Constantinopel [Istanbul], Turkey; syntypes: NMW 34032-062, 49912 [36])

27.7.6 Neogobius syrman (Nordmann, 1840) Gobius Syrman Nordmann, 1840: 419, pl. 12 fig. 1 (type locality: near Odessa and lagoon communicating with river Dniester, Ukraine; lectotype: the specimen illustrated by Nordmann, designated by Pinchuk, 1976: 546 [possibly among the following syntypes: MNHN A.1126 ? [1], Bauchot et al., 1991: 40, NMW 30099 ? [1]])

Gobius Trautvetteri Kessler, 1859b: 244 (type locality: limans of river Bug in Nikolaev and limans of river Dniester, Ukraine; syntypes: ZISP ?, NMW 30125 [1], 30127 [1])

Gobius hybridus Iljin, 1956: 190 (type locality: Kertch, Crimea, Ukraine; holotype: ZISP 55a)

\subsubsection{Padogobius bonelli (Bonaparte, 1846)}

Gobius fluviatilis Nardo, 1824: 229, 230 [7, 8 of reprint] (type locality: not stated [streams draining to the Adriatic Sea, possibly near Pavia, Italy; syntypes: LU; junior primary homonym of Gobius fluviatilis Pallas, 1814: 162)

Gobius fluviatilis Valenciennes, in Cuvier \& Valenciennes, 1837: 52 (type locality: streams of "Sardegna" [probably Piemonte, then included in Kingdom of Sardegna; Tortonese, 1975: 312], lake Aviglia [Avigliana, Torino] and lake Maggiore, Italy; syntypes: MNHN 2729 [2], A.1335 [2], A.1336 [3], A.1337 [1], A.1338 [1], A.1368 [4], Bauchot et al., 1991: 32; junior primary homonym of G. fluviatilis Pallas, 1814: 162 and Gobius fluviatilis Nardo, 1824: 229)
Gobius bonelli Bonaparte, 1846: 95 (replacement name for G. fluviatilis Bonelli [in Cuvier \& Valenciennes, 1837])

Gobius martensii Gïnther, 1861: 15 (type locality: river Ticino, Italy; syntypes: BMNH [2])

Systematic notes. See Gandolfi \& Tongiorgi (1974: 97) for a redescription of this species.

Nomenclatural notes. Gandolfi \& Tongiorgi (1973: 262; 1974: 97) considered that Gobius bonelli had to be rejected as a nomen oblitum according to ICZN because it had not been used in the previous 60 years. Actually there is (was) no such obligation: this merely was an option given by the 1964 Code. Anyway, this was only possible between 6 November 1961 and 1 January 1973 (see ICZN art. 79(c)(iii), Glossary 'nomen oblitum'). As Gandolfi \& Tongiorgi's papers appeared in 1973 and 1974, the rejection of $G$. bonelli was no longer possible; thus it is the oldest available and valid name for the present species.

Bonaparte (1846: 95) proposed G. bonelli as a replacement name for 'G. fluviatilis Bonelli' [in p. 64, he listed it as G. fluviatilis Bonelli, but in his addenda, p. 95, he added G. fluviatilis Pallas, 1814 , stating that it is not Bonelli's species which he recognises under the name $G$. bonelli]. The species has regularly been referred to as 'Gobius fluviatilis Bonelli, in Cuvier \& Valenciennes, 1846'. Valenciennes (in Cuvier \& Valenciennes, 1837: 52) considered Bonelli as author of the name; he probably had received the specimens from Bonelli under that unpublished name; it was a common practice of that time to use unpublished names and to attribute the authorship to the person who created it, even if unpublished. Under present rules of nomenclature (ICZN art. 50(a)), Valenciennes is the only author of the name.

Tortonese (1940: 144) considered three specimens (MZUT 848) as probably part of the type series and he attributed the authorship of the species to Bonelli. As the species has been described by Valenciennes and as there is no evidence that Valenciennes had examined the specimens in MZUT, these specimens cannot be part of the type series.

\subsubsection{Padogobius nigricans (Canestrini, 1867)}

Gobius fluviatilis nigricans Canestrini, 1867: 11 (type locality: river Arno, Italy; syntypes: LU [4])

Gobius avernensis Canestrini, 1868: 169 (type locality: river Arno, Italy; syntypes: LU [at least 4])

Systematic notes. See Gandolfi \& Tongiorgi 
(1974: 105) and Pirisinu \& Natali (1980) for redescriptions of this species.

\subsubsection{Pomatoschistus bathi Miller, 1982}

Pomatoschistus bathi Miller, 1982: 6, figs. 1-2 (type locality: Erdek, Bandirma, Sea of Marmara, Turkey; holotype: SMF 16431)

\subsubsection{Pomatoschistus canestrinii (Ninni,} 1883 )

Gobius Canestrinii Ninni, 1883: 276, pl. 15 figs. 15 (type locality: Estuario di Venezia, Italy and river Jadro, Salona [near Split], Dalmatia [Croatia]; syntypes: NMW 28817-818 [2], 29943-945 [3])

Gobius jadrensis Giglioli, in Ninni, 1883: 276 (nomen nudum)

Systematic notes. See Gandolfi et al. (1982) for a redescription of the species.

\subsubsection{Pomatoschistus marmoratus (Risso, 1810)}

Atherina marmorata Risso, 1810: 339 (type locality: Nice, France; type material: NT, Bauchot et al., 1991: 51)

Gobius reticulatus Valenciennes, in Cuvier \& Valenciennes, 1837: 50 (type locality: Sicilia, Italy and Nice, France; syntypes: MNHN A.1247 [1], 205 [1], Bauchot et al. 1991: 39; junior homonym of $G$. reticulatus Eichwald, 1831: 77)

Gobius leopardinus Nordmann, 1840: 436, pl. 13 fig. 4 (type locality: near Sevastopol, Crimea, Ukraine; holotype: MNHN A.1402 [1], Bauchot et al., 1991: 35)

Gobius rhodopterus Günther, 1861: 16 (replacement name for Gobius reticulatus Valenciennes. in Cuvier \& Valenciennes, 1837)

Gobius ferrugineus Kolombatovic, 1891: 18 (type locality: Spalato [Split], Dalmatia, Croatia; syntypes: NMW 29363 [12], 37509-519 [11], 77868 [15])

Syrrhothonus Charrieri Chabanaud, 1933: 1249 (type locality: Tanger, Morocco; holotype: LU, Bauchot et al., 1991: 61)

Nomenclatural notes. The specimens listed as the holotype and paratype of Gobius reticulatus by Bauchot et al. (1991: 39) are actually syntypes. The specimen they list (1991: 35) as potential syntype of $G$. leopardinus is actually the potential holotype as Nordmann (1840: 436) explicitly stated that he had a single specimen [but the locality data differ slightly: Tauria, vs. Sevastopol; Tauria is a former name for Crimea]

\subsubsection{Pomatoschistus microps (Krøyer,} 1838)

Gobius microps Krøyer, 1838: 416 (type locality: Hirtsholmene, northern Kattegat, Denmark; holotype: ZMUC 72, Nielsen, 1974: 79)

Gobius pusillus Lowe, 1874: 32 (type locality: [King's] Lynn, Norfolk, England; syntypes: LU; junior homonym of G. pusillus Canestrini, 1861) from Miller, in Hureau \& Monod, 1973: 509

Gobius minutus var. minor Heincke, 1880: 317 (type locality: river Schlei, bay of Kiel, Korsör, and Dassow, Germany; syntypes: LU)

Gobius laticeps Moreau, 1881: vol. 2, 215, figs. 103-104 (type locality: St. Valéry-en-Craux. Manche, France; holotype: MNHN 1898-395, Bauchot et al., 1991: 35)

Gobius Parnelli Day, 1881: 167, pl. 52 fig. 5 (type locality: Weston-super-Mare, Somerset, England; syntypes: BMNH ?)

Gobius microps var. puckensis Lawacz, 1965: 141, fig. 7 (infrasubspecific [ICZN art. 45 (g) (i)], name not available; locality: Baltic Sea, Puck Bay near Gdansk, Poland)

\subsubsection{Pomatoschistus minutus (Pallas, 1770)}

Gobius minutus Pallas, 1770: 4 (available by indication to Gronovius, 1763: 81, n. 276; type locality: "maris Belgici" [seas of Belgium]; syntypes: LU)

Gobius gracilis Jenyns, 1835a: 25 (nomen nudum); Yarrell, 1835: vol. 1: 260 (type locality: Colchester [Essex, U.K.]; syntypes: LU); Jenyns, 1835b: 387 (locality: Colchester [Essex, U.K.])

Gobius unipunctatus Parnell, 1838b: 243, pl. 29 (type locality: Firth of Forth in the neighbourhood of South Queensferry / Solway Frith / Exmouth on coast of Devon, U.K., syntypes: LU); also 1839a: 138, pl. 5

Gobius Ekströmi Günther, 1861: 57 (type locality: river Gotha [Göta älv], Sweden; holotype: $\mathrm{BMNH})$

Gobius elongatus Canestrini, 1861: 150, pl. 8 fig. 5 (type locality: Golf of Genova, Italy; syntype [total 4]: MSNG 12646 [1], Tortonese, 1963: 343)

Gobius gracilis Cabrera, in Steindachner, 1868: 401 (nomen nudum; junior homonym of G. gracilis Jenyns, 1835b)

Gobius cobitiformis Kessler, 1874b: 211 (type locality: Sevastopol, Crimea, Ukraine; syntypes: ZISP ?) from Miller, in Hureau \& Monod, 1973: 506

Gobius Taalmankipii Hubrecht, 1878: 17 (type locality: North Sea; syntypes: LU [5]) 
Gobius minutus var. major Heincke, 1880: 313 (type locality: Bay of Kiel, Germany; syntypes: LU)

Gobius minutus var. Guitelli Le Danois, 1913: 88 . figs. 147-148 (type locality: brackish waters in estuary of river Penzé [Roscoff], France; syntypes: LU)

Nomenclatural notes. See Nomenclatural notes under Gasterosteus aculeatus for publication dates of Gobius gracilis in Jenyns (1835a-b) and Yarrell (1835-36). Jenyns (1835b) refers to Yarrell (183536) with exact page numbers, so I conclude that Yarrell appeared first.

\subsubsection{Pomatoschistus tortonesei Miller, 1968}

Pomatoschistus tortonesei Miller, 1968: 221, fig. 1, pl. 1 (type locality: brackish water, Marsala, Sicilia, Italy; holotype: MSNG 41682)

\subsubsection{Proterorhinus marmoratus (Pallas,} 1814)

Gobius marmoratus Pallas, 1814: 161 (type locality: portum Ctenuntem, seu Sevastopolitanum Tauriae [Sevastopol], Crimea, Ukraine; syntypes: LU)

Gobius quadricapillus Pallas, 1814: 159 (type locality: sinu Theodosiano et littore Chersonesi [Feodosiya and Kherson], Crimea, Ukraine; syntypes: LU)

Gobius semilunaris Heckel, 1837: 152, pl. 8 figs. 5-6 (type locality: river Marizza [Maritsa] near Philippopoli in Rumelia [southern Bulgaria]; holotype: NMW 58144)

Gobius macropterus Nordmann, 1840: 434, pl. 13 fig. 2 (type locality: southern Crimea, Ukraine; syntypes: LU)

Gobius nasalis Filippi, 1863: 390 (type locality: Caspian Sea near Baku; syntypes: MZUT 672
[7], Tortonese, 1940: 144, MSNG 12655, 36228

[5], Tortonese, 1963b: 344, NMW 33894-896 [3]) Gobius rubromaculatus Kriesch, 1873: 371, pl. 6

(type locality: Altofner Therme, draining to river Danube [near Budapest], Hungary; syntypes: LU)

Gobius blennioides Kessler, 1877a: 12, pl. 1 fig. 4 (type locality: Baku Bay, Caspian Sea [Berg, 1949: 1100]; syntypes: LU)

Gobius marmoratus var. nasalis pontica Smitt, 1899: 544 (infrasubspecific, name not available; locality: Black Sea)

\subsubsection{Zosterisessor ophiocephalus (Pallas,} 1814)

Gobius ophiocephalus Pallas, 1814: 153 (type locality: coasts of Crimea, Ukraine; syntypes: LU)

? Gobius viridis Otto, 1821a: 1, 1821b: 7 (type locality: Nice, France; type material: NT ?)

? Gobius filamentosus Risso, 1826: 284 (type locality: not stated, probably around Nice, France; type material: NT)

Gobius reticulatus Eichwald, 1831: 77 (type locality: Black Sea near Odessa, Ukraine; syntypes: LU)

Gobius lota Valenciennes, in Cuvier \& Valenciennes, 1837: 27 (type locality: Bologna and Sicilia, Italy / Martigues, France; syntypes: MNHN A.1040 [1], A.1039 [3], A.1171 [2], Bauchot et al., 1991: 35)

Gobius gous Chiereghini, in Nardo, 1847: 119 (type locality: lagoon of Venezia, Italy; syntypes: LU)

Gobius venetiarum Nardo, 1847: 120 (listed in synonymy, name not available)

Gobius venetiarum Nardo, 1860: 79 (nomen nudum; locality: Venezia, Italy)

Gobius ophiocephalus var. citrina Ninni, 1938: 154 (type locality: Sea of Marmara; syntypes: LU)

\section{Citharidae}

28.1.1 Citharus linguatula (Linnaeus, 1758) Pleuronectes linguatula Linnaeus, 1758: 270 (based on Artedi [1738, gen. [spec.] 17, syn. 31, Pleuronectes oculis a dextra ...] and Gronovius [1754: 15, n. 41, idem]; type locality: "in M. Europaeo"; type material: NT)

Pleuronectes macrolepidotus Bloch, 1787: 34, pl. 190 (type locality: Brasilian Sea; syntype: ZMB 7491 [1])
Pleuronectes citharus Spinola, 1807: 374 (type locality: Gulf of Genova, Italy; syntypes: LU)

Pleuronectes patarachia Nardo, 1847: 121 (listed in synonymy, name not available)

Systematic notes. Included on the basis of Ivanovic's (1973: 124) record from lake Skadar (FYROM and Albania). Additional records are needed to confirm the regular occurrence of this species in freshwaters. 


\section{Pleuronectidae}

29.1.1 Platichthys flesus (Linnaeus, 1758)

Pleuronectes Flesus Linnaeus, 1758: 270 (based on Linnaeus [1746: 112, n. 300, Pleuronectes ... lateralis aspera], Artedi [1738: gen. [spec.] 17, syn. 33, Pleuronectes oculis a dextris ... spec. [gen.] 59, Pleuronectes linea lateralis aspera ...], Gronovius [1754: 15, n. 40, idem] and Linnaeus [1751: 326, idem]; type locality: "in M. Europaeo"; potential syntypes: BMNH 1853.11.12:132-134 [3], NRM 20 [1], ? UUMZ 191 [1], Wheeler, 1958: 244, 1991: 189, Fernholm \& Wheeler, 1983: 272)

Pleuronectes Passer Linnaeus, 1758: 271 (based on Artedi [1738: gen. [spec.] 18, syn. 32, Pleuronectes ... laterali aculeata]; type locality: "in Oceano Europaeo"; type material: NT)

Pleuronectes flesoides Pontoppidan, 1765: 188, pl. 15 (type locality: Denmark; type material: NT ?)

Pleuronectes Roseus Shaw, 1803: vol. 4 (2): 302, pl. 43 (type locality: river Thames, U.K.; holotype: "Leverian Museum")

Pleuronectes luscus Pallas, 1814: 427 (type locality: Theodosia [Feodosiya], Crimea; syntypes: LU)

Platessa carnaria Brown, 1830: 99, pl. 2 (type locality: Prestonpans, Scotland, U.K.) from Costa Pereira, pers. comm.

Platessa glabra Rathke, 1837: 352 (Kerch, Crimea) from Berg, 1949: 1190

Platichthys flesus var. marmorata Nordmann, 1840: 534, pl. 28 fig. 1 (type locality not stated [Black Sea]) from Costa Pereira, pers. comm.

Pleuronectes italicus Günther, 1862b: 452 (type locality: Dalmatia [Croatia]; holotype: BMNH 1842.6.7:3, Nielsen, in Hureau \& Monod, 1973: 626)

Flesus vulgaris Moreau, 1881: vol. 3: 299 (unnecessary replacement name for Pleuronectes flesus Linnaeus, 1758)

Pleuronectes flesus var. trachurus Duncker, 1892: 280, 291 (type locality: Baltic Sirit at mouth of river Trave, Germany; syntypes: ? ZMH)

Pleuronectes flesus var. leiurus Duncker, 1892: 281, 291 (type locality: river Elbe in Hamburg, Germany; syntypes: ? ZMH)

Pleuronectes Bogdanovii Sandeberg, 1878: 236, figs. 1-2 (type locality: White Sea; syntypes: LU)

Pleuronectes flesus race septentrionalis Suvorov, 1925: 280 (infrasubspecific, name not avail- able; Kola Bay, Russia) from Berg, 1949: 1189

Pleuronectes flesus race baltica Suvorov, 1925: 280 (infrasubspecific, name not available; Baltic Sea) from Berg, 1949: 1186

Pleuronectes flesus caninensis Suvorov, 1929: 111 (type locality: rivers falling into Chosha Bay, Arctic Ocean, Russia) from Berg, 1949: 1189

Pleuronectes flesus septentrionalis Berg, 1949: 1189, fig. 941 (type locality: Murman and as far eastward as the Pechora Bay, enters rivers, e.g., river Tuloma in Murman, Russia; syntypes: ZISP)

\subsubsection{Pleuronectes glacialis Pallas, 1776}

Pleuronectes glacialis Pallas, 1776: 706 (type locality: Arctic Ocean [p. 32: Kara Bay, Berg, 1949: 1181]; syntypes: LU)

Pleuronectes cicatricosus Pallas, 1814: 424 (type locality: mari inter Camtschatcam et Americam [Bering Sea]; syntypes: LU)

Platessa dwinensis Lilljeborg, 1850: 5 (type locality: river Dwina near its estuary in Archangel, Russia; syntypes: LU); also in 1851: 306, pl. 20 Pleuronectes glacialis caninensis Suvorov, 1929: 119 (Chosha Bay, Arctic Ocean) from Berg, 1949: 1181

Liopsetta glacialis knipowitschi Esipov, 1939: 171, fig. 2 (Gulf of $\mathrm{Ob}$ at Cape Drovyanyi, Siberia, Arctic Ocean) from Berg, 1949: 1181

Systematic notes. Formerly placed in genus Liopsetta. Generic position follows Sakamoto (1985: 209).

\subsubsection{Pleuronectes platessa Linnaeus, 1758}

Pleuronectes platessa Linnaeus, 1758: 269 (based on Linnaeus [1746: 113, n. 301, Pleuronectes oculis dextris ...], Artedi [1738, gen. [spec.] 17, syn. 31, Pleuronectes oculis dextris \& tuberculis ...], Linnaeus, 1747: 179, Pleuronectes oculis dextris ...; 1751: 326, Pleuronectes Slatrvar] and Gronovius [1754: 14, n. 36, Pleuronectes oculis dextris \& tuberculis ...); type locality: "in M. Europaeo"; syntypes: BMNH 1853.11.12:130-131 [2], Wheeler, 1958: 243)

Platessa vulgaris Cloquet, 1826: 403 (unnecessary replacement name for Pleuronectes platessa Linnaeus, 1758)

Pleuronectes borealis Faber, 1828: 244 (type locality: Iceland, North and West Jutland and Kattegat not south of the island Anholt, Denmark; type material: NT ?)

Platessa latus Cuvier, 1829: 339 (type locality: 
"sur nos côtes" [France]; syntypes: ? MNHN)

Pleuronectes platessa variet. baltica Nilsson, 1855:

616 (type locality: Sund, Baltic Sea [Berg, 1949:

1179]; syntypes: LU)
Systematic notes. Formerly placed in genus Liopsetta. Generic position follows Sakamoto (1985: 209).

\section{Hybrids and incertae sedis}

The following nominal species have later been considered to be hybrids. The list does not include names made of the combination of two generic and two specific names (e.g., Squalio-alburnus cephalolucidus). Names explicitly proposed for hybrids are not available (ICZN art. 1(b)(3)); names proposed for fishes which have later been considered as hybrids are available but cannot be used (ICZN arts $17(1), 23(\mathrm{~h}))$; names proposed for fish considered as hybrids by an earlier author but considered a distinct species by the author proposing the name are available (e.g., Chondrostoma lemmingi steindachneri Berg, 1932) even if these fishes are later considered again as hybrids.

\subsubsection{Acipenseridae}

Acipenser brandtii Günther, 1870: 336 (type locality: Black and Caspian Seas; syntypes: material described and illustrated by Brandt \& Ratzeburg, 1833: 350, pl. 1a fig. 2; hybrid Huso huso $\times$ Acipenser nudiventris, Berg, 1911: 169)

Acipenser Helenae Duméril, 1870: 258 (type locality: Russia; syntypes: MNHN [2]; ? hybrid Acipenser ruthenus $\times$ Acipenser gueldenstaedti, Berg, 1911: 239)

Acipenser primigenius Chalikov, 1944: 47 (northern Caspian Sea; hybrid $A$. ruthenus $\times A$. gueldenstaedti, Berg, 1949: 77)

\subsubsection{Cyprinidae}

Abramis cenomanensis Selys-Longchamps, 1870: 113 (listed in synonymy and proposed for an hybrid, name not available; locality: river Sarthe, France; hybrid Blicca bjoerkna $\times$ Scardinius erythrophalmus; author listed as Anjubault, but Selys-Longchamps is actual author)

Abramis dobuloides Günther, 1857: 51, pl. 2 (type locality: river Neckar / stream Blaulach, Tuibingen, Germany; syntypes: LU; hybrid Leuciscus dobula $\times$ Alburnus alburnus, Günther, 1868: 223)

Abramis Heckelii Selys-Longchamps, 1842: 217, pl. 8 (type locality: vicinity of Bruxelles and river Meuse, Belgium / Abbeville, Somme, France; syntypes: ISNB 110 [1], NMW 5525:3 ? [3]; hybrid Abramis brama $\times$ Rutilus rutilus, Günther,
1868: 214)

Abramis Leuckartii Heckel, 1836: 229, pl. 20 fig. 5 (type locality: river Danube near Fischament, downriver of Wien, Austria; syntypes: NMW 55331 [1]; hybrid Rutilus rutilus $\times$ Abramis brama, Guinther, 1868: 214, Berg, 1949: 531)

Abramis rhinosimus Linstow, 1878: 248 (proposed for an hybrid, name not available; hybrid $R u$ tilus rutilus $\times$ Vimba melanops, Linstow, 1878: 248)

Alburnus Leydigi Knauthe, 1893a: 416, 1893b: 488 (proposed for an hybrid, name not available; locality: Schlaupitz, Kr. Reichenbach u. d. Eule, Schlesien [now Poland]; hybrid Leucaspius delineatus $\times$ Alburnus alburnus, Berg, 1912: 333)

Alburnus rosenhaueri Jäckel, 1866: 20 (proposed for an hybrid, name not available; locality: river Altmühl at Herrieden, Bayern, Germany; hybrid Scardinius erythrophthalmus $\times$ Blicca bjoerkna, Berg, 1912: 285)

Alburnus tauricus Kessler, 1859a: 534 (type locality: market in Simpheropol, Crimea, Ukraine, said to be from river Solghir; syntypes: ZISP ? [2]; hybrid Leuciscus cephalus $\times$ Chalcalburnus chalcoides, Berg 1949: 557)

Aspius margaritaceus Siebold, 1863a: 168 (proposed for an hybrid, name not available; hybrid Scardinius erythrophthalmus $\times$ Alburnus alburnus, ? Benecke, 1884: 229)

Bliccopsis alburniformis Siebold, 1863a: 168 (proposed for an hybrid, name not available; locality: Koenigsberg [now Kaliningrad, Russia]; hybrid Blicca bjoerkna $\times$ Alburnus alburnus Selys-Longchamps, 1887: 1078)

Bliccopsis erythrophthalmoides Jäckel, 1864: 49 (proposed for an hybricl, name not available; localities: rivers Altmühl and Wieseth, Bayern, Germany: hybrid Scardinius erythrophthalmus $\times$ Blicca bjoerkna)

Brama isognathus Bleeker, 1862: 235 (type locality: river Rhine in Leiden, Netherlands; holotype: RMNH ?; hybrid Scardinius erythrophthalmus $\times$ Blicca bjoerkna, Berg, 1912: 285)

Chondrostoma lemmingi steindachneri Berg, 
1932a: 144 (available by indication to Steindachner [1866c: 21, pl. 4 fig. 2, "bastard von Ch. polylepis und Leuciscus (Leucos) arcasii"]; type locality: river Tera, Spain; syntypes: NMW 52569 [5]; hybrid Chondrostoma polylepis $\times$ Rutilus arcasii, Elvira, 1987: 138)

Chondrostoma Reiseri Steindachner, 1893: 239 (type locality: Busko Blato near Zupanjac, south of Livno, Bosnia; holotype: NMW 55524; hybrid Chondrostoma phoxinus $\times$ Phoxinellus alepidotus, Elvira, 1987: 138)

Carpio Sieboldii Jäckel, 1864: 21 (proposed for an hybrid, name not available; hybrid Cyprinus carpio $\times$ Carassius carassius

Cyprinus abramo-rutilus Holandre, 1837a: 3 (type locality: river Moselle downriver of Malroy, France; syntypes: LU; hybrid Abramis blicca $\times$ Rutilus rutilus, Giinther, 1868: 215)

Cyprinus Buggenhagii Bloch, 1784: 137, pl. 95 (type locality: river Pene and related lakes in Swedish Pommern [now Mecklenburg-Vorponmern, Germany]; holotype: ZMB 4106; hybrid Abramis brama $\times$ Rutilus rutilus, Günther, 1868: 214)

Cyprinus Carpio var. latus Fitzinger, 1832: 334 (indication to Marsilii [1726: 61, pl. 21, Cyprinus III; "circa Albim, circa Danubium"]; proposed for an hybrid, name not available; junior primary homonym of Cyprinus latus Gmelin, 1788)

Cyprinus dolabrata Holandre, 1837a: 3 (type locality: river Moselle, France; syntypes: LU; hybrid Leuciscus dobula $\times$ Alburnus alburnus, Gïnther, 1868: 223)

Cyprinus Kollarii Heckel, 1836: 223, pl. 19 fig. 2 (type locality: lake Neusiedlersee, Austria; syntypes: NMW 56166 [1], 56169 [1], 56171 [1], 56172 [1]; hybrid Cyprinus carpio $\times$ Carassius carassius, Günther, 1868: 31)

Cyprinus striatus Holandre, 1837a: 2 (type locality: ditches of the city of Metz, river Moselle, ponds of Belletanche, France; syntypes: LU [2]; hybrid Cyprinus carpio x Carassius carassius, Günther, 1868: 31)

Leuciscus affinis Valenciennes, in Cuvier \& Valenciennes, 1844: 150 (type locality: Gand, Belgium; holotype: MNHN 3354, Almaça, 1969: 1119; hybrid Rutilus rutilus $\times$ Scardinius erythrophthalmus, Günther, 1868: 214, Almaça 1969, Fang, 1942a: 169)

Leuciscus alburnolucidus Linstow, 1878: 247 (proposed for an hybrid, name not available; locality: Hameln ?, Germany; hybrid Rutilus rutilus $\times$ Alburnus alburnus)

Leuciscus carii Knauthe, 1893a: 418 (proposed for an hybrid, name not available; hybrid Leucaspius delineatus $\times$ Rutilus rutilus)

Leuciscus dvinensis Plater, 1861 (proposed for an hybrid, name not available [ICZN art. 1(b)(3)] river Dïna, Latvia; parent species not stated) from Dybowski, 1862: 95

Leuciscus Lapacinus Steffani, Serra, Loffredo \& Fossa, 1987: 15, pl. 1 (type locality: Lago di S. Croce, Alpago, Italy; holotype: Istituto di Zoologia, Universita, Cagliari; hybrid Alburnus alburnus $\times$ Leuciscus cephalus, Bianco, 1988a: 152)

Scardinopsis alburniformis Benecke, 1884: 228 (proposed for an hybrid, name not available; locality: Deutsch-Eylau [now Ilawa, Poland]; hybrid Scardinius erythrophthalmus $\times$ Blicca bjoerkna, Berg, 1912: 285)

Scardinopsis amphigenus Selys-Longchamps, 1870 : 112 (proposed for an hybrid, name not available: locality: Belgium [Longchamps-sur-Geer; SelysLongchamps, 1887: 1078]; hybrid Rutilus rutilus $\times$ Scardinius erythrophthalmus)

Scardiniopsis anceps Jäckel, 1864: 64 (proposed for an hybrid, name not available; localities: river Altmühl, Bayern, Germany; hybrid Rutilus rutilus $\times$ Scardinius erythrophthalmus)

Squalius Anjubaulti Selys-Longchamps, 1870: 113 (proposed for an hybrid, name not available; locality: river Loire bassin [river Sarthe near Le Mans; Selys-Longchamps, 1887: 1081], France; hybrid Leuciscus leuciscus $\times$ Alburnus alburnus)

\subsubsection{Incertae sedis}

The following names are either not identifiable with any known species (usually because their 'description' is very laconic, inaccurate or simply missing) or are not available because they are based on theoretical concept (that is hypothetical taxa)

Cobitis pannonica Heckel, 1848: 194 (nomen nudum; locality: lake Neusiedler, Hungary [and Austria])

Cyprinus asper Schmidt, 1795: 67 (nomen nudum; locality: river Moldau [Vltava], Czech Republic)

Cyprinus ellicola Hermann, 1804: 318 (type locality: not stated [Strasbourg, France?]; holotype: $\mathrm{LU}$ )

Knipowitschia mrakovcici Mrakovcic, Misetic \& Povz, 1995: 185 (nomen nudum; locality: river Krka, Croatia)

Leuciscus scaverde Bonaparte, 1845: 10 (nomen nudum)

Phoxinellus italicus Bonaparte, 1845: 6 (type locality: southern Italy; syntypes: LU) 
Salmo trutta mediterranea Balon, 1968: 12; Karakousis \& Triantaphyllidis, 1989: 302 (based on an hypothetical concept, name not available, ICZN art. 1(b)(1))

\section{Epilogue}

An unanswered question is very often a question which has not been asked properly.

\section{Acknowledgments}

This work would not have been possible without the help of many persons who assisted in obtaining literature, translated, provided information or unpublished manuscripts, gave specimens and allowed me access to material under their care: Petru Bacalu (Bucuresti, Romania), Petru Bănărescu (Bucuresti, Romania), Louis Bernatchez (Québec, Canada), Patrick Berrebi (Montpellier, France), Pier Giorgio Bianco (Napoli, Italy), Péter Bíró (Tihany, Hungary), Nina Bogutskaya (St. Petersburg, Russia), Bernard Buttiker (Saint-Sulpice, Switzerland), Brian W. Coad (Ottawa, Canada), Bruce B. Collette (Washington, U.S.A.), Nuno da Costa Pereira (Lisboa, Portugal), Marlis Douglas (Zürich, Switzerland), Christophe Dufour (Neuchâtel, Switzerland), Guy Duhamel (Paris, France), Frank Dyrhovden (Oslo, Norway), Panos Economidis (Thessaloniki, Greece), Roland Egli (Saint-Ursanne, Switzerland), Benigno Elvira (Madrid, Spain), Ronald Fricke (Stuttgart, Germany), Jean-Pierre Gosse (Jambes, Belgium), Kurt Grossenbacher (Bern, Switzerland), Jürgen Hartmann (Langenargen, Germany), Karol Hensel (Bratislava, Slovakia), Barbara Herzig (Wien, Austria), Juraj Holčík (Bratislava, Slovakia), Jean-Claude Hureau (Paris, France), Isaäc Isbrücker (Amsterdam, Netherlands), Arthur Kirchhofer (Bern, Switzerland), Sven O. Kullander (Stockholm, Sweden), Tomasz Linkowski (Gdynia, Poland), Monique Margout (Paris, France), Richard L. Mayden (Tuscaloosa, U.S.A.), Rudolf Müller (Kastanienbaum, Switzerland), Sonia Muller (Genève, Switzerland), Peter K. L.
Ng (Singapore), Jørgen Nielsen (København, Denmark), Aevar Petersen (Reykjavik, Iceland), Zupancic Primoz (Dolsko, Slovenia), Christian Ruhlé (St. Gallen, Switzerland), Michel Sartori (Lausanne, Switzerland), Sigurdur S. Snorrason (Reykjavik, Iceland), Neal M. Teitler (Tokyo, Japan), Carlo Violani (Pavia, Italy), Alvise Vittori (San Michele al Adige, Italy), Claude Weber (Genève, Switzerland), Alwyne Wheeler (Epping, U.K.), Tony Whitten (Cambridge, U.K.), Georges Zarfitsian (Porrentruy, Switzerland) and the staff of Bibliothèque cantonale, Porrentruy, Universitätsbibliothek, Basel, and Bibliothèque centrale, Muséum national d'Histoire naturelle, Paris.

Barbara Herzig provided catalogue numbers of all types in NMW and numerous additional information. Hans-J. Paepke (MZB), Sven Kullander (NRM) and Jean-Pierre Gosse (ISNB) provided catalogue numbers of types in their institutions. Figure 2 is by C. Ratton (MHNG).

Pier Giorgio Bianco, Nina Bogutskaya, Nuno da Costa Pereira, Panos Economidis; Benigno Elvira, Barbara Herzig, Juraj Holč́́k, Sven O. Kullander, Peter K. L. Ng, Hans-J. Paepke, Rohan Pethiyagoda, Claude Weber, and Alwyne Wheeler read, commented and discussed various versions of the manuscript. This does not imply that they share my opinion or that I convinced them; the remaining idiosyncrasy only reflects my stubborn difficulty to follow advice and all errors are still mine.

Antoinette Kottelat-Kloetzli assisted with library work. 


\section{Literature cited}

Titles of publications in languages using the Latin alphabet appear as on original. For publications using other alphabets, if a translation of the title in a language using the Latin alphabet is given in the paper, this translation is used; if no translation is given, the title has been translated into English. For some publications which I have not seen, the titles appear as in the available bibliographic references, using the quoted language or transcription. For journal names in languages not using the Latin alphabet, a translation is used only if it is printed on the journal; otherwise I use a transcription. Publication years in bold indicate publications not seen by me and quoted from bibliographies.

Agassiz, L. 1828. Beschreibung einer neuen Species aus dem Genus Cyprinus Linn. Isis (von Oken), 21 (10): 1046-1049, pl.12.

- 1835a. Remarks on the different species of the genus Salmo which frequent the various rivers and lakes of Europe. Rep. Meet. Brit. Assoc. Adv. Sci., 1834 [1835]: 617-623.

- 1835b. Description de quelques espèces de cyprins du Lac de Neuchâtel, qui sont encore inconnues aux naturalistes. Mém. Soc. Sci. Nat. Neuchâtel, 1: 33-48, 2 pls.

- 1839. Histoire naturelle des poissons d'eau douce de l'Europe centrale. Vol. 1. Neuchâtel, 27 pls.

- 1856. [The glanis of Aristotle]. Proc. Amer. Acad. Sci., 3: 325-333.

Ahnelt, H. 1991. A new species of Knipowitschia (Teleostei: Gobiidae) from Corfu, Western Greece. Ichthyol. Explor. Freshwat., 2: 265-272.

Ahnelt, H. \& P. G. Bianco. 1990. Orsinigobius milleri n. sp., a new species of freshwater goby from W-Greece (Pisces: Gobiidae). Ann. Naturhist. Mus. Wien, 91: 1-6.

Aldrovandri, U. 1613. De piscibus libri v, et de cetis lib. unus. Bononiae, iv $+732+26 \mathrm{pp}$.

Allardi, J. \& P. Keith. 1991. Atlas préliminaire des poissons d'eau douce de France. Secrétariat Faune Flore, Muséum National d'Histoire Naturelle, Paris, 234 pp.

Alm, G. 1939. Undersökningar över tillväxt $\mathrm{m}$. $\mathrm{m}$. hos olika Laxöringformer [Investigations on growth etc. by different forms of trout]. Meddelanden från Statens Undersökning- och Försöksanstalt Sötvattensfisket, 15: 1-93, 6 pls.

- 1949. Influence of heredity and environment on various forms of trout. Rep. Inst. Freshwat. Res. Drottningholm, 29: 29-34.

Almaça, C. 1967. Estudo das populações portuguesas do gén. Barbus Cuvier, 1817 (Pisces, Cyprinidae). Rev. Fac. Cienc. Univ. Lisboa, Cienc. Nat., 14: 151-400.

- 1969. Révision critique de quelques types de cyprinidés d'Europe et d'Afrique du Nord des collections du Muséum National d'Histoire Naturelle. Bull. Mus. Natn. Hist. Nat. Paris, Sér. 2, 40 (1968 [1969]): 1116-1144.

- 1990. Neogene circum-mediterranean paleogeography and Euro-Mediterranean Barbus biogeography. Arq. Mus. Bocage, N. S., 1: 585-611.

- 1991. Evolutionary, biogeographical, and taxonomic remarks on Mesopotamian species of Barbus s.s. Arq. Mus. Bocage, N.S., 2: 63-78.

- 1995. Fish species and varieties introduced into Portuguese inland waters. Museu Nacional de Historia Natural, Lisboa, 29 pp.

d'Ancona, U. 1934. Le species mediterranee del genere Syngnathus. Memorie R. Comitato Tallasogr. Ital., 210: 1-79, 3 pls.

Andersson, L., N. Ryman \& G. Ståhl. 1983. Protein loci in the Arctic charr, Salvelinus alpinus L.: electrophoretic expression and genetic variability patterns. J. Fish Biol., 23: 75-94.

Anikin, V. P. 1902. [Report on a scientific trip to the Narym Province in summer 1900]. Izvestiya Tomskogo Imperatorskogo Universiteta, 22: 1122. [also distributed as a separate publication] [Russian]

- 1903. Beiträge zur Kenntniss der Fische von Westsibirien. Sammlung zur Erinnerung an E. G. Saliscev, Tomsk, pp. 119-138.

- 1905. Descriptions of new Asiatic fish species. Izvestiya Tomskogo Universiteta, 1905: $17 \mathrm{pp}$.

Anonym. 1883. Sweden - Special catalogue. Great International Fisheries Exhibition, London, 209 pp.

- 1895. [On the fishery in the Turuchansk Province]. Vestnik Rybopromyshlennosti, St. Petersburg, 10: 78-81.

- 1964. International code of zoological nomenclature, adopted by the 15 th International Congress of Zoology. International Trust for Zoological Nomenclature, London, xvii+176 pp.

- 1985. International code of zoological nomenclature - third edition - adopted by the XX general assembly of the International Union of Biological Sciences. International Trust for Zo- 
ological Nomenclature, London, $\mathrm{xx}+338 \mathrm{pp}$.

Anslijn, N. 1828. Systematische beschrijvingder der voor ons meest belangrijke voortbrengselen uit de drie Rijken der Natuur. Dierenrijk. IV. Deel. Visschen. Du Mortier, Leyden, 345 pp.

Antipa, G. 1904. Die Clupeinen des westlichen

Teiles des Schwarzen Meeres und der Donaumündungen. Anz. Akad. Wiss. Wien, 41: 299303.

- 1905. Die Clupeinen des westlichen Teiles des Schwarzen Meeres und der Donaumündungen. Denkschr. Kais. Akad. Wiss., Math. Naturwiss. Kl., 78: 1-56, pls. 1-2.

- 1909. Fauna ichtiologica a Romaniei. Acad Romana, Publicatiunile Fondului Vasile Adamachi, 16: ix +294 pp., 31 pls.

- 1936. Ziele und Wege ichthyologischer Forschung im Schwarzen Meer. Bull. Sect. Scient. Acad. Roumaine, 18: 161-169.

Apostolidis, A., Y. Karakousis \& C. Triantaphyllidis. 1996. Genetic divergence and phylogenetic relationships among Salmo trutta L. (brown trout) populations from Greece and other European countries. Heredity, 76: 551-560.

Arai, R. \& Y. Akai. 1988. Acheilognathus melanogaster, a senior synonym of A. moriokae, with a revision of the genera of the subfamily Acheilognathinae (Cypriniformes, Cyprinidae). Bull. Natn. Sci. Mus., Ser. A., Zool., 14: 199213.

Artedi, P. 1738. Ichthyologia sive opera omnia de piscibus scilicet: Bibliotheca ichthyologica. Philosophia ichthyologica. Genera piscium. Synonymia specierum. Descriptiones specierum. Omnia in hoc genere perfectiora quam antea ulla posthuma vindicavit, recognovit, coaptavit et edidit Carolus Linnaeus. Wishoff, Lugduni Batavorum.

Ascanius, [P.]. 1772-1806. Icones rerum naturalium, ou figures enluminées d'histoire naturelle du nord. Copenhague, 1 (1767): 22 pp., pls. 1-9, 2 (1772): 8 pp., pls. 11-20, 3 (1775): 6 pp., pls. 21-30, 4 (1776): 6 pp., pls. 331-340, 5 (1805): 8 pp., pls. 41-50.

Asso [y del Rio], I. de. 1784. Introductio in oryctographiam et zoologiam Aragoniae accedit enumeratio stirpium in eadem regione noviter detectarum. Amsterdam, 192 pp, pls.

- 1801. Introduccion á la ichthyología oriental de España. An. Cienc. Nat., Madrid, 4 (10): 28-52, pls. 33-35.

d'Aubenton, F., J. Daget \& J. Spillmann. 1971. Classification numérique des blageons Leuciscus (Telestes) soufia (Pisces, Cyprinidae) ( $8^{\mathrm{e}}$ note). Bull. Mus. Natn. Hist. Nat. Paris, Sér. 2, 42
(1970 [1971]): 839-848.

Ausonius, D. M. ca. 370. Mosella. [See Dean, B. 1923, vol. 3, p. 211, for bibliographic details, translations, etc.; edition cited by Valenciennes is on p. 192 in: Wernsdorf, J. C. 1780. Poetarum latinorum minorum. Altenburg]

Avise, J. C. 1983. Protein variation and phylogenetic reconstruction, p. 103-130. In: G. S. Oxford \& D. Rollinson (eds), Protein polymorphism: adaptive and taxonomic significance. Academic Press, London.

- 1990. Flocks of African fishes. Nature, 347: 512-513.

- 1994. Molecular markers, natural history, and evolution. Chapman \& Hall, New York, 511 pp.

Babushkin, N. Y. 1942. [Towards the taxonomy of the Caspian beluga]. Izvestiya Azerbaidzhanskoi Rybokhozyaistvennoi Stantsii, 7: 115-131.

Bacescu [Bacesco], M. 1943. Deux poissons nouveaux pour la faune de la Roumanie: Cobitis aurata balcanica Karaman et Cobitis caspia romanica n. ssp. Bull. Sect. Scient. Acad. Roum., 26: 133-141, 4 pls.

- 1962a. Données nouvelles sur les loches (Cobitis) d'Europe, avec références spéciales sur les cagnettes de l'Italie du Nord. Trav. Mus. Hist. Nat. Gr. Antipa, 3: 281-301, pls. 1-4.

- 1962b. Contribution à la systématique du genre Cobitis. Description d'une espèce nouvelle, Cobitis calderoni, provenant de l'Espagne. Rev. Biol., Bucuresti, 6 (1961 [1962]): 435-448, 4 pls.

Bacescu, M. \& L. Bacescu-Mester. 1964. Cottus petiti sp. n., un chabot nouveau récolté en France. Considérations zoogéographiques et données comparatives sur d'autres Cottus d'Europe. Vie Milieu, Suppl., 17: 431-446, 2 pls.

Bacescu, M. \&.R. Mayer [Maier]. 1956. Cercetari asupra ghidrinilor (Gasterosteus aculeatus) din apele rominesti. Buletinul Institutului de Cercetari Piscicole, 15 (2): 19-36.

- 1969. [The Cobitis of the Don and Volga]. Vopr. Ikhtiol., 9 (1): 51-60. [Russian, translated in Probl. Ichthyol., 9 (1): 38-44]

Bacescu-Mester, L. 1967. Contribution to the study of the genus Noemacheilus (Pisces Cobitidae). Trav. Mus. Hist. Nat. Gr. Antipa, 7: 357-370, 2 pls.

Bade, E. 1901-1902. Die Mitteleuropäischen Süsswasserfische; ihre Naturgeschichte, Lebensweise und ihr Fang. Walther, Berlin, 1: 182 pp., 2: 176 pp., 36 pls.

Bailey, R. M. 1980. Comments on the classification and nomenclature of lampreys - an alternative view. Can. J. Fish. Aquat. Sci., 37: 1626-1629. - 1982. Reply [to Vladykov \& Kott, 1982]. Can. 
J. Fish. Aquat. Sci., 39: 1217-1220.

Bakker, T. C. M. \& P. Sevenster. 1988. Plate morphs of Gasterosteus aculeatus Linnaeus (Pisces: Gasterosteidae): comments on terminology. Copeia, 1988: 659-663.

Balon, E. K. 1962. [Age and growth of the spawning shoal of Thymallus thymallus (Linnaeus, 1758) from riverine lake on Hnilec river]. Zool. Listy, 11: 145-154.

- 1968. Notes to the origin and evolution of trouts and salmons with special reference to the Danubian trouts. Vestn. Ceskoslov. Společ. Zool., 32: 1-21.

- 1974a. Domestication of the carp Cyprinus carpio L. Life Sci. Contr. Roy. Ontario Mus., Misc. Publ., pp. 1-37.

- 1974b. [Review of:] European inland water fish. A multilingual catalogue. By M. Blanc, P. Bănărescu, J.-L. Gaudet and J.-C. Hureau. Copeia, 1974: 802-804.

- 1976. Comments on Drs. Bănărescu and Hureau's reply to my review of their book "European inland water fish - a multilingual catalogue". Copeia, 1976: 416-417.

- 1989. The epigenetic mechanisms of bifurcation and alternative life-history styles, p. 467-501. In: M. N. Bruton (ed.), Alternative life-history styles of animals. Kluwer, Dordrecht.

- 1995. The common carp, Cyprinus carpio; its wild origin, domestication in aquaculture, and selection as colored nishikigoi. Guelph Ichthyol. Rev., 3: 1-55.

Balon, E. K., S. S. Crawford \& A. Lelek. 1987. Are there sympatric forms of Vimba Fitzinger 1873 in the Danube near the future connection of the Main River ? (Pisces: Cyprinidae). Senck. Biol., 67 (1986 [1987]): 231-248.

- 1992. Die Fische der oberen Donau: taxonomische Merkmale, Grössen- und Gewichtsvariablen vor der Vollendung des Rhein-Main-DonauKanals. Courier Forsch.-Inst. Senckenberg, 148: $1-52$.

Balon, E. K. \& T. Penczak. 1980. The dwarfed charr of Dösener See, an alpine lake in Austria, p. 773-794. In: E. K. Balon (ed.), Charrs Salmonid fishes of the genus Salvelinus. Junk, Den Haag.

Bamber, R.N. \& P. A. Henderson. 1985. Morphological variation in British atherinids, and the status of Atherina presbyter Cuvier (Pisces: Atherinidae). Biol. J. Linn. Soc., 25: 61-76.

Bănărescu, P. 1953. Variatia geografica, filogenia si ecologia cyprinidului Gobio kessleri. Studii Cerc. Stiint. Cluj., 4: 297-337.

- 1957. Die rassiale Zugehörigkeit einiger rumä- nischer Süsswasser-Fischarten. Izd. Inst. Piscicult. R. P. Macédoine, 2: 59-80, 1 pl.

- 1960. Einige Fragen zur Herkunft und Verbreitung der Süsswasserfischfauna der europäischmediterranen Unterregion. Arch. Hydrobiol., 57: 16-134.

- 1961a. Pozitia sistematica a lui Chalcalburnus (Pisces, Cyprinidae) din Dunarea inferioara. Comunicarile Acad. Rep. Pop. Rom., 11: 14891495.

- 1961b. Weitere systematische Studien über die Gattung Gobio (Pisces, Cyprinidae), insbesondere im Donaubecken. Vestn. Ceskoslov. Společ. Zool., 25: 318-346, 4 pls.

- 1964. Fauna Republicii Populare Romîne. Pisces - Osteichthyes. Acad. Rep. Pop. Romîne, Bucuresti, 962 pp.

- 1968. Revision of the Indo-Burmanese genus Salmostoma Swainson (Pisces, Cyprinidae) with description of a new subspecies. Rev. Roum. Biol., Zool., 13: 3-14.

- 1970. Données sur la forme nominale de Gobio uranoscopus (Pisces, Cyprinidae). Bull. Mus. Natn. Hist. Nat. Paris, Sér. 2, 42: 165-169.

- 1981. Ihtiofauna bazinului Crisurilor in cadrul general al ihtiofaunei bazinului Dunarean. Nymphaea, 8-9: 475-481.

- 1991. Comparison of the fish faunas of the Danube and Nistru (= Dniester) river basins. Analele Stiintifice ale Universitatii "Al. I. Cuza" din Iasi, Ser. 2a Biol., 37: 291-303.

- 1992a. A critical updated checklist of Gobioninae (Pisces, Cyprinidae). Trav. Mus. Hist. Nat. Gr. Antipa, 32: 303-330

- 1992b. Zoogeography of fresh waters. Vol. 2. Distribution and dispersal of freshwater animals in North America and Eurasia. Aula, Wiesbaden, Germany, pp. 519-1091.

- 1993. Considerations on the threatened freshwater fishes of Europe. Ocrotirea Naturii si a Mediului Inconjurător, 37: 87-98.

- 1994a. On the presumed origin of Gobio albipinnatus Lukasch in the upper Danube by sympatric speciation (Pisces: Cyprinidae). Senck. Biol., 73: 49-55.

- 1994b. The present-day conservation status of the fresh water fish fauna of Romania. Ocrotirea Naturii şi a Mediului Înconjurător, 38: 5-20.

Bănărescu, P. \& J.-C. Hureau. 1976. European inland water fish, a multilingual catalogue - a reply. Copeia, 1976: 414-416.

Bănărescu, P., G. Müller \& T. T. Nalbant. 1960. Noi contributiuni la studiul ihtiofaunei de apa dulce a Republicii Populare Romine. Comunicari de Zoologie, Societaea de Stiinte 
Naturale si Geografie din R. P. R., 1957-1959 [1960]: 111-126.

Bănărescu, P. \& T. T. Nalbant. 1957. Eidonomische und taxionomische Untersuchungen an $\mathrm{Co}$ bitis elongata (Pisces, Cobitidae). Senck. Biol., 38: $283-294$.

- 1964. Süsswasserfische der Türkei. 2. Teil. Cobitidae. Mitt. Hamburg. Zool. Mus. Inst., 61: 159-201, pls. 5-8.

- 1973. Pisces, Teleostei. Cyprinidae (Gobioninae). Das Tierreich, 93: 1-304.

- 1979. Zingel streber nerensis subspecies nova from South Banat, Romania (Pisces, Percidae). Trav. Mus. Hist. Nat. Gr. Antipa, 20: 353-359.

Bănărescu, P., T. T. Nalbant \& S. Balik. 1978. Süsswasserfische der Türkei. 11. Teil. Die Gattung Orthrias in der Türkei und in Südbulgarien (Pisces, Cobitidae, Noemacheilinae). Mitt. Hamb. Zool. Mus. Inst., 75: 255-266, pl. 20.

Bănărescu, P., T. T. Nalbant \& S. Chelmu. 1972. Revision and geographical variation of Sabanejewia aurata in Romania and the origin of $S$. bulgarica and S. romanica (Pisces, Cobitidae). Annot. Zool. Bot., 75: 1-49.

Bănărescu, P., M. Papadopol \& L. Mikhailova. 1970. [Systematics], p. 23-70. In: P. Zayanchkauskas et al. (eds), [Biology and fisheries of Vimba in Europe]. Mintis Press, Vilnius, 516 pp. [Russian, English summary]

Bănărescu, P., M. Papadopol \& G. Müller. 1963. Le genre Vimba (Pisces, Cyprinidae) dans le bassin du Danube. Trav. Mus. Hist. Nat. Gr. Antipa, 4: 381-400.

Barach, G. P. 1934. [On the systematics and geographical distribution of the Caucasian chubs. 1. Historical note on study of the Caucasian chubs]. Trudy Zoologicheskogo Sektora Gruzinskogo Otdela Zakavkazskogo Filiala Akad. Nauk, Tiflis, 1: 91-133.

- 1941. [Fauna of Georgia. Vol. 1. Freshwater fishes]. Izd. Akad. Nauk Gruz. SSR, Tbilisi, 287 pp.

Barbieri, C. 1907. Le alose del Mediterraneo e gli agoni dei laghi Lombardi. Studio preliminare. Rivista Mensile di Pesca, Milano, 9: 69-82, 125143, pl. 1.

Baruš, V. \& J. Libosvárský. 1983. Umbra krameri (Umbridae, Pisces), a revisional note. Folia Zool., 32: 355-364, 2 pls.

Bath, H. 1977. Revision der Blenniini (Pisces: Blenniidae). Senck. Biol., 57 (1976 [1977]): 167234.

Battalgil, F. 1940. Eine neue Cyprinidenart. Istambul Univ. Fen. Fak. Mecm., Ser. B, 5: 74-77.

- 1941. Les poissons des eaux douces de la
Turquie (Collections de l'Institut de Zoologie de l'Université d'Istanbul). Istambul Univ. Fen. Fak. Mecm., Ser. B, 6: 170-186.

- 1942. Contribution à la connaissance des poissons des eaux douces de la Turquie. Istambul Univ. Fen. Fak. Mecm., Ser. B, 7: 287-306.

- 1944. Nouveaux poissons des eaux douces de la Turquie. Istambul Univ. Fen. Fak. Mecm., Ser. B, 9: 126-133.

Bauchot, M.-L., J. Daget \& R. Bauchot. 1990. L'ichtyologie en France au début du XIXè siècle: l'histoire naturelle des poissons de Cuvier \& Valenciennes. Bull. Mus. Natn. Hist. Nat., Paris, Sect. A, Sér. 4, 12 (Suppl.): 1-142.

Bauchot, M.-L., M. Desoutter \& P. H. J. Castle. 1993. Catalogue critique des types de poissons du Muséum national d'Histoire naturelle (suite). Ordres des Anguilliformes et des Saccopharyngiformes. Cybium, 17: 91-151.

Bauchot, M.-L., M. Desoutter, D. F. Hoese \& H. K. Larson. 1991. Catalogue critique des types de Poissons du Muséum national d'Histoire naturelles (Suite). Sous-ordre des Gobioidei. Bull. Mus. Natn. Hist. Nat. Paris, Sér. 4, 13A: $1-82$.

Beaumont, A. R., J. Bray, J. M. Murphy \& I. J. Winfield. 1995. Genetics of whitefish and vendace in England and Wales. J. Fish Biol., 46: 880-890.

Behnke, R. J. 1968. Süsswasserfische der Türkei. 6. Teil. A new subgenus and species of trout, Salmo (Platysalmo) platycephalus, from Southcentral Turkey, with comments on the classifikation [sic] of the subfamily Salmonidae. Mitt. Hamburg. Zool. Mus. Inst., 66: 1-15.

- 1972. The systematics of salmonid fishes of recently glaciated lakes. J. Fish. Res. Board Canada, 29: 639-671.

- 1980. A systematic review of the genus Salvelinus, p. 441-481. In: E. K. Balon (ed.), Charrs - Salmonid fishes of the genus Salvelinus. Junk, Den Haag.

Beling, D. 1927. [Materialen zur Ichthyofauna des südlichen Bug]. Travaux de la Station Biologique du Dniepre, Kiev, 2: 333-356 [Mém. Cl. Sci. Phys. Math. Acad. Sci. Ukraïne, 3 (7): 333-356]. [Ukrainian, German summary]

Beling, D. \& B. Iljin. 1927. Benthophiloides brauneri n. g., n. sp., ein für das Schwarzmeerbassin neuer Vertreter der Familie der Gobiidae. Travaux de la Station Biologique du Dniepre, Kiev, 2: 309-325, 2 pls. [Mém. Cl. Sci. Phys. Math. Acad. Sci. Ukraïne, 3 (7): 309-325, 2 pls.] Belon, P. 1553. De aquatilibus libri duo. Cum eiconibus ad vivam ipsorum effigiem, quoad 
eius fieri potuit, expressis. Parisiis, $448 \mathrm{pp}$ [translated: 1555. La nature et diversité des poissons, avec leurs pourtraicts, representez au plus pres du naturel. Estienne, Paris, 448 pp].

Belyaev, V. 1929. [Abramis brama bergi subsp. nov. A subspecies of the white-eye bream from the south Caspian]. Izvestiya Bakinskoi Ikhtiologicheskoi Laboratorii, 2 (2): 80-97.

Benecke, B. 1881. Fische, Fischerei und Fischzucht in Ost- und Westpreussen. Hartung, Königsberg in Pr., 514 pp.

- 1884. Ein neuer Cyprinoidenbastard. Zool. Anz., 7: 228-230.

Bennett, E. T. 1831. [...] The collection of fishes formed at the Mauritius by Charles Telfair. Proc. Zool. Soc. London, 1 (1831): 165-169.

- 1835. The fishes forwarded by Mr. Keith Abbott [from Trebizond]. Proc. Zool. Soc. London, 3: 91-92.

Berg, L. S. 1898. [.... about the collection of fish from Bessarabia ...; in Anonym, Report on the meeting of 16 March 1897]. Dnevnik Zoologicheskogo Otdeleniya Obshchestva i Zoologicheskogo Muzeya, 2 (8): 34.

- 1903. [On the systematics of the Cottidae from the Lake Baical]. Annu. Mus. Zool. St. Petersburg, 8: 99-113. [Russian, English diagnosis]

- 1905. [Die Fische von Turkestan]. Mitt. Turkestan. Abt. Kaiserl. Russ. Geogr. Ges., 4 (6): i-xvi+1-260, pls. 1-6.

- 1907a. [Note sur quelques espèces paléarctiques du genre Phoxinus]. Annu. Mus. Zool. Acad. Imp. Sci. St. Petersburg, 11 (1906 [1907]): 196 213. [Russian]

- 1907b. Einige Worte über die Notiz von V Gratzianow: "die Neunaugen des Russischen Reiches". Trav. Soc. Imp. Nat. St. Petersburg, 37: 367-370.

- 1908a. [A list of fishes from the Ob basin]. Annu. Mus. Zool. Acad. Imp. Sci. St. Petersburg, 13: 221-228. [Russian]

- 1908b. [Sur le saumon de la mer d'Aral (Salmo trutta aralensis subsp. nov.)]. Annu. Mus. Zool. Acad. Imp. Sci. St. Petersburg, 13: 315-323.

- 1910. [Report on mission to the Caucasus in 1909]. Annu. Mus. Zool. Acad. Imp. Sci. St. Petersburg, 15: 0153-0170

- 1911. Faune de Russie. Poissons. Vol. 1. Marsipobranchii, Selachii, Chondrostei. Izd. Akad. Nauk, St. Petersburg, iii+337 pp., 8 pls.

- 1912-1914. Faune de Russie. Poissons. Vol. 3. Ostariophysi. Izd. Akad. Nauk, St-Petersburg, 1 (1912): 1-336, 2 pls. 2: (1914) 337-704, 4 pls.

- 1916a. Les poissons d'eau douce de la Russie. Moskva, xxvii+563 pp. 1 pl.
- 1916b. [On the distribution of the fish Myoxocephalus quadricornis (L.), of the family Cottidae, and on some related problems]. Izv. Imper. Akad. Nauk [Bull. Acad. Imp. Sci.], (6) 10: 1343-1360.

- 1923. [Freshwater fishes of Russia, 2nd edition]. Gos. Izd., Moskva, xxx+535 pp.

- 1924. [Alburnoides bipunctatus rossicus Berg, subsp. nov.]. Izvestiya Otdela Prikladnoi Ikhtiologii i Nauchno-Promyslovykh Issledovanii, 2: 56

- 1931. A review of the lampreys of the northern hemisphere. Annu. Mus. Zool. Acad. Sci. URSS, 32: $87-116$, pls. $1-8$.

- 1932a. Uebersicht der Verbreitung der Süsswasserfische Europas. Zoogeographica, 1: 107208, pl. 2.

- 1932b. Ueber Carassius carassius und C. gibelio. Zool. Anz., 98: 15-18.

- 1932c-1933. [Fresh water fishes of the U.S.S.R. and adjacent countries, 3rd edition]. Leningrad, 1 (1932): 1-543; 2 (1933): 545-903.

- 1934. [Summer and winter races in anadromous fishes]. Izv. Akad. Nauk SSSR, Otdela Phys. Math. Nauk, 1934: 711-732

- 1935. Sur les unités taxonomiques chez les poissons. Bull. Mus. Natn. Hist. Nat., Sér. 2, 7: 79-84.

- 1948-1949. [Freshwater fishes of the U.S.S.R. and adjacent countries]. Izd. Akad. Nauk SSSR, Moskva \& Leningrad, vol. 1 (1948), vols. 2-3 (1949). [translation: Israel Program for Scientific Translations, Jerusalem, 1965].

Berg, L. S. \& A. Popov. 1932. A review of the forms of Myoxocephalus quadricornis (L.). C. R. Acad. Sci. URSS, 6: 152-160.

Berg, O. K. 1985. The formation of non-anadromous populations of Atlantic salmon, Salmon [sic] salar L., in Europe. J. Fish Biol., 27: 805815.

Bernardi, G., C. Fernández-Delgado, M. GomezChiarri \& D. A. Powers. 1995. Origin of a Spanish population of Fundulus heteroclitus inferred by cytochrome $b$ sequence analysis. J. Fish Biol., 47: 737-740.

Bernatchez, L. 1995. A role for molecular systematics in defining evolutionary significant units (ESU) in fishes. Amer. Fish. Soc. Symp., 17: 114-132.

Bernatchez, L. \& J. J. Dodson. 1994. Phylogenetic relationships among palearctic and nearctic whitefish (Coregonus sp.) populations as revealed by mitochondrial DNA variation. Can. J. Fish. Aquat. Sci., 51 (Suppl. 1): 240-251.

Bernatchez, L., R. Guyomard \& F. Bonhomme. 
1992. DNA sequence variation of the mitochondrial control region among geographically and morphologically remote European brown trout Salmo trutta populations. Molec. Ecol., 1: 161173.

Bernatchez, L. \& A. Osinov. 1995. Genetic diversity of trout (genus Salmo) from its most eastern native range based on mitochondrial DNA and nuclear gene variation. Molec. Ecol., 4: 285-297.

Berrebi, P. 1994a. Analyse génétique des truites marbrées de la Soca (Slovénie). Marqueurs enzymatiques. Rapport 1993. Laboratoire Génome et Populations, Université Montpellier 2, 13 pp.

- 1994b. Etude génétique des truites de Corse. Rapport final 1994. Laboratoire Génome et Populations, Université Montpellier 2, 16 pp.

- 1995. Speciation of the genus Barbus in the North Mediterranean basin: recent advances from biochemical genetics. Biol. Cons., 72: $237-$ 249.

Bertin, L. 1925. Recherches bionomiques, biométriques et systématiques sur les épinoches (gastérostéidés). Annls. Inst. Océanogr. Monaco, 2: 1-204.

- 1945. Les ouvrages d'ichthyologie et les types de poissons de Risso au Muséum de Paris. Bull. Mus. Natn. Hist. Nat. Paris, Sér. 2, 17: 373-378.

Bertin, L. \& R. Estève. 1948. Catalogue des types de poissons du Muséum national d'Histoire naturelle. 4e partie. Ostariophysaires (Cypriniformes). Muséum National d'Histoire Naturelle, Paris, 117 pp.

Betta, E. de. 1862. Ittiologia Veronese ad uso populare e per servire alla introduzione della piscicultura nella provinzia. Mem. Acad. Agric. Comm. Arti Verona, 41: 259-409. [also reprint: Tipografia Vicentini e Franchini, Verona, 153 pp.]

Bianco, P. G. 1980. Remarks on the genera Alburnus and Alburnoides and description of a neotype for Alburnus albidus (Costa, 1838), senior synonymous of Alburnus vulturius (Costa, 1838) (Pisces, Cyprinidae). Cybium, 3: 31-44.

- 1983. Leuciscus lucumonis n. sp. from Italy (Pisces: Cyprinidae). Senck. Biol., 64: 81-87.

- 1986. The zoogeographic units of Italy and western Balkans based on cyprinid species ranges (Pisces). Biol. Gallo-Helen., 12: 291299 .

- 1988a. I pesci d'acqua dolce d'Italia: note su un recente contributo. Atti Soc. Ital. Sci. Nat. Mus. Civ. Stor. Nat. Milano, 129: 146-158.

- 1988b. Leuciscus cephalus (Linnaeus), with records of fingerling adult males, Leuciscus pleurobipunctatus (Stephanidis) and their hy- brids from western Greece. J. Fish Biol., 32: 116.

- 1990a. Potential role of the palaeohistory of the Mediterranean and Paratethys basins on the early dispersal of Euro-Mediterranean freshwater fishes. Ichthyol. Explor. Freshwat., 1: 167-184

- 1990b. Vanishing freshwater fishes in Italy. J. Fish Biol., 37 (Suppl. A): 235-237.

- 1992. Zoogeographical implications of a first record of Lethenteron zanandreai on the Adriatic slope of central Italy (Cyclostomata: Petromyzontidae). Ichthyol. Explor. Freshwat., 3: 183-183.

- 1994a. The distribution of freshwater fishes of southern Italy. p. 71. In: VIII Congress Societas Europaea Ichthyologorum. Fishes and their environment. Program and abstracts. Oviedo, 26 Sept. to 2 Oct. 1994.

- 1994b. L'ittiofauna continentale dell'Appennino umbro-marchigano, barriera semipermeabile allo scambio di componenti primarie tra gli opposti versanti dell'Italia centrale. Biogeographia, 17 (1993 [1994]): 427-485.

- 1995a. Mediterranean endemic freshwater fishes of Italy. Biol. Cons., 72: 159-170.

- 1995b. A revision of the Italian Barbus species (Cypriniformes: Cyprinidae). Ichthyol. Explor. Freshwat., 6: 305-324.

- 1995c. Factors affecting the distribution of freshwater fishes especially in Italy. Cybium, 19: 241-259.

Bianco, P. G., A. M. Bullock, P. J. Miller \& F. R. Roubal. 1987. A unique teleost dermal organ in a new European genus of fishes (Telostei: Goboidei). J. Fish Biol, 31: 797-803.

Bianco, P. G. \& B. Knežević. 1987. The Leuciscus cephalus complex (Pisces, Cyprinidae) in the western Balkanic area. Proc. V Congr. Europ. Ichthyol., Stockholm 1985, pp. 49-55.

Bianco, P. G. \& P. J. Miller. 1990. Yugoslavian and other records of the Italian freshwater goby, Padogobius martensii, and a character polarization in gobioid fishes. J. Nat. Hist., 24: 1289-1302.

Bianco, P. G. \& R. R. Miller. 1989. First record of Valencia letourneuxi (Sauvage, 1880) in Peloponnese (Greece) and remarks on the mediterranean family Valenciidae (Cyprinodontiformes). Cybium, 13 (1989 [1990]): 385-387.

Bianco, P. G. \& F. Recchia. 1983. The Leuciscinae of the squalius species complex in Italy (Pisces, Cyprinidae). Boll. Zool., 50: 15-19.

Bianco, P. G. \& T. Taraborelli. 1984. Gobio gobio benacensis (Pollini, 1816) sottospecie valida per 
l'Italia (Pisces, Cyprinidae). Boll. Mus. Civ. Stor. Nat. Verona, 11: 525-536.

- 1985. Contributo alla conoscenza del genere Rutilus Rafinesque in Italia e nei Balcani occidentali (Pisces, Cyprinidae). Boll. Mus. Reg. Sci. Nat. Torino, 3: 131-172.

- 1988. I pesci rinvenibili in acqua dolce nelle isole Mediterranee e presenza di Gasterosteus aculeatus del fenotipo semiarmatus in Sardegna. Bull. Ecol., 2-3: 247-254.

Bielz, E. A. 1853. Uebersicht der lebenden Fische Siebenbürgens. Verh. Mitt. Siebenbürgischen Ver. Naturwiss. Hermannstadt, 4: 172-185.

Bíró, P. \& G. Paulovits. 1994. Evolution of fish fauna in Little Ballaton Water Reservoir. Verh. Intern. Ver. Limnol., 25: 2164-2168.

Blainville, H. [Ducrotay de]. 1825. Vertébrés. Classe V. Poissons. In: L.-P. Vieillot et al., 1820-1830. Faune francaise ou histoire naturelle générale et particulière des animaux qui se trouvent en France, constamment ou passagèrement, à la surface du sol, dans les eaux qui le baignent, et dans le littoral des mers qui le bornent. Levrault, Paris \& Strasbourg.

Blanc, M., P. Bănărescu, J.-L. Gaudet \& J.-C. Hureau. 1977. European inland water fish. A multilingual catalogue. Fishing News, London, $22+1131+$ xix pp.

Blanc, M. \& J.-C. Hureau. 1971. Catalogue critique des types de poissons du Muséum national d'Histoire naturelle (suite) (Mugiliformes et Polynémiformes). Bull. Mus. Natn. Hist. Nat. Paris, Sér. 3, Zool., 15: 673-734.

Blanchard, E. 1866. Les poissons des eaux douces de la France. Baillière, Paris, xvi+656 pp.

Bleeker, P. 1862. Beschrijving van eene nieuwe soort van Brama (Abramis) uit de omstreken van Leiden. Versl. Akad. Amsterdam, 15: 235238. Also in: Ned. Tijdschr. Dierk., 1 (1863): 371-373.

- 1863a. Mémoire sur les poissons de la côte de Guinée. Natuurk. Verk. Holland. Maatsch. Wetensch., 18 (2): 1-136, 28 pls.

- 1863b. Notice sur les noms de quelques genres de la famille des Cyprinoïdes. Versl. Akad. Amsterdam, 15: 261-264.

- 1863c. Systema cyprinoideorum revisum. Ned. Tijdschr. Dierk., 1: 187-218.

- 1863d. Atlas ichthyologique des Indes Orientales Néêrlandaises. Tome III. Cyprins. Muller, Amsterdam, 150 pp., pls. 102-144.

Bloch, M. E. 1779. Naturgeschichte der Maräne. Beschäft. Berlin. Ges. Naturforsch. Freunde, 4: 60-94, pl. 4.

- 1782. Oeconomische Naturgeschichte der Fis- che Deutschlands. 1. Theil. Berlin, 258 pp., pls. $1-37$.

- 1783. Oeconomische Naturgeschichte der Fische Deutschlands. 2. Theil. Berlin, 192 pp., pls. 38-72.

- 1784. Oeconomische Naturgeschichte der Fische Deutschlands. 3. Theil. Berlin, 234 pp., pls. 73-108.

- 1787. Naturgeschichte der Ausländische Fische. Dritter Theil. Morino, Berlin, xii+146 pp., pls. 181-216.

- 1792. Naturgeschichte der Ausländische Fische. Sechster Theil. Morino, Berlin, 126 pp., pls. 289-324.

- 1794. Naturgeschichte der Ausländische Fische. Achter Theil. Morino, Berlin, iv+174 pp., pls. 361-396.

- 1795. Naturgeschichte der Ausländische Fische (Neunter Theil). Morino, Berlin, 192 pp., pls, 397-429

Bock, M. \& C. D. Zander. 1986. Osteological characters as tool for blenniid taxonomy - a generic revision of European Blenniidae (Percomorphi; Pisces). Ztschr. Zool. Syst. Evol. Forsch., 24: 138-143.

Bodaly, R. A., J. Vuorinen, R. D. Ward, M. Luczynski \& J. D. Reist. 1991. Genetic composition of New and Old World coregonid fishes. J. Fish Biol., 38: 37-51.

Boeseman, M. 1962. Triodon macropterus versus Triodon bursarius: an attempt to establish the correct name and authorship. Zool. Meded., 38: $77-85$.

Bogutskaya, N. G. 1992. A revision of species of the genus Pseudophoxinus (Leuciscinae, Cyprinidae) from Asia Minor. Mitt. Hamburg. Zool. Mus. Inst., 89: 261-290.

- 1994. A description of Leuciscus lepidus (Heckel, 1843) with comments on Leuciscus and leuciscine - aspinine relationships. Ann. Naturhist. Mus. Wien, 96B: 599-620.

- 1995. Leuciscus kurui, a new cyprinid fish from the Upper Tigris (Dicle) system. Mitt. Hamb. Zool. Mus. Inst., 92: 149-154.

Böhlke, E. B. 1984. Catalog of type specimens in the ichthyological collection of the Academy of Natural Sciences of Philadelphia. Acad. Nat. Sci. Philad., Spec. Publ., 14: 1-246.

- (ed.) 1989. Fishes of the western North Atlantic. Part nine. Volume one: Orders Anguilliformes and Saccopharyngiformes). Mem. Sears Found. Mar. Res., 1 (9, 1): 1-655.

Boisneau, P., C. Mennesson-Boisneau \& R. Guyomard. 1992. Electrophoretic identity between allis shad, Alosa alosa (L.)., and twaite shad, 
A. fallax (Lacépède). J. Fish Biol., 40: 731738.

Bonaparte, C. L. 1832-1841. Iconografia della Fauna italica per le quattro classi degli animali vertebrati. Tomo 3, Pesci. Roma. [pages and plates unnumbered; dates of publication follow Salvadori, 1888]

- 1842. Descrizione di un nuovo leuciscino veduto per la prima volta nella peschiera dello Scoglietto del Durazzo in Genova. Nuovi Annali delle Scienze Naturali, Anno 4, Tomo 7: 148-151.

- 1844. Rilievi sul volume XVII dell' "Istoria naturale dei pesci" di Valenciennes che contiene i Leuciscini. Atti Sci. Ital., 1844: 431-438.

- 1845. Catalogo metodico dei ciprinidi d'Euro$\mathrm{pa}$, e rilievi sul volume XVII dell'istoria naturale dei pesci del sig. Valenciennes. Pirola, Milano, 1845,20 pp.

- 1846. Catalogo metodico dei pesci Europei. Atti della Settima Adunanza degli Scienzati Italiani tenuta in Napoli. Parte Seconda. Napoli, 96 pp. [also distributed as a reprint; in 1845 ?]

Bonnaterre, [J. P.]. 1788. Tableau encyclopédique et méthodique des trois règne de la nature ... Ichthyologie. Panckrouke, Paris, vi+215 pp., 102 pls.

Borcea, I. 1934. Note préliminaire sur les clupéides de la région littorale roumaine de la mer Noire et des eaux intérieures. Ann. Scient. Univ. Jassy, 19: $382-390$.

- 1936. Les caspialoses de la mer Noire. C. R. Acad. Sci. Roum., 1: 26-28.

Borisov, P. G. 1924. [Pereslav lake cisco]. Trudy Instituta Rybnogo Khozyaistva, Moskva, 1: 51119.

- 1928. [Fishes of the river Lena]. Trudy Kommissii po izucheniyu Yakutskoi Avtonomnoi Sovetskoi Socialisticheskoi Respubliki [Works of the Commission for Research in the Yakutian Autonom SSR]. Tom 9. Akademiya Nauk SSSR, Leningrad, ii+181 pp., $6+1$ pls.

Borlase, W. 1758. The natural history of Cornwall. The air, climate, waters, rivers, lakes, sea ... rare birds, fishes, shells, reptiles, and quadrupeds, ... Oxford, xix +326 pp.

Borodin [Borodine], N. 1896. [Notes sur les Clupea des mers Caspienne et Noire]. Annu. Mus. Zool. Acad. Imp. Sci. St. Petersburg, 1: 81-94.

Boron, A. 1992. Karyotype study of diploid and tetraploid Cobitis taenia (Pisces, Cobitididae) from Vistula river basin. Cytobios, 72: 201206.

Borzenko, M. P. 1950. Materialy po sistematike, biologii e promyslu kurinskogo shipa. Trudy
Kaspiiskogo Basseinovogo Filiala VNIRO, 11: 9-46.

Boulenger, G. A. 1896. On freshwater fishes from Smyrna. Ann. Mag. Nat. Hist., Ser. 6, 18: 153154.

- 1907. The fishes of the Nile. Rees, London, 578 pp., 97 pls.

Brandt, J. F. 1851. Ueber Albinismus und eine abweichende Farbenspielart des Sterläd (Acipenser ruthenus). Bull. Cl. Physico-Math. Acad. Imp. Sci. St. Pétersbourg, 10 (1850 [1851]): 13$16,1 \mathrm{pl}$.

Brandt, J. F. \&. J. T. C. Ratzeburg. 1833. Medizinische Zoologie oder getreue Darstellung und Beschreibung der Thiere, die in der Arzneimittellehre in Betracht kommen, in systematischer Folge herausgeben. Trowitzsch und Sohn, Berlin, 2: 1-30, 349-355, pls. 1-3.

Brenner, T. 1980. The arctic charr, Salvelinus alpinus salvelinus, in the prealpine Attersee, Austria, p. 765-772. In: E. K. Balon (ed.), Charrs - Salmonid fishes of the genus Salvelinus. Junk, Den Haag.

Brothers, D. J. 1985. Species concepts, speciation, and higher taxa. In: Vrba, E. S. (ed.), Species and speciation. Transvaal Mus. Monogr., 4: 3542.

Brown, T. 1830. Description of a new species of British fish. Edinburgh J. Nat. Geogr. Sci., 2: 99, pl. 2.

Brusina, S. 1902. Nase jesetre. Rada Jugoslavenske Akademije Znanosti i Umjetnosti, 149 (Mat.Prirodosl. Razr. 31): 1-69, 1 pl.

Bruton, M. N. 1989. The ecological significance of alternative life-history styles, p. 503-553. In: M. N. Bruton (ed.), Alternative life-history styles of animals. Kluwer, Dordrecht.

de Buen, F. 1928. El Gobius niger L. en aguas atlánticas y mediterráneas de Europa. Notas Resúm. Inst. Esp. Oceanogr., 2 (2): 1-32.

- 1930. Notas sobre la fauna ictiologica de nuestras aguas dulces. Notas Resum. Inst. Esp. Oceanogr., Ser. 2, 46: 1-62.

- 1935. Fauna ictiológica. Catálogo de los peces ibéricos: de la planicie continental, aguas dulces, pelágicos y de los abismos próximos. Primera parte. Notas Inst. Esp. Oceanogr, Ser. 2, 88-89: 1-173, pls. 1-53.

Buresch, R. 1925. Studien am Seesaibling mehrerer Alpenseen. Ein Beitrag zur Kenntnis der Seesaiblingsfauna Österreichs. Ztschr. Fischerei Hilfswiss., 23: 99-119.

Burmakin, E. V. 1941. [Food resources of the Gydan Bay and the adjacent water bodies]. Trudy Instituta Polyargono Zemledeliya, Ser. 
Promyslovoe Khozyajstvo, Leningrad \& Moskva, $15: 159-177$.

Buth, D. G. \& R. L. Mayden. 1981. Taxonomic status and relationships among populations of Notropis pilsbryi and N. zonatus (Cypriniformes: Cyprinidae) as shown by the glucosephosphate isomerase, lactate dehydrogenase, and phosphoglucomutase enzyme systems. Copeia, 1981: 583-590.

Buttiker, B. \& G. Matthey. 1986. Migration de la truite lacustre (Salmo trutta lacustris L.) dans le Léman et ses affluents. Schweiz. Ztschr. Hydrol., 48: 153-160.

Buttiker, B., G. Matthey, J. Bel \& P. Durand. 1987. Age et croissance de la truite lacustre (Salmo trutta lacustris L.) du Léman. Schweiz. Ztschr. Hydrol., 49: 3315-328.

Cain, A. J. 1957. Logic and memory in Linnaeus's system of taxonomy. Proc. Linn. Soc. London, 169: $144-163$

Calderoni, P. 1981. I Rutilus Rafinesque Italiani. II. Ulteriori indagini sul Rutilus Raf. del Lago Trasimeno ("Laschetta" del Trasimeno - Cypriniphormes, Cyprinidae). Riv. Idrobiol. 19: 391-468.

Canestrini, G. 1861. I gobii del Golfo di Genova Arch. Zool. Anat. Fisiol., 1: 121-157, pls. 7-11.

- 1864. Note ittiologie. I. Sopra una nuova specie di Mesites. II. Sopra una nuova specie di Gobius d'acqua dulce. III. Sopra une varietà dell'Alburnus aborella de-Fil. IV. Nota sul genere Scardinius. V. Nota sul genere Telestes. VI. Nota sul genere Barbus. Arch. Zool. Anat. Fisiol., 3: 100-112.

- 1866. Prospetto critico dei pesci d'acqua dolce d'Italia. Arch. Zool. Anat. Find., 4: 47-187, pl. 4.

- 1867. Due note ittiologiche. I. Sopra alcuni pesci dell'Arno. II. Effetti di dimagramento osservati nel Gobius fluviatilis Bon. Ann. Soc. Natur. Modena, 2: 7-13.

- 1868. Nuove specie italiane di animali. I. Sopra una nuova specie di Gobius. Comment. Fauna Flora Gea, Venezia, 1: 169-173.

- 1871-1872. Pesci. Parte I, 1871, pesci delle acque dolci: 4-36. Parte II, 1872, Pesci marini: 37-208. In: E. Cornalia, 1870-1874, Fauna d'Italia, Parte terza. Milano.

Cantoni, E. 1882. Sulla variabilita del cobite fluviale (Cobitis taenia, L.). Rendic. R. Istit. Lomb., Ser. 2, 15: 362-367, pl. 50.

Capello, F. de Brito. 1869. Appendice ao catalogo dos peixes de Portugal que existem no Museu de Lisboa. J. Sci. Phys. Nat. Lisboa, 2 (7): 223228.
- 1880. Catalogo dos peixes de Portugal Memoria apresentada á Academia Real das Sciencias de Lisboa. Lisboa, 73 pp.

Caporiacco, L. di. 1948. I Syngnathidae italiani. Boll. Pesca Piscic. Idrobiol., N.S., 24 (Nuova Serie 3): 71-95.

Cataudella, S., L. Sola, R. Accame Muratori \& E. Capanna. 1977. The chromosomes of 11 species of Cyprinidae and one Cobitidae from Italy, with some remarks on the problem of polyploidy in the cypriniformes. Genetica, 47: 161-171.

Cavender, T. M. 1980. Systematics of Salvelinus from the North Pacific basin, p. 295-322. In: E. K. Balon (ed.), Charrs - Salmonid fishes of the genus Salvelinus. Junk, Den Haag.

Cavicchioli, G. 1965. Contributo allo studio dei Cobitidae italiani: descrizione di una nuova sottospecie del genere Cobitis: Cobitis (Cobitis) taenia zanandreai. Boll. Zool., 32: 1151-1161.

Cawdery, S. A. H. \& A. Ferguson. 1988. Origins and differentiation of three sympatric species of trout (Salmo trutta L.) in Lough Melvin. Polsk. Arch. Hydrobiol., 35: 267-277.

Chabanaud, P. 1933. Un nouveau type de poissons de la famille des gobiidés (Syrrhothonus Charrieri, n. g., n. sp.). C. R. Acad. Sci. Paris, 197: 1249-1251.

Chalikov, B. G. 1944. [Acipenser primigenius sp. nov. - a new species of the genus Acipenser from the North Caspian]. Zool. Zh., 23: 47-50.

Champigneulle, A., B. Buttiker, M. Melhaoui, P. Durand \& J. P. Pilotto. 1993. Synthèse des principales études sur la truite de lac dans le Léman et quelques affluents. Les cahiers de la pêche, Office Fédéral de l'Environnement, des Forêts et du Paysage, Berne, 51: 29-53.

Chandler, C. R. \& M. H. Gromko. 1989. On the relationship between species concepts and speciation processes. Syst. Zool., 38: 116-125.

Changeux, T. \& D. Pont. 1995. Current status of the riverine fishes of the French Mediterranean basin. Biol. Cons., 72: 137-158.

Charleton, G. [W.]. 1668. Onomasticon zoicon, plerorumque animalium, differentias \& nomina propria, pluribus linguis exponens. Cui accedunt mantissa anatomica; \& quaedam de variis fossilium generibus. Londini.

Chen, J. 1981. [A study of the classification of the subfamily Cobitinae of China]. Trans. Chinese Ichthyol. Soc., 1: 21-32. [Chinese, English summary]

Chen, T. R. \& F. H. Ruddle. 1970. A chromosome study of four species and a hybrid of the killifish genus Fundulus (Cyprinodontidae). Chromosoma, 29: 255-267. 
Chen Y.-R. 1986. [A revision of fishes of the cyprinid genus Anabarilius]. Acta Zootax. Sinica, 11: 429-438. [Chinese, English summary]

Chereshnev, I. A. 1982. The taxonomic status of sympatric diadromous chars of the genus Salvelinus (Salmonidae) from eastern Chukotka. J. Ichthyol., 22 (6): 22-38. [translated from Vopr. Ikhthiol., 22: 922-936]

Chiappi, T. 1924. Le trote del Lago della Posta. Tipographia del Senato, Roma, 8 pp.

- 1933. Note su alcuni stadi di sviluppo dell'agone introdotto nei laghi laziali, e della cheppia del Tevere. Boll. Pesca Piscic. Idrobiol., 9: 10521061.

Chichkoff, G. 1929. Sur un nouveau poisson du genre Gobio Cuvier Gobio similis n. sp. Annu. Univ. Sofia, Fac. Phys.-Math., Sér. 1, Math. Phys., 25 (2): 159-174, 1 pl.

- 1932. [Nouveaux poissons d'eau douce de la Bulgarie]. Trav. Soc. Bulg. Sci. Nat., 15-16: 355-377, pl. 1. [Bulgarian, French summary]

- 1934. [Sur un nouveau spirlin - Alburnoides bipunctatus tzanevi subsp. nova]. Izvestiya na Bulgarskoto Geografsko Druzhestvo, 1 (1933 [1934]): 375-383. [Bulagrian, French summary]

- 1935. [Description d'un barbeau nouveau Barbus barbus bergi n. subsp.]. Annu. Univ. Sofia, Fac. Physico-Math., 31 (Livre 3, Sci. Nat.): 305-314, pl. 1. [Bulgarian, French summary]

- 1937. Sur les goujons habitant les eaux douces de la Bulgarie. Annu. Univ. Sofia, Fac. Phys.Math., 33 (Livie 3, Sci. Nat.): 227-289. [Bulgarian, French summary]

- 1939. [Poissons nouveaux et peu connus des eaux douces de la Bulgarie]. Annu. Univ. Sofia, Fac. Physico-Math., 35 (Livre 3, Sci. Nat.): 91199, pls. 1-4. [Bulgarian, French summary]

- 1940. [Etude morphologique sur les spirlins des eaux douces de la Bulgarie]. Annu. Univ. Sofia, Fac. Physico-Math., 36 (Livre 3, Sci. Nat.): 75 144, 2 pls. [Bulgarian, French summary]

Child, A. R. 1984. Biochemical polymorphism in charr (Salvelinus alpinus L.) from three Cumbrian lakes. Heredity, 53: 249-257.

Cisternas, R. 1877. Ensayo descriptivo de los peces de agua dulce que habitan en la provincia de Valencia. An. Soc. Esp. Hist. Nat., Madrid, 6: 69-138.

Cligny, A. 1907. Migrations marines de la truite commune. C. R. Hebd. Séances Acad. Sci., Paris, 145: 1302-1305.

Cloquet, H. 1826. Plie, Platessa, p. 401-405. In: Dictionnaire des sciences naturelles, dans lequel on traite méthodiquement des différens êtres de la nature, considérés soit en euxmêmes, d'après l'état actuel de nos connoissances, soit relativement a l'utilité qu'en peuvent retirer la médecine, l'agriculture, le commerce et les arts. Suivi d'une biographie des plus célèbres naturalistes. Levrault, Strasbourg \& Paris, \& Le Normant, Paris, vol. 41, 558 pp., $1 \mathrm{pl}$.

Coad, B. W. 1980. A provisional, annotated checklist of the freshwater fishes of Iran. J. Bombay Nat. Hist. Soc., 76: 86-105.

- 1982. A re-description and generic re-assignment of Kosswigobarbus kosswigi (Ladiges, 1960), a cyprinid fish from Turkey and Iran. Mitt. Hamburg. Zool. Mus. Inst., 79: 263-265.

- 1991. Fishes of the Tigris-Euphrates basin: a critical checklist. Syllogeus, 68: 1-49.

Coad, B. W. \& M. Sarieyyüpoglu. 1988. Cobitis elazigensis, a new species of cobitid fish from Anatolia, Turkey. Japan. J. Ichthyol., 34: 426430.

Cocco, A. 1885. Indice ittiologico del mare di Messina. Naturalista Sicil., 4: 85-88, 238-240.

Coelho, M. M. 1985. The straight mouth Portuguese Chondrostoma Agassiz, 1835. II-Taxonomic position and geographic distribution of Ch. polylepis Steindachner, 1865 and Ch. willkommi Steindachner, 1866 with the description of a new subspecies - Ch. polylepis duriensis. Arq. Mus. Bocage, Ser. A, 3: 13-37, 1 pl.

- 1992. Genetic differentiation of the Iberian cyprinids Chondrostoma polylepis Steind., 1865 and Ch. willkommii Steind., 1866. Arch. Hydrobiol., 125: 487-498.

Coelho, M. M., R. M. Brito, T. R. Pacheco, D. Figueiredo \& A. M. Pires. 1995. Genetic variation and divergence of Leuciscus pyrenaicus and L. carolitertii (Pisces, Cyprinidae). J. Fish Biol., 47 (Suppl. A): 243-258.

Collares-Pereira, M. J. 1980a. Les Chondrostoma à bouche arquée de la péninsule ibérique (avec la description de Ch. lusitanicum nov. sp.) (Poissons, Cyprinidae). C. R. Acad. Sci. Paris, Sér. D, 291: 275-278.

- 1980b. Contribution to the knowledge of the Iberian cyprinid Chondrostoma lemmingil (Steind., 1866) and its affinities with Chondrostoma arrigonis (Steind., 1866). Arq. Mus. Bocage, Ser. 2, 7: 151-178.

- 1984. The "Rutilus alburnoides (Steindachner, 1866) complex" (Pisces, Cyprinidae). I. Biometrical analysis of some Portuguese populations. Arq. Mus. Bocage, Ser. A, 2: 111-143.

— 1985. The "Rutilus alburnoides (Steindachner, 1866) complex" (Pisces, Cyprinidae). II. First 
data on the karyology of a well-established diploid-triploid group. Arq. Mus. Bocage, Ser. A, 3: 69-89.

Collette, B. B. \& P. Bănărescu. 1977. Systematics and zoogeography of the fishes of the family Percidae. J. Fish. Res. Board Canada, 34: 14501463.

Conci, C. \& M. Michelangeli. 1974. Catalogo dei tipi del Museo Civico di Storia Naturale di Milano. I. I tipi dei pesci, con note storiche sulla collezione ittiologica. Atti Soc. Ital. Sci. Nat. Mus. Civ. Stor. Nat. Milano, 115: 213-237.

Costa, O. G. 1830-1857. Fauna del regno di Napoli, ossia enumerazione di tutti gli animali che abitano le diverse regioni di questo regno e le acque che le bagnano contenente la descrizione de nuovi o poco esattamente conosciuti. Pesci, parte prima. Azzolino, Napoli. [see Sherborn, 1937, for dates of publication]

Costa Pereira, N. da. 1995. The freshwater fishes of the Iberian Peninsula. Arq. Mus. Bocage, N. S., 2: 473-538.

Cottiglia, M. 1963a. Studi sull'ittiofauna dulciacquicola della Sardegna. I - L'alosa migratrice del Tirso. Boll. Pesca Piscic. Idrobiol., 39 (Nuova Serie 18): 15-38.

- 1963b. Studii sull'ittiofauna dulciacquicola della Sardegna. II - L'agone del Lago Omodeo. Boll. Pesca Piscic. Idrobiol., 18: 125-141.

- 1968. La distribuzione della ittiofauna dulciacquicola in Sardegna. Riv. Idrobiol., 7: 63-115.

- 1970. Nuovi datti sulla biologia e sulla sistematica di Alosa fallax Lac. in Sardegna. Rc. Semin. Fac. Sci. Univ. Cagliari, 39 (1969 [1970]): $351-365$.

Couch, J. 1832. Fishes new to the British fauna, contained in Couch's "History of the fishes of Cornwall". Mag. Nat. Hist., 5: 15-24.

- 1859. Memoir of the natural history of the salmon tribe; from materials chiefly collected in Cornwall and the West of England. Annual Rep. Roy. Cornwall Polytechnic Society, 27: 1-29.

- 1862-1865. A history of the fishes of the British Islands. Groombridge, London, 1 (1862): vii+ 245 pp., pls. $1-57$; 2 (1863): 265 pp., 63 pls.; 3 (1864): 208 pp., 48 pls.; 4 (1865): iv+439 pp., 73 pls.

Cracraft, J, 1982. Geographic.differentiation, cladistics, and vicariance biogeography: reconstructing the tempo and mode of evolution. Amer. Zool., 22: 411-424.

- 1983. Species concepts and speciation analysis. Curr. Ornithol., 1: 159-187.

- 1987. Species concepts and the ontology of evolution. Biology and Philosophy, 2: 329-346.
- 1989. Speciation and its ontology: the empirical consequences of alternative species concepts for understanding patterns and processes of differentiation, p. 28-59. In: D. Otte \& J. A. Endler (eds), Speciation and its consequences. Sinauer Associates, Sunderland, MA.

Creech, S. 1991. An electrophoretic investigation of populations of Atherina boyeri Risso, 1810 and $A$. presbyter Cuvier, 1829 (Teleostei: Atherinidae): genetic evidence in support of the two species. J. Fish Biol., 39: 807-816.

Crespon, J. 1844. Faune méridionale ou description de tous les animaux vertébrés vivans et fossiles, sauvages ou domestiques qui se rencontrent toute l'année ou qui ne sont que de passage dans la plus grande partie du midi de la France suivie d'une méthode de taxidermie ou l'art d'empailler les oiseaux. Vol. 2. Nîmes, ix + $355 \mathrm{pp}$.

Crivelli, A. \& E. Rosecci. 1994. Status and characteristics of endemic freshwater fishes in the northern Mediterranean region: an overview of a thretened ( $\mathrm{sic}$ ) fauna, p. 14. In: Symposium on the conservation of endangered freshwater fish in Europe, 20-22 July 1994, Bern. Program, abstracts, participants.

Crozier, W. W. \& A. Ferguson. 1986. Electrophoretic examination of the population structure of brown trout, Salmo trutta L., from the Lough Neagh catchment, Northern Ireland. J. Fish Biol., 28: 459-477.

Cuvier, G. 1816. Le règne animal distribué d'après son organisation, pour servir de base à l'histoire naturelle des animaux et d'introduction à l'anatomie comparée. Deterville, Paris, 2: xvii +532 pp. [see Roux, 1976, for publication date]

- 1822. Das Thierreich eingetheilt nach dem Bau der Thiere als Grundlage ihrer Naturgeschichte und der vergleichenden Anatomie. Mit vielen Zusätzenversehen von H. R. Schinz. Cotta, Stuttgart \& Tübingen, 2: xvi +835 pp.

- 1829. Le règne animal distribué d'après son organisation, pour servir de base à l'histoire naturelle des animaux et d'introduction à l'anatomie comparée. Déterville, Paris, 2: xv + 406 Pp.

- 1838-1843. Le règne animal, distribué d'après son organisation, pour servir de base à l'histoire naturelle des animaux et d'introduction à l'anatomie comparée. 3ème edition ["édition des disciples"]. 4. Poissons [by A. Valenciennes]. Paris. 1838: 1-40; 1839: 41-48; 1840: 49-120; 1841: $121-176 ; 1842$ : 177-360; 1843: 361-392; 120 pls.

Cuvier, G. \& A. Valenciennes. 1828a. Histoire 
naturelle des poissons. Tome premier. Levrault, Paris, xvi+576 pp., pls. 1-8.

- 1828b. Histoire naturelle des poissons. Tome second. Levrault, Paris, xxi+490 pp., pls. 9-40.

- 1829a. Histoire naturelle des poissons. Tome troisième. Levrault, Paris, xxviii +500, pls. 4171.

- 1829b. Histoire naturelle des poissons. Tome quatrième. Levrault, Paris, xxvi+518 pp., pls. 72-99.

- 1830. Histoire naturelle des poissons. Tome sixième. Levrault, Paris, xxvi+559 pp., pls. 141-169.

- 1836. Histoire naturelle des poissons. Tome onzième. Levrault, Paris, $\mathrm{xx}+506$ pp., pls. $307-$ 343.

- 1837. Histoire naturelle des poissons. Tome douzième. Levrault, Paris, xxiv +507 pp., pls. 344-368.

- 1842. Histoire naturelle des poissons. Tome seizième. Bertrand, Paris, $\mathrm{xx}+472$ pp., pls. 456 487.

- 1844. Histoire naturelle des poissons. Tome disseptième. Bertrand, Paris, xxiii+497 pp., pls. $487-519$.

- 1846. Histoire naturelle des poissons. Tome dixhuitième. Bertrand, Paris, xix +505 pp., pls. 520-553.

- 1847. Histoire naturelle des poissons. Tome vingtième. Bertrand, Paris, xviii+472 pp., pls. 591-606.

- 1848. Histoire naturelle des poissons. Tome vingt et unième. Bertrand, Paris, xiv +536 pp., pls. 607-633.

Czernay, A. 1851. Beobachtungen gesammelt auf Reisen im Charkowschen und den anliegenden Gouvernements in den Jahren 1848 und 1849. Bull. Soc. Imp. Nat. Moscou, 24 (1): 279-282, pl. 7 .

Dadikyan, M. G. 1970. [A new subspecies of white bream [Blicca bjoerkna derjavini, subsp. n.] from the Sevdzhur River]. Vopr. Ikhtiol., 10: 740-742. [Russian, translated in J. Ichthyol., 10 (4): $550-552]$

- 1972. [A new subspecies of the European riffle minnow Alburnoides bipunctatus armeniensis subsp. n.]. Vopr. Ikhtiol., 12: 566-569 [Russian, translated in J. Ichthyol., 12 (3): 519-522]

Daget, J. 1983. Vers une conception bio-génétique du genre chez les poissons. Bull. Mus. Natn. Hist. Nat. Paris, Sér. 4, Sect. A, 5: 299-406.

Daget, J., P. S. Economidis \& J. Louis. 1977. Sousespèces d'Alburnoides bipunctatus (Pisces, Cyprinidae) de la Grèce continentale. Cybium, Sér. 3, 2: $7-23$.
Daget, J., J. P. Gosse \& D. F. E. Thys van den Audenaerde. 1984. Check-list of the freshwater fishes of Africa. I. ORSTOM, Paris \& Musée Royal de l'Afrique Centrale, Tervuren, xviii+410 pp.

- 1986. Check-list of the freshwater fishes of Africa. II. Institut Royal des Sciences Naturelles, Bruxelles, Musée Royal de l'Afrique Centrale, Tervuren \& ORSTOM, Paris, xiv + 520 pp.

Danilevskii, N. Y. 1873. [A report of the expedition for examination of fisheries in the northwestern lakes (Lake Onega, Lake Ladoga, Lake Il'men. Conclusion)]. Sel'skoje Khozya'stvo i Lesovodstvo, Osobyje Prilozheniya [Special Supplements (supplements printed at the end of each fascicles)], 113 (May): 61-108, (June): 109-170, (July): 171-222. [Russian]

Day, F. 1880-1884. The fishes of Great Britain and Ireland. Williams \& Norgate, London \& Edinburgh, 1: cxii+336 pp., pls. 1-93, 2: 388 pp., pls. 94-179. [for publication dates, see Wheeler, 1966]

- 1887. British and Irish Salmonidae. Williams \& Norgate, London \& Edinburgh, viii+299 pp., 12 pls.

Dean, B. 1916-1923. A bibliography of fishes. American Museum of Natural History, New York, 3 vols.

De la Pylaie. 1835. Recherches en France sur les poissons de l'Océan. Congrès Scientifique de France, Poitiers, pp. 524-534.

Delaroche, M. 1809. Suite du mémoire sur les espèces de poissons observées à Iviça. Ann. Mus. Hist. Nat., 13: 313-361, pls. 20-25.

Delgado, J. V., A. Alonso, M. E. Camacho, J. Lobillo \& J. M. Selma. 1991. Cytotaxonomy of a cyprinodontid (Teleostei, Cyprinodontidae) population in southwestern Spain. Doñana Acta Vertebrata, 18: 251-257.

Delyamure, S. L. 1937. K vopr. sistemat. poloj. krymskich peskarej. [from Bănărescu \& Nalbant, 1973, p. 119]

Derjavin, A. N. 1926 [Fishes of the Karasu River]. Izvestiya Bakinskoi Ikhtiologicheskoi Laboratorii, 2 (1): 161-182.

Dianov, P. A. 1955. [The lake Zaisan perch]. Avtoref. Diss., Inst. Zool., Akad. Nauk Kazakhstan S.S.R. [from Svetovidov \& Dorofeeva, 1963]

Dimovski, A. \& R. Grupce. 1974. [Etudes morphologico-systématiques du genre Gobio (Pisces, Cyprinidae) en Macédoine. I. Gobio kessleri banarescui n. ssp. de la rivière Vardar]. Acta Mus. Maced. Scient. Nat., 14: 69-92. [Macedonian, French summary] 
- 1976a. [Etudes morphologico-systématiques du genre Gobio (Pisces, Cyprinidae) en Macédoine. III. Gobio uranoscopus stankoi n. ssp. du bassin de la rivière Vardar]. Annu. Fac. Biol. Univ. 'Kiril et Metodij', Skopje, Biol., 29: 73-92. [Macedonian, French summary]

- 1976b. [Contribution à la systématique de certaines espèces du genre Gobio (Pisces, Cyprinidae) des eaux de la partie méridionale de la péninsule des Balkans]. Posebno Izdanie, Musei Macedonici Scientiarum Naturalium, 7: 39-52. [Macedonian, French summary]

- 1977. [Etudes morphologico-systématique du genre Gobio (Pisces, Cyprinidae) en Macédoine. IV. Gobio gobio balcanicus n. ssp. de la rivière Vardar]. Annu. Fac. Biol. Univ. 'Kiril et Metodij', Skopje, Biol., 30: 79-106. [Macedonian, French summary]

Dikson, B. I. 1909. [Fisheries on the territory of the 8th fishery district]. Rybolovstvo $\mathrm{v}$ basseine Volgi Vyche Saratova, 8: 1-107. [Russian]

Doadrio, I. 1980. Descripción de un nuevo género y de una nueva especie Iberocypris palaciosi n. gen. n. sp. (Pisces, Cyprinidae). Dóñana Acta Vertebrata, 7: 5-16.

- 1988a. Leuciscus carolitertii n. sp. from the Iberian Peninsula (Pisces: Cyprinidae). Senck. Biol., 68: 301-309.

- 1988b. Sobre la taxonomía de Barbus comiza Steindachner, 1865 (Ostariophysi: Cyprinidae). Doñana Acta Vertebrata, 15: 19-28.

- 1990. Phylogenetic relationships and classification of western palaearctic species of the genus Barbus (Osteichthyes, Cyprinidae). Aquat. Living Resour., 3: 265-282, 4 pls.

- 1994. Freshwater fish fauna of North Africa and its biogeography. Ann. Mus. R. Afr. Centr., Sci. Zool., 275: 21-34.

Doadrio, I., B. Elvira \& P. Garzon. 1988. Revisión taxonómica y distribución de Cobitis maroccana Pellegrin, 1929 (Osteichthyes, Cobitidae). Doñana Acta Vertebrata, 15: 156-161.

Doadrio, I. \& P. Garzón. 1987. Sobre la distribución de Barbus haasi (Ostariophysi: Cyprinidae). Doñana Acta Vertebrata, 14: 123-125.

Dodson, J. J., F. Colombani \& P. K. L. Ng. 1995. Phylogeographic structure in mitochondrial DNA of a South-East Asian freshwater fish, Hemibagrus nemurus (Siluroidei; Bagridae) and Pleistocene sea-level changes on the Sunda shelf. Mol. Ecol., 4: 331-346.

Donndorff, J. A. 1798. Zoologische Beytäge zur XIII. Ausgabe des Linneischen Natursystems. Dritter Band. Amphibien und Fische. Weidmann, Leipzig, $981 \mathrm{pp}$.

Donoghue, M. J. 1985. A critique of the biological species concept and recommendations for a phylogenetic alternative. Bryologist, 88: 172181.

Donovan, E. 1802-1808. The natural history of British fishes, including scientific and general descriptions of the most interesting species, and an extensive selection of accurately finished coloured plates, taken entirely from original drawings, purposely made from the specimens in a recent state, and for the most part whilst living. Donovan and Rivington, London, 1 (1802): 24 pls., 2 (1803): pls. 25-48, 3 (1804): pls. 49-72, 4 (1806): pls. 73-96, 5 (1808): pls. 97-120.

Dörfel, H.-J. 1974. Untersuchungen zur Problematik der Saiblingspopulationen (Salvelinus alpinus L.) im Überlinger See (Bodensee). Arch. Hydrobiol., Suppl., 47: 80-105.

Dorofeyeva [Dorofeeva], E. A. 1967. [Comparative morphological principles of the systematics of the east European salmon]. Vopr. Ikhtiol., 7: 317. [Russian]

- 1982. Divergence of the Sevan trout, Salmo ischan, in the light of karyological and morphological data. J. Ichthyol., 22 (1): 23-36. [translated from Vopr. Ikhtiol., 22: 36-48]

Dorofeyeva [Dorofeeva], E. A., M. Sidorovski \& N. Petrovski. 1983. [Osteological peculiarities of Ohrid trouts (Salmo letnica) with reference to their taxonomy]. Zool. Zh., 62: 1691-1700. [Russian]

Dorofeyeva, E. A., Y. A. Zinovyev, V. A. Klyukanov, Y.S. Reshetnikov, K. A. Savvaitova \& G. K. Shaposhnikova. 1980. The present state of research into the phylogeny and classification of Salmonoidei. J. Ichthyol., 20 (5): 1-20. [translated from Vopr. Ikhtiol., 20: 771-791]

Dottrens, E. 1950. Le corégone actuel du Léman. Rev. Suisse Zool., 57: 789-813.

- 1959. Systématique des corégones de l'Europe occidentale, basée sur une étude biométrique. Rev. Suisse Zool., 66: 1-66.

Dottrens, E. \& A. Quartier. 1949. Les corégones du lac de Neuchâtel - étude biométrique. Rev. Suisse Zool., 56: 689-730.

Doyle, J. J. 1992. Gene trees and species trees: molecular systematics as one-character taxonomy. Syst. Bot., 17: 144-163.

Drensky, P. 1926. [Neue und seltene Fische aus Bulgarien.]. Trav. Soc. Bulg. Sci. Nat., 12: 121150. [Bulgarian, German summary]

1928. [Die Fische der Familie Cobitidae in Bulgarien (nach der Sammlung der Königl. Natur-Historischen Museums in Sofia)]. Bull. Inst. R. Sci. Nat. Sofia, 1: 156-181. [Bulgarian, German summary] 
- 1930. Zur Kenntnis der Süsswasserfischfauna Bulgariens. Zool. Jahrb., Abt. Syst. Ökol. Geogr. Tiere, 59: 663-680.

- 1934. [Alosa bulgarica nov. sp., ein bis jetzt unbekannter Fisch aus den Flüssen der bulgarischen Küste des Schwarzen Meeres, südlich von Burgas]. Mitt. K. Naturwiss. Inst. Sofia, 7: 7987 [Bulgarian, German summary].

- 1943. [Chalcalburnus chalcoides Güld. in Bulgarien. I.] Annu. Univ. Sofia, Fac. PhysicoMath., Sér. 3, Sci. Nat., 31: 343-360, 3 pls. [Bulgarian, German summary]

Drjagin, P. A. 1932. [Belozero cisco and the acclimatization of whitefishes in lake Beloe]. Izvestiya Instituta Ozernogo i Rechnogo Rybnogo Khozyaistva, 16: 22-39

Dubalen, P.-E. 1878. Note sur un poisson mal connu du bassin de l'Adour. Bulletin de la Société de Borda, Dax, Sér. 2, 3: 157-158, 1 pl.

- 1913. Nouvelle espèce de poisson d'eau douce. Actes Soc. Linn. Bordeaux, 67 (procès verbaux): $76-79$.

Dubois, A. 1982. Les notions de genre, sous-genre et groupe d'espèces en zoologie à la lumière de la systématique évolutive. Monit. Zool. Ital., N.S., 16: 9-65.

Duhamel du Monceau, H. L. 1769-1782. Traité général des pesches et histoire des poissons qu'elles fournissent tant pour la subsistance des hommes que pour plusieurs autres usages qui ont rapport aux arts et au commerce. Paris, 4 vols.

Duméril, A. 1858a. Note sur une truite d'Algérie (Salar macrostigma, A. Dum.). C. R. Acad. Sci. Paris, 47: 160-162.

- 1858b. Note sur une truite d'Algérie (Salar macrostigma, A. Dum.). Rev. Mag. Zool. 10: 396-399, pl. 10.

- 1858c. Note sur des truites d'une espèce nouvelle (Salar macrostigma A. Dum.), récemment envoyées d'Algérie à la Société. Bull. Soc. Imp. Zool. Acclim. Paris, 5: 444-446.

- 1867. Prodrome d'une monographie des esturgeons et description des espèces de l'Amérique du Nord qui appartiennent au sous-genre Antaceus. Nvelles Arch. Mus. Nat. Hist. Paris, 3: 131-188, pls. 11-16.

- 1870. Histoire naturelle des poissons ou ichthyologie générale. Vol. 2. Ganoïdes, dipnés, lophobranches. Roret, Paris, 624 pp., 26 pls.

Dumitrescu, M., P. Bănărescu \& N. Stoica. 1957. Romanichthys valsanicola nov. gen. nov. sp. (Pisces, Percidae). Trav. Mus. Hist. Nat. Gr. Antipa, 1: 225-244.

Duncker, G. 1892. Der Elbbutt, eine Varietät der
Flunder (Pleuronectes flesus L. var. leiurus). Schrift. Naturwiss. Ver. Schleswig-Holstein, 9: 275-291.

Dybowski, B. N. 1862. Versuch einer Monographie der Cyprinoiden Livlands nebst einer synoptischen Aufzählung der europäischen Arten dieser Familie. Thesis, Dorprat. Also published in 1864 in: Arch. Naturk. Liv-, Est- und Kurlands, Dorprat, Sect. Biol., Ser. 2, 6: 133-362.

- 1869. Vorläufige Mittheilungen über die Fischfauna des Ononflusses und des Ingoda in Transbaikalien. Verh. K. K. Zool. Bot. Ges. Wien, 19: 945-958, pls. 14-18.

- 1872. Zur Kenntniss der Fischfauna des Amurgebietes. Verh. Zool. Bot. Ges. Wien, 22: 208222 .

- 1874. Die Fische des Baical-Wassersystemes. Verh. Zool. Bot. Ver. Wien, 24: 383-394.

- 1877. [Fishes of the Amur water system]. Izvestiya Sibirskogo Otdeleniya Russkogo Geograficheskogo Obshchestva, 8 (1-2): 1-29.

- 1916. Systematyka ryb: Teleostei Ostariophysi [Systematics of fishes]. Pamietnik Fizyjograficzny, Warsaw, 23 (2): 84-126, 3 pls.

Echelle, A. E. \& I. L. Kornfield (eds). 1984. Evolution of fish species flocks. University of Maine at Orono Press, Orono, 257 pp.

Economidis, P. S. 1986. Chalcalburnus belvica (Karaman, 1924) (Pisces, Cyprinidae), nouvelle combinaison taxinomique pour la population provenant du lac Petit Prespa (Macédoine, Grèce). Cybium, 10: 85-90.

- 1991. Check list of freshwater fishes of Greece. Recent status of threats and protection. Hellenic Society for the Protection of Nature, Athens, 48 pp.

- 1992. Fishes, p. 43-81. In: M. Karandinos \& L. Paraschi (eds), The red data book of threatened vertebrates of Greece. Hellenic Zoological Society \& Hellenic Ornithological Society, Athens. [Greek]

- 1995. Endangered freshwater fishes of Greece. Biol. Cons., 72: 201-211.

Economidis, P. S. \& P. M. Bănărescu. 1991. The distribution and origins of freshwater fishes in the Balkan Peninsula, especially in Greece. Int. Revue Ges. Hydrobiol., 76: 257-283.

Economidis, P. S. \& P. J. Miller. 1990. Systematics of freshwater gobies from Greece (Teleostei: Gobiidae). J. Zool., 221: 125-170.

Economidis, P. S. \& T. T. Nalbant. In press. A study of the loaches of the genera Cobitis and Sabanejewia (Pisces, Cobitidae) of Greece, with description of six new taxa. Trav. Mus. Hist. Nat. Gr. Antipa, 36 
Economidis, P. S. \& A. I. Sinis. 1982. Les poissons du système des lacs Koronia et Volvi (Macédoine, Grèce) - Considérations systématiques et zoogéographiques. Biol. Gallo-Hellenica, 9: 291316.

- 1986. Situation taxinomique et comparaisons des aloses (Pisces, Clupeidae) provenant des lacs Volvi et Vistonis (Grèce). Description d'une nouvelle sous-espèce: Alosa caspia vistonica. J. Nat. Hist., 20: 723-734.

Economou, A. N., C. Daoulas \& P. S. Economidis. 1991. Observations on the biology of Leuciscus 'svallize' in the Kremasta reservoir (Greece). Hydrobiologia, 213: 99-111.

Eichwald, E. 1831. Zoologia specialis quam expositis animalibus tum vivis, tum fossilibus potissimum rossiae in universum, et poloniae in specie, in usum lectionum publicarum in Universitate Caesarea Vilnensis. Vol. 3. Pars posterior - De spondylozois. J. Zawadzki, Vilnius, 404 pp., 1 $\mathrm{pl}$.

- 1838. Faunae Caspii maris primitiae. Bull. Soc. Imp. Nat. Moscou, 11: 125-174.

- 1871. Analecten aus der Paläontologie und Zoologie Russlands. Zur 100-jährigen Feier der Geburt Gotthelf Fisher's von Waldheim. Universitätsbuchhandlung, Moskva, 4, 23 pp.

Ekström, C. U. 1832. Fiskarne i Mörkö Skärgård. Kongl. Vetensk. Acad. Handl., Stockholm, 1831 [1832]: 270-321, pl. 2.

- 1835. Die Fische in dem Scheeren von Mörkö ... aus dem Schwedischen übersetzt und mit einigen Anmerkungen versehen von Dr. F. C. H. Creplin. Berlin, 269 pp., 6 pls.

Elvira, B. 1987. Taxonomic revision of the genus Chondrostoma Agassiz, 1835 (Pisces, Cyprinidae). Cybium, 11: 111-140.

Endler, J. A. 1989. Conceptual and other problems in speciation, p. 625-648. In: D. Otte \& J. A. Endler (eds), Speciation and its consequences. Sinauer Associates, Sunderland, Ma.

Erk'akan, F. \& M. Kuru. 1982. Systematical researchs on the Sakarya basin fishes (Pisces). Hacettepe Bull. Nat. Sci. Engineer., 11: 15-24.

Eschmeyer, W. N. 1990. Catalog of the genera of recent fishes. California Academy of Sciences, San Francisco, 697 pp.

Esipov, V. K. 1939. [On the systematics of the polar sole (Liopsetta glacialis) in the Barents and Kara Seas]. Sbornik Posvyashchionnyj Nauchnoj Deyatelnosti N. M. Knipovicha, Moskva, pp. $167-176$

Etnier, D. A. \& R. M. Bailey. 1989. Etheostoma (Ulocentra) flavum, a new darter from the
Tennessee and Cumberland River drainages Occ. Pap. Mus. Zool. Univ. Mich., 717: 1-23.

Etnier, D. A. \& W. C. Starnes. 1986. Etheostoma lynceum removed from the synonymy of $E$. zonale (Pisces, Percidae). Copeia, 1986: 832836.

Faber, F. 1828. Kort efterretning om en zoologisk rejse til det nordligste Jylland i sommeren 1827 . Tidskr. Naturv. Kjøbenh., 5: 243-256.

- 1829. Naturgeschichte der Fische Islands. Frankfurt-am-Main, $206 \mathrm{pp}$.

Fabricius, O. 1780. Fauna Groenlandica, systematice sistens animalia groenlandiae occidentalis hactenus indagata, quoad nomen specificum, triviale, vernaculumque; [. . . ]. Hafniae et Lipsiae, xvi +452 pp., 1 pl.

Fahlberg, A. 1750. Rön, angående Åhl-fiskens alstrande och förökelse. Kongl. Svenska Vetenskaps Academiens Handlingar För År 1750, 11: 194-196.

Fang, P. W. 1942a. Sur certains types peu connus de cyprinidés des collections du Muséum de Paris. Bull. Mus. Paris, Sér. 2, 14: 169-172.

- 1942b. Sur certains types peu connus de cyprinidés des collections du Muséum de Paris (II). Bull. Soc. Zool. Fr., 67: 165-169.

- 1943. Sur certains types peu connus de cyprinidés des collections du Muséum de Paris (III). Bull. Mus. Paris, Sér. 2, 15: 399-405.

Fatio, V. 1882. Faune des vertébrés de la Suisse. IV. Histoire naturelle des poissons. 1re partie. I. Anarthropterygiens, II. Physostomes Cyprinidés. Georg, Genève \& Bâle, xiv+786 pp., 5 pls.

- 1885. Les corégones de la Suisse (féras diverses) classification et conditions de frai. Recueil Zool. Suisse, Sér. 1, 2: 649-665, pls. 22-23.

- 1888a. Sur un nouveau corégone français (Coregonus Bezola) du lac du Bourget. C. R. Acad. Sci. Paris, 106: 1541-1544.

- 1888b. Un nouveau corégone français Coregonus bezola du lac du Bourget. Arch. Sci. Phys. Nat., 20: 180-185.

- 1890. Faune des vertébrés de la Suisse. V. Histoire naturelle des poissons. II. Physostomes (suite et fin), anacanthiens, chondrostéens, cyclostomes. Georg, Genève \& Bâle, lxxx+576+13 pp., 4 pls. .

Fehlmann, W. 1936. Felchen im Rhein. Der Fischer, 1936 (10): 3-8.

Ferguson, A. 1974. The genetic relationships of the coregonid fishes of Britain and Ireland indicated by electrophoretic analysis of tissue proteins. J. Fish Biol., 6: 311-315.

- 1981. Systematics of Irish charr as indicated 
by electrophoretic analysis of tissue proteins Biochem. Syst. Ecol., 9: 225-232.

- 1986. Lough Melvin - a unique fish community. Roy. Dublin Soc., Occ. Pap. Irish Sci. Technol., 1: $1-17$.

- 1989. Genetic differences among brown trout, Salmo trutta, stocks and their importance for the conservation and management of the species. Freshwat. Biol., 21: 35-46.

Ferguson, A. \& C. C. Fleming. 1983. Evolutionary and taxonomic significance of protein variation in the brown trout (Salmo trutta L.) and other salmonid fishes, p. 85-99. In: G. S. Oxford \& D. Rollinson (eds), Protein polymorphism: adaptive and taxonomic significance. Academic Press, London.

Ferguson, A., K.-J. M. Himberg \& G. Svärdson. 1978. Systematics of the Irish pollan (Coregonus pollan Thompson): an electrophoretic comparison with other Holarctic Coregoninae. J. Fish Biol., 12: 221-233.

Ferguson, A. \& F. M. Mason. 1981. Allozyme evidence for reproductively isolated sympatric populations of brown trout Salmo trutta L. in Lough Melvin, Ireland. J. Fish Biol., 18: 629642 .

Ferguson, A. \& J. B. Taggart. 1991. Genetic differentiation among the sympatric brown trout (Salmo trutta) populations of Lough Melvin, Ireland. Biol. J. Linn. Soc., 42: 221-237.

Fernholm, B. \& A. Wheeler. 1983. Linnaean fish specimens in the Swedish Museum of Natural History, Stockholm. Zool. J. Linn. Soc., 78: 199286.

Fernández-Delgado, C., J. A. Hernando, M. Herrera \& M. Bellido. 1986. Sobre el status taxonómico del género Valencia Myers, 1928 en el suroeste de Iberia. Doñana Acta Vertebrata, 13: 161-163.

Fernández-Pedrosa, V., A. Latorre \& A. González. 1996. Evidence from mtDNA RFLP analysis for the introduction of Fundulus heteroclitus to southwestern Spain. J. Fish Biol., 48: 12781282 .

Ferrero, L. 1951. Studio comparativo sulle cheppie del Mediterraneo e gli agoni delle acque interne d'Italia. Boll. Pesca Piscic. Idrobiol., 27 (N.S. 6): 108-133.

Filippi, F. de 1844. Cenni sui pesci d'acqua dolce della Lombardia. Not. Nat. Civ. Lombardia, 1: 389-406.

- 1859. Nouvelle espèce de poisson d'eau douce du Piémont. Rev. Mag. Zool., 1859: 50-51.

- 1861. Cobitis larvata De Fil. Mem. Reale Acad. Sci. Torino, Ser. 2, 19: lxxi.
- 1863. Nuove o poco note specie di animali vertebrati raccolte in um viaggio in Persia nell'estate dell'anno 1862. Arch. Zool. Anat. Fisiol., 2: 377-394

- 1865. Note d'un viaggio in Persia nel 1862 Milano, viii+396 pp.

Fisher, P. B. \& J. W. Rachlin. 1972. Karyotypic analysis of 2 sympatric species of fish, Fundulus heteroclitus L. and F. majalis. J. Fish Biol., 4: $67-71$.

Fitzinger, L. J.. 1832. Ueber die Ausarbeitung einer Fauna des Erzherzogthumes Oesterreich, nebst einer systematischen Aufzählung der in diesem Lande vorkommenden Säugethiere, Reptilien und Fische, als Prodrom einer Fauna derselben. Beiträge zur Landeskunde Oesterreich's unter der Enns, 1: 280-340.

Fitzinger, L. J. \& J. J. Heckel. 1836. Monographische Darstellung der Gattung Acipenser. Ann. Wien. Mus. Naturgesch., 1: 261-326, pls. 25-30.

Fleming, J. 1822. The philosophy of zoology. Vol 2. Constable, Edinburgh, 618 pp.

- 1828. A history of British animals exhibiting the descriptive characters and systematical arrangement of the genera and species of quadrupeds, birds, reptiles, fishes, mollusca, and radiata of the United Kingdom. Bell \& Bradfute, Edinburgh, xxiii +565 pp.

Focant, B., M. Trabelsi, F. Kartas \& J.-P. Quignard. 1992. Caractérisation biochimique de trois populations d'Atherina boyeri des milieux lagunaires méditerranéens. Rapp. Comm. Int. Mer Médit., 33: 94.

Fors[s]kål, P. 1775. Descriptiones animalium avium, amphibiorum, piscium, insectorum, vermium; quae in itinere orientali observavit. Post mortem auctoris edidit Carsten Niebuhr. Möller, Hauniae, 20+xxxiv+164 pp., 43 pls.

Forster, J. R. 1767. Specimen historiae naturalis volgensis. Phil. Trans. [R. Soc. London], 57: 312-357.

Fortunatov, M. A. 1926. [Fishes and fisheries and Lake Sevan]. Izvestiya Otdela Prikladnoi ikhtiologii i nauchno-promyslovykh issledovanii, 4: 240-246. [Russian]

- 1927. [Trouts of lake Sevan. Conspecies Salmo ischchan Kessler. Part I. Taxonomy]. Trudy Sevanskoi Ozernoi Stantsii [Rep. Lake Sevan Station], 1 (2): 1-131, 4 pls.

Fowler, H. W. 1972. A catalog of world fishes (XVII). Quart. J. Taiwan Mus., 25: 157-198.

- 1973. A catalog of world fishes (XIX). Quart. J. Taiwan Mus., 26: 217-346.

- 1974. A catalog of world fishes (XX). Quart. J. Taiwan Mus., 27: 1-132. 
- 1976. A catalog of world fishes (XIV). Quart. J. Taiwan Mus., 29: 1-110.

- 1977. A catalog of world fishes (XXVI). Quart. J. Taiwan Mus., 30: 1-88.

Frankiewicz, P. 1985. [Golden loach, Cobitis aurata (Fil.) in the drainage basin of the Odra River]. [Polish, English summary]. Przeglad Zool., 29: 331-337.

Freidenfeldt, T. 1928. Om de viktigaste sikformerna och om sikfisket i Vänern. Ny Svensk Fiskeritidskrift, 37 (10): 91-93.

Fridriksson, A. 1939. Um murtuna i Thingvallavatni med hlidsjón af ödrum silungstegundum í vatninu [On the murta in Thingvellir Lake in relation to the char and trout in that lake]. Náttúrufraedingurinn, 9: 1-30.

Fricke, R. 1995. Types in the fish collection of the Staatliches Museum für Naturkunde in Stuttgart. Part 3. Types of fishes described in 1850-1994. Stuttgarter Beitr. Naturk., Ser. A, Biol., 524: 1-30.

Friend, G. F. 1956. A new sub-species of charr from Loch Eck. Glasgow Naturalist, 17 [1955 (1956)]: 219-220.

- 1959. Subspeciation in British charrs. Syst. Ass. Publ., 3: 121-129.

Frost, D. R. \& D. M. Hillis. 1990. Species in concept and practice: herpetological applications. Herpetologica, 46: 87-104.

Frost, W. E. 1966. Breeding habits of Windermere charr, Salvelinus willughbii (Günther), and their bearing on speciation of these fish. Proc. R. Soc. Lond., Ser. B, Biol. Sci., 163: 232-284.

Fuhrmann, O. 1903. [... l'omble chevalier des zones profondes, Salvelinus salvelinus var. profundus ...]. Arch. Sci. Phys. Nat., (4) 16: 331332 [also in: Bull. Soc. Neuchâtel. Sci. Nat., 31 (1903): 382-384].

Furnestin, J. \& A. Vincent. 1958. Variation des branchiospines dans le genre Alosa. C. R. Acad. Sci. Paris, 246: 3376-3379.

Gaimard, J. P. 1842. Voyage en Islande et au Groenland. Publié par ordre du roi sous la direction de M. Paul Gaimard. Atlas zoologique, médical et géographique. Bertrand, Paris.

Gamper, M. \& J. Suter. 1982. Postglaziale Klimageschichte der Schweizer Alpen. Geographica Helvetica, 37: 105-114.

Gandolfi, G., A. Marconato \& P. Torricelli. 1985. Posizione sistematica e biologia di un ghiozzo delle acque dolci italiane: Orsinigobius (gen. nov.) punctatissimus (Canestrini, 1864) (Pisces, Gobiidae). Boll. Mus. Civ. Stor. Nat. Verona, 12: $367-380$.

Gandolfi, G. \& P. Tongiorgi. 1973. Considerazioni sulla posizione systematica delle due specie di ghiozzi (Osteichthyes, Gobiidae) presenti nelle acque dolci italiane. Ateneo Parmense, Acta Naturalia, 9: 261-265.

- 1974. Taxonomic position, distribution and biology of the gobies present in the Italian fresh-waters, Padogobius martensi (Günther) and Gobius nigricans Canestrini (Osteichthyes, Gobiidae). Ann. Mus. Civ. Stor. Nat. Genova, 80: $92-118$.

Gandolfi, G., P. Torricelli \& A. Cau. 1982. Osservazioni sulla biologia del ghiozzetto cenerino, Pomatoschistus canestrinii (Ninni) (Osteichthyes, Gobiidae). Nova Thalassia, 5: 97-123.

Gandolfi, G. \& S. Zerunian. 1987. I pesci delle acque interne italiane: aggiornamento e considerazioni critiche sulla sistematica e la distribuzione. Atti Soc. Ital. Sci. Nat. Mus. Civ. Stor. Nat. Milano, 128: 3-56.

- 1991. I pesci delle acque interne italiane: chiarimenti sulle critiche ad un nostro recente contributo. Atti Soc. Ital. Sci. Nat. Mus. Civ. Stor. Nat. Milano, 131: 293-307.

Gandolfi, G., S. Zerunian, P. Torricelli \& A. Marconato. 1991. I pesci delle acque interne italiane. Istituto Poligrafico e Zecca dello Stato, Roma, xvi+617 pp.

Garman, S. 1890. Silurus (Parasilurus) aristotelis, Glanis aristotelis Ag., 1856, Pr. Amer. Acad., 333 (named). Bull. Essex Inst., 22 (1-3): 8-11.

Gasowska, M. 1960. Genus Coregonus L. discussed in connection with a new systematic feature that of shape and proportion of os maxillare and os supramaxillare. Annales Zoologici, 18: 471-490, pls. 21-32.

- 1964. Coregonid classification discussed on the basis of Coregonus pollan Thompson from Lough Neagh (Northern Ireland). Annales Zoologici, 22: 413-419.

- 1965. A preliminary taxonomic revision of the British whitefish with especial reference to the gwyniad of Llyn Tegid (Bala). Proc. Zool. Soc. London, 145: 1-8.

- 1979. Osteological revision of the genus Phoxinus Raf., sensu Bănărescu 1964, with description of a new genus, Parchrosomus gen. n. (Pisces, Cyprinidae). Annal. Zool., 34: 371-413, 2 pls.

Gatti, M. A. 1896. Il Chondrostoma genei, Bp. nella provincia di Teramo. Boll. Soc. Romana Stud. Zool., 5: 211-217.

Geiger, W. 1961. Die Forellenschläge der verschiedenen Gewässer unseres Landes. Schweiz. Fischerei-Zeitung, 69: 300-302, 326-329.

Geldiay, R. \& S. Balik. 1988. Türkiye tatlisu balik- 
lari (ders kitabi). Ege Úniversitesi Fen Fakültesi Kitaplar Serisi, Ege Úniversiesi Basimevi, Izmir, 97: i-xviii + 1-520.

Geoffroy Saint-Hilaire, E. 1808. Poissons du Nil, pls. 1-17. In: Description de l'Egypte ou recueil des observations et des recherches qui ont été faites en Egypte pendant l'expédition de l'armée française. Planches. Histoire naturelle. Tome premier. Panckoucke, Paris.

- 1817. Poissons [de la Mer Rouge et de la Méditerranée], pls. 18-27. In: Description de l'Egypte ou recueil des observations et des recherches qui ont été faites en Egypte pendant l'expédition de l'armée française. Planches. Histoire naturelle. Tome premier, Panckroucke, Paris.

Georgi, J. G. 1775. Bemerkungen einer Reise im Russischen Reich in den Jahren 1773 und 1774. Zweyter Band. Kayserlische Akademie der Wissenschaften, St Petersburg, pp. 507-920.

Gerdeaux, D. 1993. Etat actuel des connaissances sur le corégone du Léman. Les cahiers de la pêche, Office Fédéral de l'Environnement, des Forêts et du Paysage, Berne, 51: 21-28.

Gesner, C. 1556. De piscibus et aquatilibus omnibus libelli III novi. I. Scholia et emendationed in Halieuticon P. Ovidii Nasonis. II. Aquatilium animantium enumeratio juxta Plinium, emendata et explicata, serie literarum. III. Eorundem nomenclator Germanicus longe copiossissimus. Et alia quaedam ad piscium historiam pertinentia. Froschover, Tiguri [Zürich], vi+280 pp., 18 pls.

- 1558. Historiae animalium. Liber III qui est de piscium et aquatilium animantium natura. Froschoverum, Tiguri, 1297 pp.

- 1560. Nomenclator aquatilium animantium. Icones animalium aquatilium in mari \& dulcibus aquis degentium, plus quam Dcc. cum nomenclaturis singulorum Latinis, Greçis, Italicis, Hispanicis, Gallicis, Germanis, Anglicis, alijsq; interdum per certos ordines digestae. C. Froschoverus, Tiguri [Zürich], 374 pp.

- 1563. Fischbuch, das ist ein kurtze doch volkomne Beschreybung aller Fischen so in dem Meer und süssen Wasseren, Seen, Flïssen oder anderen Bächen ir Wohnung habend, sampt irer waaren Conterfactur [...]. Froschower, Zürych, $\mathrm{ix}+404 \mathrm{pp}$. [also 1575,1598 and 1670 editions]

- 1604. Historiae animalium. Liber IV. Qui est de piscium \& aquatilium animantium natura. Cambieri, Francofurti, $1052+38$ pp.

Ghiselin, M. T. 1975. A radical solution to the species problem. Syst. Zool., 23: 536-544.

Gianferrari, L. 1927. Diagnosi preliminare di due nuove specie ittiche di Rodi. Atti Soc. Ital. Sci. Nat. Mus. Civ. Stor. Nat. Milano, 66: 123-125. - 1929. Ricerche faunistiche nelle isole italiana dell'Egeo. Pesci. Arch. Zool. Ital., 13: 37-44.

- 1932. Un nuovo Cyprinodon sirtico. Atti Soc. Ital. Sci. Nat. Mus. Civ. Stor. Nat. Milano, 71: 214-216.

Gill, T. N. 1896. Lipophrys a substitute for Pholis. Amer. Nat., 30: 498.

- 1903. On some fish genera of the first edition of Cuvier's Règne Animal and Oken's names. Proc. U. S. Natn. Mus., 26: 965-967.

Girard, C. 1858. Fishes. In: General report upon the zoology of the several Pacific railroad routes, 1857. Explorations and surveys for a railroad route from the Mississippi River to the Pacific Ocean. War Department, Washington, 10 (4): $1-400$

Gisler, N. 1753. Anmärkingar om sik-fisket uti Norrdländska älfver och Skärgårdar. K. Wetensk. Akad. Handl., 1753: 195-209.

Gistel, J. \& C. Bromme. 1850. Handbuch der Naturgeschichte aller drei Reiche, für Lehrer und Lernende, für Schule und Haus. Hoffmann, Stuttgart, 626 pp.

Giuffra, E., L. Bernatchez \& R. Guyomard. 1994. Mitochondrial control region and protein coding genes sequence variation among phenotypic forms of brown trout Salmo trutta from northern Italy. Molec. Ecol., 3: 161-171.

Giuffra, E., R. Guyomard \& G. Forneris. 1996. Phylogenetic relationships and introgression patterns between incipient parapatric species of Italian brown trout (Salmo trutta L. complex). Molec. Ecol., 5: 207-220.

Gmelin, C. C. 1818. Gemeinnuitzige systematische Naturgeschichte der Fische für gebildete Leser nach dem Linnéischen Natursystem entworfen. Neydeck, Mannheim, $\mathrm{x}+10+384+22$ pp., 113 pls.

Gmelin, J. F. 1788. Caroli a Linné systema naturae per regna tria naturae, secundum classes, ordines, genera, species cum characteribus, differentiis, synonymis, locis. Beer, Lipsiae, vol. 1, pars 3: 1033-2224.

- 1793. Caroli a Linné systema naturae per regna tria naturae, secundum classes, ordines, genera, species cum characteribus, differentiis, synonymis, locis. Beer, Lipsiae, vol. 3: 1-476, 3 pls.

Gmelin, S. G. 1774. Reise durch Russland, zur Untersuchung der drey Natur-Reiche. Dritter Theil. Reise durch das nordliche Persien, in den Jahren 1770, 1771, bis im April 1772. Kayserliche Academie der Wissenschaften, St. 
Petersburg, 508 pp.

- 1784. Reise durch Russland, zur Untersuchung der drey Natur-Reiche. Vierter Theil. Reise von Astrachan nach Zarizyn und von da durch die kumanische Steppe, über Mosdok zurük; imgleichen zweyte Persische Reise: in den Jahren 1772 und 1773 bis im Frühling 1774. Nebst dem Leben des Verfassers. Kayserliche Academie der Wissenschaften, St. Petersburg, xxvi+218 pp., 18 pls.

Goldfuss, G. A. 1820. Handbuch der Zoologie. Zweite Abtheilung. Schrag, Nürnberg, 510 pp., 4 pls.

Goll, H. 1883. Contribution à l'histoire naturelle des corégones du lac de Neuchâtel. Arch. Sci. Phys. Nat. Genève, Sér. 3, 10: 341-343.

Gomez Caruana, F., S. Peiro Gomez \& A. Sanchez Artal. 1984. Descripcion de una nueva especie de pez continental iberico, Valencia lozanoi n. sp. (Pisces, Cyprinodontidae). Bol. Estación Centr. Ecol., 13: 67-74.

Gratzianow, V. 1904. [Beitrag zur Ichthyofauna des russischen Nordens]. Zapiski Geograficheskogo Obshchestva po Obshchei Geografii, 41: 171-249, 2 pls.

- 1906. Die Neunaugen des russischen Reiches]. Dnevn. Zool. Otd. Obshch. Lyub. Jest. Moskva, 3 (7-8): 18. [Russian]

- 1907a. Uebersicht der Süsswassercottiden des russischen Reiches. Zool. Anz., 31: 654-660.

- 1907b. [Versuch einer Übersicht der Fische des Russischen Reiches in systematiker und geographischer Hinsicht]. Trudy Otdela Ikhtiologii, 4: $\mathrm{xxx}+567 \mathrm{pp}$.

Gray, J. E. 1851. List of the specimens of fish in the collection of British Museum. Part I Chondropterygii. British Museum, London, 160 pp., 2 pls.

Grib, A. V. \& M. F. Vernidub. 1935. [Taxonomy and biology of the bream Abramis brama L. from the eastern part of the Gulf of Finland]. Uchenye Zapiski Leningradskogo Universiteta, Seriya Biologicheskaya, 1: 105-120.

Griffiths, G. C. D. 1974. On the foundations of biological systematics. Acta Biotheoretica, 23: 85-131.

Grimm, O. 1896. [Der Fischfang im Kaspi-See und in der Wolga]. St. Petersburg, 154 pp. [Russian]

- 1901. [Herrings of the Azov Sea]. Vestnik Rybopromyshlennosti, St. Petersburg, 16 (2): 57-70 [Russian] [also in Nikolskogo Rybovodnogo Zavoda, St. Petersburg, 1901 (4): 17-31]

Gronovius, J. F. 1746. Pisces Belgii seu piscium in Belgio natantium, et a se observatorum catalogus. Acta Societatis Regiae Scientiarum Upsaliensis, 1.741 [1746]: 67-76.

- 1748. Pisces Belgii descripti. Acta Societatis Regiae Scientiarum Upsaliensis, 1742 [1748] 79-107, pl. 3.

- 1751. Perca pinnis dorsalibus distinctis, secundae radiorum quatuordecim. Acta Societatis Regiae Scientiarum. Upsaliensis, 1750 [1751]: $39-42$, pl. 4.

Gronovius, L. T. 1754-1756. Museum ichthyologicum, sistens piscium indigenorum e quorundam exoticorum, qui in museuo Laurentii Theodori Gronovii, adservantur, descriptiones, ordine systematico; accedunt nonnullorum exoticorum piscium icones, aeri incisae. Haak, Lugduni Batavorum [Leiden], vol. 1 (1754): 70 pp., pls. 1-4, vol. 2 (1756): 88 pp., pls. 5-7.

- 1763-1764. Zoophylacii Gronoviani fasciculus primus exhibens animalia quadrupeda, amphibia atque pisces, quae in museo suo adservat, rite examinavit, systematice disposuit, descripsit atque iconibus illustravit Laur. Theod. Gronovius. Lugduni Batavorum [Leiden], 1 (1763): 1-140, pls. 1-17; 2 (1764): 141-236.

Gronow, L. T. 1854. Catalogue of fish collected and described by Laurence Theodore Gronow, now in the British Museum [edited by E. J. Gray]. British Museum, London, 196 pp.

Gross, H. P. 1979. Geographic variation in European ninespine sticklebacks, Pungitius pungitius. Copeia, 1979: 405-412.

Grossinger, J. B. 1794. Universa historia physica regni Hungariae secundum tria naturae regna digesta. Tomus III. Ichthyologia, sive historia piscium, et amphibiorum. Weber, Posonii \& Comaromii, xiv $+400+8$ pp.

Grupče [Gruptche], R. \& A. Dimovski. 1984. [Recherches comparatives sur les caractères méristiques et morphométriques de Scardinius erythrophthalmus scardafa (Bonaparte) des lacs d'Ohrid et Skadar et Scardinius erythrophthalmus dojranensis Karaman du lac de Döran]. Acta Mus. Mac. Scient. Nat., 17: 81-101. [Macedonian, French summary]

Gueldenstaedt, A. I. 1772. Salmo leucichthys et Cyprinus chalcoides descripti. Nov. Comment. Acad. Petropol., 16 (1771 [1772]): 531-547, pl. 16.

- [Güldenstaedt] 1778. Cyprinus barbus et Cyprinus capito descripti. Acta Acad. Imp. Sci. Petropol., 2: 239-260, pls. 8-10.

Guichenot, A. 1850. Histoire naturelle des reptiles et des poissons. In: Exploration scientifique de l'Algérie pendant les années 1840, 1841, 1842. Sciences Physiques, Zoologie, Volume V. 
Imprimerie Nationale, Paris, iv+146 pp., 8 pls. - 1859. Notice sur deux espèces nouvelles de poissons du genre Cyprinodon. Rev. Mag. Zool. Pure Appl., Sér. 2, 11: 377-380.

Gulia, G. 1861. Tentamen ichthyologiae melitensis sistens methodo naturali stirpium objectis nonnulis observationibus genera ac species, a recentioribus de re zoologica scriptoribus admissas piscium insularum Melitae. Typographia Anglica, Melitae, $71 \mathrm{pp}$.

Gundrizer, A. N. 1967. K izuchenyu ryb Tuvy. Uchenye Zapisky Tomskogo Universiteta, 53.

- 1979. [Ecomorphological feature of large-mouth gugdeon Gobio gobio magnicapitata Gundriser, 1967 from the Enisei basin], p. 9-14. In: Novye Dannye o Faune i Flore Sibiri. Izd. Tomsk. Univ., Tomsk.

Günther, A. 1857. Über einen neuen Fisch des Neckars. Jahresheft. Ver. Vaterl. Naturk. Wuirttemberg, 13: 50-54, pl. 2.

- 1861a (14 December). Catalogue of the acanthopterygian fishes in the collection of the British Museum. British Museum, London, $\mathrm{xxv}+586 \mathrm{pp}$.

- 1861b. On the British species of Mugil, or grey mullets. Ann. Mag. Nat. Hist., Ser. 3, 7: 345352.

- 1862a. Contribution to the knowledge of the British charrs. Proc. Zool. Soc. London, 1862: $37-54$, pls. $5-7$.

- 1862b. Catalogue of the Acanthopterygii Pharyngognathii and Anacanthini in the collection of the British Museum. British Museum, London, xxii+534 pp.

- 1863a. Contribution to the knowledge of the British charrs. Part II. Proc. Zool. Soc. London, 1863: 6-16, pls. 1-2.

- 1863b. On the European species of the genus Labrax. Ann. Mag. Nat. Hist., Ser. 3, 12: 174175 .

- 1865a. Contribution to the knowledge of the British charrs. Part III. Proc. Zool. Soc. London, 1865: 698-699, pl. 40.

- 1865b. Salmo cambricus. Ann. Mag. Nat. Hist., Ser. 3, 15: 75 .

- 1866. Catalogue of the fishes in the British Museum. 6. British Museum, London, $x v+368$ pp.

- 1868. Catalogue of the fishes in the British Museum. 7. British Museum, London, $\mathrm{xx}+512$ pp.

- 1870. Catalogue of the fishes in the British Museum. 8. British Museum, London, xxv+549 pp.

- 1876. Remarks on fishes, with descriptions of new species in the British Museum, chiefly from the Southern Seas. Ann. Mag. Nat. Hist., Ser. 4, 17: 389-402.

Guyomard, R. 1989. Diversité génétique de la truite commune. Bull. Fr. Pêche Piscic., 314: 118-135.

- 1994. La diversité génétique des populations de saumon atlantique, p. 141-151. In: J. Guégen \& P. Prouzet (eds), Le saumon atlantique en France, aménagement et gestion de la ressource. IFREMER \& Bordas, Paris, 330 pp.

Guyomard, R., C. Grévisse, F. X. Oury \& P. Davaine. 1984. Evolution de la variabilité génétique inter et intra-populations de populations de salmonidés issues de mêmes pool géniques. Can. J. Fish. Aquat. Sci., 41: 1024-1029.

Haack, H. 1882. Einiges aus der Praxis. Circulare Deutschen Fischerei-Vereins, Jahre 1881, pp. 126-127.

Hadzisce, S. 1960. Zur Kenntnis der Gattung Salmothymus Berg zugleich ein Beitrag zur Systematik der Familie der Salmoniden (Pisces) (Vorläufige Mitteilung). Izdanija Zavoda za Ribarstvo NRM (Skopje), 3 (2): 39-56.

- 1961. Zur Kenntnis des Salmothymus ohridanus (Steindachner) (Pisces, Salmonidae). Verh. Intern. Ver. Limnol., 14: 785-791.

Haempel, O. 1924. Studien am Seesaibling mehrerer österreichischer Alpenseen. Verh. Intern. Ver. Limnol., 2: 129-135.

- 1930. Fischereibiologie der Alpenseen. Vol. 10 in: A. Thienemann (ed.), Die Binnengewässer - Einzeldarstellungen aus der Limnologie und ihren Nachbargebieten. Schweizerbart, Stuttgart, $259 \mathrm{pp}$.

Haglund, T. R., D. G. Buth \& R. Lawson. 1992a. Allozyme variation and phylogenetic relationships of Asian, North American, and European populations of the threespine stickleback, Gasterosteus aculeatus. Copeia, 1992: 432-443.

- 1992b. Allozyme variation and phylogenetic relationships of Asian, North American, and European populations of the ninespine stickleback, Pungitius pungitius, p. 438-452. In: R. L. Mayden (ed.), Systematics, historical ecology, and North American freshwater fishes. Stanford University Press, Stanford.

Hamilton, K. E., A. Ferguson, J. B. Taggart, T Tomasson, A. Walker \& E. Fahy. 1989. Postglacial colonisation of brown trout, Salmo trutta L.: Ldh-5 as phylogeographic marker locus. J. Fish Biol., 35: 651-664.

Hammer, C. 1775. Forsog til en norsk naturhistorie. I deel. Sander, Kobenhavn, 13+248 pp. Hancock, J. 1830. Observations on the mullets of 
the coast of Guiana, and the grey mullet of the British coast: with incidental remarks on the airbladder and stomach in fishes. Quart. J. Litt. Sci. Arts, London, 1: 125-139.

Hankó, B. 1922. Explorationes zoologica ab E. Csiki in Albania peractae. I. Fische. Magyar Tudom. Ak. Balk.-Kutat. Tudom. Ered., Budapest, 1: 1-6, $1 \mathrm{pl}$.

- 1923. Über den Hundfisch Umbra lacustris (Grossinger) (=U. krameri Fitz.). Zool. Anz., 57: 89-95.

- 1924. Fische aus Klein-Asien. Ann. Hist. Nat. Mus. Natn. Hungar., 21: 137-158, pl. 3.

Hantke, R. 1978-1983. Eiszeitalter. Otto, Thun, 1 (1978): 468 pp.; 2 (1980): 703 pp.; 3 (1983): 730 pp.

- 1993. Flussgeschichte Mitteleuropas. Skizzen zu einer Erd-, Vegetations- und Klimageschichte der letzten 40 Millionen Jahre. Enke, Stuttgart, $\mathrm{xx}+459 \mathrm{pp}$.

Hardin, S. 1862. Om Venerns laxarter. Öfversigt af Kongl. Vetenskaps-Akademiens Förhandlingar, 18 (1861 [1862]): 381-384.

Hartmann, G. L. 1827. Helvetische Ichthyologie, oder ausführliche Naturgeschichte der in der Schweiz sich vorfindenden Fische. Orell and Füssli, Zurich, xii+240 pp.

Hartmann, J. 1994. Hilft der Saiblingsbesatz den Saiblingsarten des Bodensee ? Österreichs Fischerei, 47: 46-50.

Hasselqvist, F. 1757. Iter Palestinum, eller resa til Heliga Landet förrättad infrän år 1749 til 1752, med beskrifningar, rön, anmärkningar, öfver de märkvärdigaste naturalier, på Hennes Kongl. Maj.'ts befallning utgifven af Carl Linnaeus. Salvii, Stockholm, xiv +619 pp.

Hatta, S. 1911. Ueber die Variabilität und den Dimorphismus des japanischen Neunauges. Annot. Zool. Japon., 7: 263-270, pl. 9.

Heckel, J. J. 1836. Úber einige neue, oder nicht gehörig unterschiedene Cyprinen, nebst einer systematischen Darstellung der europäischen Gattungen dieser Gruppe. Ann. Wiener Mus. Naturgesch., 1: 221-234, pls. 19-21.

- 1837. Ichthyologische Beiträge zu den Familien der Cottoiden, Scorpaenoiden, Gobioiden und Cyprinoiden. Ann. Wien. Mus. Naturgesch., 2: 143-164, pls. 8-9. [publication date follows Paxton et al., 1989: 450]

- 1841a. Ueber eine neue Gattung (Genus) von Süsswasserfischen in Europa. Bull. Sci. Acad. Imp. Sci. St.-Pétersbourg, 8: 384.

- 1841b. On a new European genus of freshwater fish. Ann. Mag. Nat. Hist., Ser. 1, 7: 522-523.

- 1842. [... Beschreibung eines ... in Dalma- tien im Herbst 1840 entdeckten neuen Fischen ...]. Amtlicher Bericht über die neunzehnte Versammlung deutscher Naturforscher und Aerzte zu Braunschweig im September 1841, pp. 73-74.

- 1843. Ichthyologie, p. 991-1099. In: J. Russegger, Reisen in Europa, Asien und Afrika mit besonderer Rücksicht auf die naturwissenschaftlichen Verhältnisse der betreffenden Länder, unternommen in den Jahren 1835 bis 1841. Erster Band. Reise in Griechenland, Unteregypten, im nördlichen Syrien und südöstlichen Kleinasien. Schweizerbart, Stuttgart.

- 1848. U̇ber die Fische Ungarns. Ber. Mitt. Freund. Naturwiss. Wien, 3: 194-195.

- 1849. Nachtrag zur Charakteristik und Classifikation der Cyprineen-Gattungen, p. 273290. In: J. Russegger, Reisen in Europa, Asien und Afrika, mit besonderer Rücksicht auf die naturwissenschaftlichen Verhältnisse der betreffenden Länder, unternommen in den Jahren 1835 bis 1841. Zweiter Band. Reise in Egypten, Nubien und Ost-Sudan. Dritter Theil. Zweite Reise duch Nubien und Egypten. Naturhistorischer Anhang. Schweizerbart, Stuttgart.

- 1851a. Ueber die in den Seen Oberösterreichs vorkommenden Fische. Sitz-Ber. Österr. Akad. Wiss., Math.-Naturw. Kl., Abt. 1, 6: 145-149.

- 1851b. Bericht einer auf Kosten der kais. Akademie der Wissenschaften durch Oberösterreich nach Salzburg, München, Innsbruck, Botzen, Verona, Padua, Venedig und Triest unternommenen Reise. Sitz-Ber. Akad. Wiss. Wien, Math.-Naturwiss. Cl., 7: 281-333.

- 1851c. Stör-Arten der Lagunen bei Venedig. Sitz-Ber. Math. Naturwiss. Cl. Kaiserl. Akad. Wiss., Abt. 1, 7: 547-563, pls. 23-24.

- 1852a. Beiträge zu den Gattungen Salmo, Fario, Salar, Coregonus, Chondrostoma und Telestes. Sitz-Ber. Akad. Wiss. Wien, Math.Natwiss. Cl., 8: 347-391, pls. 7-14.

- 1852b. Über die zu den Gattungen Idus, Leuciscus und Squalius gehörigen Cyprinen. Sitz-Ber. Akad. Wiss. Wien, Math.-Natwiss. Cl., 9: 49-123, pls. 6-13.

- 1852c. Verzeichniss der Fische des Donaugebietes in der ganzen Ausdehnung des Österreichischen Kaiserstaates. Verh. Zool. Bot. Ges. Wien, 2 (1853 [1852]): 28-33

Heckel, J. J. \& R. Kner. 1858. Die Süsswasserfische der Österreichischen Monarchie mit Rücksicht auf der angrenzenden Länder. Engelmann, Leipzig, xii +388 .

Heese, T. 1992. Systematics of Polish populations of European whitefish, Coregonus lavaretus (L.), based on skull osteology. Polsk. Arch. 
Hydrobiol., 39: 491-500

Heincke, F. 1880. Die Gobiidae und Syngnathidae der Ostsee nebst biologischen Bemerkungen. Arch. Naturgesch., 46 (1): 301-354.

Hensel, K. 1971. Some notes on the systematic status of Carassius auratus gibelio (Bloch, 1782) with further record of this fish from the Danube River in Czechoslovakia. Vestn. Českoslov. Společ. Zool., 35: 186-198, pls. 1-4.

- 1978. Morphology of lateral-line canal system of the genera Abramis, Blicca and Vimba with regards to their ecology and systematic position. Acta Univ. Carol., Biol., 1975-1976 [1978]: 105-153.

Hensel, K. \& J. Holčík. 1983. On the identity of Hucho hucho and Hucho taimen (Pisces, Salmonidae). Folia Zool., 32: 67-83.

Hermann, J. 1804. Observationes zoologicae quibus novae complures, aliaeque animalium species describuntur et illustrantur. Opus posthumum edidit F. L. Hammer. Pars prior. Observationum quatuor centurias continens. Koenig, Argentorati [Strasbourg] \& Paris, viii + 332 pp

Hertel, R. 1978. U̇ber die "Ichthyographie der Elbe" des Johannes Kentmann - eine Studie über die ältesten sächsischen Fischfaunen (Pisces). Zool. Abhandl. Dresden, 35: 75-100, pls. $1-13$.

Hervey, G. F. \& J. Hems. 1968. The goldfish Faber \& Faber, London, 271 pp.

Heuschmann, O. 1957. Die Weissfische (Cyprinidae), p. 23-199. In: R. Demoll, H. N. Maier \& H. H. Wundisch. 1957-1962. Handbuch der Binnenfischerei Mitteleuropas. Band III B. Schweizerbart, Stuttgart, 352 pp., 8 pls.

Hewitt, G. M. 1989. The subdivision of species by contact zones, p. 85-110. In: D. Otte \& J. A. Endler (eds), Speciation and its consequences. Sinauer Associates, Sunderland, MA.

Hildegarde de Pinguia [Hildegard vom Bingen]. 1533. Physica S. Hildegardis elementorum, fluminum aliquot Germaniae, metallorum, leguminum, fructuum et herbarum, arborum et arbustorum, piscium, denique, volatilium et animantium terrae naturas et operationes, libris IV, mirabili experientia posteritati tradens. Argentorati [Strasbourg].

Hindar, K. \& B. Jonsson. 1982. Habitat and food segregation of dwarf and normal arctic charr (Salvelinus alpinus) from Vangsvanet Lake, western Norway. Can. J. Fish. Aquat. Sci., 39: 1030-1045.

- 1993. Ecological polymorphism in Arctic charr. Biol. J. Linn. Soc., 48: 63-74.

Hindar, K., N. Ryman \& G. Stahl. 1986. Genetic differentiation among local populations and morphotypes of Arctic charr, Salvelinus alpinus Biol. J. Linn. Soc., 27: 269-285.

Hoestlandt, H. (ed.). 1991. The freshwater fishes of Europe. Vol. 2. Clupeidae, Anguillidae. Aula, Wiesbaden, $447 \mathrm{pp}$.

Hoffmann, R. C. 1995. Environmental change and the culture of common carp in medieval Europe. Guelph Ichthyol. Rev., 3: 57-85.

- 1996. Economic development and aquatic ecosystems in medieval Europe. American Historical Review, 101: 631-669.

Holandre, J. 1837a. Notice sur plusieurs espèces non décrites de poissons du genre cyprin, observées dans le département de la Moselle; communiquée à la Société d'Histoire naturelle de Metz, le 27 avril 1837. 4 pp.

- 1837b. Faune du département de la Moselle. Thiel, Metz, viii $+282+59$ pp. [publication date given as 1836, apparently published in 1837, after previous paper; see p. viii and Holandre, 1837a: 1, 4]

Holčík, J. 1959. [Golchik, Y.] [Systematic status of the European bitterling, Rhodeus sericeus amarus (Bloch, 1783)]. Vopr. Ikhtiol., 13: 3950. [Russian]

- 1963. Notes on the Czechoslovakian lampreys with description of Lampetra (Eudontomyzon) vladykovi (Oliva et Zanandrea), 1959. Vestn. Českoslov. Společ. Zool., 27: 51-61.

- 1969. A note on the occurence and taxonomy of brown trout - Salmo trutta Linnaeus, 1758 in the Danube River. Acta Soc. Zool. Bohemoslov. 33: 223-228.

- 1986. (ed.) The freshwater fishes of Europe. Vol. 1, Part I. Petromyzontiformes. Aula, Wiesbaden, $313 \mathrm{pp}$.

- 1989. (ed.) The freshwater fishes of Europe. Vol. 1, Part II. General introduction to fishes - Acipenseriformes. Aula, Wiesbaden, 469 pp.

- 1995. Geographische Verbreitung von Neunaugen (Petromyzontiformes) in der mittleren und unteren Donau (zwischen Österreich und dem Schwarzen Meer). Fischökologie, 8: 23-30

Holčík, J. \& J. J. Duyvené de Wit. 1964. Systematic status of the bitterlings from Asia Minor and notes on the geographical variability of Rhodeus sericeus (Pallas, 1776) in the area of its distribution. Ztschr. Wiss. Zool., 169: 396-412.

Holčík, J. \& K. Hensel. 1974. A new species of Gymnocephalus (Pisces: Percidae) from the Danube, with remarks on the genus. Copeia, 1974: 471-486.

Holčík, J., K. Hensel, J. Nieslanik \& L. Skácel 1988. The Eurasian huche. Junk, Dordrecht, 239 pp.

Holčík, J. \& L. Jedlička. 1994. Geographical vari- 
ation of some taxonomically important characters in fishes: the case of the bitterling Rhodeus sericeus. Env. Biol. Fish., 41: 147-170.

Holčík, J., V. Mišík, I. Bastl \& A. Kirka. 1965. [Ichthyological investigation of the Carpathian Mountains. 3. Fishes of the Orava Valley reservoir and its tributaries]. Acta Rer. Nat. Mus. Natn. Slov., 11: 93-139.

Holčík, J. \& Š. Nagy. 1987. Burbot (Lota lota) from the river Turiec. Folia Zool., 36: 85-96.

Holčík, J. \& K. Pivnička. 1969. Notes on a collection of fishes from Mongolia with description of Microphysogobio tungtingensis anudarini ssp. $\mathrm{n}$. and discovery of some new or little know fishes. Annot. Zool. Bot., 56: 1-25.

Holčík, J. \& V. Skořepa. 1971. Revision of the roach, Rutilus rutilus (Linnaeus, 1758), with regard to its subspecies. Annot. Zool. Bot., 64: $1-60,9$ pls.

Hollberg, L. 1822. Beskrifning öfver Bohuslänske Fiskarne uigifven af Kongl. Vettenskaps och Vitterhs. Samhället i Götheborg. III Haftet. Götheborgs Kongl. Wettenskaps och Witterhets Samhållets nya Handlingar, 5: 1-70.

Holly, M. 1929. Zoologische Forschungsreise nach den Jonischen Inseln und dem Peloponnes von Max Beier (Wien). VI. Teil Pisces. Sitz-Ber. Akad. Wiss. Wien, Math.-Naturwiss. Kl., Abt. 1, 138: 487-489.

Howes, G. J. 1981. Anatomy and phylogeny of the Chinese major carps Ctenopharyngodon Steind., 1866 and Hypophthalmichthys Blkr., 1860. Bull. Brit. Mus. Nat. Hist., Zool., 41: 152.

- 1985. A revised synonymy of the minnow genus Phoxinus Rafinesque, 1820 (Teleostei: Cyprinidae) with comments on its relationships and distribution. Bull. Br. Mus. Nat. Hist., Zool., 48: 57-74.

- 1987. The phylogenetic position of the Yugoslavian cyprinid fish genus Aulopyge Heckel, 1841, with an appraisal of the genus Barbus Cuvier \& Cloquet, 1816 and the subfamily Cyprininae. Bull. Br. Mus. Nat. Hist., Zool., 52: 165-196.

- 1991. Systematics and biogeography: an overview, p. 1-33. In: I. J. Winfield \& J. S. Nelson (eds), Cyprinid fishes - systematics, biology and exploitation. Chapman \& Hall, London.

Hoyer, H. \& W. Michalski. 1915. Das Lymphgefässsystem bei Forellenembryonen (Salmo fario L.). Bull. Intern. Acad. Sci. Cracovie, Cl. Sci. Math. Nat., Sér. B, Sci. Nat., 1915: 212-216.

Holzhauser, H. 1982. Neuzeitliche Gletscherschwankungen. Geographica Helvetica, 37: 115126.
Hsü, K., L. Montadert, D. Bernouilli, M. B. Cita, A. Erikson, R. E. Garrison, R. B., Kidd, F. Mélières, C. Müller \& R. Wright. 1977. History of the Mediterranean salinity crisis. Nature, 267: 399-403.

Hsü, K., W. B. F. Ryan \& M. B. Cita. 1973. Late Miocene dessication of the Mediterranean. Nature, 242: 240-244.

Hubbs, C. L. 1955. Hybridization between fish species in nature. Syst. Zool., 4: 1-20.

Hubs, C. L. \& K. F. Lagler. 1947. Fishes of the great Lakes region. Cranbrook Inst. Sci. Bull., 26: 1-213.

Hubbs, C. L. \& I. C. Potter. 1971. Distribution, phylogeny and taxonomy, p. 1-65. In: M. W. Hardisty \& I. C. Potter (eds), The biology of lampreys. Academic Press, London, 2 vols.

Hubbs, C. L. \& L. P. Schultz. 1941. Contributions to the ichthyology of Alaska, with descriptions of two new fishes. Occ. Pap. Mus. Zool. Univ. Mich., 431: 1-31.

Hubrecht, A. A. W. 1878. Over eene nieuwe Gobiussoort uit de Noordzee, alsmede opgrave van eenige zeldzamere vischsoorten op den tocht met loodsschoener "Vlissingen $\mathrm{n}^{\circ}$. 10" in Juli 1877 gevangen. Jaarverslag Zoölogisch Station Nederlandsche Dierkunde Vereeniging, 2: 15-20.

Hull, D. L. 1976. Are species really individuals? Syst. Zool., 25: 174-191.

Humboldt, A. de \& A. Valenciennes. 1817-1821. Recherches sur les poissons fluviatiles de l'Amérique équinoxiale, p. 145-216, pls. 45-51. In: A. de Humboldt \& A. Bonpland (1813-1832). Voyage aux régions équinoxiales du nouveau continent, fait en 1799, 1800, 1801, 1802, 1803 et 1804. Deuxième partie. Recueil d'observations de zoologie et d'anatomie comparées faites dans l'Océan Atlantique, dans l'intérieur du nouveau continent et dans la mer du sud pendant les années 1799, 1800, 1801, 1802, 1803 et 1804. Vol. 2. Schoell, Paris.

Hureau, J.-C. \& T. Monod (eds). 1973. Check-list of the fishes of the north-eastern Atlantic and of the Mediterranean [CLOFNAM]. UNESCO, Paris, 1: 683 pp; 2: $394 \mathrm{pp}$.

Hynes, R. A., A. Ferguson \& M. A. McCann. 1996. Variation in mitochondrial DNA and postglacial colonization of north western Europe by brown trout. J. Fish Biol., 48: 54-67.

Iliadou, K., J. Sourdis, P. S. Economidis \& A Wheeler. 1996. Morphological differentiation among species of the genus Scardinius (Pisces: Cyprinidae) in Greece. J. Nat. Hist., 30: 459473.

Iljin [Il'jin], B. S. 1949. [Short review of the Black 
Sea gobiids (Pisces, Gobiidae]. Byull. Mosk. Obshch. Ispyt. Prir., Otd. Biol., 54 (3): 16-30.

- 1956. [Remarks on and alterations to the Gobioidei in L. S. Berg's "Freshwater fishes of the USSR and adjacent countries", 4th edition, 1948-1849, pp. 1055-1125]. Vopr. Ikhtiol, 7: 185-192. [Russian]

International Commission on Zoological Nomenclature. 1926. Opinion 93. Twelve generic name placed in the Official List, by suspension of the rules. Smithson. Misc. Coll., 73 (4): 5-11.

- 1955. Opinion 345. Rejection of a proposal for the use of the plenary powers for the benefit of the generic name "Rhina" Latreille, [1802-1803] (Class Insecta, Order Coleoptera) and addition of the generic name "Rhina" Schneider, 1801 (Class Elasmobranchii) to the "Official List of Generic Names in Zoology": designation under the plenary powers for the genera "Rhinostomus" Rafinesque, 1815, and "Magdalis" Germar, 1817 (Class Insecta, Order Coleoptera) of type species in harmony with accustomed usage: validation, under the plenary powers, of the specific name "barbicornis" Latreille, [18031804], as published in the combination "Rhina barbicornis" (Class Insecta, Order Coleoptera). Opinions and Declaration of the Intern. Comm. Zool. Nom., 10: 353-388.

- 1968a. Opinion 859. Alosa fallax (Lacépède, 1803): preserved as the name for the twaite shad (Pisces). Bull. Zool. Nom., 25: 92-93.

- 1968b. Opinion 860. Gobius lenkoranicus Kessler, 1877 (Pisces): suppressed under the plenary powers. Bull. Zool. Nom., 25: 94-95.

- 1988. Opinion 1500. Cobitis Linnaeus, 1758 (Osteichthyes, Cypriniformes): Cobitis taenia Linnaeus, 1758 designated as the type species, and the original spelling of the family-group name Cobitidae Swainson, 1839 confirmed. Bull. Zool. Nom., 45: 178-179.

- 1993. Opinion 1715. Homalopteridae Bleeker, 1859 (Osteichthyes, Cypriniformes): not given precedence over Balitoridae Swainson, 1839. Bull. Zool. Nom., 50: 92-93.

Ioganzen [Johansen], B. G. 1935. Zametka o minogakh vostochnoi Azii. Sistematicheskie zametki po materialem Zool. Muzeya Biol. Inst. Pri Tomskom Gos. Univ. im V.V. Kuibysheva 2: 14.

- 1945. [New forms of fishes of west Sibiria]. Zametki po Faune i Flore Sibiri, Tomskogo Gosudarstvennogo Universiteta im. V. V. Kuzbisheva, Biol. Fak., 6: 1-16. [Russian, English summary]

- 1946. [Sterlet of the Ob river basin]. Trudy
Tomskogo Gosudarstvennogo Universiteta, 97: 151-182.

Issatschenko, W. 1925. Zur Erforschung der Heringe Genus Caspialosa des nordwestlichen Schwarzmeer-Bassins. Trudy Chernomorsko-Azovskoi Stantsii [Arb. All-Ukrain wissensch.praktisch. Staats-Stat. Schwarzen und Asowschen Meeres], 1: 103-131.

Ivanova-Berg, M. M. 1966. [Morphological distinctions of Ladoga and Neva lampreys]. Vopr. Ikhtiol., 6: 561-565. [Russian]

Ivanovic, B. M. 1973. Ichthyofauna of Skadar lake. Biological Station, Titograd, 146 pp.

Ivasik, V. M. 1968. The center of distribution of the carp and its parasite fauna. Vopr. Ikhtiol., 8: 342-349. [Russian, translated in Probl. Ichthyol., 8 (2): 269-275]

Jäckel, A. J. 1864. Die Fische Bayerns. Abhandl. Zool.-Miner. Ver. Regensburg, 9 (2): 1-104.

- 1866. Alburnus Rosenhaueri, ein neuer Fischbastard. Zool. Garten, Ztschr. Beobacht. Pflege Zucht Thiere, 7: 20-25.

Jardine, W. 1831. Observations upon rare or little known British fishes. No. 1. The vendace or vendis of the Lochmaben lochs. Edinburgh J. Nat. Geogr. Sci. Edinburgh, 3: 1-4, pl. 1.

- 1835a. Observations upon the Salmonidae met with during an excursion to the north-west of Sutherlandshire, in June 1834. Edin. New Philos. J., 18: 46-58.

- 1835b. Observations on the Salmonidae which were met with during an excursion to the Northwest of Sutherlandshire in June 1834. Rept. Meeting Brit. Ass. Adv. Sci., 4: 613-615.

-1840-1841. Salmons of England, Scotland and Ireland drawn from nature. Parts 1 \& 2 . Edinburgh, 12 pls.

Järvi, T. H. 1928. Über die Arten und Formen der Coregonen s. str. in Finnland. Acta Zool. Fennica, 5: 1-259, 48 pls.

- 1943. Zur Kenntnis der Coregonen-Formen Nord-Finnlands insbesondere des Kuusamo-Gebietes. Acta Zool. Fenn., 40: 1-91, pls. 1-28.

Jarocki, F. 1822. Zoologiia czyli zwierzetopismo ogolne podlug nainowszego Systematu. 4. Drukarni Latkiewicza, Warsaw, 464 pp.

Jaszfalusi, L. 1951. Die endemischen Cobitis- und Gobio-Arten der Tisza, sowie ihrer Nebenflüsse. Ann. Hist. Nat. Mus. Natn. Hung., 1: 113-125, pl. 1.

Jeitteles, L. H. 1862. Prodromus faunae vertebratorum Hungariae Superioris. Beiträge zur näheren Kenntniss der Wirbelthiere Ungarn's. Verh. Zool. Bot. Ges. Wien, 12: 244-314, pl. 2. - 1863. Die Fische der March bei Olmuitz. I. 
Abtheilung. Jahres-Ber. Kaiserl. Königl. Gymnasium Olmütz, 1863: 3-33.

Jenkins, R. E. \& N. M. Burkhead. 1994. Freshwater fishes of Virginia. American Fisheries Society, Bethesda, Ma., xxii+1080 pp., 40 pls.

Jenyns, L. 1835a. A systematic catalogue of British vertebrate animals. Deighton, Stevensons and Longman, London, iv + [19] pp.

- 1835b. A manual of British vertebrate animals. Cambridge \& London, xxxii+359 pp.

Joensen, J. S. \& A. V. Tåning. 1970. Marine and freshwater fishes, p. 1-241. In: R. Spärck \& S. L. Tuxen (eds), The zoology of the Faroes. Vol. 3, Part 1. Høst, Copenhagen.

Johnson, L. 1980. The arctic charr, Salvelinus alpinus, p. 15-98, In: E. K. Balon (ed.), Charrs - Salmonid fishes of the genus Salvelinus. Junk, Den Haag.

Jonsson, B. \& K. Hindar. 1982. Reproductive strategy of dwarf and normal arctic charr (Salvelinus alpinus) from Vangsvatnet Lake, western Norway. Can. J. Fish. Aquat. Sci., 39: 1404-1413.

Jonsson, B., S. Skúlason, S. S. Snorrason, O. T. Sandlund, H. J. Malmquist, P. M. Jonasson, R. Gydemo \& T. Lindem. 1988. Life history variation of polymorphic arctic charr (Salvelinus alpinus) in Thingvallavatn, Iceland. Can. J. Fish. Aquat. Sci., 45: 1537-1547.

Jonston, J. 1649. Historia naturalis de piscibus et cetis libri v, cum aeneis figuris. Francoforti ad Moenum, 228 pp., 47 pls.

Jordan, D. S., B. W. Evermann \& H. W. Clark. 1930. Check list of the fishes and fishlike vertebrates of North and Middle America north of the northern boundary of Venezuela and Colombia. Rep. U. S. Comm. Fish., 1928 (2): $1-670$.

Jordan, D. S. \& C. H. Gilbert. 1883. A synopsis of the fishes of North America. Bull. U. S. Natn. Mus., 16: i-lvi + 1-1018.

Jordan, D. S. \& C. L. Hubbs. 1925. Records of fishes obtained by David Starr Jordan in Japan, 1922. Mem. Carnegie Mus., 10: 93-346, pls. 512.

Jurine, L. 1825-1826. Histoire abrégée des poissons du lac Léman. Mém. Soc. Phys. Hist. Nat. Genève, 3: 133-235, 15 pls. [text published in 1825 , plates in 1826]

Kaganovskii, A. G. 1933. [Commercial fishes of the Anadyr river and liman]. Vestnik Dalnevostochnogo Filiala Akad. Nauk SSSR, 1-3: 3-4.

Kaj, J. 1953. Rozprzestrzenienie i zmiennosc, rasowa ryb z gatunku Phoxinus percnurus Pall. na terenie Polski. Polskie Archivum Hydrobiologii,
1: 49-78. [Polish, English summary]

Kalashnikov, Y. Y. 1968. [The many gill-raker sig [whitefish] of lake Oron in the Vitim River basin]. Vopr. Ikhthiol., 8:637-645. [Russian, translated in Probl. Ichthyol., 8 (4): 511$518]$

Kamenskii, S. N. 1897. [On the ichthyology of the Caucasus]. Trudy Kharkovskogo Obshchestva Ispytatelei Prirody, 31: 79-100, pls. 3-4.

- 1901. Die Cypriniden der Kaukasusländer und ihrer angrenzenden Meere. 2te Lieferung. Izdanie Kavkazskogo Muzeya, Tiflis, 2: 1-192, pls. 7-12. [Russian and German]

Karakousis, Y., J. Paschos \& C. Triantaphyllidis, 1992. Chromosomal studies in brown trout (Salmo trutta L.) populations. Cytobios, 72: 117-124, 2 figs.

Karakousis, Y., C. Peios, P. S. Economidis \& C. Triantaphyllidis. 1993. Multivariate analysis of the morphological variability among Barbus peloponnesius (Cyprinidae) populations from Greece and two populations of $B$. meridionalis meridionalis and B. meridionalis petenyi. Cybium, 17: 229-240.

Karakousis, Y. \& C. Triantaphyllidis. 1989. Genetic structure and differentiation among Greek brown trout (Salmo trutta L.) populations. Heredity, 64: 297--304.

Karakousis, Y., C. Triantaphyllidis \& P. S. Economidis. 1991. Morphological variability among seven populations of brown trout, Salmo trutta L., in Greece. J. Fish Biol., 38: 807-817.

Karaman, M. S. 1963. Eine neue Unterart des Steinpeitzgers, Cobitis aurata bosniaca n. ssp. (Cobitidae, Pisces), aus Jugoslawien. Int. Rev. Ges. Hydrobiol., 48: 629-632.

- 1966. Beitrag zur Kenntnis der Salmoniden Suideuropas. Hydrobiologia, 28: 1-41.

- 1971. Süsswasserfische der Türkei. 8. Revision der Barben Europas, Vorderasiens und Nordafrikas. Mitt. Hamburg. Zool. Mus. Inst., 67: $175-254$.

- 1972. Süsswasserfische der Türkei. 9. Revision einiger kleinwüchsiger Cyprinidengattungen Phoxinellus, Leucaspius, Acanthobrama usw. aus Südeuropa, Kleinasien, Vorder-Asien und Nordafrika. Mitt. Hamburg. Zool. Mus. Inst., 69: 115-155, pl. 1.

- 1974a. Eudontomyzon vladykovi stankokaramani n. ssp. a new subspecies of lamprey from tributaries of the Ohrid - Drim - Skadar system in west Balkan Peninsula. Folia Balcanica, 3: $1-14$.

- 1974b. Beitrag zur Kenntniss der Untergattung Romanogobio Bănărescu (Pisces, Cyprini- 
dae) in Mazedonie und Griechenland. Izdanija, Skopje, 4 (10): 1-18.

Karaman. S. 1922. Über eine neue Cobitis-Art aus Jugoslavien, Cobitis balcanica n. sp. Glasnik. Kroat. Naturwiss. Ges. Zagreb, 34: 307310.

- 1924a. Anofeli makedonije i njihovo suzbijanje [Die Anopheliden Mazedoniens und ihre Bekämpfung]. Glasnik Min. Nar. Zdravlja, Beograd, 22: 1-47 [Serbocroat, German summary]

-- 1924b. Pisces macedoniae. Hrvatska Stamparija, Split, 90 pp.

- 1927. [Les salmonidés des Balkans]. Glasn. Skopskog Naucnog Drustva [Bull Soc. Scient. Skoplje], 2 (1926 [1927]): 253-268. [Serbocroat, French summary]

- 1928. [Beiträge zur Ichthyologie von Jugoslavien. I]. Glasn. Skopskog Nauknog Drustva [Bull. Soc. Sci. Skoplje], 6 (Odel. Prirod. Nauk [Sect. Sci. Nat.] 2): 147-176. [Serbocroat, German summary]

- 1929. Über einige neue Fische aus Jugoslavien. Zool. Anz., 80: 171-173.

- 1932. Novi prilozi poznavanju nasih Salmonida. Ribarski List, Sarajevo, 7 (9-10): 1-3.

- 1933. Prilozi ihtiologiji Crne Gore. Ribarski List, Sarajevo, 8 (9-10): 104-106.

- 1936. [10. Beitrag zur Kenntnis der Süssswasserfische Jugoslaviens]. Glasn. Skopskog Naucnog Drustva [Bull. Soc. Scient. Skoplje], 17 (Odel. Prirod. Nauk [Sect. Sci. Nat.] 5): 55-64. [Serbocroat, German summary]

- 1938. 11. Beitrag zur Kenntnis der Süsswasserfische Jugoslaviens. Glasn. Skopskog Naucnog Drustva [Bull. Soc. Scient. Skoplje], 18 (Odel. Prirod. Nauk [Sect. Sci. Nat.] 6) (1936 [1938]): 131-139.

- 1955. Die Fische der Strumica (Struma-System). Acta Mus. Maced. Scient. Nat., 3: 181208.

- 1957. [The Radika River trouts]. Folia Balcanica, 1: 57-70. [Serbocroat, English summary]

Karasev, G. L. 1987. [The fishes of Transbaicalia]. Nauka, Sibirskoe Otdelenie, Novosibirsk, 296 pp.

Károli, J. 1881. Umbra canina [Marsili]. Természetrajzi Füzetek, 5: 188-191.

Karsten, D. L. G. 1789. Museum Leskeanum. Müller, Lipsiae [Leipzig], 2 vols.

Kartas, F. \& M. Trabelsi. 1990. Sur le polymorphisme de l'athérine Atherina boyeri Risso, 1810 (Atherinidae) des eaux littorales tunisiennes. Cybium, 14: 295-311.

Kashchenko, N. F. 1899. [Results of the Altai zoological expedition in 1898. Vertebrata]. Izvestiya
Tomskogo Universiteta (Izd. Kononova i Skulinskogo), $158 \mathrm{pp}$.

Kattoulas, M. E., A. Stephanidis \& P. S. Economidis. 1973. The fish fauna of the Aliakmon River system (Macedonia, Greece). I. The species of the genus Gobio Cuvier, 1817 (Pisces. Cyprinidae). Biol. Gallo-Helen., 4: 175-187.

Kaup, J. 1856a. Uebersicht der Aale. Arch. Naturges., Berlin, 22 (1): 41-77.

- 1856b. Catalogue of the Lophobranchiate fish in the collection of the British Museum. British Museum, London, iv +80 pp.

- 1856 [1857]. Catalogue of the apodal fish in the collection of the British Museum. British Museum, London, 163 pp., 19 pls.

Kawraisky, T. T. 1896-97. Die Lachse der Kaukasusländer und ihrer angrenzenden Meere. Izdanie Tifliskogo Museya, Tiflis, 1 (1896): 1-87, 9 pls; 2 (1897): 1-79, 5 pls. [Russian and German]

Kessler, K. 1856a. [Natural history of the provinces belonging to the Kiev educational district. Fishes]. Kiev, 98 pp. [Russian]

- 1856b. Zur Ichthyologie des südwestlichen Russlands. Bull. Soc. Imp. Nat. Moscou, 29 (1) 335-393.

- 1857. Nachträge zur Ichthyologie des südwestlichen Russlands. Bull. Soc. Imp. Nat. Moscou. 30 (2): 453-481.

- 1859a. Auszüge aus dem Berichte über eine an die nordwestlichen Küsten des schwarzen Meeres und durch die westliche Krym unternommene Reise. Bull. Soc. Imp. Nat. Moscou, $32(2): 520-546$

- 1859b. Auszuige aus dem Berichte über eine an die nordwestlichen Küsten des schwarzen Meeres und durch die westliche Krym unternommene Reise. Bull. Soc. Imp. Nat. Moscou, 32 (3): 186-268.

- 1860. Puteshestvie, s zoologicheskoi tsel'yu, k severnomu beregu Chernogo morya i v Krym v 1858 godu. Kiev, 248 pp.

- 1864. [Descriptions of the fishes which are met with in the freshwaters of the St.-Petersburg's government]. In: [Natural history exploration of the St. Petersburg government, undertaken by the members of the Russian Entomological Society in St. Petersburg]. Russkoe Entomol. Obsh., St. Petersburg, 1 (sect 1, Zool. Explor.): 1-240, pls. 1-3. [in Russian]

- 1868. [Materials to the study of Lake Onega and the Onega District]. Trudy Isezd. Russikh Estets. St. Petersburg, Suppl., pp. 1-144.

- 1872. [Ichthyological fauna of Turkestan.]. Izvestiya Imp. Obshchestva Lyubitelei Estestvoz- 
naniya, Antropologii i Etnografii, 10: 47-76, pls. 6-12. [Russian]

- 1874a. [Fishes]. In A.P. Fedchenko's travel to Turkestan. Izvestiya Obshchestva Lyubitelei Estestvoznaniya, Antropologii i Etnografii, 11 (3): $1-63,8$ pls.

- 1874b. [A description of fishes belonging to the families common to both the Black and the Caspian Seas]. Trudy Sankt-Petersburgskogo Obshchestva Estestvoispytatelei, 5: 191-324, 1 $\mathrm{pl}$.

- 1876. [Descriptions of the fishes collected by Col. Przewalski in Mongolia], p. 1-36, 3 pls. In: N. Przewalski, [Mongolia, and the land of the Tanguts]. St. Petersburg, vol. 2, part 4. [Russian]

- 1877. [Fishes of the Aralo-Caspio-Pontian region]. Trudy Aralo-Kasp. Eksped., 4: xxviii+ 360 pp., 8 pls.

- 1879. Beiträge zur Ichthyologie von CentralAsien. Bull. Acad. Imp. Sci. St.-Pétersbourg, 25: 282-310.

Kevan, D. K. M. 1973. The place of classical taxonomy in modern systematic entomology, with particular reference to orthopteroid insects. Can. Entomol., 105: 1211-1222.

Keyserling, E. 1861. Neue Cypriniden aus Persien. Ztschr. Gesamm. Naturwiss., 17: 1-27, pls. 1-9.

Kiener, A. \& C. J. Spillmann. 1969. Contribution à l'étude systématique et écologique des athérines des côtes françaises. Mém. Mus. Hist. Nat. Paris, Nouy. Sér., Sér. A, Zool., 40: 33-74.

Kimura, S., T. Mori, T. Kataoka, K. Hosoya, Wu H.-L. \& K. Suzuki. 1992. Preliminary report on a collection of freshwater fishes from the Altai District, northern Xinjiang, China. Bull. Fac. Bioresources, Mie Univ., 7: 91-105, 5 pls.

Kirchhhofer, A. 1990. Limnologische und ichthyologische Untersuchungen im Brienzersee unter besonderer Berücksichtigung der Differenzierung der sympatrischen Felchenpopulationen. Thesis, Universität Bern, 100 pp. 6 pls.

Kirillov, F. N. 1956. Vodoemy Yakutii i ikh ryby. Yakutsk.

- 1958. [Lenok]. Nauchn. Soobshch. Yakutskogo Filiala Siberskogo Otdeleniya Akad. Nauk. SSR, 1.

- 1962. [Ichtyofauna of the Vilyui River basin]. Trudy Instituta Biologii, Yakutskii Filial Sibirskogo Otdeleniya Akad. Nauk SSSR, 8: 5-71. [Russian]

-- 1972. [Fishes of Yakutia]. Nauka, Moskva, 360 pp. [Russian]

Klausewitz, W. 1955. Cobitis taenia haasi, eine iberische Unterart der Dorngrundel, (Pisces,
Cobitidae). Senck. Biol., 36: 41-43.

Klemetsen, A. \& P. E. Grotnes. 1980. Coexistence and immigration of two sympatric arctic charr, p. 757-763. In: E. K. Balon (ed.), Charrs Salmonid fishes of the genus Salvelinus. Junk, Den Haag.

Klunzinger, C. B. 1884. Ueber die Felchenarten des Bodensees. Jahresheft Ver. Vaterl. Naturk. Württemberg, 40: 105-128.

Knauthe, K. 1893a. Zwei fortpflanzungsfähige Cyprinidenbastarde. Zool. Anz., 16: 416-418.

- 1893b. U̇ber einen neuen Fortpflanzungsfähigen Cypriniden aus Mittelschlesien. Alburnus Leydigii mihi, sp. nov. (Alb. lucidus $\times$ Leucaspius delineatus). Zool. Anz., 16: 448-450.

Knox, R. 1854. Fish and fishing in the lone glens of Scotland, with a history of the propagation, growth and metamorphoses of the salmon. London, 144 pp.

- 1855. Some observations on the Salmo estuarius or estuary trout. Zoologist, 13: 4662-4673.

Koch, K. L., A. Herrich-Schäffer \& F. Forster. 1840. Fauna Ratisbonensis, oder Uebersicht der in der Gegend um Regensburg einheimischen Thiere. Manz, Regensburg, xv +478 pp.

Köhler, C. \& A. Lelek. 1992. Die Fischfauna des Rheins: Analyse der Artengemeinschaften sowie Daten zur Morphometrie und Meristic der Vorkommenden Arten. Courier Forsch. Senckenberg, 148: 53-153.

Koli, L. 1969. Geographical variation of Cottus gobio L. (Pisces, Cottidae) in northern Europe. Ann. Zool. Fenn., 6: 353-390.

Koller, O. 1926a. Eine neue Barbus-Art aus Albanien. Anz. Math.-Naturw. Kl. Österr. Akad. Wiss., 62: 178-179.

- 1926b. Drei Fischarten aus Albanien, darunter eine bisher unbeschriebene Varietät von Barbus meridionalis Risso. Zool. Anz., 65: 315-320.

- 1926c. Eine kritische Übersicht über die bisher beschriebenen mittel- und südeuropäischen Arten der Cyprinidengattung Barbus Cuv. SitzBer. Math. Naturwiss. Cl. Kaiserl. Akad. Wiss., Abt. 1, 135: 167-201, pls. 1-2.

Kolombatovic, J. 1890. Notizie ittiologiche. I. Seconda trotta marina pescata nel mare di Vranjic Trutta adriatica n. sp. II. Cattura del Lophotes Cepedianus (Giorna), nelle acque di Trappano (Dalmazia). III. Sui Mullus dell'Adriatico. Glasnika Hrvatskoga Naravoslovnoga Druztva, 5: 165-174.

- 1891. Glamoci (Gobii) spljetskog pomorskog okruzja u Dalmaciji. Spljetu [Split], 29 pp.

Kottelat, M. 1984. Catalogue des types du Musée d'Histoire Naturelle de Neuchâtel. I. Pisces. 
Bull. Soc. Neuchâtel. Sci. Nat., 107: 143-153.

- 1986. Cobitis Linnaeus, 1758 (Osteichthyes, Cypriniformes): proposed designation of Cobitis taenia Linnaeus, 1758 as type species and request for a ruling on the stem of the familygroup name Cobitididae Swainson, 1839. Bull. Zool. Nom., 43: 360-362.

- 1987. Nomenclatural status of the fish names created by J. C. van Hasselt (1823) and of some related cobitoid genera. Japan. J. Ichthyol., 33: 368-375.

- 1988a. Authorship, dates of publication, status and types of Spix and Agassiz's Brazilian fishes. Spixiana, 11: 69-93.

- 1988b. Indian and Indochinese species of $\mathrm{Ba}$ litora (Osteichthyes: Cypriniformes) with descriptions of two new species and comments on the family group names Balitoridae and Homalopteridae. Rev. Suisse Zool., 95: 487-504.

- 1990a. Indochinese nemacheilines - A revision of nemacheiline loaches (Pisces: Cypriniformes) of Thailand, Burma, Laos, Cambodia and southern Viet Nam. Pfeil, München, 262 pp.

- 1990b. Sailfin silversides (Pisces: Telmatherinidae) of Lakes Towuti, Mahalona and Wawontoa (Sulawesi, Indonesia) with descriptions of two new genera and two new species. Ichthyol. Explor. Freshwat., 1: 227-246.

- 1991. Sailfin silversides (Pisces: Telmatherinidae) of Lake Matano, Sulawesi, Indonesia, with descriptions of six new species. Ichthyol. Explor. Freshwat., 1: 321-344.

- 1995. Systematic studies and biodiversity: the need for a pragmatic approach. J. Nat. Hist., 29: $565-569$.

- 1996. Nomenclatural status of Cyprinus clupeoides Bloch, 1795 (Osteichthyes: Cyprinidae). J. South Asian Nat. Hist., 2: 61-62.

Kottelat, M. \& X.-L. Chu 1988. Revision of Yunnanilus with descriptions of a miniature species flock and six new species from China (Cypriniformes: Homalopteridae). Env. Biol. Fish., 23: 65-93.

Kottelat, M. \& K. K. P. Lim. 1995. Hemibagrus hoevenii, a valid name for the Sundaic blacktailed catfish (Teleostei: Bagridae). Malay. Nat. J., 49: 41-47.

Kottelat, M. \& P. K. L. Ng. 1994. Diagnoses of five new species of fighting fishes from Banka and Borneo (Teleostei: Belontiidae). Ichthyol. Explor. Freshwat., 5: 65-78.

Kottelat, M., J. G. Nielsen \& H. Nijssen. 1993. Survey of ichthyological resources in European museums and collections. Societas Europaea Ichthyologorum, 24 pp.
Kottelat, M. \& E. Sutter. 1988. Catalogue des types de poissons du Musée d'Histoire naturelle de Bâle (Naturhistorisches Museum Basel). Verh. Naturf. Ges. Basel, 98: 51-57.

Kottelat, M. \& A. Wheeler. In press a. Lebias Goldfuss, 1820 (Pisces, Cyprinodontidae): proposed change of the type species. Bull. Zool. Nom.

- In press b. Acipenser naccarii Bonaparte, 1836 (Pisces, Acipenseridae): proposed conservation of the species name. Bull. Zool. Nom.

Kramer, W. H. 1756. Elenchus vegetabilium et animalium per Austriam inferiorem observatorum, sistens ea in classes et ordines, genera et species redacta. Trattner, Viennae, Pragae \& Tergesti.

Krauss, F. 1879. Beiträge zur Fauna Württembergs. 5. Eine varietät der Nase (Chondrostoma Nasus L.). Jahreshefte Ver. Vaterl. Naturk. Württ., 35: 348-349.

- 1882. Beiträge zur Fauna Württembergs. 1. Barbus fluviatilis Ag. var. alba. Jahreshefte Ver. Vaterl. Naturk. Wuirtt., 38: 346.

Krieg, F. \& R. Guyomard. 1985. Population genetics of French brown trout (Salmo trutta L): large geographical differentiation of wild populations and high similarity of domesticated stocks. Génét. Sél. Evol., 17: 225-242.

Kriesch, J. 1873. Ein neuer Gobius. Verh. Zool. Bot. Ges. Wien, 23: 369-376, pl. 6.

Krögius, F. V. 1926. [Materials on the age and rate of growth of the whitefish of Lake Imandra]. Murmanskaya Biologicheskaya Stantsiya [Rep. Biol. Stat. Murmansk], 2: 77-87. [Russian]

Kröyer, H. N. 1838-1853. Danmarks Fiske. Kjobenhavn, 1 (1838): 616 pp.; 2 (1843-1844): 644 pp.; 3 (1846): 320 pp.; (1849): 321-640; (1852): 641960; (1853): 961-1279.

Krupka, I. \& J. Holčík. 1976. On the occurrence of Barbus plebejus in the Poparad River (Vistula basin, Czechoslovakia) with regard to its assumed hybrid origin. Vestn. Ceskoslov. Společ. Zool., 40: 163-178, 2 pls.

Krynicki, J. 1832. Schilus Pallasii descriptus et icones illustratus. Nouv. Mém. Soc. Imp. Nat. Moscou, 2: 441-446, pl. 25.

Kulmatycki, W. J. 1927 Studien an Coregonen Polens. Archiwum Hydrobiol. i Rybactwa, 1: 275-336.

- 1928. Beitrag zur Kenntnis der Coregonen Polens. Arch. Hydrobiol., 19: 37-49.

Kuru, M. 1971. The fresh-water fish fauna of eastern Anatolia. Istambul Univ. Fen. Fak. Mecm., Ser. B, 36: 137-147.

Kux, Z. 1965. [Lampetra gracilis, eine neue nichtparasitische Art des Neunauges aus Ostslowakei. 
Vorläufige Mitteilung]. Acta Mus. Moraviae, Sci. Nat., 50: 293-302. [Czech, German summary]

Kux, Z. \& J. Libosvárský. 1957. Zur Verbreitung und Rassenzugehörigkeit der Europäischen Hundsfische (Umbra krameri Walbaum 1792 = Lacustris Gross. 1794). Folia Zool., 6: 215-224, pl. 11.

Kuznetsov, I. D. 1888. [Percarina and Benthophilus of the Sea of Azov]. Trudy SanktPeterburgskogo Obshchestva Estestvoispytatelei, 19: 189-212.

La Blanchère, H. de 1872. Sur une espèce nouvelle de Chondrostome, déterminée dans les eaux du Rouergue (Chondrostoma Peresi La Bl.). C. R. Acad. Sci. Paris, 75: 1632-1636.

- 1872b. Sur une vandoise nouvelle déterminée dans les eaux du Rouergue (Squalius oxyrrhis, La Bl.). C. R. Hebd. Séances Acad. Sci., 76: 662-665.

La Cepède, [E.]. 1798-1803. Histoire naturelle des poissons. Paris. 1 (1798): $8+$ cxlvii +532 pp., 25 pls.; 2 (1800): lxiv+632 pp., 20 pls.; 3 (1801): lxvi+558 pp., 34 pls.; 4 (1802): xliv+728 pp., 16 pls.; 5 (1803): xlviii+803 pp., 21 pls.

Ladiges, W. 1960. Süsswasserfische der Türkei. 1. Teil. Cyprinidae. Mitt. Hamburg. Zool. Mus. Inst., 58: 105-150.

Ladiges, W., G. von Wahlert \& E. Mohr. 1958. Die Typen und Typoide der Fischsammlung des Hamburgischen Zoologischen Staatsinstituts und Zoologischen Museums. Mitt. Hamburg. Zool. Inst., 56: 155-167.

Lamby, K. 1941. Zur Fischereibiologie des Mývatn, Nord-Island. Ztschr. Fisch. Hilfswiss., 39: 749805.

Largiadèr, C. R. \& A. Scholl. 1995. Effects of stocking on the genetic diversity of brown trout populations of the Adriatic and Danubian drainages in Switzerland. J. Fish Biol., 47 (Suppl. A): 209-225.

Largiadèr, C. R., A. Scholl \& R. Guyomard. 1996. The role of natural and artificial propagation on the genetic diversity of brown trout (Salmo trutta L.) of the upper Rhône drainage, p. 181-197. In: A. Kirchhofer \& D. Hefti (eds), Conservation of endangered freshwater fish in Europe. Birkhäuser, Basel, 341 pp.

Larson, A. 1989. The relationship between speciation and morphological evolution, p. 579-598. In: D. Otte \& J. A. Endler (eds), Speciation and its consequences. Sinauer Associates, Sunderland, Ma.

Lauzanne, L. 1982. Les Orestias (Pisces, Cyprinodontidae) du Petit Lac Titicaca. Rev.
Hydrobiol. Trop., 15: 39-70, 4 pls.

Lawacz, W. 1965. An analysis of variations in two populations of Gobius microps Kr. depending on the salinity of the habitat. Ekol. Polsk., Ser. A, 13: 125-142.

Lazara, K. J. 1993. Dating and authorship of new species described in Humboldt and Valenciennes' "Recherches sur les poissons fluviatiles de l'Amérique équinoxiale". Copeia, 1993: 11601161.

- 1995. History of the genera Lebia Oken 1817 and Lebias Goldfuss 1820 (Teleostei: Cyprinodontidae) with designation of a type species for Lebias. Copeia, 1995: 501-503.

Lechler, H. 1929a. Untersuchungen über die Reinanke des Attersees. (I.). Ztschr. Fischerei Hilfswiss., 27: 407-415.

- 1929b. Untersuchungen über die Reinanke des Mondsees. (I.). Ztschr. Fischerei Hilfswiss., 27: 547-560.

- 1930a. Untersuchungen über den Kröpfling des Attersees. (I.). Ztschr. Fischerei Hilfswiss., 28: 83-100.

- 1930b. Untersuchungen ïber die Reinanke des Mondsees. (II.). Ztschr. Fischerei Hilfswiss., 28: 485-493.

Le Danois, E. 1913. Contribution à l'étude systématique et biologique des poissons de la Manche Occidentale. Annls. Inst. Océanogr. Monaco, 5 (5): 1-124.

Leiblein, 1853. Versuch einer Aufzählung der Fische des Main-Gebietes (Schluss). Korr.-Bl. Zool.-Miner. Ver. Regensburg, 7: 113-127.

Lelek, A. 1987a. The freshwater fishes of Europe. Vol. 9. Threatened fishes of Europe. Aula, Wiesbaden, 343 pp.

- 1987b. Notes on the reproductive ecology of the feral form of the common carp, Cyprinus carpio carpio, in the Rhine River, pp. 169-173. In: Proc. V Congr. Europ. Ichthyol., Stockholm. - 1988. Vorkommen, Taxonomie und Massnahmen zur Erhaltung der Forelle Salmo trutta labrax Pallas 1811 in der NO-Türkei. Courier Forsch. Senckenberg, 131: 1-44.

Lepechin, I. 1771a. Descriptiones avium. Novi Commentarii Academiae Scientiarum Imperialis Petropolitanae, 15 (1770 [1771]): 485-493.

- 1771b-1805. [Travel journals about various provinces of the Russian state in 1768 and 1769]. St.-Petersbourg, 1 (1771), 2(1772), 3 (1780), 4 (1805).

- 1795. Varietas acipenseris stellati oppido rara descripta. Nova Acta Academiae Scientiarum Imperialis Petropolitanae, 9 (Histoire): 35-38. Leske, N. G. 1774. Ichthyologiae Lipsiensis speci- 
men. Siegfried Lebrecht Crusium, Lipsiae, iv+ $82 \mathrm{pp}$.

Lesniewski, P. E. 1837. Rybactwo krajowe czyli historyja naturalna ryb krajowych. I. Kalendarz Rybacki. Zawadzki \& Wecki, Warszawa, 360 pp., 7 pls.

Leviton, A. E. \& R. H. Gibbs. 1983. Standards in herpetology and ichthyology. Standard symbolic codes for institution resource collections in herpetology and ichthyology. Supplement No. 1: additions and corrections. Copeia, 1988: 280282

Leviton, A. E., R. H. Gibbs, E. Heal \& C. E. Dawson. 1985. Standards in herpetology and ichthyology: part I. Standard symbolic codes for institutional resource collections in herpetology and ichthyology. Copeia, 1985: 802-832.

Lilljeborg, W. 1850. Ny art af fiskslägtet Platessa. Öfvers. Kongl. Vetensk. Akad. Förhandl., 7: 56.

- 1851. Bidrag till Norra Rysslands och Norriges fauna, samlade under en vetenskaplig resa i dessa länder 1848. Kongl. Vetensk. Akad. Handl., 1850 [1851]: 233-341, pls. 19-20.

- 1884-1891. Sveriges och Norges fiskar. Schultz, Upsala, 3 vols. 1 (1884): xxii+782 pp.; 2 (1891): 830 pp.; 3 (1891): 788 pp.

Lim, K. K. P., P. K. L. Ng \& M. Kiottelat. 1990 On a collection of freshwater fishes from EndauRompin, Pahang-Johore, Peninsular Malaysia. Raffles Bull. Zool., 38: 31-54.

Linnaeus, C. [C. von Linné]. 1732. Iter Lapponicum. Platen \& Wydow, Stockholm. [reprint 1975 by Wahlström \& Widstrand, Stockholm]

- 1746. Fauna Svecica sistens animalia Sveciae regni. Wishoff, Lugduni Batavorum, 411 pp., 2 pls.

- 1747. Wästgöta-resa, pă riksens högloflige Ständers befallning förrättad àr 1746. Salvii, Stockholm, xii $+284+19$ pp., 5 pls.

- 1748. Systema naturae sistens regna tria naturae systematice proposita per classes ordines, genera, \& species. Editio sexta. Kiesewetteri, Stockholmiae, ii $+224+30$ pp., 8 pls.

- 1751. Skănska resa på höga öfwerhetens befallning förrättad àr 1749. Salviani, Stockholm, xiv +434 pp., 6 pls

- 1754. Museum S. R. M. Adolphi Friderici Regis Suecorum. Typographia regia, Stockholmiae, 133 pp., 32 pls.

- 1758. Systema naturae per regna tria naturae. secundum classes, ordines, genera, species, cum characteribus, differentiis, synonymis, locis. Tomus I. Editio decima, reformata. Salvius, Holmiae, 1: $824 \mathrm{pp}$.
- 1766. Systema naturae per regna tria naturae, secundum classes, ordines, genera, species cum characteribus, differentiis, synonymis, locis. Editio duodecima, reformata. Salvius, Holmiae. 1: $532 \mathrm{pp}$.

Linstow, [O. F. B.] von. 1878. Ichthyologische Notizen. Arch. Naturgesch., 44 (1): 246-250.

Lityński, A. 1923. Sieja i sielawa w jeziorach suwalskich i augustowskich [Die grosse und die kleine Maräne in den Seen des Suwalkier Seengebietes]. Sprawodz. Stac. Hydrobiol. na Wygrach, 1 (2-3): 91-108, pl. 6. [Polish, German summary] Lönnberg, E. 1900. Contributions to the ichthyology of the Caspian Sea. Bihang Kungl. Svenska Vet.-Akad. Handlingar., Afd. 4, 26 (8): 1-38.

- 1919. Några ord om Vänerns hornsimpa. Fauna och Flora, 1919: 163-172.

- 1932a. Some remarks on the relict forms of Cottus quadricornis L. in Swedish freshwater lakes. Ark. Zool., Ser. A, 24 (7): 1-23, pl. 1.

- 1932b. Notes on some relict races of Cottus quadricornis living in lakes in Finland. Ark. Zool., Ser. A, 24 (12): 1-16, pl. 1.

- 1939. Notes on some relict forms of Cottus quadricornis L. recently found in Swedish freshwater lakes. Ark. Zool., Ser. B, 31 (2): 1-5.

Lorec, Z. \& T. Wolski. 1910. Nowy gatunek z rodzaju strzebla (Phoxinus Agas.). Strzebla przekopowa (Phoxinus Dybowskii spec. nov.?). Sprawozdania z posiedzen Towarzysta Naukowego Warszawskiego [C. R. Séances Soc. Scient. Varsovie], 3: 114-120.

Lovetzky [Lovetskii], A. 1828. O Rybakh, k rodu osetra prinadler vazhikh. 4. Bielujii chiph (AC. nudiventris, Lovetzky). Novyi Magazin Estestvennoi istorii, fizikii, khimii i svedenii ekologischeskikh, Moskva, 2 (2): 78-79, pl. 6. [summary in Bull. Sci. Nat. (Férussac, 1830), 23: 131-134; from Sokolov \& Vasilev, in Holčík (1989): 225]

- 1834. Diagnosis piscium ad genus Acipenserinum pertinentium, praeprimis eorum, qui habitant in aquis Imperii Rossici. Nouv. Mem. Soc. Imp. Nat. Moscou, 3: 253-263, pls. 15-19.

Lowe, R. T. 1838. A synopsis of the fishes of Madeira, with the principal synonyms, Portuguese names and characters of the new genera and species. Trans. Zool. Soc. London, 2: 173-200 [see Whitehead et al. (1988): 527 for publication date].

- 1839. A supplement to a synopsis of the fishes of Madeira. Proc. Zool. Soc. London, 7: 76-92.

- 1874. Fauna and flora of Norfolk. Trans. Norfolk and Norwich Nat. Soc., 1: 21-56.

Lozano Rey, L. 1929. Los clupeidos de la Peninsula Ibérica y del Rif. Mem. Real Soc. Esp. Hist. 
Nat., 15 (2): 647-666, pls. 30-31.

Lukash, A. V. 1925. [Fishes of the Vyatka River basin upstream of Slobodskoe]. Trudy Vyatskogo Nauchno-Issled. Inst. Kraevedenya, Vyatka, 1: 1-50.

- 1929. [Fishes of the upper reaches of the Kama River]. Trudy Vyatskogo Nauchno-Issledovatelskogo Instituta Kraevedeniya, Vyatka, 5: 36 pp [offprint]

- 1933. [Fishes of the lower Vyatka River]. Trudy Vyatskogo Nauchno-Issledovatelskogo Instituta Kraevedeniya, Vyatka, 6: 5-110.

- 1939. [Investigations on the fisheries of lake Vodlozero]. Rybnoe khozyaistvo Karelii. Vol 5.

Lundberg, J. G. 1992. The phylogeny of ictalurid catfishes: a synthesis of recent work, p. 392-420. In: R. L. Mayden (ed.), Systematics, historical ecology and North American freshwater fishes. Stanford University Press, Stanford.

Lunel, G. 1874. Histoire naturelle des poissons du bassin du Léman. Georg, Genève, 212 pp., 20 pls.

MacCulloch, J. 1819. A description of the western islands of Scotland, including the Isle of Man: comprising an account of their geological structure; with remarks on their agriculture, scenery, and antiquities. Vol. II. Constable, Edinburgh and Hurst, Robinson, London, $589 \mathrm{pp}$.

Machordom, A., I. Doadrio \& P. Berrebi. 1995. Phylogeny and evolution of the genus Barbus in the Iberian peninsula as revealed by allozyme electrophoresis. J. Fish Biol., 47: 211-236.

Magnusson, K. P. \& M. M. Ferguson. 1987. Genetic analysis of four sympatric morphs of arctic charr, Salvelinus alpinus, from Thingvallavatn, Iceland. Env. Biol. Fish., 20: 67-73.

Maiorova, A. A. 1934. [A new herring species from lake Paleostom]. Trudy Nauchnoi Rybokhozyaistvennoi i Biologicheskoi Stantsii Gruzii, 1 (1): 21-30.

Maisch, M. 1982. Zur Gletscher- und Klimageschichte des alpinen Spätglazials. Geographica Helvetica, 37: 93-104.

Maitland, J. P. G. 1881. A new char. The Field, 8 Oct. 1881: 516.

Maitland, P. S. 1970. The origin and present distribution of Coregonus in the British Isles, p. 99-114. In: C. C. Lindsey \& C. S. Woods (eds), Biology of coregonid fishes. University of Manitoba Press, Winnipeg, 560 pp.

- 1976. Hamlyn guide to freshwater fishes. Hamlyn, London. [data based on 1977 French translation, Les poissons des lacs et rivières d'Europe en couleurs. Elsevier-Séquoia, Paris, 255 pp.]

Mäklin, F. W. 1869. En för Finland ny form af siklöja. Öfversigt af Finska VetenskapsSocietetens Förhandlingar, 11: 19-23.

Maksunov, V. A. 1961. [Materials for morphobiological characteristics of fishes of Farkhad reservoir]. Trudy Inst. Zool. y Parazitol. Akad. Nauk Tadzhikistan SSR, Dushanbe, 23: 1-159. [Russian]

Malfer, F. 1927. Il Benaco. Parte I e II. Oroidrografia ed ittiologia. Accad. Agric. Sci. Lett., Verona, $415 \mathrm{pp}$.

Malm, A. W. 1861. Raekke af fiske, krebsdyr og bløddyr, som ere nye for den skandinaviske Fauna. Forhandlinger ved de Skandinaviske Naturforskeres Ottende Møde i Kiøbenhavn, 8 (1860 [1861]): 616-624.

- 1877. Göteborgs och Bohusläns fauna, Ryggradsdjuren. Göteborg, 674 pp., 9 pls.

Malmgren, A. J. 1863. Kritisk öfversigt af Finlands fisk-fauna. Akademisk Afhandling [Dissertation], Helsingfors, xii +75 pp.

- 1864. Kritische Uebersicht der Fisch-Fauna Finlands. Aus dem Schwedischen von C. F. Frisch. Arch. Naturges., Berlin, 30 (1): 259-351.

Malmquist, H. J., S. S. Snorrason, S. Skúlason, B. Jonsson, O. T. Sandlund \& P. M. Jonasson. 1992. Diet differentiation in polymorphic arctic charr in Thingvallavatn, Iceland. J. Anim. Ecol., 61: 21-35.

Marfin, J.-P. 1982. Les problèmes liés au polymorphisme de l'espèce Atherina boyeri Risso, 1810. Cybium, 6 (4): 19-26.

Maric, D. 1989. The species revision of genus Rutilus Rafinesque, 1820 (Pisces) from western part of Balkan peninsula. Glasnik Republicklog Zavoda za Zastitu Prirode I Prirodujackog Muzeju u Titogradu, 21 (1988 [1989]): 55-79.

Marinov, B. T. \& C. I. Dikov. 1986. Cottus gobio haemusi subsp. n. (Pisces, Cottidae) from Bulgaria. Acta Zool. Bulg., 31: 18-23.

Markun, M. I. 1936. [On the taxonomy and biology of the Kama river burbot]. Izvestiya Permskogo Biologicheskogo Instituta, 10 (6): 211-236.

Marsili, A. F. 1726. Danubius Pannonico-Mysicus, observationibus geographicis, astronomicis, hydrographicis, historicis, physicis perlustratus et in sex tomos digestus. Tomus quartus. De piscibus in acquis Danubii viventibus. Gosse, Alberts and de Hondt, Hagae Comitum and Uytwerf \& Changuion, Amstelodami, ii $+92+2$ pp., 33 pls. [French translation in 1744].

Martens, E. von. 1857. Ueber einige Fische und Crustaceen der süssen Gewässer Italiens. Arch. Naturges., Berlin, 23 (1): 149-204, pls. 9-10.

- 1868. Ueber einige ostasiatische Süsswasser- 
thiere. I. Fische. Arch. Naturges., Berlin, 34: 117.

Marti, V. Y. 1940. [Taxonomy and life history of the Russian sturgeon at the Caucasian coast of the Black Sea]. Zool. Zh., 19: 865-872.

Mauduyt [père]. 1849. Ichthyologie de la Vienne. Bull. Soc. Acad. Agric. Belle Lettres Sci. Arts Poitiers, Nouv. Sér., 3: 8-49.

Mayden, R. L. 1997. A hierarchy of species concepts: the denouement in the saga of the species problem, p. 381-424. In: M. F. Claridge, H. A. Dawah \& M. R. Wilson (eds), The units of biodiversity - species in practice. Chapman \& Hall, London.

Mayden, R. L. \& B. R. Kuhajda. 1996. Systematics, taxonomy, and conservation status of the endangered Alabama sturgeon, Scaphirhynchus suttkusi Williams \& Clemmer (Actinopterygii, Acipenseridae). Copeia, 1996: 241-273.

Mayden, R. L. \& R. M. Wood. 1995. Systematics, species concepts, and the evolutionarily significant unit in biodiversity and conservation biology. Amer. Fish. Soc. Symp., 17: 58-113.

Mayr, E. 1952. Comments on evolutionary litterature. Evolution, 6: 248-252.

- 1969. Principles of systematic zoology. McGraw-Hill, New York, 428 pp.

- 1982a. Of what use are subspecies ? Auk, 99: 593-595.

- $1982 \mathrm{~b}$. The growth of biological thought. Diversity, evolution, and inheritance. Harvard University Press, Cambridge, MA, 974 pp.

Mayr, E. \& P. D. Ashlock. 1991. Principles of systematic zoology. McGraw-Hill, New York, xx +475 pp.

McClelland, J. 1842. On the freshwater fishes collected by William Griffith during his travels from 1835 to 1842 . Calcutta J. Nat. Hist. Misc. Arts Sci. India, 2: 560-589.

M'Coy, F. 1841. On some new and rare fish occuring on the coast of Ireland. Ann. Mag. Nat. Hist., Ser. 1, 6: 402-408.

McKitrick, M. C. \& R. M. Zink. 1988. Species concepts in ornithology. The Condor, 90: 1-14.

McVeigh, H. P., R. A. Hynes \& A. Ferguson. 1995. Mitochondrial DNA differentiation of sympatric populations of brown trout, Salmo trutta L., from Lough Melvin, Ireland. Can. J. Fish. Aquat. Sci., 52: 1617-1622.

Meidinger, C. von. 1785-1794. Icones piscium Austriae indigenorum quos collegit vivisque coloribus expressos edidit Carolus Lib. Baro a Meidinger. Decuria I. 1785. Sumtibus, Viennae, pls. 1-10. Decuria II. 1786. Wappler, Viennae, pls. 11-20. Decuria III. 1788. Wappler, Viennae, pls. 21-30. Decuria IV. 1790. Wappler, Viennae. pls. 31-40. Decuria V. et ultima. 1794. Wappler, Viennae, pls. 41-50.

Mela, A. J. 1882. Vertebrata fennica. Suomen luurankoiset, eli luonnontieteellisen Suomen luurankois-eläimistö. Holmin, Helsingissä, 426 pp.

Melyantsev, V. G. 1948. [Burbot of lake Novoe Vygozero]. Uchennye Zapiski Karelo-Finskogo Universiteta, 2 (3) (1947 [1948]): 90-106.

Menshikov, M. I. 1939. [On the ichthyofauna of lake Marka-Kul]. Uchennye Zapiski Permskogo Universiteta, 3 (2): 119-142.

Mertens, R. 1924. Fische aus dem nördlichen und östlichen Spanien. Arch. Naturgesch., Berlin, 90 (A 11): 1-4.

Meyer, A. 1987. Phenotypic plasticity and heterochrony in Cichlasoma managuense (Pisces, Cichlidae) and their implications for speciation in cichlid fishes. Evolution, 41: 1357-1369.

Meyer, A., T. D. Kocher, P. Basasibwaki \& A. C. Wilson. 1990. Monophyletic origin of Lake Victoria cichlid fishes suggested by mitochondrial DNA sequences. Nature, 347: 550-553.

Meyer, J. D. 1748-1756. Angenehmer und nützlicher Zeit-Vertreib mit Betrachtung curioser Vorstellungen allerhand kriechender, fliegender, und schwimmender, auf dem Land und im Wasser sich befindender und nährender Thiere, sowohl nach ihrer Gestalt und äusserlichen Beschaffenheit als auch nach der accuratest davon verfertigten Structur ihrer Scelete oder Bein-Körper / nebst einer deutlichen so physicalisch und anatomisch besonders aber osteologisch und mechanischen Beschreibung derselben nach der Natur gezeichnet, gemahlet, in Kupfer gestochen. Meyer, Nürnberg, 3 vols., 240 pls.

Michahelles, C. W. 1829. Fische des adriatischen Meers. Isis (von Oken), 22 (9): 1011-1015.

Mihai-Bardan, A. 1984. Le catalogue de la collection de poissons de Roumanie gardée au Muséum d'Histoire naturelle "Grigore Antipa". Trav. Mus. Hist. Nat. Gr. Antipa, 25: 445-472.

Miller, P. J. 1968. A new species of Pomatoschistus (Teleostei: Gobiidae) from western Sicily. Ann. Mus. Civ. Stor. Nat. G. Doria, 77: 221-231, pl. 1.

- 1972. Gobiid fishes of the Caspian genus Knipowitschia from the Adriatic sea. J. Mar. Biol. Ass. U. K., 52: 145-160.

- 1982. A new Pomatoschistus from the Mediterranean and redescription of $P$. tortonesei Miller 1968 (Pisces: Gobiidae). Senck. Biol., 62 (1981 [1982]): 5-19.

Miller, R. R. \& C. L. Hubbs. 1969. Systematics of Gasterosteus aculeatus, with particular ref- 
erence to intergradation and introgression along the Pacific coast of North America: a commentary on a recent contribution. Copeia, 1969: 5269.

Minelli, A. 1993. Biological systematics - The state of the art. Chapman \& Hall, London, 387 pp.

Mishler, B. D., Donoghue, M. J. 1982. Species concepts: a case for pluralism. Syst. Zool., 31: 491-503.

Mišík, V. 1957a. Porovnanie plotíc z dunajského systému (Rutilus rutilus carpathorossicus Vladykov) s ploticou z labskej tône Poltruba $(R u-$ tilus rutilus frici ssp. n.). Acta Rer. Nat. Mus. Natn. Slov., 3 (4): 1-27.

- 1957b. Biometrika plotice lesklej Dunajskej Rutilus pigus (Lacépède 1804) virgo (Heckel, 1852) [Biometrik des Frauenfisches aus der Donau Rutilus pigus (Lacépède, 1804) virgo (Heckel, 1852)]. Biológia, Bratislava, 12: 177191.

- 1958. Biometrika Dunajského kapra (Cyprinus carpio carpio L.) z Dunajského systému na Slovensku. Biol. Práce, 4 (6): 55-125.

- 1959. Pstruh obyčajný čiernomorský forma potočná (Salmo trutta labrax Pallas 1811 morpha fario Linné, 1758) z údolnej nádrže na Hnilci pri obci Dedinky [The brown trout (Salmo trutta labrax Pallas 1811 morpha fario Linné 1758) from the dam lake on Hnilec River near Dedinky]. Biológia, Bratislava, 14: 763-773.

Miyamoto, M. M. \& J. E. Cracraft (eds). 1991. Phylogenetic analysis of DNA sequences. Oxford Univ. Press, NY, x+358 pp.

Mohr, E. 1927. Mugiliden-Studien. Zool. Jahrb., Abt. Syst., 54: 177-202.

Mohr, N. 1786. Førsog til en Islandsk naturhistorie, med adskillige oekonomiske samt andre anmaertkninger. Holm, Kjøbenhavn, xvi+413 pp., 7 pls.

Molin, R. 1853. Acipenser Vallisnerii. Nuova specie descritta. Riv. Period. Lavori Acad. Sci. Lett. Arti Padova, 1 (1851-53): 366-371.

Moran, P., I. Kornfield \& P. N. Reinthal. 1994. Molecular systematics and radiation of the haplochromine cichlids (Teleostei: Perciformes) of Lake Malawi. Copeia, 1994: 274-288.

Moreau, E. 1881. Histoire naturelle des poissons de la France. Masson, Paris, 1: vii+480 pp., 2: 572 pp., 3: 697 pp.

- 1891. Le syngnathe à nageoire pectorale courte (Syngnathus microchirus, n. sp. ). Bull. Soc. Zool. Fr., 16: 187-190.

Movchan, Y. V. 1967. [Acipenser güldenstädti Brandt of the north-west part of the Black Sea and the Kuban River]. Vestn. Zool., 6: 26-32. [Russian, English summary]

Mrakovcic, M., M. Kerovec, S. Misetic \& D. Schneider. 1994. New freshwater species of goby in the ichthyological region of Dalmatia, Croatia, p. 46. In: Symposium on the conservation of endangered freshwater fish in Europe. 20-22 July 1994, Bern. Program, abstracts, participants.

- 1996. Description of Knipowitschia punctatissima croatica, (Pisces: Gobiidae), a new freshwater goby from Dalmatia, Croatia, p. 311-319. In: A. Kirchhofer \& D. Hefti (eds), Conservation of endangered freshwater fish in Europe. Birkhäuser, Basel, 341 pp.

Mrakovcic, M., S. Misetic \& M. Povz. 1995. Status of freshwater fish in Croatian Adriatic river systems. Biol. Cons., 72: 179-185.

Mukhomediyarov, F. B. 1948. [Least cisco (Coregonus sardinella baunti subsp. nova) from the Tsipo-Tsipikan system of lakes in the Vitim river basin]. Doklady na Pervoi Nauchnoi Sessii Yakutskoi Bazy Akad. Nauk SSSR, Yakutsk, 1948, pp. 270-280.

Müller, G. 1958. Scardinius racovitzai n. sp. (Pisces, Cyprinidae), eine reliktäre Rotfeder aus Westrumänien. Senck. Biol., 39: 165-168.

Miinzing, J. 1962. Ein neuer semiarmatus-Typ von Gasterosteus aculeatus L. (Pisces) aus dem Izniksee. Mitt. Hamburg. Zool. Mus. Inst., 60: 181-194.

- 1963. The evolution of variation and distributional patterns in European populations of the three-spined stickleback, Gasterosteus aculeatus. Evolution, 17: 320-332.

- 1969. Variabilität, Verbreitung und Systematik der Arten und Unterarten in der Gattung Pungitius Coste, 1848 (Pisces, Gasterosteidae). Ztschr. Zool. Syst. Evol. Forsch., 7: 208-233.

Muus, B. J. \& P. Dahlström. 1967. Europas ferskvandsfisk. Gads, København. [data based on 1968 French translation, Guide des poissons d'eau douce et pêche. Delachaux \& Niestlé, Neuchâtel, 243 pp.]

Myers, G. S. 1960. The endemic fish fauna of Lake Lanao, and the evolution of higher taxonomic categories. Evolution, 14: 323-333.

Nagelkerke, L. A. J., F. A. Sibbing, J. G. M. van den Boogaart, E. H. R. R. Lammens \& J. W. M. Osse. 1994. The barbs (Barbus spp.) of lake Tana: a forgotten species flock? Env. Biol. Fish., 39: $1-22$.

Nakabo, T. (ed.). 1994. [Fishes of Japan with pictorial keys to the species]. Tokai University Press, Tokyo, 1474 pp. [Japanese] 
Nalbant, T. T. 1957. Cobitis aurata vallachica, eine neue Unterart des Balkan-Steinpeitzgers (Pisces, Cobitidae). Senck. Biol., 38: 209-312.

- 1993. Some problems in the systematics of the genus Cobitis and its relatives (Pisces, Ostariophysi, Cobitidae). Rev. Roum. Biol., Biol. Anim., 38: 101-110.

Nardo, D. [G. D., J. D.] 1824. Osservazioni ed aggiunte all' Adriatica Ittiologia pubblicata dal sig. Cav. Fortunato Luigi Naccari presentate dal sig. Domenico Nardo al sig. Giuseppe Cernazai di Udine. Giorn. Fis. Chim. St. Nat. Med. Arti Pavia, Decade 2, 7 (17): 222-234, 249-263.

- 1827a. Prodromus observationum et disquisitionum ichthyologiae Adriaticae. Isis (von Oken), 20 (6): 472-489.

- 1827b. Prodromus observationum et disquisitionum Adriaticae ichthyologiae. Giornale di Fisica, Chimica, Storia naturale, Medicina ed Arti, Pavia, Decade Seconde, 10: 22-40.

- 1847. Sinonimia moderna delle specie registrate nell'opera intitolata: Descrizione de' crostacei, de' testacei e de' pesci che abitano le lagune e golfo veneto rappresentati in figure, a chiaroscuro ed a colori dall'Abate Stefano Chiereghini ven. Clodiense. Antonelli, Venezia, xi+128 pp.

- 1860. Prospetti sistematici degli animali delle provincie Venete e del mare Adriatico e distinzione delle specie in gruppi relative alla loro geografia fisica ed all'interesse economico statistico che presentano. Antonelli, Venezia, 104 pp.

- 1866. [...] Relazione sopra un opusculo donato all'I. R. Istituto dal sig. prof. Giovanni Canestrini di Modena intitolata Note ittiologiche, con relative sue illustrazioni. Atti Ist. Veneto Sci., Lett. Arti, Ser. 3, 11: 139-145.

Nau, B. S. 1791. Bemerkungen zu des Herrn Prof. Sanders Beyträge zur Naturgeschichte der Fische im Rhein. Der Naturforscher, 25: 24-34.

Nei, M. 1978. Estimation of average heterozygosity and genetic distance from small numbers of individuals. Genetics, 89: 583-590.

Neill, P. 1808. A list of fishes found in the Frith of Forth, and rivers and lakes near Edinburgh, with remarks. Mem. Wernerian Nat. Hist. Soc. Edinburgh, 1: 526-555.

Neresheimer, E. 1937. Die Lachsartige (Salmonidae). 1. Teil, p. 219-365. In: R. Demoll \& H. N. Maier (eds), Handbuch der Binnenfischerei Mitteleuropas. Band IIIA. Schweizerbart, Stuttgart.

Neyelov [Neelov], A. V. 1979. The sensory line system and classification of cottoid fishes (Cottidae: Myoxocephalinae, Artediellinae). Nauka, Leningrad, 208 pp.
Ng, P. K. L. \& H. H. Ng. 1995. Hemibagrus gracilis, a new species of large riverine catfish (Teleostei: Bagridae) from Peninsular Malaysia. Raffles Bull. Zool., 43: 133-142.

Nichols, J. T. 1925. The two Chinese loaches of the genus Cobitis. Amer. Mus. Novit., 170: 1-4.

Nielsen, J. G. 1974. Fish types in the Zoological Museum of Copenhagen. Zoological Museum, Copenhagen, 115 pp.

Nikolski, A. M. 1923. Novyj vid sel'di iz Chernogo morya. Byulleten Gos. Chernnomorsko-Azovskoi Nauchnoprom. Opitn. Stantsii, 8-9: 4-6.

Nikolski, G. W. 1933. Zur Systematik der mittelasiatischen Vertreter der Plötze [Rutilus rutilus (L.) Pisces Cyprinidae]. Zool. Anz., 102: 260266.

- 1936. [Sur la variabilité géographique de Gobio gobio (L. ) du nord-est de Kazachstan et de la Sibérie occidentale]. Trav. Inst. Zool. Acad. Sci. URSS, 3: 457-473. [Russian, French summary]

Nilsson, N.-A. \& O. Filipsson. 1971. Characteristics of two discrete populations of Arctic char (Salvelinus alpinus L.) in a north Swedish Lake. Rep. Inst. Freshwat. Res. Drottningholm, 51: 90-108.

Nilsson, S. 1832. Prodromus ichthyologiae scandinavicae. Berlingianis, Lund, 124 pp.

- 1855. Skandinavisk fauna. Fjerde delen [Fourth part]: Fiskarna. Gleerups, Lund, xxxiv +768 pp

Ninni, A. P. 1872. Rivista critica delle specie di pesci adriatici descritte nell' opera manoscritta dell' Abate Stefano Chiereghini di Chioggia. Fascicolo primo. Antonelli, Venezia, $63 \mathrm{pp}$.

- 1882. Nuova specie di Gobius. Atti Soc. VenetoTrentina Sci. Nat. Padova, 8: 276-279, pl. 15.

- 1930. Sopra la Trutta adriatica (Kolomb.). Bull. Soc. Zool. Fr., 55: 446-451.

- 1938. I Gobius del mari e delle acque interne d'Italia. Memorie R. Comitato Talassogr. Ital., 242: 1-169, pls. 1-19.

Nordeng, H. 1961. On the biology of char (Salmo alpinus L.) in Salangen, North Norway. I. Age and spawning frequency determinated from scales and otoliths. Nytt Magasin for Zoologi, Oslo, 10: 67-123.

- 1983. Solution to the "char problem" based on Arctic char (Salvelinus alpinus) in Norway. Can. J. Fish. Aquat. Sci., 40: 1372-1387.

Nordmann, A. de. 1840-1842. Prodrome de l'ichthyologie pontique, p. 353-549, 32 pls. In: A. de Sainson, F. Le Play, A. Huot, J. H. Léveillé, D. A. M. Raffet, L. Rousseau, A. de Nordmann \& A. du Ponceau, Voyage dans la Russie méridionale et la Crimée, par la Hongrie, la Vallachie et la Moldavie, exécuté 
en 1837, sous la direction de M. Anatole de de Démidoff. Tome troisième. Observations sur la faune pontique. Bourdin, Paris, 756 pp. [text dated 1840, plates dated 1842].

Novikov, P. I. 1947. [Characteristics of the fish resources of lake Keret' in the White Sea basin]. Bulleten' Rybnogo Khozyajstva Karelo-Finskoy SSR. Trudy 1 Nauchno-Tekhnicheskoy Konferentsii po Rybnoj Promyshlennosti KareloFinskoy SSR, Petrozavodsk, 1: 239-248. [Russian]

Niimann, W. 1953. Artanalyse und Wachstumsuntersuchungen an Salmo lacustris und Salmo carpio. Ztschr. Fisch. Hilfswiss., N. F., 2: 271-316.

- 1967. Markierungsversuche an Bach- und Regenbogenforellen im Bodensee als Beitrag zur systematischen Eingliederung von Forellen. Schweiz. Ztschr. Hydrol., 29: 269-283.

- 1972. The Bodensee: effects of exploitation and eutrophication on the salmonid community. J. Fish. Res. Board Canada, 29: 833-847.

- 1978. Versuch einer Klassifizierung der Bodenseecoregonen durch Vergleich kombinierter Merkmale und die Eingliederung des sogenannten Braunfelchen. Arch. Hydrobiol., 82: 500521

Niisslin, O. 1882a. Beiträge zur Kenntnis der Coregonus-Arten des Bodensees und einiger anderer nahegelegener nordalpiner Seen. II. Eine wissenschaftlich neue Coregonspecies. Zool. Anz., 5: 164-169, 182-189.

1882b. Beiträge zur Kenntnis der CoregonusArten des Bodensees und einiger anderer nahgelegener nordalpiner Seen. III. Muthmassliche Varietäten des Coregonus macrophthalmus Niissl. Zool. Anz., 5: 207-212.

- 1882c. Beiträge zur Kenntnis der CoregonusArten des Bodensees und einiger anderer nahgelegener nordalpiner Seen. IV. Coregonus Sulzeri nova species, Pfäffikoner Albuli. Zool. Anz., 5: $253-258$

- 1882d. Beiträge zur Kenntniss der CoregonusArten des Bodensees und einiger anderer nahgelegener nordalpiner Seen. V. Coregonus Steindachneri, nova species, der Rheinankel oder die Rheinanke des Traunsees. Zool. Anz., 5: 279306

- 1903. Die Schweizer Coregonenspecies. Eine Erwiderung auf Dr. V. Fatio's "Deux mots à propos du Coregonus macrophthalmus de Nüßlin". Zool. Anz., 36: 393-406.

Nybelin, O. 1940. Nytt västsvenskt fynd av relikt hornsimpa. Fauna och Flora, 35: 71-79.

Nyman, L. 1972. A new approach to the taxonomy of the "Salvelinus alpinus species complex".
Rep. Inst. Freshw. Res. Drottningholm, 52: 103131.

Nyman, L., J. Hammar \& R. Gydemo. 1981. The systematics and biology of landlocked populations of arctic char from northern Europe. Rep. Inst. Freshw. Res. Drottningholm, 59: 128-141.

Odenwall, E. 1927. Cottus gobio L. var. roseus n var. Mem. Soc. Fauna Flora Fennica, 2 (19251926 [1927]): $56-57$.

Ogérien, 1863. Histoire naturelle du Jura et des départements voisins. Tome III. Zoologie vivante. Masson, Paris, $\mathrm{xx}+570 \mathrm{pp}$.

Okada, Y. 1959-1960. Studies on the freshwater fishes of Japan. J. Fac. Fish. Pref. Univ. MieTsu, 4: 1-860, 61 pls.

Oken, L von. 1817. Cuviers und Okens Zoologien neben einander gestellt. Isis (von Oken), 8: $1145-1184$.

Olafsen, E. 1772. Vice-Lavmand Eggert Olafsens of Land-Physici Biarne Povelsens Reise igiennem Island. Lindgren, Sorse, 1: 1-618, 51 pls.

- 1774. Des Vice-Lavmands Eggert Olafsens und des Landphysici Biarne Povelsens Reise durch Island, veranstaltet von der königlichen Societat der Wissenschaften in Kopenhagen. Aus dem Dänischen Übersetzt. Heinecke \& Faber, Kopenhagen \& Leipzig, 328 pp., 25 pls.

Oliva, O. 1961. Bemerkungen uiber einige Fischarten aus Albanien. Acta Soc. Zool. Bohemoslov., 25: 39-54.

- 1963. The Gdansk swamp-minnow Phoxinus percnurus gdaniensis Berg, 1932 its rediscovery and systematic position. Acta Biol. Medica Soc. Scient. Gedariensis, 7: 307-337, pls. 1-4.

Oliva, O., L. Hanel \& V. Safránek. 1989. On the systematics of the perch (Perca fluviatilis) (Pisces, Perciformes). Vestn. Ceskoslov. Společ. Zool., 53: 214-225.

Oliva, O. \& G. Zanandrea. 1959. Posizione sistematica di una lampreda di cilistovo (Cecoslovacchia). Doriana, 2 (98): 1-5.

O'Maoileidigh, N., S. Cawderi, J. J. Bracken \& A. Ferguson. 1988. Morphometric, meristic character and electrophoretic analysis of two Irish populations of twaite shad, Alosa fallax (Lacépède). J. Fish Biol., 32: 355-366.

Opalatenko, L. K. 1967. [On morphological and ecological peculiarities of Abramis sapa of the upper Dniester]. Vestn. Zool., 6: 33-37. [Russian, English summary]

O'Reilly, B. 1813. Catalogue of the subjects of natural history, in the Museum of the Right Hon. and Hon. The Dublin Society. Dublin.

Ortí, G., M. A. Bell, T. E. Reimchen \& A. Meyer. 1994. Global survey of mitochondrial 
DNA sequences in the threespine stickleback: evidence for recent migrations. Evolution, 608622 .

Osbeck, P. 1756. Beskrifning öfver fisken Mal. Kongl. Svenska Vetenskaps Academiens Handlingar [för År 1756], 17: 34-39, pl. 3.

- 1757. Dagbok öfvers en Ostindisk resa ăren 1750-52, med anmärkningar uti naturkundigheten, främmande folkslags språk [...]. Stockholm, vi+376 pp., 12 pls.

- 1765. Reise nach Ostindien und China. Koppe, Rostock, xxvi+552pp, 13 pls.

Osinov, A. G. 1984. [Zoogeographical origins of brown trout, Salmo trutta (Salmonidae): data from biochemical genetic markers]. Vopr. Ikhtiol., 24: 11-24. [Russian, English translation in: J. Ichthyol., 24: 10-23]

- 1989. [Low level of genetic variability and differentiation in ecological forms of Sevan trout Salmo ischchan Kessler]. Genetika, 25: 18171835. [translated in 1990 in Soviet Genetics, 25: 1210-1216]

Otterstrøm, C. V. 1922. Heltling (Coregonus albula L.) og helt (Coregonus lavaretus L.) i Danmark. Undersøgelser af de Ferske Vandes Fiskeriforhold, Kolding, 2: 1-50.

Otto, A. G. 1821. Animalium quorandum maritimorum mondum editorum. Pars Prior. Isis (von Oken), 1821 (2) (9, Beilage 18): 1-5.

- 1821b. Conspectus animalium quorundam maritinorum nondum editorum. Inaug. Dissert., Vratislaviae, $20 \mathrm{pp}$.

Paepke, H.-J. 1982. Phänogeographische Strukturen in den Süsswasserpopulationen von Gasterosteus aculeatus L. (Pisces, Gasterosteidae) in der DDR und ihre evolutionsbiologischen Aspekte. Mitt. Zool. Mus. Berlin, 58: 269-328.

- 1983. Die Stichlinge - Gasterosteidae. Neue Brehm Bücherei. Ziemsen, Wittenberg, 144 pp.

Paepke, H.-J. \& R. Fricke. 1992. Kritischer Katalog der Typen der Fischsammlung des Zoologischen Museums Berlin. Teil 4: Scorpaeniformes. Mitt. Zool. Mus. Berlin, 68: 267-293.

Paepke, H.-J. \& K. Schmidt. 1988. Kritischer Katalog der Typen der Fischsammlung des Zoologischen Museums Berlin. Teil 2: Agnatha, Chondrichthyes. Mitt. Zool. Mus. Berlin, 64: 155-189, pls. 7-8.

Paepke, H.-J. \& L. Seegers. 1986. Kritischer Katalog der Typen und Typoide der Fischsammlung des Zoologischen Museums Berlin. Teil 1: Atheriniformes. Mitt. Zool. Mus. Berlin, 62: 135-186.

Pallas, P. S. 1767-1778. Spicilegia zoologica, quibus novae imprimis ezt obscurae animalium species iconibus, descriptionibus atque comentariis illustrantur. Berlini, 2 vols. [pisces: vol 1, (7) 1769: 1-42, 6 pls; (8) 1770: 1-54, 5 pls.]

- 1771. Reise durch verschiedene Provinzen des Russischen Reichs. Erster Theil. Kaiserlichen Academie der Wissenschaften, St. Petersburg, 504 pp.

- 1773. Reise durch verschiedene Provinzen des Russischen Reichs. Zweyter Theil. Kayserlichen Academie der Wissenschaften, St. Petersburg, 744 pp., pls.

- 1776. Reise durch verschiedene Provinzen des Russischen Reichs. Dritter Theil. Kayserlichen Academie der Wissenschaften, St. Petersburg, 760 pp., pls.

- 1814. Zoographia rosso-asiatica, sistens omnium animalium in extenso Imperio Rossico et adjacentibus maribus observatorum recensionem, domicilia, mores et descriptiones anatomen atque icones plurimorum. Vol. 3. Animalia monocardia seu frigidi sanguinis imperii rosso-asiatici recensente P. S. Pallas. Supplendis quirusdam ranarum descriptionibus et iconibus imprimis piscium camtschaticorum auxit et locupletavit Guil. Theophil. Tilesius. Academia Scientiarum, Petropolis. 428 pp. [See Svetovidov, 1978, 1981 for history of this book; most plates referred to in text have not been published; this volume is often reported as published in 1811, but the publication date for nomenclatural purposes is 1814 , see Opinion 212 of International Commission on Zoological Nomenclature]

Parenti, L. R. 1984. A taxonomic revision of the Andean killifish genus Orestias (Cyprinodontiformes, Cyprinodontidae). Bull. Amer. Mus. Nat. Hist., 178: 107-214.

Parnell, R. 1838a. Observations on the Coregoni of Loch Lomond. Ann. Mag. Nat. Hist., Ser. 1, 1: 161-165, pl. .

- 1838b. Prize essay on the natural and economical history of the fishes, marine, fluviatile and lacustrine of the river district of the Firth of Forth. Memoirs of the Wernerian Natural History Society, Edinburgh, 7 (1831-1837 [1838]): 161-460, 515-520, pls. 18-44. [apparently also distributed as a separate volume with independent pagination]

- 1839a. An account of three new species of British fishes, with some remarks on twenty others new to the coast of Scotland. Trans. Roy. Soc. Edinburgh, 14: 137-145, pls. 4-5.

- 1839b. Account of a new species of British bream, and of an undescribed species of skate: to which is added a list of the fishes of the 
Frith of Forth, and its tributary streams, with observations. Trans. Roy. Soc. Edinburgh, 14: 146-157, pls. 6-8.

Patarnello, T., L. Bargelloni, F. Caldara \& L. Colombo. 1994. Cytochrome $b$ and 16S rRNA sequence variation in the Salmo trutta (Salmonidae, Teleostei) species complex. Molec. Phylogen. Evol., 3: 69-74.

Paterson, H. E. H. 1985. The recognition concept of species. Transvaal Mus. Monogr., 4: 21-29.

Paula Schrank, F. von 1783. [Auszüge aus Briefen:] An den Herrn D. Bloch von Herrn Professor von Paula Schranck zu Burghausen vom 30. November 1782. Schrift. Berlin. Ges. Naturforsch. Freunde, 4: 427-429.

- 1792. Nähere Bestimmung dreyer Barscharten. Abhandlungen einer Privatgesellschaft von $\mathrm{Na}$ turforschern und Oekonomen in Oberdeutschland, 1: 98-103.

- 1798. Fauna boica: Durchgedachte Geschichte der in Bayern einheimischen und zahmen Thiere. Stein, Nürnberg, 1 vol. (in 2 parts), 720 pp.

Pavesi, P. 1877. Spigolature nel Museo Zoologico dell'Universitá di Pavia. Rendiconti Reale Ist. Lombardo Sci. Lettere, Ser. 2, 10: 494-504.

Pavlov, P. I. 1953. [Umbra from the lower Danube]. Zool. Zh., 32: 272-276.

- 1956. [Comparison between the Danubian and Dneprovian bream]. Zool. Zh., 35: 891-896, summaries (6), p. 10. [Russian, English summary]

- 1959. [The clupeid genus Alosa in the northwestern part of the Black Sea]. Izd. Akad. Nauk. USSR, Kiev, 252 pp. [Ukrainian]

- 1961. [Morphometric characteristics of the Dnepr Sea roach and its economical importance]. Zool. Zh., 40: 244-250. [Russian, English summary]

Paxton, J. R., D. F. Hoese, G. R. Allen \& J. E. Hanley. 1989. Zoological catalogue of Australia. Vol. 7. Pisces. Petromyzontidae to Carangidae. Australian Government Publishing Service, Canberra, xii+665 pp.

Payne, R. H., A. R. Child \& A. Forrest. 1971. Geographic variation in the Atlantic salmon. Nature, 231: 250-252.

Pedroli, J.-C., B. Zaugg \& A. Kirchhofer. 1991. Atlas de distribution des poissons et cyclostomes de Suisse. Centre Suisse de Cartographie de la Faune, Neuchâtel, 206 pp.

Pellegrin, J. 1924a. Les salmonidés du Maroc. C. R. Acad. Sci. Paris, 178: 970-972.

- 1924b. Le Salmo pallaryi Pellegrin, poisson du Moyen Atlas marocain. Bull. Mus. Natn. Hist. Nat., Paris, Sér. 2, 30: 181-184.
- 1927. Poissons d'Asie mineure recueillis par M. H. Gadeau de Kerville. Bull. Soc. Zool. Fr., 52: $36-37$.

- 1929. La loche du Maroc. Bull. Soc. Zool. Fr., 54: 524-526.

Pelz, G. R. 1987. Der Giebel: Carassius auratus gibelio oder Carassius auratus auratus? Natur und Museum, 117: 118-129.

Penczak, T. 1964. Three-spined stickleback from Iceland Gasterosteus aculeatus islandicus Sauvage. Annales Zoologici, 22: 441-448.

Pennant, T. 1769. British Zoology. 2nd edition. Vol. 3. White, London.

- 1776. British Zoology.4th edition. Vol. 3. Chester, London, 425 pp., 73 pls.

- 1784-1785. Arctic zoology. London, 3 vols. 1812: British zoology. 5th edition. Vol. 3. Walker et al., London, 546 pp., 487 pls.

Perdices, A., A. Machordom \& I. Doadrio. 1995. Allozyme variation of African and Iberian populations of the genus Cobitis. J. Fish Biol., 47: 707-718.

Persat, H., D. Beaudou \& J. Freyhof. 1996. The schulpin of the Lez spring (South France), Cottus petiti (Bacescu and Bacescu-Mester, 1964), one of the most threatened fish species in Europe, p. 321-328. In: A. Kirchhofer \& D. Hefti (eds), Conservation of endangered freshwater fish in Europe. Birkhäuser, Basel, 341 pp.

Perty, J. A. M. 1832. Beyträge zur Kenntniss der Fauna Monacensis. Isis (von Oken), 1832 (7): 711-733.

Peters, W. 1852. Diagnosen von neuen Flussfischen aus Mossambique. Monatsber. K. Preuss. Akad. Wiss., Berlin, 1852: 681-685.

- 1875. Über eine neue Art von Maränen, Coregonus generosus, aus der Mark Brandenburg. Monatsber. K. Preuss. Akad. Wiss., Berlin, 1874 [1875]: 790-795.

Petrov, V. V. 1926. [To the knowledge of the Caucasian bleaks]. Bull. Ichthyol. Lab. Baku, 2: 133-160.

- 1930. Die geographische Variabilität von Alburnus alburnus L. Zool. Anz., 88: 141-150.

Phillips, A. R. 1982. Subspecies and species: fundamentals, needs, and obstacles. Auk, 99: 612-615.

Pietsch, T. W. 1985. The manuscript materials for the Histoire naturelle des poissons, 1828-1849: sources for understanding the fishes described by Cuvier and Valenciennes. Arch. Nat. Hist., 12: $59-106$.

Pietschmann, V. 1937. Eine neue Lokalform von Cobitis taenia L. (Cyprinoidea), Cobitis taenia 
tesselatus. Anz. Akad. Wiss. Wien, 74: 29-30.

Pinchuk, V. I. 1976. [Systematics of the goby, genera Gobius Linné (native species), Neogobius Iljinu and Mesogobius Bleeker]. Vopr. Ikhtiol., 16: 600-609 [translation: J. Ichthyol., 16: 543$552]$.

- 1977. [The systematics of gobies of the genera Gobius Linne (native species), Neogobius Iljin and Mesogobius Bleeker]. Vopr. Ikhtiol., 17: 587-596 [translation in J. Ichthyol., 17: 517$166]$.

- 1978. [A new species of goby from the western part of the Black sea, Knipowitschia georghievi Pinchuk, sp. n. (Gobiidae, Pisces)]. Zool. Zh. 57: 796-799.

Pirola, E. 1930. L'agone, fécondation et incubation artificielles. Bull. Fr. Piscic., 22: 225-230.

Pirisinu, Q. \& M. Natali. 1980. Gobius nigricans Canestrini (Pisces, Osteichthyes, Gobiidae) endemico dell'Italia centrale. Riv. Idrobiol., 19: 593-617.

Pizzul, E., M. Specchi \& G. Valli. 1993. Gobio gobio benacensis (Pollini, 1816) (Osteichthyes, Cyprinidae) nelle acque del Friuli-Venezia Giulia. Atti Mus. Civ. Stor. Nat. Trieste, 45: 163168.

Plater, A. 1861. Opisanie hydrograficzno-statystyczne Dzwiny zachodniej, oraz ryb w niej zyjacych [Description of the West Duina river and its fishes]. Wilno, $69 \mathrm{pp}$.

Pokrovsky, V. V. 1951. [Materials on the investigation of intraspecific variation in the perch (Perca fluviatilis L.)]. Trudy Kar.-Finsk. Otd. VNII Ozern. i Rechn. Rybn. Kh-va, 3: 95149.

Poljakov, G. D., N. Filipi \& K. Basho. 1958. Peshqit e shqiperise. Universiteti Shtetébror, Tiranéb, $286 \mathrm{pp}$.

Pollini, C. 1816. Viaggio al Lago di Garda e al Monte Baldo in cui si ragiona delle cose naturali di quei luoghi aggiuntori un cenno sulle curiosita del Bolca e degli altri monte veronesi. Mainardi, Verona, $152 \mathrm{pp}$.

Polyakov, I. S. 1874. [On whitefishes]. Trudy Sankt-Petersburgskogo Obshchestva Estestvoispytatelei, 5: minutes xxix-xxxi.

Pomini, F. P. 1940. Ricerche sul Salmo macrostigma Dum. Boll. Pesca Piscic. Idrobiol., 18: 1363.

- 1941. Ricerche sui Salmo dell'Italia peninsulare. I. La trota del Sagittario (Abruzzi): Salmo ghigii (n. sp. ). Atti Soc. Ital. Sci. Nat. Mus. Civ. Stor. Nat. Milano, 80: 33-48.

Pontoppidan, E. 1765. Kurzgefasste Nachrichten, die Naturhistorie in Dännemark betreffend.
Rothen, Kopenhagen \& Bock, Hamburg, 232 pp., 17 pls.

Popescu-Gorj, A. \& M. Dimitriu. 1950. Observatiunii piscicole la Lacul Rosu (Ciuc). Buletinul Institutului de Cercetari si Proiectari Piscicole, 9: 81-99.

Popov, A. M. 1930. [Mullets of Europe (Mugilidae) with descriptions of a new species from the Pacific Ocean]. Trudy Sevastopol. Biol. Sta., 2: 47-125, pls. 1-5 [Russian, English summary]

Pravdin, I. F. 1927. [Rutilus rutilus caspius natio knipowitschi, a roach from the Astrabad Bay]. Sbornik v Chest' Prof. N. M. Knipovicha, Moskva, Izd. Akad. Nauk SSSR, pp. 83-88. [Russian]

- 1929. [The Suna whitefish Coregonus lavaretus lavaretoides Poljakow natio sunensis nova. Taxonomy]. Izvestiya Otdela Prikladnoi Ikhtiologii i nauchno-promyslovykh Issledovanii, 10 (1): 7-207, 6 pls.

- 1931a. [Whitefishes of the Lake District]. Izvestiya Ikhtiologicheskogo Instituta, 12 (1): 166-235, 12 pls.

- 1931b. [Whitefishes. Habits, varieties, breeding, and fishing]. Gos. Izd. Sel'-Khoz. Lit., Moskva, pp. 3-56.

- 1945. Uchenye Zapiski Leningradskogo Universiteta, 75 .

- 1948a. [Morphological classification and genesis of the whitefishes (genus Coregonus s. str.) of lake Ladoga]. Izv. Karelo-Finskoi Bazy Akad. Nauk SSSR, Petrozavodsk, 1947 [1948] (1-2): $75-83$.

- 1948b. [Whitefishes of Vygozero]. Uchennye Zapiski Karelo-Finskogo Universiteta, 2 (3) (1947 [1948]): 58-71.

- 1949. [Morpho-biological classification and genesis of the whitefishes (Coregonus s. str.) of lake Onega and its basin]. Izv. Karelo-Finskoi Bazy Akad. Nauk SSSR, Petrozavodsk, 1949 (1): $40-46$.

- 1950. [Morpho-biological classification of the whitefish in the White Sea basin, within the Karelo-Finskoy SSR]. Izv. Karelo-Finskoi Fil. Akad. Nauk SSSR, Petrozavodsk, 1950 (1).

- 1954. [Coregonids in Karelo-Finskoy SSR]. Akad. Nauk SSR, Moskva \& Leningrad, 324 pp [Russian]

Pravdin, I. F. \& L. S. Berg. 1948. [Fishes of the Kola Bay]. Izv. Instituta Ozërnogo i Rechnogo Rybnogo Khozya'stva, 26 (2): 3-24. [Russian]

Pravdin, I. F., F. R. Golubev \& K. I. Belyaeva. 1938. [On the systematic position of the Ladoga whitefish (Coregonus albula Linne infraspecies ladogae nova)]. Uchenye Zapiski Leningrad- 
skogo Universiteta, Ser. Biol., 5 (3) (1937 [1938]): 216-234.

Presa, P., F. Krieg, A. Estoup \& R. Guyomard. 1994. Diversité et gestion génétique de la truite commune: apport de l'étude du polymorphisme des locus protéiques et microsatellites. Génét. Sél. Evol., 26 (Suppl. 1): 183-202.

Quartier, A. 1951. Morphologie et biologie de Salvelinus alpinus dans le lac de Neuchâtel. Rev. Suisse Zool., 58: 631-637.

Quigley, D. T. G. \& K. Flannery. 1996. Endangered freshwater fish in Ireland, p. 27-34. In: A. Kirchhofer \& D. Hefti (eds), Conservation of endangered freshwater fish in Europe. Birkhäuser, Basel, 341 pp.

Quignard, J.-P. \& F. Kartas. 1977. Les aloses fintes Alosa fallax (Lacepède, 1803) poissons clupéiformes de l'Atlantique nord-est et de la Méditerranée. Etude des caractères numériques. Bull. Mus. Natn. Hist. Nat. Paris, Sér. 3, 501 (Zool. 350): 1241-1256.

Ráb, P. \& O. Slavík. 1996. Diploid-triploidtetraploid complex of the spined loach, genus Cobitis in Pšovka Creek: the first evidence of the new species of Cobitis in the ichthyofauna of the Czech Republic. Acta Univ. Carol., Biol., 39 (1995 [1996]): 201-214.]

Radde, G. 1899. Die Sammlungen des Kaukasischen Museums. Band I. Zoologie. Tiflis, 521 pp. .

Rafinesque Schmaltz, C. S. 1810a. Caratteri di alcuni nuovi generi e nuove specie di animali e piante della Sicilia, con varie osservazioni sopra i medesimi. Palermo, 105 pp., 20 pls.

- 1810b. Indice d'ittiologia siciliana ossia, catalogo metodico dei nomi latini, italiani, e siciliani dei pesci, che si rinvengono in Sicilia disposti secondo un metodo naturale eseguito da un appendice che contiene la descrizione di alcuni nuovi pesci siciliani. Messina, 70 pp., 2 pls.

- 1820. Ichthyologia Ohiensis or natural history of the fishes inhabiting the river Ohio and its tributary streams, preceded by a physical description of the Ohio and its branches. Rafinesque, Lexington, Ky., 90 pp. [also as parts 1-9 in Western Rev. Misc. Mag., vols. 1-2, 1819-1820].

Ragimov [Rahimov], D. B. 1978. [On the systematic status of some species of the genus Benthophilus (family Gobiidae) from the Caspian Sea and the Sea of Azov]. Vopr. Ikhtiol., 18: 791-798 [translation in J. Ichthyol., 18: 701708].

Rakaj, N. \& A. llloko. 1995. Conservation status of freshwater fish of Albania. Biol. Cons., 72: 195-199.
Rapp, W. von. 1854. Die Fische des Bodensees. Jahreshefte Ver. Vaterl. Naturk. Württemberg, 10: $137-175$.

Rathke, M. H. 1837. Beitrag zur Fauna der Krym. Fische. Mém. Sav. Etranger St. Pétersbourg, 3: 309-354.

Ray, J. F. 1710. Synopsis methodica avium \& piscium; opus posthumum: quod vivus recensuit \& perfecit ipse auctor, in quo multas species in ipsius ornithologia \& ichthyologia desideratas adjecit, methodumque suam piscium natuare magis convenientem reddidit. Londinum [London].

Regan, C. T. 1906. The vendaces of Lochmaben and of Derwentwater and Bassenthwaite Lakes, Coregonus vandesius and Coregonus gracilior. Ann. Mag. Nat. Hist., Ser. 7, 17: 180-182, pl. 7. - 1908a. A preliminary revision of the Irish char. Ann. Mag. Nat. Hist., Ser. 8, 2: 225-234.

- 1908b. A revision of the British and Irish fishes of the genus Coregonus. Ann. Mag. Nat. Hist., Ser. 8, 2: 482-490.

- 1909a. The char (Salvelinus) of Great Britain. Ann. Mag. Nat. Hist., Ser. 8, 3: 111-122.

- 1909b. The species of three-spined sticklebacks (Gastrosteus). Ann. Mag. Nat. Hist., Ser. 8, 4: 435-437.

- 1911a. A synopsis of the marsipobranchs of the order Hyperoartii. Ann. Mag. Nat. Hist., Ser. 8, 7: $193-204$.

- 1911b. The freshwater fishes of the British Isles. Methuen, London, xxv+287 pp., 37 pls.

- 1916. The British fishes of the subfamily Clupeinae and related species in other seas. Ann. Mag. Nat. Hist., Ser. 8, 18: 1-19, pls. 1-3. - 1926. Organic evolution. Rep. 93rd Meet. Brit. Assoc. Adv. Sci., 1925, pp. 75-86.

Reisinger, E. 1953. Zum Saiblingsproblem. Carinthia II, 143: 74-102.

Reisinger, J. 1830. Specimen ichthyologiae sistens pisces aquarum dulcium Hungariae. Universitatis Hungaricae, Buda, xii+98+3 pp.

Relyea, K. 1983. A systematic study of two species complexes of the genus Fundulus (Pisces: Cyprinodontidae). Bull. Florida State Mus., Biol. Sci., 29: 1-64.

Renaud, C. B. \& J. Holčík. 1988. Lampretra (Eudontomyzon) gracilis, a synonym of Eudontomyzon danfordi. Env. Biol. Fish., 23: 127-130.

Rendahl, H. 1935. Ein paar neue Unterarten von Cobitis taenia. Mem. Soc. Fauna Flora Fenn., 10 (1933-1934 [1935]): 329-336.

Reshetnikov, Y. S. 1980. [Ecology and systematics of the coregonid fishes]. Izd. Nauka, Moskva, 301 pp. [in Russian] 
Richardson, J. 1835. Salmones, p. lv-lviii. In: J. Ross, Appendix to the narrative of a second voyage in search of a north-west passage and of a residence in the arctic regions during the years 1829, 1830, 1831, 1832, 1833. Webster, London, $120+$ cxliv +cii pp., 20 pls.

- 1836. Part III. The fish. In: Fauna borealis Americana, or the zoology of the northern parts of British America, containing descriptions of the objects of natural history collected on the late northern land expeditions, under the command of Sir John Franklin R. N. London \& Norwich, vol. 3: xv + 327 pp., pls. 74-96.

- 1846. Report on the ichthyology of the seas of China and Japan. Rep. Meet. Brit. Assoc. Adv. Sci., 1845 [1846]: 187-320.

- 1856. On some fish from Asia Minor and Palestine. Proc. Zool. Soc. London, 24: 371-377.

Riehl, R. \& W. Meinel. 1994. Die Eier heimischer Fische. 8. Kaulbarsch - Gymnocephalus cernuus (Linnaeus, 1758) mit Anmerkungen zum taxonomischen Status von Gymnocephalus baloni (Holčík \& Hensel, 1974). Fischökologie, 7: 2533.

Riffel, M., M. Schenk \& A. Schreiber. 1994. Electrophoretic differentiation between European loach (Misgurnus fossilis L.) and Oriental weatherfish (Misgurnus mizolepis Günther), an autochtonous and a feral species of central European freshwater fish. Ztschr. Angew. Zool., 80: 473-483.

Riffel, M. \& A. Schreiber. 1995. Coarse-grained population structure in central European sculpin (Cottus gobio L.): secondary contact or ongoing genetic drift? J. Zool. Syst. Evol. Res., 33: 173-184.

Riffel, M., V. Storch \& A. Schreiber. 1995. Allozyme variability of brown trout (Salmo trutta L.) populations across the RhenanianDanubian watershed in southwest Germany. Heredity, 74: 241-249.

Riget, F. F., K. H. Nygaard \& B. Christensen. 1986. Population structure, ecological segregation, and reproduction in a population of Arctic char (Salvelinus alpinus) from Lake Tasersuaq, Greenland. Can. J. Fish. Aquat. Sci., 43: 985992.

Risso, A. 1810. Ichthyologie de Nice ou histoire naturelle des poissons du Département des Alpes Maritimes. Schoell, Paris, xxxvi+388 pp., 11 pls.

- 1820. Mémoire sur quelques poissons observés dans la mer de Nice. J. Phys. Chim. Hist. Nat., 91: 241-255.

- 1826. Histoire naturelle des principales produc- tions de l'Europe méridionale et particulièrement de celles des environs de Nice et des Alpes Maritimes. Tome troisième. Levrault, Paris, 480 pp., 16 pls.

Robins, C. R. 1961. Two new cottid fishes from the fresh waters of eastern United States. Copeia, 1961: 305-315.

Robins, C. R., R. M. Bailey, C. E. Bond, J. R. Brooker, E. A. Lachner, R. N. Lea \& W. B. Scott. 1991. Common and scientific names of fishes from the United States and Canada. Fifth edition. Amer. Fish. Soc., Spec. Publ., 20: 1183.

Rolik, H. 1960. Cobitis aurata (Filippi, 1865) koza zlotawa, nowy gatunek w zlewisku Morza Baltyckiego. Fragm. Faun., 8: 411-420.

- 1967. Materialy do ichtiofauny Strwiaza (doplyw Dniestru) ze szczegolnym uwzglednieniem Gobio gobio (L.) i Cobitis (Sabanejewia) aurata (Fil.). Fragm. Faun., 14: 133-152.

- 1970. Barbus cyclolepis waleckii ssp. n. - a new subspecies of B. cyclolepis Haeckel, 1840, from the Vistula and Dniestr basins (Pisces, Cyprinidae). Bull. Acad. Pol. Sci., Cl. II, Ser. Sci. Biol.. 7: 401-404.

Römer-Büchner, B. J. 1827. Verzeichniss der Steine und Thiere welche in dem Gebiete der freien Stadt Frankfurt und deren nächster Umgebung gefunden werden. Sauerländer, Frankfurt am Main, 88 pp., 2 pls.

Rondelet, G. 1554. Libri de piscibus marinis, in quibus verae piscium effigies expressae sunt. Quae in tota piscium historia contineantur, indicat elenchus pagina nona et decima. Bonhomme, Lugdunum, $14+583+23$ pp.

- 1555. Universae aquatilium historiae pars altera, cum veris ipsorum imaginibus. His accesserunt indices necessarii. Bonhomme, Lugduni, $10+242+9$ pp.

Rosen, D. E. 1978. Vicariant patterns and historical explanation in biogeography. Syst. Zool., 27: 159-188.

- 1979. Fishes from the uplands and intermontane basins of Guatemala: revisionary studies and comparative geography. Bull. Amer. Mus. Nat. Hist., 162: 267-376.

Rossikov, K. M. 1895. [Caspian roach as an object of exploitation in the delta of Terek]. Kavkazskoe Sel'skoe Khozyajstvo, 54-56. [cited by Berg, 1912: 52; bibliographic data checked by N. Bogutskaya, pers. comm., 1995; in Berg's library, this is a booklet with a collection of reprints of papers published in a newspaper; original pagination unknown]

Roule, L. 1902. Atherina Riqueti nov. sp. nouvelle 
espèce d'athérine vivant dans les eaux douces. Zool. Anz., 25: 262-267.

- 1923. Description de la grande truite du Rhône (Salmo trutta Linné, forma major Fatio, facies rhodanensis). Bull. Mus. Natn. Hist. Nat., 29: 291-295.

- 1924. Les aloses des eaux douces de la France. Bull. Soc. Zool. Fr., 49: 265-266.

- 1925. Les poissons des eaux douces de France. Presses Universitaires de France, Paris, xiv +228 pp., 37 pls.

Roule, L. \& G. del P. de Cardaillac de Saint-Paul. 1903. Les chevaines et les vandoises du bassin de l'Adour. Comptes-Rendus Sessions Assoc. Franç. Avanc. Sci., 31 (1902 [1903]) (2): 731733.

Roux, C. 1976. On the dating of the first edition of Cuvier's Règne Animal. J. Soc. Bibliogr. Nat. Hist., 8: 31.

Rubin, J.-F. \& B. Buttiker. 1987. Croissance et reproduction de l'omble-chevalier, Salvelinus alpinus (L.), dans le lac de Neuchâtel (Suisse). Schweiz. Ztschr. Hydrol., 49: 51-61.

Rufli, H. 1978. Die heutigen sympatrischen Felchenpopulationen (Coregonus spp. ) des Thunerund Bielersees und ihre Morphologie. Schweiz. Ztschr. Hydrol., 40: 7-31.

Ruhlé, C. 1986. Ergebnisse bisheriger Abklärungen über die systematische Beziehungen zwischen Sommeralbeli und Winteralbeli (Coregonus lavaretus (L.)) im Walensee. Schweiz. Ztschr. Hydrol., 48: 145-152

- 1988. Preliminary results of investigations on the biology of slow-growing whitefish (Coregonus lavaretus (L.)) in Lake Walenstadt, Switzerland. Finn. Fish. Res., 9: 175-182.

- 1991. Comparison of the growth pattern of autochtonous and stocked arctic char (Salvelinus alpinus (L.)) in the oligotrophic source lake (lake Lucerne) and the mesotrophic stocked lake (lake Constance). Intern. Soc. Arctic Char Fanatics, Inform. Ser., 1991: 147-150.

Ruhlé, C., J. Deufel, G. Keiz, T. Kindle, M. Klein, H. Löffler \& B. Wagner. 1984. Die Bodensee-Seeforelle. Probleme und Problemlösungen. Österreichs Fischerei, 37: 272-307.

Ruhlé, C. \& T. Kindle. 1992. Morphological comparison of river-spawning whitefish of the alpine Rhine with the whitefish of lake Constance. Polskie Archiv. Hydrobiol., 39: 403-408.

Rutty, J. 1772. An essay towards a natural history of the county of Dublin. Dublin, 1: 263-392.

Ruzskii, M. D. 1914. [On a new form of Leucaspius delineatus Heck. (L. delineatus dimorphus Ruz.)]. Protokoly Zasedanii Kazanskogo
Obshchestva Estestvoispytatelei za 1912-1913 [1914], Suppl., 287: 1-5.

- 1926. [On a new form of the minnow from the Eastern Transuralia. Phoxinus percnurus sarykul (nov. subsp.)]. Izv. Tomskogo Gosudarstvennogo Universiteta, 77 (2, Biol.): 112. [Russian]

Ryman, N., F. W. Allendorf \& G. Ståhl. 1979. Reproductive isolation with little genetic divergence in sympatric populations of brown trout (Salmo trutta). Genetics, 92: 247-262.

Řepa, P. \& K. Pivnička. 1980. Morphologische Variabilität der Elritze (Phoxinus phoxinus) (Pisces: Cyprinidae). Vestn. Ceskoslov. Společ. Zool., 44: 68-80.

Sabaneev, L. P. 1871. [Catalogue of the mammals, birds, reptiles and fishes of the middle Oural]. Bull. Soc. Imp. Nat. Moscou, 44 (1): 210-278. [Russian]

Sadovskii, A. 1934. Caspialosa tanaica paleostomi n. sp. Eine neue Unterart aus der PaläostomLagune (Rion-Mündung), West Georgien. Trudy Zoologicheskogo Sektora Zakavkazskogo Filiala Akad. Nauk, Gruzinskoe Otdelenie, Tiflis, 1: $135-141$.

Saemundsson, B. 1909. Oversigt over Islands fiske med oplysninger om deres forekomst, vigtigste biologiske forhold og $\varnothing$ konomiske betydning. Skrifter Udgivne af Kommissionen for Havundersøgelser, 5: 1-140.

Sakamoto, K. 1984. Interrelationships of the family Pleuronectidae (Pisces: Pleuronectiformes). Mem. Fac. Fish. Hokkaido Univ., 31: 95-215.

Salnikov, N. I. \& S. M. Malyatskii. 1934. [On the taxonomy of the beluga of the Azov-Black Sea basin]. Trudy Nauchnoi Rybokhozyaistvennoi i Biologicheskoi Stantsii Gruzii, 1 (1): 31-50.

Salvadori, T. 1888. Le date della pubblicazione della "Iconografia della Fauna Italica" del Bonaparte ed indice delle specie illustrate in detta opera. Boll. Mus. Zool. Anat. Comp. Univ. Torino, 3 (48): 1-25.

Salviani [Salviano], H. 1554-58. Aquatilium animalium historiae, liber primus, cum eorundem formis aere excusis. Romae, $16+256$ pp., 98 pls.

Sandeberg, H. 1878. Pleuronectes Bogdanovii descriptus. Bull. Soc. Imp. Nat. Moscou, 53 (3): 236-237.

Sander, H. 1784-1785. Heinrich Sanders kleine Schriften nach dessen Tode herausgegeben von Georg Friederich Götz. Dessau \& Leipzig, 1 (1784): 383 pp.; 2 (1785): 330 pp.

Sandlund, O. T., K. Gunnarsson, P. M. Jonasson, B. Jonsson, T. Lindem, K. P. Magnusson, H. J. Malmquist, H. Sigurjonsdottir, S. Skúlason \& S. 
S. Snorrason. 1992. The arctic charr Salvelinus alpinus in Thingvallavatn. Oikos, 64: 305-351.

Sandlund, O. T., B. Jonsson, H. J. Malmquist, R. Gydemo, T. Lindem, S. Skúlason, S. S. Snorrason \& P. M. Jonasson. 1987. Habitat use of arctic charr Salvelinus alpinus in Thingvallavatn, Iceland. Env. Biol. Fish., 20: 263-274.

Sanford, C. P. J. 1990. The phylogenetic relationships of salmonoid fishes. Bull. Brit. Mus. Nat., Hist., Zool., 56: 145-153.

Sauvage, H.-E. 1874a. Révision des espèces du groupe des épinoches. Nouv. Arch. Mus. Hist. Nat. Paris, 10: 5-38, pl. 1.

- 1874b. Notices ichthyologiques. I-VI. Rev. Mag. Zool., 1874: 332-340.

- 1880. Note sur quelques poissons recueillis par M. Letourneux, en Epire, à Corfou et dans le lac Maréotis. Bull. Soc. Philom. Paris, Sér. 7, 4: 211-215.

Savvaitova, K. A. 1980. Taxonomy and biogeography of charrs of Palearctic, p. 281-294, In: E. K. Balon (ed.), Charrs - Salmonid fishes of the genus Salvelinus. Junk, Den Haag.

- 1983. The application of the biological species concept to an evaluation of the systematic position of chars of the genus Salvelinus (Salmonidae). J. Ichthyol., 23 (6): 1-12. [translation from Vopr. Ikhtiol., 23: 883-893]

Savvaitova, K. A., E. A. Dorofeeva, V. G. Markaran \& A. I. Smolei. 1989. [The trouts of the Sevan Lake. The estimation of condition from the results of morphoecological monitoring, the perspectives of preservation and utilization]. Trudy Zool. Inst. Akad. Nauk SSSR, 204: 1180.

Sawada, Y. 1982. Phylogeny and zoogeography of the superfamily Cobitoidea (Cyprinoidei, Cypriniformes). Mem. Fac. Fish. Hokkaido Univ., 28: 65-223.

Schaeffer, J. C. 1760. Epistola ad Regio-Borussicam societatem litterariam dvisbvrgensem de studii ichthyologici faciliori ac tvtiori methodo, adiectis nonnvllis speciminibvs vna cvm tabvla aeri incisa figvras ... Montagii, Ratisbonnae, 24 pp., 1 pl.

- 1761. Piscium Bavarico-Ratisbonensium pentas, cum tabulis IV. aeri incisis icones coloribus suis distinctas exhibentibus. Montagii, Ratisbonae, 82 pp., 4 pls.

Schäfer, M. 1844. Moselfauna oder Handbuch der Zoologie, enthaltend die Aufzählung und Beschreibung der im Regierungsbezirke Trier beobachteten Thiere, mit Berïcksichtigung der Angrenzung des Moseldepartements und Belgiens. Erster Theil. Wirbelthiere: Säugethiere,
Vögel, Reptilien und Fische. Lintz, Trier, xliv+ 339 pp.

Schillinger, A. 1901. Der Tiefseesaibling. Allgemeine Fischerei-Zeitung, N. F., 16: 149-151.

Schindler, O. 1940. Die Saiblinge des Königssees. Int. Rev. Ges. Hydrobiol. Hydrogr., 39: 600627.

Schinz, H. 1840. Europäische Fauna oder Verzeichniss der Wirbelthiere Europa's. Zweiter Band. Reptilien und Fische. Schweizerbart, Stuttgart, viii+535 pp.

Schmidt, F. W. 1795. Versuch eines Verzeichnisses aller in Böhmen bisher bemerkten Thiere, p. 1104. In: Sammlung physikalisch-ökonomischer Aufsätze zur Aufnahme der Naturkunde und deren damit verwandten Wissenschaften in Böhmen. Vol. 1. Salve, Prag, 375 pp.

Schmidt-Ries, H. 1943. Die Fische Griechenlands (eine kritische U̇bersicht). I. Die Süsswasserfische. Ztschr. Fisch. Hilfswiss., 41: 319-344.

Schnakenbeck, W. 1942. Die Entwicklung des Oberflächenpigments bei der Gattung Salmo. Zool. Anz., 140: 91-99.

Schneider, J. G. 1801. M. E. Blochii Systema Ichthyologiae iconibus cx illustratum, post obitum auctoris opus inchoatum absoluit, correxit, interpolavit Jo. Gottlob Schneider. Berlin, 584 pp., 110 pls.

Schöffmann, J. 1993. Autochthone Forellen (Salmo trutta L.) in Nordafrika. Österreichs Fischerei, 46: $146,164-169$.

- 1994. Endemische Seeforellen im Adriaraum Salmo carpio L. und Salmo letnica Karaman. Österreichs Fischerei, 47: 78, 97-99.

Schnovelde, S. A. 1624. Ichthyologia et nomenclaturae animalium marinorum, fluviatilium, lacustrium, quae in florentissimis ducatibus Slesvici et Holsatiae et Emporio Hamburgo occurrunt triviales; ac plerorumque hactenus desideratorum imagines, breves descriptiones et explicationes. Hamburgi, 85 pp., 7 pls.

Scopoli, I. A. 1786-1788. Deliciae florae et faunae insubricae seu novae, aut minus cognitae species plantarum et animalium quas in Insubria Austriaca tam spontaneas, quam exoticas vidit, descripsit et aeri incidi curavit Ioannes Antonius Scopoli. Monasterius S. Salvatoris, Ticino, 1: viii+85 pp., 25 pls.; 2: 115 pp., pls. 25 pls.; 3: 87 pp., 25 pls.

Scott, W. B. \& E. J. Crossman. 1973. Freshwater fishes of Canada. Bull. Fish. Res. Board Can., 184: 966 pp. [also French version: 1974. Poissons d'eau douce du Canada. Bull. Serv. Pêches Sci. Mer, 184: 1026 pp.]

Segre, R. 1904. Res Italicae. XII. Boll. Mus. Zool. 
Anat. Comp. Univ. Torino, 19 (472): 1-18.

Selys-Longchamps, E. de 1842. Faune belge, 1re partie, indication méthodique des mammifères, oiseaux, reptiles et poissons observés jusqu'ici en Belgique. Dessain, Liége \& Muquart, Bruxelles, xii +309 pp., 9 pls.

- 1870. [no title; on fishes of Belgium]. Congrès Scientifique de France, 36ème Session, Chartres, 1869, pp. 110-115.

- 1887. Révision des poissons d'eau douce de la faune belge. Bull. Acad. Roy. Sci. Lettres Beaux-Arts Belgique, Cl. Sci., Sér. 3, 14: 10211097.

Seppovaara, O. 1962. Zur Systematik und Ökologie des Lachses und der Forellen in den Binnengewässern Finnlands. Ann. Zool. Soc. Zool. Bot. Fenn. 'Vanamo', 24: 1-84.

Shaw, G. 1803-1804. General zoology or systematic natural history ... with plates from the first authorities and most select specimens. Kearsley, London, 14 vols. (1800-1826); 4 (1) [1803]; 4 (2) [1803]: i-xiii+187-632, pls. 26-92; 5 (1) [1804]; 5 (2) [1804]: i-viii+251-463, pls. 133-182.

Sherborn, C. D. 1922. Index animalium, sive index nominum quae A.D. 1758 generibus et specieribus animalium imposita sunt. Sectio Secunda, a kalendis Januariis 1801 usque ad finem Decembris 1850 societatibus eruditorum adjuvantivus. Part 1. Introduction, bibliography and index. British Museum of Natural History, London, cxxxi+128 pp.

- 1937. On the dates of publication of Costa (O. G.) and (A.) Fauna del Regno di Napoli, 18291886. J. Soc. Bibl. Nat. Hist., 1: 35-47.

Shutov, V. A. 1969. [Revision of the genus Blicca and some data concerning phyletic relationships between representatives of the genus Abramis (Pisces, Cyprinidae)]. Zool. Zh., 48: 1105-1107.

Siebold, C. T. von 1863a. Die Suisswasserfische von Mitteleuropa. Engelmann, Leipzig, vi+431 pp., 2 pls.

- 1863b. Ueber die Fische des Ober-Engadins. Verh. Schweiz. Naturforsch. Ges., 47: 173-190.

Sigurjónsdóttir, H. \& K. Gunnarson. 1989. Alternative mating tactics of arctic charr, Salvelinus alpinus, in Thingvallavatn, Iceland. Environ. Biol. Fish., 26: 159-176.

Simpson, G. G. 1961. Principles of animal taxonomy. Columbia University Press, New York.

Sinitzyn, D. F.1900. [Collections of the zoological cabinet of the Imperial Warsaw University. 6. List and description of the ichthyological collection]. Varshavskogo Uchebnogo Okruga (Warsaw Educational District), Warsaw, 57 pp. Sivkov, Y. S. \& I. S. Dobrovolov. 1984. A new species of Cobitis (Pisces, Cobitidae) from Bulgaria. C. R. Acad. Bulg. Sci., 37: 1673-1676.

Skaala, Ø. 1992. Genetic population structure of Norwegian brown trout. J. Fish. Biol., 41: 631646.

Skaala, Ø. \& K. E. Jørstad. 1987. Fine-spotted brown trout (Salmo trutta): its phenotypic description and biochemical genetic variation. Can. J. Fish. Aquat. Sci., 44: 1775-1779.

- 1988. Inheritance of the fine-spotted pigmentation pattern of brown trout. Polsk. Arch. Hydrobiol., 35: 295-304.

Skaala, Ø., K. E. Jørstad \& R. Borgstrøm. 1991. Fine-spotted brown trout: genetic aspects and the need for conservation. J. Fish Biol., 39 (Suppl. A): 123-130.

Skaala, Ø. \& G. Nævdal. 1989. Genetic differentiation between freshwater resident and anadromous brown trout, Salmo trutta, within watercourses. J. Fish Biol., 34: 597-605.

Skrochowska, S. 1969. Migrations of the sea-trout (Salmo trutta L.), brown trout (Salmo trutta m. fario L.) and their crosses. Polsk. Arch. Hydrobiol., 16: 125-192.

Skreslet, S. 1973. Group segregation on landlocked Arctic char, Salvelinus alpinus (L.), of Jan Mayen Island, in relation to the char problem. Astarte, 6: 55-58.

Skúlason, S., D. L. G. Noakes \& S. S. Snorrason. 1989. Ontogeny of trophic morphology in four sympatric morphs of arctic charr Salvelinus alpinus in Thingvallavatn, Iceland. Biol. J. Linn. Soc., 38: 281-301.

Slastenenko, E. P. 1934. Les goujons de l'Ukraine. Bull. Soc. Zool. Fr., 59: 346-363.

- 1938a. Les poissons de la Mer Noire et de la Mer d'Azov. Annls. Scient. Univ. Jassy, Seconde Partie, Sci. Nat., 25 (1) (1939 [1938]): 1-196.

- 1938b. [Species composition of the genus Syngnathus in the Black Sea]. Trudy Novorossiiskoi Biologicheskoi Stantsii, 2 (2): 3-6

Šlechtová, V. \& V. Šlechta. 1996. Gymnocephalus baloni - species validity and position within the genus Gymnocephalus (Pisces, Percidae): evidence from lactate dehydrogenase isozyme pattern. Acta Univ. Carol., Biol., 39 (1995 [1996]): 243-250.

Smith, G. R. \& R. F. Stearley. 1989. The classification and scientific names of rainbow and cutthroat trouts. Fisheries, 14 (1): 4-10.

Smitt, F. A. 1883. Schematisk framställning af de i Riksmuseum befintliga laxartade fiskarnes slägtskapsförhăllanden. Ofversigt af Kongl. Vetenskaps-Akademiens Förhandlingar, 39 (8) [1882 (1883)]: 31-40. 
— 1886. Kritisk förteckning öfver de i Riksmuseum befintliga Salmonider. Kongl. Svenska Vetensk.-Akad. Handl., 21 (8): 1-290, 6 pls.

- 1892-5. Skandinaviens fiskar målade af W. von Wright beskrifna av B. Fries, C.U. Ekström och C. Sundevall. Andra upplagan. Bearbetning och fortsättning. Norstedt, Stockholm, 1 (1892): 1$566+$ i-viii +2 pp.; 2 (1895): 567-1239 + 1 p.; 3 (1895): i-iii, pls. 1-53 [also translated as: A history of Scandinavian fishes, by B. Fries, C. U. Ekström, and C. Sundevall. Second edition revised and completed by ... F. A. Smitt. Norstedt, Stockholm \& Friedländer, London, 1 (1893), 2 (1895)].

- 1899. Preliminary notes on the arrangement of the genus Gobius, with an enumeration of its European species. Öfvers. Kongl. Vetensk. Akad. Förhandl., 56: 543-555.

Snorrason, S. S., S. Skúlason, B. Jonsson, H. J. Malmquist, P. M. Jonasson, O. T. Sandlund \& T. Lindem. 1994. Trophic specialization in arctic charr Salvelinus alpinus (Pisces; Salmonidae): morphological divergence and ontogenetic niche shifts. Biol. J. Linn. Soc., 52: 1-18.

Sofradžija, A. \& L. Berberovič. 1978. Diploidtriploid sexual dimorphism in Cobitis taenia taenia L. (Cobitidae, Pisces). Genetika, 10: 389397.

Sokal, R. R. \& T. J. Crovello. 1970. The biological species concept: a critical evaluation. Amer. Nat., 104: 127-153.

Sokal, R. R. \& P. H. A. Sneath. 1963. The principles of numerical taxonomy. Freeman, San Francisco, xvi+359 pp.

Soldatov, V. K. 1924. [Fishes of the Pechora river]. Trudy Severnoi Nauchno-Promyslovoi Ekspeditsii, 17: 1-73.

Sommani, E. 1948. Sulla presenza del Salmo fario L. e del S. marmoratus Cuv. nell'Italia settentrionale: loro caratteristiche ecologiche e considerazioni relativi ai ripopolamenti. Boll. Pesca Piscic. Idrobiol., 24 (N. S. 3): 136-145.

- 1960. Il Salmo marmoratus Cuv.: sua origine e distribuzione nell'Italia settentrionale. Boll. Pesca Piscic. Idrobiol., 36 (N. S. 15): 40-47, pl. 1.

Šrić, V. 1992. Osteology and taxonomic status of the genus Pachychilon Steind., 1882 (Pisces, Cyprinidae). Bios (Macedonia, Greece), 1: 4967.

Sözer, F. 1941. Les gobiidés de la Turquie. Istambul Univ. Fen. Fak. Mecm., Ser. B, 6: 128169.

Spiczakow, T. 1935. Zum Problem der Rassen und des Exterieurs beim Karpfen. Ztschr. Fischerei Hilfswiss. 33: 409-472.

Spillmann, J. 1958. Sur deux poissons des collections du Muséum, témoins de l'existence au $\mathrm{XIX}^{\mathrm{e}}$ siècle, dans le lac Léman, de corégones du type "dispersus". Bull. Mus. Natn. Hist. Nat. Paris, Sér. 2, 30: 144-145.

- 1959. Note préliminaire sur la systématique de Telestes soufia Risso, poisson de la famille des Cyprinidae. Bull. Mus. Natn. Hist. Nat. Paris, Sér. 2, 31: 491-498.

- 1961a. Faune de France. Vol. 65. Poissons d'eau douce. Lechevalier, Paris, 303 pp.

- 1961b. Sur la systématique de Telestes soufia Risso ( $2^{\mathrm{e}}$ note). Bull. Mus. Natn. Hist. Nat. Paris, Sér. 2, 32 (1960 [1961]): 411-414.

- 1962. Sur la systématique de Telestes soufia Risso. Variation de certains caractères numériques et métriques de l'espèce ( $3^{\mathrm{e}}$ note). Bull. Mus. Natn. Hist. Nat. Paris, Sér. 2, 34: 435-452. - 1966. Sur la systématique de Telestes soufia Risso. Etude d'un lot de poissons du Paillon (Alpes-Maritimes) (5 note). Bull. Mus. Natn. Hist. Nat. Paris, Sér. 2, 37 (1965 [1966]): 962965.

- 1967. Sur la systématique de Telestes soufia Risso. Etude d'un lot de poissons de la Dourbie, affluent du Tarn ( $6^{e}$ note). Bull. Mus. Natn. Hist. Nat. Paris, Sér. 2, 39 (1967): 501-505.

Spinola, M. 1807. Lettre sur quelques poissons peu connus du Golfe de Gênes, adressée à M. Faujasde-Saint-Fond. Ann. Mus. Hist. Nat. Paris, 10: 366-380, pl. 28.

Stangenberg, M. 1938. Zmiennosc ekologiczna ploci - Die ökologische Variabilität der Plötze. Rozprawy i Sprawozdania Instytut Badawczy Lasow Panstwowych, Warszawa, Ser. A, 39: 1112.

Stankovic, S. 1953. Sur la spéciation dans le lac d'Ohrid. Verh. Int. Ver. Limnol. 12: 478-506.

- 1960. The Balkan Lake Ohrid and its living world. Junk, Den Haag, 357 pp.

Stark, J. 1831. Four-spined stickleback. Edinburg New Philos. J., 1831: 386.

Stauffer, J. R., C. H. Hocutt \& R. L. Mayden. In press. Pararhinichthys, a new monotypic genus of minnows (Teleostei: Cyprinidae) of hybrid origin from eastern North America. Ichthyol. Explor. Freshwat., 7.

Stearley, R. F. 1992. Historical ecology of Salmonidae, with special reference to Oncorhynchus, p. 623-658. In: R. L. Mayden (ed.), Systematics, historical ecology, and North American freshwater fishes. Stanford University Press, Stanford.

Stearley, R. F. \& G. R. Smith. 1993. Phylogeny of 
the Pacific trouts and salmons (Oncorhynchus) and genera of the family Salmonidae. Trans. Amer. Fish. Soc., 122: 1-33.

Stefani, R., G. P. Serra, F. Loffredo \& I. Fossa. 1987. Un nuovo cyprinide dell'Alpago Leuciscus lapacinus n. sp. (Pisces Cyprinidae). Atti Ist. Veneto Sci., Lett. Arti, 145 [1986 (1987)]: 1323, 5 pls.

Stefanovic, D. 1948. Rasna i ekoloska ispitivanja na Ohridskim salmonidima [Systematic and ecological study of the salmonids of lake Ohrid]. Posebna Izd. SAN, Beograd, 139 (Prirod. Matem Slisi 38): 1-207. [Serbian]

Steindachner, F. 1865. Catalogue préliminaire des poissons d'eau douce de Portugal conservés au Muséum d'Histoire naturelle de Lisbonne. Mem. Acad. Real Sci. Lisboa, Cl. Sci. Math. Phys. Nat., 3 (2): 1-7.

- 1866a. Ichthyologische Notizen (II). Zur Flussfischfauna von Croatien. Sitz-Ber. Math. Naturwiss. Cl. Akad. Wiss. Wien, Abt. 1, 52(1865 [1866]): 594-599, $1 \mathrm{pl}$.

- 1866b. Ichthyologischer Bericht über eine nach Spanien und Portugal unternommene Reise (Fortsetzung). Sitz-Ber. Math. Naturwiss. Cl. Akad. Wiss. Wien, Abt. 1, 53: 198-205.

- 1866c. Ichthyologischer Bericht über eine nach Spanien und Portugal unternommene Reise (Zweite Fortsetzung). Sitz-Ber. Math. Naturwiss. Cl. Akad. Wiss. Wien, Abt. 1, 54: 6-27, 6 pls.

- 1866d. Ichthyologischer Bericht über eine nach Spanien und Portugal unternommene Reise (Dritte Fortsetzung). Sitz-Ber. Math. Naturwiss. Cl. Akad. Wiss. Wien, Abt. 1, 54: 261-272, 3 pls.

- 1866e. Úber eine neue Telestes-Art aus Croatien. Sitz-Ber. Math. Naturwiss. Cl. Akad. Wiss. Wien, Abt. 1, 54: 300-302, 1 pl.

- 1868. Ichthyologischer Bericht über eine nach Spanien und Portugal unternommene Reise. Sitz-Ber. Math. Naturwiss. Cl. Akad. Wiss. Wien, Abt. 1, 57: 351, 400-403, 424, pls. 1-6.

- 1870. Ichthyologische Notizen (X.). (Schluss). Sitz-Ber. Math. Naturwiss. Cl. Akad. Wiss. Wien, Abt. 1, 61: 623-642, 5 pls.

- 1874. [...] Beschreibung einer neuen Art und Gattung aus der Familie der Pleuronectiden und einer neuen Thymallus-Art. Anz. Akad. Wiss., Math.-Naturwiss. Cl., Wien, 11: 171-172.

- 1875. Uber eine neue Gattung und Art aus der Familie der Pleuronectiden und über eine neue Thymallus-Art. Sitz-Ber. Math. Naturwiss. Cl. Akad. Wiss. Wien, Abt. I, 70: 363-371, pls. 1-2. - 1882a. Ichthyologische Beiträge (XII). Sitz-Ber.
Math. Naturwiss. Cl. Akad. Wiss. Wien, Abt. 1 , 86: 61-82, pls. $1-5$.

- 1882b. Beiträge zur Kenntniss der Fische Afrika's (II.) und Beschreibung einer neuen Paraphoxinus-Art aus der Herzegowina. Denkschr. Akad. Wiss. Wien, Math.-Naturwiss. Cl., 45: 118, pls. 1-6.

- 1892. Über einige neue und seltene Fischarten aus der ichthyologischen Sammlung des K. K. Naturhistorischen Hofmuseums. Denkschr. Akad. Wiss. Wien, Math.-Naturwiss. Cl., Abt. 1, 59: 357-383, pls. 1-6.

- 1893. Ichthyologische Beiträge (XVI.). SitzBer. Math. Naturwiss. Cl. Akad. Wiss. Wien, Abt. 1, 102: 215-243, 3 pls.

- 1894. Ichthyologische Beiträge (XVII.). SitzBer. Math. Naturwiss. Cl. Akad. Wiss. Wien, Abt. 1, 103: 443-464, pls. 1-5.

- 1896. Beiträge zur Kenntniss der Süsswasserfische der Balkan-Halbinsel. Denkschr. Akad. Wiss. Wien, Math.-Naturwiss. Cl., 63: 181-188, 2 pls.

- 1897. Bericht über die von Dr. Escherich in der Umgebung von Angora gessammelten Fische und Reptilien. Denkschr. Akad. Wiss. Wien, Math.-Naturwiss. Cl., 40: 685-699, 3 pls.

- 1901. [... uiber das Vorkommen einer bisher noch unbeschriebenen Paraphoxinus-Art ...]. Anz. Akad. Wiss., Math.-Naturwiss. Cl., Wien, 38: 197-198.

Steinmann, P. 1944. Probleme der Systematik unserer einheimischen Forelle. Schweiz. Fisch. Ztg., 52: 78-83, 164-170, 283-292.

- 1950a. Monographie der schweizerischen Koregonen. Beitrag zum Problem der Entstehung neuer Arten. Einleitung. Schweiz. Ztschr. Hydrol., 12: 109-189.

- 1950b. Monographie der schweizerischen Koregonen. Beitrag zum Problem der Entstehung neuer Arten. Spezieller Teil. Schweiz. Ztschr. Hydrol., 12: 340-491.

- 1951. Monographie der schweizerischen Koregonen. Beitrag zum Problem der Entstehung neuer Arten. Spezieller Teil [2nd part]. Schweiz. Ztschr. Hydrol., 13: 54-191.

- 1952. Polytypie und intraspezifische Evolution bei Süsswassertieren. Schweiz. Ztschr. Hydrol., 16: 313-332.

Stephanidis, A. 1937. Poissons d'eau douce, nouveaux pour l'ichthyofaune de la Grèce. Acta Inst. Mus. Zool. Univ. Atheniensis, 1: 263-268, pls. 1-2.

- 1939a. [Poissons d'eau douce de la Grèce occidentale et de l'île Kerkyra [Corfou]]. Thesis, Univ. Athens, 44 pp., 8 pls. [Greek] 
- 1939b. [Poissons d'eau douce de l'Atticobéotie]. Deltion Fysskon Epistimon, 5 (50-51): 49-60, 3 pls. [Greek]

- 1950. [Contribution à l'étude des poissons d'eau douce de la Grèce]. Prakt. Akad. Athen, 18 (1943 [1950]): 200-210. [Greek, French summary]

- 1971a. Poissons d'eau douce du Péloponnèse. Biol. Gallo-Helen., 3: 163-212.

- 1971b. [Sur quelques poissons d'eau douce de la Grèce]. Biol. Gallo-Helen., 3: 213-241. [Greek, French summary]

- 1973. Fresh waters fish from Thessaly and the valley of Sperchios River. I. The species of the genus Gobio Cuvier, 1817 (Pisces, Cyprinidae). Biol. Gallo-Helen., 4: 189-203.

- 1974a. A new species of Cobitis from Greece: Cobitis (Bicanestrinia) trichonica n. sp. (Pisces Cobitidae). Biol. Gallo-Helen., 5: 227 234 .

- 1974b. On some fishes of the Ioniokorinthian region (W. Greece etc.) - A new genus of Cyprinidae: Tropidophoxinellus n. gen. Biol. Gallo-Helen., 5: 235-257.

Sterba, G. 1987. Süsswasserfische der Welt. Urania, Leipzig, 915 pp., 320 pls.

Stiassny, M. L. J., U. Schliewen \& W. J. Dominey. 1992. A new species flock of cichlid fishes from Lake Bermin, Cameroon with a description of eight new species of Tilapia (Labroidei: Cichlidae). Ichthyol. Explor. Freshwat., 3: 311246.

van Straaten, L. M. J. U. 1971. Holocene and latePleistocene sedimentation in the Adriatic Sea. Geol. Rundschau, 60: 106-131.

Stratil, A., P. Bobák, V. Tomášek \& M. Valenta. 1983. Transferrins of Barbus barbus, Barbus meridionalis petenyi and their hybrids. Genetic polymorphism, heterogeneity and partial characterization. Comp. Biochem. Physiol., 76B: 845-850.

Strøm, H. 1762. Physisk og oekonomisk beskrivelse over Fögderiet Søndmør beliggende i Bergens stift i Norge. Oplyst med Landkort og Kobberstykker. Første Part. Ernkt, Sorøe.

- 1784. Physisk-oeconomisk beskrivelse over Eger præstegiæld i Aggerhuus-stift i Norge; tilligemed et geographisk kort over samme. Gyldendals, København, 288 pp. [facsimile reprint 1980, Grøndahl, Oslo].

Suvorov, E. K. 1925. [On the biology of the flounder (Pleuronectes flesus) in the Kola Bay]. Izv. Gosudarstvennogo Instituta Opytnoi Agronomii, $3(5-6): 280-281$.

- 1929. [On the fish fauna of the Chosha Bay].
Trudy Instituta po Izucheniyu Severa, 43: 101131.

Svärdson, G. 1957. The coregonid problem. VI. The Palearctic species and their intergrades. Rep. Inst. Freshwat. Res. Drottningholm, 38: 267-356.

- 1970. Significance of introgression in coregonid evolution, p. 33-59. In: C. C. Lindsey \& C. S. Woods (eds), Biology of coregonid fishes. University of Manitoba Press, Winnipeg, 560 pp.

- 1979. Speciation of Scandinavian Coregonus. Rep. Inst. Freshw. Res. Drottningholm, 57: 195.

Svärdson, G. \& O. Filipsson. 1994. Nygammal fisk till Norrbotten. Fauna och Flora, 1994 (2): 4750.

Svarvar, P.-O. \& R. Müller. 1982. Die Felchen des Alpnachersees. Schweiz. Ztschr. Hydrol., 44: 295-314

Svedäng, H. 1990. Genetic basis of life-history variation of dwarf and normal Arctic charr, Salvelinus alpinus (L.), in Stora Rösjön, central Sweden. J. Fish Biol., 36: 917-932.

Svetovidov, A. N. 1934. On the geographical variability of Coregonus lavaretus pidschian. Dokl. Akad. Nauk SSSR, 4: 343-347.

- 1936. Graylings, genus Thymallus Cuvier, of Europe and Asia. Trudy Zool. Inst. Akad. Nauk SSSR, 3: 183-301, pls. 1-5.

- 1945. Chalcalburnus chalcoides iranicus subsp. nova from the Caspian coast of Iran, and some zoogeographical problems of the southern part of this sea. Dokl. Akad. Nauk SSSR, N. S., 48: 142-144. [translation; apparently pp. 149-152 in original version]

- 1952. Fauna of U.S.S.R. Fishes. Vol. 2 (1). Clupeidae. Akad. Nauk SSSR, Leningrad [English translation. Israel Program for Scientific Translation, Jerusalem, 1963, 428 pp.]

- 1964. [Fishes of the Black Sea]. Nauka, Moskva. $551 \mathrm{pp}$.

- 1966. Gobius lenkoranicus Kessler, 1877 (Pisces): proposed suppression as a nomen dubium. Bull. Zool. Nom., 23: 55-56.

-1978. [The types of the fish species described by P. S. Pallas in "Zoographia rosso-asiatica" (with a historical account of publication of this book)]. Nauka, Leningrad, 34 pp., 27 pls. [Russian, English abstract]

- 1981. The Pallas fish collection and the Zoographia Rosso-Asiatica: an historical account. Arch. Nat. Hist., 10: 45-64.

Svetovidov, A. N. \& E. A. Dorofeeva. 1963. [Systematics, origin, and history of the distribution 
of the Eurasian and North American perches and pikeperches (genera Perca, Lucioperca, and Stizostedion)]. Vopr. Ikhtiol., 3: 625-651 [Russian]

Swainson, W. 1839. The natural history of fishes, amphibians, and reptiles, or monocardian animals. Vol. 2. Longman, Orme, Brown, Green and Longmans \& Taylor, London.

Taggart, J. B., E. Verspoor, P. T. Galvin, P. Morán \& A. Ferguson. 1995. A minisatellite DNA marker for discriminating between European and North American Atlantic salmon (Salmo salar). Can. J. Fish. Aquat. Sci., 52: 2305-2311.

Taler, Z. 1950. Visovacka jezerska pastrva (Salmo visovacensis n. sp.) Glasnik Bioloske Sekcije, Hrvatsko Prirodoslovno Drustvo, Ser. 2B, 2-3 (1948-49 [1950]): 118-158.

Taranec, A. 1933. Some new fresh-water fishes from far east of USSR. Dokl. Akad. Nauk SSSR, N. S., 1933: 83-85.

Teitler, N. M. \& K. Fujita. 1993. The genus Carassius: an overview. p. 47. In: Fourth IndoPacific Fish Conference, Bangkok, Program and abstracts.

Temminck, C. J. \& H. Schlegel. 1842-1850. Pisces. In: Fauna japonica, sive descriptio animalium quae in itinere per Japoniam suscepto annis 1823-1830 collegit, notis observationibus et adumbrationibus illustravit P. F. de Siebold conjunctis studiis C. J. Temminck et H. Schlegel pro vertebratis atque $\mathrm{W}$. de Haan pro invertebratis elaborata. Lugdunum Batavorum [Leiden], 1 (1842): $1-20 ; 2-4$ (1843): 21-72; 5-6 (1844): $73-112 ; 7-9$ (1845): 113-172, pls. 1-196 + A; 10-14 (1846): 173-269; (1850): 270-324.

Templeton, A. R. 1981. Mechanisms of speciation - a population genetic approach. Ann. Rev. Ecol. Syst., 12: 23-48.

Thienemann, A. 1912. Die Silberfelchen des Laacher Sees. Zool. Jahrb., Abt. Syst. Geogr. Biol. Tiere, 32: 173-220, pls. 2-4.

- 1916. Die Unterschiede zwischen der grossen Maräne des Madüsees und des Selentersees. Zool. Anz., 48: 97-101.

- 1921. Ủber einige schwedische Coregonen mit Bemerkungen über die Systematik der Gattung Coregonus und die Wege und Ziele der kïnftigen Coregonenforschung. Arch. Naturgesch., Berlin, Abt. A, 87 (2): 170-195.

- 1922. Weitere Untersuchungen an Coregonen. Arch. Hydrobiol., 13: 415-470, pls. 5-8.

- 1928. U̇ber die Edelmaräne (Coregonus lavaretus forma generosus Peters) und die von ihr bewohnten Seen. Arch. Hydrobiol., 19: 1-36.
- 1933. Coregonus albula lucinensis, eine Tiefenform der kleinen Maräne aus einem Norddeutschen See (Zugleich ein Beitrag zur Rassenbildung bei Coregonus albula). Ztschr. Morph. Ökol. Tiere, 27: 654-683.

Thomson, J. 1873. Peculiar trout of Loch Islay. Hardwicke's Science Gossip, 1872 [1873]: 85-86.

Thompson, W. 1835. [...] Numerous specimens of birds and fishes, some which were new to the British, and many to the Irish Fauna. Proc. Zool. Soc. London, 3: 77-82.

- 1837. [...] Notes, relating chiefly to the natural history of Ireland. Proc. Zool. Soc. London, 5: $52-63$.

- 1839. Observations on several British fishes, including description of a new species. Ann. Mag. Nat. Hist., Ser. 1, 2: 402-423, pl. 16.

- 1856. The natural history of Ireland. Vol. IV. Mammalia, reptiles, and fishes, also invertebrata. Bohn, London, xxxii+516 pp.

Tilesius [Tilesius von Tilenau, W. G.]. 1811. Piscium camtschaticorum descriptiones et icones. Mém. Acad. Sci. St. Pétersbourg, 3: 225-285, pls. 8-13.

Togan, I., A. Z. Fidan, E. Yain, A. Ergüven \& Y Emre. 1995. Genetic structure of two Turkish trout populations. J. Fish Biol., 47 (Suppl. A): 164-169.

Tortonese, E. 1940. Elenco clei tipi esistenti nella collezione ittiologica del R. Museo di Torino. Boll. Mus. Zool. Univ. Torino, Ser. 3, 48: 133144.

- 1954. The trouts of Asiatic Turkey. Istamb. Univ. Fen. Fak. Hidrobiol, Ser. B, 2: 1-46.

- 1961. Catalogo dei tipi di pesci del Museo Civico di Storia Naturale di Genova (Parte I). Ann. Mus. Civ. Stor. Nat. Genova, 72: 179-191. 1963a. Catalogo dei tipi di pesci del Museo Civico di Storia Naturale di Genova (Parte II). Ánn. Mus. Civ. Stor. Nat. Genova, 73: 306-316. - 1963b. Catalogo dei tipi di pesci del Museo Civico di Storia Naturale di Genova (Parte III). Ann. Mus. Civ. Stor. Nat. Genova, 73: 333-350. - 1970a. Fauna d'Italia. Vol. 10. Osteichthyes (pesci ossei). Parte prima. Calderini, Bologna, $565 \mathrm{pp}$.

- 1970b. Aphanius fasciatus Nardo, 1827: nome valido per il ciprinodontide delle coste italiane (Pisces). Doriana, 4 (189): 1-3.

- 1975. Fauna d'Italia. Vol. 11. Osteichthyes (pesci ossei). Parte seconda. Calderini, Bologna, 636 pp., 8 pls.

Tortonese, E. \& J.-C. Hureau. 1979. Clofnam, supplément 1978. Cybium, (3) 1979 (5): 5-66 [also as pp. 333--394 of Hureau \& Monod, 1973]. 
Trabelsi, M., J.-P. Quignard \& J. Kartas. 1994. Atherina boyeri: première mention en Méditerranée de deux populations marines sympatriques. Cybium, 18: 457-459.

Trautschold, H. von. 1884. [title unknown]. Mem. Soc. Nat. Moscou, 15 (1).

Trewavas, E. 1971. The type-species of the genera Phoxinellus, Pseudophoxinus and Paraphoxinus (Pisces, Cyprinidae). Bull. Brit. Mus. Nat. Hist., Zool., 21: 359-361.

Tseeb, Y. 1930. [A study of the Crimean shemaya Alburnus chalcoides mentoides]. Trudy Krymskogo Nauchno-Issledovatelskogo Instituta, Simferopol, 3 (1): 133-166.

Tseeb, Y. \& S. L. Delyamure. 1938. [Material on the freshwater fish fauna of the Crimea]. Izv. Krymskogo Pedagogicheskogo Instituta, Simferopol, 7: 143-148.

Tsigenopoulos, C. 1996. Phylogéographie mitochondriale du genre Barbus au nord de la Mediterranée. Une approche de la spéciation des poissons d'eau douce d'Europe occidentale. DEA Thesis, Université de Montpellier, 38 pp.

Tsigenopoulos, C. \& Y. Karakousis. 1996. Phylogenetic relationships of Leuciscus keadicus, an endemic cyprinid species from Greece, with other Greek species in the genus Leuciscus. Folia Zool., 45: 87-93.

Turdakov, F. A. \& K. V. Piskarev. 1955. [Chuisk gudgeon]. Trudy Inst. Zool. Parasitol. Kirgiz. Acad. Sci., 3: 83-91.

Tyapkin, P. N. 1939. [Abramis sapa aralensis subsp. n. - a new subspecies of white-eye bream from the Aral Sea]. Sbornik Trudov Zoologicheskogo Museya Moskovskogo Universiteta, 5: 144-150.

Uiblein, F. \& H. Winkler. 1994. Morphological variability among Vimba in Austrian waters: quantitative examination of a taxonomic and a functionnal hypothesis (Pisces: Cyprinidae). Senck. Biol., 73: 57-65.

Valenciennes, A. 1837-1844. Ichthyologie des îles Canaries, ou histoire naturelle des poissons rapportés par MM. Webb et Berthelot. In: B. Parker-Webb \& S. Berthelot, Histoire naturelle des îles Canaries, Paris, 2 (2): 1-109, 26 pls. [plates: 1837: 1, 3-4, 8, 11-12; 1938: 7, 10, 13, 14, 17; 1839: 5, 9, 15-16, 18; 1840: 2; 1841: 6; 1842: 19; 1843: 20-23; 1844: 24-26. Text: 1842: 1-8; 1843: 9-96; 1844: 97-109; according to Hureau \& Monod, 1973: 157.]

Vallot, J.-N. 1837. Ichthyologie française, ou histoire naturelle des poissons d'eau douce de la France. Frantin, Dijon, 321 pp.

Valmont de Bomare, J. C. 1765. Dictionnaire raisonné d'histoire naturelle, contenant l'histoire des animaux, des végétaux et des minéraux ... Paris, 5 vols. [2nd edition, 1768, Paris, 4 vols.; 4th edition, 1791, Lyon, 8 vols.]

Van Beneden, P.-J. 1857. Un nouveau poisson du littoral de Belgique (Petromyzon Omalii). Bull. Acad. Roy. Sci. Lettr. Beaux-Arts Belgique, Sér. 2, 2: 549-554, 1 pl.

Vásárhelyi, S. 1940. Trutta Ungeri n. sp., eine neue Forellenart in der ungarischen Fauna. Zool. Anz., 132: 187-190.

Vasiljev, V. P. \& E. D. Vasiljeva. 1994. The karyological diversity in spined loaches from genera Cobitis and Sabanejewia, p. 67. In: VIII Congress Societas Europaea Ichthyologorum. Fishes and their environment. Program and abstracts. Oviedo, 26 Sept. - 2 Oct. 1994.

Vasiljeva, E. D., M. J. Collares-Pereira \& J. M. Madeira. 1992. [Variability, divergence and taxonomy of loach of the Iberian Peninsula]. Vopr. Ikhtiol., 32: 41-55. [Russian, translation in J. Ichthyol., 32 (4): 69-84]

Vasiljeva, E. D. [Vasil'yeva, Y. D.] \& P. Ráb. 1992. [Golden spiny loach, Sabanejewia aurata (Cobitidae), from the Laborec River]. Vopr. Ikhtiol., 32 (2): 176-181. [Russian, translation in J. Ichthyol., 32 (6): 138-144]

Vasiljeva [Vasil'eva], E. D. \& V. P. Vasiljev [Vasil'ev]. 1988. [Study of the intraspecific structure of Sabanejewia aurata (Cobitidae) with description of a new subspecies, $S$. aurata kubanica]. Vopr. Ikhthiol., 28: 192-212. [Russian, translation in J. Ichthyol., 28 (6): 15-35]

- 1996. The description of Neogobius iljini sp. nov. within former $N$. kessleri (Gobiidae, Pisces). Acta Univ. Carol., Biol., 39 (1995 [1996]): 261-270.

Veesenmayer, [G.] 1884. Beiträge zur Fauna Württembergs. I. Barbus fluviatilis Ag. var. aurata. Jahreshefte Ver. Vaterl. Naturk. Württ., 40: 325-326.

Velikokhatko, F. D. 1940. [New forms of Vimba vimba L. from the Dnieper and the Bug]. Izv. Akad. Nauk SSSR, Biol., 1940 (2): 270-275.

Verga, D. 1841. [ ... Descrizione d'un Gobius frequente nelle lagune di Comacchio...]. Atti della Terza Riunione degli Scienziati Italiani tenuta in Firenze, p. 379

Villwock, W., A. Scholl \& P. Labhart. 1982. Die Gattung Valencia Myers, 1928 (Pisces: Cyprinodontidae), ein Beitrag zur Verbreitung und Taxonomie. Mitt. Hamburg. Zool. Mus. Inst., 79: 273-280, pl. 1.

Vinciguerra, D. 1921. Descrizione di tre nuove specie di pesci delle acque dolci di Grecia. Ann. 
Mus. Civ. Stor. Nat. Genova, Ser. 3, 9: 322-331, pl. 4.

1933. Pesci di Albania raccolti dal Dr. Pietro Parenzan nel 1930. Ann. Mus. Civ. Stor. Nat. Genova, 46: 303-310.

Vladimirov, V. J. 1950. O systematicheskom polozhenii azovskoy i chernomorskoy tyul'ki Clupeonella delicatula (Nordmann). Dokl. Akad. Nauk. SSSR, 70: 125-128.

Vladykov, V. [V. D.] 1925a. Über einige neue Fische aus der Tschechoslowakei (Karpathorussland). Zool. Anz., 64: 248-252.

- 1925b. Úber eine neue Cobitis-Art aus der Tschechoslovakei: Cobitis montana n. sp. Zool. Jahrb., Abt. Syst. Geogr. Biol. Tiere, 50: 320338 , pls. $7-8$.

- 1930. Sur une nouvelle forme de gardon de la Russie sous-carpathique (bassin du Danube) Rutilus rutilus carpathorossicus subsp. n. Bull. Soc. Zool. Fr., 55: 103-107.

- 1931. Poissons de la Russie sous-carpathique (Tchécoslovaquie). Mém. Soc. Zool. Fr., 29: $217-374$.

- 1955. Lampetra zanandreai, a new species of lamprey from northern Italy. Copeia, 1955: 215223, 4 pls.

Vladykov, V. D. \& E. Kott. 1982. Comment on Reeve M. Bailey's view of lamprey systematics. Can. J. Fish. Aquat. Sci., 39: 1215-1217.

Vladykov, V. [V. D.] \& G. Petit. 1930. Sur quelques poissons d'eau douce d'Albanie. Bull. Soc. Zool. Fr., 55: 383-409.

Vladykov, V. D., C. B. Renaud, E. Kott \& P. S. Economidis. 1982. A new nonparasitic species of Holarctic lamprey, genus Eudontomyzon Regan, 1911 (Petromyzontidae), from Greece. Can. J. Zool., 60: 2897-2915.

Vogt, C. \& B. Hofer. 1908-1909. Die Süsswasserfische von Mittel-Europa. Engelmann, Leipzig, xxiv +558 pp. (1909), 31 pls (1908).

Vukovic, T. 1963. Prilog poznavanju rasprostranjenja Leuciscus souffia Risso u vodama Jugoslavije i opis podvrste Leuciscus souffia montenegrinus n. ssp. Godisnjak Bioloskog Instituta Univerziteta u Sarajevu, 16: 205-207.

- 1965. Leuciscus souffia montenegrinus n. ssp. (Cyprinidae, Pisces) eine neue Unterart aus dem Flusse Moraca. Bull. Scient. Conseil Acad. RSF Yougosl., Sect. A. Sci. Nat., Techn. Med., 10: 217 .

Vukovic, T. \& B. Ivanovic. 1971. Slast kovodne ribe Jugoslavije. Zemaljski Muzeh, Sarajevo, 268 pp.

Vuorinen, J., M. J.-K. Himberg \& P. Lankinen, P. 1981. Genetic differentiation in Coregonus albula (L.) (Salmonidae) populations in Finland.
Hereditas, 94: 113-121.

Wagenitz, G. 1963. Taxonomie und Evolutionforschung im Bereich höheren Kategorien. Ber. Dtsch. Bot. Ges., 76 (Sondernummer): 91-97.

Wagler, E. 1930a. Die Coregonen in den Seen des Voralpengebietes. II. Die Schwebrenke des Tegernsees. Arch. Hydrobiol., 21: 455-469, pls. $23-26$.

- 1930b. Die Coregonen in den Seen des Voralpengebietes. III. Die Schwebrenke des Ammersees. Arch. Hydrobiol., 21: 470-482, pls. 1416.

- 1932. Die Coregonen in den Seen des Voralpengebietes. VI. Die Schwebrenken des Chiemsees. Arch. Hydrobiol., 24: 282-300, pls. 9-11.

- 1933. Die Coregonen in den Seen des Voralpengebietes. VII. Der Kilch des Bodensees (Coregonus acronius von Rapp). Int. Rev. Ges. Hydrobiol. Hydrogr., 30: 1-48, 2 pls.

- 1937. Die Coregonen in den Seen des Voralpengebietes. IX. Die Systematik der Voralpencoregonen. Int. Rev. Ges. Hydrobiol. Hydrogr., 35: 345-446, 7 pls.

- 1941. Die Lachsartigen (Salmonidae). II. Teil. Coregonen, p. 371-501. In: R. Demoll \& H. N. Maier (eds), Handbuch der Binnenfischerei Mitteleuropas. Vol. IIIA. Schweizerbart, Stuttgart.

- 1950. Die Coregonen in den Seen des Voralpengebietes. XI. Herkunft und Einwanderung der Voralpencoregonen. Veröffentl. Zool. Staatssamml. München, 1: 3-62, 8 pls.

Wagner, R. 1828. Beyträge zur Kenntniss der Gattung Lebias Cuvier und der verwandten Gattungen, nebst Beschreibung zweyer neuen in Sardinien entdeckten Arten. Isis (von Oken), 21 (10): 1050-1158, pl. 12.

Wajgel, L. 1884. Die Zusammenziehung der zwei Arten von Petromyzon ( $P$. Planeri und $P$. fluviatilis) in Eine. Verh. Zool.-Bot. Ges. Wien, 33 (1883 [1884]): 311-319, pl. 17.

Walbaum, J. J. 1792. Petri Artedi sueci Genera piscium in quibus systema totum ichthyologiae proponitur cum classibus, ordinibus, generum characteribus, specierum differentiis, observationibus plurimis. A. F. Rose, Grypeswaldiae, 723 pp., 3 pls.

Walecki, A. 1863. Materyaly do fauny ichtyologicznej Polski. Biblioteka Warszawska, 1863 [1864] (3): 325-364. [date 1864 printed on reprint]

- 1864. Materyaly do fauny ichtyjologicznej Polski. II. Systematyczny przeglad ryb Krajowych. Warszawa, $115 \mathrm{pp}$.

Walker, A. F., R. B. Greer \& A. S. Gardner. 
1988. Two ecologically distinct forms of Arctic charr Salvelinus alpinus (L. ) in Loch Rannoch, Scotland. Biol. Cons., 43: 43-61.

Walker, J. 1812. Essays on natural history and rural economy. Longman, Hurst, Rees and Orme, London \& Guthrie \& Anderson, Edinburgh, ii+629 pp.

Wang Y. 1979. [On the classification, distribution, origin and evolution of the fishes referred to the subfamily Cyprininae of China, with description of a new species]. Acta Hydrobiol. Sinica, 6: 419-438, pl. 1. [Chinese, English summary]

Warpachovski, N. 1886a. Eine neue Phoxinus-Art. Zool. Anz., 9: 76-77.

- 1886b. [Fishes of lake Ilmen and the Volkhov river]. Zapiski Akad. Nauk, 53: 31-68.

- 1887. Notiz über die in Russland vorkommenden Arten der Gattung Phoxinus. Bull. Acad. Imp. Sci. St. Petersbourg, 31: 533-536.

- 1889a. [Short data on the ichthyofauna of Russia-in-Asia]. Zapiski Akad. Nauk, 59: 1-21.

- 1889b. [A key to the fishes of the Volga basin].

- 1892. [Fish collection at the All-Russian Fisheries Exhibition]. Vestnik Rybopromyshlennosti 7: 145-157.

- 1895. [Some data on the fish fauna of the eastern Transcaucasia]. Russkoe Sudokhodstvo, 158: 25-35.

- 1897. [Sur la faune ichthyologique du bassin du fleuve Obi. I]. Annu. Mus. Zool. Acad. Imp. Sci. St. Petersburg, 2: 241-271, pls. 11-12.

- 1900. [Die Fische des Telétzki-Sees]. Annu. Mus. Zool. Acad. Imp. Sci. St. Petersburg, 5: 412-427, pl. 13.

Warren, M. L. 1992. Variation of the spotted sunfish, Lepomis punctatus complex (Centrarchidae): meristics, morphometrics, pigmentation and species limits. Bull. Alabama Mus. Nat. Hist., 12: 1-47.

Wartmann, B. 1777. Beschreibung und Naturgeschichte des Blaufelchen. Beschäft. Berlin. Ges. Naturforsch. Freunde, 3: 184-213.

- 1783. [Ausziige aus Briefen:] Vom Herrn D. Wartmann vom 9ten Jan. 1783. Schrift. Berlin. Ges. Naturforsch. Freunde, 4: 431-433.

- 1783. Von dem Rheinanken oder Illanken, Salmo Illanca. Schrift. Berlin. Ges. Naturforsch. Freunde, 4: 55-68.

Werner, F. 1931. Ergebnisse einer zoologischen Forschungsreise nach Marokko. II. Fische. SitzBer. Akad. Wiss. Wien, Abt. 1, 140: 261-270, 3 pls.

Wheeler, A. 1958. The Gronovius fish collection: a catalogue and historical account. Bull. Brit.
Mus. Nat. Hist., Hist. Ser., 1: 185-249, pls. 2634.

- 1966. A note on Francis Day's "The fishes of Great Britain and Ireland". J. Soc. Bibl. Nat. Hist., 4: 252.

- 1985. The Linnaean fish collection in the Linnean Society of London. Zool. J. Linn. Soc., 84: 1-76.

— 1988. [Review of:] The freshwater fishes of Europe. Vol. 9. Threatened fishes of Europe. By A. Lelek. Copeia, 1988: 1099-1100.

- 1991. The Linnaean fish collection in the Zoological Museum of the University of Uppsala. Zool. J. Linn. Soc., 103: 145-195.

- 1993. [Review of:] The freshwater fishes of Europe. Volume 2. Clupeidae, Anguillidae. By H. Hoestlandt. Copeia, 1993: 249-250.

Wheeler, A. \& K. Easton. 1978. Hybrids of chub and roach (Leuciscus cephalus and Rutilus rutilus) in English rivers. J. Fish Biol., 12: 167171, 2 pls.

Whitehead, P. J. P. 1967. The clupeoid fishes described by Lacepède, Cuvier \& Valenciennes. Bull. Brit. Mus. Nat. Hist., Zool., Suppl., 2: 1180.

- 1985. Clupeoid fishes of the world (Suborder Clupeoidei). An annotated and illustrated catalogue of the herrings, sardines, pilchards, sprats, shads, anchovies and wolf-herrings. Part 1 Chirocentridae, Clupeidae and Pristigasteridae. FAO Fish. Synops., 125 (vol. 7, part 1): 1-303.

- 1990. Systematics: an endangered species. Syst. Zool., 39: 179-184.

Whitehead, P. J. P., M.-L. Bauchot, J.-C. Hureau, J. Nielsen \& E. Tortonese. 1986. Fishes of the north-eastern Atlantic and the Mediterranean. UNESCO, Paris, 3 vols., 1473 pp.

Whitehead, P. J. P., G. J. Nelson \& T. Wongratana. 1988. Clupeoid fishes of the world (Suborder Clupeoidei). An annotated and illustrated catalogue of the herrings, sardines, pilchards, sprats, shads, anchovies and wolfherrings. Part 2 - Engraulididae. FAO Fish. Synops., 125 (vol. 7, part 2): 305-579.

Widegren, A. H. 1863. Bidrag till kännedomen om Sveriges salmonider. Oefversigt af Kongl. Vetenskaps-Akademiens Förhandlingar, 19 (1862 [1863]): 517-594, pls. 4-12.

Wiley, E. O. 1978. The evolutionary species concept reconsidered. Syst. Zool., 27: 17-26.

- 1981. Phylogenetics, the theory and practice of phylogenetic systematics. Wiley, New York, $\mathrm{xv}+439 \mathrm{pp}$.

Wilkens, H. 1977. Die Typen der Ichthyologischen Sammlung des Zoologischen Instituts und Zoo- 
logischen Museums der Universität Hamburg (ZMH). Teil III. Mitt. Hamburg. Zool. Mus. Inst., 74: 155-163.

Willughby, F. 1686. De historia piscium libri quatuor. [...] Totum opus recognovit, coaptavit, supplevit, librum etiam primum \& secundum integros adjecit Johannes Raius e Societate Regiae. Oxonii, iii+343+31+11 pp., 186 pls.

Wimberger, P. H. 1992. Plasticity of fish body shape. The effects of diet, development, family and age in two species of Geophagus (Pisces: Cichlidae). Biol. J. Linn. Soc., 45: 197-218.

- 1994. Trophic polymorphisms, plasticity, and speciation in vertebrates, p. 19-43. In: D. J. Stouder, K. L. Fresh \& R. J. Feller (eds), Theory and application of fish feeding ecology. University of South Carolina Press, Columbia, $\mathrm{SC}$.

Winistörfer, J. 1990. Paysage et écosystèmes, p. 41-70. In: J.-B. Racine \& C. Raffestin (eds), Nouvelle géographie de la Suisse et des Suisses, vol. 1. Payot, Lausanne.

Witkowski, A. 1979. A taxonomic study on freshwater sculpins of genus Cottus Linnaeus, 1758 (Cottus gobio L. and Cottus poecilopus Heck.) in Poland. Acta Univ. Wratislav., 458 (Prace Zool. 10): 1-95.

- 1994. Morphological characteristics of Sabanejewia aurata (De Filippi, 1865) from the Odra River basin, with description of a new subspecies (Teleostei: Cypriniformes: Cobitidae). Zool. Abhandl. Staatl. Mus. Tierkunde Dresden, 48: 23-51.

- 1995. Phenotypic variability of Cottus gobio Linnaeus, 1758 in Polish waters (Teleostei: Scorpaeniformes: Cottidae). Zool. Abhandl. Staatl. Mus. Tierkunde Dresden, 48: 177-183.

Witte, F. 1984. Consistency and functional significance of morphological differences between wild-caught and domestic Haplochromis squamipinnis (Pisces, Cichlidae). Neth. J. Zool., 34: 596-612.

Wu, H.-W. 1977. [The cyprinid fishes of China. Vol. 2]. Technical Printing House, Shanghai, pp. 229-598, 109 pls. [Chinese]

Wulff, J. C. 1765. Ichthyologia, cum amphibiis regni borussici. Methodo linneana disposita. Kanter, Regiomonti, 60 pp.

Yakovlev, V. E. 1870. [On the new and littleknown species of fish found in the mouths of the Volga]. Protokoly Zasedanii Obshchestva Estestvoispytatelei pri Kazanskom Universitete, 1869-1870: 101-111.

- 1873. [A note on the Caspian vobla]. Priroda, (1) 2 (2): $332-345$.
Yarrell, W. [1833]. Descriptions of three British species of fresh-water fishes belonging to the genus Leuciscus Klein. Trans. Linn. Soc. London, 17 [1833-1837]: 5-10, pl. 2.

- 1835-1839. A history of British fishes, illustrated by nearly 400 woodcut, in two volumes. Van Voorst, London, 1 (1835-1836): xxxvii+408 pp.; 2 (1836): 472 pp.; suppl. (1839): vi+48 pp.

Yang J.-X. \& X.-L. Chu. 1987. [Phylogeny of the cyprinid genus Anabarilius (Pisces: Cyprinidae)]. Zool. Res., 8: 261-276. [Chinese, English summary]

Yashchenko, A. 1895. [Catalogue of the collection of the zoological cabinet of the St. Petersburg University. 1. Pisces]. Trudy SanktPeterburgskogo Obshchestva Estestvoispytatelei, Otd. Zoologii i Fisiologii, 25 (2): 59-99. [Russian]

Zanandrea, G. 1959. Lamprede parassite e non parassite nel bacino del Danubio e la nuova entità sistematica Eudontomyzon danfordi vladykovi. Arch. Zool. Ital., 44: 215-249, 2 pls.

Zava, B., T. Beller, P. Chiari, P. A. Nardi, C. Violani \& F. Bernini. 1996. Note faunistiche e tassonomiche su Salmo (trutta) macrostigma (Dum.) della Sicilia (Osteichthyes, Salmonidae). Atti Congressuali, $4^{\circ}$ Convegno Nazionale A.I.I.A.D. 1991, pp. 413-421.

Zerunian, S. 1982. A new cyprinid from Italy: Rutilus erythrophthalmus n. sp., $\mathrm{N}^{\circ}$ 346. In: Fourth Congress of European Ichthyologists. Abstracts. Hamburg.

- 1984. Il problema sistematico dei Rutilus italiani (Pisces, Cyprinidae). Boll. Mus. Civ. Stor. Nat. Verona, 11: 217-236.

- 1991. A proposito del nome scientifico del triotto (Osteichthyes, Cyprinidae). Atti Soc. Ital. Sci. Nat. Mus. Civ. Stor. Nat. Milano, 131: 285-289.

Zerunian, S. \& G. Gandolfi. 1990. Salmo fibreni n. sp. (Osteichthyes, Salmonidae) endemica nel bacino del Fibreno (Italia centrale). Riv. Idrobiol., 29: 521-531.

Zhou W. 1989. [Phylogeny of the subfamily Cyprininae (Pisces: Cyprinidae)]. Acta Zootax. Sinica, 14: 247-256. [Chinese, English summary] Zhou W. \& X.-L. Chu. 1986. [Systematic study of the genus Cyprinus (Pisces: Cyprinidae) in Yunnan, China]. Zool. Res., 7: 297-310. [Chinese, English summary]

Zhukov, P. I. 1965a. [Fishes of Belarus]. Nauka i Tekhnika, Minsk, 415 pp. [Russian]

- 1965b. [Distribution and evolution of freshwater lampreys in the BSSR waters]. Vopr. Ikhtiol., 5: 240-244. [Russian] 
- 1968. [The routes of penetration of PontoCaspian chthyofauna into the rivers of the Baltic Sea basin]. Zool. Zh., 47: 1417-1419. [Russian, English summary]

- 1975. Puti formirovaniya dannye ikhtiofauny presnykh vodoemov vostochnoi chasti basseina Baltiiskogo morya. Ichthyologia, 7: 85-91.
Zill. M. 1858. Note sur l'existence de la truite en Algérie. Ann. Sci. Nat., Zool., Sér. 4, 9: 126-128. Zubovich, P. O. 1925. [On the Black Sea Gobiidae. I. Mesogobius gymnotrachelus otschakovinus subsp.]. Trudy Gosudarstvennoi Chernomorsko-Azovskoi Opytnoi Stantsii, 1: 189-202. 


\section{INDEX}

Subspecies and infrasubspecific names are listed under generic name in alphabetical sequence and with omission of species and other intercalate names or words (e.g. Acipenser güldenstaedti var. golis is listed as Acipenser golis). Accidental misspelling of generic names are corrected (e.g. Acipenser for Acipense) misspellings of species names are not corrected. Unless involved in nomenclatural discussions, introduced taxa are not indexed.

- acutirostris 2.1.1

- affinis 2.1 .4

- alba 2.1.4

- albinea 2.1 .4

- albula 2.2.1

- aleutensis 2.1 .4

- attilus 2.1.6

- baschmakovae 2.1.4

- beluga 2.2.1

- birostrata 2.1 .4

- brandtii 30.1 .1

- brevirostris 2.1.1, 2.1.4

- colchica 2.1.1

- cyrensis 2.1.5

- danubialis 2.1 .5

- danubica 2.1.1

- derjavini 2.1.3

- donensis 2.1 .5

- dubius 2.1.4

- ducissae 2.1.6

- erythraea 2.1.4

- europaeus 2.1.6

- fitzingeri 2.1.6

- glaber 2.1.3

- gmelini 2.1.4

- golis 2.1.1

- grisescens 2.1.4

- gueldenstaedtii 2.1.1

- güldenstädtii 2.1.1

- heckelii 2.1.2

- helenae 30.1.1

- helops 2.1.5

- hospitus 2.1.6

- illyricus 2.1 .5

- jenisensis 2.1.4

- kamensis 2.1.4

- kankreni 2.1.4

- kostera 2.1.4

- ladanus 2.1.2

- latirostris 2.1.6

- leucotica 2.1.4

- lichtensteinii 2.1.6

- longirostris 2.1.1

- lovetzkyi 2.1.4

- lutescens 2.1.2

- macrophthalmus 2.1.1

- marsiglii 2.1.4

- medius 2.1.1

- naccarii 2.1.2

- nardoi 2.1.2

- nasus 2.1.2 
- nehelae 2.1.6

- nudiventris 2.1 .3

- obtusirostris 2.1.4.

- platycephalus 2.1.2

- podapos 2.1.6

- primigenius 30.1.1

- pygmaeus 2.1.1, 2.1.4

- ratzeburgii 2.1.5

- rostratus 2.1 .5

- ruthenicus 2.1.4

- ruthenus 2.1.4

- ruzskyi 2.1.4

- schypa 2.1.3, 2.2.1

- scobar 2.1.1

- septemcarinata 2.1.4

- seuruga 2.1.5

- shyp 2.2 .1

- stellatus 2.1.5

- sterlet 2.1.4

- sterleta 2.1.4

- sturio 2.1.6

- sturioides 2.1.6

- sturionaster 2.1.2

- sturionellus 2.1.2

- tanaica 2.1.1

- thompsonii 2.1.6

- tücka 2.1.1

- turritus 2.1.3

- valenciennii 2.1.6

- vallisnerii 2.2.1

- yarrellii 2.1.6

acronius, Coregonus 13.1.19

aculeatus, Acipenser 2.1.1

aculeatus, Gasterosteus 21.1.1

acuminatus, Cyprinus 5.11.1

acus, Syngnathus 22.2.2

acutipinnatus, Gobio 5.13.5

acutirostris, Acipenser 2.1.1

acutirostris, Anguilla 3.1.1

acutirostris, Coregonus 13.1.21

acutus, Alburnus 5.3.2

adriatica, Trutta 14.3.4, 14.3.14

adriaticus, Petromyzon 1.5.1

adspersus, Leucos 5.20.1

adspersus, Phoxinellus 5.20.1

aegyptia, Atherina 17.1.1

aegyptiaca, Anguilla 3.1.1

aeliani, Thymallus 14.6.1

aeolicus, Syngnathus 22.2.1

aestivalis, Salmo 14.3.1

affinis, Acipenser 2.1.4

affinis, Cottus 23.1.2

affinis, Gobius 27.7.5

affinis, Leuciscus 30.1.2

africana, Alosa 4.1.2

agassii, Leuciscus 5.17 .16

agassiz, Syngnathus 22.2.1

agassizii, Leuciscus 5.17.16

agdamicus, Squalius 5.17.4

agone, Alosa 4.1.1

agone, Cyprinus 4.1.1

Alausa vulgaris 4.1.3 alba, Acipenser 2.1.4

alba, Barbus 5.7.2

alba, Salmo 14.3.10

albanicus, Barbus 5.7.1

albanicus, Gobio 5.13.5

albellus, Coregonus 13.1.9

albescens, Gobius 27.4.2

albicans, Chondrostoma 5.10.2

albicoloris, Cobitis 6.3.2

albidus, Alburnus 5.3.1

albidus, Leuciscus 5.3.1

albiensis, Leuciscus 5.17.4

albinea, Acipenser 2.1.4

albipinnatus, Gobio 5.13.1

albor, Cyprinus 5.3.2

Alborella maxima 5.3.2

alborella, Alburnus 5.3.2

albula, Acipenser 2.2.1

albula, Coregonus 13.1.28

albula, Cyprinus 5.17.4

albula, Salmo 13.1.28

alburnellus, Leuciscus 5.3.2

alburniformis, Bliccopsis 30.1.2

alburniformis, Scardinopsis 30.1.2

Alburnoides armeniensis 5.2.1

- bipunctatus 5.2.1

- kubanicus 5.2.1

- rossicus 5.2.1

- smyrnaea 5.2.1

- strymonicus 5.2.1

- thessalicus 5.2.1

- tzanevi 5.2.1

alburnoides, aspius 5.3.2

alburnoides, Leuciscus 5.24.1

alburnoides, Rutilus 5.24.1

alburnolucidus, Leuciscus 30.1.2

Alburnus acutus 5.3.2

- albidus 5.3.1

- alborella 5.3.2

- alburnu 5.3.2

- angustior 5.3.2

- arquatus 5.3.2

- augustior 5.3.2

- ausonii 5.3.2

- avola 5.3.2

- belvica 5.9.1

- breviceps 5.3.2

- carinatus 5.9.2

- colobocephala 5.3.2

- dagestanicus 5.3.2

- danubicus 5.9.2

- derjugini 5.9.2

- eichwaldii 5.2.1

- elata 5.3.2

- elongata 5.3.2

- fabraei 5.3.2

- fracchia 5.3.2

- gracilis 5.3.2

- iblioides 5.5.1

- ilmenensis 5.3.2

- istanbulensis 5.9.2

- lacustris 5.3.2 
- lateristriga 5.3.2

- latior 5.3.2

- latissimus 5.9.2

- leydigi 30.1.2

- linnéi 5.3.2

- longissimus 5.9.2

- lucidus 5.3.2

- macedonicus 5.3.2

- maculatus 5.2.1

- mentoides 5.9.2

- mirandella 5.3.2

- nicaeensis 5.9.2

- obtusus 5.3.2

- ohridanus 5.2.1

- oxycephala 5.3.2

- prespensis 5.2.1

- rosenhaueri 30.1.2

- sapancae 5.9.2

- scoranza 5.3.2

- scoranzoides 5.3.2

- strigio 5.3.2

- strumicae 5.3.2

- tauricus 30.1.2

- thessalicus 5.3.2

alburnus, Alburnus 5.3.2

alburnus, Cyprinus 5.3.2

albus, Leuciscus 5.17.4

albus, Salmo 14.3.23.1

alepidotus, Cyprinus 5.11.1

alepidotus, Phoxinellus 5.20.2

aleutensis, Acipenser 2.1.4

alfiensis, Rutilus 5.17.13

algarbiensis, Gobius 27.4.1

algeriensis, Alosa 4.1.2

algeriensis, Gasterosteus 21.1

algeriensis, Syngnathus 22.2.1

Alosa africana 4.1.2

- agone 4.1.1

- algeriensis 4.1.2

- alosa 4.1.3

- asovi 4.1.9

- benacensis 4.1.1

- bolivari 4.1.2

- borysthenis 4.1 .5

- bulgarica 4.1.9

- caspia 4.1.9

- ceresio-verbana 4.1.1

- communis 4.1.3

- cuvierii 4.1 .3

- danubii 4.1.5

- fallax 4.1.4

- ficta 4.1.4

- gracilis 4.1.1

- immaculata 4.1.5

- issatschenkovi 4.1.5

- kessleri 4.1.5

- killarnensis 4.1.6

- lacustris 4.1.1

- lariana 4.1.1

- macedonica 4.1.6

- maeotica 4.1 .8

- minor 4.1.4
- moreac 4.1.5

- moriac 4.1.5

- nigrescens 4.1 .5

- nordmanni 4.1.9

- rhodanensis 4.1.4

- rusa 4.1.3

- russac 4.1.5

- tanaica 4.1.9

- vistonica 4.1.10

- volgensis 4.1.5

alosa, Alosa 4.1.3

alosa, Clupea 4.1.3

alpestris, Blennius 26.1.1

alpino-stagnalis, Salmo 14.5.1

alpinus, Coregonus 13.1.10, 13.1.11

alpinus, Salmo 14.5.1

alpinus, Salvelinus 14.5.1

alternans, Syngnathus 22.2.2

altior, Coregonus 13.1.42

altirostris, Anguilla 3.1.1

altus, Leuciscus 5.24.3

altus, Phoxinus 5.12.1

amarus, Cyprinus 5.8.3, 5.23.1

amarus, Rhodeus 5.23.1

americanus, Salmo 14.3.19

ammerensis, Coregonus 13.1.27

Ammocoetes communis 1.3.1

amnipetens, Coregonus 13.1.29

amphigenus, Scardinopsis 30.1.2

Anaecypris hispanica 5.4.1

anarensis, Coregonus 13.1.37

anatoliae, Cobitinula 6.1.10

anatolicus, Cyprinus 5.11.1

anceps, Scardiniopsis 30.1.2

ancidda, Anguilla 3.1.1

angermanensis, Coregonus 13.1.45

anglicana, Nemacheilus 7.1.1

angorensis, Chondrostoma 5.10.4

Anguilla acutirostris 3.1.1

- aegyptiaca 3.1.1

- altirostris 3.1.1

- ancidda 3.1.1

- anguilla 3.1.1

- bibroni 3.1.1

- brevirostris 3.1.1

- callensis 3.1.1

- canariensis 3.1.1

- capitone 3.1.1

- cloacina 3.1.1

- cuvieri 3.1.1

- eurystoma 3.1.1

- fluviatilis 3.1.1

- hibernica 3.1.1

- kieneri 3.1.1

- lacustus 3.1.1

- latirostris 3.1.1

- limnéi 3.1.1

- marginata 3.1.1

- marina 3.1.1

- mediorostris 3.1.1

- melanochir 3.1.1

- microptera 3.1.1 
- migratoria 3.1.1

- morena 3.1.1

- nilotica 3.1.1

- oblongirostris 3.1.1

- platycephala 3.1.1

- platyrhynchus 3.1.1

- savignyi 3.1 .1

- septembrina 3.1.1

- vulgaris 3.1.1

anguilla, Anguilla 3.1.1

anguilla, Muraena 3.1.1

anguillicaudatus, Misgurnus 6.2.1

anguisigola, Syngnathus 22.2.1

angulatus, Cyprinus 5.11.1

angustior, Alburnus 5.3.2

anjubaulti, Squalius 30.1.2

annectus, Coregonus 13.1.14

annoni, Cyprinus 5.2.1

anomalum, Petromyzon 1.3.2

anterina, Atherina 17.1.1

anticolus, Blennius 26.1.1

antipai, Gobio kessleri 5.13.6

Aphanius fasciatus 20.1.1

- iberus 20.1.2

- nanus 20.1.1

aphelios, Salmo 14.3.1

aphnitis, Vimba 5.28.3

Aphya lacustris 11.1.1

aphya, Cyprinus 5.21.1

apollonitis, Leuciscus 5.25.2

apron, Aspro 25.5.1

arahthoensis, Cobitis 6.1.15

aralensis, Abramis 5.1.4

aralensis, Chalcalburnus 5.9.2

aralensis, Cobitis 6.3.1

aralensis, Cyprinus 5.11.1

aralensis, Rutilus 5.24.16

aralensis, Salmo 14.3.28

aralensis, Silurus 9.1.2

aralychensis, Leuciscus 5.17 .4

arborella, aspius 5.3.2

arcasii, Leucos 5.24.2

arcasii, Rutilus 5.24.2

ardebilicus, Leuciscus 5.17.4

arenicolus, Coregonus 13.1.18

argentatissimus, Gasterosteus 21.1.3

argentatus, Syngnathus 22.2.4

argenteus, Fario 14.3.23.1

argenteus, Leuciscus 5.17.8

argenteus, Petromyzon 1.3.1

argenteus, Salmo 14.3.20

argenteus, Squalius 5.17.8

argyreus, Abramis 5.1.3

argyroleuca, Blicca 5.1.2

argyropomus, Gasterosteus 21.1.3

aristotelis, Glanis 9.1.1

aristotelis, Silurus 9.1.1

armeniensis, Alburnoides 5.2.1

arnoldi, Coregonus 13.1.31

arquatus, Alburnus 5.3.2

arrigonis, Chondrostoma 5.10.1

arrigonis, Leuciscus 5.10.1 artedii, Coregonus 13.1.42

arurensis, Coregonus 13.1.10

arvensis, Salmo 14.3.23.2.1

ascanii, Salmo 14.5.1

ashanteënsis, Mugil 16.3.3

asianus, Abramis 5.28.2

asiatica, Lota 15.1.1

asovi, Alosa 4.1.9

Asper verus 25.5.3

asper, Cyprinus 30.1.3

asper, Perca 25.5.1

asper, Zingel 25.5.1

asperi, Coregonus 13.1.17

Asperulus ratisbonensium 25.5.4

Aspius alburnoides 5.3.2

- arborella 5.3.2

- aspius 5.5.1

- erytrostomus 5.5.1

- fasciatus 5.2.1

- heckelii 5.9.2

- jaxartensis 5.5.1

- linnéi 5.5.1

- margaritaceus 30.1.2

- mento 5.9.2

- ochrodon 5.3.2

- ovsianka 5.16.1

- phragmiteti 5.5.1

- transcaucasicus 5.5.1

- vulgaris 5.5.1

aspius, Aspius 5.5.1

aspius, Coregonus 13.1.33

aspius, Cyprinus 5.5.1

aspius, Leuciscus 5.5.1

Aspro apron 25.5.1

- balcanicus 25.5.2

- streber 25.5.3

- vulgaris 25.5.1

asundensis, Cottus 23.2.1

athaerina, Atherina 17.1.2

Atherina aegyptia 17.1.1

- anterina 17.1.1

- athaerina 17.1.2

- bonapartii 17.1.1

- boyeri 17.1.1

- caspia 17.1.1

- hepsetus 17.1.2

- hyalosoma 17.1.1

- lacustris 17.1.1

- marmorata 27.9.3

- mochon 17.1.1

- pontica 17.1.1

- presbyter 17.1.3

- riqueti 17.1 .1

- risso 17.1.1

- sarda 17.1.1

athurensis, Squalius 5.17.4

atrox, Esox 10.1.1

atterensis, Coregonus 13.1.23

attilus, Acipenser 2.1.6

Aturius dufourii 5.17.2

augustior, Alburnus 5.3.2

aula, Rutilus 5.24.3 
aula, Squalius 5.24.3

Aulopyge huegelii 5.6.1

aurata, Barbus 5.7.2

aurata, Cobitis 6.3.1

aurata, Leuciscus 5.24.16

aurata, Liza 16.2.1

aurata, Perca 25.2.1

auratus, Carassius 5.8.1

auratus, Chondrostoma 5.10.4

auratus, Cyprinus 5.26.1

auratus, Leuciscus 5.8.1, 5.17.5, 5.24.16

auratus, Mugil 16.2.1

aurea, Perca 25.2.1

aurea, Tinca 5.26.1

ausonii, Alburnus 5.3.2

ausonii, Salar 14.3.23.1

austriaca, Coregonus 13.1.27

autumnalis, Coregonus 13.1.42

autumnalis, Salvelinus 14.5.21

avernensis, Gobius 27.8.2

avola, Alburnus 5.3.2

azovi, Clupeonella 4.2.1

\section{B}

baerii, Coregonus 13.1.37

baicalensis, Esox 10.1.1

baicalensis, Squalidus 5.17.8

bailloni, Gasterosteus 21.1.3

bailloni, Salar 14.3.23.1

balcanica, Cobitis 6.3.1

balcanica, Sabanejewia 6.3.1

balcanica, Trutta 14.3.2

balcanicus, Aspro 25.5.2

balcanicus, Gobio 5.13.5

balcanicus, Salmo 14.3.2

balcanicus, Zingel 25.5.2

balchaschana, Phoxinus 5.21.1

baldneri, Leuciscus 5.2 .1

balleoides, Coregonus 13.1.14

balleropsis, Abramis 5.1.4

ballerus, Abramis 5.1.1

ballerus, Cyprinus 5.1.1

balleus, Coregonus 13.1.10

baloni, Gymnocephalus 25.1.1

balookee, Salmostoma 5.19.1

baltica, Coregonus 13.1.29

baltica, Pleuronectes 29.1.1, 29.2.2

baltica, Sabanejewia 6.3.1

banarescui, Gobio 5.13.2

banaticus, Gobio 5.13.6

Barbatula barbatula 7.1.1

- brandti 7.1.2

- bureschi 7.1 .2

- samantica 7.1.2

- simavika 7.1.2

barbatula, Barbatula 7.1.1

barbatula, Cobitis 7.1.1

barbatulus, Nemacheilus 7.1.1

barbatulus, Orthrias 7.1.1

Barbus alba 5.7.2

- albanicus 5.7.1

- aurata 5.7.2
- barbus 5.7 .2

- bergi 5.7.6

- bocagei 5.7 .3

- borysthenicus 5.7 .2

- canalii 5.7.20

- caninus 5.7.4

- cholorematicus 5.7.6

- comiza 5.7.5

- comizo 5.7.5

- communis 5.7.2

- cyclolepis 5.7.6

- eques 5.7.16

- ercisianus 5.7.16

- euboicus 5.7.7

- fluviatilis 5.7.2

- fucini 5.7 .20

- gallicus 5.7.2

- graecus 5.7.8

- graellsii 5.7.9

- guiraonis 5.7.10

- haasi 5.7.11

- kosswigi 5.7.16

- lacerta 5.7.16

- macedonicus 5.7.12

- mayori 5.7.2

- meridionalis 5.7.4, 5.7.13

- microcephalus 5.7.14

- microphthalmus 5.7.2

- peloponnesius 5.7.15

- pergamonensis 5.7.6

- petenyi 5.7.15

- petenyii 5.7.15

- plebeius 5.7.16

- plebejus 5.7.16

- polylepis 5.7.6

- prespensis 5.7.17

- rebeli 5.7.15

- scincus 5.7.16

- sclateri 5.7.18

- sperchiensis 5.7.6

- steindachneri 5.7.19

- strumicae 5.7.6

- thessalus 5.7.12

- tyberinus 5.7.20

- tyrasensis 5.7.2

- vulgaris 5.7.2

- waleckii 5.7.15

barbus, Barbus 5.7.2

barbus, Cyprinus 5.7.2

basak, Leucos 5.24.4

basak, Rutilus 5.24.4

baschmakovae, Acipenser 2.1.4

bathi, Pomatoschistus 27.9.1

batrachocephalus, Gobius 27.6.1

batrachocephalus, Mesogobius 27.6.1

baunti, Coregonus 13.1.36

bavarica, Coregonus 13.1.24

bavaricus, Coregonus 13.1.24

bavarorum, Bürstel 25.2.1

bearnensis, Squalius 5.17.2

belingi, Gobio 5.13.1

beluga, Acipenser 2.2.1 
belvica, Alburnus 5.9.1

belvica, Chalcalburnus 5.9.1

benacensis, Alosa 4.1.1

benacensis, Gobio 5.13.3

benaci, Salmo 14.3.3

Benthophiloides brauneri 27.1.1

Benthophilus maeotica 27.2.1

- monstrosus 27.2.1

- nudus 27.2.1

- ponticus 27.2.1

- stellatus 27.2.1

beoticus, Pseudophoxinus 5.22.1

beoticus, Rutilus 5.22.1

bergi, Abramis 5.1.3, 5.1.4

bergi, Barbus 5.7.6

bergi, Coregonus 13.1.29

bergi, Esox 10.1.1

bergi, Lampetra 1.1.1

bergi, Vimba 5.28 .3

bergianus, Coregonus 13.1.45

bergiellus, Coregonus 13.1.35

bezola, Coregonus 13.1.1

biarmatus, Gasterosteus 21.1.1

bibroni, Anguilla 3.1.1

bicolor, Petromyzon 1.3.2

biennis, Salmo 14.3.19

bilineata, Cobitis 6.1.1

bipunctatus, Alburnoides 5.2.1

bipunctatus, Cyprinus 5.2.1

birostrata, Acipenser 2.1.4

bithynicus, Cyprinus 5.11.1

bjoerkna, Abramis 5.1.2

bjoerkna, Cyprinus 5.1.2

blackiana, Nemacheilus 7.1.1

bleekeri, Hucho 14.2.1

blennioides, Gobius 27.10.1

Blennius alpestris 26.1.1

- anticolus 26.1.1

- cagnota 26.1.1

- fluviatilis 26.1.1

- frater 26.1.1

- inaequalis 26.1.1

- lupulus 26.1.1

- petteri 26.1.1

- sujefianus 26.1.1

- vulgaris 26.1.1

Blicca argyroleuca 5.1.2

- derjavini 5.1.2

- intermedia 5.1.2

- transcaucasica 5.1.2

blicca, Cyprinus 5.1.2

Bliccopsis alburniformis 30.1 .2

- erythrophthalmoides 30.1 .2

blochii, Cyprinus 5.2.1

bocagei, Barbus 5.7.3

bodanensis, Coregonus 13.1.21

bodensis, Coregonus 13.1.20, 13.1.18

bodensis, Salmo 14.3.23.2.2

bogdanovii, Pleuronectes 29.1.1

bolivari, Alosa 4.1.2

bolmeniensis, Coregonus 13.1.31 bolmensis, Coregonus 13.1.31

bolmensis, Leuciscus 5.24 .16

bonapartii, Atherina 17.1.1

bondella, Coregonus 13.1.5

bonelli, Gobius 27.8.1

bonelli, Padogobius 27.8.1

borealis, Coregonus 13.1.31

borealis, Petromyzon 1.4.1

borealis, Pleuronectes 29.2.2

borkensis, Cottus 23.2.1

borysthenica, Chondrostoma 5.10.4

borysthenica, Vimba 5.28 .3

borysthenicum, Chondrostoma 5.10.4

borysthenicus, Barbus 5.7.2

borysthenicus, Leuciscus 5.17.1

borysthenicus, Squalius 5.17.1

borysthenis, Alosa 4.1.5

bosniaca, Cobitis 6.3.1

boyeri, Atherina 17.1.1

brachycentrus, Gasterosteus 21.1.3

brachypoma, Salmo 14.3.23.1

brachyrhynchus, Syngnathus 22.2.2

Brama isognathus 30.1.2

brama, Abramis 5.1.3

brama, Cyprinus 5.1.3

branchialis, Petromyzon 1.3.1

brandti, Barbatula 7.1.8

brandtii, Acipenser 30.1.1

brauneri, Benthophiloides 27.1.1

brauneri, Caspialosa 4.1.8

breviceps, Alburnus 5.3.2

breviceps, Gasterosteus 21.2.2

breviceps, Mugil 16.2.1

brevicirri, Cyprinus 5.11.1

brevicirris, Gobio 5.13.5

brevipes, Salmo 14.3.19

brevirostris, Acipenser 2.1.1, 2.1.4

brevirostris, Anguilla 3.1.1

brevirostris, Leptocephalus 3.1.1

brevis, Coregonus 13.1.28

britannicus, Mugil 16.2.2

brittanicus, Gobius 27.4.2

brutius, Leuciscus 5.17 .4

Bubyr kosswigii 27.5.1

bucculentus, Syngnathus 22.2.1

bucephalus, Carassius 5.8.3

bucharensis, Rutilus 5.24.16

buggenhagii, Cyprinus 30.1.2

bulgarica, Alosa 4.1.9

bulgarica, Cobitis 6.3.2

bulgarica, Gobio 5.13.5

bulgarica, Sabanejewia 6.3.2

bullarensis, Coregonus 13.1.31

Bungia nigrescens 5.13.5

buosega, Mugil 16.1.1

burdigalensis, Leuciscus 5.17.2

bureschi, Barbatula 7.1.8

bureschi, Nemacheilus 7.1.8

burgundianus, Gasterosteus 21.2.2

burmeisteri, Gobius 27.7.3

Bürstel bavarorum 25.2.1 


\section{C}

cabeda, Leuciscus 5.17.4

caecifer, Salmo 14.3.23.1

caecus, Petromyzon 1.3.2

caecutiens, Myxus 16.3.3

caerulescens, Chondrostoma 5.10.4

caerulescens, Salmo 14.3.19

caeruleus, Cyprinus 5.23.2

cagnota, Blennius 26.1.1

calaritana, Poecilia 20.1.1

calderoni, Cobitis 6.1.2

callensis, Anguilla 3.1.1

cambricus, Salmo 14.3.23.1, 14.5.14

camtschaticum, Lethenteron 1.4.1

camtschaticus, Petromyzon 1.4.1

canalii, Barbus 5.7.20

canariensis, Anguilla 3.1.1

candidus, Coregonus 13.1.5

canestrinii, Gobius 27.9.2

canestrinii, Pomatoschistus 27.9.2

canina, Umbra 11.1.1

caninensis, Pleuronectes 29.1.1, 29.2.1

caninus, Barbus 5.7.4

capito, Cyprinus 5.17.4

capito, Mugil 16.2.2

capitone, Anguilla 3.1.1

Carassius auratus 5.8.1

- bucephalus 5.8.3

- carassius 5.8.2

- charax 5.8.2

- ellipticus 5.8.3

- encobia 5.8.1

- gibbosus 5.8.2

- gibelio 5.8.3

- humilis 5.8.2

- jacuticus 5.8.2

- kolenty 5.8.3

- lacustris 5.8.2

- linnaei 5.8.2

- linnéi 5.8 .2

- minutus 5.8.2

- moles 5.8.2

- oblongus 5.8 .2

- piscinarum 5.8.2

- subventrosus 5.8.2

- ventrosus 5.8 .3

- vovkii 5.8 .3

- vulgaris 5.8.2

carassius, Carassius 5.8.2

carassius, Cyprinus 5.8.2

carbonarius, Salmo 14.5.1

carii, Leuciscus 30.1.2

carinata, Pygosteus 21.2.4

carinatus, Alburnus 5.9.2

carinatus, Cyprinus 5.28.3

carnaria, Platessa 29.1.1

carneus, Salmo 14.5.20

carolitertii, Leuciscus 5.17.3

carpathicus, Gobio 5.13.5

carpathicus, Phoxinus 5.21.1

carpathorossicus, Gobio 5.13.6

carpathorossicus, Rutilus 5.24.16
Carpio sieboldii 30.1.2

carpio, Cyprinus 5.11.1

carpio, Salmo 14.3.3

caspia, Alosa 4.1.9

caspia, Atherina 17.1.1

caspia, Clupeonella 4.2.1

Caspialosa brauneri 4.1.8

- chtamalocephala 4.1.5

- elongata 4.1.8

- etemi 4.1.9

- hypselocephala 4.1.5

- palaeostomi 4.1.9

- palaeostomica 4.1.9

caspicus, Cyprinus 5.11.1

caspicus, Huso 2.2.1

caspicus, Leuciscus 5.24.16

Caspiomyzon wagneri 1.5 .1

caspius, Leuciscus 5.24 .5

caspius, Salmo 14.3.28

caspius, Syngnathus 22.2.1

caucasica, Gobio 5.13.5

caucasica, Knipowitschia 27.5.1

caucasicus, Gasterosteus 21.2.3

caucasicus, Gobius 27.5.1

caucasicus, Leucaspius 5.16.1

caucasicus, Pomatoschistus 27.5.1

caustelus, Mugil 16.2.2

cavedanus, Leuciscus 5.17.4

cenerinus, Salmo 14.3.4

cenisophius, Leucos 5.24.16

cenomanensis, Abramis 30.1.2

Centropomus mullus 24.1.1

- sandat 25.4.1

cepedii, Coregonus 13.1.40

cephalarges, Gobius 27.7.5

cephalopsis, Squalius 5.17.4

cephalotus, Mugil 16.3.3

cephalus, Cyprinus 5.17.4

cephalus, Leuciscus 5.17.4

cephalus, Mugil 16.3.3

ceresio-verbana, Alosa 4.1.1

Cernua fluviatilis 25.1.2

cernua, Perca 25.1.2

cernuus, Gymnocephalus 25.1.2

cestellae, Tinca 5.26.1

cettii, Salmo 14.3.5

Chalcalburnus aralensis 5.9.2

- belvica 5.9.1

- chalcoides 5.9.2

- iranicus 5.9 .2

- longicephala 5.9.2

- macedonicus 5.9.2

- mandrensis 5.9.2

- schischkovi 5.9.2

chalcoides, Chalcalburnus 5.9.2

chalcoides, Cyprinus 5.9.2

chalybeius, Squalius 5.17.8

charax, Carassius 5.8.2

charrieri, Syrrhothonus 27.9.3,

chelo, Mugil 16.1.1

Chelon labrosus 16.1.1

chibinae, Coregonus 13.1.31 
chilo, Gobius 27.7.5

cholorematicus, Barbus 5.7.6

Chondrochylus nasicus 5.10 .9

Chondrostoma albicans 5.10.2

- angorensis 5.10.4

- arrigonis 5.10.1

- auratus 5.10.4

- borysthenica 5.10 .4

- borysthenicum 5.10.4

- caerulescens 5.10.4

- dremaei 5.1010

- duriensis 5.10.6

- genei 5.10 .2

- hernadiensis 5.10 .4

- jaculum 5.10.2

- knerii 5.10.3

- lumi 5.10.4

- lusitanicum 5.24.9

- macrolepidotus 5.10.4

- miegii 5.1010

- nasus 5.10.4

- ohridanum 5.10.4

- peresi 5.1010

- phoxinus 5.10.5

- polylepis 5.10.6

- prespense 5.10 .7

- prespensis 5.10 .7

- reiseri 30.1.2

- rhodanensis 5.1010

- rysela 5.10.9, 5.17.16

- scodrense 5.10 .8

- scodrensis 5.10.8

- seva 5.10 .9

- soetta 5.10 .9

- steindachneri 30.1.2

- taeniata 5.10 .4

- toxostoma 5.1010

- turiensis 5.10 .1

- vardarense 5.1011

- vardarensis 5.1011

- willkommii 5.1012

chrysitis, Tinca 5.26 .1

chrysoprasius, Cyprinus 5.21.1

chtamalocephala, Caspialosa 4.1.5

chub, Cyprinus 5.17.4

cicatricosus, Pleuronectes 29.2.1

cii, Leuciscus 5.17.4

Ciprinoides nanus 20.1.1

cirrosus, Cyprinus 5.11.1

cisalpinus, Leucos 5.24 .3

ciscaucasicus, Salmo 14.3.28

Citharus linguatula 28.1.1

citharus, Pleuronectes 28.1.1

citrina, Gobius 27.11.1

clathratus, Squalius 5.17.4

cleveza, Cyprinus 5.1.4

cloacina, Anguilla 3.1.1

Clupea alosa 4.1.3

- cultriventris 4.2 .1

- delicatula 4.2.1

- eichwaldii 4.1.5

- elongata 4.1.4
- fallax 4.1.4

- finta 4.1.4

- macedonica 4.1.7

- maeotica 4.1.8

- major 4.1 .1

- minor 4.1.1

- nilotica 4.1.4

- parvula 4.1.1

- pontica 4.1 .5

- rufa 4.1.4

- sardinella 4.1.4

- tanaica 4.1.9

- ziga 5.19 .1

clupeoides, Coregonus 13.1.40

clupeoides, Cyprinus 5.9.2, 5.19.1

clupeoides, Salmostoma 5.19.1

Clupeonella azovi 4.2.1

- caspia 4.2.1

- cultriventris 4.2 .1

- tscharchalensis 4.2.1

cobitiformis, Gobius 27.9.5

Cobitinula anatoliae 6.1.10

Cobitis albicoloris 6.3.2

- arahthoensis 6.1.15

- aralensis 6.3.1

- aurata 6.3.1

- balcanica 6.3.1

- barbatula 7.1.1

- bilineata 6.1 .1

- bosniaca 6.3.1

- bulgarica 6.3 .2

- calderoni 6.1.2

- conspersa 6.3.3

- dalmatina 6.1.10

- danubialis 6.1.4

- elongata 6.1.3

- elongatoides 6.1.4

- fossilis 6.2 .2

- fundulus 5.13 .5

- furstenbergii 7.1.1

- haasi 6.1.7

- hellenica 6.1.14

- hohenackeri 6.3.1

- larvata 6.3.3

- maroccana 6.1.7

- megaspila 6.1.5

- meridionalis 6.1.6

- montana 6.3.1

- narentana 6.1.10

- ohridana 6.1.10

- paludica 6.1.7

- paludicola 6.1.7

- pannonica 30.1.3

- parisiensis 7.1.1

- peschevi 6.1.9

- pictava 7.1.1

- pironae 7.1.1

- punctilineata 6.1.13

- puta 6.1.1

- radnensis 6.3.1

- romanica 6.3.4

- septa 6.1.1 
- spilura 6.1.10

- stephanidisi 6.1 .8

- strumicae 6.1.9

- taenia 6.1.10

- taenioides 6.1.10

- tanaitica 6.1.10

- tesselatus 6.3.2

- toni 7.1 .1

- trichonica 6.1.11

- vallachica 6.3.1

- vardarensis 6.1.12

- variabilis 7.1.1

- zanandreai 6.1.1

cobitis, Gobius 27.4.1

coeruleus, Coregonus 13.1.21

cognatus, Coregonus 13.1.4

colchica, Acipenser 2.1.1

colchicus, Phoxinus 5.21.1

colii, Salmo 14.5.2

colii, Salvelinus 14.5.2

colobocephala, Alburnus 5.3.2

comes, Leuciscus 5.17.12

comiza, Barbus 5.7.5

comizo, Barbus 5.7.5

communis, Alosa 4.1.3

rommunis, Ammocoetes 1.3.1

communis, Barbus 5.7.2

communis, Leuciscus 5.24.16

communis, Lota 15.1.1

communis, Tinca 5.26.1

compactus, Coregonus 13.1.15

compressus, Cyprinus 5.23.2

confusus, Coregonus 13.1.6

conspersa, Cobitis 6.3.3

cordilla, Leuciscus 5.3.1

Coregonus acronius 13.1.19

- acutirostris 13.1.21

- albellus 13.1.9

- albula 13.1.

- alpinus 13.1.10, 13.1.11

- altior 13.1.42

- ammerensis 13.1.27

- amnipetens 13.1.29

- anarensis 13.1.37

- angermanensis 13.1.45

- annectus 13.1.14

- arenicolus 13.1.18

- arnoldi 13.1.31

- artedii 13.1.42

- arurensis 13.1.10

- asperi 13.1.17

- aspius 13.1.33

- atterensis 13.1.23

- austriaca 13.1.27

- autumnalis 13.1.42

- baerii 13.1.37

- balleoides 13.1.14

- balleus 13.1.10

- baltica 13.1.29

- baunti 13.1.36

- bavarica 13.1.24

- bavaricus 13.1.24
- bergi 13.1.29

- bergianus 13.1.45

- bergiellus 13.1 .35

- bezola 13.1.1

- bodanensis 13.1.21

- bodensis 13.1.20, 13.1.18

- bolmeniensis 13.1.31

- bolmensis 13.1.31

- bondella 13.1.5

- borealis 13.1 .31

- brevis 13.1.28

- bullarensis 13.1.31

- candidus 13.1.5

- cepedii 13.1.40

- chibinae 13.1.31

- clupeoides 13.1.40

- coeruleus 13.1.21

- cognatus 13.1.4

- compactus 13.1.15

- confusus 13.1.6

- crassirostris 13.1.13

- cuvieri 13.1.4

- danica 13.1.45

- danneri 13.1.25

- dispar 13.1.17

- dispersus 13.1.8

- dolosus 13.1.17

- duplex 13.1.17

- elegans 13.1.42

- euryrhynchus 13.1.45

- exiguus 13.1.20, 13.1.33

- exilis 13.1.33

- fatioi 13.1.11

- fera 13.1.2

- feritus 13.1 .8

- finnica 13.1.28

- generosus 13.1.32

- gracilior 13.1.44

- gracilis 13.1.37

- gutturosus 13.1.11

- hartmannianus 13.1.19

- heglingus 13.1.16

- helveticus 13.1 .10

- hiemalis 13.1.3

- hoferi 13.1.26

- holsata 13.1.37

- humilis 13.1.31

- hypselorhynchus 13.1.45

- imandrae 13.1.45

- inarensis 13.1.35

- intermedia 13.1.17

- järvii 13.1.45

- jurassica 13.1.7

- karelica 13.1.45

- kessleri 13.1.37

- kilone 13.1.45

- knipowitschi 13.1.45

- lacepedei 13.1.40

- lacus-lunae 13.1.23

- lacustris 13.1.31

- ladogae 13.1.28, 13.1.37

- ladogensis 13.1.28 
- lapponica 13.1.45

- lapponicus 13.1.29

- lavaretoides 13.1.31

- lavaretus 13.1.4

- lindimacensis 13.1.17

- lloydii 13.1.29

- longiusculus 13.1.33

- lovensis 13.1 .45

- lucernensis 13.1.14

- lucinensis 13.1.38

- ludoga 13.1.37

- macrophthalmus 13.1.20

- manocentrus 13.1.32

- maraena 13.1.29

- maraenoides 13.1.17, 13.1.45

- maxillaris 13.1.30

- mediospinatus 13.1.29

- megalops 13.1.31

- melga 13.1.45

- microcephalus 13.1.40, 13.1.45

- microps 13.1.45

- multispinatus 13.1.33

- musta-siiska 13.1.31

- nasutus 13.1 .33

- neschka 13.1.45

- nilssoni 13.1.32

- nobilis 13.1.13

- nordmanni 13.1.45

- norvegica $13.1 .28,13.1 .45$

- nüsslini 13.1.20

- okoniensis 13.1.37

- olonensis 13.1.28, 13.1.31

- onegi 13.1.37

- orientalis 13.1 .45

- oronensis 13.1.31

- oxyrinchus 13.1 .39

- pachycephalus 13.1.31

- palaea 13.1.7

- pallasii 13.1.33

- palloni 13.1.45

- pelagicola 13.1.45

- pelagicus 13.1.45

- peled 13.1.34

- pennantii 13.1.41

- perejaslawica 13.1.28

- pereslavicus 13.1.28

- pereslawicus 13.1.28

- pidschian 13.1.35

- pidschianoides 13.1.35

- poljakovi 13.1.37

- pollan 13.1.42

- polonica 13.1.37

- pommerana 13.1.29

- prawdini 13.1 .45

- pulsensis 13.1.45

- pycnocentrus 13.1.32

- reisingeri 13.1.27

- renke 13.1.27

- restrictus 13.1 .8

- rhodanensis 13.1.2

- ringsjöensis 13.1.31

- riusensis 13.1.14
- rondeleti 13.1 .4

- rudolphianus 13.1.34

- sanctibenedicti 13.1.5

- scallensis 13.1.37

- schinzii 13.1.2

- schuensis 13.1.31

- sieboldi 13.1.17

- sikus 13.1.35

- smitti 13.1.35

- soldatovi 13.1.35

- steckbornensis 13.1.20

- steindachneri 13.1.27

- stenorhynchus 13.1.45

- stigmaticus 13.1.43

- suecica 13.1.37

- suidteri 13.1.14

- sulzeri 13.1.17

- sunensis 13.1.31

- swirensis 13.1.37

- tapinorhynchus 13.1.45

- telekinae 13.1.29

- thienemanni 13.1.45

- thunensis 13.1.10

- tigurinus 13.1.17

- topozeri 13.1.28

- trybomi 13.1.36

- tscholmugensis 13.1.37

- umbrae 13.1.45

- vaetterensis 13.1.29

- vandesius 13.1.44

- verchoswirka 13.1.37

- vessicus 13.1.28

- vigrensis 13.1.29

- vimbaeformis 13.1.45

- vodlosericus 13.1.28

- vogti 13.1.14

- voronjensis 13.1.45

- vygrensis 13.1.29

- wagleri 13.1.27

- wartmanni 13.1.21

- werchoswirka 13.1.37

- widegreni 13.1.37

- willughbeii 13.1.44

- zuerichensis 13.1.17

- zugensis 13.1.14, 13.1.15

- zürichensis 13.1.17

coriaceus, Cyprinus 5.11.1

cornubiensis, Salmo 14.3.23.1

corrugatus, Mugil 16.1.1

costatus, Salmo 14.5.20

Cottus affinis 23.1.2

- asundensis 23.2.1

- borkensis 23.2.1

- ferrugineus 23.1.3

- frykenensis 23.2.1

- gobio 23.1.2

- haemusi 23.1.2

- hispaniolensis 23.1.2

- jaxartensis 23.1.2

- kallavesensis 23.2.1

- koshewnikowi 23.1.2

- kuznetzovi 23.1.4 
- latifrons 23.2.1

- lönnbergi 23.2.1

- macrostomus 23.1.2, 23.1.4

- microcephalus 23.1.4

- microstomus 23.1.2, 23.1.4

- milvensis 23.1.2

- oernensis 23.2.1

- pellegrini 23.1.2

- petiti 23.1.3

- poecilopus 23.1.4

- pygmaeus 23.2.1

- quadricornis 23.2.1

- relicta 23.2.1

- roseus 23.1.2

- szanaga 23.1.4

- vaenernensis 23.2.1

- vermelnensis 23.2.1

- volki 23.1.4

crassa, Leuciscus 5.24.16

crassirostris, Coregonus 13.1.13

crenobionta, Gasterosteus 21.1.2

crenobiontus, Gasterosteus 21.1.2

croatica, Knipowitschia 27.5.2

croaticus, Phoxinellus 5.20.3

crocophthalmus, Scardinius 5.23.2

cryptocheilos, Mugil 16.2.1

csikii, Phoxinus 5.21.1

cultratus, Cyprinus 5.19.1

cultratus, Pelecus 5.19.1

cultriventris, Clupea 4.2.1

cultriventris, Clupeonella 4.2.1

cumberland, Salmo 14.3.23.1

curensis, Huso 2.2.1

curtus, Mugil 16.1.1

cuvieri, Anguilla 3.1.1

cuvieri, Coregonus 13.1.4

cuvieri, Syngnathus 22.2.1

cuvierii, Alosa 4.1.3

cyanogaster, Cyprinodon 20.1.1

Cyclocheilichthys kosswigi 5.7.16

cyclolepis, Barbus 5.7.6

cynocephalus, Gobio 5.13.5

Cyprinodon cyanogaster 20.1.1

- desioi 20.1.1

- doliatus 20.1.1

- hammonis 20.1.1

- iberus 20.1.2

- umbra 11.1.1

Cyprinoides nanofasciatus 20.1.1

cyprinoides, Salmo 13.1.34

Cyprinus abramo-rutilus 30.1.2

- acuminatus 5.11.1

- agone 4.1.1

- albor 5.3.2

- albula 5.17.4

- alburnus 5.3.2

- alepidotus 5.11.1

- amarus 5.8.3, 5.23.1

- anatolicus 5.11.1

- angulatus 5.11.1

- annoni 5.2.1

- aphya 5.21.1
- aralensis 5.11.1

- asper 30.1.3

- aspius 5.5.1

- auratus 5.26.1

- ballerus 5.1.1

- barbus 5.7.2

- benacensis 5.13.3

- bipunctatus 5.2.1

- bithynicus 5.11.1

- bjoerkna 5.1.2

- blicca 5.1.2

- blochii 5.2.1

- brama 5.1.3

- brevicirri 5.11.1

- buggenhagii 30.1 .2

- caeruleus 5.25.2

- capito 5.17.4

- carassius 5.8.2

- carinatus 5.28.3

- carpio 5.11.1

- caspicus 5.11.1

- cephalus 5.17.4

- chalcoides 5.9.2

- chrysoprasius 5.21.1

- chub 5.17 .4

- cirrosus 5.11.1

- cleveza 5.1.4

- clupeoides 5.9.2, 5.19.1

- compressus 5.25.2

- coriaceus 5.11.1

- cultratus 5.19.1

- dobula 5.17.8

- dolabrata 30.1.2

- elatus 5.11.1

- ellicola 30.1.3

- elongatus 5.11.1

- erythrophthalmus 5.25.2

- erythrops 5.25 .2

- farenus 5.1.1

- festetitzii 5.11.1

- fischeri 5.16.1

- fulvus 5.24.16

- fuscus 5.25.2

- galian 5.21.1

- gibbosus 5.1.2, 5.11.1

- gibelio 5.8.3

- gieben 5.1.2

- gobio 5.13.5

- graining 5.17 .8

- grislagine 5.17.8

- gronovii 5.2.1

- haematopterus 5.11.1

- hungaricus 5.11.1

- idbarus 5.17.5

- idus 5.17 .5

- isetensis 5.21.1

- jaculus 5.24.16

- jeses 5.17 .5

- kietaibeli 5.17.4

- kleweza 5.1.4

- kollarii 30.1.2

- lacustris 5.11.1, 5.24.16 
- lancastriensis 5.17.8

- lanceolatus 5.3.2

- laskyr 5.1.2

- latus 5.1.2, 30.1.2

- leuciscus 5.17 .8

- longicirri 5.11.1

- lugdunensis 5.17.4

- lumaireul 5.21.1

- macrolepidotus 5.11.1

- meckel 5.1.2

- microlepidotus 5.17.5

- moles 5.8.2

- monstrosus 5.11.1

- morella 5.21.1

- mugilis 5.17 .8

- muraenula 13.1.28

- nasus 5.10 .4

- nordmannii 5.11.1

- notha 5.2.1

- nudus 5.11.1

- oblongus 5.11.1

- orfus 5.17 .5

- orthonotus 5.17.4

- pallasii 5.16.1

- perenurus 5.12.1

- persa 5.28.3

- phoxinus 5.21.1

- pigus 5.24.13, 5.24.16

- plestya 5.1.2

- punctatus 5.2.1

- rapax 5.5.1

- regina 5.11.1

- regius 5.11.1

- rex 5.11.1

- rex cyprinorum 5.11.1

- riemling 5.2.1

- rivularis 5.21 .1

- rondeletii 5.11.1

- rubellio 5.24.16

- rufus 5.17.4

- rutilus 5.24.16

- salax 5.17.8

- salmoneus 5.17.4

- sanderi 5.2.1

- sapa 5.1.4

- scardula 5.25.2

- serta 5.28.3

- simus 5.17.8, 5.24.16

- specularis 5.11.1

- spirlin 5.2.1

- squalus 5.17 .8

- striatus 30.1 .2

- taeniatus 5.5.1

- thermalis 5.11.1

- tinca 5.26.1

- tincauratus 5.26.1

- tincaurea 5.26.1

- toxostoma 5.1010

- umbra 5.17.8

- uranoscopus 5.13.7

- vimba 5.28 .3

- vimpa 5.28 .3
- viridescens 5.11.1

- vulgaris 5.11.1

- wiresuba 5.24 .5

- xanthopterus 5.24.16

- zeelt 5.26.1

- zerta 5.28 .3

cyrensis, Acipenser 2.1.5

czekanowskii, Acerina 25.1.2

czernayi, Owsianka 5.16.1

\section{D}

dagestanicus, Alburnus 5.3.2

dalmatina, Cobitis 6.1.10

danfordi, Eudontomyzon 1.1.1

danica, Coregonus 13.1.45

danneri, Coregonus 13.1.25

danubialis, Acipenser 2.1.5

danubialis, Cobitis 6.1.4

danubica, Acerina 25.1.2

danubica, Acipenser 2.1.1

danubica, Gasterosteus 21.2.3

danubicus, Alburnus 5.9.2

danubicus, Rhodeus 5.23.1

danubicus, Salmo 14.3.10

danubiensis, Perca 25.1.3

danubii, Abramis 5.1.3

danubii, Alosa 4.1.5

daugawensis, Leuciscus 5.24.16

dauricus, Phoxinus 5.12.1

decipiens, Leuciscus 5.24.16

decorus, Thymallus 14.6.1

delalandi, Syngnathus 22.2.2

delicatula, Clupea 4.2.1

delineatus, Leucaspius 5.16.1

delineatus, Squalius 5.16.1

dentex, Salar 14.3.6

dentex, Salmo 14.3.6

dergle, Scardinius 5.23.2

derjavini, Acipenser 2.1.3

derjavini, Blicca 5.1.2

derjugini, Alburnus 5.9.2

desioi, Cyprinodon 20.1.1

Dicentrarchus labrax 24.1.1

- punctatus 24.1.2

dimorphus, Leucaspius 5.16.1

dispar, Coregonus 13.1.17

dispersus, Coregonus 13.1.8

distichus, Salmo 14:5.20

dobula, Cyprinus 5.17.8

dobuloides, Abramis 30.1.2

doiranica, Sabanejewia 6.3.1

dojranensis, Rutilus 5.24.16

dojranensis, Scardinius 5.23.2

dolabrata, Cyprinus 30.1.2

doliatus, Cyprinodon 20.1.1

Doliichthys stellatus 27.2.1"

dolosus, Coregonus 13.1.17

donensis, Acipenser 2.1.5

dono-kubanensis, Rutilus 5.24.6

dorsatus, Petromyzon 1.5.1

dremaei, Chondrostoma 5.10.10

dubahra, Mugil 16.2.2 
dubius, Acipenser 2.1.4

ducissae, Acipenser 2.1.6

dufourii, Aturius 5.17.2

duplex, Coregonus 13.1.17

duriensis, Chondrostoma 5.10.6

dvinensis, Leuciscus 30.1.2

dwinensis, Platessa 29.2.1

dybowskii, Phoxinus 5.12.1

\section{E}

Economidichthys pygmaeus 27.3.1

- trichonis 27.3.2

eichwaldii, Alburnus 5.2.1

eichwaldii, Clupea 4.1.5

ekströmi, Gobius 27.9.5

elata, Alburnus 5.3.2

elata, Leuciscus 5.24.16

elata, Squalius 5.17 .8

elatus, Cyprinus 5.11.1

elatus, Squalius 5.24.3

elegans, Coregonus 13.1.42

elegans, Gasterosteus 21.1.3

elimeius, Gobio 5.13.4

ellicola, Cyprinus 30.1.3

ellipticus, Carassius 5.8.3

elongata, Alburnus 5.3.2

elongata, Caspialosa 4.1.8

elongata, Clupea 4.1.4

elongata, Cobitis 6.1.3

elongata, Leuciscus 5.24.16

elongata, Perca 24.1.1

elongata, Squalius 5.17.8

elongata, Vimba 5.28.1

elongatoides, Cobitis 6.1.4

elongatus, Abramis 5.28.1

elongatus, Cyprinus 5.11.1

elongatus, Gobius 27.9.5

encobia, Carassius 5.8.1

eperlano-marinus, Salmo 12.1.1

Eperlanus schonfieldii 12.1.1

- schonfoldii 12.1.1

- vulgaris 12.1 .1

eperlanus, Osmerus 12.1.1

eperlanus, Salmo 12.1.1

epiroticus, Paraphoxinus 5.20.4

epiroticus, Phoxinellus 5.20.4

eques, Barbus 5.7.16, 5.7.20

ercisianus, Barbus 5.7.16

eriox, Salmo 14.3.23.1

ernstii, Petromyzon 1.4.1

erythraea, Acipenser 2.1.4

erythraea, Leuciscus 5.24.16

erythrinna, Nemacheilus 7.1.1

erythrophthalmoides, Bliccopsis 30.1.2

erythrophthalmus, Cyprinus 5.23.2

erythrophthalmus, Rutilus 5.24.3

erythrophthalmus, Scardinius 5.23.2

erythrops, Cyprinus 5.23.2

erythropterus, Abramis 5.1.2

erytrostomus, Aspius 5.5.1

Esox atrox 10.1.1

- baicalensis 10.1.1
- bergi 10.1.1

- lucius 10.1.1

- variegatus 10.1.1

- wiliunensis 10.1.1

essipovi, Acerina 25.1.2

estuarius, Salmo 14.3.23.1

etemi, Caspialosa 4.1.9

ethon, Syngnathus 22.2.1

euboicus, Barbus 5.7.7

Eudontomyzon danfordi 1.1.1

- hellenicus 1.1.2

- mariae 1.1.3, 1.1.6

- stankokaramani 1.1.3, 1.1.4

- vladykovi 1.1.3 1.1.5

Eupalasella perenurus 5.12.1

europaeus, Acipenser 2.1.6

europaeus, Salmo 14.3.19

eurycephalus, Gobius 27.7.1

eurycephalus, Neogobius 27.7.1

euryrhynchus, Coregonus 13.1.45

eurystoma, Anguilla 3.1.1

exanthematosus, Gobius 27.7.5

excelsa, Salmo 14.3.10

exiguus, Coregonus 13.1.20, 13.1.33

exilis, Coregonus 13.1.33

ezenami, Salmo 14.3.28

F

fabraei, Alburnus 5.3.2

fallax, Alosa 4.1.3

fallax, Clupea 4.1.3

farenus, Cyprinus 5.1.1

Fario argenteus 14.3.23.1

- marsilii 14.3.10

fario, Salmo 14.3.10, 14.3.23.1, 14.3.28

farioides, Salmo 14.3.7

faroensis, Salvelinus 14.5.3

fasciata, Lebias 20.1.1

fasciatus, Aphanius 20.1.1

fasciatus, Aspius 5.2.1

fatioi, Coregonus 13.1.11

fecunda, Salmo 14.3.23.1

fera, Coregonus 13.1.2

feraeensis, Gobio 5.13.5

ferganensis, Abramis 5.1.4

feritus, Coregonus 13.1.8

ferox, Salmo 14.3.8

ferra, Salmo 13.1.21

ferrugineus, Cottus 23.1.3

ferrugineus, Gobius 27.9.3

festetitzii, Cyprinus 5.11.1

fibreni, Salmo 14.3.9

ficta, Alosa 4.1.3

filamentosus, Gobius 27.11.1

fimbriatus, Salvelinus 14.5.4

finnica, Coregonus 13.1.28

finta, Clupea 4.1.3

fischeri, Acerina 25.1.2

fischeri, Cyprinus 5.16.1

fischeri, Leucaspius 5.12.1

fitzingeri, Acipenser 2.1.6

flava, Lebias 20.1.1 
flavescens, Syngnathus 22.2.1

flesoides, Pleuronectes 29.1.1

Flesus vulgaris 29.1.1

flesus, Platichthys 29.1.1

flesus, Pleuronectes 29.1.1

fluviatilis, Anguilla 3.1.1

fluviatilis, Barbus 5.7.2

fluviatilis, Blennius 26.1.1

fluviatilis, Cernua 25.1.2

fluviatilis, Gobio 5.13.5

fluviatilis, Gobius 27.7.2, 27.8.1

fluviatilis, Lampetra 1.3.1

fluviatilis, Leuciscus 5.24.16

fluviatilis, Lota 15.1.1

fluviatilis, Neogobius 27.7.2

fluviatilis, Perca 25.2.1

fluviatilis, Petromyzon 1.3.1, 1.4.1

fluviatilis, Salaria 26.1.1

fluviatilis, Trutta 14.3.23.1

fluviatilna, Anguilla 3.1.1

fontinalis, Phoxinellus 5.20.5

forestensis, Salmo 14.3.23.1

fossilis, Cobitis 6.2.2

fossilis, Misgurnus 6.2.2

foveolata, Trematina 10.1.1

fracchia, Alburnus 5.3.2

frater, Blennius 26.1.1

frici, Gobio 5.13.7

frici, Rutilus 5.24.16

frigidus, Leuciscus 5.17.4

frisii, Gardonus 5.24.5

frisii, Rutilus 5.24.5

frivaldszkyi, Abramis 5.28.3

frykenensis, Cottus 23.2.1

fucini, Barbus 5.7.20

fucini, Leuciscus 5.24.15

fuliginosus, Gobius 27.4.2

fulvus, Cyprinus 5.24.16

Fundulus heteroclitus 18.1.1

- letourneuxi 19.1.2

fundulus, Cobitis 5.13.5

furcata, Liza 16.2.3

furstenbergii, Cobitis 7.1.1

fuscus, Cyprinus 5.23.2

G

gadoïdes, Salmo 14.3.23.1

Gadus lota 15.1.1

gaimardi, Salar 14.3.23.1

galian, Cyprinus 5.21.1

gallicus, Barbus barbus 5.7.2

gallivensis, Salmo 14.3.23.1

ganzirriensis, Syngnathus 22.2.1

Gardonus pigulus 5.24.16

- ruboculus 5.24.16

- wyrozub 5.24.5

Gasterosteus aculeatus 21.1.1

- algeriensis 21.1

- argentatissimus 21.1.3

- argyropomus 21.1.3

- bailloni 21.1.3

- biarmatus 21.1.1
- brachycentrus 21.1 .3

- breviceps 21.2.2

- burgundianus 21.2.2

- caucasicus 21.2.3

- crenobionta 21.1.2

- crenobiontus 21.1.2

- danubica 21.2.3

- elegans 21.1.3

- globiceps 21.2.2

- gymnurus 21.1.3

- hologymnus 21.1.3

- islandicus 21.1.4

- kessleri 21.2.3

- laevis 21.2.2

- leiurus 21.1.3

- lotharingus 21.2.2

- messinicus 21.1.3

- nemauensis 21.1.3

- neustrianus 21.1.3

- niger 21.2.3

- platygaster 21.2.3

- ponticus 21.1.1

- pungitius 21.2.4

- quadrispinosa 21.1.3

- semiarmatus 21.1.3

- semiloricatus 21.1.3

- spinulosus 21.1.1

- tetracanthus 21.1.3

- tetraculeatus 21.1.1

- trachurus 21.1.1

- vulgaris 21.2.2

gdaniensis, Phoxinus 5.12.1

gehini, Abramis 5.1.3

genei, Chondrostoma 5.10.2

genei, Leuciscus 5.10.2

generosus, Coregonus 13.1.32

genitalis, Rhodeus 5.23.1

genivittatus, Salar 14.3.14

geoktshaicus, Rutilus 5.24.16

georghievi, Knipowitschia 27.5.4

germanorum, Hucho 14.2.1

ghetaldii, Paraphoxinus 5.20.6

ghetaldii, Phoxinellus 5.20.6

ghigii, Ladigesocypris 5.15 .1

ghigii, Leucaspius 5.15 .1

ghigii, Salmo 14.3.5

gibba, perca 25.2.1

gibbosus, Carassius 5.8.2

gibbosus, Cyprinus 5.1.2, 5.11.1

gibbosus, Gobius 27.4.1

gibelio, Carassius 5.8.3

gibelio, Cyprinus 5.8.3

gieben, Cyprinus 5.1.2

glaber, Acipenser 2.1.3

glacialis, Liopsetta 29.2.1

glacialis, Pleuronectes 29.2.1

Glanis aristotelis 9.1.1

glanis, Silurus 9.1.2

globiceps, Gasterosteus 21.2.2

gmelini, Acipenser 2.1.4

Gobio acutipinnatus 5.13.5

- albanicus 5.13.5 
- albipinnatus 5.13.1

- antipai 5.13.6

- balcanicus 5.13.5

- banarescui 5.13.2

- banaticus 5.13.6

- belingi 5.13 .1

- benacensis 5.1:3

- brevicirris 5.13.5

- bulgarica 5.13 .5

- carpathicus 5.13.5

- carpathorossicus 5.13.6

- caucasica 5.13.5

- cynocephalus 5.13.5

- elimeius 5.13.4

- feraeensis 5.13.5

- fluviatilis 5.13.5

- frici 5.13 .7

- gobio 5.13.5

- gymnostethus 5.13.5

- holurus 5.13.5

- insuyanus 5.13 .5

- intermedius 5.13 .5

- katopyga 5.13.5

- kesslerii 5.13.6

- kovatschevi 5.13.5

- krymensis 5.13.5

- latus 5.13.5

- lepidolaemus 5.13.5

- lindbergi 5.13.5

- longicirris 5.13.5

- lutescens 5.13.3

- magnicapitata 5.13.5

- microlepidotus 5.13.5

- muresia 5.13.5

- nikolskyi 5.13 .5

- obtusirostris 5.13.5

- ohridana 5.13 .5

- phoxinoïdes 5.13 .5

- pollinii 5.13.3

- prosopyga 5.13.5

- sarmaticus 5.13.5

- saxatilis 5.13.5

- sibiricus 5.13.5

- similis 5.13.6

- skadarensis 5.13.5

- soldatovi 5.13 .5

- stankoi 5.13.4

- uranoscopus 5.13.7

- venatus 5.13 .3

- vladykovi 5.13 .1

- vulgaris 5.13 .5

gobio, Cottus 23.1.2

gobio, Cyprinus 5.13.5

gobio, Gobio 5.13.5

Gobius affinis 27.7.5

- albescens 27.4.2

- algarbiensis 27.4.1

- avernensis 27.8.2

- batrachocephalus 27.6.1

- blennioides 27.10.1

- bonelli 27.8.1

- brittanicus 27.4 .2
- burmeisteri 27.7.3

- canestrinii 27.9.2

- caucasicus 27.5.1

- cephalarges 27.7.5

- chilo 27.7.5

- citrina 27.11.1

- cobitiformis 27.9.5

- cobitis 27.4.1

- ekströmi 27.9.5

- elongatus 27.9.5

- eurycephalus 27.7 .1

- exanthematosus 27.7.5

- ferrugineus 27.9.3

- filamentosus 27.11.1

- fluviatilis 27.7.2, 27.8.1

- fuliginosus 27.4.2

- gibbosus 27.4.1

- gorgione 27.4.2

- gous 27.11.1

- gracilis 27.9.5

- grossholzii 27.7.5

- guitelli 27.9.5

- guttatus 27.4.1

- gymnotrachelus 27.7.3

- hispanicus 27.4.2

- hybridus 27.7.6

- jadrensis 27.9.2

- jozo 27.4 .2

- kessleri 27.7.4

- lacteus 27.7.2

- laticeps 27.9.4

- lenkoranicus 27.5.1

- leopardinus 27.9.3

- limbatus 27.4.1

- longecaudata 27.5.4

- longiradiatus 27.4 .2

- lota 27.11.1

- lugens 27.7.5

- macropterus 27.10.1

- macropus 27.7.3

- major 27.4.2, 27.9.5

- marmoratus 27.10.1

- martensii 27.8.1

- melanio 27.7.5

- melanostomus 27.7.5

- microps 27.9.4

- minor 27.4.2, 27.9.4

- minutus 27.9.5

- nasalis 27.10 .1

- niger 27.4.2

- nigerrimus 27.4.2

- nigra 27.7.2

- nigrescens 27.4 .2

- nigricans 27.8.2

- ophiocephalus 27.11.1

- panizzae 27.5.6

- parnelli 27.9.4

- platycephalus 27.7.4

- pontica 27.4.2, 27.10.1

- prevesicus 27.3.1

- puckensis 27.9.4

- punctatissimus 27.5.7 
- punctulatus 27.4 .2

- pusillus 27.9.4

- pygmaeus 27.3.1

- quadricapillus 27.10.1

- reticulatus 27.9.3, 27.11.1

- rhodopterus 27.9.3

- rubromaculatus 27.10.1

- semilunaris 27.10.1

- sordidus 27.7 .2

- spilogonurus 27.4.1

- steveni 27.7 .2

- sulcatus 27.7.5

- syrman 27.7.6

- taalmankipii 27.9.5

- thessalus 27.5.8

- trautvetteri 27.7 .6

- unipunctatus 27.9.5

- venetiarum 27.11.1

- virescens 27.7.5

- viridis 27.4.2, 27.11.1

goedenii, Salmo 14.3.19

goerneri, Knipowitschia 27.5.3

golis, Acipenser 2.1.1

goplensis, Rutilus 5.24.16

gorgione, Gobius 27.4.2

gorlap, Neogobius 27.7.4

gous, Gobius 27.11.1

gracilior, Coregonus 13.1.44

gracilis, Alburnus 5.3.2

gracilis, Alosa 4.1.1

gracilis, Coregonus 13.1.37

gracilis, Gobius 27.9.5

gracilis, Lampetra 1.1.1

gracilis, Perca 25.2.1

gracilis, Salmo 14.3.19

gracillimus, Salvelinus 14.5.6

graecus, Barbus 5.7.8

graecus, Scardinius 5.25.3

graellsii, Barbus 5.7.9

graining, Cyprinus 5.17.8

grayi, Salmo 14.5.5

grayi, Salvelinus 14.5.5

grisescens, Acipenser 1.1.3

grislagine, Cyprinus 5.17.8

gronovii, Cyprinus 5.2.1

grossholzii, Gobius 27.7.5

gueldenstaedtii, Acipenser 2.1.1

guiraonis, Barbus 5.7.10

guitelli, Gobius 27.9.5

güldenstädtii, Acipenser 2.1.1

guttatus, Gobius 27.4.1

gutturosa, Salmo 13.1.19

gutturosus, Coregonus 13.1.11

Gymnocephalus baloni 25.1.1

- cernuus 25.1.2

- schraetser 25.1.3

gymnogaster, Thymallus 14.6.1

gymnostethus, Gobio 5.13.5

gymnothorax, Thymallus 14.6.1

gymnotrachelus, Gobius 27.7.3

gymnotrachelus, Neogobius 27.7.3

gymnurus, Gasterosteus 21.1.3
H

haasi, Barbus 5.7.11

haasi, Cobitis 6.1.7

haematopterus, Cyprinus 5.11.1

haemusi, Cottus 23.1.2

hamatus, Salmo 14.3.19

hammonis, Cyprinodon 20.1.1

hardinii, Salmo 14.3.19

hartmannianus, Coregonus 13.1.19

hautin, Tripteronotus 13.1.39

heckelii, Abramis 30.1.2

heckelii, Acipenser 2.1.2.

heckelii, Aspius 5.9.2

heckelii, Leuciscus 5.24.6

heckelii, Rutilus 5.24.6

heegeri, Leuciscus 5.25 .5

heegeri, Rutilus 5.25.5

Heegerius typus 5.25.5

heglingus, Coregonus 13.1:16

helenae, Acipenser 30.1.1

hellenica, Cobitis 6.1.14

hellenicus, Eudontomyzon 1.1.2

hellenicus, Pungitius 21.2.1

hellenicus, Rutilus 5:27.1

hellenicus, Tropidophoxinellus 5.27.1

helops, Acipenser 2.1.5

helvetica, Perca 25.2.1

helveticus, Coregonus 13.1.10

henle, Leuciscus 5.24.3

hepsetus, Atherina 17.1.2

hernadiensis, Chondrostoma 5.10.4

hesperidicus, Scardinius 5.23.2

heterandrius, Leuciscus 5.17:1

heteroclitus, Fundulus 18.1.1

hexagonus, Tiphle 22.2.4

hibernica, Anguilla 3.1.1

hiemalis, Coregonus 13.1.3.

hispanica, Anaecypris 5.4.1

hispanica, Hydargyra 19.1.1

hispanica, Nemacheilus 7.1.1

hispanica, Phoxinus 5.4.1

hispanica, Valencia 19.1.1

hispanicus, Gobius 27.4.2

hispaniolensis, Cottus 23.1.2

hoferi, Coregonus 13.1.26

hohenackeri, Cobitis 6.3.1

Holocentrus post 25.1.2

- schraizer 25.1.3

hologymna, Pygosteus 21.2.2

hologymnus, Gasterosteus 21.1.3

holsata, Coregonus 13.1.37

holurus, Gobio 5.13.5

Hoplerythrinus unitaeniatus 5.17.4

hospitus, Acipenser 2.1.6

Hucho bleekeri 14.2.1

- germanorum 14.2.1

- hucho 14.2.1

- ishikawai 14.2.1

- perryi 14.2.1

- taimen 14.2.1

hucho, Hucho 14.2.1

hucho, Salmo 14.2.1 
huegelii, Aulopyge 5.6.1

humilis, Carassius 5.8.2

humilis, Coregonus 13.1.31

hungaricus, Cyprinus 5.11.1

Huso caspicus 2.2.1

- curensis 2.2.1

- huso

- ichthyocolla 2.2 .1

- maeoticus 2.2.1

- occidentalis 2.2.1

- orientalis 2.2.1

- ponticus 2.2.1

huso, Acipenser 2.2.1

huso, Huso

hyalosoma, Atherina 17.1.1

hybridus, Gobius 27.7.6

hybridus, Salmo 14.5.1

Hydargyra hispanica 19.1.1

hypselocephala, Caspialosa 4.1.5

hypselorhynchus, Coregonus 13.1.45

\section{I}

Iberocypris palaciosi 5.14 .1

iberus, Aphanius 20.1.2

iberus, Cyprinodon 20.1.2

iblioides, Alburnus 5.5.1

ichthyocolla, Huso 2.2.1

Ichthyocoris pollinii 26.1.1

Ictalurus pannonicus 8.1.1

idbarus, Cyprinus 5.17.5

Idus melanotus 5.17 .5

- miniatus 5.17.5

- orientalis 5.17 .5

- stagnalis 5.17 .8

idus, Cyprinus 5.17.5

idus, Leuciscus 5.17.5

iljini, Neogobius 27.7.4

illanca, Salmo 14.3.23.2.2

illyricus, Acipenser 2.1.5

illyricus, Leuciscus 5.17 .6

illyricus, Squalius 5.17.6

ilmenensis, Alburnus lucidus 5.3.2

imandrae, Coregonus 13.1.45

immaculata, Alosa 4.1.5

inaequalis, Blennius 26.1.1

inarensis, Coregonus 13.1.35

inframundus, Salvelinus 14.5.7

insuyanus, Gobio 5.13.5

intermedia, Blicca 5.1.2

intermedia, Coregonus 13.1.17

intermedius, Gobio 5.13.5

intermedius, Perca 25.2.1

iranicus, Chalcalburnus 5.9.2

irideus, Ladigesocypris 5.15.1

ischan, Salmo 14.3.1

isetensis, Cyprinus 5.21.1

ishikawai, Hucho 14.2.1

islandicus, Gasterosteus 21.1.4

islayensis, Salmo 14.3.23.1

isognathus, Brama 30.1.2

issatschenkovi, Alosa 4.1.5

istanbulensis, Alburnus 5.9.2 istanbulensis, Vimba 5.28.3

italica, Perca 25.2.1

italica, Tinca 5.26.1

italicus, Phoxinellus 30.1.3

italicus, Pleuronectes 29.1.1

italicus, Rutilus 5.24.15

$\mathbf{J}$

jaculum, Chondrostoma 5.10.2

jaculus, Cyprinus 5.24.16

jacuticus, Carassius 5.8.2

jadrensis, Gobius 27.9.2

japonicum, Lethenteron 1.4.1, 1.5.1

japonicus, Petromyzon 1.4.1

järvii, Coregonus 13.1.45

jaxartensis, Aspius 5.5.1

jaxartensis, Cottus 23.1.2

jelskii, Phoxinus 5.12.1

jenisensis, Acipenser 2.1.4

jeses, Cyprinus 5.17.5

jozo, Gobius 27.4.2

jurae, Petromyzon 1.3.1

jurassica, Coregonus 13.1.7

jurinii, Leuciscus 5.24 .16

K

kallavesensis, Cottus 23.2.1

kamensis, Acipenser 2.1.4

kamensis, Lota 15.1.1

kamensis, Thymallus 14.6.1

kameraticus, Petromyzon 1.4.1

kankreni, Acipenser 2.1.4

karamani, Rutilus 5.24.7

karasuensis, Vimba 5.28.3

karelica, Coregonus 13.1.45

katopyga, Gobio 5.13.5

kaznakovi, Leuciscus 5.17.4

keadicus, Leuciscus 5.17.7

kessleri, Alosa 4.1.5

kessleri, Coregonus 13.1.37

kessleri, Gasterosteus 21.2.3

kessleri, Gobius 27.7.4

kessleri, Lethenteron 1.4.1

kessleri, Neogobius 27.7.4

kesslerii, Gobio 5.13.6

kieneri, Anguilla 3.1.1

kietaibeli, Cyprinus 5.17.4

killarnensis, Alosa 4.1.6

killinensis, Salmo 14.5.8

killinensis, Salvelinus 14.5.8

kilone, Coregonus 13.1.45

kirgisorum, Leuciscus 5.17 .8

kleweza, Cyprinus 5.1.4

knerii, Chondrostoma 5.10.3

knipowitschi, Coregonus 13:1.45

knipowitschi, Liopsetta 29.2.1

knipowitschi, Pomatoschistus 27.5.4

knipowitschi, Rutilus 5.24.16

Knipowitschia caucasica 27.5.1

- croatica 27.5.2

- georghievi 27.5.4

- goerneri 27.5.3 
- longecaudata 27.5.4

- milleri 27.5.5

- mrakovcici 30.1.3

- panizzae 27.5.6

- punctatissima 27.5.7

- thessala 27.5.8

kolenty, Carassius 5.8.3

kollarii, Cyprinus 30.1.2

koshewnikowi, Cottus 23.1.2

kosswigi, Barbus 5.7.16

kosswigi, Cyclocheilichthys 5.7.16

kosswigii, Bubyr 27.5.1

kostera, Acipenser 2.1.4

kovatschevi, Gobio 5.13.5

krameri, Umbra 11.1.1

krkensis, Trutta 13.4.1

krymensis, Gobio 5.13.5

kubanica, Sabanejewia 6.3.1

kubanicus, Alburnoides 5.2.1

kurensis, Rutilus 5.24.16

kutum, Leuciscus 5.24.5

kuznetzovi, Cottus 23.1.4

\section{L}

Labrax linnéi 24.1.1

- lupus 24.1.1

- orientalis 24.1.2

labrax, Dicentrarchus 24.1.1

labrax, Perca 24.1.1

labrax, Salmo 14.3.10

labrosus, Chelon 16.1.1

labrosus, Mugil 16.1.1

lacepedei, Coregonus 13.1.40

lacerta, Barbus 5.7.16

lacteus, Gobius 27.7.2

lacus-lunae, Coregonus 13.1.23

lacustus, Anguilla 3.1.1

lacustris, Alburnus 5.3.2

lacustris, Alosa 4.1.1

lacustris, Aphya 11.1.1

lacustris, Atherina 17.1.1

lacustris, Carassius 5.8.2

lacustris, Coregonus 13.1.31

lacustris, Cyprinus 5.11.1, 5.24.16

lacustris, Salmo 14.3.7, 14.3.10, 14.3.14, 14.3.19, 14.3.23.2.2, 14.3 .28

lacustris, Thymallus 14.4.1

ladanus, Acipenser 2.1.2

Ladigesocypris ghigii 5.15.1

- irideus 5.15.1

- mermere 5.15.1

ladogae, Coregonus 13.1.28, 13.1.37

ladogensis, Coregonus 13.1.28

ladogensis, Lampetra 1.3.1

ladogensis, Osmerus 12.1.1

laevis, Gasterosteus 21.2.2

laevis, Phoxinus 5.21.1

laevis, Pungitius 21.2.2

laevis, Salmo 14.5.1

Lampetra bergi 1.1.1

- fluviatilis 1.3.1
- gracilis 1.1.1

- ladogensis 1.3.1

- major 1.4.1

- mariae 1.1.3

- minor 1.1.1

- mitsukurii 1.4.1

- opisthodon 1.3.1

- planeri 1.3.1, 1.3.2

- praecox 1.3.1, 1.4.1

- septentrionalis 1.4.1

- variegata 1.4 .1

- zanandreai 1.4.2

lampetra, Petromyzon 1.5.1

lancastriensis, Cyprinus 5.17.8

lanceolatus, Cyprinus 5.3.2

lapacinus, Leuciscus 30.1.2

lapasseti, Salmo 14.3.5

lapponica, Coregonus 13.1.45

lapponicus, Coregonus 13.1.29

lapponicus, Leuciscus 5.17.5

lariana, Alosa lacustris 4.1.1

larvalis, Petromyzon 1.3.2

larvata, Cobitis 6.3.3

larvata, Sabanejewia 6.3.3

lascha, Leuciscus 5.24.15

laskyr, Cyprinus 5.1.2

lateristriga, Alburnus 5.3.2

lateristriga, Squalius 5.17.8

laticeps, Gobius 27.9.4

latifrons, Cottus 23.2.1

latifrons, Leuciscus 5.17.4

latior, Alburnus 5.3.2

latirostris, Acipenser 2.1.6

latirostris, Anguilla 3.1.1

latissimus, Alburnus 5.9.2

latus, Cyprinus 5.1.2, 30.1.2

latus, Gobio 5.13.5

latus, Platessa 29.2.2

latus, Salmo 13.1.39

lavaretoides, Coregonus 13.1.31

lavaretus, Coregonus 13.1.4

lavaretus, Salmo 13.1.4

Lebias fasciata 20.1.1

- flava 20.1.1

- lineo-punctata 20.1.1

- nigropunctata 20.1.1

- rhomboidalis 20.1.1

- sarda 20.1.1

leiurus, Gasterosteus 21.1.3

leiurus, Pleuronectes 29.1.1

lemanus, Salmo 14.3.23.2.1

lemmingii, Leuciscus 5.24.8

lemmingii, Rutilus 5.24.8

lenkoranicus, Gobius 27.5.1

leonhardi, Pseudobarbus 5.7.15

leopardinus, Gobius 27.9.3

lepechini, Salmo 14.5.1

lepidolaemus, Gobio 5.13.5 ,

Leptocephalus brevirostris 3.1.1

leptorhinus, Squalius 5.17.8

leptura, Lota 15.1.1 
lepusculus, Squalius 5.17.8

Lethenteron camtschaticum 1.4.1

- japonicum 1.4.1, 1.5.1

- kessleri 1.4.1

- zanandreai 1.4.2

letnica, Salmo 14.3.11

letnica, Trutta 14.3.11

letourneuxi, Fundulus 19.1.2

letourneuxi, Valencia 19.1.2

Leucaspius abruptus 5.16.1

- caucasicus 5.16.1

- delineatus 5.16.1

- dimorphus 5.16.1

- fischeri 5.12.1

- ghigii 5.15.1

- marathonicus 5.22.3

- prosperi 5.15.1

- prosperoi 5.15 .1

- relictus 5.16.1

- threspoticus 5.22.3

Leuciscus affinis 30.1.2

- agassii 5.17.16

- agassizii 5.17.16

- albidus 5.3.1

- albiensis 5.17.4

- alburnellus 5.3.2

- alburnoides 5.25.1

- alburnolucidus 30.1.2

- albus 5.17.4

- altus 5.25.3

- apollonitis 5.25.2

- aralychensis 5.17 .4

- ardebilicus 5.17.4

- argenteus 5.17.8

- arrigonis 5.10.1

- aurata 5.24.16

- auratus 5.8.1, 5.17.5, 5.24.16

- baldneri 5.2.1

- bolmensis 5.24 .16

- borysthenicus 5.17.1

- brutius 5.17.4

- burdigalensis 5.17.2

- cabeda 5.17.4

- carii 30.1 .2

- carolitertii 5.17.3

- caspicus 5.24.16

- caspius 5.24.5

- cavedanus 5.17.4

- cephalus 5.17.4

- cii 5.17 .4

- comes 5.17.12

- communis 5.24.16

- cordilla 5.3.1

- crassa 5.24.16

- daugawensis 5.24 .16

- decipiens 5.24.16

- dvinensis 30.1.2

- elata 5.24.16

- elongata 5.24.16

- erythraea 5.24.16

- fluviatilis 5.24.16

- frigidus 5.17.4
- frisii 5.24 .5

- fucini 5.24 .15

- genei 5.10.2

- heckelii 5.24.6

- heegeri 5.25.5

- henle 5.24.3

- heterandrius 5.17.1

- idus 5.17 .5

- illyricus 5.17.6

- jurinii 5.24.16

- kaznakovi 5.17.4

- kearlicus 5.17.7

- kirgisorum 5.17.8

- kutum 5.24.5

- lapacinus 30.1.2

- lapponicus 5.17 .5

- lascha 5.24.15

- latifrons 5.17.4

- lemmingii 5.24.8

- leuciscus 5.17 .8

- lividus 5.24.16

- lucumonis 5.17.9

- macedonicus 5.17.4, 5.18.1

- macrolepidotus 5.24.10

- majalis 5.17.8

- marrochius 5.25.5

- meidingeri 5.24.11

- microlepis 5.17.10

- montenegrinus 5.17.11

- montenigrinus 5.17.11

- moreoticus 5.17.4

- muticellus 5.17.12

- neglectus 5.17 .5

- nothulus 5.17.4

- obtusus 5.21.1

- orientalis 5.17 .4

- pagellus 5.24.3

- pallens 5.24.16

- pamvoticus 5.17.4

- parvulus 5.28.3

- pauperum 5.24.3

- pausingeri 5.24.16

- peloponensis 5.17.4

- pictava 5.17.4

- pigmeus 5.16.1

- pleurobipunctatus 5.17.13

- polylepis 5.17.14

- prasinus 5.24.16

- pursakensis 5.17.4

- pyrenaicus 5.17.15

- risae 5.17 .4

- rissoi 5.17 .4

- rodens 5.17 .8

- roseus 5.24 .13

- rostratus 5.17 .8

- roulei 5.17.8

- rubella 5.24 .15

- rubilio 5.24.15

- ruffoi 5.17 .4

- rutiloides 5.24.16

- ryzela 5.24 .13

- saltator 5.17 .8 
- sardella 5.24 .15

- scardafa 5.25 .5

- scardinus 5.24 .3

- scarpata 5.25 .5

- scarpetta 5.25.5

- scaverde 30.1.3

- selysii 5.24 .16

- sibiricus 5.17 .5

- souffia 5.17 .16

- squalius 5.17.4

- squalus 5.17 .4

- stymphalicus 5.22 .3

- svallize 5.17 .17

- teletzkensis 5.17.8

- terekensis 5.24 .16

- thracicus 5.17.4

- trasimenicus 5.24.15

- turskyi 5.17 .18

- ukliva 5.17.19

- virgo 5.24 .13

- vobla 5.24 .16

- vulgaris 5.17.8

- vulturius 5.3.1

- wjatkensis 5.17.4

- wobla 5.24 .16

- zangicus 5.17 .4

- zrmanjae 5.17.20

leuciscus, Cyprinus 5.17.8

leuciscus, Leuciscus 5.17.8

leuckartii, Abramis 30.1.2

leucoides, Telestes 5.17.1

Leucos adspersus 5.20.1

- arcasii 5.25.2

- basak 5.24.4

- cenisophius 5.24.16

- cisalpinus 5.24 .3

- ohridana 5.24 .12

- pigulus 5.24.16

- prespensis 5.24.14

- rubellicus 5.24 .3

leucotica, Acipenser 2.1.4 levenensis, Salmo 14.3.23.1 levis, Salmo 14.5.1 leydigi, Alburnus 30.1.2 lichtensteinii, Acipenser 2.1.6 likana, Trutta 14.3.22 limbatus, Gobius 27.4.1 limosa, Tinca 5.26.1 lindbergi, Gobio 5.13.5 lindimacensis, Coregonus 13.1.17 lineo-punctata, Lebias 20.1.1 linguatula, Citharus 28.1.1 linguatula, Pleuronectes 28.1.1 linnaei, Carassius 5.8.2 linnéi, Alburnus 5.3.2 linnéi, Anguilla 3.1.1 linnéi, Aspius 5.5.1

linnéi, Carassius 5.8.2

linnéi, Labrax 24.1.1

linnéi, Lota 15.1.1

linnéi, Lucioperca 25.4.1

linnéi, Tinca 5.26.1
Liopsetta glacialis 29.2.1

- knipowitschi 29.2.1

- platessa 29.2.2

littoralis, Scyphius 22.1.1

lividus, Leuciscus 5.24.16

Liza aurata 16.2.1

- furcata 16.2.3

- ramada 16.2.2

- saliens 16.2.3

lloydii, Coregonus 13.1.29

loënsis, Salmo 14.3.23.1

longecaudata, Gobius 27.5.4

longecaudata, Knipowitschia 27.5.4

longicephala, Chalcalburnus 5.9.2

longicirri, Cyprinus 5.11.1

longicirris, Gobio 5.13.5

longiradiatus, Gobius 27.4.2

longirostris, Acipenser 2.1.1

longissimus, Alburnus 5.9.2

longiusculus, Coregonus 13.1.33

lönnbergi, Cottus 23.2.1

lönnbergi, Myoxocephalus 23.2.1

lonsdalii, Salvelinus 14.5.9

Lota asiatica 15.1.1

- communis 15.1.1

- fluviatilis 15.1.1

- kamensis 15.1.1

- leptura 15.1.1

- linnéi 15.1.1

- lota 15.1.1

- marmorata 15.1.1

- obensis 15.1.1

- onegensis 15.1.1

- vulgaris 15.1.1

lota, Gadus 15.1.1

lota, Gobius 27.11.1

lota, Lota 15.1.1

lotharingus, Gasterosteus 21.2.2

lotreganus, Mugil 16.2.1

lovensis, Coregonus 13.1.45

lovetzkyi, Acipenser 2.1.4

lozanoi, Valencia 18.1.1

Luccius vorax 10.1.1

lucernensis, Coregonus 13.1.14

lucidus, Alburnus 5.3.2

lucifuga, Umbra 11.1.1

lucinae, Rhodeus 5.23.1

lucinensis, Coregonus 13.1.38

Lucioperca linnéi 25.4 .1

- lucioperca 25.4.1

- sandra 25.4.1

lucioperca, Lucioperca 25.4.1

lucioperca, Perca 25.4.1

lucioperca, Sander 25.4.1

lucioperca, Stizostedion 25.4.1

lucius, Esox 10.1.1

lucumonis, Leuciscus 5.17.9

ludoga, Coregonus 13.1.37

lugdunensis, Cyprinus 5.17.4

lugens, Gobius 27.7.5

lumaireul, Cyprinus 5.21.1

lumbricalis, Petromyzon 1.4 .1 
lumi, Chondrostoma 5.10.4

lumi, Salmo 14.3.12

lupulus, Blennius 26.1.1

lupus, Labrax 24.1.1

luscus, Pleuronectes 29.1.1

lusitanicum, Chondrostoma 5.24.9

lusitanicus, Rutilus 5.24.9

lutescens, Acipenser 2.1.2.

lutescens, Gobio 5.13.3

\section{M}

macedonica Clupea 4.1.7

macedonica, Alosa 4.1.7

macedonica, Perca 25.2.1

macedonica, Trutta 14.3.13

macedonicum, Pachychilon 5.18.1

macedonicus, Alburnus 5.3.2

macedonicus, Barbus 5.7.12

macedonicus, Chalcalburnus 5.9.2

macedonicus, Leuciscus 5.17.4, 5.18.1

macedonicus, Salmo 14.3.13

macrocephala, Muraena 3.1.1

macrogaster, Micromugil 20.1.1

macrolepidotus, Chondrostoma 5.10.4

macrolepidotus, Cyprinus 5.11.1

macrolepidotus, Leuciscus 5.24.10

macrolepidotus, Pleuronectes 28.1.1

macrolepidotus, Rutilus 5.24.10

macrophthalmus, Acipenser 2.1.1

macrophthalmus, Coregonus 13.1.20

macrophthalmus, Scardinius 5.23.2

macrops, Petromyzon 1.3.1

macroptera, Trutta 14.3.13

macropterus, Gobius 27.10.1

macropus, Gobius 27.7.3

macrostigma, Salmo 14.3.5

macrostomus, Cottus 23.1.2, 23.1.4

maculata, Muraena 3.1.1

maculata, Perca 25.2.1

maculata, Tinca 5.26.1

maculatus, Alburnus 5.2.1

maculosus, Petromyzon 1.5.1

maderensis, Mugil 16.2.1

maeotica Clupea 4.1.8

maeotica, Alosa 4.1.8

maeotica, Benthophilus 27.2.1

maeoticus, Huso 2.2.1

maeoticus, Syngnathus 22.2.1

magnicapitata, Gobio 5.13.5

majalis, Leuciscus 5.17.8

major, Clupea 4.1.1

major, Gobius 27.4.2, 27.9.5

major, Lampetra 1.4.1

major, Petromyzon 1.3.1

major, Salmo 14.3.23.1

mallochi, Salvelinus 14.5.10

mandrensis, Chalcalburnus 5.9.2

manocentrus, Coregonus 13.1.32

mantschuricus, Phoxinus 5.12.1

maraena, Coregonus 13.1.29

maraena, Salmo 13.1.29

maraenoides, Coregonus 13.1.17, 13.1.45 maraenula, Coregonus 13.1.28

marathonicus, Leucaspius 5.22.3

margaritaceus, Aspius 30.1.2

marginata, Anguilla 3.1.1

mariae, Eudontomyzon 1.1.3, 1.1.6

mariae, Lampetra 1.1.3

marina, Anguilla 3.1.1

marina, Trutta 14.3.23.1

marinus, Petromyzon 1.5.1

mariza, Rutilus 5.24.16

marmorata, Atherina 27.9.3

marmorata, Lota 15.1.1

marmorata, Trutta 14.3.4, 14.3.14

marmoratus, Gobius 27.10.1

marmoratus, Pomatoschistus 27.9.3

marmoratus, Proterorhinus 27.10.1

marmoratus, Salmo 14.3.14

maroccana, Cobitis 6.1.7

maroccensis, Myxus 16.2.2

marrochius, Leuciscus 5.25.5

marsiglii, Acipenser 2.1.4

marsilii, Fario 14.3.10

marsilii, Phoxinus 5.21.1

marsilii, Salmo 14.5.20

martensii, Gobius 27.8.1

mas, Salmo 14.3.19

maxillaris, Coregonus 13.1.30

maxillaris, Salvelinus 14.5.11

maxima, Alborella 5.3.2

maximus, Petromyzon 1.5.1

mayori, Barbus 5.7.2

meckel, Cyprinus 5.1.2

media, Abramis 5.28.2

media, Coregonus 13.1.19

media, Salmo 13.1.19

mediorostris, Anguilla 3.1.1

mediospinatus, Coregonus 13.1.29

mediterranea, Salmo 30.1.3

medius, Acipenser 2.1.1

megalophthalmus, rutilus 5.27.2

megalops, Coregonus 13.1.31

megaspila, Cobitis 6.1.5

mehdem, Squalius 5.17.8

meidingeri, Leuciscus 5.24.11

meidingeri, Rutilus 5.24.11

melaenus, Abramis 5.1.3

melanio, Gobius 27.7.5

melanochir, Anguilla 3.1.1

melanops, Abramis 5.28.2

melanops, Vimba 5.28.2

melanostomus, Gobius 27.7.5

melanostomus, Neogobius 27.7.5

melanostomus, Salvelinus 14.5.1

melanotus, Idus 5.17.5

melga, Coregonus 13.1.45

menschikowi, Rutilus 5.24.16

mento, Aspius 5.9.2

mentoides, Alburnus 5.9.2

meridionalis, Barbus 5.7.4, 5.7.13

meridionalis, Cobitis 6.1.6

meridionalis, Rhodeus 5.23.1

meridionalis, Salmo 14.3.4 
meridionalis, Squalius 5.17.4

mermere, Ladigesocypris 5.15.1

Mesogobius batrachocephalus 27.6.1

- otschakovinus 27.7.3

messinicus, Gasterosteus 21.1.3

metohiensis, Paraphoxinus 5.20.7

metohiensis, Phoxinellus 5.20.7

meunier, Squalius 5.17.4

microcephalus, Barbus 5.7.14

microcephalus, Coregonus 13.1.40, 13.1.45

microcephalus, Cottus 23.1.4

microchirus, Syngnathus 22.2.1

microlepidotus, Abramis 5.1.3

microlepidotus, Cyprinus 5.17.5

microlepidotus, Gobio 5.13.5

microlepis, Leuciscus 5.17 .10

microlepis, Salmo 14.3.10

microlepis, Squalius 5.17.10

microlepis, Thymallus 13.4.1

Micromugil macrogaster 20.1.1

- timidus 20.1.1

microphthalmus, Barbus 5.7.2

microps, Coregonus 13.1.45

microps, Gobius 27.9.4

microps, Pomatoschistus 27.9.4

microps, Salmo 14.3.23.2.3

microptera, Anguilla 3.1.1

micropteryx, Abramis 5.1.2

microstomus, Cottus 23.1.2, 23.1.4

miegii, Chondrostoma 5.10.10

migratoria, Anguilla 3.1.1

migratorius, Rutilus 5.24.6

mikrosquamatus, Phoxinus 5.21.1

milleri, Knipowitschia 27.5.5

milleri, Orsinigobius 27.5.5

milvensis, Cottus 23.1.2

miniatus, Idus 5.17.5

minor, Alosa 4.1.3

minor, Clupea 4.1.1

minor, Gobius 27.4.2, 27.9.4

minor, Lampetra 1.1.1

minor, Petromyzon 1.3.2

minor, Salmo 14.3.23.1

minor, Squalius 5.17.8

minutus, Carassius 5.8.2

minutus, Gobius 27.9.5

minutus, Paraphoxinus 5.22.2

minutus, Pomatoschistus 27.9.5

minutus, Pseudophoxinus 5.22.2

mirabilis, Acanthobrama 5.28.3

mirandella, Alburnus 5.3.2

Misgurnus anguillicaudatus 6.2.1

- fossilis 6.2 .2

- mizolepis 6.2.1

mistops, Salmo 14.3.23.1

mitsukurii, Lampetra 1.4.1

mizolepis, Misgurnus 6.2.1

mochon, Atherina 17.1.1

moles, Carassius 5.8.2

moles, Cyprinus 5.8.2

monostichus, Salmo 14.5.20

monstrosus, Benthophilus 27.2.1 monstrosus, Cyprinus 5.11.1

montana, Cobitis 6.3.1

montana, Salmo 14.3.23.1

montanus, Phoxinus 5.21.1

montenigrina, Trutta 13.4.1

montenegrinus, Leuciscus 5.17.11

montenigrinus, Leuciscus 5.17.11

montenigrinus, Phoxinellus 5.22.2

moreac, Alosa 4.1.5

morella, Cyprinus 5.21.1

morena, Anguilla 3.1.1

moreoticus, Leuciscus 5.17.4

moriac, Alosa 4.1.5

mrakovcici, Knipowitschia 30.1.3

Mugil ashanteënsis 16.3.1

- auratus 16.2.1

- breviceps 16.2.1

- britannicus 16.2 .2

- buosega 16.1.1

- capito 16.2 .2

- caustelus 16.2.2

- cephalotus 16.3.1

- cephalus 16.3.1

- chelo 16.1 .1

- corrugatus 16.1.1

- cryptocheilos 16.2.1

- curtus 16.1.1

- dubahra 16.2.2

- labrosus 16.1.1

- lotreganus 16.2.1

- maderensis 16.2.1

- octo-radiatus 16.2.1

- öur 16.3.1

- petherici 16.2.2

- provensalis 16.3.1

- ramada 16.2 .2

- saliens 16.2.3

- septentrionalis 16.1.1

- tang 16.3.1

- verselata 16.2.3

- vulpinus 16.3.1

mugilis, Cyprinus 5.17.8

mullus, Centropomus 24.1.1

multispinatus, Coregonus 13.1.33

Muraena anguilla 3.1.1

- macrocephala 3.1.1

- maculata 3.1.1

- ornithorhyncha 3.1.1

- oxycephala 3.1.1

- oxyrhina 3.1.1

- platyrhina 3.1.1

- platyura 3.1 .1

muraenula, Salmo 13.1.28

muresia, Gobio 5.13.5

murta, Salmo 14.5.12

murta, Salvelinus 14.5.12

musta-siiska, Coregonus 13.1.31

muticellus, Leuciscus 5.17.12

Myoxocephalus lönnbergi 23.2.1

- onegensis 23.2.1

- quadricornis 23.2.1

Myxus caecutiens 16.3.1 
nobilis, Coregonus 13.1.13

nobilis, Salmo 14.3.19

$\mathbf{N}$

naccarii, Acipenser 2.1.2

nanofasciatus, Cyprinoides 20.1.1

nanus, Aphanius 20.1.1

nanus, Ciprinoides 20.1.1

nardoi, Acipenser 2.1.2

narentana, Cobitis 6.1.10

nasalis, Gobius 27.10.1

nasicus, Chondrochylus 5.10.9

nasus, Acipenser 2.1.2

nasus, Chondrostoma 5.10.4

nasutus, Coregonus 13.1.33

nasutus, Squalius 5.17 .8

neglectus, Leuciscus 5.17.5

nehelae, Acipenser 2.1.6

Nemacheilus anglicana 7.1.1

- barbatulus 7.1.1

- blackiana 7.1.1

- bureschi 7.1 .2

- erythrinna 7.1.1

- hispanica 7.1.1

- quignardi 7.1 .1

- sturanyi 7.1.1

- vardarensis 7.1.1

nemauensis, Gasterosteus 21.1.3

Neogobius eurycephalus 27.7.1

- fluviatilis 27.7.2

- gorlap 27.7.4

- gymnotrachelus 27.7.3

- iljini 27.7.4

- kessleri 27.7.4

- melanostomus 27.7.5

- odessicus 27.7.1

- syrman 27.7.6

nerensis, Zingel 25.5.3

Nerophis ophidion 22.1.1

neschka, Coregonus 13.1.45

neustrianus, Gasterosteus 21.1.3

nicaeensis, Alburnus 5.9.2

nicaeensis, Vimba 5.28 .3

niger, Gasterosteus 21.2.3

niger, Gobius 27.4.2

niger, Petromyzon 1.3.2

niger, Salmo 14.3.10, 14.5.18

nigerrimus, Gobius 27.4.2

nigra, Gobius 27.7.2

nigrescens, Alosa 4.1.5

nigrescens, Bungia 5.13.5

nigrescens, Gobius 27.4.2

nigrescens, Perca 25.2.1

nigricans, Gobius 27.8.2

nigricans, Padogobius 27.8.2

nigripinnis, Salmo 14.3.15

nigrolineatus, Syngnathus 22.2.1

nigropunctata, Lebias 20.1.1

nikolskyi, Gobio 5.13.5

nilotica, Anguilla 3.1.1

nilotica, Clupea 4.1.3

nilssoni, Coregonus 13.1.32

nivalis, Salmo 14.5.1 nordmanni, Alosa 4.1.9

nordmanni, Coregonus 13.1.45

nordmannii, Abramis 5.28.3

nordmannii, Cyprinus 5.11.1

norvegica, Coregonus 13.1.28, 13.1.45

notha, Cyprinus 5.2.1

nothulus, Leuciscus 5.17.4

nuda, Pygosteus 21.2.3

nudiventris, Acipenser 2.1.3

nudus, Benthophilus 27.2.1

nudus, Cyprinus 5.11.1

nüsslini, Coregonus 13.1.20

O

obensis, Lota 15.1.1

oblongirostris, Anguilla 3.1.1

oblongus, Carassius 5.8.2

oblongus, Cyprinus 5.11.1

obtusirostris, Acipenser 2.1.4

obtusirostris, Gobio 5.13.5

obtusirostris, Salar 13.4.1

obtusirostris, Salmothymus 13.4.1

obtusus, Alburnus 5.3.2

obtusus, Leuciscus 5.21.1

obtusus, Salvelinus 14.5.13

occidentalis, Huso 2.2.1

occidentalis, Phoxinus 5.12.1

ochrodon, aspius 5.3.2

ocla, Salmo 14.3.19

octo-radiatus, Mugil 16.2.1

odessicus, Neogobius 27.7.1

oernensis, Cottus 23.2.1

ohridana, Acantholingua 14.1.1

ohridana, Cobitis 6.1.10

ohridana, Gobio 5.13.5

ohridana, Leucos 5.24.12

ohridana, Scardinius 5.23.2

ohridanum, Chondrostoma 5.10.4

ohridanus, Alburnus 5.2.1

ohridanus, Rutilus 5.24.12

ohridanus, Salmo 14.1.1

okoniensis, Coregonus 13.1.37

olonensis, Coregonus 13.1.28, 13.1.31

omalii, Petromyzon 1.3.1

omiscomaycus, Salmo 14.3.19

Oncocottus quadricornis 23.2.1

onegensis, Lota 15.1.1

onegensis, Myoxocephalus 23.2.1

onegi, Coregonus 13.1.37

ophidion, Nerophis 22.1.1

ophidion, Syngnathus 22.1.1

ophiocephalus, Gobius 27.11.1

ophiocephalus, Zosterisessor 27.11.1

opisthodon, Lampetra 1.3.1

orcadensis, Salmo 14.3.23.1

orfus, Cyprinus 5.17.5

orientalis, Abramis 5.1.3

orientalis, Coregonus 13.1.45

orientalis, Huso 2.2.1

orientalis, Idus 5.17 .5 
orientalis, Labrax 24.1.2

orientalis, Leuciscus 5.17 .4

orientalis, Salmo 14.3.28

Orisinigobius milleri 27.5.5

ornithorhyncha, Muraena 3.1.1

oronensis, Coregonus 13.1.31

orthonotus, Cyprinus 5.17.4

Orthrias barbatulus 7.1.1

- pindus 7.1.3

Osmerus eperlanus 12.1.1

- ladogensis 12.1.1

- schonfoldi 12.1.1

otschakovinus, Mesogobius 27.7.3

öur, Mugil 16.3.3

ovsianka, Aspius 5.16.1

Owsianka czernayi 5.16.1

oxianus, Salmo 14.3.28

oxianus, Squalius 5.17 .5

oxycephala, Alburnus 5.3.2

oxycephala, Muraena 3.1.1

oxyrhina, Muraena 3.1.1

oxyrhynchus, Salmo 13.4.1

oxyrinchus, Coregonus 13.1.39

oxyrinchus, Salmo 13.1.39

oxyrrhis, Squalius 5.17.2

\section{$\mathbf{P}$}

pachycephalus, Coregonus 13.1.31

Pachychilon macedonicum 5.18.1

- pictum 5.18.2

Padogobius bonelli 27.8.1

- nigricans 27.8.2

pagellus, Leuciscus 5.24.3

palaciosi, Iberocypris 5.14.1

palaea, Coregonus 13.1.7

palaeostomi, Caspialosa 4.1.9

palaeostomica, Caspialosa 4.1.9

palja, Salmo 14.5.1

pallaryi, Salmo 14.3.5

pallasii, Coregonus 13.1.33

pallasii, Cyprinus 5.16.1

pallasii, Salmo 14.3.10

pallasii, Schilus 25.4.2

pallayeri, Salmo 14.3.23.1

pallens, Leuciscus 5.24.16

pallidus, Salmo 14.5.20

palloni, Coregonus 13.1.45

paludica, Acanthopsis 6.1.7

paludica, Cobitis 6.1.7

paludicola, Cobitis 6.1.7

pamvoticus, Leuciscus 5.17.4

panizzae, Gobius 27.5.6

panizzae, Knipowitschia 27.5.6

pannonica, Cobitis 30.1.3

pannonicus, Ictalurus 8.1.1

Paraphoxinus epiroticus 5.20.4

- ghetaldii 5.20.6

- metohiensis 5.20.7

- minutus 5.22.2

- prespensis 5.20.8

- pstrossii 5.20.9

parcepunctata, Salar 14.3.23.1 pareti, Squalius 5.17.4

parisiensis, Cobitis 7.1.1

parnelli, Gobius 27.9.4

parvula, Clupea 4.1.1

parvulus, Leuciscus 5.28.3

passer, Pleuronectes 29.1.1

patarachia, Pleuronectes 28.1.1

pauperum, Leuciscus 5.24.3

pausingeri, Leuciscus 5.24.16

pavlovi, Umbra 11.1.1

pelagicola, Coregonus 13.1.45

pelagicus, Coregonus 13.1.45

pelagicus, Syngnathus 22.2.4

pelagonicus, Salmo 14.3.16

Pelecus cultratus 5.19.1

peled, Coregonus 13.1.34

peled, Salmo 13.1.34

pelet, Salmo 13.1.34

pellegrini, Cottus 23.1.2

pellegrini, Salmo 14.3.5

peloponensis, Leuciscus 5.17.4

peloponnesius, Barbus 5.7.15

pennantii, Coregonus 13.1.41

Perca asper 25.5.1

- aurata 25.2.1

- aurea 25.2.1

- cernua 25.1.2

- danubiensis 25.1.3

- elongata 24.1.1

- fluviatilis 25.2.1

- gibba 25.2.1

- gracilis 25.2.1

- helvetica 25.2.1

- intermedius 25.2.1

- italica 25.2.1

- labrax 24.1.1

- lucioperca 25.4.1

- macedonica 25.2.1

- maculata 25.2.1

- nigrescens 25.2.1

- phragmiteti 25.2.1

- schraetser 25.1.3

- volgensis 25.4 .2

- vulgaris 25.2 .1

- zaissanica 25.2.1

- zingel 25.5.4

perejaslawica, Coregonus 13.1.28

perenurus, Cyprinus 5.12.1

perenurus, Eupalasella 5.12.1

perenurus, Phoxinus 5.12.1

peresi, Chondrostoma 5.10.10

pereslavicus, Coregonus 13.1.28

pereslawicus, Coregonus 13.1.28

pergamonensis, Barbus 5.7.6

perisii, Salmo 14.5.14

perisii, Salvelinus 14.5.14

peristericus, Salmo 14.3.17

perryi, Hucho 14.2.1

persa, Cyprinus 5.28.3

peschevi, Cobitis 6.1.9

petenyi, Barbus 5.7 .15

petenyii, Barbus 5.7.15 
petherici, Mugil 16.2.2

petiti, Cottus 23.1.3

Petromyzon adriaticus 1.5.1

- anomalum 1.3.2

- argenteus 1.3.1

- bicolor 1.3.2

- borealis 1.4.1

- branchialis 1.3.1

- caecus 1.3.2

- camtschaticus 1.4.1

- dorsatus 1.5.1

- ernstii 1.4.1

- fluviatilis 1.3.1, 1.4.1

- japonicus 1.4.1

- jurae 1.3.1

- kameraticus 1.4.1

- lampetra 1.5.1

- larvalis 1.3.2

- lumbricalis 1.4.1

- macrops 1.3.1

- maculosus 1.5.1

- major 1.3.1

- marinus 1.5.1

- maximus 1.5.1

- minor 1.3.2

- niger 1.3 .2

- omalii 1.3.1

- planeri 1.3.2

- plumbeus 1.3 .2

- ponticus 1.1.3

- pricka 1.3.1

- ruber 1.5.1

- sanguisuga 1.3.1

- septocuille 1.3.2

- septoeuil 1.3.2

- variegatus 6.2.2

petteri, Blennius 26.1.1

phinoc, Salmo 14.3.23.1

Phoxinellus adspersus 5.20.1

- alepidotus 5.20.2

- croaticus 5.20 .3

- epiroticus 5.20 .4

- fontinalis 5.20 .5

- ghetaldii 5.20.6

- italicus 30.1.3

- metohiensis 5.20.7

- montenigrinus 5.22.2

- prespensis 5.20 .8

- pstrossii 5.20.9

- thracicus 5.16.1

phoxinoïdes, Gobio 5.13.5

Phoxinus altus 5.12.1

- balchaschana 5.21.1

- carpathicus 5.21.1

- colchicus 5.21.1

- csikii 5.21.1

- dauricus 5.12.1

- dybowskii 5.12.1

- gdaniensis 5.12.1

- hispanica 5.4.1

- jelskii 5.12 .1

- laevis 5.21.1
- mantschuricus 5.12.1

- marsilii 5.21.1

- mikrosquamatus 5.21.1

- montanus 5.21.1

- occidentalis 5.12.1

- phoxinus 5.21.1

- posnaniensis 5.12.1

- punctatus 5.12.1

- sabanejewi 5.12.1

- sachalinensis 5.12.1

- saposchnikowi 5.21.1

- sarykul 5.12.1

- sedelnikowi 5.21.1

- stagnalis 5.12 .1

- strandjae 5.21.1

- ujmonensis 5.21.1

- variabilis 5.12.1

- varius 5.21.1

phoxinus, Chondrostoma 5.10.5

phoxinus, Cyprinus 5.21.1

phoxinus, Phoxinus 5.21.1

phragmiteti, Aspius 5.5.1

phragmiteti, Perca 25.2.1

phragmiteti, Rutilus 5.24.16

pictava, Cobitis 7.1.1

pictava, Leuciscus 5.17.4

pictum, Pachychilon 5.18.2

pictus, Leuciscus 5.18.2

pidschian, Coregonus 13.1.35

pidschian, Salmo 13.1.35

pidschianoides, Coregonus 13.1.35

pigmeus, Leuciscus 5.16.1

pigulus, Leucos 5.24.16

pigus, Cyprinus 5.24.13, 5.24.16

pigus, Rutilus 5.24.13

pindus, Orthrias 7.1.9

pironae, Cobitis 7.1.1

piscinarum, Carassius 5.8.2

planeri, Lampetra 1.3.1, 1.3.2

planeri, Petromyzon 1.3.2

Platessa carnaria 29.1.1

- dwinensis 29.2.1

- latus 29.2.2

- vulgaris 29.2 .2

platessa, Liopsetta 29.2.2

platessa, Pleuronectes 29.2.2

Platichthys flesus 29.1.1

platizza, Scardinius 5.23.2

platycephala, Anguilla 3.1.1

platycephala, Squalius 5.17.4

platycephalus, Acipenser 2.1.2

platycephalus, Gobius 27.7.4

platygaster, Gasterosteus 21.2.3

platygaster, Pungitius 21.2.3

platyrhina, Muraena 3.1.1

platyrhynchus, Anguilla 3.1.1

platyura, Muraena 3.1.1

plebeius, Barbus 5.7.16

plebejus, Barbus 5.7.16

plestya, Cyprinus 5.1.2

pleurobipunctatus, Leuciscus 5.17.13

pleurobipunctatus, Rutilus 5.17.13 
Pleuronectes baltica 29.1.1, 29.2.2

- bogdanovii 29.1.1

- borealis 29.2 .2

- caninensis 29.1.1, 29.2.1

- cicatricosus 29.2.1

- citharus 28.1.1

- flesoides 29.1.1

- flesus 29.1.1

- glacialis 29.2.1

- italicus 29.1.1

- leiurus 29.1.1

- linguatula 28.1.1

- luscus 29.1.1

- macrolepidotus 28.1.1

- passer 29.1.1

- patarachia 28.1.1

- platessa 29.2.2

- roseus 29.1.1

- septentrionalis 29.1.1

- trachurus 29.1.1

plotizza, Scardinius 5.23.2

plumbeus, Petromyzon 1.3.2

podapos, Acipenser 2.1.6

Poecilia calaritana 20.1.1

poecilopus, Cottus 23.1.4

polcur, Salmo 13.1.35

poljakovi, Coregonus 13.1.37

pollan, Coregonus 13.1.42

pollinii, Gobio 5.13.3

pollinii, Ichthyocoris 26.1.1

polonica, Coregonus 13.1.37

polylepis, Barbus 5.7.6

polylepis, Chondrostoma 5.10.6

polylepis, Leuciscus 5.17.14

polylepis, Telestes 5.17 .14

polyosteus, Salmo 14.3.23.1

Pomatoschistus bathi 27.9.1

- canestrinii 27.9.2

- caucasicus 27.5.1

- knipowitschi 27.5.4

- marmoratus 27.9.3

- microps 27.9.4

- minutus 27.9.5

- tortonesei 27.9.6

pommerana, Coregonus 13.1.29

pontica, Abramis 5.28.3

pontica, Atherina 17.1.1

pontica, Clupea 4.1.5

pontica, Gobius 27.4.2, 27.10.1

ponticus, Benthophilus 27.2.1

ponticus, Gasterosteus 21.1.1

ponticus, Huso 2.2.1

ponticus, Petromyzon 1.1.3

ponticus, Syngnathus 22.2.4

posnaniensis, Phoxinus 5.12.1

post, Holocentrus 25.1.2

praecox, Lampetra 1.3.1

praecox, Lampetra 1.4.1

prasinus, Leuciscus 5.24.16

prawdini, Coregonus 13.1.45

presbyter, Atherina 17.1.3

prespense, Chondrostoma 5.10.7 prespensis, Alburnus 5.2.1

prespensis, Barbus 5.7.17

prespensis, Chondrostoma 5.10.7

prespensis, Leucos 5.24.14

prespensis, Paraphoxinus 5.20.8

prespensis, Phoxinellus 5.20.8

prespensis, Rutilus 5.24.14

prespensis, Squalius 5.17.4

prevesicus, Gobius 27.3.1

pricka, Petromyzon 1.3.1

primigenius, Acipenser 30.1.1

profundicola, Salvelinus 14.5.1

profundus, Salmo 14.3.23.2.1, 14.5.15

profundus, Salvelinus 14.5.15

prosopyga, Gobio 5.13.5

prosperi, Leucaspius 5.15.1

prosperoi, Leucaspius 5.15.1

Proterorhinus marmoratus 27.10.1

provensalis, Mugil 16.3.3

Pseudobarbus leonhardi 5.7.15

Pseudophoxinus beoticus 5.22.1

- minutus 5.22.2

- stymphalicus 5.22.3

pstrossii, Paraphoxinus 5.20.9

pstrossii, Phoxinellus 5.20.9

puckensis, Gobius 27.9.4

pulsensis, Coregonus 13.1.45

punctata, Sciaena 24.1.2

punctatissima, Knipowitschia 27.5.7

punctatissimus, Gobius 27.5.7

punctatus, Cyprinus 5.2.1

punctatus, Dicentrarchus 24.1.2

punctatus, Phoxinus 5.12.1

punctatus, Salmo 14.5.1, 14.6.1

punctilineata, Cobitis 6.1.13

punctulatus, Gobius 27.4.2

Pungitius hellenicus 21.2.1

- laevis 21.2.2

- platygaster 21.2.3

- pungitius 21.2.4

pungitius, Gasterosteus 21.2.4

pungitius, Pungitius 21.2.4

pursakensis, Leuciscus 5.17.4

pusillus, Gobius 27.9.4

puta, Cobitis 6.1.1

pycnocentrus, Coregonus 13.1.32

pygmaeus, Acipenser 2.1.1, 2.1.4

pygmaeus, Cottus 23.2.1

pygmaeus, Economidichthys 27.3.1

pygmaeus, Gobius 27.3.1

Pygosteus carinata 21.2.4

- hologymna 21.2.2

- nuda 21.2.3

- semiarmata 21.2.4

- trachura 21.2.4

pyrenaicus, Leuciscus 5.17.15

pyrois, Syngnathus 22.2.4

Q

quadricapillus, Gobius 27.10.1

quadricornis, Cottus 23.2.1

quadricornis, Myoxocephalus 23.2.1 
quadricornis, Oncocottus 23.2.1 quadricornis, Triglopsis 23.2.1 quadrispinosa, Gasterosteus 21.1.3 quignardi, Nemacheilus 7.1.1

\section{$\mathbf{R}$}

racovitzai, Scardinius 5.25.4

radnensis, Cobitis 6.3.1

ramada, Liza 16.2.2

ramada, Mugil 16.2.2

rapax, Cyprinus 5.5.1

rappii, Salmo 14.3.23.2.2

ratisbonensium, Asperulus 25.5.4

ratisbonensium, Schraitser 25.1.3

ratisbonensium, Streber 25.5.3

ratisbonensium, Zindel 25.5.4

ratzeburgii, Acipenser 2.1.5

rebeli, Barbus 5.7.15

regina, Cyprinus 5.11.1

regius, Cyprinus 5.11.1

reiseri, Chondrostoma 30.1.2

reisingeri, Coregonus 13.1.27

relicta, Cottus 23.2.1

relicta, Trutta 14.3 .19

relictus, Leucaspius 5.16.1

relictus, Salmo 14.3.19

renatus, Salmo 14.3.19

renke, Coregonus 13.1.27

renke, Salmo 13.1.27

restrictus, Coregonus 13.1.8

reticulatus, Gobius 27.9.3, 27.11.1

rex cyprinorum, Cyprinus 5.11.1

rex, Cyprinus 5.11.1

rhenana, Salmo 14.3.23.1

rhinosimus, Abramis 30.1.2

rhodanensis, Alosa 4.1.3

rhodanensis, Chondrostoma 5.10.10

rhodanensis, Coregonus 13.1.2

rhodanensis, Salmo 14.3.18

Rhodeus amarus 5.23.1

- danubicus 5.23.1

- genitalis 5.23 .1

- lucinae 5.23.1

- meridionalis 5.23.1

- sericeus 5.23 .1

- strumicae 5.23.1

- svetovidovi 5.23.1

rhodopterus, Gobius 27.9.3

rhomboidalis, Lebias 20.1.1

riemling, Cyprinus 5.2.1

rilla, Salmo 14.3.19

ringsjöensis, Coregonus 13.1.31

riqueti, Atherina 17.1.1

risae, Leuciscus 5.17.4

risso, Atherina 17.1.1

rissoi, Leuciscus 5.17.4

riusensis, Coregonus 13.1.14

rivularis, Cyprinus 5.21.1

rodens, Leuciscus 5.17.8

romanica, Cobitis 6.3.4

romanica, Sabanejewia 6.3.4

Romanichthys valsanicola 25.3.1 romanovi, Salmo 14.3.28

rondeleti, Coregonus 13.1.4

rondeletii, Cyprinus 5.11.1

rondeletii, Syngnathus 22.2.4

rosenhaueri, Alburnus 30.1.2

roseus, Cottus 23.1.2

roseus, Leuciscus 5.24.13

roseus, Pleuronectes 29.1.1

rossicus, Alburnoides 5.2.1

rostratus, Acipenser 2.1.5

rostratus, Leuciscus 5.17.8

rostratus, Salmo 13.1.39

rotundatus, Syngnathus 22.2.4

roulei, Leuciscus 5.17 .8

rubella, Leuciscus 5.24 .15

rubellicus, Leucos 5.24.3

rubellio, Cyprinus 5.24.16

ruber, Petromyzon 1.5.1

rubescens, Syngnathus 22.2.2

rubilio, Leuciscus 5.24 .15

rubilio, Rutilus 5.24 .15

ruboculus, Gardonus 5.24.16

rubromaculatus, Gobius 27.10.1

rudolphianus, Coregonus 13.1.34

rufa, Clupea 4.1.3

ruffoi, Leuciscus 5.17.4

rufus, Cyprinus 5.17.4

rusa, Alosa 4.1.3

russac, Alosa 4.1.5

ruthenicus, Acipenser 2.1.4

ruthenus, Acipenser 2.1.4

rutiloides, Leuciscus 5.24.16

rutiloides, Scardinius 5.23.2

Rutilus alburnoides 5.25.1

- alfiensis 5.17.13

- aralensis 5.24.16

- arcasii 5.25.2

- aula 5.24 .3

- basak 5.24.4

- beoticus 5.22 .1

- bucharensis 5.24.16

- carpathorossicus 5.24.16

- dojranensis 5.24.16

- dono-kubanensis 5.24.6

- erythrophthalmus 5.24 .3

- frici 5.24.16

- frisii 5.24 .5

- geoktshaicus 5.24 .16

- goplensis 5.24 .16

- heckelii 5.24 .6

- heegeri 5.25.5

- hellenicus 5.27.1

- italicus 5.24.15

- karamani 5.24 .7

- knipowitschi 5.24.16

- kurensis 5.24.16

- lemmingii 5.24 .8

- lusitanicus 5.24 .9

- macrolepidotus 5.24.10

- mariza 5.24.16

- megalophthalmus 5.27.2

- meidingeri 5.24.11 
- menschikowi 5.24.16

- migratorius 5.24.6

- ohridanus 5.24.12

- phragmiteti 5.24.16

- pigus 5.24.13

- pleurobipunctatus 5.17.13

- prespensis 5.24.14

- rubilio 5.24.15

- rutilus 5.24.16

- schelkovnikovi 5.24 .16

- spartiaticus 5.27 .2

- sucharensis 5.24 .16

- tscharchalensis 5.24.16

- uzboicus 5.24.16

- vegariticus 5.24 .16

- velecensis 5.24 .5

- vukovici 5.24.7

- ylikiensis 5.24 .17

rutilus, Cyprinus 5.24.16

rutilus, Rutilus 5.24.15

rutilus, Salmo 14.5.1

ruzsky, Acipenser 2.1.4

rysela, Chondrostoma 5.10.9, 5.17.16

rysela, Telestes 5.17 .16

ryzela, Leuciscus 5.24 .13

\section{$\mathrm{S}$}

sabanejewi, Phoxinus 5.12.1

Sabanejewia balcanica 6.3.1

- baltica 6.3.1

- bulgarica 6.3.2

- doiranica 6.3.1

- kubanica 6.3.1

- larvata 6.3.3

- romanica 6.3 .4

- thrakica 6.3.1

sachalinensis, Phoxinus 5.12.1

saimensis, Salmo 14.3.19

Salar ausonii 14.3.23.1

- bailloni 14.3.23.1

- dentex 14.3.6

- gaimardi 14.3.23.1

- genivittatus 14.3.14

- obtusirostris 14.4.1

- parcepunctata 14.3.23.1

- semipunctata 14.3.23.1

- spectabilis 14.3.23.1

salar, Salmo 14.3.19

Salaria fluviatilis 26.1.1

Salarias varus 26.1.1

salax, Cyprinus 5.17.8

saliens, Liza 16.2.3

saliens, Mugil 16.2.3

salmanata, Trutta 14.3.23.1

salmarinus, Salmo 14.5.20

Salmo abanticus 14.3.28

- aestivalis 14.3.1

- alba 14.3.10

- albula 13.1.

- albus 14.3.23.1

- alpino-stagnalis 14.5.1

- alpinus 14.5.1
- americanus 14.3.19

- aphelios 14.3.1

- aralensis 14.3.28

- argenteus 14.3.20

- arvensis 14.3.23.2.1

- ascanii 14.5.1

- balcanicus 14.3.2

- benaci 14.3.3

- biennis 14.3.19

- bodensis 14.3.23.2.2

- brachypoma 14.3.23.1

- brevipes 14.3.19

- caecifer 14.3.23.1

- caerulescens 14.3.19

- cambricus 14.3.23.1, 14.5.14

- carbonarius 14.5.1

- carneus 14.5.20

- carpio 14.3.3

- caspius 14.3.28

- cenerinus 14.3.4

- cettii 14.3.5

- ciscaucasicus 14.3.28

- colii 14.5.2

- cornubiensis 14.3.23.1

- costatus 14.5.20

- cumberland 14.3.23.1

- cyprinoides 13.1.34

- danubicus 14.3.10

- dentex 14.3.6

- distichus 14.5.20

- eperlano-marinus 12.1 .1

- eperlanus 12.1.1

- eriox 14.3.23.1

- estuarius 14.3.23.1

- europaeus 14.3.19

- excelsa 14.3.10

- ezenami 14.3.28

- fario $14.3 .10,14.3 .23 .1,14.3 .28$

- farioides 14.3.7

- fecunda 14.3.23.1

- ferox 14.3.8

- ferra 13.1.21

- fibreni 14.3.9

- forestensis 14.3.23.1

- gadoïdes 14.3.23.1

- gallivensis 14.3.23.1

- ghigii 14.3.5

- goedenii 14.3.19

- gracilis 14.3.19

- grayi 14.5 .5

- hamatus 14.3.19

- hardinii 14.3.19

- hucho 14.2.1

- hybridus 14.5 .1

- illanca 14.3.23.2.2

- ischan 14.3.1

- islayensis 14.3.23.1

- killinensis 14.5.8

- labrax 14.3.10

- lacustris 14.3.7, 14.3.10, 14.3.14, 14.3.19, 14.3.23.2.2, 14.3 .28

- laevis 14.5.1 
- lapasseti 14.3.5

- latus 13.1.39

- lavaretus 13.1.4

- lemanus 14.3.23.2.1

- lepechini 14.5.1

- letnica 14.3.11

- levenensis 14.3.23.1

- levis 14.5.1

- loënsis 14.3.23.1

- lumi 14.3.12

- macedonicus 14.3.13

- macrostigma 14.3.5

- major 14.3.23.1

- maraena 13.1.29

- maraenula 13.1.28

- marmoratus 14.3.14

- marsilii 14.5.20

- mas 14.3.19

- media 13.1.19

- mediterranea 30.1.3

- meridionalis 14.3.4

- microlepis 14.3.10

- microps 14.3.23.2.3

- minor 14.3.23.1

- mistops 14.3.23.1

- monostichus 14.5.20

- montana 14.3.23.1

- murta 14.5.12

- niger 14.3.10, 14.5.18

- nigripinnis 14.3.15

- nivalis 14.5.1

- nobilis 14.3.19

- ocla 14.3.19

- ohridanus 14.1.1

- omiscomaycus 14.3.19

- orcadensis 14.3.23.1

- orientalis 14.3 .28

- oxianus 14.3.28

- oxyrhynchus 14.4 .1

- oxyrinchus 13.1.39

- palja 14.5.1

- pallaryi 14.3 .5

- pallasii 14.3.10

- pallayeri 14.3.23.1

- pallidus 14.5.20

- pelagonicus 14.3.16

- peled 13.1.34

- pelet 13.1.34

- pellegrini 14.3 .5

- perisii 14.5.14

- peristericus 14.3.17

- phinoc 14.3.23.1

- pidschian 13.1.35

- polcur 13.1.35

- polyosteus 14.3.23.1

- profundus 14.3.23.2.1, 14.5.15

- punctatus 14.5.1, 14.6.1

- rappii 14.3.23.2.2

- relictus 14.3 .19

- renatus 14.3.19

- renke 13.1.27

- rhenana 14.3.23.1
- rhodanensis 14.3.18

- rilla 14.3 .19

- romanovi 14.3.28

- rostratus 13.1 .39

- rutilus 14.5.1

- saimensis 14.3.19

- salar 14.3.19

- salmarinus 14.5 .20

- salmo 14.3 .19

- salmulus 14.3.19

- salvelino-stagnalis 14.5.1

- salvelinus 14.5.20

- saxatilis 14.3.10, 14.3.23.1

- schiefermuelleri 14.3.20

- schrankii 14.5.20

- septentrionalis 14.3.23.1

- spirinchus 12.1 .1

- spurius 14.3.28

- sterilis 14.3.23.1

- stomachicus 14.3.21

- stomachius 14.3.21

- striatus 14.6.1

- stroemii 14.3.23.1

- struanensis 14.5.17

- sylvaticus 14.3.23.1

- taleri 14.3.22

- taurinus 14.3.23.1

- thingvallensis 14.5 .18

- thymallus 14.6.1

- thymus 14.6 .1

- trutta 14.3.23.1

- truttula 14.3.23.2.3

- typicus 14.3.11

- umbla 14.5.20

- venernensis 14.3.23.2.3

- ventricosus 14.5.1

- vimba 13.1.

- visovacensis 14.3.24

- wartmanni 13.1.21

- willoughbii 14.5.21

- willughbii 14.5.21

- zetensis 14.4 .1

- zrmanjaensis 14.3.25

salmo, Salmo 14.3.19

salmonata, Trutta 14.3.23.1

salmoneus, Cyprinus 5.17.4

Salmostoma balookee 5.19.1

- clupeoides 5.19.1

Salmothymus obtusirostris 14.4 .1

salmulus, Salmo 14.3.19

salonitana, Trutta 13.4.1

saltator, Leuciscus 5.17.8

salvelino-stagnalis, Salmo 14.5.1

Salvelinus alpinus 14.5.1

- autumnalis 14.5.21

- colii 14.5.2

- faroensis 14.5 .3

- fimbriatus 14.5.4

- gracillimus 14.5.6

- grayi 14.5 .5

- inframundus 14.5.7

- killinensis 14.5.8 
- lonsdalii 14.5.9

- mallochi 14.5.10

- maxillaris 14.5.11

- melanostomus 14.5.1

- murta 14.5.12

- obtusus 14.5.13

- perisii 14.5.14

- profundicola 14.5.1

- profundus 14.5.15

- scharffi 14.5.16

- struanensis 14.5 .17

- thingvallensis 14.5.18

- trevelyani 14.5.19

- umbla 14.5.20

- vernalis 14.5.21

- willoughbii 14.5.21

- youngeri 14.5.22

salvelinus, Salmo 14.5.20

samantica, Barbatula 7.1.8

sanctibenedicti, Coregonus 13.1.5

sandat, Centropomus 25.4.1

Sander lucioperca 25.4.1

- volgensis 25.4.2

sanderi, Cyprinus 5.2.1

sandra, Lucioperca 25.4.1

sanguisuga, Petromyzon 1.3.1

sapa, Abramis 5.1.4

sapa, Cyprinus 5.1.4

sapancae, Alburnus 5.9.2

sapancae, Vimba 5.28.3

saposchnikowi, Phoxinus 5.21.1

sarda, Atherina 17.1.1

sarda, Lebias 20.1.1

sardella, Leuciscus 5.24.15

sardinella, Clupea 4.1.3

sarmaticus, Gobio 5.13.5

sarykul, Phoxinus 5.12.1

savignyi, Anguilla 3.1.1

savigny, Telestes 5.17.12

saxatilis, Gobio 5.13 .5

saxatilis, Salmo 14.3.10, 14.3.23.1

scallensis, Coregonus 13.1.37

scardafa, Leuciscus 5.25.5

scardafa, Scardinius 5.25.5

Scardiniopsis anceps 30.1 .2

Scardinius acarnanicus 5.25.1

- achrus 5.25.2

- crocophthalmus 5.25 .2

- dergle 5.25 .2

- dojranensis 5.25 .2

- erythrophthalmus 5.25.2

- graecus 5.25.3

- hesperidicus 5.25 .2

- macrophthalmus 5.25.2

- ohridana 5.25.2

- platizza 5.25.2

- plotizza 5.25.2

- racovitzai 5.25.4

- rutiloides 5.25.2

- scardafa 5.25.5

Scardinopsis alburniformis 30.1.2

- amphigenus 30.1.2 scardinus, Leuciscus 5.24.3

scardula, Cyprinus 5.23.2

scarpata, Leuciscus 5.25.5

scarpetta, Leuciscus 5.25:5

scaverde, Leuciscus 30.1.3

scharffi, Salvelinus 14.5.16

schelkovnikovi, Rutilus 5.24.16

schiefermuelleri, Salmo 14.3.20

Schilus pallasii 25.4.2

schinzii, Coregonus 13.1.2

schischkovi, Chalcalburnus 5.9.2

schonfieldii, Eperlanus 12.1.1

schonfoldi, Osmerus 12.1.1

schonfoldii, Eperlanus 12.1.1

schraetser, Gymnocephalus 25.1.3

Schraitser ratisbonensium 25.1.3

schraizer, Holocentrus 25.1.3

schrankii, Salmo 14.5.20

schreibersii, Abramis 5.1.4

schuensis, Coregonus 13.1.31

schypa, Acipenser 2.1.3, 2.2.1

Sciaena punctata 24.1.2

scincus, Barbus 5.7.16

sclateri, Barbus 5.7.18

scobar, Acipenser 2.1.1

scodrense, Chondrostoma 5.10 .8

scodrensis, Chondrostoma 5.10.8

scoranza, Alburnus 5.3.2

scoranzoides, Alburnus 5.3.2

Scyphicus teres 22.1.1

Scyphius littoralis 22.1.1

- violaceus 22.1.1

sedelnikowi, Phoxinus 5.21.1

selysii, Leuciscus 5.24.16

semiarmata, Pygosteus 21.2.4

semiarmatus, Gasterosteus 21.1.3

semiloricatus, Gasterosteus 21.1.3

semilunaris, Gobius 27.10.1

semipunctata, Salar 14.3.23.1

septa, Cobitis 6.1.1

septembrina, Anguilla 3.1.1

septemcarinata, Acipenser 2.1.4

septentrionalis, Lampetra 1.4.1

septentrionalis, Mugil 16.1.1

septentrionalis, Pleuronectes 29.1.1

septentrionalis, Salmo 14.3.23.1

septocuille, Petromyzon 1.3.2

septoeuil, Petromyzon 1.3.2

sericeus, Rhodeus 5.23.1

serta, Cyprinus 5.28.3

seuruga, Acipenser 2.1.5

seva, Chondrostoma 5.10.9

shyp, Acipenser 2.2.1

sibiricus, Gobio 5.13.5

sibiricus, Leuciscus 5.17.5

sibiricus, Sterledus 2.1.4

sieboldi, Coregonus 13.1.17

sieboldii, Carpio 30.1.2

sikus, Coregonus 13.1.35

Silurus aralensis 9.1.2

- aristotelis 9.1.1

- glanis 9.1.2 
- silurus 9.1.2

silurus, Silurus 9.1.2

simavika, Barbatula 7.1.8

similis, Gobio 5.13.6

simus, Cyprinus 5.17.8, 5.24.16

sinegorensis, Abramis 5.1.3

skadarensis, Gobio 5.13.5

smitti, Coregonus 13.1.35

smyrnaea, Alburnoides 5.2.1

soetta, Chondrostoma 5.10.9

soldatovi, Coregonus 13.1.35

soldatovi, Gobio 5.13.5

sordidus, Gobius 27.7.2

souffia, Leuciscus 5.17.16

spartiaticus, Rutilus 5.27.2

spartiaticus, Tropidophoxinellus 5.27.2

spectabilis, Salar 14.3.23.1

specularis, Cyprinus 5.11.1

sperchiensis, Barbus euboicus 5.7.6

spilogonurus, Gobius 27.4.1

spilura, Cobitis 6.1.10

spinulosus, Gasterosteus 21.1.1

spirinchus, Salmo 12.1.1

spirlin, Cyprinus 5.2.1

spurius, Salmo 14.3.28

Squalidus baicalensis 5.17 .8

Squalius agdamicus 5.17.4

- anjubaulti 30.1.2

- argenteus 5.17.8

- athurensis 5.17.4

- aula 5.24.3

- bearnensis 5.17.2

- borysthenicus 5.17.1

- cephalopsis 5.17.4

- chalybeius 5.17 .8

- clathratus 5.17.4

- delineatus 5.16.1

- elata 5.17 .8

- elatus 5.24.3

- elongata 5.17.8

- illyricus 5.17 .6

- lateristriga 5.17.8

- leptorhinus 5.17 .8

- lepusculus 5.17 .8

- mehdem 5.17.8

- meridionalis 5.17.4

- meunier 5.17.4

- microlepis 5.17.10

- minor 5.17.8

- nasutus 5.17 .8

- oxianus 5.17 .5

- oxyrrhis 5.17 .2

- pareti 5.17.4

- pictus 5.18.2

- platycephala 5.17.4

- prespensis 5.17.4

- suworzewi 5.17.8

- svallize 5.17.17

- tenellus 5.17 .10

- turcicus 5.17.4

- turskyi 5.17.18

- tyberinus 5.17.4
- ukliva 5.17.19

- vardarensis 5.17.4

- vulgaris 5.17 .8

- zrmanjae 5.17.20

squalius, Leuciscus 5.17.4

squalus, Cyprinus 5.17.8

squalus, Leuciscus 5.17 .4

stagnalis, Idus 5.17.8

stagnalis, Phoxinus 5.12.1

stankoi, Gobio 5.13.4

stankokaramani, Eudontonyzon 1.1.3, 1.1.4

steckbornensis, Coregonus 13.1.20

steindachneri, Barbus 5.7.19

steindachneri, Chondrostoma 30.1.2

steindachneri, Coregonus 13.1.27

stellatus, Acipenser 2.1.5

stellatus, Benthophilus 27.2.1

stellatus, Doliichthys 27.2.1

stenorhynchus, Coregonus 13.1.45

stephanidisi, Cobitis 6.1.8

sterilis, Salmo 14.3.23.1

Sterledus sibiricus 2.1.4

sterlet, Acipenser 2.1.4

sterleta, Acipenser 2.1.4

steveni, Gobius 27.7.2

stigmaticus, Coregonus 13.1.43

Stizostedion lucioperca 25.4.1

stomachicus, Salmo 14.3.21

stomachius, Salmo 14.3.21

strandjae, Phoxinus 5.21.1

Streber ratisbonensium 25.5.3

streber, Aspro 25.5.3

streber, Zingel 25.5.3

striatus, Cyprinus 30.1.2

striatus, Salmo 14.6.1

strigio, Alburnus 5.3.2

stroemii, Salmo 14.3.23.1

struanensis, Salmo 14.5.17

struanensis, Salvelinus 14.5.17

strumicae, Alburnus 5.3.2

strumicae, Barbus 5.7.6

strumicae, Cobitis 6.1.9

strumicae, Rhodeus 5.23.1

strymonicus, Alburnoides 5.2.1

sturanyi, Nemacheilus 7.1.1

sturi, Acipenser 2.1.6

Sturio accipenser 2.1.6

- vulgaris 2.1.6

sturioides, Acipenser 2.1.6

sturionaster, Acipenser 2.1.2

sturionellus, Acipenser 2.1.2

stymphalicus, Leuciscus 5.22.3

stymphalicus, Pseudophoxinus 5.22.3

subventrosus, Carassius 5.8.2

sucharensis, Rutilus 5.24.16

suecica, Coregonus 13.1.37

suidteri, Coregonus 13.1.14

sujefianus, Blennius 26.1.1

sulcatus, Gobius 27.7.5

sulzeri, Coregonus 13.1.17

sunensis, Coregonus 13.1.31

suworzewi, Squalius 5.17.8 
svallize, Leuciscus 5.17.17

svallize, Squalius 5.17.17

svetovidovi, Rhodeus 5.23.1

swirensis, Coregonus 13.1.37

sylvaticus, Salmo 14.3.23.1

Syngnathus abaster 22.2.1

- acus 22.2.2

- aeolicus 22.2.1

- agassiz 22.2.1

- algeriensis 22.2.1

- alternans 22.2.2

- anguisigola 22.2.1

- argentatus 22.2.4

- brachyrhynchus 22.2 .2

- bucculentus 22.2.1

- caspius 22.2.1

- cuvieri 22.2.1

- delalandi 22.2 .2

- ethon 22.2.1

- flavescens 22.2.1

- ganzirriensis 22.2.1

- maeoticus 22.2.1

- microchirus 22.2.1

- nigrolineatus 22.2.1

- ophidion 22.1.1

- pelagicus 22.2.4

- ponticus 22.2.4

- pyrois 22.2.4

- rondeletii 22.2.4

- rotundatus 22.2 .4

- rubescens 22.2.2

- taenionotus 22.2.3

- temminckii 22.2.2

- typhle 22.2 .4

- viridis 22.2 .4

syrman, Gobius 27.7.6

syrman, Neogobius 27.7.6

Syrrhothonus charrieri 27.9.3

szanaga, Cottus 23.1.4

\section{T}

taalmankipii, Gobius 27.9.5

taenia, Cobitis 6.1.10

taeniata, Chondrostoma 5.10.4

taeniatus, Cyprinus 5.5.1

taenioides, Cobitis 6.1.10

taenionotus, Syngnathus 22.2.3

taimen, Hucho 14.2.1

taleri, Salmo 14.3.22

taleri, Trutta 14.3.22

tanaica, Acipenser 2.1.1

tanaica, Alosa 4.1.9

tanaica, Clupea 4.1.9

tanaitica, Cobitis 6.1.10

tang, Mugil 16.3.3

tapinorhynchus, Coregonus 13.1.45

tauricus, Alburnus 30.1.2

taurinus, Salmo 14.3.23.1

telekinae, Coregonus 13.1.29

Telestes leucoides 5.17.1

- polylepis 5.17.14

- rysela 5.17 .16
- savigny 5.17 .12

teletzkensis, Leuciscus 5.17 .8

temminckii, Syngnathus 22.2.2

tenellus, Abramis 5.28.3

tenellus, Squalius 5.17.10

terekensis, Leuciscus 5.24.16

teres, Scyphicus 22.1.1

tesselatus, Cobitis 6.3.2

tetracanthus, Gasterosteus 21.1.3

tetraculeatus, Gasterosteus 21.1.1

thermalis, Cyprinus 5.11.1

thessala, Knipowitschia 27.5.8

thessalicus, Alburnoides 5.2.1

thessalicus, Alburnus 5.3.2

thessalus, Barbus 5.7.12

thessalus, Gobius 27.5.8

thienemanni, Coregonus 13.1.45

thingvallensis, Salmo 14.5.18

thingvallensis, Salvelinus 14.5.18

thompsonii, Acipenser 2.1.6

thracicus, Leuciscus 5.17.4

thracicus, Phoxinellus 5.16.1

thrakica, Sabanejewia 6.3.1

threspoticus, Leucaspius 5.22.3

thunensis, Coregonus 13.1.10

Thymallus aeliani 14.6.1

- decorus 14.6.1

- gymnogaster 14.6.1

- gymnothorax 14.6.1

- kamensis 14.6.1

- lacustris 14.4.1

- microlepis 14.4.1

- thymallus 14.6.1

- umbrosus 14.6.1

- vexillifer 14.6.1

- vulgaris 14.6.1

thymallus, Salmo 14.6.1

thymallus, Thymallus 14.6.1

thymus, Salmo 14.6.1

tigurinus, Coregonus 13.1.17

timidus, Micromugil 20.1.1

Tinca aurea 5.26.1

- cestellae 5.26.1

- chrysitis 5.26 .1

- communis 5.26.1

- italica 5.26.1

- limosa 5.26.1

- linnéi 5.26.1

- maculata 5.26.1

- tinca 5.26.1

- vulgaris 5.26 .1

tinca, Cyprinus 5.26.1

tinca, Tinca 5.26.1

tincauratus, Cyprinus 5.26.1

tincaurea, Cyprinus 5.26.1

Tiphle hexagonus 22.2.4

toni, Cobitis 7.1.1

topozeri, Coregonus 13.1.28

tortonesei, Pomatoschistus 27.9.6

toxostoma, Chondrostoma 5.10.10

toxostoma, Cyprinus 5.10.10

trachura, Pygosteus 21.2.4 
trachurus, Gasterosteus 21.1.1 trachurus, Pleuronectes 29.1.1 transcaucasica, Blicca 5.1.2 transcaucasicus, Aspius 5.5.1 trasimenicus, Leuciscus 5.24.15 trautvetteri, Gobius 27.7.6

Trematina foveolata 10.1.1 trevelyani, Salvelinus 14.5.19

trichonica, Cobitis 6.1.11

trichonis, Economidichthys 27.3.2

Triglopsis quadricornis 23.2.1

Tripteronotus hautin 13.1.39

Tropidophoxinellus hellenicus 5.27.1

- spartiaticus 5.27.2

Trutta adriatica $14.3 .4,14.3 .14$

- balcanica 14.3.2

- fluviatilis 14.3.23.1

- krkensis 14.4.1

- letnica 14.3.11

- likana 14.3.22

- macedonica 14.3.13

- macroptera 14.3.13

- marina 14.3.23.1

- marmorata 14.3.4, 14.3.14

- montenigrina 14.4.1

- relicta 14.3.19

- salmanata 14.3.23.1

- salmonata 14.3.23.1

- salonitana 14.4.1

- taleri 14.3.22

- ungeri 14.3.10

- variabilis 14.3.23.2.1

trutta, Salmo 14.3.23.1

truttula, Salmo 14.3.23.2.3

trybomi, Coregonus 13.1.36

tscharchalensis, Clupeonella 4.2.1

tscharchalensis, Rutilus 5.24.16

tscholmugensis, Coregonus 13.1.37

tücka, Acipenser 2.1.1

turcicus, Squalius 5.17.4

turiensis, Chondrostoma 5.10.1

turritus, Acipenser 2.1.3

turskyi, Leuciscus 5.17.18

turskyi, Squalius 5.17.18

tyberinus, Barbus 5.7.20

tyberinus, Squalius 5.17.4

typhle, Syngnathus 22.2.4

typicus, Salmo 14.3.11

typus, Heegerius 5.25.5

tyrasensis, Barbus 5.7.2

tzanevi, Alburnoides 5.2.1

\section{$\mathrm{U}$}

ujmonensis, Phoxinus 5.21.1

ukliva, Leuciscus 5.17.19

ukliva, Squalius 5.17.19

umbla, Salmo 14.5.20

umbla, Salvelinus 14.5.20

Umbra canina 11.1.1

- krameri 11.1.1

- lucifuga 11.1.1

- pavlovi 11.1.1 umbra, Cyprinodon 11.1.1

umbra, Cyprinus 5.17.8

umbrae, Coregonus 13.1.45

umbrosus, Thymallus 14.6.1

ungeri, Trutta 14.3.10

unipunctatus, Gobius 27.9.5

unitaeniatus, Hoplerythrinus 5.17.4

uranoscopus, Cyprinus 5.13.7

uranoscopus, Gobio 5.13.7

uzboicus, Rutilus 5.24.16

V

vaenernensis, Cottus 23.2.1

vaetterensis, Coregonus 13.1.29

Valencia hispanica 19.1.1

- letourneuxi 19.1.2

- lozanoi 18.1.1

valenciennii, Acipenser 2.1.6

vallachica, Cobitis 6.3.1

vallisnerii, Acipenser 2.2.1

valsanicola, Romanichthys 25.3.1

vandesius, Coregonus 13.1.44

vardarense, Chondrostoma 5.10.11

vardarensis, Chondrostoma 5.10.11

vardarensis, Cobitis 6.1.12

vardarensis, Nemacheilus 7.1.1

vardarensis, Squalius 5.17.4

variabilis, Cobitis 7.1.1

variabilis, Phoxinus 5.12.1

variabilis, Trutta 14.3.23.2.1

variegata, Lampetra 1.4.1

variegatus, Esox 10.1.1

variegatus, Petromyzon 6.2.2

varius, Phoxinus 5.21.1

varus, Salarias 26.1.1

vegariticus, Rutilus 5.24.16

velecensis, Rutilus 5.24.5

venatus, Gobio 5.13.3

venernensis, Salmo 14.3.23.2.3

venetiarum, Gobius 27.11.1

ventricosus, Salmo 14.5.1

ventrosus, Carassius 5.8.3

verchoswirka, Coregonus 13.1.37

vermelnensis, Cottus 23.2.1

vernalis, Salvelinus 14.5.21

verselata, Mugil 16.2.3

verus, Asper 25.5.3

vessicus, Coregonus 13.1.28

vetula, Abramis 5.1.3

vexillifer, Thymallus 14.6.1

vigrensis, Coregonus 13.1.29

Vimba abulyontis 5.28.3

- aphnitis 5.28.3

- bergi 5.28 .3

- borysthenica 5.28 .3

- elongata 5.28 .1

- istanbulensis 5.28.3

- karasuensis 5.28.3

- melanops 5.28.2

- nicaeensis 5.28 .3

- sapancae 5.28.3

- vimba 5.28 .3 
vimba, Cyprinus 5.28.3

vimba, Salmo 13.1.28

vimba, Vimba 5.28.3

vimbaeformis, Coregonus 13.1.45

vimpa, Cyprinus 5.28.3

violaceus, Scyphius 22.1.1

virescens, Gobius 27.7.5

virgo, Leuciscus 5.24 .13

viridescens, Cyprinus 5.11.1

viridis, Gobius 27.4.2, 27.11.1

viridis, Syngnathus 22.2.4

visovacensis, Salmo 14.3.24

vistonica, Alosa 4.1.10

vladykovi, Eudontomyzon 1.1.3, 1.1.5

vladykovi, Gobio 5.13.1

vobla, Leuciscus 5.24.16

vodlosericus, Coregonus 13.1.28

vogti, Coregonus 13.1.14

volgensis, Alosa 4.1.5

volgensis, Perca 25.4.2

volgensis, Sander 25.4.2

volki, Cottus 23.1.4

vorax, Luccius 10.1.1

voronjensis, Coregonus 13.1.45

vovkii, Carassius 5.8.3

vukovici, Rutilus 5.24.7

vulgaris, Abramis 5.1.3

vulgaris, Acerina 25.1.2

vulgaris, Alausa 4.1.3

vulgaris, Anguilla 3.1.1

vulgaris, Aspius 5.5.1

vulgaris, Aspro 25.5.1

vulgaris, Barbus 5.7.2

vulgaris, Blennius 26.1.1

vulgaris, Carassius 5.8.2

vulgaris, Cyprinus 5.11.1

vulgaris, Eperlanus 12.1.1

vulgaris, Flesus 29.1.1

vulgaris, Gasterosteus 21.2.2

vulgaris, Gobio 5.13.5

vulgaris, Leuciscus 5.17.8

vulgaris, Lota 15.1.1

vulgaris, Perca 25.2.1

vulgaris, Platessa 29.2.2

vulgaris, Squalius 5.17.8

vulgaris, Sturio 2.1.6

vulgaris, Thymallus 14.6.1

vulgaris, Tinca 5.26.1

vulpinus, Mugil 16.3.3

vulturius, Leuciscus 5.3.1

vygrensis, Coregonus 13.1.29
W

wagleri, Coregonus 13.1.27

wagneri, Caspiomyzon 1.5.1

waleckii, Barbus 5.7.15

wartmanni, Coregonus 13.1.21

wartmanni, Salmo 13.1.21

werchoswirka, Coregonus 13.1.37

widegreni, Coregonus 13.1.37

wiliunensis, Esox 10.1.1

willkommii, Chondrostoma 5.10.12

willoughbii, Salmo 14.5.21

willughbeii, Coregonus 13.1.44

willughbii, Salmo 14.5.21

wiresuba, Cyprinus 5.24.5

wjatkensis, Leuciscus 5.17.4

wobla, Leuciscus 5.24 .16

wyrozub, Leuciscus 5.24 .5

\section{X}

xanthopterus, Cyprinus 5.24.16

Y

yarrellii, Acipenser 2.1.6

ylikiensis, Rutilus 5.24.17

youngeri, Salvelinus 14.5.22

$\mathbf{Z}$

zaissanica, Perca 25.2.1

zanandreai, Cobitis 6.1.1

zanandreai, Lampetra 1.4.2

zanandreai, Lethenteron 1.4 .2

zangicus, Leuciscus 5.17.4

zeelt. C'vprinus 5.26 .1

zerta, Cyprinus 5.28.3

zetensis, Salmo 13.4.1

ziga, Clupea 5.19.1

Zindel ratisbonensium 25.5.4

Zingel asper 25.5.1

- balcanicus 25.5.2

- nerensis 25.5.3

- streber 25.5.3

- zingel 25.5.4

zingel, Perca 25.5.4

zingel, Zingel 25.5.4

Zosterisessor ophiocephalus 27.11.1

zrmanjae, Leuciscus 5.17.20

zrmanjae, Squalius 5.17.20

zrmanjaensis, Salmo 14.3.25

zuerichensis, Coregonus 13.1.17

zugensis, Coregonus 13.1.14, 13.1.15

zürichensis, Coregonus 13.1.1 